\title{
A User's Manual for the BNW-II Optimization Code for Dry/Wet-Cooled Power Plants
}

May 1978

Prepared for the U.S. Department of Energy under Contract No. EY-76-C-06-1830

Pacific Northwest Laboratory Operated for the U.S. Department of Energy by 
NOTICE

This repon was prepared as an account of work sponsored by the United States Government. Neither the United States nor the Department of Energy, nor any of their employees, nor any of their contractors; subcontractors, or their employees, makes any warranty, express or implied, or assumes any legal liability or responsibility for the accuracy, completeness of usefulness of any information, apparatus, product or process disclosed, or represents that its use would not infringe privately owned rights:

The views, opinions and conclusions contained in this report are those of the contractor and do not necessarily represent those of the United States Government or the United States Department of Energy.

\author{
PACIFIC NORTHWEST LABORATORY \\ operated by \\ BATTELLE \\ for the \\ UNITED STATES DEPARTMENT OF ENERGY \\ Under Contract EY-76-C-06-7830
}

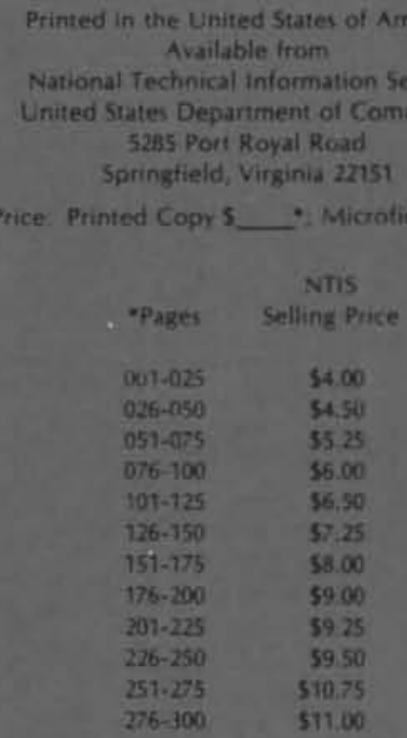




\section{3}

A USER'S MANUAL FOR THE

BNW-I I OPTIMIZATION CODE

FOR DRY/WET-COOLED POWER PLANTS

VOLUME I I

Daniel J. Braun

Judith A. Bamberger

David J. Braun

Duane W. Faletti

Lawrence $E$. Wiles

May 1978

Prepared for the U.S. Department of Energy under Contract No. EY-76-C-06-1830

Pacific Northwest Laboratory Richland, Washington 99352 


\section{PREFACE}

This document has been issued in two volumes to facilitate handling. Volume I is a narrative description of the code's algorithms, as well as logic, input and output information. Volume II is an appendix of Volume I, providing a listing of the BNW-II dry/wet ammonia heat rejection optimization code. 
$<$

. 
APPENDIX I

BNW-I I COMPUTER CODE LISTING 
- 


\section{APPENDIX I \\ BNW-I I COMPUTER CODE LISTING}

Figure I. 1 is a subroutine linkage chart indicating which subroutines may call or may be called by others. This chart should be of assistance in using the listing making up the remainder of this appendix. 


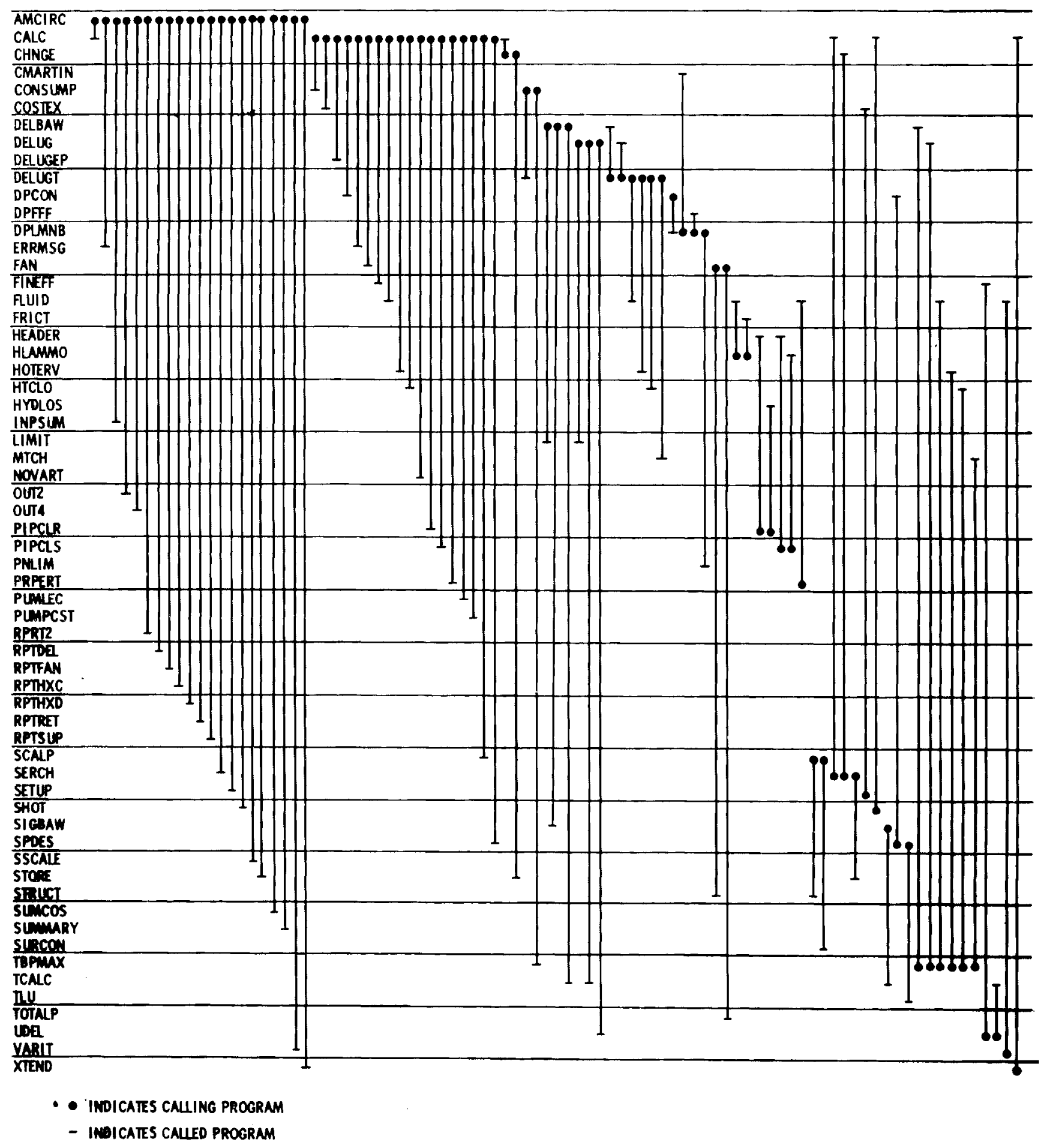

FIGURE I.1. Dry/Wet Ammonia $\left(\mathrm{NH}_{3}\right)$ Model Subroutine Linkages 
PROGRAM AMCIRC (INPUT, OUTPUT, TAPE5EINPUT, TAPEG $=$ OUTPUT)

BNW1B

PROGRAM AMCIRC IS A PROGRAM WHICH DETERMINES THE OPTIMUM DESIGN OF A WET/DRY COOLED POWER PLANT WITH AMMONIA AS THE INTERMIDIATE FLUID. THE OPTIMUM DESIGN IS DETERMINED BY REPEATING THE DESIGN OF THE COOLING SYSTEM OVER A RANGE OF DESIGN CONDITIONS INORDER TO FIND THE COOLING SYSTEM WITH THE SMALLEST INCREMENTAL COST. THIS IS ACCOMPLISHED RY VARYING FIVE PARAMETERS OF THE PLANT AND COOLING SYSTEM OVER RANGES OF VALUES. THESE PARAMETERS ARE VARIED SYSTEMATICALLY ACCORDING TO TECHNIQUES THAT PERFORM PATTERN AND GRADIENT SEARCHES.

THE DRY COOLING SYSTEM OPTIMIZED BY PROGRAM AMCIRC IS COMPOSED OF A CONDENSER/REROILER ICONDENSATION OF STEAM AND BOILING OF AMMONIA). PIPING SYSTEM (TRANSPORTS AMMONIA VAPOR OUT AND AMMONIA LIOUID FROM THE WET/DRY COOLING TOWERS), AND POLYGONAL SHADED TOWER SYSTEM (HORIZONTAL ONE-PASS HOTERV HEAT EXCHANGERS FORM?NG THE LENGTH OF THE SIDES OF THE TOWERS WITH COCURRENT AMMONIA FLOW IN THE TURES OF THE HEAT EXCHANGER

DIMENSION PCST (16)

LNGICAL FIRST, FRST, FXTEMP, FXTVAR,FXTTTD,FXTLNG COMYON

S AFTR, ALPHA, ANG (3)

* BDLIM(16)

5 , CAP̈F, CSSPKW, CONF, CONL, COSTL, CAPCHG, CONMAT, CONMAZ, CAF

5 , ERJ. CTURB, CDANG, CLUVR, CHAILS, CVM

5 . DFIN.DEEPL, DESVEL. DESVELV.DESVELD. DENSIF

5 ,EFFD, EFS

S ,FCR , FIRST, FIXL, FCOS , FFHX, FXTEMR, FACTOR, FRST, FACTORD, FSHOT , FDELUG

S,FIXV,FIXTTD,FXTVAR,FXTTTO,FXTLNG

S. GREFF

S. HXNP,HPCST, HCD

I. ITMAX, ITMIN

$\$$. JCONS

S. KCONV, KALEXT

5. MXEXT

S, NTA,NSIDES, NBUNHI COMMON

$S O D R$

\$. PSIZE, PER, PWCOS, PLANC, PFACT, POMDPL

5 ,OOHAAF, POHFAN, POHLEC, POHCIR, POHCND, POHSTC, POHSCL

5 - DRP.REDUCE, REDUCV, ROOFL, RHOUT

S OREJ, OREDUCE, OREDUCV

S. SIGMAG, SAAF, SEGL

S, TH, PPO(4), TEFF, TLIM, THFIN, TLPRA, TFIX

s, TCẼ. TW

5 , Ues, IJWS

S WEV̈. WATCONA, WATCOST

5. VAS(5)

S:XDEPA, XW, XD COMMON/SINK, VAR (5), CPLNI, OPIPI, CCOSI.

5 OINI, CLANI, REAIl, Zl.HRFAZS, WAI, UI, DEPWI, PPOWI,

5 FPOWI, DEFCI, WIDTI, ELENI, VAIRI, VWATI, HII, HOI, T4I,

5 RFWA1, NWI, NTI, NPI,ZPLNC2,ZSIZ12, ZSPEP, SW41, SW31,

5 SPCI, PTOTI,PLNC3I, AFROI, AAIRI, YFPMIN, SPCDI, SPSI3,WTZMIN,

S BPIMIN,NWZMIN, TCOSI, DEPAI, OPWTI, DPWCI,CONGAI, XNTSI, VELDI

BNWIS

BNWIS

BNWIB

BNWIB

BNWIB

BNWI5

$B N W 13$

BNWIB

BNWIB

BNWIB

BNWIB

BNWI3

BNWIB

BNWII

BNW! 3

BNWIB

BNWIB

BNWIB

BNWIB

BNWIB

BIGCOM

BIGCOM

BIGCOM

BIGCOM

SI GCOM

BIGCOM

BIGCOM

BIGCOM

BI GCOM

BIGCOM

BI GCOM

BIGCOM

BIGCOM

BIGCOM

BIGCOM

BIGCOM

BIGCOM

BIGCOM

BIGCOM

IIGCOM

BI GCOM

BIGCOM

BIGCOM

BI GCOM

BIGCOM

BIGCOM

BI GCOM

BI SCOM

BIGCOM

BI GCOM

SINK

SINK

SINK

SINK

SINK

SINK 
5. TLA1, UCON1, PMCSI, FMCS1, FBACl, EFFCl, CHI, CA1, CSI F, CLTCl, CFCl, CPCCI,FITCOI, CSCI,CEPREI,DEPIDI, ASTI,OLDCSI \$, CADC51, XNFAN1,BLDANI,CPLENI, DDFELI, WHI, WTTUI, ADII, ABLI

\$, WTHDI. WTFRI,STRUCI, CFMPF I, APPFI,TOTCFI, TPI,EFFINI, ANTUI 5. AIPFFl, WATFF1, XNMODI, TCTPFI, FDCPF1, FMTRCI, ACTTPI, ADJTPI 5, $A C T B P 1, \triangle D J B P I, A C T V H 1, A D J V H I, ~ A D J P P I, T H P A I l, T C T F !, ~ A F C S T I$

5. CSRI, WATERI, WSTRCI, CFOUNI, HUBDII. TOWLEI, NUMTOI, CTOWDI

\$, SORJ1, SORJS1, ATUBCI, CBAI, TTDII, TTD2I, DELPSI, CSTLVI, PIDCTDI

* CSTHSI, CYLRNI, CFANEL, PSTACI, PUFITI, PMDCSI, RPTPLI, SEPCSI

\$.PWD 1 (16),WATCWDI (16), DEPER! (16), DEPERG (16)

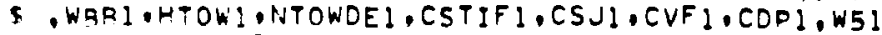

CAMMON /SCALER/HRFACI, ATUB, CDERA, SSCD,PLANCI, CASSSI,

s GAIF, FAIR, HPAIR, HPWAT, WLRTP, PLANCTI

COMMON /SUPPLY, VAL (S), CPLN, CPIP, CCOS.

$\$$ QIN, CLAND, REAIR, Z,HRFACE, W4,

FDCW, DELFC, WIDTH, ELENG, VAIR, VWAT:

5 REAT, NW, NT, NP,PLANCZ,PSIZIz SDBP, HO, T4,

$\$$ SPC,PTOTAL, PLANC3,SAFRON, AAIR, YFP, SPCO,PSIZI3, WTZ,

$\$$ RP?, NWZ, TEOS, DELPA, DELPWT, DELPWC, CONBAS, XNTSX, VELDX

- TLAX, UCON, PMCST, FMCST, FBACC, EFFC, CH, CA, CS

\$: CLTC, CFC, CPCC, FITCO, CSC,CEPREP,DELPIP, AST,OLDCST

5,CADCST, XNFAN,BLDANG, CPLEN, DPFEL, WHX, WTTUB, ADIA, ABLN

5. WTMDR, WTFRM, STRUCC, CFPERF, HPPERF, TOTCF, TDC. EFFIN, ANTU

5, $\triangle I R F F$, WATFF, XNMOD, TCTPF, FDCPF, FMTRC, ACCTTP, $\triangle D J T P$

5. $\triangle C T R D D$, $\triangle D J B P D$, ACTVH, ADJVH, ADJPPF, THDAIR, TCTF, AFCST

S, CSRD, WATERW, WSTRCT, CFOUND. HUBDIA, TOWLEN, NUMTOW, CTOWD

5 , SODJ.SORJS,ATUBC, CBA, TTDI,TTDZ,DELPS, CSTLVR,PIDCSTO

\$ . CSTHS, CYLRNG, CFANEL, PSTACS, PUFITC, PMPCST, RPTPL, SEPCST

- PWO (16).WATCWD(16),DEPER(16), DEPERQ(26)

5 WBR,HTOW,NTOWDE, CSTIF, CSJ,CVF, CDP,W5

COMUNN /VARVAR, TPER(16), TA(16); TAW8116

COMMON /INDIS/ DIST

COMMON /TINE/ XNS, TLA, XNTS, ARPS, TTDZOE, LINOR, XNP, XOUALY

81 FORMAT ("I TO USED IN THE FOLLOWING CALCULATIONS IS EIFB.3)

930 FORMATITO2,"AMBIENT TURBINE", T28, "HEAT", T83, "COST TO".

5 T93,"DOWER COST", T105."PCT TIME",TI15."WORTION OF")

931 FORMATTTO4, "TEMPU, T:O, "OUTLET", T2O, "BACK", T2B, "RATE", T50, "HX"

S.T57,"HX", T64,"POWER", T72,"CHANGE IN", 783,"REPLACE",

8.T93."AT AMBIENT", TI04."AT AMBIENT", T115, INCREMENTAL"

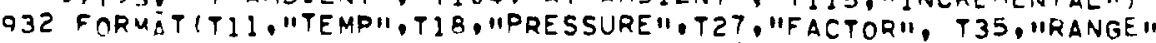

\$,T42, "TTDP", T49,"LMTD", T57,"ITD", T62,"GENERATEO"

S.T72." HELL COST". T93."LOST CAP", T95,"TEMP". TIO6."TEMPI

S.T1IE."POWER COST")

933 FORMAT! T02,13!("-"), T9," ",T17," ", T26," ", T33," ", T40," " 5.T4\%," "T54," ", T61," ", T7 ," "T81," ", T92," ", T103," S. Tु广स," ")

934 FCRMATI TO3, "DEG F", TII,"DEG F", TI9,"IN, HG", T35, "DEG F"

S.T41, "DEG F", T48, "DEG F", T56, "DEG F", T62, "MEGAWATTS"

5, TY2,"MILLS/KWH", TS2,"MILLS/KWH", T93, "MILLS/KWH"

\$.TI G." "PERCENT", TI15, "MILLS/KWH")

944 FORMATIIHO.T91, IF INAL INCREMENTAL COST", G 11.5 !

945 FORMATIIHO, T9G"CAPAC!TY CHARGE S".G15.9)

946 FORMAT I/,T?."PERCENT OF",T13,"DERCENT OF", /,T2,"HX DELUGED",TI3. I"HEAT REJECT", /.T13, "BY WET HX",T26"WATER EVAPORATED";

947 FORMATIT2,4?(11-11),T11," ", T24," "1

Q 48 FOR $4 A T$ (T26, "ACRE FEET")

949 FORMATITZ,F10.4,T13,F10.4,T26,F10.4)

SINK

SINK

SINK

$\operatorname{SinK}$

SINK

SINK

SINK

SINK

SINK

SINK

SINK

SCALER

SCALER

SUPPLY

SUPPLY

SUPPEY

SUPPIYY

SUPPLY

SUPPLY

SUPPLY

SUPPLY

SUPP:Y

SUPPLY

SUPPLY

SUPPLY

SUPPLY

SUPPLY

SUPDLY

SUPPLY

SUPPLY

BNWIS

BNWIB

BNWIB

BNWIB

BNWIB

BNWIB

BNAIS

BNWIS

BNWIS

BNWIS

BNWIB

BNWIB

BNWIB

BNWIB

BNW 18

BNWIS

BNWIB

BNWIS

BNWIB

ENWIB

BNWIB

BNWIB

BNWIB

BNWIB

BNWIB

BNW19

BNWIS 


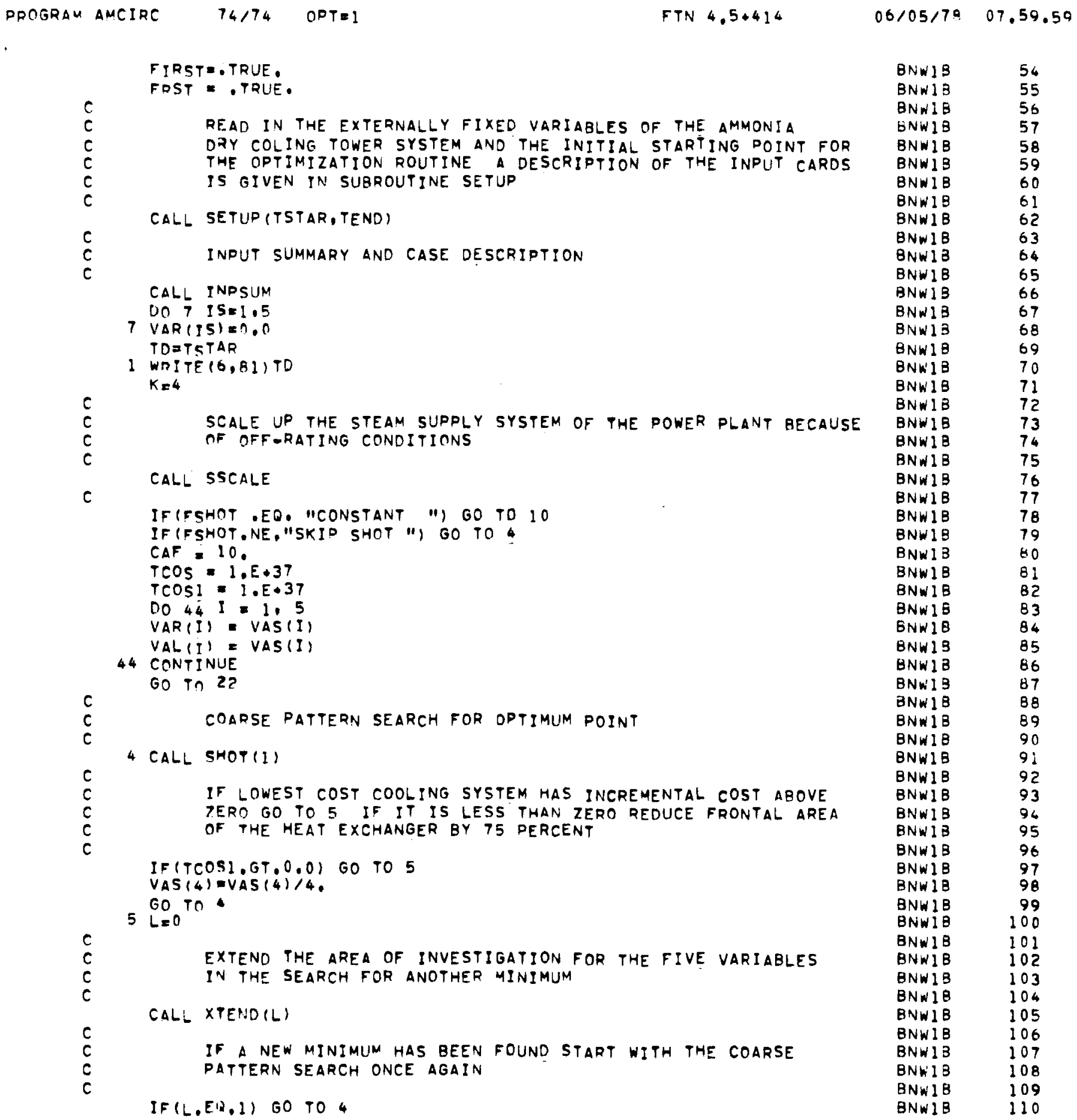


c

FINE PATTERN SEARCH FOR OPTIMUM POINT

C

CALL SHOT(2)

DO $2 \bar{I} I=1.5$

VALII) = VAR(I)

2I CONTINUE

22 CALL CALC (VAL (1), VAL (2), VAL (3), VAL (4), VAL (5), 1)

CALL STORE

$c$
$c$
$c$

2 JCONS $=0$$$
c
$$$$
\text { GRADIENT SEARCH FOR OPTIMUM POINT }
$$

CAL: SERCH

COSTl = COSTS

COST2 - TCOS1

$c$
$c$

If SERCH HAS DETERMINED AN OPTIMUN GO TO 10 BUT IF IT HAS NOT FOUND AN OPTIMUM CALL SERCH AGAIN

IFIJCONS EO, O) 60 TO 20

$K=K-1$

IF $(K, G T, 0)$ GO TO 2

IF (ARS (COSTI- COST2)/COST2.LT. 0.00) GO TO 20

$K=1$

GO TO ?

10 CONTINUE

CALL CALCITFIX,FIXV,FIXTTD,FIXL,DEEPL,I)

CALL STORE

$c$

20 CONTINUE

c

PRINTOUT OF OPTIMUM DESIGN DF THE COOLING SYSTEM

CALL OUTZ (RBP, HRFACI, SSCD,CASSSI, PLANCTI,PLANCI, CTURB, TCD)

CALL OUT4 (1)

CALL OUTA 2

CAL! RPTHXD

CALL RPTHXC (AFROI)

CALL RPTDEL

CALL RPTSUP(DESVELV, NUMTOI, CTOWDI, WBBI, HTOWI, NTOWDE I)

CALL RPTRET (CTOWDI, DOHCIR, PSTACI, PUFITI, DMPCSI, RPTPLI,

S SEDCSI,PIPCTDII

CALL PPRTZ (EFFCI, CONMAT, CONMAZ)

CALL RPTFAN

CALL ERRMSG (3)

WRITE $(5,100)$

c

IOO FORMAT (IHI)

PRINTOUT OF OPERATING PARAMETERS AND CONDITIONS OF THE

PLANT VERSUS AMBIENT TEMPERATURE ALSO DRINTOUT OF INCREMENTAL POSER PRODUCTION COSTS ATTRIBUTED TO EACH AMRIENT

TEMPERATURE AND THE TOTAL INCREMENTAL POWER PRODUCYION COSTS

WRITE $(6,930)$

WPITE $(5.931)$

WPITE $(6,932)$

WRITE $(6,933)$

BNWl

112

BNW1B $\quad 113$

BNWIS 114

BNWIB 115

BNWIB $\quad 116$

BNWIS 117

BNWIB IIB

BNWIB 119

BNWIB 120

BNWIB 121

BNWIS 122

BNWIB 123

BNWIB $12 \%$

BNWIB 125

BNWIS 126

BNWIB $\quad 127$

BNWIB $\quad 128$

BNWIB 129

BNWIB 130

BNWIB 13!

BNWIS 132

BNWIB 133

BNWIB $\quad 134$

BNWIB $\quad 135$

BNWI3 $\quad 136$

BNWIB 137

BNWIB 138

BNWIB 130

BNWIB $\quad 140$

BNWIB 141

BNWIB 142

BNWIS $\quad 143$

BNWIB 144

BNWIB $\quad 145$

BNWIB 146

BNWIB 147

BNWI 148

BNWIB 149

BNWIB 150

BNWIS 15!

BNWIB 152

BNWIB $\quad 153$

BNWIB 154

BNWIB 155

BNWIB $\quad 156$

BNWIB $\quad 157$

BNWIS 158

BNWIB 159

BNWIS 160

BNWIS 161

BNNIS 162

BNwlB $\quad 163$

BNWIB $\quad 164$

BNWIS 165

BNWIB $\quad 166$

BNWIB 167 
PROGRAN AMCIRC $14 / 74 \quad$ OPTEI

WRITE $(6.934)$

WRITE $(6,933)$

$C L S I J M=0.0$

FCSIJM $=0.0$

DO $31=1 . N T A$

3 CALL VARITITA(I),TPER (I),PCST (I) I, CLSUM,FCSUM)

WPITE $(6,946)$

WRITE $(6,947)$

WRITE $(6,948)$

WDITE $(6,947)$

DO Q I = I. NTA

WPITE $(6,049)$ DEPER (I) 100 . DEPERQ1 (I)*100..WATCWDI(I)/2719015.

9 CONTINUE

$A C O 5=0.0$

DO $a$ IEI, NiA

ACOSZACOS $P$ DST II:

8 CONTINUE

WRITE (6.944) ACOS

WRITE $(6,945)$ CAPCSI

$T D=T D \cdot 5$.

FIRST = TRUE.

KALEXT = 0

IF (TR.LE, TEND) GOTO:

6 CONTINIJE

$c$
$c$
$c$
$c$

DRINTOUT OF THE CAPITAL AND UNIT ENERGY COSTS OF THE DRY COOLING TOWER SYSTEM

BNWIB $\quad 168$

BNMI3 169

BNWIB 170

BNWIB 171

BNwla 172

BNWIB 173

BNWIB 17

BNW!B 175

BNWIB I76

BNWIS 177

BNWIB 178

BNWIS 179

BNWIS 180

BNAIB 181

BNWIS 182

BNWIB 183

BNWIB 164

BNWIB 185

BNWIE $\quad 186$

BNWIB 187

BNWIB $\quad 188$

BNWIB 180

BNWIS $\quad 190$

BNWIB 191

BNWIB $\quad 192$

BNW!S 193

BNWIB 194

BNWIB 195

CALL SUMCOS IFCR, PSIZE, CAPF, CCOSI, CPIPI, CONBAI, ATUBCI, CPLENI BNWI3 196

S FBACI. FMCSI, STRUCI, CFOUNI, CAPCSI, SPCDI, SSCD, HRFACI B BNWIB I97

9. HRFAZS, YFPMIN, PLANC, CLANI, CLSUM, FCSUM, COSMI, CSTLVI BNH1B 198

\& ,CSTHSI, CFANE I XNFANI/(YFPMIN+1,), CYLRNI XNFANI, WATCONA , WATCOST) CALL SUMMARY 


$$
74 / 74 \text { OPT } \times]
$$

SUBROLITINE CALCTI, FVAIR,TTD,ELENH, ZD,M)

SUBPOUTINE CALC IS USEO FOR THE ANALYSIS OF THE AMMONIA

WET/DRY COOLING SYSTEM. "IN THIS SUBROUTINE THE CONDENSER/

REROILER, PIPING SYSTEM, HEAT EXCHANGER, TOWERS, AND DELUGE

SYSTEM ARE DESIGNED. THE COSTS FOR ALL OF THESE SYSTEMS ARE

FOUND ALONG WITH THE INCREMENTAL COST FOR OPERATING THE PLANT

WITH THE ORY/WET COOLING SYSTEM OVER THE ENTIRE YEAR.

THE FOLLOWING FIVE VARIABLES ARE THE FIVE INDEPENDENT

VARIABLES THAT ARE USED TO DESIGN THE AMMONIA COOLING SYSTEM

$T 1$

TTDI

- TURBINE OUTLET TEMPERATURE (DEg F)

- TEMPERATURE DIFFERENCE BETWEEN THE OUTLET TEMPERATURE OF THE TURBINE AND THE TEMPERATURE OF THE AMMONIA IN THE CONOENSER/REBOILER (DEG F)

FVAIR

- VELOCITY (FT/SEC)

ELENH

- Length of the heat exchanger tuBes (FT)

ZD

- heAT EXCHANGER DEPTH IN NUMBER OF TUBES (OIMENSIONLESS)

OEFINITION OF VARIABLES

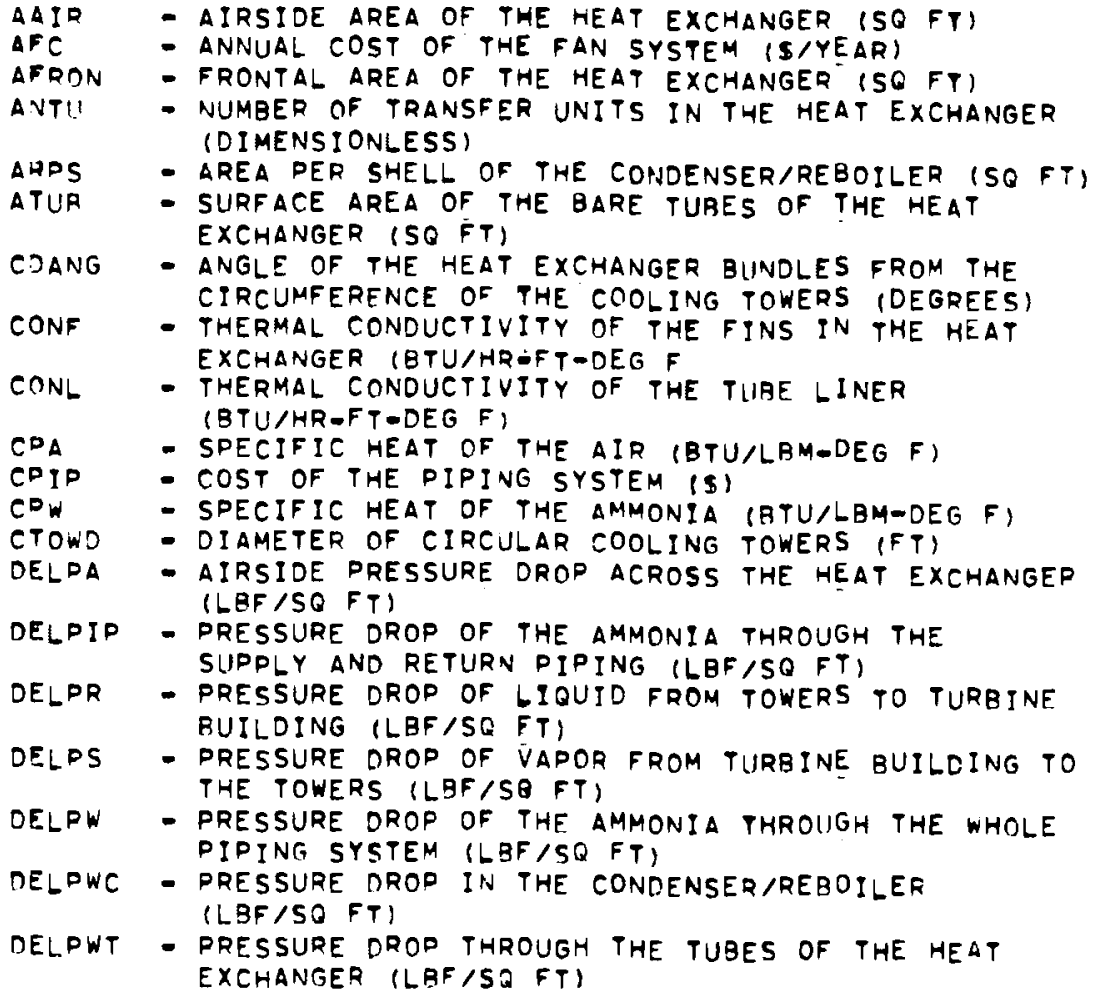

$06 / 05 / 78$

07.59 .59

AMCALC

AMCALC

AMCALE

AMCALC

AMCALC

AMCALC

AMCALC

AMCALC

AMCALC

AMCALC

AMEALC

AMCALC

AMCALC

AMCALC

AMCALC

AMCALC

AMCALC

AMCALC

AMCALC

AMEALC

AMCALC

AMCALC

AMCALC

AMCALC

AMCALC

AMCALC

AMCALC

AMEALC

$\triangle M E A L C$

$\triangle M C A L C$

AMCALC

AMCALC

$\triangle M C A L C$

AMCALC

AMCALC

AMCALC

AMCALC

AMCALC

AMCALC

AMCALC

AMCALC

AMCALC

$\triangle M C A L C$

AMCALC

AMCALC

AMCALC

AMCALC

AMCALC

AMCALC

AMCALC

AMCALC

AMCALC

AMCALC

AMCALC

AMEALC

AMCALC

AMCALC 
$74 / 74$ OPTE1 FTN $4.5+414 \quad 06 / 05 / 78 \quad 07.59 .59$

DENAV - aVERAGE DENSITY OF THE AIR IN THE HEAT EXCHANGER (LBF/SQ FT)

DENIN - DENSITY OF THE AIR ENTERING THE HEAT EXCHANGER (LBM/CU FT)

DENOUT - DENSITY OF THE AIR LEAVING THE hEAT EXCHANGER (LBM/CU FT)

DENSIF - DENSITY ÖF THE AIR ENTERING THE HEAT EXCHANGER (LBM/CU FT)

DENSIV - DENSITY OF AMMONIA VAPOR (LBM/CU FT)

DENSIW - DENSITY OF AMMONIA LIQUID (LBM/CU FT)

DEPTH - DEPTH OF THE HEAT EXCHANGER (FT)

DESVEL - DESIGN VELOCITY OF AMMONIA LIQUID (FT/SEC)

DESVELD - DESIGN VELOCITY OF THE DELUGE WATER IN THE DELUGE PIPING (FT/SEC)

DESVELV - DESIGN VELOCITY DF AMMONIA VAPOR (FT/SEC)

DFIN - FIN DIAMETER OF THE TUBES IN THE HEAT EXCHANGER (IN)

DI - INSIDE DIAMETER OF THE TUBES IN THE HEAT EXCHANGE? (IN)

DIST - DISTANCE FROM TURBINE BUILDING TO COOLING TOWERS (FT)

DPTINW - AIRSIDE PRESSURE DROP ACROSS THE HEAT EXCHANGER (IN HZO)

AMCALC $\quad 59$

AMCALC 60

AMCALC 61

AMCALC 62

AMCALC 63

AMCALC 64

AMCALC 65

AMCALC $\quad 66$

AMCALC 67

AMCALC 68

AMCALC 69

AMCALC $\quad 70$

AMCALC 71

AMCALC 72

$\triangle M C A L C \quad 73$

AMCALC 74

AMCALC 75

AMCALC 76

AMCALC 77

AMCALC 78

AMCALC 79

AMCALC 80

AMCALC \&

EFF - EFFECTIVENESS OF THE HEAT EXCHANGER (DIMENSIONLESS)

EFFIN - FIN EFFECTIVENESS OF THE TUBES IN THE HEAT EXCHANGER (DIMENSIONLESS)

EFFP - PUMP EFFICIENCY TOIMENSIONLESSI

EFFS - OVERALL SURFACE EFFICIENCY OF THE FINNED TUBES (DI MENSIONLESS)

ELENG - LENGTH OF THE HEAT EXCHANGER TUBES (FT)

ELEV - ELEVATION OF THE PLANT SITE (FT)

ENGTH - LENGTH OF THE HEAT EXCHANGER TUBES (IN)

FACTORD - RATIO OF THE AMOUNT DF DELUGE WATER PUT ON THE HEAT EXCHANGER TO THE AMOUNT OF WATER EVAPORATED (DIMENSIONLESS)

FAIR - FRICTION FACTOR OF THE PRESSURE DROP THROUGH THE HEAT EXEHANGER (DIMENSIONLESS)

GAIR - MASS FLUX OF THE AIR IN THE HEAT EXCHANGER (LBM/HR-SQ FT)

GWAT - MASS FLUX OF THE AMMONIA ON THE INSIDE OF THE TUBES IN THE HEAT EXCHANGER (LBM/HR-SO FT)

HFG - HEAT OF VAPORIZATION OF THE AMMONIA (BTULLBM)

- aIRSIDE PRESSURE DROP OF THE hEAT EXCHANGER AT THE REFERENCE TEMPERATURE OF TO DEG $F$ (IN H2O)

HI

- INSIDE HEAT TRANSFER COEFFICIENT OF THE TUBES OF THE HEAT EXCHANGER (BTU/HR-SQ FT -DEG F)

HO - OUTSIDE HEAT TRANSFER COEFFICIENT OF THE HEAT EXCHANGER (RTU/HR-SR FT-DEG F)

HPWAT - HORSEPOWER LOSS FOR THE TOTAL PIPING SYSTEM (HP)

HPWATO - HORESDOWER LOSS THROUGH THE DELUGE PIPING (HP)

HPWATI - HORSEPOWER LOSS THROUGH THE SUPPLY AND RETURN PIPING $\triangle N$ THE TJUES OF THE HEAT EXCHANGEF (HD)

HOWATZ - HORSEDOWER LOSS THROUGH THE TUAING OF THE CONDENSER/REBOILER (HP)

HRFAC2 - HEAT RATE FACTOR OF THE TURBINE (DIMENSIONLESS)

HTOW

- heIRHT OF THE COOLING TOWERS (FT)

AMCALC 83

AMCALC $\quad 54$

AMCALC B5

$\triangle M E A L C, \quad 86$

AMCALC 87

AMCALC 88

AMCALC 89

AMEALC 90

AMCALC 91

AMCALC 92

AMCALC 93

AMCALC

AMCALC 95

AMCALC 96

AMCALC 97

AMCALC 9

AMCA:C 9

AMCALC 100

AMCALC 101

AMCALC 102

AMCALC 103

AMCALC 104

AMCALC 105

AMCALC 106

AMCALC $\quad 107$

AMCALC 108

AMCALC 109

AMCALC 110

AMCALC 111

AMCALC III

AMCALC 113

AMCALC II4

AMCALC 115 
$74 / 74 \quad$ OPT $=1 \quad$ FTN $4.5 .416 \quad 06 / 05 / 79 \quad 07.59 .59$

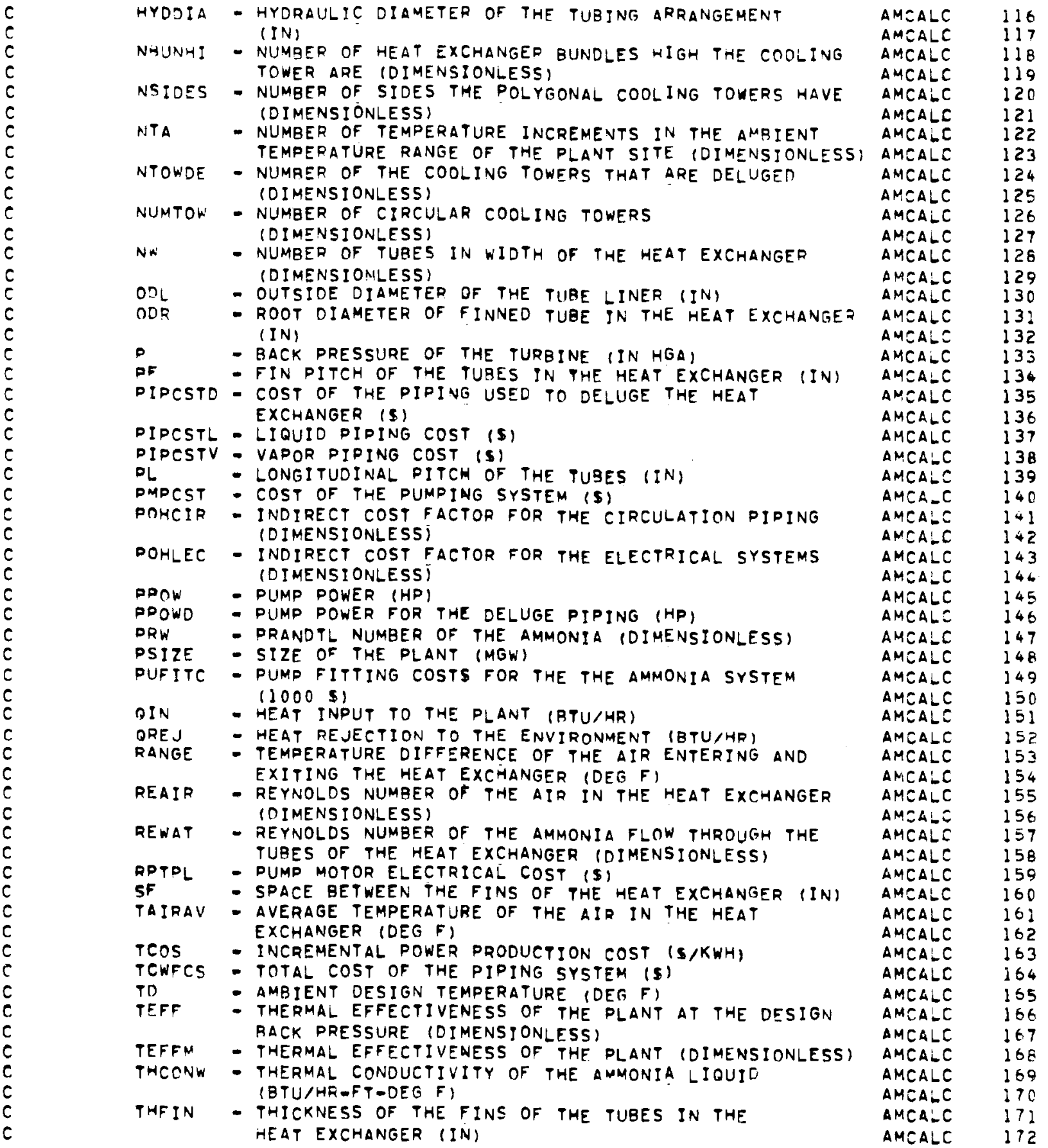




\begin{tabular}{|c|c|c|c|}
\hline & FTN $4.5+414$ & $5 / 78$ & \\
\hline in & THE AMMONIA ENTERING THE SUPPLY & $A M \subset A L C$ & 173 \\
\hline I NHX & $\begin{aligned} & \text { PIPING (DEG F) } \\
& \text { - TEMPERATURE OF AMMONIA ENTERING THE HEAT }\end{aligned}$ & $\begin{array}{l}\text { AMCALC } \\
\text { AMCALC }\end{array}$ & \\
\hline T & $\begin{aligned} & \text { EXCHANGER IDEG F) } \\
& \text { - LENGTH OF THE TUBES IN THE CONDENSER/REBOILER (FT) } \\
& \text { - TEMPERATURE DIFFEAENCE BETWEEN THE SATURATED }\end{aligned}$ & & $\begin{array}{l}176 \\
177 \\
178\end{array}$ \\
\hline 102 & $\begin{array}{l}\text { STEAM AND AMMONIA IN THE CONDENSER/REBOILER (DEG F) } \\
\text { - TEMPERATURE DROP OF THE AMMONIA IN THE SUPPLY } \\
\text { PIPING (DEG F) }\end{array}$ & $\begin{array}{l}\text { AMCALC } \\
\text { AMCALC } \\
\text { AMCALC }\end{array}$ & $\begin{array}{l}179 \\
180 \\
181\end{array}$ \\
\hline WATAV & $\begin{array}{l}\text { - TEMPERATURE OF THE AMMONIA IN THE CONDENSER/ } \\
\text { REBOILER (DEG F) }\end{array}$ & & \\
\hline 4 & $\begin{array}{l}\text { - TEMPERATURE OF THE AIR EXITING THE HEAT EXCHANGER } \\
\text { (DEG F) }\end{array}$ & & \\
\hline & $\begin{array}{l}\text { - OVERALL HEAT TRANSFER COEFFICIENT OF THE HEAT } \\
\text { EXCHANGER (RTU/HR=SO FT-DEG F) }\end{array}$ & & \\
\hline VELD & $\begin{array}{l}\text { - VELOCITY OF THE AIR IN THE HEAT EXCHANGER (FT/SEC) } \\
\text { - INLET VELOCITY OF THE AMMONIA ENTERING } \\
\text { THE CONDENSER/REBOILER (FT/SEC) }\end{array}$ & $\begin{array}{l}\text { DALC } \\
\text { CALC } \\
\text { CALC }\end{array}$ & $\begin{array}{l}188 \\
189 \\
190\end{array}$ \\
\hline VELDD & $\begin{array}{l}\text { - OUTLET VELOCITY OF THE AMMONIA LEAVING THE } \\
\text { CONDENSER/REBOILER (FT/SEC } \\
\text { - VISCOSITY OF AIR IN THE HEAT EXCHANGER (LBM/FT-HR) }\end{array}$ & $\begin{array}{l}\text { AMCALC } \\
\text { AMCALC } \\
\text { AMCALC }\end{array}$ & $\begin{array}{l}191 \\
192 \\
193\end{array}$ \\
\hline $\begin{array}{l}\operatorname{lis} 5 \\
\operatorname{lis} 5\end{array}$ & $\begin{array}{l}\text { - VISCOSITY OF AMMOAIA VAPOR (LBM/FT-HR) } \\
\text { - VISCOSITY OF THE AMMONIA (LBM/FT-HR) }\end{array}$ & & \\
\hline $\begin{array}{ll}\text { VOL } \\
\text { VWAT }\end{array}$ & $\begin{array}{l}\text { - VOLUME OF THE HEAT EXCHANGER ICU FT) } \\
\text { - VAPOR VELOCITY OF THE AMMONIA IN THE ENTRANCE OF } \\
\text { THE TUBES OF THE MEAT EXCHANGER (FT/SEC) }\end{array}$ & $\begin{array}{l}A M C A L C \\
\text { AMCALC } \\
\text { AMCALC }\end{array}$ & \\
\hline DNA & $\begin{array}{l}\text { - AMOUNT OF WATER ALLOWED TO BE EVAPORATED OVER THE } \\
\text { WHOLE YEAR WHEN DELUGING THE HEAT EXCHANGER } \\
\text { (LBM/YEAR) }\end{array}$ & & \\
\hline 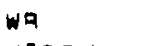 & - BUNDLE WIDTH OF HEAT EXCHANGER (FT) & CALC & \\
\hline IOTH & - WIOTH OF THE HEAT EXCHANGER (FT) & MCAL & 03 \\
\hline & $\begin{array}{l}\text { - WIDTH LENGTH RATIO OF THE HEAT EXCHANGER (FT/FT) } \\
\text { - MASS FLON RATE OF THE AMMONIA (LBM/HR) }\end{array}$ & & \\
\hline 14 & - MASS FLOW RATE OF THE AIR (LBM/HR) & CALC & 206 \\
\hline W5 & $\begin{array}{l}\text { - MAXIMUM AMOUNT OF WATER PUMPED THROUGH THE DELUGED } \\
\text { PIPING SYSTEM (GPM) }\end{array}$ & & $\begin{array}{l}207 \\
208\end{array}$ \\
\hline D & $\begin{array}{l}\text { - LONGITUDINAL PITCH OF THE TUBES IN THE HEAT } \\
\text { EXCHANGER (IN) }\end{array}$ & & \\
\hline$X D G$ & $\begin{array}{l}\text { - DIAGONAL PITCH OF THE TUBES IN THE HEAT EXCHANGER } \\
\text { (IN) }\end{array}$ & & \\
\hline NP & $\begin{array}{l}\text { - NUMBER OF PASSES IN THE CONDENSER/REBOILER } \\
\text { (DIMENSIONLESS) } \\
\text { - NUMBER OF SHELLS IN THE CONDENSER/REBOILER }\end{array}$ & $\begin{array}{l}\text { AMCALC } \\
\text { AMCALC } \\
\text { AMCALC }\end{array}$ & \\
\hline & $\begin{array}{l}\text { (DIMENSIONLESS) } \\
\text { - NUMBER OF TUBES PER SHELL (OIMENSIONLESS) }\end{array}$ & & \\
\hline DUALY & $\begin{array}{l}\text { - EXIT QUALITY OF THE AMMONIA LEAVING THE } \\
\text { CONDENSER/REBOILEQ (OIMENSIONLESS) } \\
\text { - TRANSVERSE PITCH OF THE TUAES (IN) } \\
\text { - TUBE DEPTH OF THE HEAT EXCHANGER (DIMENSIONLESS) }\end{array}$ & $\begin{array}{l}\operatorname{LC} C \\
\operatorname{LC} C \\
L C\end{array}$ & \\
\hline-12108 & ION OF ARRAYS & & $\begin{array}{l}223 \\
224\end{array}$ \\
\hline NG & $\begin{array}{l}\text { - THE ANGLE OF THE HEAT EXCHANGER TUBES FROM THE } \\
\text { HORIZONTAL (DEG) AND THE SINE AND COSINE OF THAT } \\
\text { ANGLE (DIMENSIONLESS) }\end{array}$ & & \\
\hline & $\begin{array}{l}\text { - FRACTION OF THE HEAT EXCHANGER THAT IS DELUGED } \\
\text { FOR EACH AMBIENT TEMPERATURE INCREMENT }\end{array}$ & & $\begin{array}{l}228 \\
229\end{array}$ \\
\hline
\end{tabular}


(OIMENSIONLESSI

TPER

TPO

WATCWD (DIMENSI ONLESS) FRACTION OF THE YEAR THAT THE AMBIENT AND WET BULS TEMPERATURE CURVES EXIST (DIMENSIONLESS)

- ARRAY OF COEFFICIENTS FOR THE CUBIC CURVE FIT OF HEAT RATE AS A FUNCTION OF TURBINE RACK PRESSURE

- WATER CONSUMPTION OF THE HEAT EXCHANGER OVER THE WHOLE YEAR FOR EACH AMBIENT TEMPERATURE INCREMENT (LBM/YEAR)

LOGICAL FIRST,FRST, FXTEMP, FXTVAR,FXTTTD,FXTLNG COMMON

S AFTR, ALPHA, ANG(3)

- adim(16)

5. CAOF, CSSPKW, CONF, CONL, COSTL, CAPCHG, CONMAT, CONMAZ,CAF

5 CRJ, CTURB, CDANG, CLUVR, CHAILS, CVM

$\$$.DFIN,DEEPL, DESVEL, DESVELV, DESVELD,DENSIF

S EFFP, EFS

S .FCË,FIRST, FIXL, FCOS, FFHX, FXTEMP, FACTOR, FRST , FACTORD, FSHOT, FOELUG

S. FIXV,FIXTTO,FXTVAR,FXTTTD, FXTLNG

$5 . G R F F F$

5 . HXNP, HPCST,HCD

5 . ITMAX, ITMIN

5 - JCONS

S, KCONV, KALEXT

S. MXEXT

5 NTA NSTOES, NBUNHI COMMON

S. ODR

5 . PSIZE, PER, PWCOS, PLANC, PFACT, POMDPL

5 , POHAAF, POHFAN, POHLEC, POHCIR, POHCND, POHSTC, POHSCL

5 - RRP,REDUCE, REDUCV, ROOFL, RHOUT

S DREJ, QREDUCE, OREDUCV

S. STGMAG, SAAF, SEgL

5 ,TD, TPO(4), TEFF, TLIM, THFIN, TLPRA, TFIX

3.TCD. TW

5 ,UES, UWS

S WFV. WATCONA, WATCOST

S. VAS (5)

$5, X O F P A, X W, X O$

COMMON / SUPPLY, VAL (5), CPLN, CPIP, CCOS, W3, COSM, EFF,

S OIN. CLAND. REAIR. NW, NT.

NP, PLANEZ,PSIZI?

U. DELPW.

HI. HO. TH,

T4, SPC,PTOTAL,PLANC3,SAFRON, AAIR, YFP, SPCD,PSIZ13, WT2, BP?, NWZ, TCOS, DELPA,DELPWT, DELPWC, CONBAS, XNTSX, VELDX

5 - PLAX, UCON, PMCST, FMCST, FBACC, EFFC, CH, CA, CS 5 : CLTC: CFC, CPCC, FITCO, CSC,CEPREP,DELPIP. AST,OLOCST S,CADCST, XNFAN,BLDANG, CPLEN, DPFEL, WHX, WTTUB, ADIA, ABLN

S. WTHDR, WTFRM, STRUCC, CFPERF, HPPERF, TOTCF, TPC, EFFIN, ANTU

5. $\triangle$ TRFF, WATFF, XNMOD, TCTPF, FDCPF, FMTRC,

5. $A C T P P D, ~ A D J B P D$, ACTVH, ADJVA, ADJPPF, THDAIR, TCTF, AFCST

S, CSRD, WATERW, WSTRCT, CFOUND, HUBDIA, TOWLEN, NUMTOW, CTOWD

\$ STPJ,SRRJS, ATUBC, CRA,TTDI, TTDZ,DELPS, CSTLVR,PIPCSTD

S. CSTHS, CYLRNG, CFANEL, PSTACS, PUFITC, PMPCST, RPTPL, SEPCST

5. PWT (16). WATCWD(16). DEPER (16). DEPERD(16)

\$ WAR, HTOW, NTOWDE, CSTIF, CSJ,CVF, CDP, W5

VAR (5), CPLN], CPIPI, CCOSI.

W31, COSMl, EFFl.

AMCALC 230

AMCALC 231

AMCALC 232

AMCALC 233

AMCALC 234

AMCALC 235

AMCALC 236

AMCALC 237

AMEALC 238

BIGCOM

BI GCOM

BI GCOM

BI GCOM

BIGCOM

BI SCOM

BIGCOM

BI GCOM

BIGCOM

BIGCOM

BIGCOM

BI GCOM

BIGCDY

BIGCOM

BIGCOM

BIGCOY

BIGCOM

BIGCOM

BIGCOM

BI GCOM

BIGCOM

BI GCOM

BIGCOM

BIGCOM

BI GCOM

BIGCOM

BI GCOM

BIGCOM

BIGCOM

BIGCOM

SUPPLY

SUPPLY

SUPPEY

SUPPLY

SUPPLY

SUPPLY

SUPPLY

SUPPLY

SUPPLY

SUPPLY

SUPPLY

SUPPLY

SUPPLY

SUPPLY

SUPPLY

SUPPLY

SUPP:Y

SINK

3
3
4
5
6
7
8
9
10
11
12
13
14
15
16
17
18
19
20
21
22
23
24
25
26
27
28
29
30
31
2
- 
s GAIR, FAIR, HPAIR. HPWAT, WLRTP, PLANCTI

COMMON/DJB/DI, XDG, SF

COMMON /INDIS/ DIST

COMMON /LACALL, SRN

COMMON /TINE/ XNS, TLA, XNTS, ARPS, TTDZOE, LINOR, XNP, XQUALY

COMMON /HEADEX, CHH,CN,CHM,CMW,CRJ,CWJ,CST,CMO,CSR,CSM,PITCHF,CPM,

1 PRESS, HEDMAT, HEDTYP, NPASS, WB, TSP, CSH, CSHP

COMMON /SURFEX, ODL.DUMLST (15)

COMMON/HXO/AI, ATOT,SFA,RFA,ACS

LOGICAL: LOOPER

COMMON/FAN/ FANDAP (153,10), FANDAH (153,10),FMCPF,FBCPF,PZ,R,,

I VELREC, AFC: ELEV, EHR, LOOPER, CTMAX

COMMON, PROP, CPW, THCONW, DENSIW, VISW, HFG,

-

CPV. THCONV, DENSIV, VISV,SIGMA,

$*$

CPA, THCONA, DENSIA, VISA

COMMON/VARVAR/TPER (16), TA (16):TAWB (16)

DATA DI/3.14159/, GC/32.0/

1002 FORMAT 15X, 11AI, PF, SFA, EFA,RFA E"1,5G12,6/5X, "ATOT, HXVOL,ART,AF, ACS

$1 ", 56 I 2: 6 / 5 X, " A F T R, A L P H A, S I G M A G=", 3612.61$

KCONVEO

LOODER = FALSE.

CTMAX $=0.0$

IF (FXTLNG) ELENH $=F I X L$

$T T D Y=T T D$

WAB $=$ WR

COUNT - COUNT $\cdot 1.0$

HEAT REJECTED BY THE TURBINE

BACK PRESSURE OF TURBINE AS A FUNCTION OF THE SATURATION TEMPERATURE $P=E \times P(17.168-9240 . /(T) \cdot 460)$. B०2:5

HEAT RATE FACTOR OF THE PLANT AT THE DESIGN BACK PRESSURE

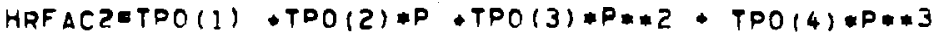

SINK

SINK

SINK

SINK

5 INK

SINK

SINK

SINK

SINK

SINK

SINK

S I NK

SINK

SINK

SINK

SCALER

SCALER

AMCALC

AMCALC

AMCALC

AMCALC

AMCALC

AMCALC

AMCALC

AMCALC

F ANDEK

FANDEK

F ANDEK

AMCALC

AMCALC

AMCALC

AMCALC

AMCALC

AMCALC

AMCALC

AMCALC

AMCALC

AMCALC

AMCALC

AMCALC

AMCALC

AMCALC

AMCALC

AMCALC

AMCALC

AMCALC

AMCALC

AMCALC

AMCALC

AMCALC

AMCALC

AMCALC

$\triangle M C A L C$

AMCALC

AMCA:C

AMCALC 
AMCALC 280

AMEALC 281

AMCALC 282

AMCALC 283

AMCALC 284

AMCALC 285

AMCALC 286

AMEALC 287

AMCALC 288

$\triangle M E A L C \quad 289$

AMCALC 290

AMCALC 291

$\triangle M C A L=\quad 292$

$\triangle M E A L C \quad 293$

AMCALC 294

AMCALC 295

AMCALC 296

AMCALC 297

AMCALC 296

AMCALC 299

AMCALC 300

AMCALC 301

AMCALC 302

AMCALC 303

AMCALC 304

AMCALC 305

AMCALC 306

AMEALC 307

AMCALC 308

AMCALE 309

AMCALC 310

AMCALC 311

AMCALC 312

AMEALC $3: 3$

AMCALC $\quad 314$

AMCALC 315

AMCALC $\quad 316$

AMCALC 317

AMCALC 328

AMCALC 319

AMCALC 320

AMCALC 321

AMCALC 322

AMCALC 323

$\triangle M C A L C \quad 324$

AMCALC 325

AMCALC $\quad 326$

AMCALC 327

AMCALC 328

AMEALC 329

AMCALC 330

AMCALC 331

AMCALC 332

AMCAIC 333

AMCALC 334

AMCALC 335

AMCALC 336 
$\begin{array}{llllll}\text { SUROUTINE CALC } & 74 / 74 & \text { OPT }=1 & \text { FTN } 4.5+414 & 06 / 05 / 78 & 07.59 .59\end{array}$

\begin{tabular}{|c|c|c|c|}
\hline & $\begin{array}{l}\text { IF (KDEBUG,NE,O) WRITE }(6,1002) \text { AI, PF, SFA, EFA, RFA, ATOT, HXVOL, } \\
\text { SART, AF, ACS, AFTR, ALPHA, SIGMAG } \\
\text { DESIGN OF THE CONOENSER, REBOILER } \\
\text { CALL SPDES(TI, TTDI, XNS,XNP, QREJ, TLA,XOUALY, VELD,VELDO, ARPS, XNTS, } \\
\text { IOELOWC,LINOR) }\end{array}$ & $\begin{array}{l}\text { AMCALC } \\
\text { AMCALC } \\
\text { AMCALC } \\
\text { AMCALC } \\
\text { AMCALC } \\
\text { AMCALC } \\
\text { AMCALC }\end{array}$ & $\begin{array}{l}337 \\
338 \\
339 \\
340 \\
341 \\
342 \\
343\end{array}$ \\
\hline & $\begin{array}{l}\text { CHECK IF NUMBER OF TUBES IN DEPTH IS TO BE HELD CONSTANT } \\
\text { IF (DEEPL.GT.0.00) ZD = DEEPL }\end{array}$ & $\begin{array}{l}\text { AMCALC } \\
\text { AMCALC } \\
\text { AMCALC } \\
\text { AMCALC } \\
\text { AMCALC }\end{array}$ & $\begin{array}{l}344 \\
345 \\
346 \\
347 \\
348\end{array}$ \\
\hline & $\begin{array}{l}\text { GUESS THE TEMPERATURE DROP IN THE PIPING FROM THE } \\
\text { CONDENSER/REBOILER TO THE HEAT EXCHANGER }\end{array}$ & $\begin{array}{l}\text { AMCALC } \\
\text { AMCALC } \\
\text { AMCALC }\end{array}$ & $\begin{array}{l}349 \\
350 \\
351\end{array}$ \\
\hline & 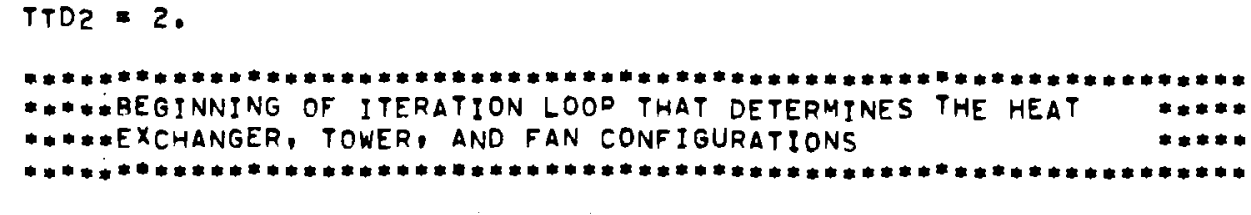 & $\begin{array}{l}\text { AMCALC } \\
\text { AMCALE } \\
\text { AMCALC } \\
\text { AMCALC } \\
\text { AMCALC } \\
\text { AMCALC } \\
\text { AMCALC }\end{array}$ & $\begin{array}{l}352 \\
353 \\
354 \\
355 \\
356 \\
357 \\
358\end{array}$ \\
\hline & 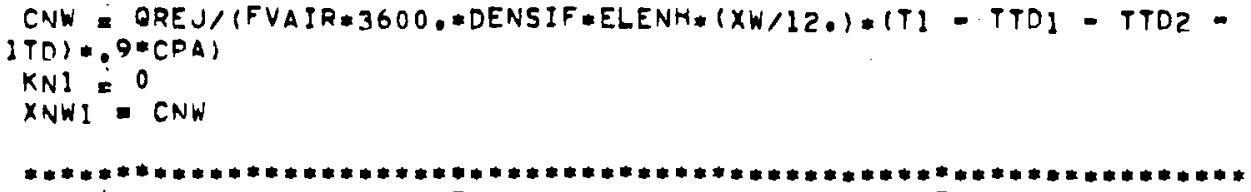 & $\begin{array}{l}\text { AMCALC } \\
\text { AMCALC } \\
\text { AMCALC } \\
\text { AMCALC } \\
\text { AMCALC } \\
\text { AMCALC }\end{array}$ & $\begin{array}{l}359 \\
360 \\
361 \\
362 \\
363 \\
364\end{array}$ \\
\hline & 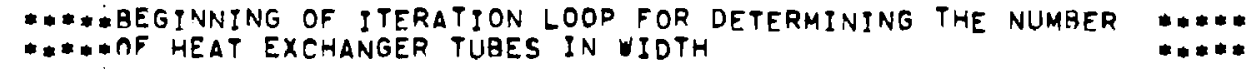 & $\begin{array}{l}\text { AMCALC } \\
\text { AMCALC }\end{array}$ & \\
\hline & 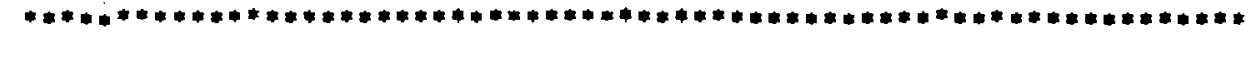 & $\begin{array}{l}\text { AMCALC } \\
\text { AMCALC }\end{array}$ & $\begin{array}{l}367 \\
368\end{array}$ \\
\hline 170 & RANGE $=$ RREJ/IFVAIR*DENSIF *ELENH*CNW*XW*CPA*3600./12.) & $\begin{array}{l}\text { AMCALC } \\
\text { AMCALC }\end{array}$ & $\begin{array}{l}369 \\
370\end{array}$ \\
\hline & 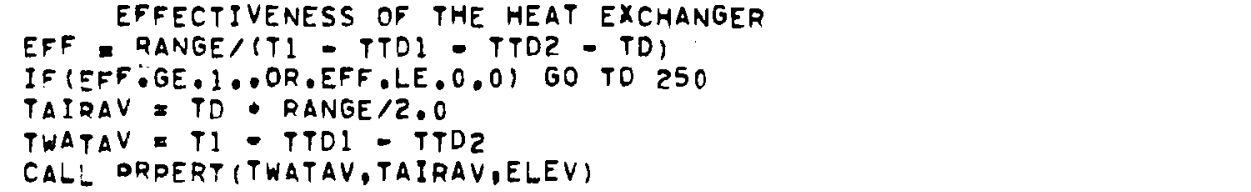 & $\begin{array}{l}\text { AMCALC } \\
\text { AMCALC } \\
\text { AMCALC } \\
\text { AMCALC } \\
\text { AMCALC } \\
\text { AMCALC }\end{array}$ & $\begin{array}{l}371 \\
372 \\
373 \\
374 \\
375 \\
376\end{array}$ \\
\hline & $\begin{array}{l}\text { NUMBER OF TRANSFER UNITS IN THE HEAT EXCHANGER } \\
\text { ANTU }=- \text { ALOG }(1.0 \text { - EFF })\end{array}$ & $\begin{array}{l}\text { AMCALC } \\
\text { AMCALC } \\
\text { AMCALC }\end{array}$ & $\begin{array}{l}377 \\
378 \\
379\end{array}$ \\
\hline & $\begin{array}{l}\text { DEPTH OF THE HEAT EXCHANGER } \\
\text { IF }(20: E Q . O Q \text { GO TO } 250 \\
\text { DFPTH }=20 . \times 0 / 12,0\end{array}$ & $\begin{array}{l}\text { AMCALC } \\
\text { AMCALC } \\
\text { AMCALC } \\
\text { AMCALC }\end{array}$ & $\begin{array}{l}380 \\
381 \\
382 \\
383\end{array}$ \\
\hline & 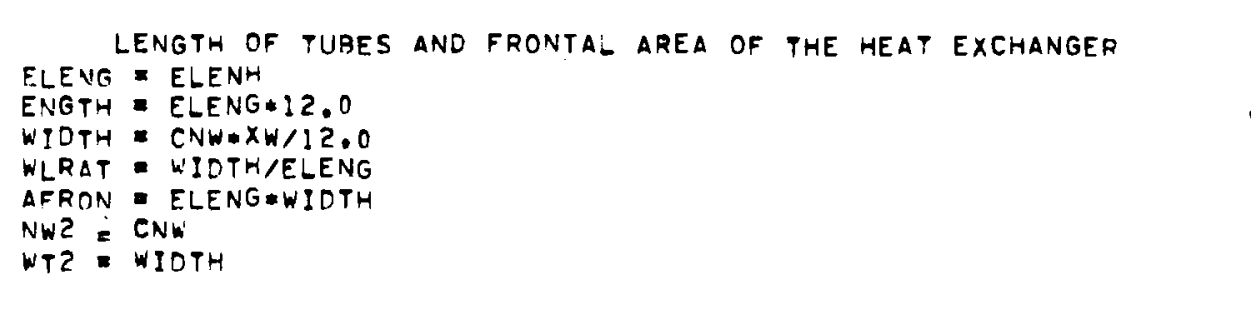 & $\begin{array}{l}\text { AMCALC } \\
\text { AMCALC } \\
\text { AMCALC } \\
\text { AMCALC } \\
\text { AMCALC } \\
\text { AMCALC } \\
\text { AMCALC } \\
\text { AMCALC } \\
\text { AMCALC } \\
\text { AMCALC }\end{array}$ & $\begin{array}{l}384 \\
385 \\
386 \\
387 \\
388 \\
389 \\
390 \\
391 \\
392 \\
393\end{array}$ \\
\hline
\end{tabular}




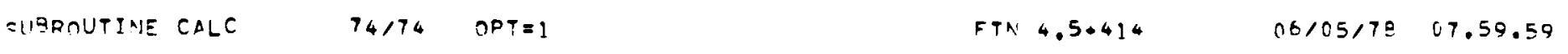

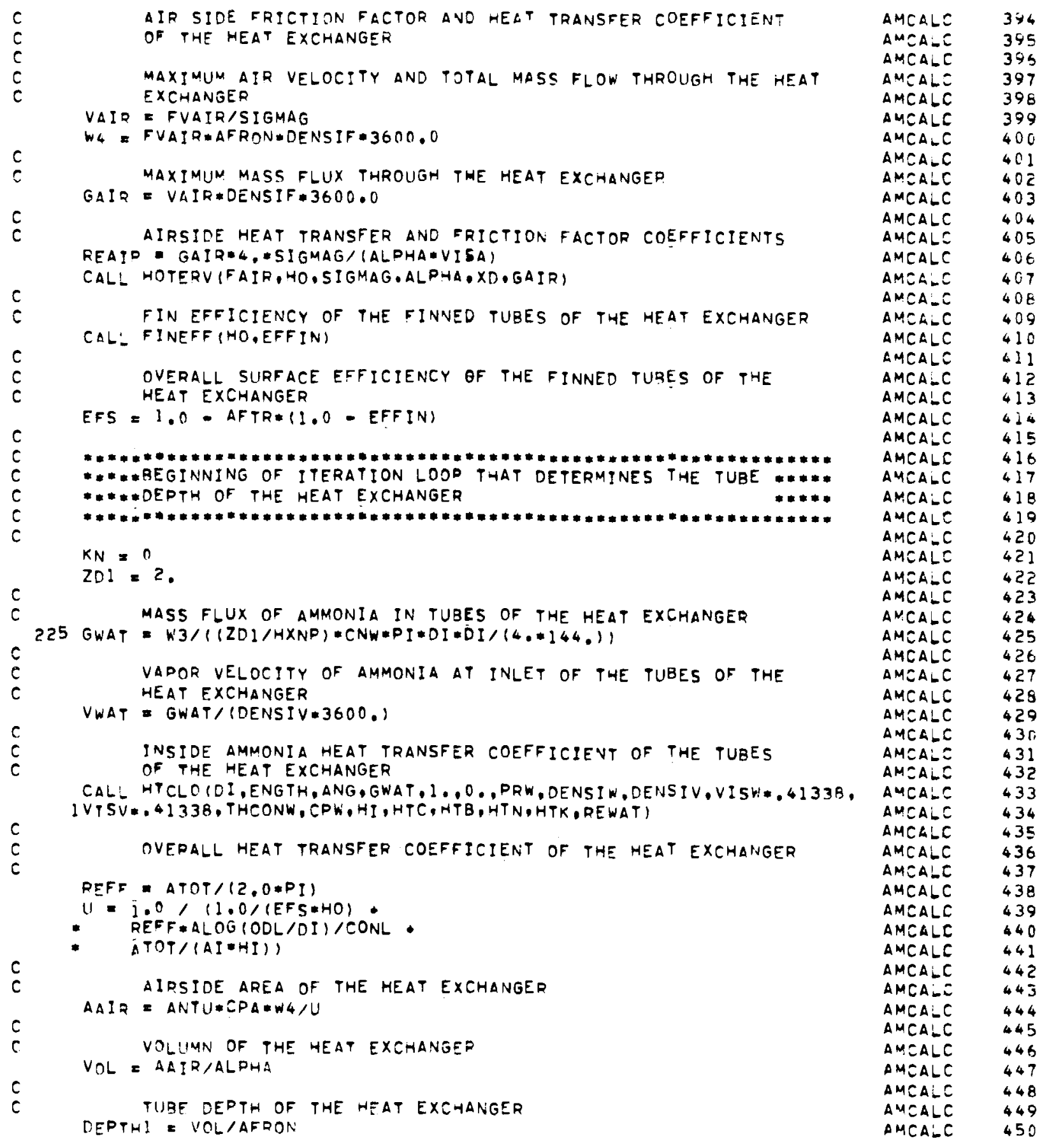




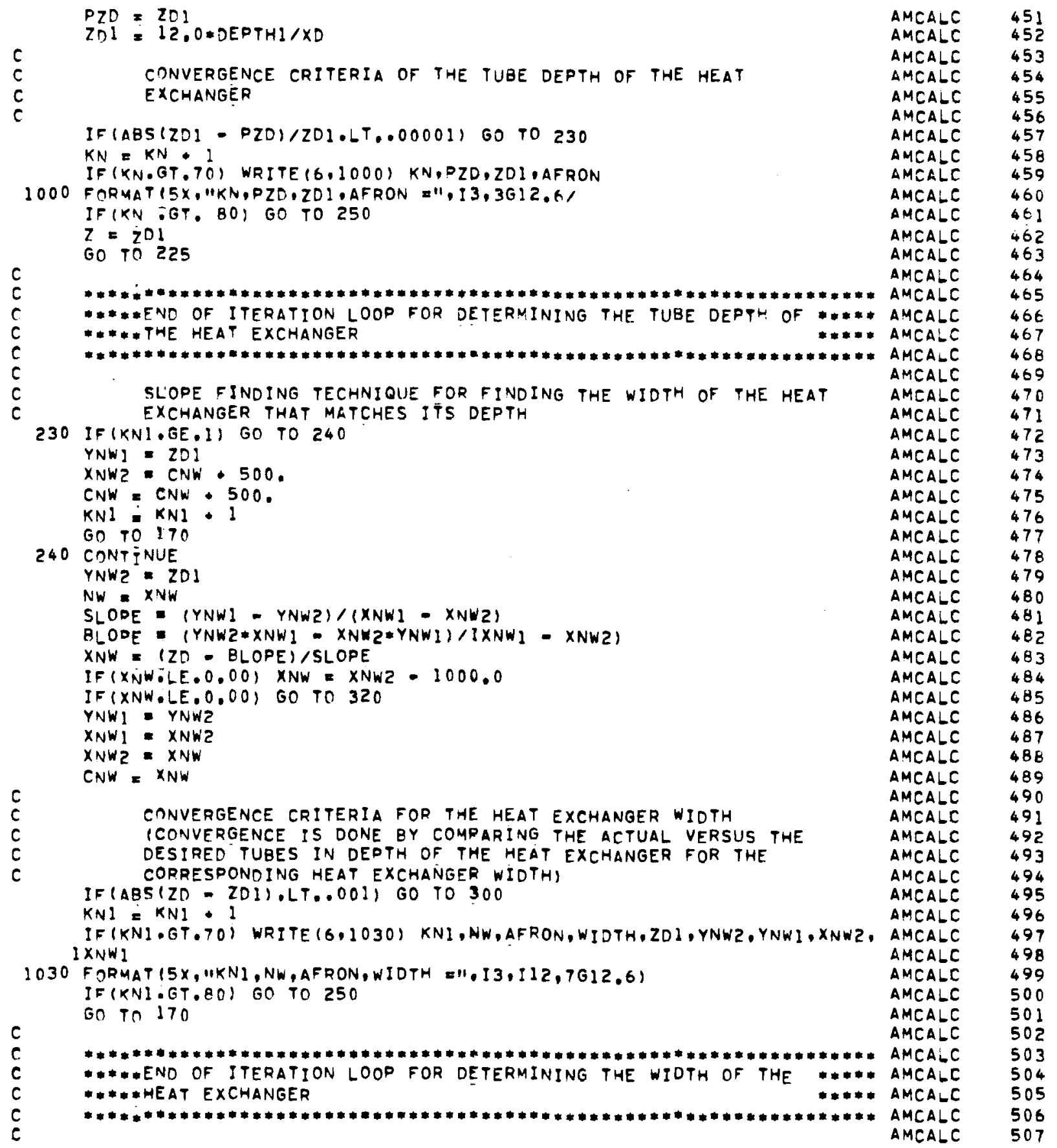




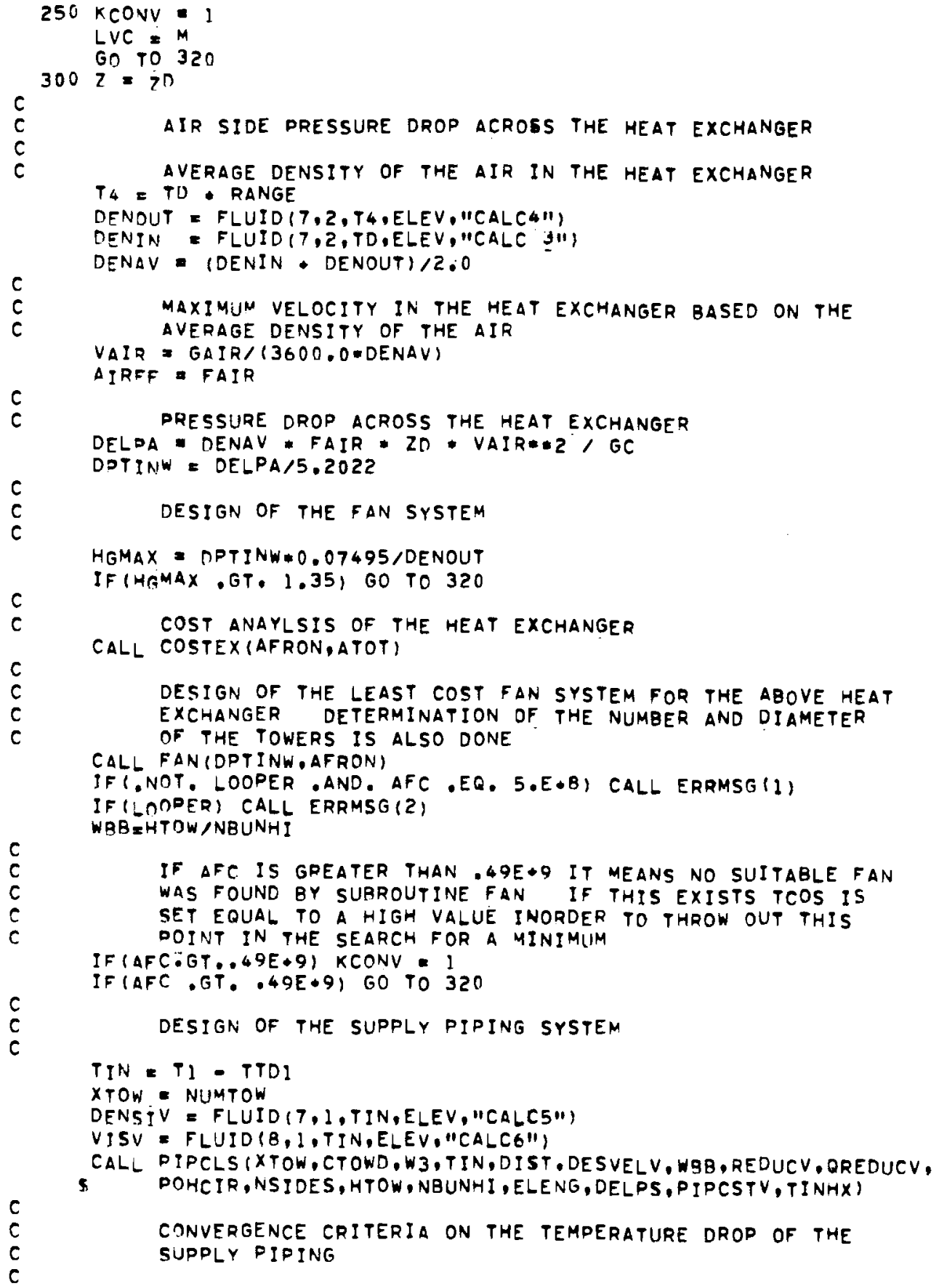

AMCALC

AMCALC

AMCALC

AMCALC

AMCALC

AMCALC

AMCALC

AMCALC

AMCALC

AMCALC

AMCALC

AMCALC

AMCALC

AMEALC

AMCALC

AMEALC

AMCALC

AMCALC

AMCALC

AMCALC

AMCALC

$\triangle M C A L C$

AMCALC

AMCALC

AMCALC

AMCALC

AMCALC

AMCALC

AMCALC

AMCALC

AMCALC

AMCALC

AMCALC

AMCALC

AMCALC

AMCALC

AMCALC

AMCALC

AMCALC

AMCALC

AMCALC

AMCALC

AMCALC

AMCALC

AMCALC

AMCALC

AMCALC

AMCALC

AMCALC

AMCALC

AMCALC

AMCALC

AMCALC

$\triangle M C A L C$

$\triangle M C A L C$

AMCALC

AMCALC
508

509

510

511

512

513

514

515

516

$5 ! 7$

518

519

520

521

522

523

524

525

526

527

528

529

530

531

532

533

534

535

536

537

538

539

540

541

542

543

544

545

546

547

548

549

550

$55 !$

552

553

554

555

556

557

558

559

560

561

562

563

564 
310 CONTINUE

c

DESIGN OF THE RETURN PIPING SYSTEM

CALL DYPCLRIXTOW, CTOWD,W3,DIST, DESVEL,WB, REDUCE, QREDUCE, POHCIP, NSIDES, HTOW, NRUNHI, ELENG, DELPR, PIPCSTL)

PRESSURE DROP OF THE AMMONIA THROUGH THE TUBES OF THE HEAT EXCHANGER

CALL DPCONIGOSW, OI, ENGTH, ANG, GWAT, 1 , O . DENSIW, DENSIV, VISW*,41338, SVISV*.41338, DELPWT, DPF, DPG,DPM, XLM, PHIL, PHILZ,VF, WATFF, RL,RV)

CALL PUMPCSTIW3, DELPS, DELPR, DELPWT, DELPWC, XQUALY, DESVEL, S PMPCST, PUFITCI
AMCALC $\quad 565$

AMCALC $\quad 566$

AMCALC $\quad 567$

AMCALC 568

AMCALC 569

AMCALC $\quad 570$

AMCALC 571

AMCALC 572

AMCALC 573

AMCALC 574

AMCALC 575

AMCALC $\quad 576$

AMCALC $\quad 577$

AMCALC 578

AMCALC $\quad 579$

AMCALC 580

AMCALC 58?

AMCALC 582

AMCALC 583

AMCALC 584

AMCALC 585

AMCALC 586

AMCALC 587

AMCALC 588

AMCALC $5 \overline{8} 9$

AMCALC 590

591

592

593

594

595

596

597

598

599

600

601

602

603

604

605

606

607

$60 B$

609

610

611

612

613

614

615

616

617

618

619

620

621 


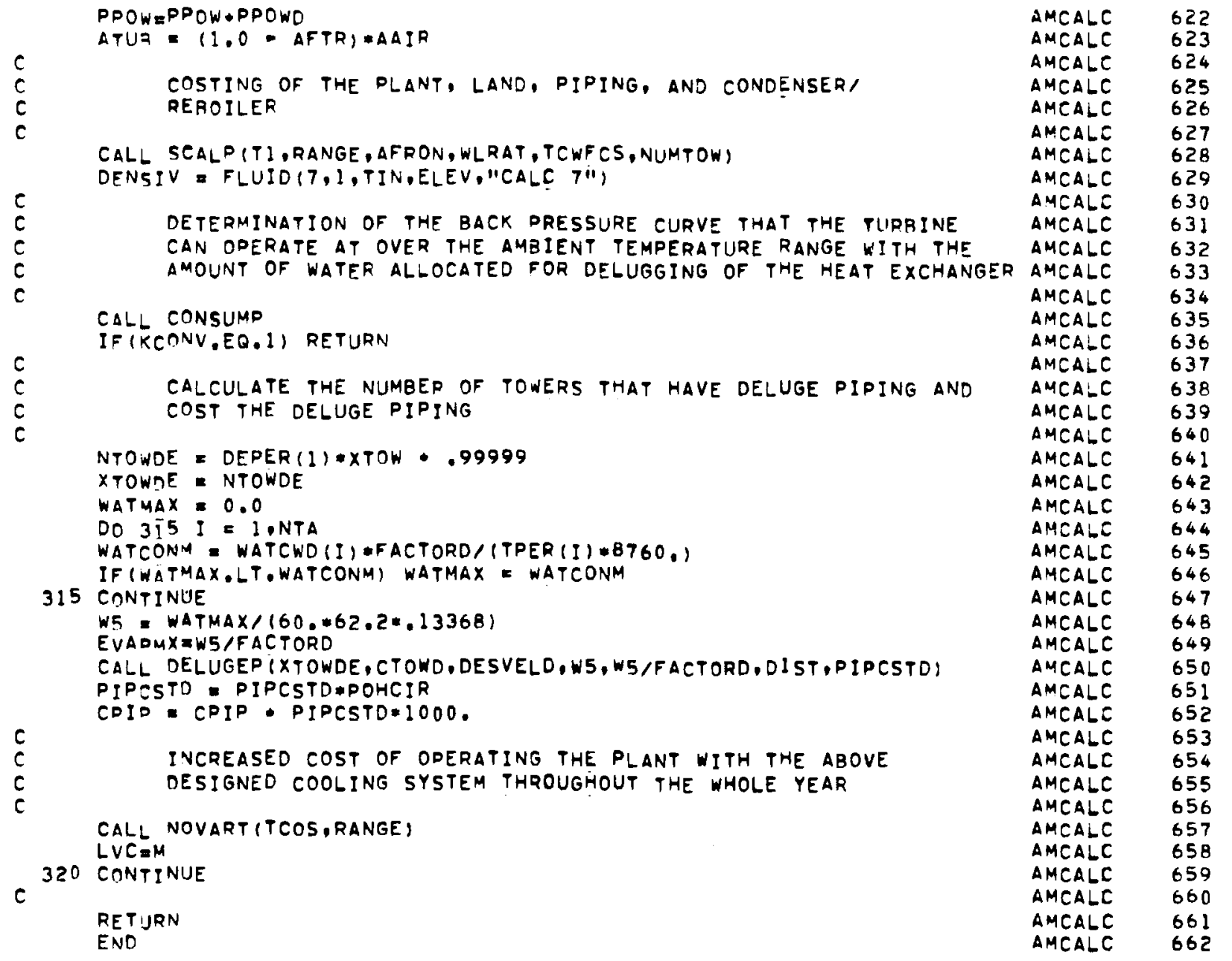


THIS ROUTINE IS CALLED BY SUBROUTINE SERCH WHEN PRESSURE DROP RESTRICTION IS ENCOUNTERED. IT SEARCHES BY CHANGING A VARIABLE OTHER THAN THAT SELECTED BY SUBROUTINE SERCH.

DIMENSION DP (5), DV (5), VAJ (5)

COMMON /SCALER/HRFACI. ATUB, CPERA, SSCD,PLANCI, CASSSI,

S GAIR, FAIR, HPAIR, HPWAT, WLRTP, PLANCTI

LOGICAL FIRST, FRST, FXTEMP, FXTVAR OFXTTTD, FXTLNG COMMON

5 SETR, ALPHA, ANG (3)

- Bdim(16)

- CADF, CSSPKW, CONF, CONL, COSTL, CAPChg, CONMAT, CONMAz, CAF

5 , CRJ, CTURB, CDANG, CLUVR, CHAILS, CVM

\$ DFIN, DEEPL, DESVEL, DESVELV, DESVELD. DENSIF

$\$$ EFFP, EFS

S IFCE, FIRST, FIXL, FCOS, FFHX, FXTEMR, FACTOR, FRST, FACTORO, FSHOT, FDELUG

\$. FIXV,FIXTTD,FXTVAR,FXTTTD,FXTLNG

S. GBEFF

S HXNP:HPCST IHCD

S. ITMAX, ITMIN

5 JEONS

- KCONV, KALEXT

\$. MXEXT

5. NTA,NSIDES, NBUNHI

COMMON

$5 O D R$

3. PSizE, PER, PWCOS, PLANC, PFACT, POMDPL

5 ,POHRAF, POHFAN, POHLEC, POHCIR, POHCNO, POHSTC, POHSCL

\$ RRP,REDUCE, REDUCV, ROOFL, RHOUT

- QREJ, QREDUCE, QREDUCV

S.SIGMAG, SAAF, SEGL

S.TD. TPO(4), TEFF, TLIM, THFIN, TLPRA, TFIX

S ,TCD̃. TW

$\$$.UES, UWS

* WFü. hatcona, watcost

5 VAS (5)

S, XDFDA, XW, XD COMMON /SUPPLY, VAL (5), CPLN, CPIP, CCOS, W3, COSM, EFF,

- DIN. CLAND. REAIR. CPLN, CPIO, CCOS,

\$ DIN, CLAND, REAIR, ZLE, ZRFACZ, WH, WE, DELPW, PPOW, REWAT. NW, NT, NP,PLANCZ,PSIZI2, SPAP, SW4, SW3: SPC.PTOTAL,PLANC3,SAFRON, AAIR, YFP, SPCD,PSIZI3, WTZ, BP2, NW2, TCOS, DELPA, OELPWT, DELPWC, CONBAS, XNTSX, VELDX

- TLAX, UCON, PMCST, FMCST, FBACC, EFFC, CH, CA, CS $\$$ CLTC. CFC. CPCC. FITCO, CSC.CEPREP,DELPIP, AST,OLDCST S. CADCST, XNFAN, BLDANG, CPLEN, DPFEL, WHX, WTTUB, ADIA, ABLN

\$, WTHDR, WTFRM, STRUCC,CFPERF, HDPERF, TOTCF, TPC, EFFIN, ANTU

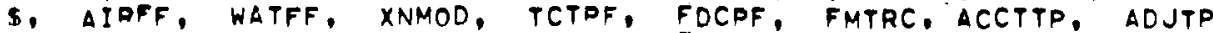

5. ACTEPD, ADJAPD, ACTVH, ADJVH, ADJJPPF, THPAIR, TCTF, AFCST

5. CSRD, WATERW, WSTRCT, CFOUND. HUBDIA. TOWLEN, NUMTOW, CTOWD

S. SOEJ J.SORJS, ATUBC, CBA, TTDI, TTOZ, DELPS, CSTLVR, PIPCSTD

\$ CSTHS, CYLRNG. CFANEL, PSTACS, PUFITC, PMPCST, RPTPL, SEPCST

1.PWD(16), WATCWD (16) ,DEPER (16), JEPERQ (16)

I WRR,HTOW, NTOWDE. CSTIF,CSJ,CVF,CDO,W5

COMMON / SINK, VAR(5), CPLNI, CPIPI, CCOSI,

W31, COSMl, EFFl,

CHNGE

CHNGE

CHNGE

CHNGE

CHNGE

CHNGE

SCALER

SCALER

BIGCOM

BIGCOM

BI GCOM

BIGCOM

BI GCOM

BI GCOM

B I GCON

BIGCOM

BIGCOM

BIGCOM

BI GCOM

BIGCOM

BI GCOM

BI GCOM

BI GCOM

BIGCOM

BIGCOM

BIGCOM

BIGCOM

BI GCOM

8I GCOM

B I GCOM

BIGCOM

BIGCOM

BIGCOM

BI GCOM

BIGCOM

BIGCOM

BI GCOM

BIGCOM

SUPPLY

SUPPLY

SUPPLY

SUPPLY

SUPPLY

SUPPLY

SUPP!Y

SUPPLY

SUPPLY

SUPPLY

SUPPLY

SUPPLY

SUPPLY

SUPPLY

SUPPLY

SUPPLY

SUDPLY

SINK 


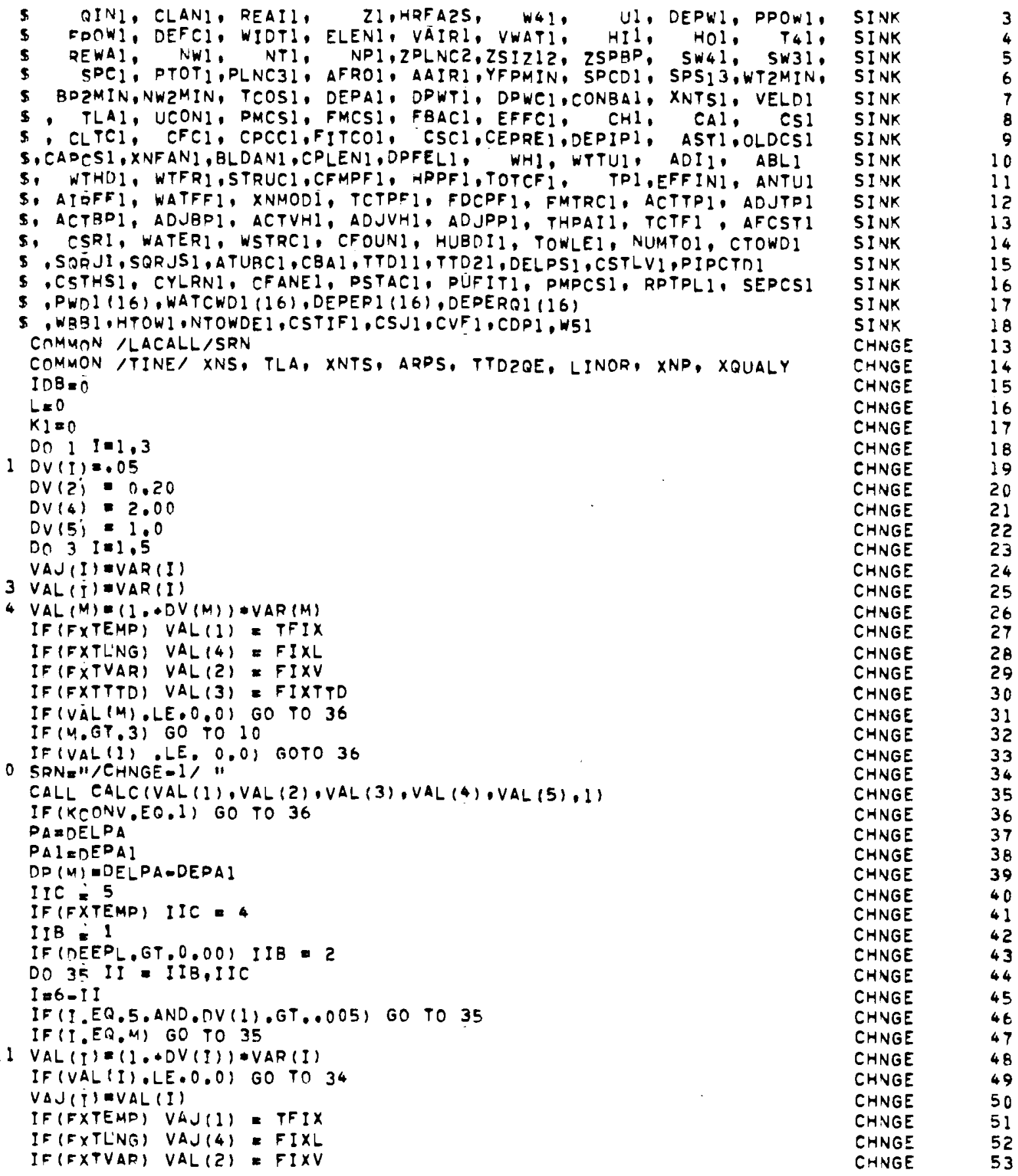


IF (FXTTTD) VAL (3) = FIXTTO

IFII.GT.3) GO TO 17

IF(VAJ(I) .LE. 0.0$)$ GOTO 12

GO TO 17

12 VAJ $(Y)=V_{A R}(I)$

GO TO 34

$17 \mathrm{~J}=\mathrm{I}$

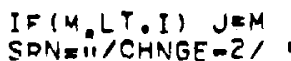

CALL CALC(VAJ (1), VAJ (2) VAJ (3) , VAJ (4), VAJ (5), J)

$\operatorname{VAU}(I)=V A R(I)$

IF (KCONV.EO.1) GO TO 34

$P A=D E L P A$

$P A I=\cap E D A\}$

$D P(1)=D E L D A=D E P A]$

$E=0$.

$C=1$.

$D=1$.

18 VAL $(j)=(1 \cdots O V V(I) *(E-D P(M)) / D P(I)) * V A R(I)$

IF (IXTEMD) VAL (I) $=$ TFIX

IF(EXTLNG) VAL $(4)=F I X L$

IF (FXTVAR) VAL (2) = FIXV

IF (FXTPTD) VAL (3) = FIXTTO

IF(VAL II).LE.0.0) GO TO 34

IF (I.GT.3) GO TO 24

IF (VALI (I) .LE. 0.0$)$ GOTO 34

24 SRNE"/CHNGE-3/"

CALL CALC (VAL (1), VAL (2), VAL (3), VAL (4) VAL (5),J)

IF (KCONV.EQ.1) GO TO 34

IF IDELPA.GE,XDEPA.ANO.DELPA.LE.1.01*XDEPA GO TO 26

IF (DELPA,GE. ..01*XDEPA) GO TO 25

$C=0 / 10$ :

IF (C.LT..0OI) GO TO 34

$E=E+C$

GO TO 18

$25 \mathrm{D}=\mathrm{C} / \mathrm{J} 0$ :

IF(O.LT..0OI) GO TO 34

$E=E-\bar{n}$

GO TO 18

26 IF (Tत̈OS.GE.TCOS1) GO TO 34

CALL STORE

$L=1$

DO $27 \quad \mathrm{~J}=1.5$

27 VAUIIJIEVAR(IJ)

$V A L(M)=(1)+O V(M))=V A R(M)$

IF(FXTEMD) VAJ(I) \& TFIX

IF (FXTEMP) VAL (1) \& TFIX

IF (FXTLING) VAL (4) $=F I X L$

IF (FXTVAR) VAL (Z) $=$ FIXV

IF(EXTPTD) VAL (3) = FIXTTD

IF (VALIM).LE.0.0) GO TO 36

IF (M.GT.3) GO TO 33

IF(VAL (1),GE.170..OR.VAL (1).LT.110.) GO TO 36

33 SRNE"/CHNGE-4/"

CALL CALC(VAL (1), VAL (2), VAL (3), VAL (4), VAL (5), M)

IF (KCONV.EQ.1) GO TO 36

PAI $=D E P A I$

$\begin{array}{ll}\text { CHNGE } & 54 \\ \text { CHNGE } & 55 \\ \text { CHNGE } & 56 \\ \text { CHNGE } & 57 \\ \text { CHNGE } & 58 \\ \text { CHNGE } & 59 \\ \text { CHNGE } & 60 \\ \text { CHNGE } & 61 \\ \text { CHNGE } & 62 \\ \text { CHNGE } & 63 \\ \text { CHNGE } & 64 \\ \text { CHNGE } & 65 \\ \text { CHNGE } & 66 \\ \text { CHNGE } & 67 \\ \text { CHNGE } & 68 \\ \text { CHNGE } & 69 \\ \text { CHNGE } & 70 \\ \text { CHNGE } & 71 \\ \text { CHNGE } & 72 \\ \text { CHNGE } & 73 \\ \text { CHNGE } & 74 \\ \text { CHNGE } & 75 \\ \text { CHNGE } & 76 \\ \text { CHNGE } & 77 \\ \text { CHNGE } & 78 \\ \text { CHNGE } & 79 \\ \text { CHNGE } & 80 \\ \text { CHNGE } & 81 \\ \text { CHNGE } & 82 \\ \text { CHNGE } & 83 \\ \text { CHNGE } & 84 \\ \text { CHNGE } & 85 \\ \text { CHNGE } & 86 \\ \text { CHNGE } & 87 \\ \text { CHNGE } & 88 \\ \text { CHNGE } & 89 \\ \text { CHNGE } & 90 \\ \text { CHNGE } & 91 \\ \text { CHNGE } & 92 \\ \text { CHNGE } & 93 \\ \text { CHNGE } & 94 \\ \text { CHNGE } & 95 \\ \text { CHNGE } & 96 \\ \text { CHNGE } & 97 \\ \text { CHNGE } & 98 \\ \text { CHNGE } & 99 \\ \text { CHNGE } & 100 \\ \text { CHNGE } & 101 \\ \text { CHNGE } & 102 \\ \text { CHNGE } & 103 \\ \text { CHNGE } & 104 \\ \text { CHNGE } & 105 \\ \text { CHNGE } & 106 \\ \text { CHNGE } & 107 \\ \text { CHNGE } & 108 \\ \text { CHNGE } & 109 \\ \text { CHNGE } & 110 \\ \text { CHNG } & \end{array}$


SUBROUTINE CHNGE $\quad 74 / 74 \quad$ OPTEI

$P A=D E L P A$

$D P(M)=D E L P A-D E P A]$

GO TO 11

34 VAL II) $=V A R(I)$

35 CONTINUE

36 DO $37 \quad I=1.5$

37 DVII)=nD (I)

$K I=K 1+1$

IF $(K \bar{I}, E Q .1)$ GO TO 4

$K I=0$

IF (DV (1).LE..005) GO TO 39

DO $38 \quad I=1,5$

38 DV $(I)=D V(I) / 5$. GO Tn

39 IF (L.EQ.1.AND,IDB.NE.0) RETURN

103 FRRMATPU

RETURN

AND CHANGES MADE")

END
FTN $4.5 \cdot 414$

$06 / 05 / 78 \quad 07.59 .59$

$\begin{array}{ll}\text { CHNGE } & 111 \\ \text { CHNGE } & 112 \\ \text { CHNGE } & 113 \\ \text { CHNGE } & 11 \\ \text { CHNGE } & 115 \\ \text { CHNGE } & 116 \\ \text { CHNGE } & 117 \\ \text { CHNGE } & 118 \\ \text { CHNGE } & 119 \\ \text { CHNGE } & 120 \\ \text { CHNGE } & 121 \\ \text { CHNGE } & 122 \\ \text { CHNGE } & 123 \\ \text { CHNGE } & 124 \\ \text { CHNGE } & 125 \\ \text { CHNGE } & 126 \\ \text { CHNGE } & 127 \\ \text { CHNGE } & 128\end{array}$


FUNCTIDN CMARTIN (XMART , JMART, KMART)

FUNCTION CMARTIN SUPPLIES THE MARTINELLI COEFFICIENTS FOR TWO-DHASE FLOW

DIMENSION PHI $(9,6,2)$

DEFINITION OF MARTINELLI INDICES FOR DATA SUPPLY

DHI (IMART, JMART, KMART)

IMART - COEFFICIENTS OF CURVE FITS 11.91

JMART - FLOW REGIME (I - 4$)$

I - VISCOUS - VISCOUS

2 - TURBULENT - VISCOUS

3 -. VISCOUS - TURBULENT

-- TURBULENT - TURBULENT

HOLDUP CORRELATIONS $(5-6)$

5 -- MARTINELLI PARAMETER ( $X$ ) LESS THAN ONE

6 -- MARTINELLI PARAMETER (X) GREATER THAN ONE

CMART

CMART

CMART

CMART

CMART

CMART

CMART

CMART

CMART

CMART

CMART

CMART

CMART

CMART

CMART

CMART

CMART

CMART

CMART

KMART - FLUID

1 LIOUIO

2 -. VAPOR

CMART

CMART

CMART

CMART

MARTINELLI FUNCTIONAL COEFFICIENTS FOR THE LIQUID

CMART

CMART

CMART

DATA(IDHI IMART, JMART, I), IMART $=1,9)$, JMART $=1,6)$,

CMART VISCOUS - VISCOUS

CMART

$.0 .07995,-0.42951,0.09563,-0.00547,0.00142,0.00011$, CMART

* $0.00000,0.00000,0.00000$. CMART TURBULENT: VISCOUS

CMART

$-1.24907,-0.44314,0.06680,-0.00521,-0.00057,0.00012$, CMART

- $0.00000,0.00000,0.00000$.

C

* $1.23807,-0.46844,0.07189 \cdot-0.00464,-0.00070,0.00012$,

CMART 35

CMART

* $0.00000,0.00000,0.00000$.

$1.44065,-0.50445,0.06212,0.00106,-0.00101,0.00003$, CMART - $0.00000 \cdot 0.00000,0.00000$.

c

RL HOLDUP CORRELATION - MARTINELLI PARAMETER LESS CMART THAN ONE

$=0.22206 \cdot 0.04251 \cdot=0.00127 \cdot 0.00002 \cdot 0.00000 \cdot 0.00000$.

- $0.00000 \cdot 0.00000 \cdot 0.00000$.

c GREATER THAN ONE

- $0.22918,0.11270,0.01113,-0.00431,0.00050,0.00009$ cMART

$-0.00013 \cdot-0.00007 \cdot 0.00001 /$

$c$
$c$
$c$
$c$

MARTINELLI FUNETIONAL COEFFICIENTS FOR THE VAPOR

CMART

CMART

CMART

CMART

CMART

DATA $($ OHI (IMART,JMART,2), IMART $=1,9)$, JMART $=1,611$ CMART VISCOUS - VISEOUS

CMART

$=1.10310,1.49456,-0.01132,0.00014,0.00000,0.00000$, CMART

* $0.00000,0.00000 \cdot 0.00000$ ?

c

TURBULENT - VISCOUS

CMART 
$c$

$-1.24906,-0.55672$

$0.00000,0.00000,0.00000$ :

VISCOUS - TURBULENT

* $1.33750,0.53139,0.07181,-0.00442,-0.00069,0.00012$,

* $0.00000,0.00000,0.00000$.

$c$

C

$+1.44105,0.4954,0.06153,-0.00113,=0.00095,0.00003$,

* $0.00002,0.00000,0.00000$ :

c

$-0.25522,-0.20583,-0.02893,-0.00884,0.00000,0.00000$, RG HOLDUP
THAN ONE
10583

C

* $-0.25522,-0.10573,-0.02893,00.00884,0.00000,0.00000$,

C - $0.00000,0.00000,0.00000 \%$

C

IMARTं $=0$

$J=$ JMART

$K=$ KMAPT

$S U M=0.00$

IF ( J EO. I AND. K.EO. 2) GO TO 75

IFIJ.EQ. 5 .AND. K.EQ. I) GO TD 75

DO $5 \cap I=$ I. IMART

SIIM $=\operatorname{SUM} *$ PHI $(I, J, K) \triangle A L O G(X M A R T) * I I-1)$

50 CONTENIIE

CMARTIN = EXP(SUM)

IFIJ.EQ. 6. AND.K.EO. 1 I CMARTIN = SUM RETIIRN

75 DO 100 I E I. IMART

SIIM $=$ SUM + PHI $(I, J, K) * X M A R T *(I-1)$

100 CONTINUE

CMADTIN = SUM

RETURN

END
59

60

CMART

CMART

CMART

CMART

CMART

CMART

CMART

CMART

CMART

CMART

CMART

CMART

CMART

CMART

CMART

CMART

CMART

CMART

CMART

CMART

CMART

CMART

CMART

CMART

CMART

CMART

CMART

CMART

CMART

CMART

CMART

CMART

CMART

CMART

CMART 
SURROUTINE CONSUMP IS USED TC DETERMINE THE BACK PRESSURE CURVE THAT THE TURBINE CAN OPERATE AT OVFR THE ENTIRE YEAR WITH THE TOTAL AMOUNT OF ALLOCATED WATER EVAPORATED IN DELUGING THE WET/DRY TOWERS. IF DELUGING THE TOWER DOES NOT CONSUME ENOUGH WATER TO MAKE IT ABOVE THE AMOUNT ALLOWED TO BE CONSUMED THE DESIGN IS TERMINATED. THE SAME OCCURS IF THERE IS NOT ENOUGH WATER TO KEEP THE BACK PRESSURE AT THE RACK PRESSURE LIMIT CURVE.

\section{DEFINITION OF VARIABLES} BDLZ - STORAGE VARIABLE USED IN THE SLOPE FINDING
TECHNIQUE FOR THE CORRECT WET/DRY BÁCK PRESSURE (IN HGA;

BPLII - STORAGE VARIABLE, USED IN THE SLOPE FINDING TECHNIQUE FOR THE CORRECT WET/DRY BACK PRESSURE (IN HGA)

R'LIII - STORAGE VAIABLE, UEEO IN THE SLOPE FINDING TECHNIQUE FOR THE CORRECT WET/DRY BACCK PRESSURE (IN HGA)

NTA - NUMBER OF TEMPERATURE DIVISIONS IN THE YEARLY TEMPERATURE PROFILE (DIMENSIONLESS)

WATCONA- AMOUNT OF WATER THAT CAN BE EVAPORATED DURING THE YEAR (LBM/YEAR)

WATCWOT- STORAGE VARIABLE, USED IN THE SLOPE FINDING TECHNIOUE FOR THE CORRECT WET/DRY SACK PRESSLIRE (LBM/YEAR)

WATCIZ - WATER EVAPORATED gY THE HEAT EXCHANGER OVER THE WHOLE YEAR WHEN THE BACK PRESSURE IS AT THE BACK PRESSURE SEPARATING ZONE I AND ZONE 2 (LBM/YEAR)

WATC1Z3- WATER EVAPORATED BY THE HEAT EXCHANGER OVER THE WHOLE YEAR WHEN THE BACK PRESSURE IS AT THE RACK PRESSURE SEPARATING ZONE I PROM ZONE 3 OR ZONE 2 FROM ZONE 3 (LBM/YEAR)

WATDET - AMOUNT OF WATER CONSUMED IF THE HEAT EXCHANGER IS DELUGEO COMPLETELY YEAR AROUND (LBM/YEAR)

WATWDTI- STORAGE VARIABLE, USED IN THE SLOPE FINDING TECHNIQUE FOR THE CORRECT WETIORY BACK PRESSURE (LBM/YEAR)

DEFINITION OF ARRAYS

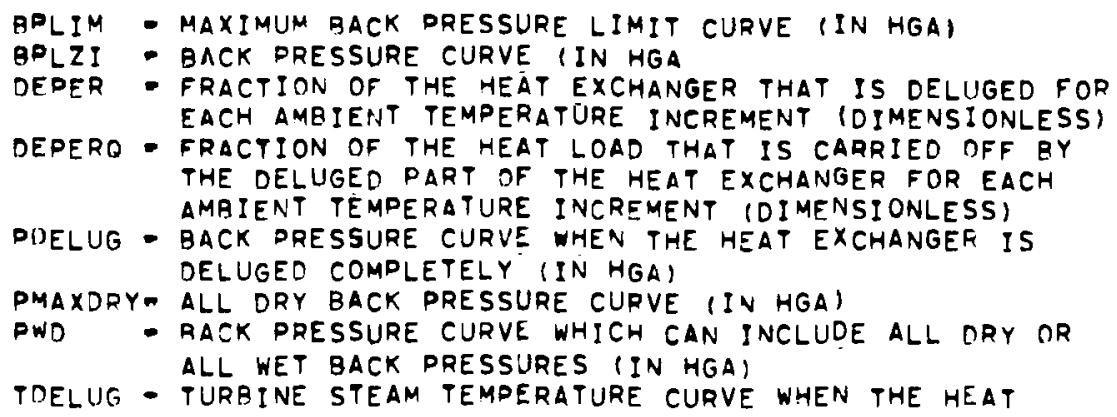

CONSUMP

CONSUMP

CONSUMP

CONSUMP

CONSUMP

CONSUMP

CONSUMP

CONSUMP

CONSUMP

CONSUMP

CONSUMP

CONSUMP

CONSUMD

CONSUMP

CONSUMP

CONSUMP

CONSUMP

CONSUMP

CONSUMO

CONSUMO

CONSUMP

CONSUMP

CONSUMP

CONSUMP

CONSUMP

CONSUMP

CONSUMP

CONSUMP

CONSUMP

CONSUMP

CONSUMP

CONSUMD

CONSUMD

CONSUMP

CONSUMP

CONSUMP

CONSUMP

CONSUMP

CONSUMO

CONSUMP

CONSUMO

CONSUMO

CONSUMP

CONSUMP

CONSUMP

CONSUMP

CONSUMP

CONSUMP

CONSUMP

CONSUMP

CONSUMP

CONSUMP

CONSUMP

CONSUMP

CONSJMP

CONS JMP 


$$
74 / 74 \text { OPT }=1 \quad \text { FTN } 4.5 .414 \quad 06 / 05 / 78 \quad 07.59 .59
$$

CONSUMP 59

CONSJMP 60

CONSUMD 61

CONSUMP 62

CONSUMP 63

CONSUMP 64

CONSUMP 65

CONSUMP 66

CONSUMD 67

CONSUMD 68

CONSUMD 69

BIGCOM

BIGCOM

PI GCOM

BIGCON

BI GCOM

BIGCOM

BIGCOM

BIGCOM

BI GCOM

BIECOM

BIGCOM

RI GCOM

BI SCOM

BI $6 \mathrm{CO}_{4}$

BISCOM

BISCOM

BI SCOM

BIGCOM

BIGCOM

BIGCOM

BI GCOM

BIGCOM

BI GCOM

BI SCOM

BIGCOM

BI GCOM

BISCOM

BIGCOM

BI GCOM

BI GCOM

SUPPLY

SUPPLY

SUPPIY

SUPPLY

SUPPLY

SUPPLY

SUPPLY

SUPPLY

SUPPLY

SIJPPLY

SUPPLY

SUPPLY

SUPPLY

SUPPLY

SUPPLY

SUPPEY 


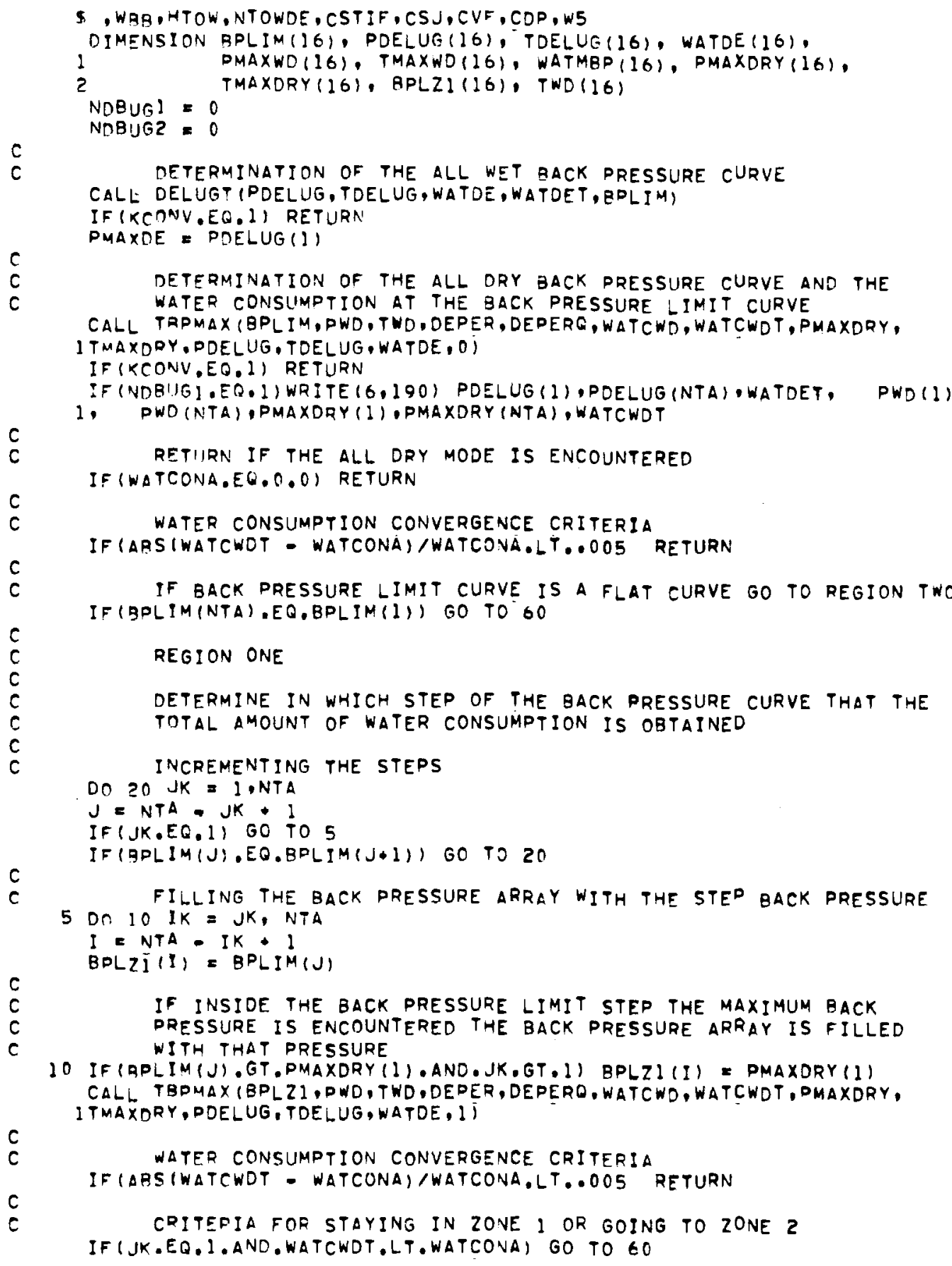

SUPPLY I8

CONSUMP 72

CONSUMP 73

CONSUMD 74

CONSUMP 75

CONSUMP $\quad 76$

CONSUMP 77

CONSUMP $\quad 78$

CONSUMP 79

CONSUMP 80

CONSUMD 81

CONSUMP 82

CONSUMD 83

CONSJMP $\quad 84$

CONSUMP 85

CONSUMP 86

CONSUMP 87

CONSUMP $\quad 88$

CONSUMP $\quad 39$

CONSUMP 90

CONSUMP 91

CONSUMP 92

CONSUMP 93

CONSUMP 94

CONSUMP 95

CONSUMP 96

CONSUMP 97

CONSUMP 98

CONSUMP 99

CONSUMP $\quad 100$

CONSUMP 101

CONSUMP 102

CONSUMD 103

CONSUMP 104

CONSUMP 105

CONSUMP 106

CONSUMP 107

CONSUMP 108

CONSUMP 109

CONSUMP 110

CONSUMP III

CONSUMP 112

CONSUMP 113

CONSUMP $\quad 114$

CONSUMP 215

CONSUMP 116

CONSUMP 117

CONSUMP 118

CONSUMP 119

CONSUMP 120

CONSUMD 121

CONSUMO 122

CONSUMP 123

CONSUMP 124

CONSUMP 125

CONSUMP 126

CONSJUP 127 
:IJ7ROUTINE CONSUMP $\quad 74 / 74 \quad$ OPT $=1 \quad$ FTN $4.5 .414 \quad 06 / 05 / 78 \quad 07.59 .59$

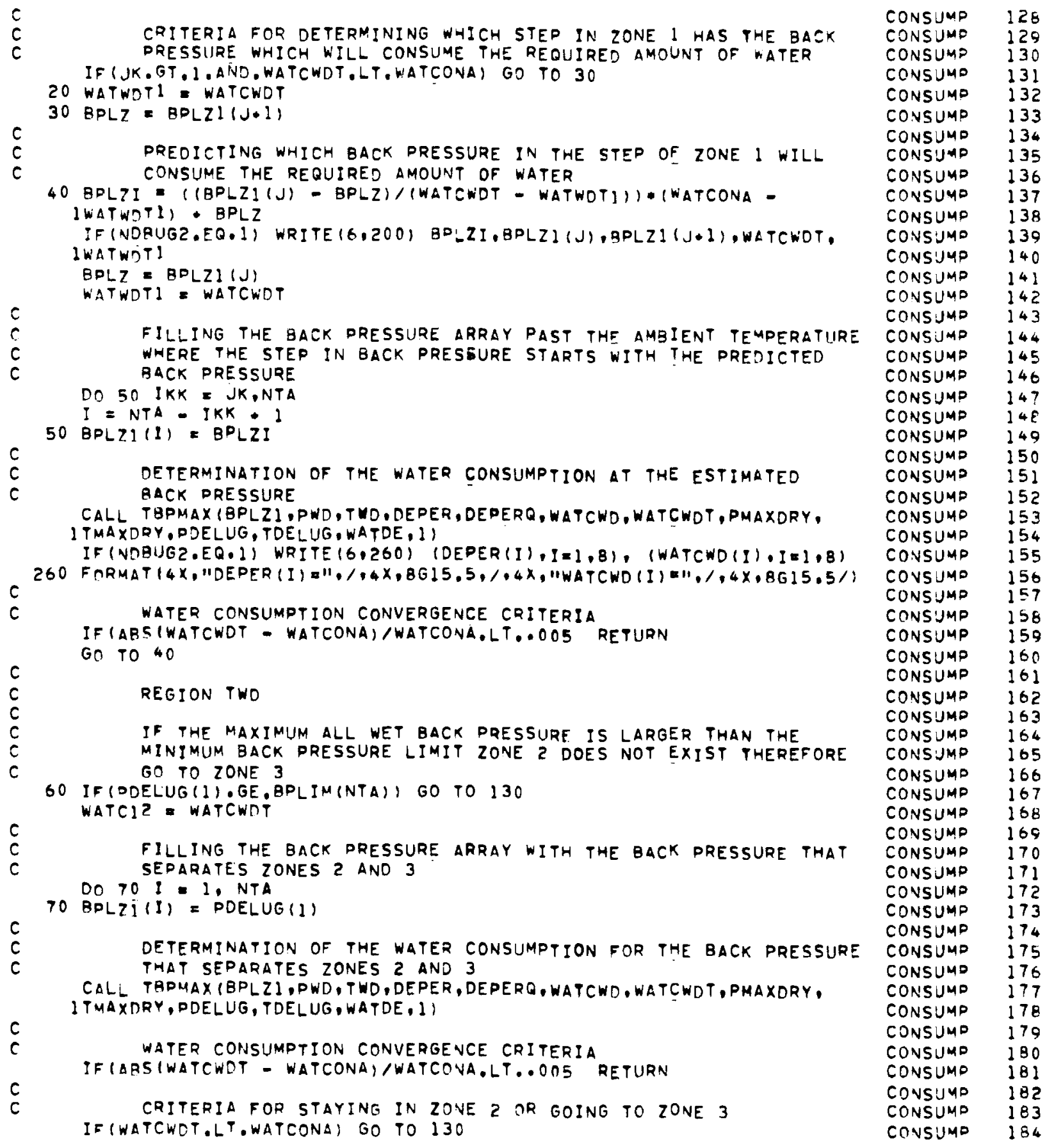




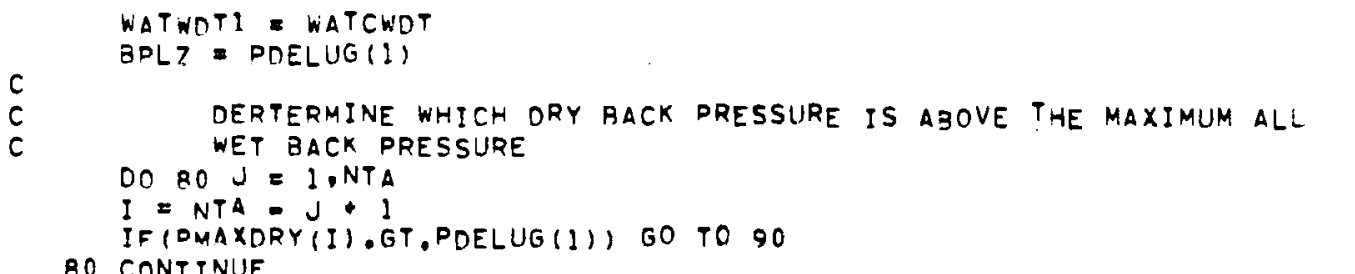

CONSUMP 195

CONSJMD 186

CONSUMP 187

CONSUMP 188

CONSUMP 189

CONSUMP 190

CONSUMD 191

CONSUMP 192

CONSUMP 193

CONSUMP 194

CONSUMP 195

0000100 II = I. NTA

100 BDLZI III) = PMAXORY (I)

BPLZI E DMAXDRY (I)

$c$
$c$
$c$

DETERMINATION OF THE WATER CONSUMPTION FOR THE DRY BACK PRESSURE

CALL TAPMAX (BPLZ), PWD, TWD, DEPER, DEPERQ, WATCWD, WATCWOT, PMAXORY,

ITMAXORY, D JELUG, TOELUG,WATDE, J)

c

$c$

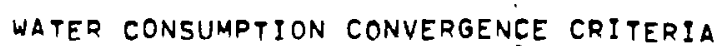

CRITERIA FOR DETERMINING WHICH DRY BACK PRESSURE STEP CONTAINS THE BACK PRESSURE WHICH WILL REQUIRE THE ALLOCATED AMOUNT OF WATER

IF (WATCWDT.LT.WATCONA) GO TO 110

WATWNT I WATCWDT

$c$

BDLZ = BPLZI

C INCREMENTING TO THE NEXT DRY BACK PRESSURE

$I=I=I$

$c$
$c$

SETYING THE WATER CONSUMPTION AND BACK PRESSURE AT THE

UPPPER LIMIT OF ZONE ?

IF (DMAXDQY (I), GT,APLIM(NTA)) WATCWDT = WATCI?

IF (DMAXDRY II).GT.BPLIM(NTA)) BPLZI = BPLIM(NTA)

IF (DMAXDRY (I) , GT.BPLIM(NTA)) GO TO 110

$c$

GO TO 90

C PREDICTING WHICH BACK PRESSURE IN THE ORY BACK PRESSURE STED

WILL CONSUME THE REOUIRED AMOUNT OF WATER

110 BPLZII = (IBPLZI - BPLZ)/WATCWDT - WATWDT $)$ ) (WATCONA - WATWDTI) $1+R P_{L} Z$

IF(NDB!IGZ,EO.1) WRITE(6,2Z0) BDLZII,BPLZI,BPLZ,WATCWDT,WATWDTI

$B D L Z$ a BOLZI

BPLZI = BPLZII

WATWDTI: WATCWDT$$
\begin{aligned}
& c \\
& c \\
& c
\end{aligned}
$$

FILLING THE BACK PRESSURE ARRAY WITH THE PREDICTED BACK PRESSURE $D \cap 120 I I=1, N T A$ c 120 RPLZI II $=$ BPLZII

c

DETERMINATION OF THE WATER CONSUMPTION AT THE ESTIMATED BACK DRESSURE

CALL THPMAXIBPLZI,PWD, YWD.DEPER, DEPERG,WATCWD,WATCWDT,PMAXDPY, CONSJMP

CONSUMD 197

CONSUMP 198

CONSUMP 199

CONSUMP 200

CONSUMP 201

CONSUMP 202

CONSUMP 203

CONSUMP 204

CONSUMP 205

CONSUMP 206

CONSUMP 207

CONSUMP 208

CONSUMP 209

CONSUMP 210

CONSUMD 211

CONSUMP 212

CONSUMP 213

CONSUMP 214

CONSUMP 215

CONSUMP 216

CONSUMP 217

CONSUMD 218

CONSUMP 219

CONSUMP 220

CONSUMP 221

CONSUMP 222

CONSUMP 223

CONSUMP 224

CONSUMP 225

CONSUMP 226

CONSUMP 227

CONSJMP 228

CONSUMP 229

CONSUMP 230

CONSUMP 231

CONSUMP 232

CONSUMD 233

CONSUMP 234

CONSUMP 235

CONSUMP 236

CONSUMP 237

CONSUMP 238

CONSUMP 239

CONSUMD 240 


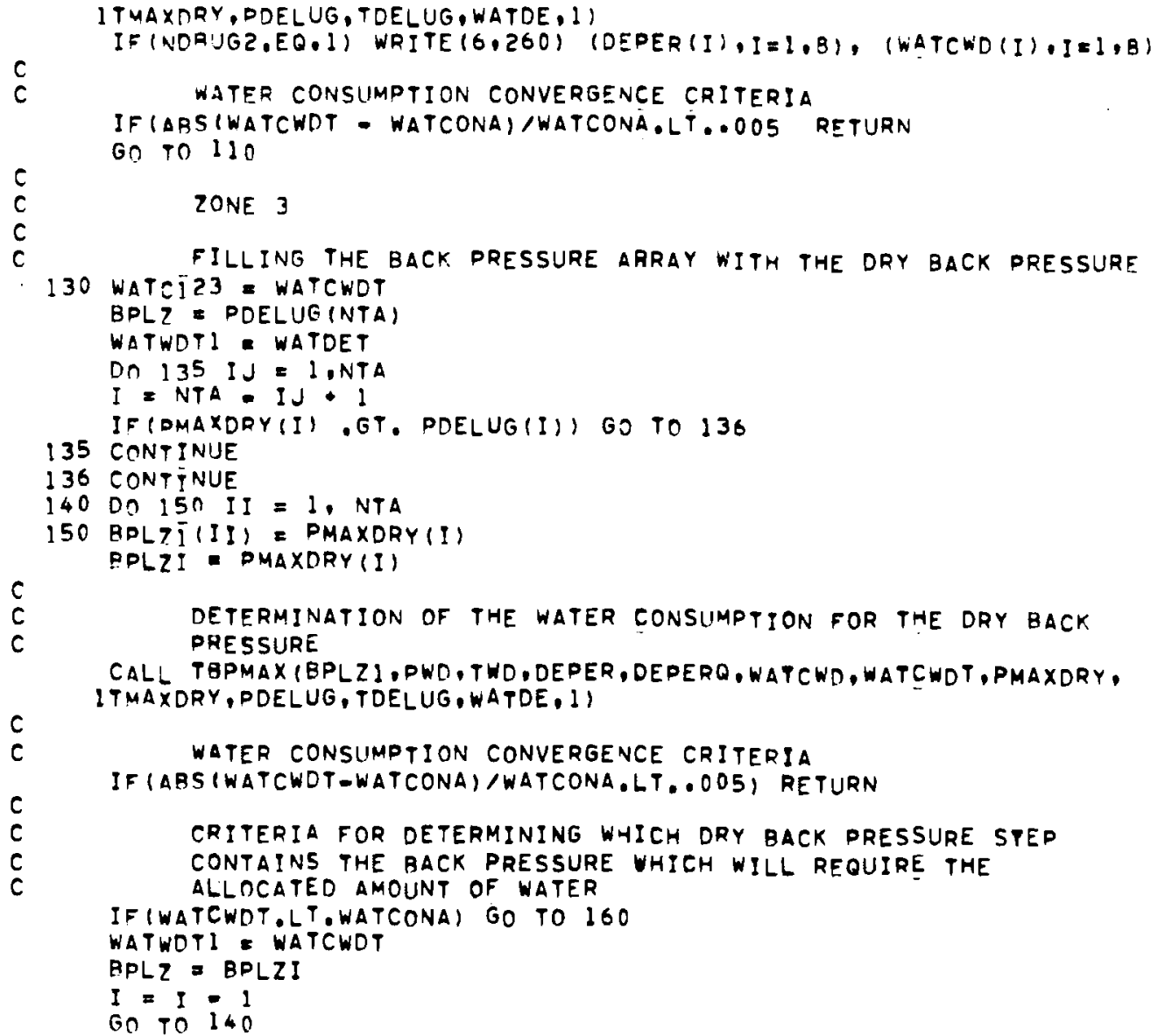

CONSUMP

CONSUMP

CONSUMP

CONSUMP

CONSUMD

CONSUMP

CONSUMO

CONSUMP

CONSUMP

CONSUMP

CONSIJM

CONSUMP

CONSJMP

CONSJMD

CONSUMP

CONSUMP

CONSUMP

CONSUMD

CONSUMP

CONSUMP

CONSUMD

CONSUMP

CONSUMP

CONSUMP

CONSUMP

CONSUMP

CONSUMP

CONSUMP

CONSUMD

CONSUMP

CONSUMP

CONSUMP

CONSUMP

CONSUMP

CONSUMP

CONSUMP

CONSUMP

CONSUMP

CONSUMP

CONSUMP

CONSUMP

CONSUMD

CONSUMP

CONSUMP

CONSUMP

CONSUMP

CONSUMP

CONSUMP

CONSUMP

CONSUMP

CONSUMP

CONSUMP

CONSUMP

CONSUMP

CONSUMP

CONSUMP

CONSUMP 
SUPPOUTINE CONSUMP $\quad 74 / 74 \quad$ OPT=1 FTN $4.5+414 \quad 06 / 05 / 79 \quad 07.59 .59$

\begin{tabular}{|c|c|c|c|}
\hline & IF (NDPUG2,EO.1) WRITE $(6,260)$ (DEPER(I),I=1,8), (WATCWD $(I), I=1,8)$ & CONSUMD & 299 \\
\hline & $\begin{array}{l}\text { WATER CONSUMPTION CONVERGENCE CRITERIA } \\
\text { IF (ARSIWATCWDT - WATCONA)/WATCONA.LT.0OO RETURN } \\
\text { GO TO I6O }\end{array}$ & $\begin{array}{l}\text { CONSUMP } \\
\text { CONSUMP } \\
\text { CONSUMP } \\
\text { CONSUMP } \\
\text { CONSUMP }\end{array}$ & $\begin{array}{l}300 \\
301 \\
302 \\
303\end{array}$ \\
\hline $\begin{array}{l}190 \\
200 \\
220 \\
240\end{array}$ & 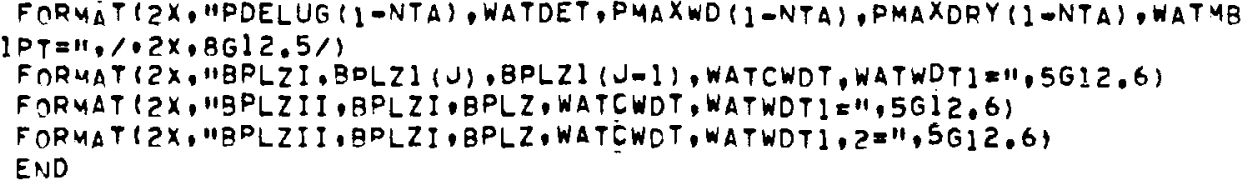 & $\begin{array}{l}\text { CONSUMP } \\
\text { CONSUMP } \\
\text { CONSUMP } \\
\text { CONSUMP } \\
\text { CONSUMP } \\
\text { CONSUMD }\end{array}$ & $\begin{array}{l}304 \\
305 \\
306 \\
307 \\
308 \\
309\end{array}$ \\
\hline
\end{tabular}

\footnotetext{
SEVERITY DETAILS DIAGNOSIS OF PROBLEM

I BPLIM PREVIOUSLY DIMENSIONED ARRAY, FIRST DIMENSIONS WILL BE RETAIVED.
} 
$74 / 74 \quad$ OPT $=1$

FTN $4.5+414$

$06 / 05 / 78$

07.59 .59

SUBROUTINE COSTEX (AFRON.ATOT)

COSTEX

COSTEX

SURROUTINE COSTEX DETERMINES THE COST OF THE HEAT EXCHANGER

COSTEX

RUNDLES OF THE DRY COOLING TOWERS. THE COST OF THE HEAT

EXCHANGER BUNDLE IS BROKEN DOWN INTO SEVERAL SUBCOMPONENTS

1. HEADER

3. TUBING SEALANT

4. SPACERS

5. BUNDLE FRAME

6. BUNDLE ASSEMBLY

PHE WEIGHT OF THE HEAT EXCHANGER BUNDLE AND THE WATER ARE ALSO CALCULATED.

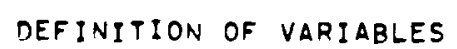

$\operatorname{costEx}$

COSTEX

COSTEX

COSTEX

COSTEX

COSTEX

COSTEX

COSTEX

COSTEX

COSTEX

COSTEX

COSTEX

COSTEX

COSTEX

COSTEX

COSTEX

COSTEX

COSTEX

COSTEX

COSTEX

COSTEX

COSTEX

COSTEX

COstex

$\cos T E x$

COSTEX

COSTEX

COSTEX

COSTEX

COSTEX

COSTEX

COSTEX

COSTEX

COSTEX

COSTEX

COSTEX

COSTEX

Costex

COSTEX

COSTEX

COSTEX

COSTEX

COSTEX

COSTEX

COSTEX

COSTEX

COSTEX

COSTEX

COSTEX

COSTEX

COSTEX

COSTEX

COSTEX

COSTEX 
$74 / 74 \quad O D T=1$

FTN $4.5 \cdot 414$

$06 / 05 / 78$

07.59 .59
COSTEX

COSTEX

COSTEX

COSTEX

COSTEX

COSTEX

COSTEX

COSTEX

COSTEX

COSTEX

COSTEX

COSTEX

COSTEX

COSTEX

COSTEX

COSTEX

COSTEX

COSTEX

COSTEX

COSTEX

COSTEX

COSTEX

COSTEX

COSTEX

COSTEX

COSTEX

COSTEX

COSTEX

COSTEX

COSTEX

COSTEX

COSTEX

COSTEX

COSTEX

COSTEX

COSTEX

COSTEX

COSTEX

COSTEX

COSTEX

COSTEX

COSTEX

COSTEX

COSTEX

COSTEX

COSTEX

COSTEX

COSTEX

COSTEX

COSTEX

COSTEX

COSTEX

COSTEX

COSTEX

COSTEX

COSTEX

COSTEX
59

60

61

62

63

64

65

66

67

68

69

70

71

72

73

74

75

76

77

78

79

80

81

82

83

84

85

86

86

89

90

91

92

93

94

95

96

97

98

99

100

101

102

103

104

105

106

107

108

109

110

111

112

113

114

115 


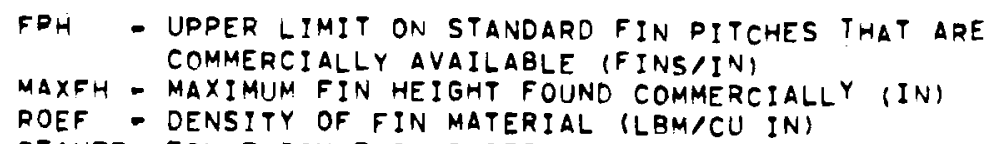

COSTEX

COSTEX

COSTEX

COSTEX

COSTEX

COSTEX

COSTEX

REAL NTUB

COSTEX

COSTEX

BIGCOM

LOGICAL FIRST,FRST, FXTEMP,FXTVAR,FXTTTD,FXTLNG

BIGCOM COMUNN

5 AETR, ALPHA, ANG(3)

* BPLIM (16)

\$ CADF. CSSPKW, CONF, CONL, COSTL, CAPCHG, CONMAT, CONMAZ,CAF

BIGCOM

BIGCOM

BIGCOM

BIGCOM

$\$$. DFIN.DEEPL, DESVEL, DESVELV, OESVELD, DENSIF

\$. EFFP, EFS

S ,FCR,FIRST,FIXL, FCOS ,FFHX, FXTEMP, FACTOR, FRST, FACTORD, FSMOT, FDELUG

$\$$, FIXV,FIXTTD,FXTVAR,FXTTTD,FXTLNG

$S$ GQEFF

S HXNP, HPCST, HCD

S. ITMAX, ITMIN

5 . JCONS

SCONV, KALEXT

S. MXEXT

\$.NTA,NSIDES, NBUNHI COMMON

$S O D R$

5. PSIZE, PER, PWCOS, PLANC, PFACT, POMDPL

5 . POHBAF, POHFAN, POHLEC, POHCIR, POHCND. POHSTC, POHSCl

5 : RQD, REDUCE, REDUCV, ROOFL, RHOUT

5 - QREJ, QREDUCE, QREDUCV

S. SIGMAG, SAAF, SEgL

\$, TD, PPO(4), TEFF, TLIM, THFIN, TLPRA, TFIX

\$.TED. TW

SUES, UWS

S WFV. WATCONA, WATCOST

5 , VAS (5)

S, XDEPA, XW, XD

COMMON /SUPPLY, VAL (5), CPLN, CPIP, CCOS, W3, COSM, EFF,

BIGCOM

BIGCOM

BIGCOM

BIGCOM

BISCOM

BIGCOM

BIGCOM

BIGCOM

BIGCOM

BIGCOM

BIGCOM

BIGCOM

BIGCOM

8I GCOM

BIGCOM

BI GCOM

BIGCOM

BI GCOM

BIGCOM

BI GCOM

BIGCOM

BIGCOM

BIGCOM

BIGCOM 


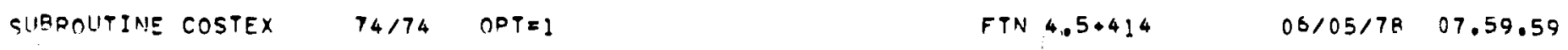

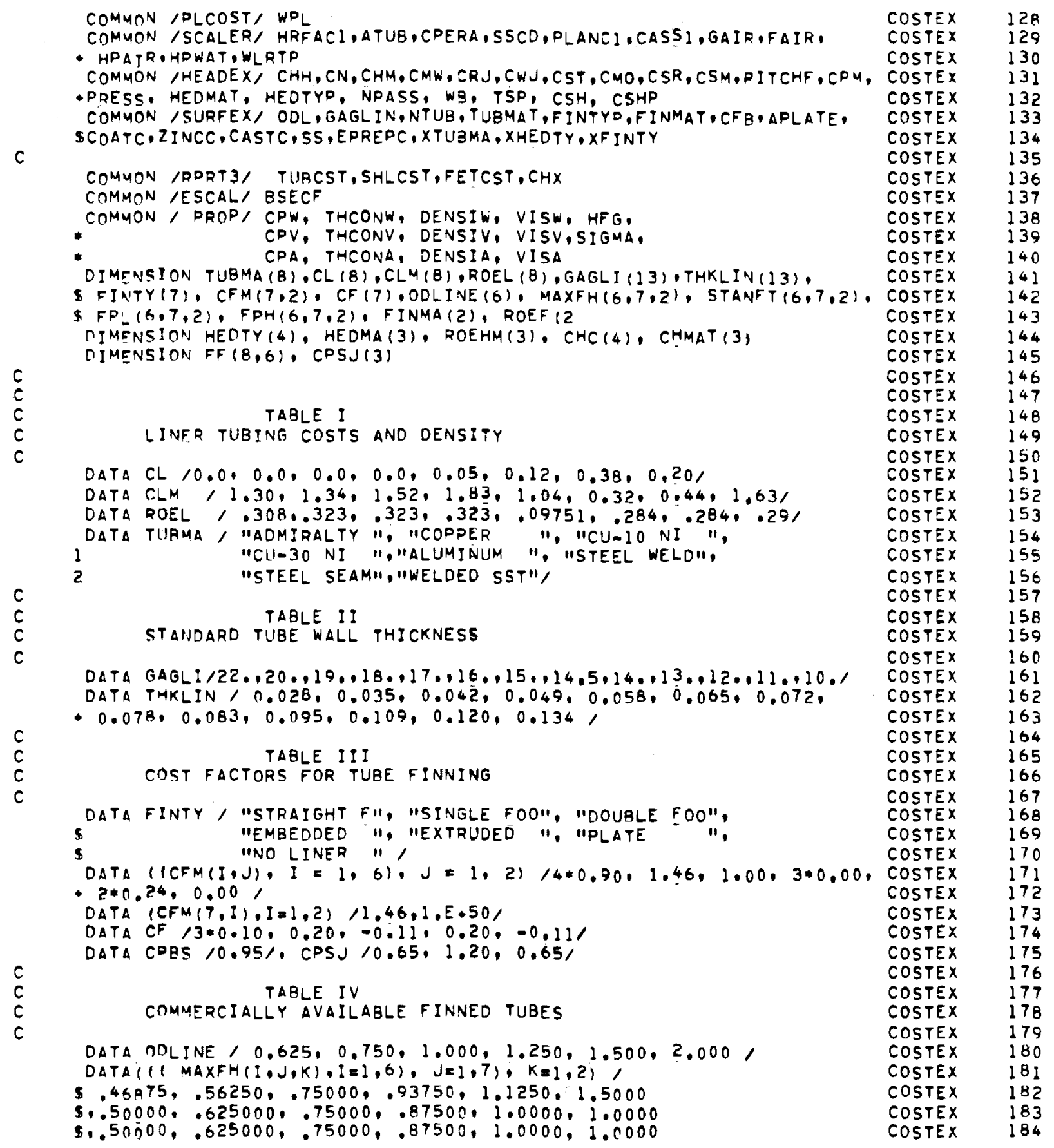




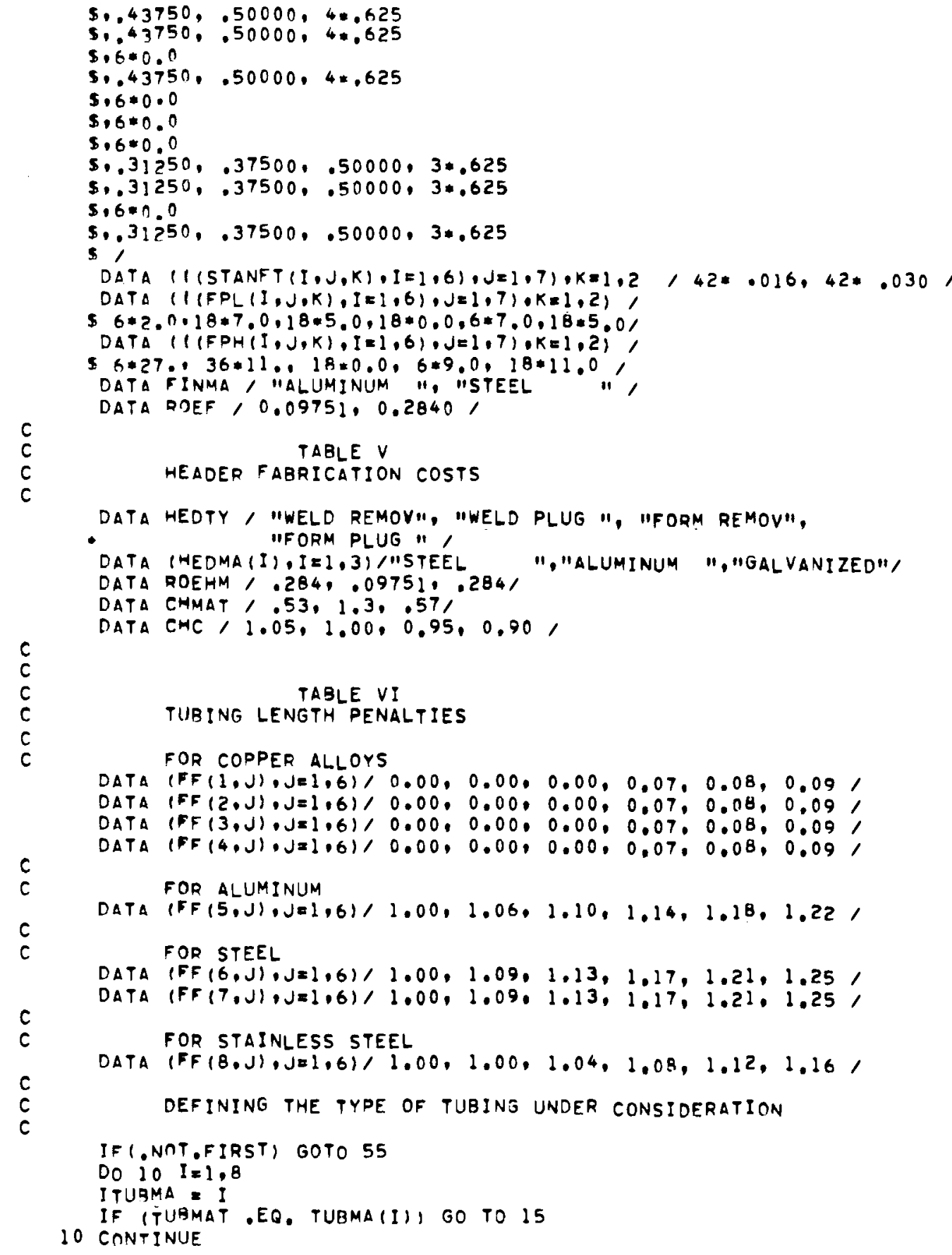

\begin{tabular}{|c|c|}
\hline $\begin{array}{l}\text { COSTEX } \\
\text { COSTEX } \\
\text { COSTEX } \\
\text { COSTEX }\end{array}$ & $\begin{array}{l}185 \\
186 \\
187 \\
188\end{array}$ \\
\hline $\begin{array}{l}\text { COSTEX } \\
\text { COSTEX }\end{array}$ & $\begin{array}{l}189 \\
190\end{array}$ \\
\hline $\begin{array}{l}\text { COSTEX } \\
\text { COSTEX }\end{array}$ & $\begin{array}{l}191 \\
192\end{array}$ \\
\hline COSTEX & 193 \\
\hline COSTEX & 194 \\
\hline COSTEX & 195 \\
\hline COSTEX & 196 \\
\hline COSTEX & 197 \\
\hline COSTEX & 198 \\
\hline COSTEX & 199 \\
\hline COSTEX & 200 \\
\hline COSTEX & 201 \\
\hline COSTEX & 202 \\
\hline COSTEX & 203 \\
\hline COSTEX & 204 \\
\hline COSTEX & 205 \\
\hline COSTEX & 206 \\
\hline COSTEX & 207 \\
\hline COSTEX & 208 \\
\hline COSTEX & 209 \\
\hline COSTEX & 210 \\
\hline COSTEX & 211 \\
\hline COSTEX & 212 \\
\hline COSTEX & 213 \\
\hline COSTEX & 214 \\
\hline COSTEX & 215 \\
\hline COSTEX & 216 \\
\hline COSTE & 211 \\
\hline COSTEX & 219 \\
\hline COSTEX & 220 \\
\hline COSTEX & 221 \\
\hline COSTEX & 222 \\
\hline COSTEX & 223 \\
\hline COSTEX & 224 \\
\hline COSTEX & 225 \\
\hline $\begin{array}{l}\text { COSTEX } \\
\text { COSTEX }\end{array}$ & \\
\hline COSTEX & 228 \\
\hline COSTEX & \\
\hline COSTEX & 230 \\
\hline COSTEX & 231 \\
\hline COSTEX & 232 \\
\hline COSTEX & 233 \\
\hline COSTEX & 234 \\
\hline COSTEX & 235 \\
\hline COSTEX & 236 \\
\hline COSTEX & 237 \\
\hline COSTEX & 238 \\
\hline COSTEX & 239 \\
\hline COSTEX & 240 \\
\hline COSTEX & $24 !$ \\
\hline
\end{tabular}




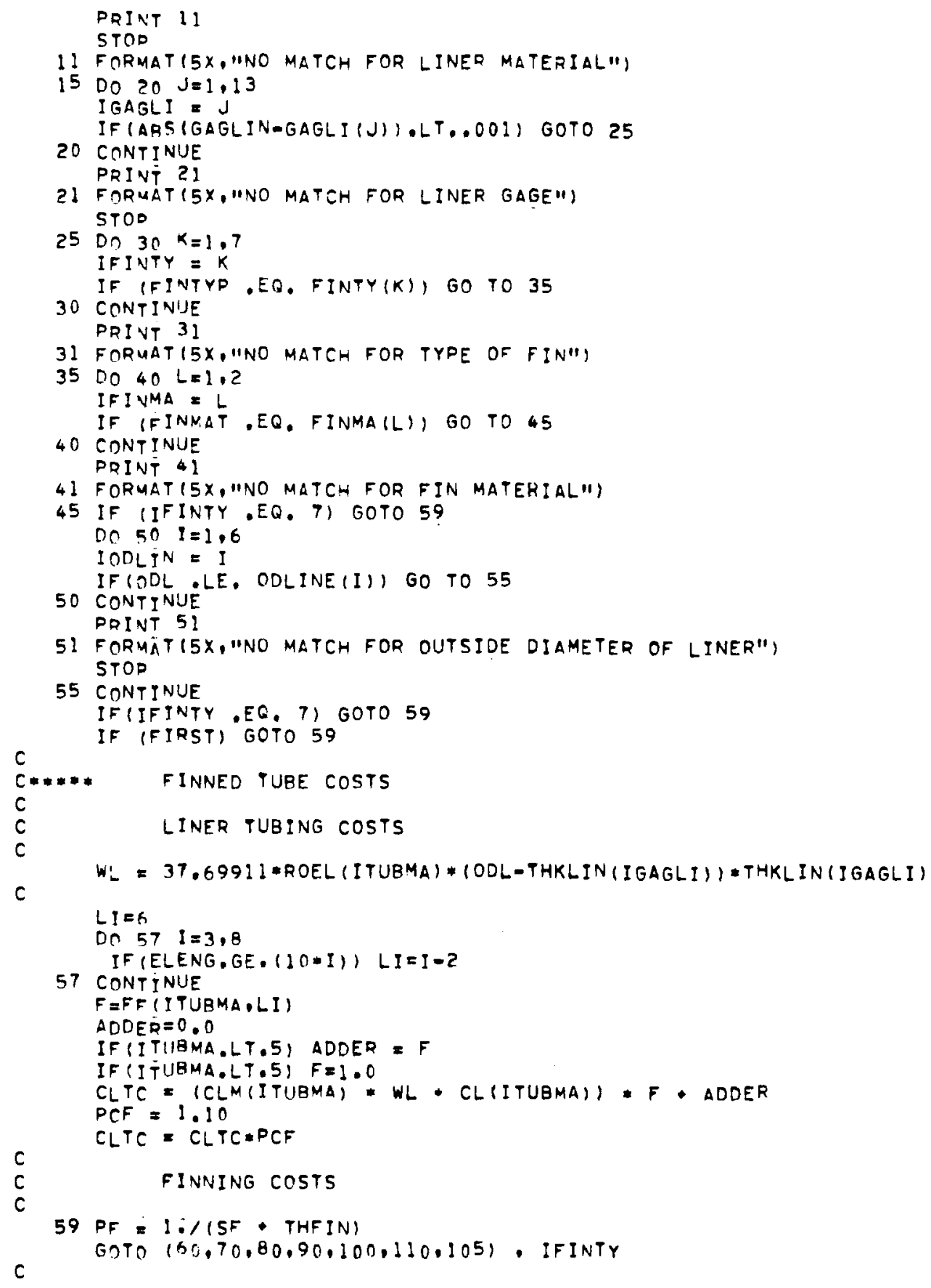

$\cos T E x$

COSTEX

$\cos 5 x$

$\cos T E x$

COSTEX

COSTEX

COSTEX

COSTEX

COSTEX

COSTEX

COSTEX

COSTEX

COSTEX

COSTEX

COSTEX

COSTEX

COSTEX

COSTEX

COSTEX

COSTEX

COSTEX

COSTEX

COSTEX

COSTEX

COSTEX

COSTEX

COSTEX

COSTEX

COSTEX

COSTEX

COSTEX

COSTEX

COSTEX

COSTEX

COSTEX

COSTEX

COSTEX

COSTEX

COSTEX

COSTEX

COSTEX

COSTEX

COSTEX

COSTEX

COSTEX

COSTEX

COSTEX

COSTEX

COSTEX

COSTEX

COSTEX

COSTEX

COSTEX

COSTEX

COSTEX

COSTEX

COSTEX
242

243

244

245

246

247

248

249

250

251

252

253

254

255

256

257

258

259

260

261

262

263

264

265

266

267

268

269

270

271

272

273

274

275

276

277

278

279

280

28!

252

283

284

285

286

287

288

289

290

291

292

293

294

295

296

297
298 


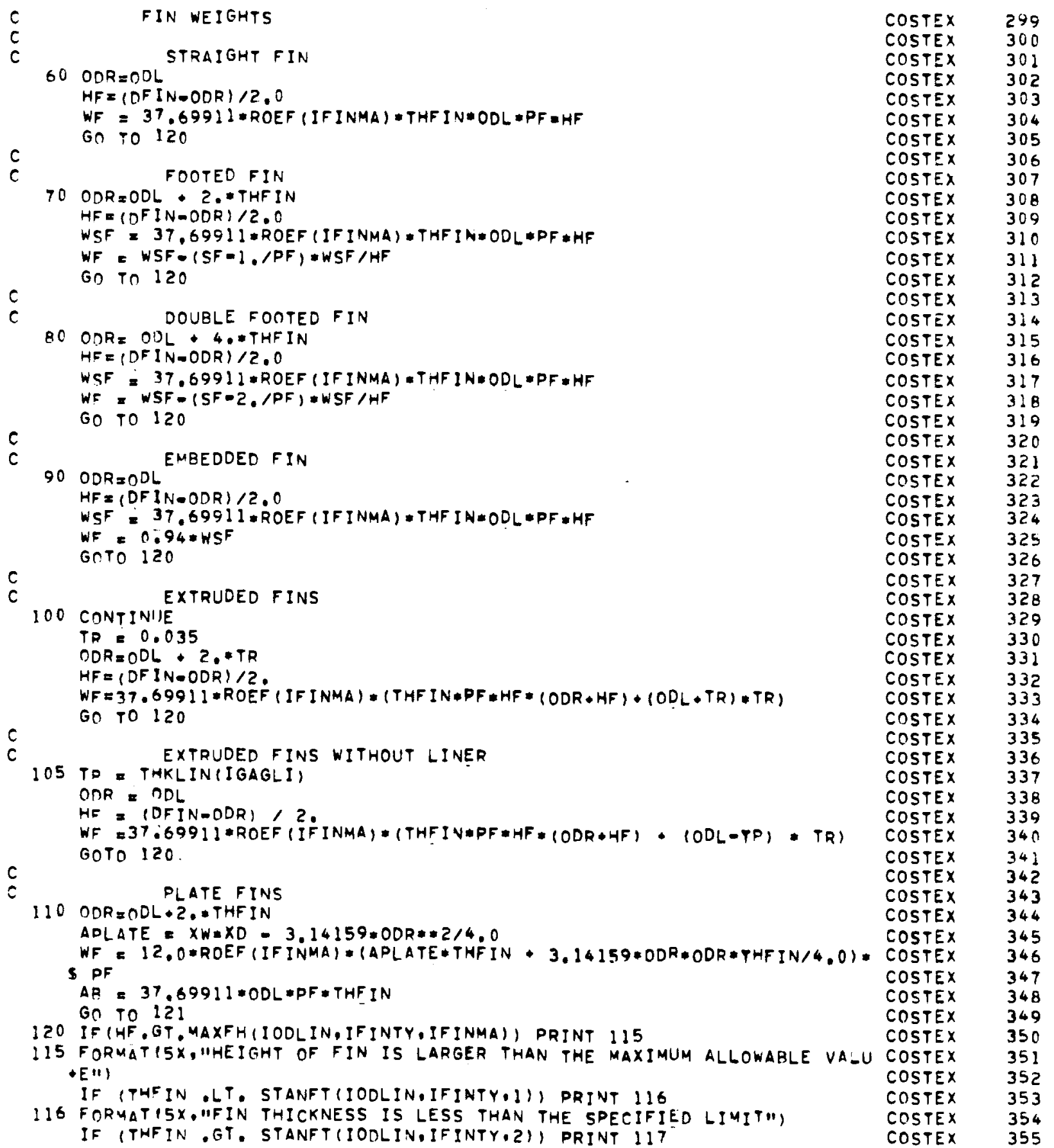


SIIBROUTINE COSTEX T4,74 OPT=1 FTN $4.5+414 \quad 06 / 05 / 78 \quad 07.59 .59$

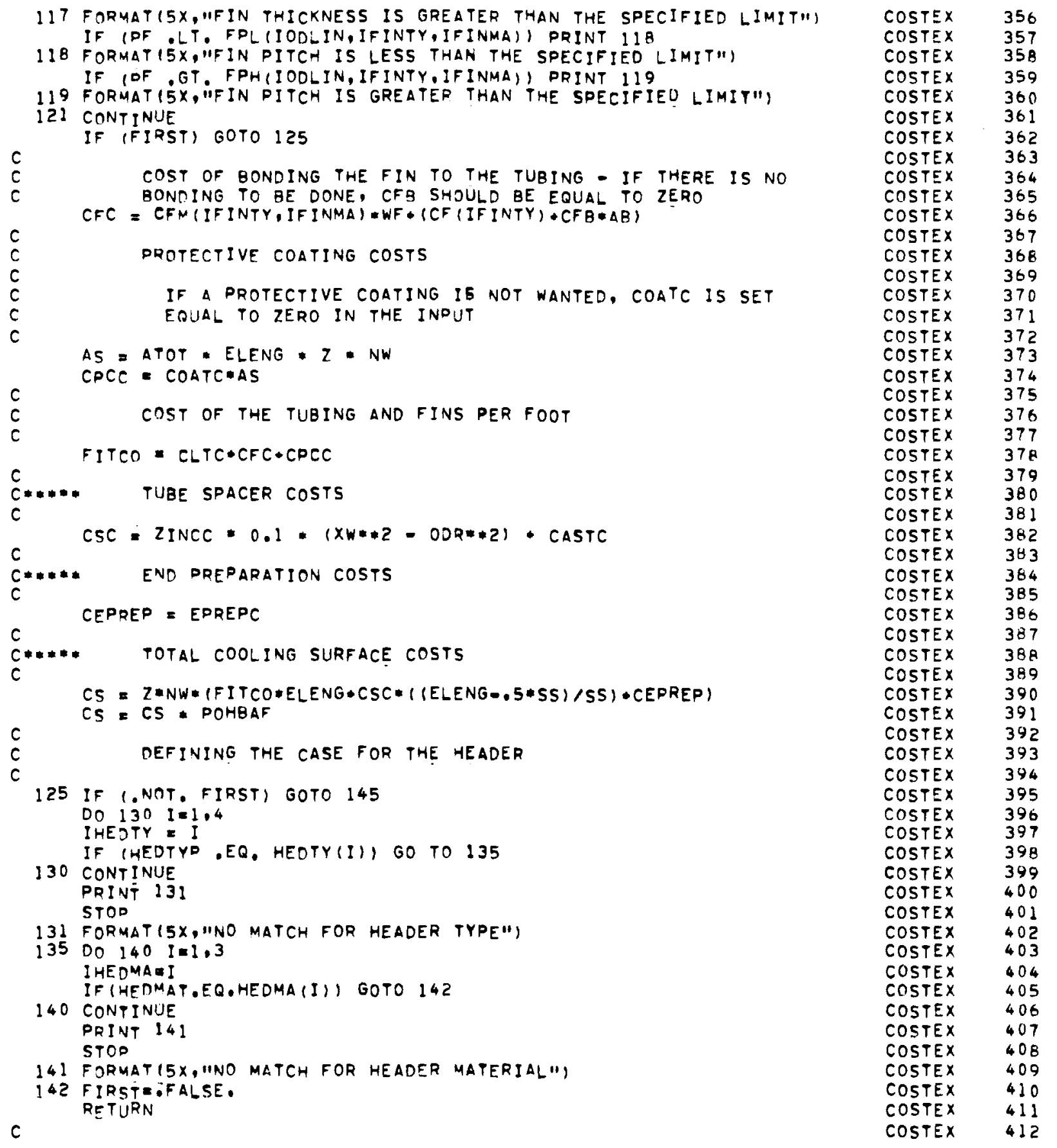




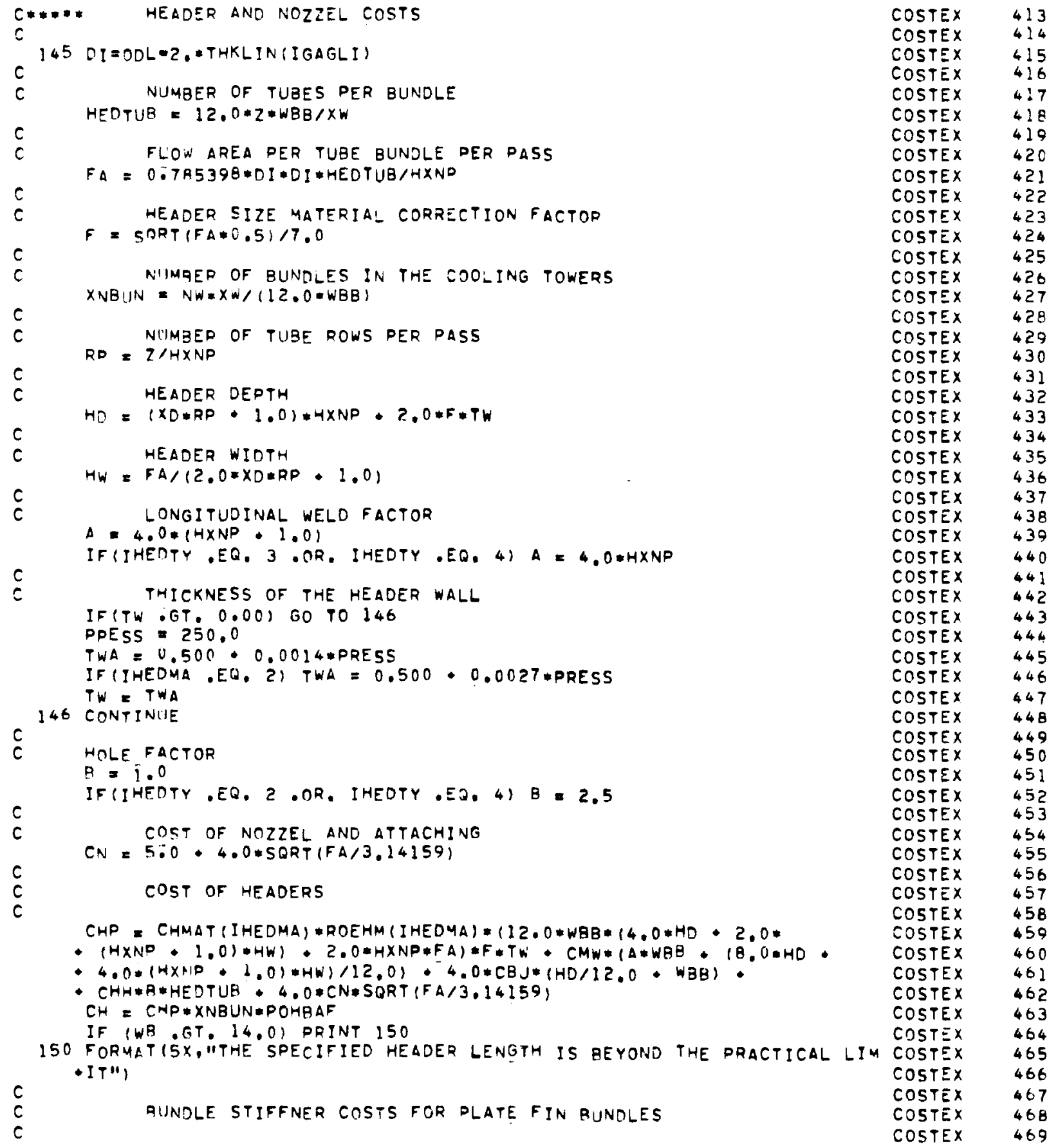

COSTEX 413

COSTEX $\quad 414$

COSTEX 415

COSTEX $\quad 416$

COSTEX $4: 7$

COSTEX 418

COSTEX 410

COSTEX 420

COSTEX 42I

COSTEX 422

COSTEX 423

COSTEX 424

COSTEX $\quad 425$

COSTEX 426

COSTEX 427

COSTEX 428

COSTEX 429

COSTEX 430

COSTEX 431

COSTEX 432

COSTEX 433

COSTEX 434

COSTEX 435

COSTEX 436

COSTEX 437

COSTEX 438

COSTEX 439

COSTEX 440

COSTEX 441

COSTEX 442

COSTEX 443

COSTEX 444

COSTEX 445

COSTEX 446

COSTEX 447

COSTEX 448

COSTEX 440

COSTEX 450

COSTEX 451

COSTEX 452

COSTEX 453

COSTEX 454

COSTEX 455

COSTEX 456

COSTEX $\quad 457$

COSTEX 458

COSTEX 450

COSTEX 460

COSTEX 46I

COSTEX 462

COSTEX 463

COSTEX 464

464
465

466

467

468

469 
FTN $4.5+414$

$06 / 05 / 78$

07.59 .59

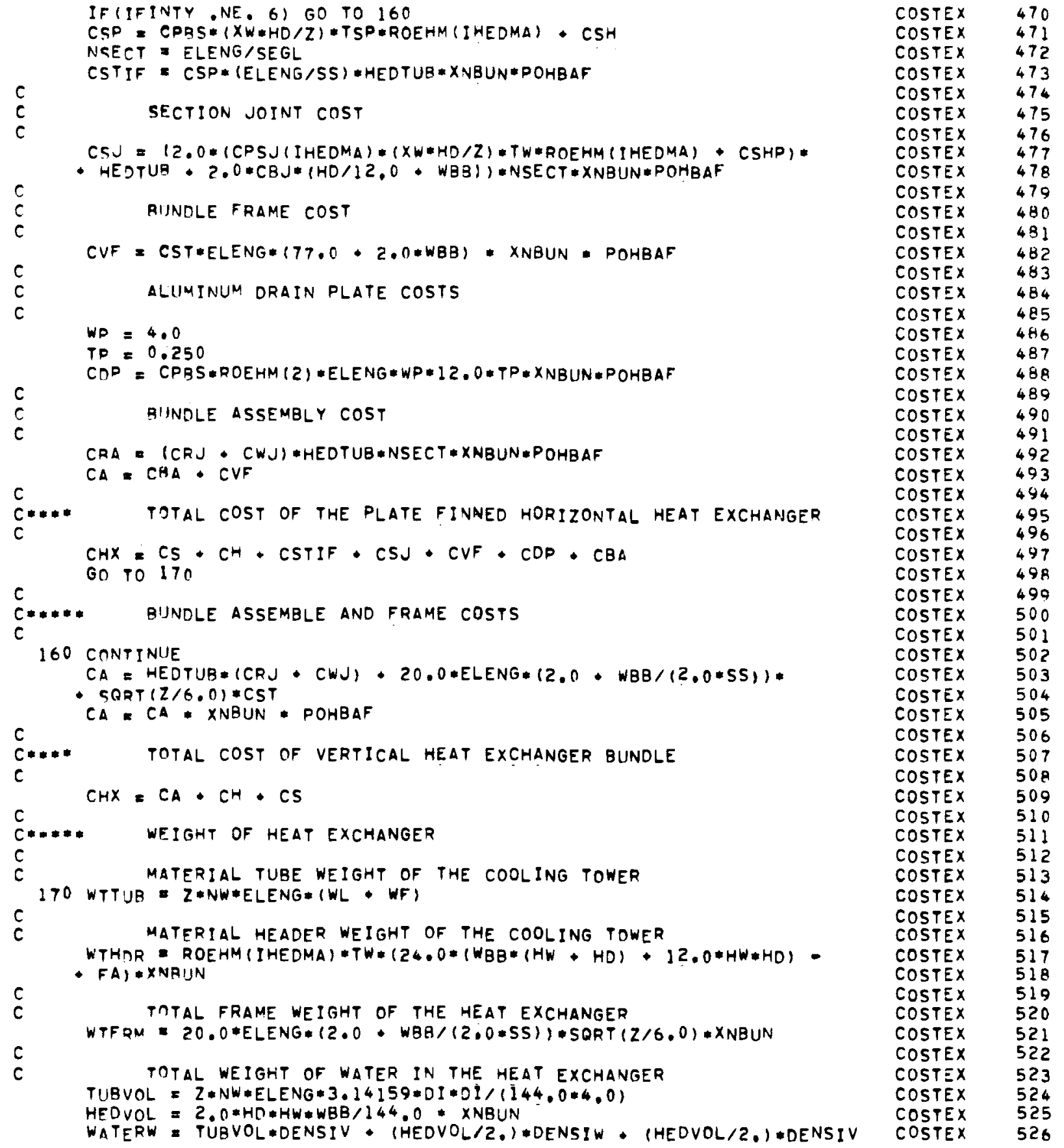




SURROUTINE COSTEX $74 / 74$ OPTEI
\[ \begin{array}{c}C \\ C \\ C\end{array} \begin{array}{l}\text { RETUR }=\text { WTTUT OF THE HEAT EXCHANGER } \\ \text { END }\end{array} \]

FTN 4.5 .414

END
$06 / 05 / 78 \quad 07.59 .59$

Cost5x 527

COSTEX 528

COSTEX 529

COSTEX 530

COSTEX 531

COSTEX 532 
$74 / 74 \quad$ OPT $=1$

SURROUTINE DELBAW CALCUALTES THE HEAT TRANSFER AND THE WATER

DELBAW

EVAPORATED FOR A DELUGEO HEAT EXCHANGER, THE B ANDW COMPUTATIONAL METHOD IS USED. THE DRIVING FORCE

USED TO CALCULATE THE HEAT PRANSFER IS THE ENTHALPY (BTU/LB OF DRY AIR) AS IS COMMONLY USED IN WET TOWER DESIGN METHODS, ALL CONDUCTIVE AND CONVECTIVE TERMS ARE APPROPRIATELY TRANSFORMED FROM A LINEAR TEMPERATURE DEPENDENCE TO A ENTHALPIC DEPENDENCE.

SUBROUTINE DELBAW OBTAINS THE HEAT TRANSFER BY TAKING THE AVERAGE OF THE NUMBER OF TRANSFER UNITS AT THE LEADING EDGE AND AT THE TRAILING EOGE OF THE HEAT EXCHANGER AND CALCULATING A HEAT EXCHANGER EFFECTIVENESS. THIS EFFECTIVENESS IS THEN USED TO DETERMINE THE HEAT TRANSFERRED AND THE OUTLET TEMPERATURE OF THE AIR. THE EVAPORATION RATE OF WATER IS OBTAINED FROM THE CHANGE IN ABSOLUTE HUMIDITY OF THE AIR AND FROM THE MASS FLOWRATE OF THE AIR.

THE COMPUTATION OF THE EXIT CONDITIONS OF THE AIR EXITING THE HEAT EXCHANGER IS CONDUCTED BY SUCCESSIVE APPROXIMATIONS.

A NOMENCLATURE OF SOME OF THE MORE FREQUENTLY USED TERMS FOLLOWS.

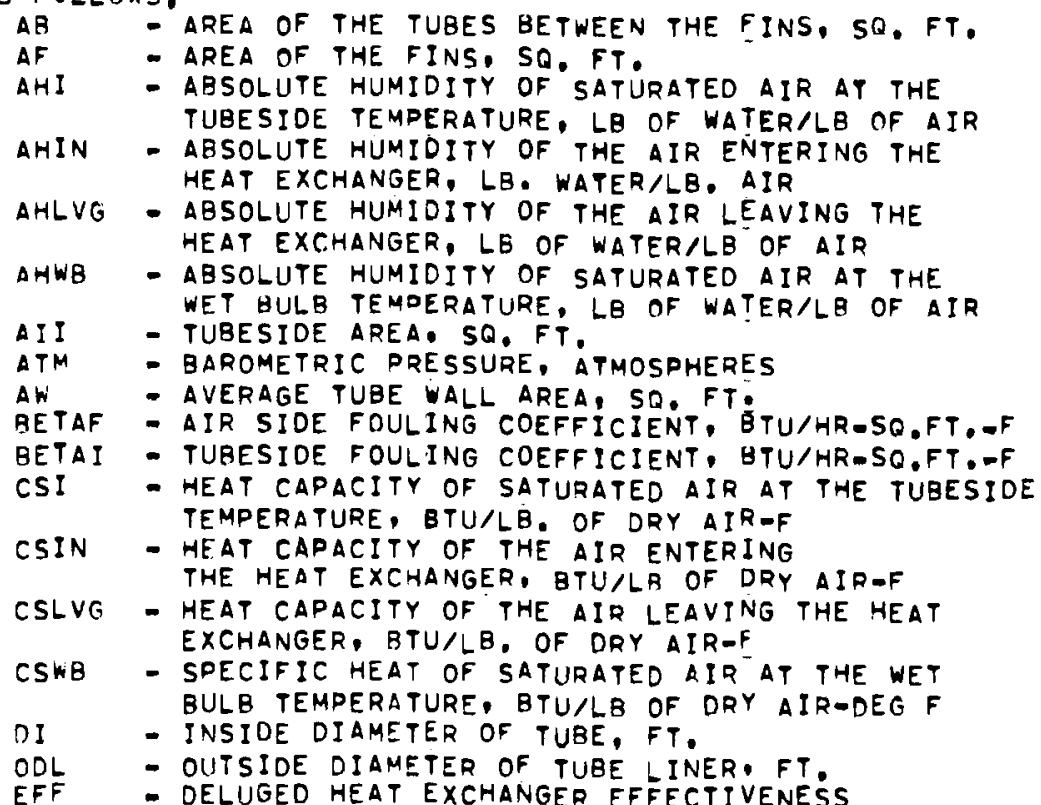




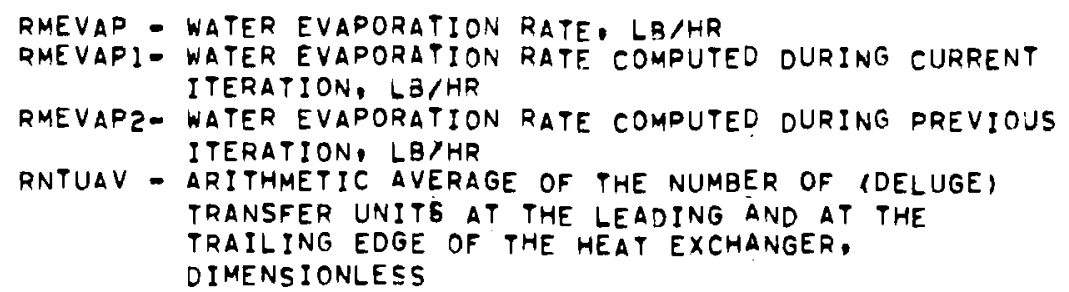

RNTUIN - NUMBER OF (DELUGE) TRANSFER UNITS AT THE LEADING EDGE OF THE HEAT EXCHANGER, DIMENSIONLESS

RNTULV - NUMBER OF (DELUGE) TRANSFER UNITS AT THE TRAILING EDGE OF THE HEAT EXCHANGER. DIMENSIONLESS

SIGIN - TUBESIDE TO AIR MASS TRANSFER COEFFICIENT EVALUATED FOR CONDITIONS AT THE LEADING EDGE OF THE HEAT EXCHANGER, LB MASS/SO.FT. OHR

SIGLVG - TUBESIDE TO AIR MASS TRANSFER COEFFICIENT EVALUATED FOR CONDITIONS AT THE TRAILING EDGE OF THE HEAT EXCHANGER. LB. OF DRY AIR/SQ,FT FT-HR

SIGMA - MASS TRANSFER COEFFICIENT BETWEEN THE DELUGEATE AND THE AIR, LB. OF DRY AIR/SO.FT.-HR.

T - TEMPERATURE, DEG, F.

TAIN - TEMPERATURE OF THE AIR ENTERING THE HEAT EXCHANGER, F

TAL - TEMPERATURE OF THE AIR LEAVING THE HEAT EXCHANGER, F

TEST - ESTIMATE OF TEMPERATURE USED AS AN A FIRST APPROXIMATION BY SUBROUTINE TCALC

THW - TUBE WALL THICKNESS,FT.

TI - TUBESIDE TEMPERATURE

TMAX - MAXIMUM TEMPERATURE ALLOWED DUE TO CONSTRAINTS UPON THE ANALOGY UPON WHICH THE HEAT TRANSFER CALCULATION IS BASED,F

TMIN - MINIMUM TEMPERATURE ALLOWED TO PREVENT POSSIBLE INSTABILITIES WITHIN THE SUBROUTINES, F

TOUT - TEMPERATURE OF AIR LEAVING THE HEAT EXCHANGER, DEG, F

TWBIN - WET BULB TEMPERATURE OF THE AIR ENTERING THE HEAT EXCHANGER, DEG, F

LOGICAL' FRSTI, FRST2, FRST3

LOGICAL FIRST,FRST, FXTEMP, FXTVAR,FXTTTD,FXTLNG COMMON

5 AFTR. $\triangle L P H A, ~ A N G(3)$

- Bplim(16)

5. CAPF. CSSPKH, CONF, CONL, COSTL, CAPCHG, CONMAT, CONMA?,CAF

, CBJ. CTURB, CDANG, CLUVR, CHAILS, CVM

\$ DEIN.DFEPL. DESVEL.DESVELY, DESVELD. DENSIF

S.EFFD, EFS

S. FCD,FIRST,FIXL,FCOS,FFHX,FXTEMP, FACTOR, FRST, FACTORD,FSHOT, FDELJG

S. FIXV,FIXTTD,FXTVAR,FXTTTD,FXTLNG

S. GPEFF

\$ HXNP,HPCST,HCD

ITMAX, ITMIN

DELBAW

DELBAW

DELBAW

DELBAW

DELBAW

DELBAW

DELBAW

DELBAW

DELBAW

DELBAW

DELBAW

DELBAW

DELBAW

DELBAW

DELBAW

DELBAW

DELBAW

DELBAW

DELBAW

DELBAW

DELBAW

DELBAW

116

117

118

119

120

121

122

123

124

125

126

127

128

129

130

131

132

133

134

135

136

137

138

139

140

141

142

143

144

145

146

147

148

149

150

151

152

153

154

155

156

157

158

DELBAW 159

AI GCOM

BI GCOM

BIGCOM

BI GCOM

BI SCOM

BI GCOM

BI GCOM

BI GCOM

BI GCOM

BI GCOM

BIGCOM

BI GCOM 


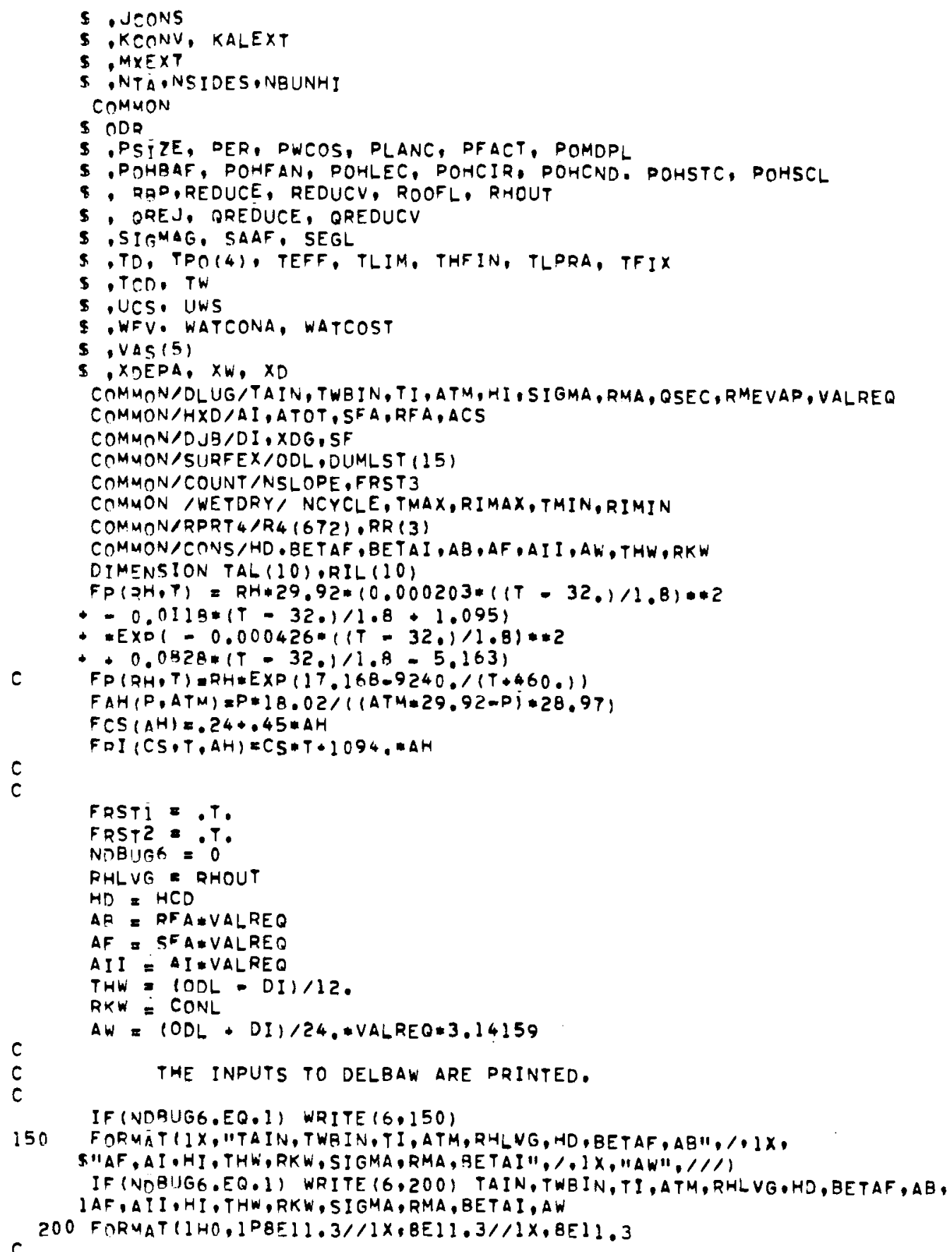


THE NUMBER OF HEAT TRANSFER UNITS (BASED ON ENTHALPY ORIVING FORCE) BASED ON CONDITIONS AT THE LEADING EOGE ARE COMPUTED.

DELBAW

DELBAW

DELBAW

DELBAW

DELBAW

DELBAW

DELBAW

DELBAW

DELBAW

DELBAW

DELBAW

DELBAW

DELBAW

DELBAW

DELBAW

DELBAW

DELBAW

DELBAW

DELBAW

DELBAW

DELBAW

DELBAW

DELBAW

DELBAW

DELBAW

DELBAW

DELBAW

DELBAW

DELBAW

DELBAW 
SURROUTINE DELBAW $\quad \mathbf{7 4 / 7 4}$ OPTE

FTN $4.5+414$

$06 / 05 / 78 \quad 07.59 .59$

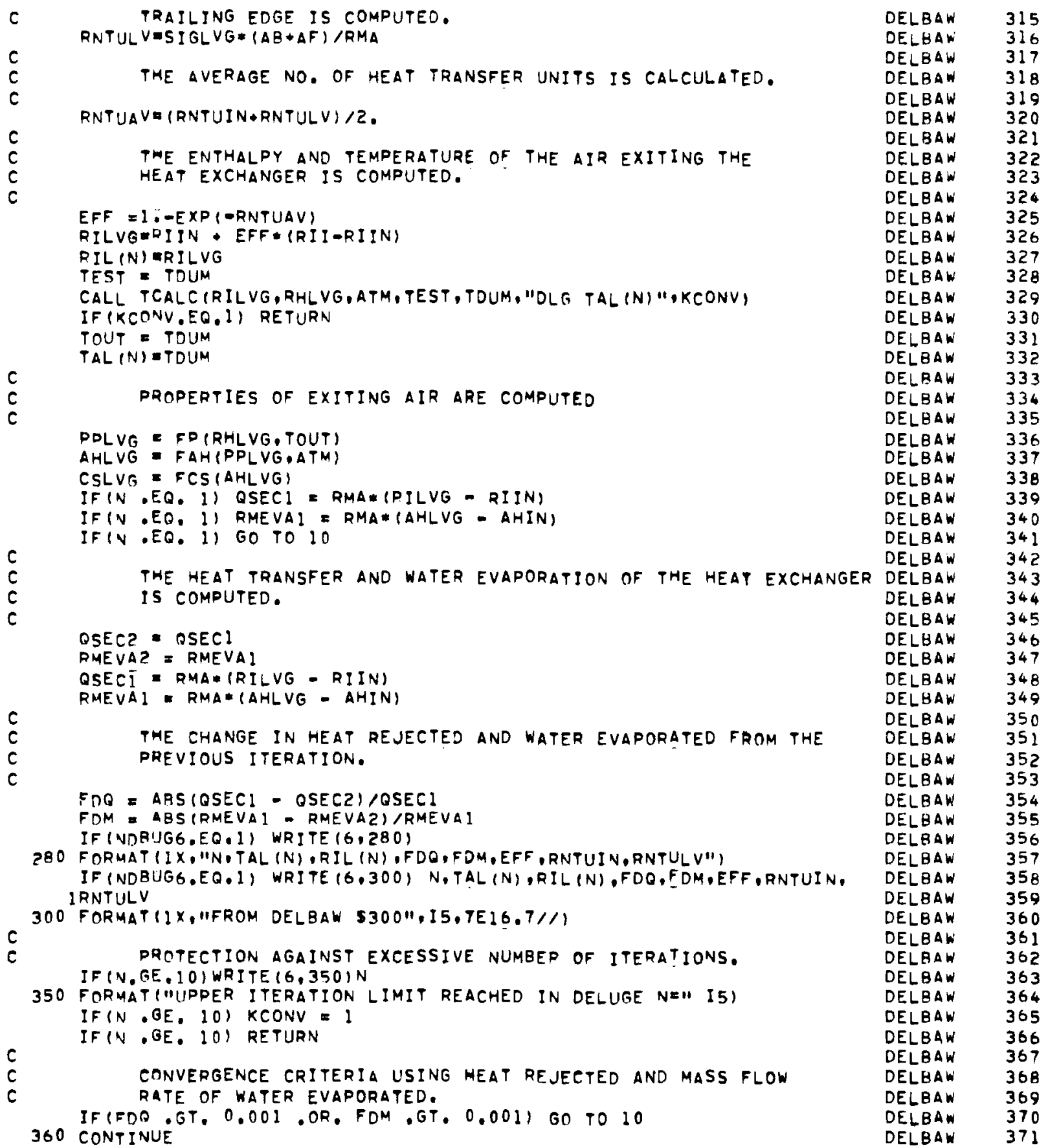


TAI = TAZ

TAZ: TAIN

370 TOLITE = TOUT

RILOII E RILVG

ISL $=$ (NSLOPE - 1)*26

R4IISL+I) E TAIN

R4(ISL + 2) = RIIN

R4 ISL +13$)=A H I N$

R4(ISL + 14) = TOUT

R4 (ISL * 15) = RILVG

R4IISL +24$)=$ OSECI

R4IISL +25$\}=$ RMEVAI

R4 (ISL + 26) = AHLVG

RR(1) $=A T M$

$R R(2)=D M A$

$R Q(3)=N S L O P E$

OSEC = QSECI

RMEVAPa RMEVAI

c

FRST3 $=$.FALSE.

c

OUTPUT STATEMENTS.

IF (NDRUG6.NE.1) RETURN

DO $300 \mathrm{~J}=1, \mathrm{~N}$

WPITE (S, 395) J,TAL (J),RIL (J)

390 CONTINUE

395 FORMATIIX,"N.TAL (N) AND RIL(N) = ".I5.1P2EI7.8) WRITF $(6,375)$

375 FORMATIIX, "N, OSEC,RMEVAP, UDIN, UDLVG, RNTUAVII)

WRI TE $(6,400) \mathrm{N}, Q$ SEC, RMEVAP, SI GIN, SI GLVG, RNTUAV

400 FORMAT (IX,"LEAVING DELBAW \$400", IP5E15.6)

WRITE $(6,450)$

450 FORMAT $\left(1 X_{1} / / /\right)$

RETUPN

END 
$74 / 74 \quad$ OPT $=1$

FTN $4.5+414$

$06 / 05 / 78$

07.59 .59

SUBROUTINE DELUG

DELUG

DELUG

DELUG

SUBROUTINE DELUG CALCULATES THE HEAT TRANSFER ANO THE WATER EVAPORATION RATES FOR A DELUGED HEAT EXCHANGER. THE COMPUTATIONAL METHOD DEVELOPED BY D.K. KRIEO AND EXTENDED TO FIN TUBES BY D. W. FALETTI IS USED. THE DRIVING FORCE USED TO CALCULATE THE HEAT TRANSFER IS THE ENTHALPY (BTU/LB OF DRY AIR) AS IS COMMONLY USED IN WET TOWER DESIGN METHOD DIVIDED EY THE HEAT CAPACITY OF AIR. ALL CONDUCTIVE AND CONVECTIVE TERMS ARE APPROPRIATELY TRANSFORMED FROM A LINEAR TEMPERATURE DEPENDENCE TO A ENTHALPIC DEPENDENCE.

SUBROUTINE DELUG OBTAINS THE HEAT TRANSFER BY TAKING THE AVERAGE OF THE NUMBER OF TRANSFER UNITS AT THE LEADING EDGE AND AT THE TRAILING EDGE OF THE HEAT EXCHANGER AND CALCULATING A HEAT EXCHANGER EFFECTIVENESS. THIS EFFECTIVENESS IS THEN USED TO DETERMINE THE HEAT TRANSFERRED AND THE OUTLET TEMPERATURE OF THE AIR. THE EVAPORATION RATE OF WATER IS OBTAINED FROM THE CHANGE IN ABSOLUTE HUMIDITY OF THE AIR AND FROM THE MASS FLOWRATE OF THE AIR.

THE COMPUTATION OF THE CONOITIONS OF THE AIR EXITING THE HEAT EXCHANGER IS CONDUCTED BY SUCCESSIVE APPDOXIMATIONS.

A NOMENCLATURE OF SOME OF THE MORE FREQUENTLY USED TERMS FOLLOWS.

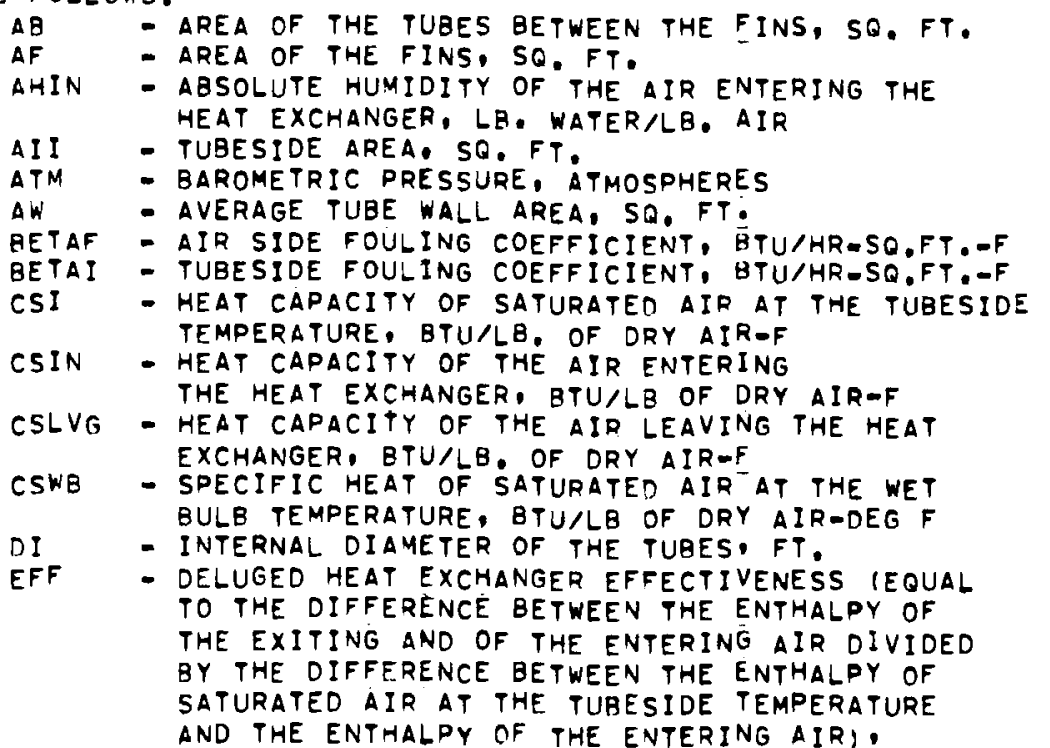

DELUG

DELUG

DELUG

DELUG

DELUG

DELUG

DELUG

DELUG

DELUE

DELUG

DELUG

DELUG

DELUG

DELUG

DELUG

DELUG

DELUG

DELUG

DELUG

DELUS

DELUG

DELUG

DELUG

DELUG

DELUG

DELUG

OELUG

OELUG

DELUG

DELUG

DELUG

DELUG

DELUG

DELUG

DELUG

DELUG

DELUS

DELUG

DELUG

DELUG

DELUG

DELUG

DELUG

DELUG

DELUG

DELUG

DELUG

DELUG

DELUG

DELUG

DELUG

DELUG

DELUG

DELUG 
$74 / 74 \quad$ OPT $=1$

FTN $4.5+414$

$06 / 05 / 78$

07.59 .59

DIMENSIONLESS

DELUG

DELUS

EFFIN - FIN EFFICIENCY, DIMENSIONLESS

FDM - QUANTITY USED FOR CONVERGENCE TEST, EQUAL

TO THE DIFFERENCE IN HEAT TRANSFER COMPUTED

DELUG

BY THE CURRENT AND BY THE PREVIOUS ITERATION

DELUG

DIVIDED BY THE HEAT TRANSFER COMPUTED BY THE

DELUG

DELUG CURRENT ITERATION, DIMENSIONLESS

FDQ - QUANTITY USED FOR CONVERGENCE TEST, EQUAL TO

DELUG

THE DIFFERENCE OF THE WATER EVAPORATION RATE

DELUS COMPUTED BY TME CURRENT AND BY THE PREVIOUS

DELUG

ITERATION, DIVIDED BY THE WATER EVAPOEATION

DELUG

DELUG RATE COMPUTED BY THE CURRENT ITERATION, DIMENSIONLESS

HI - TUBESIDE HELT TRANSFER COEFFICIENT,BTU/HR-SO.

DELUS

DELUS

DELUG $F T,-F$

ho - metal to delugeate heat transfer coefFicient. BTU/HR-SO.FT OF

ODL - OUTSIOE DIAMETER OF THE LINER, FT.

PPI - PARTIAL PRESSURE OF WATER AT SATURATION AT THE TUBESIDE TEMPERATURE, IN. HG.

DPIN - PARTIAL PRESSURE OF WATER IN THE ENTERING AIR, IN. HG.

PPLVG - PARTIAL PRESSURE OF WATER IN THE AIR LEAVING THE HEAT EXCHANGER, IN, HG.

PPWB - PARTIAL PRESSURE OF WATER AT. THE WET BULE TEMPERATURE OF THE ENTERING AIR (SATURATED CONDITIONS), IN.HG.

PSATIN - PARTIAL PRESSURE OF SATURATED AIR AT THE TEMPERATURE OF THE ENTERING AIR, IN,HG. OF THE ENTERIÑG AIR, IN HG.

OSECI - HEAT TRANSFER RATE COMPUTEE BY CURRENT ITERATION, LB/HR

QSECZ - HEAT TRANSFER RATE COMPUTED BY PREVIOUS ITERATION, LB/HR

QSEC - HEAT TRANSFER FROM THE HEAT EXCHANGER SECTION DEF INED BY AB AND AF, BTU/HR

RHIN - RELATIVE HUMIDITY OF THE AIR ENTERING THE HEAT EXCHANGER, DIMENSIONLESS

RHOUT - RELATIVE HUMIDITY OF THE AIR LEAVING THE HEAT EXCHANGER, DIMENSIONLESS

RII

- ENTHALPY OF SATURATED AIR AT THE TUBESIDE TEMPERATURE, BTU/LB OF DRY AIR

DELUG

DELUG

DELUS

DELUG

DELUG

DELUG

DELUG

DELUG

DELUG

DELUG

DELUG

DELUG

DELUG

DELUG

DELUS

DELUG

DELUG

DELUG

DELUS

DELUG

DELUG

DELUG

DELUG

DELUG

DELUG

DELUG

DELUS

RIIN - ENTHALPY OF TME AIR ENTERING THE HEAT EXCHANGER DELUG QTU/LB. OF DRY AIR

RILOUT - ENTHALPY OF THE AIR EXITING THE HEAT EXCHANGER, BTU/LB OF DRY AIR

RILOUI - ENTHALPY OF THE AIR EXITING THE HEAT EXCHANGER, DELUG GTU/LB OF DRY AIR

DELUG

DELUG

DELUG

DELUG

RILVG - ENTHALPY OF THE AIR EXITING THE HEAT EXCHANGER, BTU/LB OF DRY AIR

RIWB - ENTHALPY OF SATURATED AIR AT TWBIN, BTULLB OF DRY AIR

RKW - THERMAL CONDUCTIVITY OF THE TUBE WALL, BTU/HRSO.FT, $-F / F T$

RMA

- MASS Flow RaTE of AIR, Lg/HR

DELUG

DELUG

DELUG

DELUG

DELUG

DELUG

DELUG

DELUG

RMEVAR - WATER EVAPORATION RATE, LB/AIR

DELUG

RMEVAPI - WATER EVADORATION RATE COMPUTED DURING CURRENT DELUG 
$74 / 74 \quad$ OPT $=1$

FTN $4,5+414$

$06 / 05 / 78$

07.59 .59

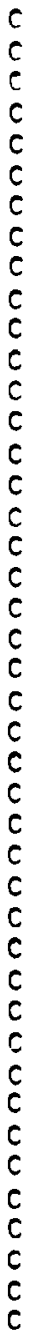

ITERATION, LB/HR DELUS 116

RMEVAPZ - WATER EVAPORATION RATE COMPUTED DURING PREVIOLIS DELUS 117

ITERATION, LB/HR DELUS 118

RNTUAV - ARITHMETIC AVERAGE OF THE NUMBER OF (DELIIGE) DELUG 119

TRANSFER UNITS AT THE LEADING AND AT THE DELUG I2O

TRAILING EDGE OF THE HEAT EXCHANGER, DELUG 121

DIMENSIONLESS DELUO I22

RNTUIN - NLMBER OF (DELUGE) HEAT TRANSFER UNITS AT THE DELUG 123

LEADING EDGE OF THE HEAT EXCHANGER. DELUG 124 DIMENISONLESS

RNTULV - NUMBER OF (DELUGE) TRANSFER UNITS AT THE

TRAILING EDGE OF THE HEAT EXCHANGER,

DELUG $\quad 125$

DELUG $\quad 126$

DELUG $\quad 127$

DELUG $\quad 128$

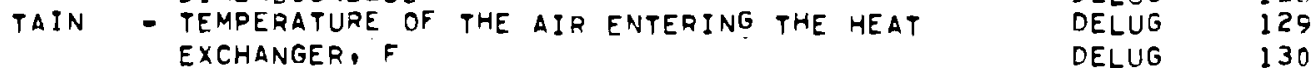

tal - temperature of the aIR leAVING the HEAT DELUg 131

EXCHANGER, $F$

THW - TUBE WALL THICKNESS,FT,

TEST - ESTIMATE OF TEMPERATURE OF AIR EXITING

DELUG $\quad 132$

DELUS 133

DELUG 134

THE HEAT EXCHANGER, DEG. F.

TI - TUBESIDE TEMPERATURE, DEG. 5

TMAX - MAXIMUM TEMPERATURE ALLOWED DUE TO CONSTRAINTS UPON THE ANALOGY UPON WHICH THE HEAT TRANSFER CALCULATION IS BASED,F

TMIN - MINIMUM TEMPERATURE ALLOWED TO PREVENT

POSSIBLE INSTABILITIES WITHIN THE SUBROUTINES, F

TOUT - TEMPERATURE OF AIR EXITING THE HEAT EXCHANGER, DEG. F,

TWBIN - WET BULB TEMDERATURE OF THE ENTERING AIR, DEG, $F$.

UDIN - TUBESIDE TO AIR MEAT TRANSFER COEFFICIENT ICONVERTED TO THE DRIVING FORCE OBTAINED EY

DELUG

DELUG

DELUG

DELUG

DELUG

DELUG

DELUG

DELUG

DELUG

DELUG

DELUS

DELUG

DELUG

DIVIDING THE ENTHALPY DIFFERENCE BY THE

DELUG

SPECIFIC HEAT OF HUMID AIR) AT THE LEADING DELUG EOGE OF THE HEAT EXCHANGER

DELUG BTU/HR-SQ.FT.DEG. F

UDLVG - TUBESIDE TO AIR HEAT TRANSFER COEFFICIENT ICONVERTED TO THE DRIVING FORCE OBTAINED BY

DELUG

DELUG

DIVIDING THE ENTHALPY DIFFERENCE BY THE

SPECIFIC HEAT OF AIRI AT THE TRAILING EDGE

DELUG

DELUG OF THE HEAT EXCHANGER,

LOGICAL FRSTI, FRST2, FRST3 FT, DEG F $F$-HR

LOGICAL FIRST, FRST, FXTEMP, FXTVAR,FXTTTD, FXTLNG

DELUG

DELUG

DELUG

BIGCOM

BIGCOM

BIGCOM

5 AFTR, ALPHA, ANG (3)

- bolim(16)

5. CAPF, CSSOKW, CONF, CONL, COSTL, CAPCHG, CONMAT, CONMAZ, CAF

BIGCOM

BIGCOM

\$ CBJ, CTURB, CDANG, CLUVR, CHAILS, CVM

$\$$, DEIN, DEEPL, DESVEL, DESVELV, DESVELD,DENSIF

BI GCOM

BIGCOM

S EFFD, EFS

5 . FCD,FIRST, FIXL, FCOS,FFHX, FXTEMP, FACTOR, FRST, FACTORD, FSHOT , FDEL UG

S, FIXV,FIXTTD,FXTVAR,FXTTTD,FXTLNG

BIGCOM

135

136

137

138

139

140

141

142

143

144

145

146

147

148

149

150

151

152

153

154

155

156

157

158

159

5 GAEFF

\$ HXNP,HPCST,HCD

S. ITMAX, ITMIN

BI GCOM

BIGCOM

BI GCOM

BIGCOM 


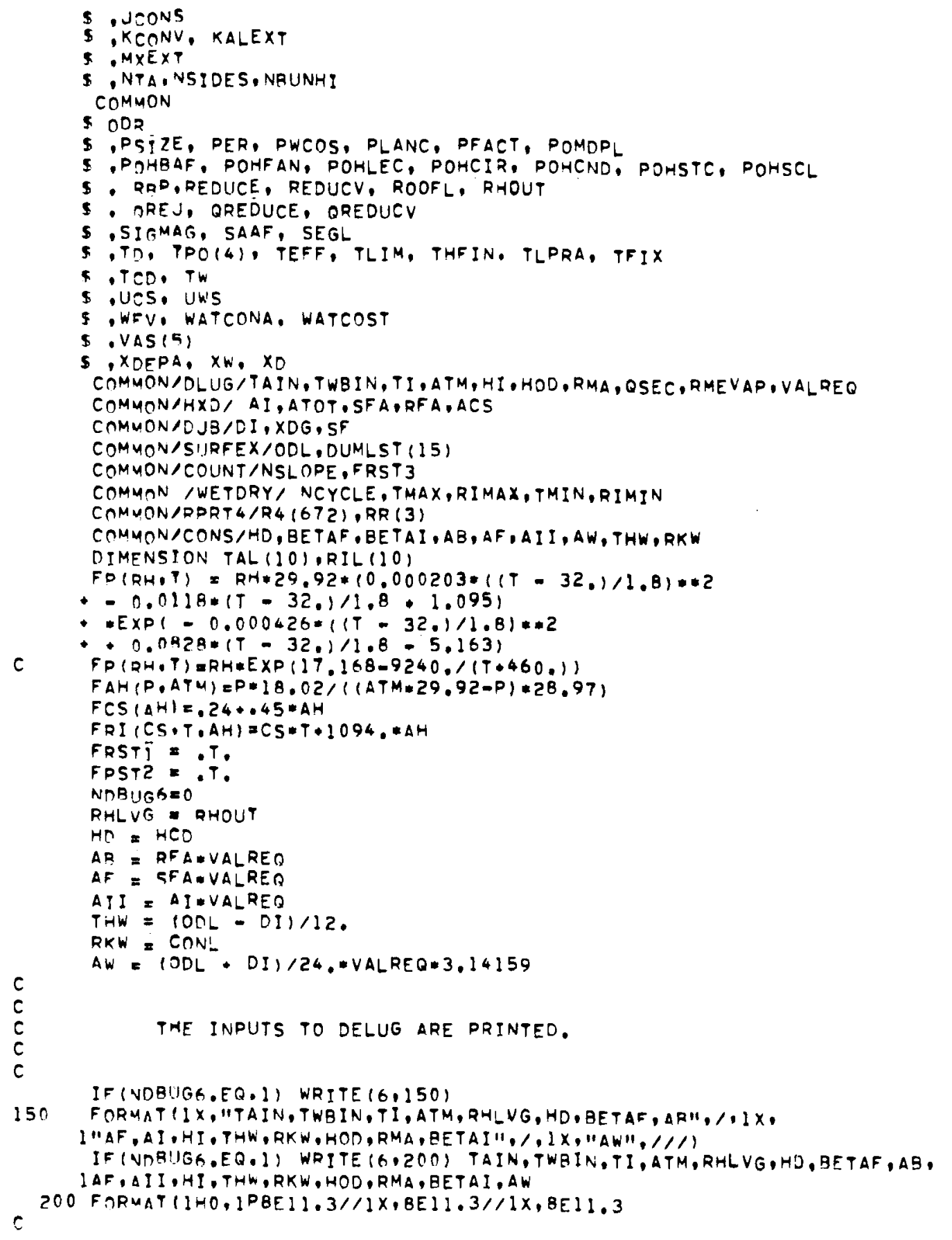

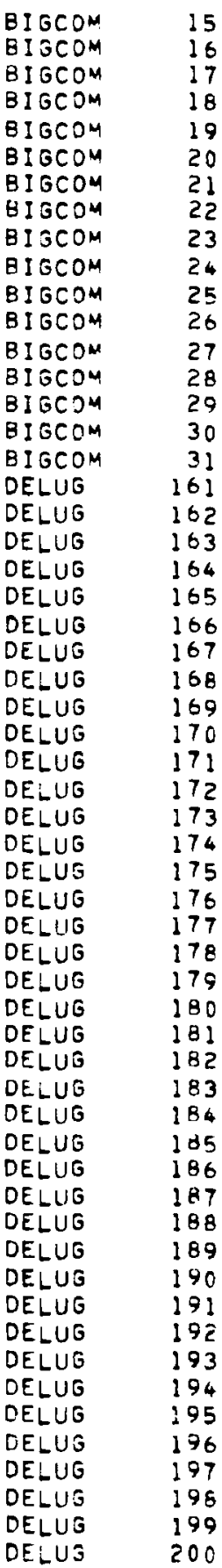


IF (FRST) CALL LIMITIATM,TMAX,RIMAX,TMIN,RIMIN)

DELUG

DELUG

FRST $=. F$.

IF (TAIN.LE.TMIN OR. TAIN.GE,TMAX) WRITE 6,5 ) TAIN

IF (TI.LE.TMIN OOP. TI OGE,TMAX) NRITE $(6,7)$ TI

IF (TI LE.TMIN,OR.TI,GE.TMAX) KCONV = I

DELUG

DELUG

DELUG

DELUG

IF (TAIN.LE,TMIN.OR.TAIN,GE,TMAX) KCONV=I

IF (KCONV.EO.1) RETURN

5 FORMAT "OTAIN IS LESS THAN TMIN DR GREATER THAN TMAX IN DELUge "

- "TAINa", IPE [3.5)

7 FORMATI"OTI IS LESS THAN TMIN OR GREATER THAN TMAX IN DELUGE."

DELUG

DELUG

DELUG

DELUG

DELUG

S."TIE". IDEI3.5)

DELUG

DELUG

DELUG

DELUG

PROPERTIES OF THE INLET AIR AND TUBESIDE FLUID (IN ENTHALPIC EQUIVALENT) ARE COMPUTED.

DELUG

DELUG

DELUG

DELUG

PPWBEFP (1, , TWBIN

DELUG

AHWBEFAH (DPWB, ATM)

CSWGEFCS (AHWB)

RIWB =FRI (CSWB, TWBIN,AHWB)

$R$ IINER IWB

AHIN=(RIIN-.24*TAIN)/1.45*TAIN+1094.)

CSINE. 24*.45*AHIN

PPINE $(A H I N * A T M * 866.78240) /(18.02+A H I N * 28.97)$

DELUG

DELUG

DELUG

DELUG

DELUG

DELUG

PSATIN = FPI I.,TAIN)

$c$
$c$
$c$
$c$

RHINEPPIN/PSATIN

DELUG

DELUG

DELUG

DELUG

DELUG

THE hEAT TRANSFER COEFFICIENT AT THE LEADING EDGE IS COMPUTED DELUG

DELUG

DELUG

$P D I=F P(1,0, T I)$

DELUG

$\triangle H I=F A H(P P I, A T M)$

DELUG

CSI =FCS $(A H I)$

RII =FRI (CSI,TI, AHI)

DELUG

CALL UDEL ITAIN,RIIN,ATM,HD,BETAF, AB, AF, AII, AW, HI, BETAI, THW,RKW, TI, -RII, HOD,UDIN,EFFIN,FRSTI, KCONV,OI

$c$
$c$
$c$
$c$
$c$

IF (KCONV.ER.I) RETURN

DELUG

DELUG

DELUG

DELUG

DELUG

PME NUMBER OF HEAT TRANSFER UNITS IBASED ON ENTHALPY DRIVING

DELUG

FORCE) BASED ON CONDITIONS AT THE LEADING EDGE ARE COMPUTED.

DELUG

DELUG

RNTUINGUDIN* (AB०EFFIN*AF) / (RMA $-C S I N)$

$N=0$

DELUG

DELUG

DELUG

ESTIMATION THE EXIT TEMPERATURE AND ENTHALPY OF THE AIR

DELUG LEAVING THE HEAT EXCHANGER

DELUG

DELUG 


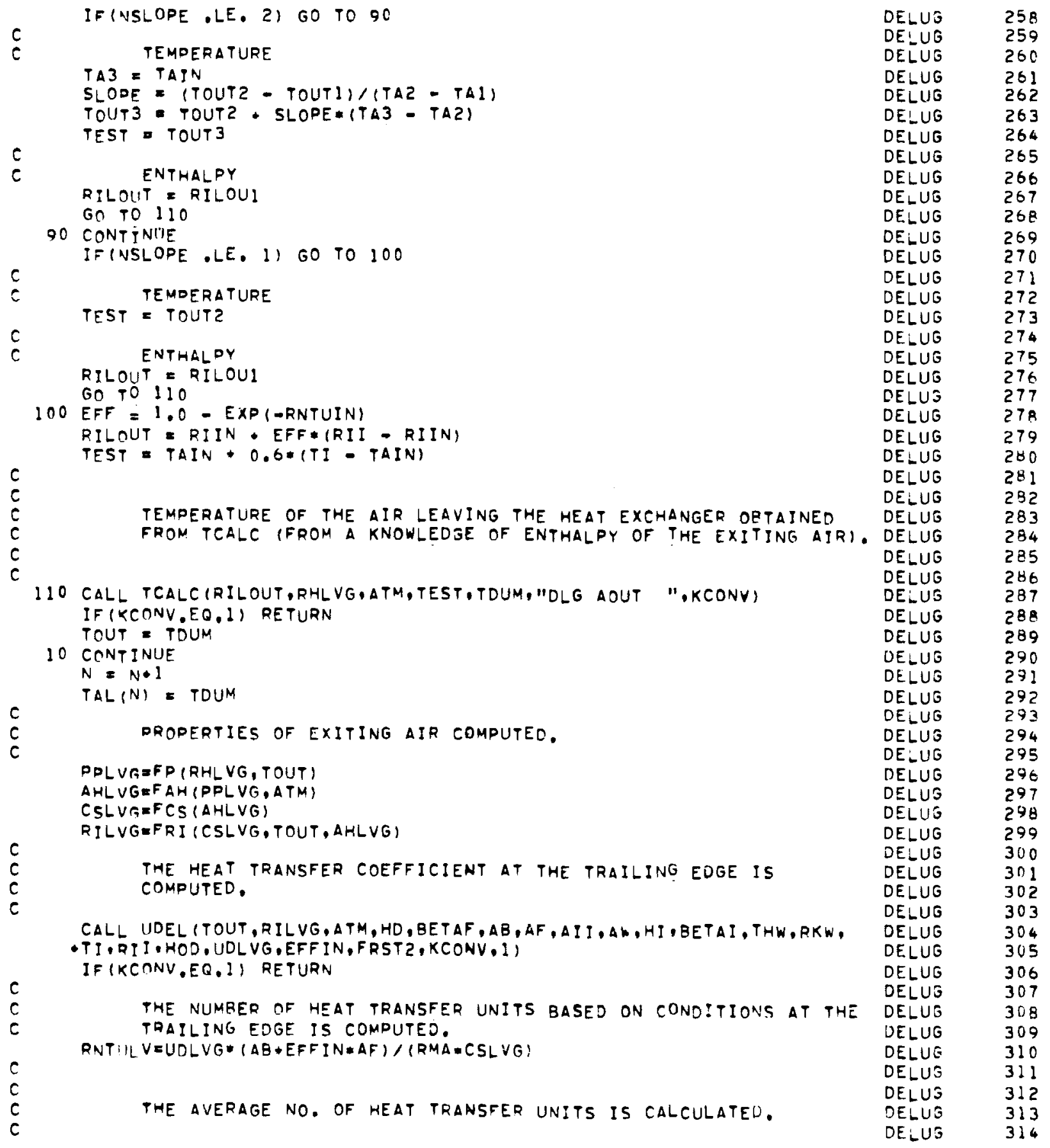




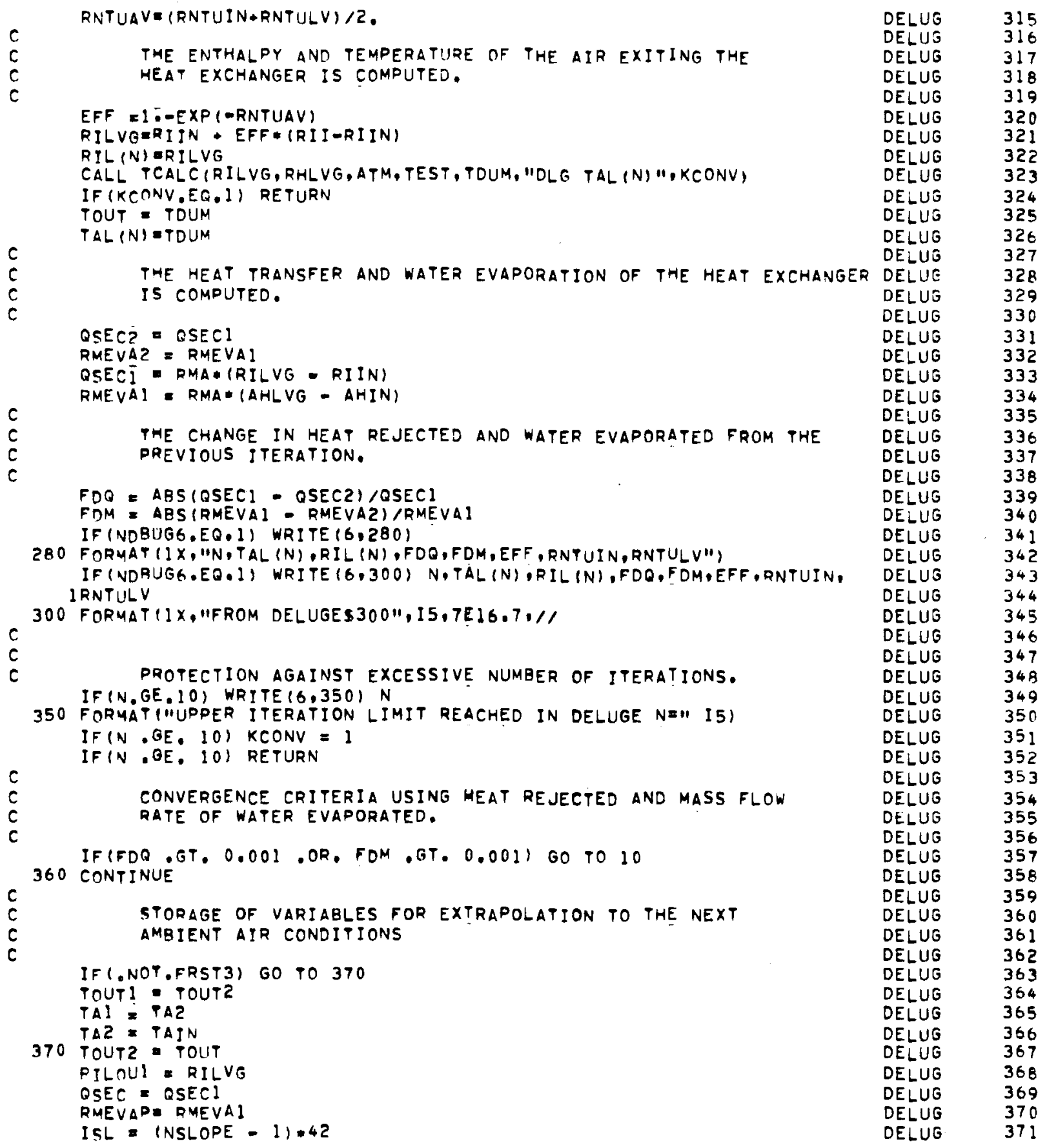




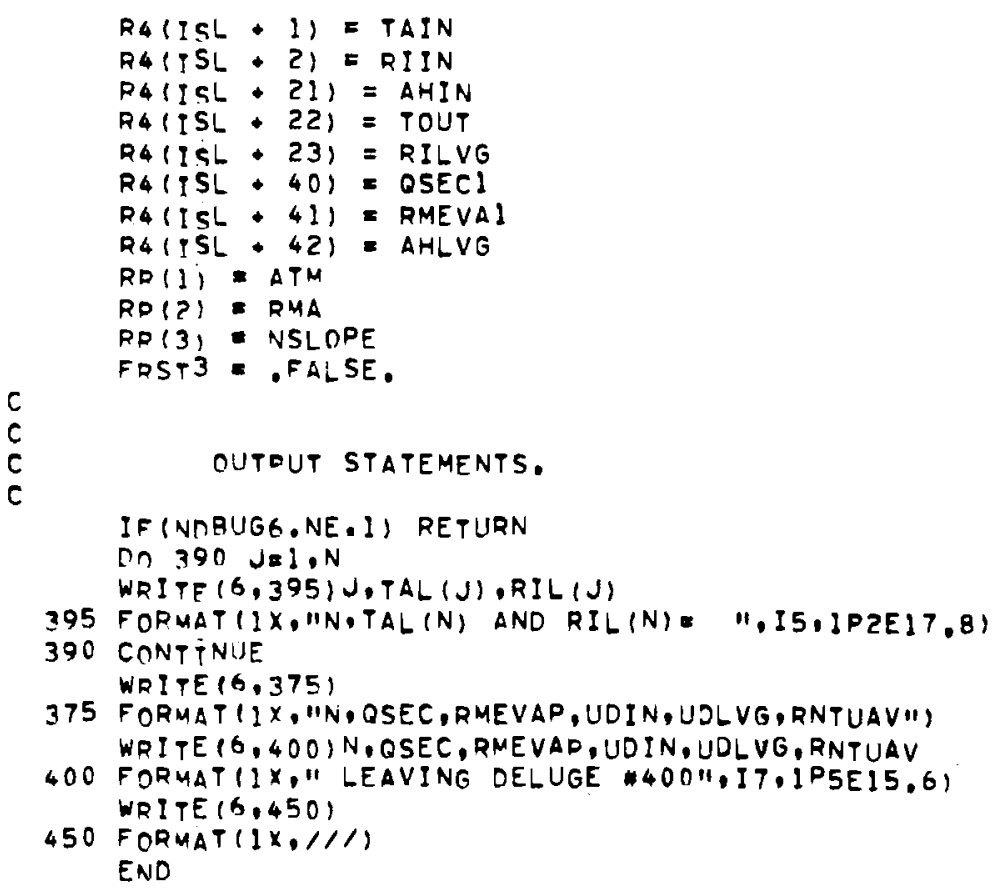

$\begin{array}{ll}\text { DELUG } & 372 \\ \text { DELUG } & 373 \\ \text { DELUG } & 374 \\ \text { DELUG } & 375 \\ \text { DELUG } & 376 \\ \text { DELUG } & 377 \\ \text { DELUG } & 378 \\ \text { DELUG } & 379 \\ \text { DELUG } & 380 \\ \text { DELUG } & 381 \\ \text { DELUG } & 382 \\ \text { DELUG } & 383 \\ \text { DELUG } & 386 \\ \text { DELUG } & 385 \\ \text { DELUG } & 386 \\ \text { DELUG } & 387 \\ \text { DELUG } & 388 \\ \text { DELUG } & 389 \\ \text { DELUG } & 370 \\ \text { DELUG } & 391 \\ \text { DELUG } & 392 \\ \text { DELUG } & 393 \\ \text { DELUG } & 394 \\ \text { DELUG } & 375 \\ \text { DELUG } & 396 \\ \text { DELUG } & 397 \\ \text { DELUG } & 398 \\ \text { DELUG } & 399\end{array}$


$74 / 74 \quad O P T=1$

FTN $4.5+414$

$06 / 05 / 78$

07.59 .59

SIJB qOUTINE DELUGEP (CTOWDEL, TOWDIA, VELDEL, DELHZO, DELMUP, DISDEL.

DELUGEP IDELCSTI

THIS SUBROUTINE APPROXIMATES THE COST OF THE DELUGE

PIPING SYSTEM. THIS IS DONE BY COSTING THE MAJOR

DORTIONS OF THE MAKEUP AND TOWER COMPONENTS. PIPE DIAMETERS

ARE DETERMINED FROM THE DELUGE FLOW RATE AND DESIGN

VELOCITY.

CONSTI - CONVERSION FACTOR

CTOWDEL - NUMBER OF DELUGED TOWERS

DELCST - TOTAL COST OF THE DELUGE SYSTEM

DELHZO - DELUGE FLOW RATE (GPM)

DELMUP - MAKEUP FLOW RATE IGPM)

DELTOW - DELUGE FLOW RATE PEA TOWER (GPM)

DIA PIPE DIAMETER (IN)

DIADEL - MAXIMUM DIAMETER OF TDWER PIPING (IN)

DIAMUP - MAXIMUM DIAMETER OF MAKEUP PIDING (IN)

DISDEL - DISTANCE FROM DELUGE WATER SUPPLY AND TOWER BOUNDARY (FT)

DISTCST - COST OF MAKEUP PIPING (SI000)

GPMCST - PUMPING COSTS (S/GPM)

IM.IT - INDICES FOR MAKEUP AND TOWER DIPING

NTOW - INTEGER NUMBER OF TOWERS DIVIDED BY ?

PIPCST - PIPE COST (SIOOO)

PIPCSTM - COST OF MAKEUP PIPING (\$1000)

PIPLEN - APPROXIMATE MAKEUP PIPE LENGTH (FT)

PMPCST - MAKEUP PUMPING COSTS (\$1000)

RMODEL - DENSITY OF DELUGE WATER (LBM/FYCU)

TOWCST - COST OF TOWER COMPONENTS (\$1OOO)

TOWDIA - TOWER DIAMETER (FT)

TOWPIP - COST OF TOWER PIPING (\$1000)

TOWPMP - TOWER PUMPING COSTS ISI000

VALVCST - VALVE COST (S100D)

VELDEL - DELUGE DESIGN VELOCITY (FT/SEC)

DIMENSION DIA 110$),$ PIPCST 110$),$ VALVCST 10

DATA DIAK

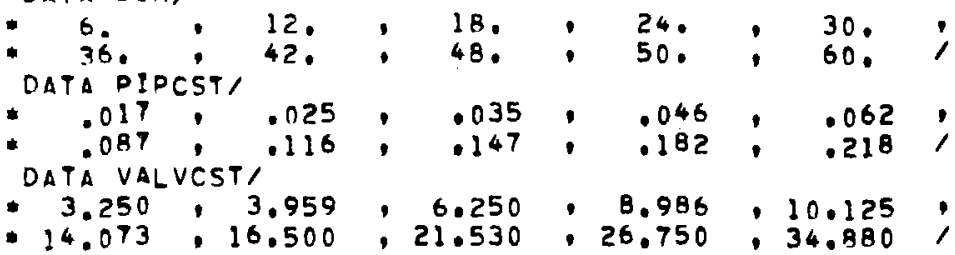

$\mathrm{C}$
$\mathrm{C}$
$\mathrm{C}$

COST THE MAKEUP PIPING.

IF (DEL H2O,EO.0.0) RETURN

CONSTI $=1.117 .481 .60 .1$

GPMCST $=2.50$

NTOW $=$ CTOWDEL/2.

DIPIENEDISDEL +.5*TOWOIA*1.5*NTOW*TOWDIA

DI AMUP = SQRT (DELMUP *CONST I/VELDEL) 12 .

DELUGEP

DELUGEP 4

DELUGEP 5

DELUGEP 6

DELUGEP 7

DELUGEP 8

DELUGEP 9

DELUGEP 10

DELUGEP 11

DELUGEP 12

DELUGEP

DELUGEP I4

DELUGEP 15

DELUGEP 16

DELUGEP 17

DELUGEP 18

DELUGEP 19

DELUGEP 20

DELUGEP 2 ?

DELUGEP 22

DELUGEP 23

DELUGEP 24

DELUGEP 25

DELUGEP 26

DELUGEP 27

DELUGEP 28

DELUGEP 20

DELUGEP 30

DELUGEP 31

DELUGEP

DELUGEP 33

DELUGEP 3

DELUGEP 35

DELUGEP 36

DELUGEP 37

DELUGEP 38

DELUGEP 39

DELUEEP 40

DELUGEP 41

DELUGEP 42

DELUGEP 43

DELUGEP $\quad 44$

DELUGEP $\quad 45$

DELUGEP

DELUGEP $\quad 47$

DELUGEP 48

DELUGEP $\quad 49$

DELUGEP 50

DELUGEP 51

DELUGE 52

DELUGEP 53

DELUGEP 54

DELUGEP 55

DELUGEP 56

DELUGEP 57

DELUGEP 58 
IF (DIAMUP.LT.DIA(I) DIAMUP $=O I A(1)$

DELUGEP

DO IO $1=1,10$

$I M=$ I

IF (OIAMUP-DIA (IM)) $11.11,10$

10 CONTINIJE

C

11 DIAMUPEDIAIIM)

TO PERCENT OF THE LARGEST DIAMETER PIPE COST WAS USED

TO DETERMINE THE AYERAGE PIPE COST

PIPESTME. TO\#PIPLEN*PIPCST (IM)

PMPCST I DELMUP $\triangle$ GPMCST $/ 1000$.

90 PERCNT OF THE MAKEUP COSTS WERE FROM PIPEOVALVE

ANO DUMP COSTS

DISTCSTE (PIPCSTM PMPCST +VALVCST IIM)) 1.90

COST THE TOWER COMPONENTS. PUMPS, VALVES AND

PIPING REPRESENT 51.5 PERCENT OF THE TOWER COSTS.

DETERMINE THE DIAMETER OF THE TOWER PIPING

DELTOWEDELHZO/CTOWDEL

DIADEL = SORT (DELTOW CONST I /VELDEL) 12 . IF (DIADEL.LT.DIA(1)) DIADEL=DIA (1)

DO 50 IEI, 10

$I T=I$

IF (DIADEL-DIA(IT)) $21,21,20$

50 CONTINUE

21 DIADELODIAIIT)

TOWD IP=3. *TOWDIA $=P$ IPCST (IT)

TOWDMD : DELTOW GPMCST 1000 .

TOWCSTE (TOWPIP.6. *VALVCST (IT) +TOWPMP)/.515

DETERMINE THE TOTAL DELUGE PIPING SYSTEM COSTS

DELCST $=D I S T C S T \cdot C T O W D E L * T O W C S T$

RETURN

END
DELUGEP

DELUGEP

DELUGEP

DELUGED

DELUGEP

DELUGED

DELUGEP

DELUGEP

DELUGEP

DELUGEP

DELUGEP

DELUGEP

DELUGEP

DELUGEP

DELUGEP

DELUGEP

DELUGEP

DELUGED

DELUGED

DELUGEP

DELUGEP

DELLGE:

DELUGEP

DELUGEP

DELUGED

DELUGEP

DELUGEP

DELUGED

DELUGED

DELUGED

DELUGEP

DELUGEP

DELUGED

DELUGEP

DELUGEP

DELUGEP

DELUGED

DELUGEP

DELUGEP

DELUGEP
59

60

61

62

63

64

66

68

69

70

71

74

75

76

77

78

80

81

82

83

84

85

87

88

89

90

91

92

93

94

95

96

97

99 
SURROUTINE DELUGT IS USED TO DETERMINE THE OPERATION OF THE

DELUGT

POWER PLANT OVER THE ENTIRE YEAR WHEN THE ENTIRE HEAT

DELUGT

EXCHANGER IS DELUGED. IT DETERMINES THE BACK PRESSURE OF

DELUGT

THE TURBINE AND THE SATURATION TEMPERATURE OF THE STEAM IN

DELUGT

THE CONDENSER/REBOILER AS A FUNCTION OF THE AMBIENT AIR

TEMPERATURE AND WET BULB AMBIENT TEMPERATURE:

DELUGT

DELUGT

DEFINITION OF VARIABLES

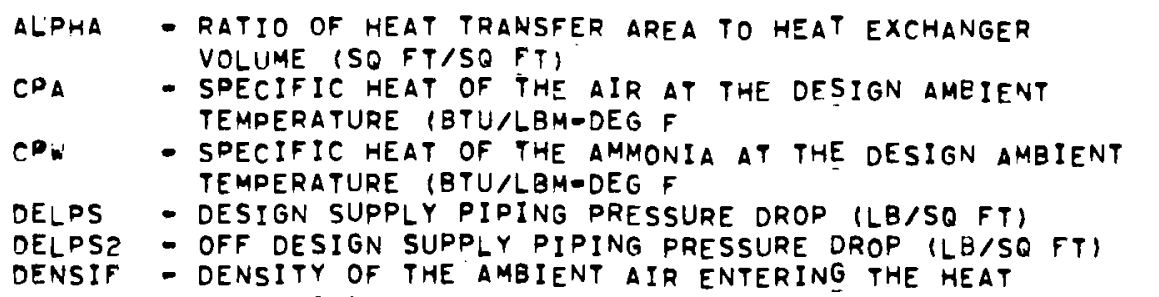

DELUGT

DELUGT

DELUGT

DELUGT

DELUGT

DELUGT

DELUGT

DELUGT

DELUGT

DELUGT

DELUGT

DENSIF

EXCHANGER (LBM/CU FT)

DELUGT

DENSIV - DENSITY OF THE AMMONIA VAPOR LEAVING THE CONDENSER,

DENSIVZ - DENSITY OF THE AMMONIA VAPOR LEAVING THE CONDENSER,

DELUGT REBOILER AT OFF OESIGN CONDITIONS (LBM/CU FT)

DENSIW - DENSITY OF THE AMMONIA LIQUID (LBM/CU FT)

DI - INSIDE DIAMETER DF THE HEAT EXCHANGER TUBES (IN)

EFF - EFFECTIVENESS OF THE HEAT EXCHANGER AT DESIGN CONDITIONS (DIMENSIONLESS)

ELENG - LENGTH OF THE HEAT EXCHANGER TUBES (FT)

EL'EV - ELEVATION OF THE PLANT SITE (FT)

FACTOR - RATIO OF THE AIR VELOCITY IN THE HEAT EXCHANGER WHEN IT IS DELUGED TO THE AIR VELOCITY IN THE HEAT EXCHANGER WHEN IT IS DRY (DIMENSIONLESS)

DELUGT

DELUGT

DELUGT

DELUGT

DELUGT

DELUGT

DELUGT

DELUGT

DELUGT

DELUGT

DELUGT

DELUGT

DELUGT

GAIRZ - MASS FLUX OF THE AIR THROUGH THE HEAT EXCHANGER WHEN IT IS DELUGED (LBM/SQ FT-HR)

gWAT2 - MASS FLUX OF THE AMMONIA THROUGH THE TUBES OF THE HEAT EXCHANGER (LAM/SO FT-HR)

HFG2 - HEAT OF VAPORIZATION OF AMMONIA AT OFF DESIGN CONDITIONS (BTU/LBM)

HI2 - INSIDE HEAT TRANSFER COEFFICIENT OF THE HEAT EXCHANGER TUBES AT OFF DESIGN CONDITIONS (BTU/HR-DEG F-SQ FT)

HO2 - AIR SIDE HEAT TRANSFER COEFFICIENT OF THE HEAT EXCHANGER AT OFF DESIGN CONOITIONS (BTU/HR -DEG F-SO FT)

HRFACI - HEAT RATE FACTOR AT RATING CONDITION (DIMENSIONLESS)

HRFAC2 - HEAT RATE FACTOR AT DESIGN CONDITIONS

HRFAC4 - HEAT RATE FACTOR AT OFF DESIGN CONDITIONS (OIMENSIONLESS)

NTA

- NUMBER OF TEMPERATURE INCREMENTS IN THE AMBIENT TEMPERATURE RANGE OF THE PLANT SITE (OIMENSIONLESS)

NW

- NUMBER OF TUBES IN THE WIDTH OF THE HEAT EXCHANGER (DIMENSIONLESS)

DELUGT

DELUGT

DELUGT

DELUGT

DELUGT

DELUGT

DELUGT

DELUGT

DELUGT

DELUGT

DELUGT

DELUGT

DELUGT

DELUGT

DELUGT

DELUGT

DELUGT

DELUGT

DELUGT

DELUGT

DELUGT

DELUGT

DELUGT 


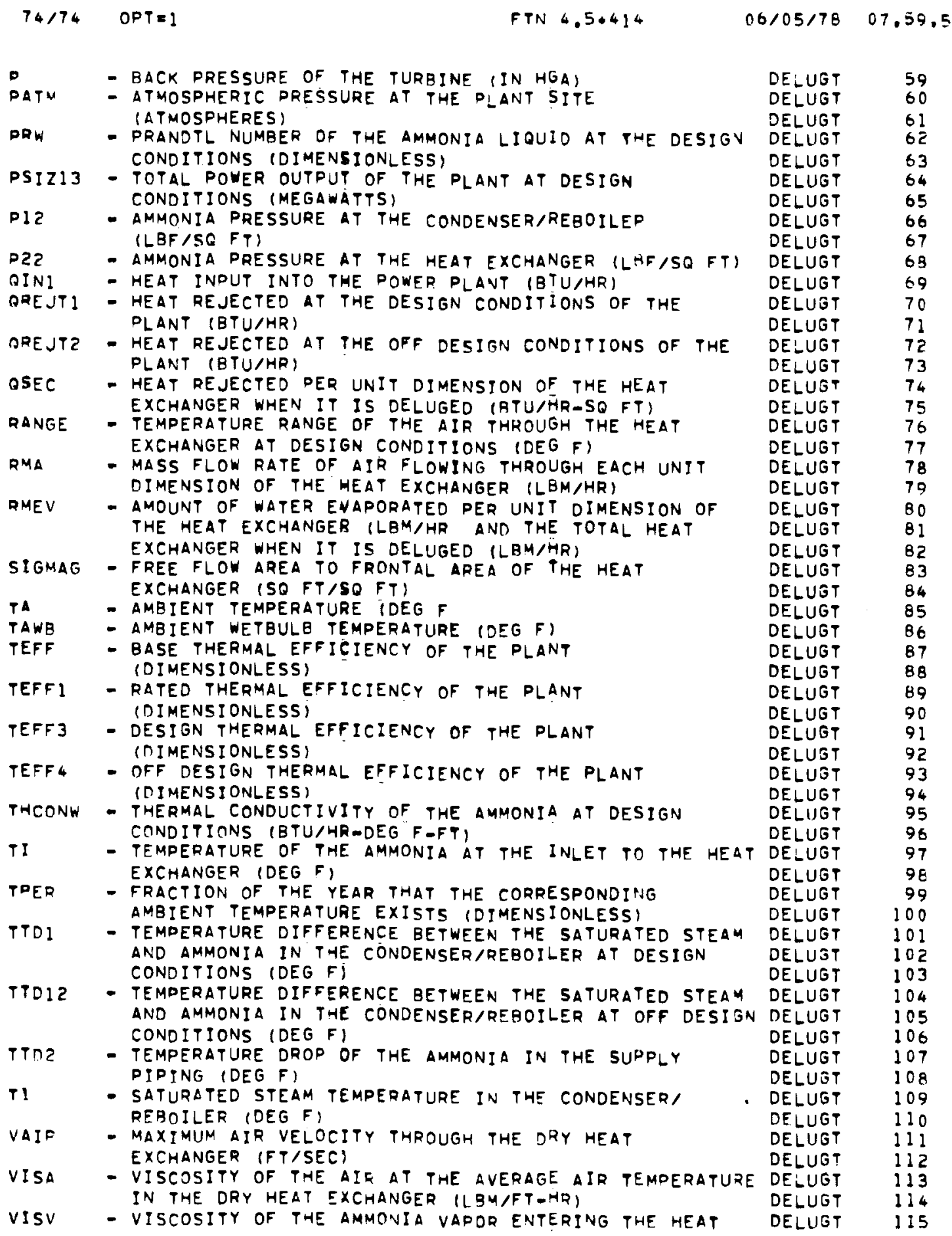


$74 / 74$ OPTE1 FTN $4.5 .414 \quad 06 / 05 / 79 \quad 07.59 .59$

EXCHANGER AT DESIGN CONDITIONS (LBM/FTIHR) DELUGT

VISW - VISCOSITY OF THE AMMONIA LIOUTD ENTERING THE HEAT DELUGT

EXCHANGER AT DESIGN CONDITIONS (LBM/FT-HR)

DELUGT 128

WATCONA - AMOUNT OF WATER ALLOWED TO BE EVAPORATED OVER THE WHOLE YEAR (LBM/YEAR)

WATCONT - AMOUNT OF WATER EVAPORATED DURING THE WHOLE YEAR

DELUGT

DELUGT

43

BY THE COMPLETELY DELUGED HEAT EXCHANGER ILBM/YEAR

W
W2

(LBM/HR)

- mass Flow rate of ammonia through the heat

EXCHANGER AT DESIGN CONDITIONS (LBM/HR)

XD - LONGITUDINAL PITCH OF THE TUBES IN THE HEAT EXCHANGER (IN)

YPP - PLANT SCALING FACTOR FOR FAN AND PUMP PONER LOSS (DIMENSIONLESS)

$Z$ - HEAT EXCHANGER DEPTH IN NUMBER OF TUBES (D I MENS I ONLESS)

20 - HEAT EXCHANGER DEPTH IN NUMBER OF TUBES (DIMENSIONLESS)

DELUG

DELUGT

DELUGT

DELUGT

DELUGT

DELUGT

DELUGT

DELUGT

DELUGT

DELUST

DELUGT

DELUGT

DELUGT

DELUGT

DELUGT

\section{DEFINITION OF ARRAYS}

ANG - THE ANGLE OF THE HEAT EXCHANGER TUBES FROM THE HORIZONTAL AND THE SINE AND COSINE OF THAT ANGLE

DELUGT

DELUGT

DELUGT

DELUGT

APL

- BACK PRESSURE LIMIT CURVE (IN HGA)

DELUGT

PSATD

- TURBINE BACK PRESSURE CURVE WHEN TH IS DELUGED COMPLETELY (IN HGA)

TPO

- ARRAY OF COEFFICIENTS FOR THE CUBIC CURVE FIT OF HEAT RATE FACTOR AS A FUNCTION OF TURBINE BACK PRESSURE (DIMENSIDNLESS)

TSATD - TURBINE STEAM SATURATION CURVE WHEN THE HEAT EXCHANGER IS DELUBED COMPLETELY (DEG F)

VTA - AMBIENT TEMPERATURE CURVE IDEG F)

VTAWB - AMBIENT MET BULB TEMPERATURE CURVE IDEG F)

VTPER - FRACTION OF THE YEAR THAT THE AMBIENT AND WET BULB TEMPERATURE INCREMENTS EXIST (DIMENSIONLESS)

WATCON

- WATER CONSUMPTION OF THE HEAT EXCHANGER WHEN IT IS COMPLETELY DELUGED OVER THE WHOLE YEAR FOR EACH AMBIENT TEMPERATURE INCREMENT (LBM/YEAR)

DELUGT

DELUGT

DELUGT

DELUGT

DELUGT

DELUGT

DELUGT

DELUGT

DELUGT

DELUGT

DELUGT

DELUGT

DELUGT

DELUGT

DELUGT

LOGICAL FRST3

LOGICAL FIRST, FRST, FXTEMP, FXTVAR, FXTTTD,FXTLNG

COMMON

S AFTR, ALPHA, ANG (3)

- Bplim(16)

S, CAPF, CSSPKW, CONF, CONL, COSTL, CAPCHG, CONMAT, CONMAZ, CAF

$\$$. CSJ, CTURB, CDANG, CLUVR, CHAILS, CVM

\$ DFIN, DEEPL , DESVEL, OESVELV, DESVELD. DENSIF

5 ,EFFP, EFS

\$. FCR, FIRST, FIXL, FCOS,FFHX, FXTEMP, FACTOR, FRST, FACTORD, FSHOT, FDELUG

S.FIXV,FIXTTD,FXTVAR,FXTTTD,FXTLNG

S.GQFFF

5.HXNP,HDCST IHCD

\$. ITMAX, ITMIN

S IJONS

BIGCOM

BIGCOM

BI GCOM

BIGCOM

BIGCOM

BI GCOM

BIGCOM

BIGCOM

BIGCOM

BI GCOM

BIGCOM

BIGCOM

BI GCOM

116

128

119

120

121

122

123

124

125

126

127

128

129

130

131

132

133

134

135

136

137

138

139

140

141

142

143

144

145

146

147

148

149

150

151

152

153

154

155

156

157

158

2

4

5

6

? 


\section{$\$$ ODR}

\$ PSIZE, PER, PWCOS, PLANC, PFACT, POMDPL

- POHBAF, POHFAN, DOHLEC, POHCIR, POHCND, POHSTC, POHSCL

F RED, REDUCE, REDUCV, ROOFL, RHOUT

\$ OREJ, QREDUCE, QREDUCV

S SIGMAG, SAAF, SEGL

S,TO, TPO(4), TEFF, TLIM, THFIN, TLPRA, TFIX

3.TCO. TW

S.UCS. UWS

\$. WEV. WATCONA, WATCOST

? VAS(5)

\$ XOEDA, XW, XC COMMON /SUPPLY, VAL (5), CPLN, CPIP, COOS.

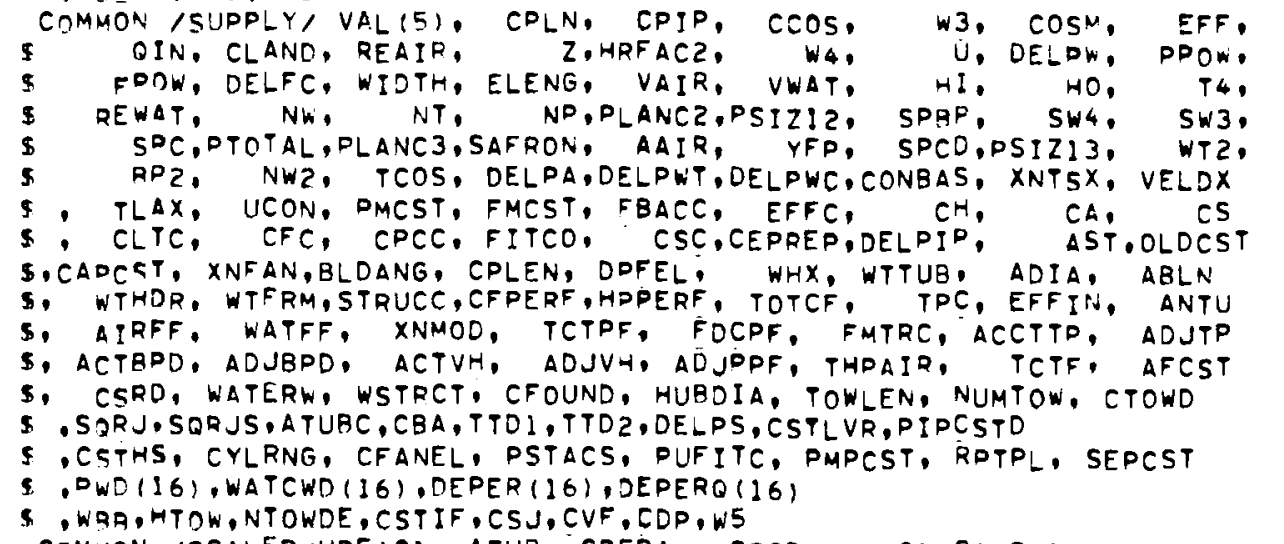

COMMON ISCALER/HRFACI. ATUB, CPERA, SSCD,PLANCI, CASSSI.

5 GATR, FAIR, HPAIR, HPWAT, WLRTP, PLANCTI

COMMON /WXD/ AI,ATOT,SFA,RFA, ACS

COMMON /VARVAR/ VTPER(16), VTA (16), VTAWB(16)

COMUON/DJS/DI, XDG, SF

COMUAN /SURFEX/ ODL.OUMLST (15)

COMMON/DLUG/TA, TAWB,TI,PATM, HIZ, HOZ, RMA, OSEC, RMEV, VALREO COMMON/COUNT/NSLOPE, FRST 3

COMMON/FAN/FANDAP (153,10), FANOAM $(153,10), F M C P F, F B C P F, P 2, R, S$,

$\$$ VE BEC, AFC,ELEV,EHR, LOOPER, CTMAX

COMMON /PROP/ CPW, THCONW. DENSIW, VISW, HFG.

* CPV, THCONV. DENSIV, VISV, SIGMA,

* CPa, thCona, densia, visa

DIMENSION PSATD(16), TSATO(16), WATCON(16, QPL (16)

NOB\JGL $=0$

WATCONT $=0.0$

NSLOPE $=0$

$Z D=Z$

VALRFQ $=Z$

DESIGN THERMAL EFFECTIVENESS OF THE DOWER PLANT TEFF3 = TEFF/HRFAC2

BI GCOM

BIGCOM

BI GCOM

BI GCOM

BI GCOM

BI GCOM

BI GCOM

BI GCOM

BI GCON

BI GCOM

BIGCOW

BIGCON

BI GCOM

BI GCOM

BIGCOM

BIGCON

SUPP $Y$

SUPPLY

SUPPLY

SUPPLY

SUPPLY

SUPPLY

SUPPLY

SUPPLY

SUPPLY

SUPP:Y

SUPPLY

SUPPLY

SUPP:Y

SUPPLY

SUDDLY

SUPPLY

SUPPLY

SCALER

SCALER

DELUGT

DELUGT

DELUGT

DELUGT

DELUGT

DELUGT

DELUST

DELUGT

DELUGT

DELUGT

DELUGT

DELUGT

DELUGT

DELUGT

DELUGT

DELUGT

DELUGT

DELUGT

DELUGT

DELUGT

OELUGT

DELUGT 


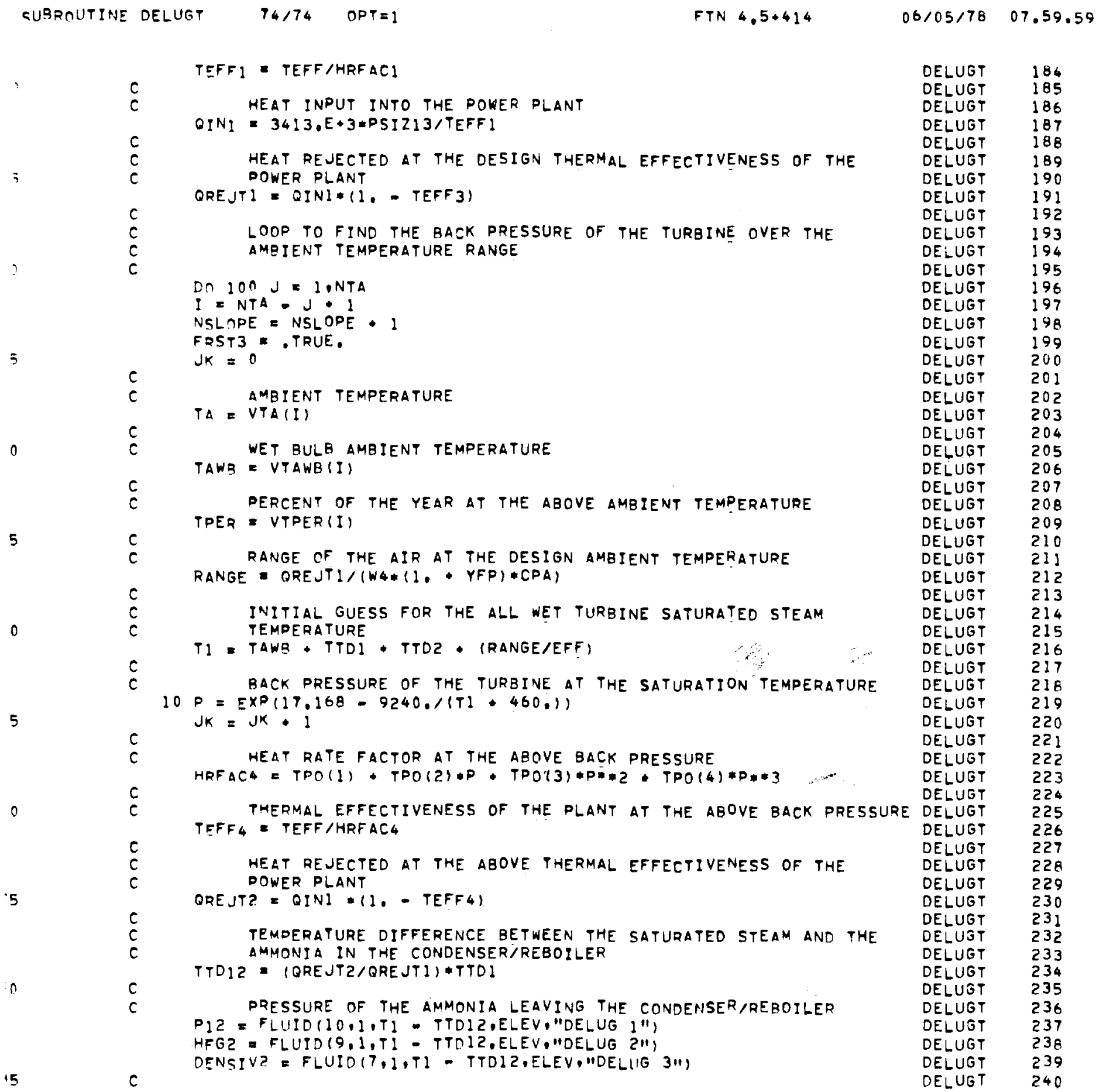

TMERMAL EFFECTIVENESS OF THE PLANT AT THE ABOVE BACK PRESSURE TEFF4 TEFF/HRFAC4

hEAT REJECTED at THE ABOVE THERMAL EFFECTIVENESS OF THE DOWER PLANT

DELUG

DELUGT

DELUGT

DELUGT

DELUGT

DELUGT

DELUGT

DELUGT

DELUGT

DELUGT

DELUGT

DELUGT

DELUGT

DELUGT

DELUGT

DELUGT

DELUGT

DELUGT

DELUGT

DELUGT

DELUGT

DELUGT

DELUGT

DELUGT

DELUGT

DELUGT

DELUGT

DELUGT

DELUGT

DELUGT

DELUGT

DELUGT

DELUGT

DELUGT

DELUGT

DELUGT

DELUGT

DELUGT

DELUGT 
TEMOERATURE DROP IN THE AMMONIA FROM THE BEGINNING TO THE END OF THE SUPPLY PIPING

30 TTD22 $=$ TI - TTO12 - TI

WRITE(S,500) TTDI, TTDI2, TTD2, TTO22, OREJT2, OREJTI,PIZ,HFG2, DENSIVZ, IDENSIV,W32, DELPS, DELPS2,P22, TI, WATCONT

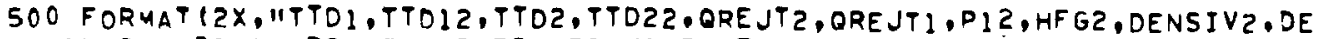
INSIV,W32, DELPS, DELPS2,P22,TI, WATCONTE", /, 2X,PG I2.5, $, 2 X, 4612.5 / 1$

INSIDE HEAT TRANSFER COEFFICIENT OF THE HEAT EXCHANGER WHEN IT IS DELUGED

GWATZ WW32/IZD*NW:3.14159*DI*0I/14.*144.)

PDW E VISW:CPW/THCONW

CALL HTCLOCDI, ELENG 12 , . ANG, GWAT2, $1, \ldots$, , PRW, DENSIW, DENSIV, VI SW *

1.41338 , VISV*.41338, THCONW, CDW, HI2,HTC,HTB,HTN,HTK,REWAT2)

AIR SIDE HEAT TRANSFER COEFFICIENT OF THE HEAT EXCHANGER WHEN IT IS DELUGED

GAIR2 = FACTOR VAIR $=D E N S I F=3600$.

IF(FDELUG.EQ."DELUG "I) CALL HOTERVIFAIRZ, HOZ, SIGMAG, ALPHA,XD. IGAIRZ,VISA)

IF (FDELUG.EQ."DELBAW ") CALL MTCH(GAIR2,HOZ)

DATH $=$ EXP $(3.39853-3,81835 E-5$ * ELEV $) / 29.92$

MASS FLOW RATE OF AIR THROUGH A UNIT PART OF THE HEAT EXCHANGER

$R M A=F A C T O R * V A I R * D E N S I F * 3600 . * A C S$

CALCULATION OF THE HEAT REJECTED FROM THE ALL WET HEAT EXCHANGER AND THE AMOUNT OF WATER USED IF IFOEL'UG.EQ. "DELUG

ii) CALL DELUG IF IFDELUG.EQ, "DELBAW

ii) CALL DELBAW IF KKCONV.EQ,1) RETURN QRE JTN = NWELENG QSEC RMEV = NWEELENG IF (NOBUG:,EQ.1) WRITE (6,60) TI,D, GWAT2, HI2,GAIR2, HOZ,TA, TAWB,TI, IPATM,RMA, QSEC, RMEV, QREJT N

$\begin{array}{ll}\text { DELUGT } & 241 \\ \text { DELUGT } & 242 \\ \text { DELUGT } & 243 \\ \text { DELUGT } & 244 \\ \text { DELUGT } & 245 \\ \text { DELUGT } & 246 \\ \text { DELUGT } & 247 \\ \text { DELUGT } & 248 \\ \text { DELUGT } & 249 \\ \text { DELUGT } & 250 \\ \text { DELUGT } & 251 \\ \text { DELUGT } & 252 \\ \text { DELUGT } & 253 \\ \text { DELUGT } & 254 \\ \text { DELUGT } & 255 \\ \text { DELUGT } & 256 \\ \text { DELUGT } & 257 \\ \text { DELUGT } & 258 \\ \text { DELUGT } & 259 \\ \text { DELUGT } & 260 \\ \text { DELUGT } & 261 \\ \text { DELUGT } & 262 \\ \text { DELUGT } & 263 \\ \text { DELUGT } & 264 \\ \text { DELUGT } & 265 \\ \text { DELUGT } & 266 \\ \text { DELUGT } & 267 \\ \text { DELUGT } & 268 \\ \text { DELUGT } & 269 \\ \text { DELUGT } & 270 \\ \text { DELUGT } & 271 \\ \text { DELUGT } & 272 \\ \text { DELUGT } & 273 \\ \text { DELUGT } & 274 \\ \text { DELUGT } & 275 \\ \text { DELUGT } & 276 \\ \text { DELUGT } & 277 \\ \text { DELUUGT } & 295 \\ \text { DELUGT } & 296 \\ \text { DELUGT } & 297 \\ \text { DELUGT } & 278 \\ \text { DELUGT } & 279 \\ \text { DELUGT } & 280 \\ \text { DELUGT } & 281 \\ \text { DELUGT } & 282 \\ \text { DELUGT } & 283 \\ \text { DELUGT } & 284 \\ \text { DELUGT } & 285 \\ \text { DELUGT } & 286 \\ \text { DELUGT } & 287 \\ \text { DELUGT } & 288 \\ \text { DELUGT } & 289 \\ \text { DELUGT } & 290 \\ \text { DELUGT } & 291 \\ \text { DEUGT } & 292 \\ \text { DE } & 293 \\ \text { DEG } & 294 \\ \text { DEG } & \end{array}$




$$
74 / 74 \quad \text { OPT }=1
$$

FTN $4.5+414$

$06 / 05 / 79$

07.59 .59

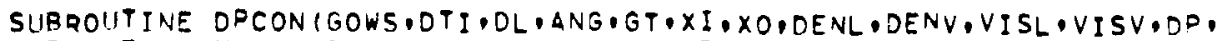
1 DPF, DPG,DPM, XLM,PHIL,PHIL , VF,FL,RL,RV)

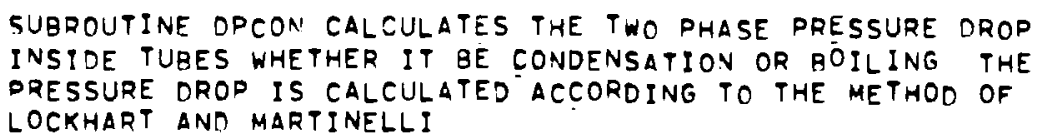

DPこOV

DPCON

DPCON

DPCON

$D P \cong O N$

DPCON

DPCON

DPCON

DPCON

DPCON

DPCON

DPCON

DPCON

DPCON

DPCON

DPCON

DPCON

DPCON

DPCON

DPEON

DPCON

DPCON

DPCON

DPCON

DPCON

DPEON

$D P C O N$

DPCON

DPCON

DPCON

DPCON

DPCON

$D P C O N$

DPCON

DPCON

DPCON

DPCON

DPCON

DPCON

DPCON

DPCON

DPCON

DPCON

DPCON

DPCON

DPCON

DPCON

DPCON

DPCON

DPCON

DPCON

DPCON

DPCON

DPCON

DPCON

DPCON

DPCON 


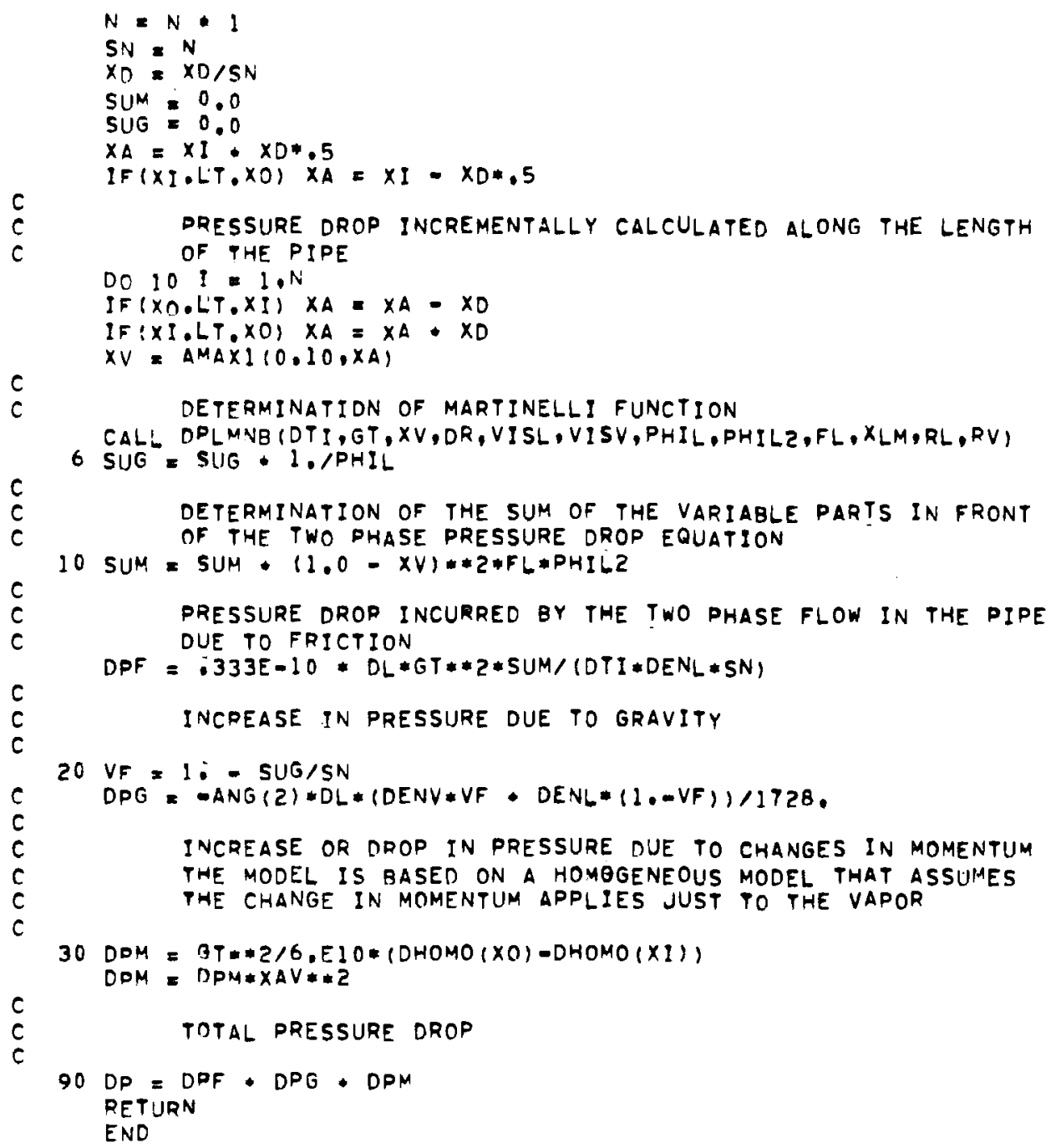




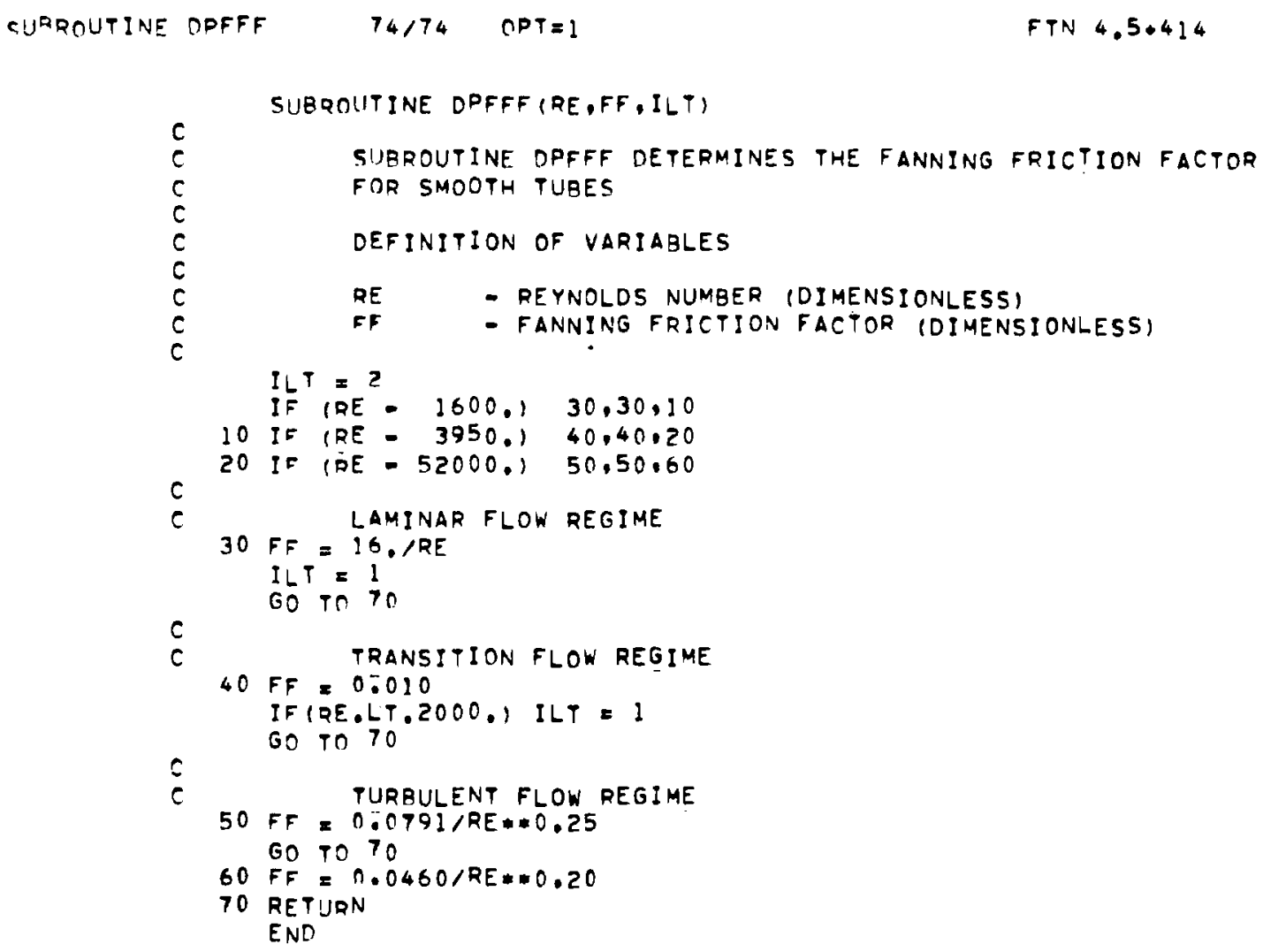

\begin{tabular}{ll} 
DPFFF & 2 \\
DPFFF & 3 \\
DPFFF & 4 \\
DPFFF & 5 \\
DPFFF & 6 \\
DPFFF & 7 \\
DPFFF & 8 \\
DPFFF & 9 \\
DPFFF & 10 \\
DPFFF & 11 \\
DPFFF & 12 \\
DPFFF & 13 \\
DPFFF & 14 \\
DPFFF & 15 \\
DPFFF & 16 \\
DPFFF & 17 \\
DPFFF & 18 \\
DPFFF & 19 \\
DPFFF & 20 \\
DPFFF & 21 \\
DPFFF & 22 \\
DPFFF & 23 \\
DPFFF & 24 \\
DPFFF & 25 \\
DPFFF & 26 \\
DPFFF & 27 \\
DPFFF & 28 \\
DPFFF & 29 \\
DPFFF & 30 \\
DPFFF & 31 \\
DPFFF & 32 \\
\hline
\end{tabular}


:UBROUTINE DPLMNE T4/74 OPTEI FTN $4.5 .414 \quad 06 / 05 / 78 \quad 07.59 .59$

SUBROUTINE OPLMNB (DTI,GT,XO,DR,VISL,VISV,PHIL,PHILZ,FL,XLM,RL,RV) DPLMNB

2

SUGROUTINE DPLMNB DETERMINES THE MARTINELLI FUNCTION FOR BIILING INSIDE OF TUBES BY CURVE FITS OF THE LOCKHARTMARTINELLI PARAMETER

DEFINITION OF VARIABLES

DR - DENSITY RATIO (LIQUID/VAPOR) (DIMENSIONLESS)

DT: - INSIDE DIAMETER OF THE TUBING (IN)

FL - LIQUID FANNING FRICTION FACTOR (DIMENSIONLESS)

F!) VAPOR FANNING FRICTION FACTOR (OIMENSIONLESS)

GP - MASS FLUX OF FLUID IN THE TUBE (LEM/(HR-SOFT))

DHIL - MARTINELLI FUNCTION (DIMENSIONLESS)

PHILZ - MARTINELLI FUNCTION SOUARED (DYMENSIONLESS)

RL - REYNODLS NUMBER LIQUID FRACTION

RV - REYNOLDS NUMBER VAPOR FRACTION

VISL - VISCOSITY OF THE LIQUID (CENTIPOSE)

VISV - VISCOSITY OF THE LIQUID VAPOR (CENTIPOSE)

XA - VAPOR QUALITY OF THE FLUID (DIMENSIONLESS)

XLM - LOCKHART-MARTINELLI PARAMETER (DIMENSIONLESS)

$X A=P N L I M(I, E-9, .9999, X 0)$

6

LIQUTD REYNOLDS NUMBER

$R L=D T I * G T *(1,-X A) /(V I S L * 29$.

VAPOR REYNOLOS NUMBER

$R V=D T I * G T * X A /(V I S V * 29$.

c

FANNING FRICTION FACTOR FOR THE LIOUID

CALL DDFFF IRL,FL,ILTL)

c

FANNING FRICTION FACTOR FOR THE VAPOR

CALL DPFFF IRV,FV.ILTV:

LOCKHART-MARTINELLI PARAMETER

$X L M=S R R T(F L /(F V * D R)) *(1,-X A) / X A$

VISCOUS FLOW LIOUIO - VISCOUS FLOW VAPOR

IF (ILTL.EQ.I.AND. ILTV.EQ.1) JMART = 1

TURBULENT FLOW LIOUID - VISCOUS FLOW VAPOR

IF(ILTL.EQ.2.AND.ILTV.EQ.I) JMART E 2

VISCOUS FLOW LIOUID - TURBULENT FLOW VAPOR

IF (ILTL,EQ.I.AND.ILTV.EQ.2) JMART $=3$

$\mathrm{c}$

I

TURBULENT FLOW LIOUID - TURBULENT FLOW VAPOR

IF (ILTL,EQ.2,AND.ILTV.EQ.2) JMART $=4$

c

MARTIMELLI FUNCTION

PHIL E CMAPTIN IXLM, JMART, I)

MARTINELLI FUNCTION SQUARED

PHIL2 = PHIL*PHIL

RETURN

DPEMNA

DPLMNB

DPLMNB

DPLMNB

DPLMNB

DPLMNB

DPLMNB

DPLMNB

DPLMNE

DPLMNB

DPLMNB

DPLMNB

DPLMNB

DPLMNG

DPLMNE

DPLMNS

DPLMNE

DPLMNB

DPLMNZ

DPLMNB

DPLMNB

DPLMNB

DPLMNB

DPLMNB

DPLMNB

DPLMNB

DPLMNB

DPLMNB

DPLMNS

DPLMNE

DPLMNB

DPLMNB

DPLMNB

DPLMNB

DPLMVB

DPLMNE

DPLMNB

DPLMNB

DPLMNB

DPLMNB

DPLMNB

DPLMNB

DPLMNB

DPLMNB

DPLMNB

DPLMNB

DPLMNB

DPLMNE

DPLMNB

OPLMNB

DPLMNE

DPLMNB

DPLMNS

DPLMNB 
URROUTINE ERRMSG $74 / 74$ OPT =

FTN $4.5+414$

$06 / 05 / 78$

07.59 .59

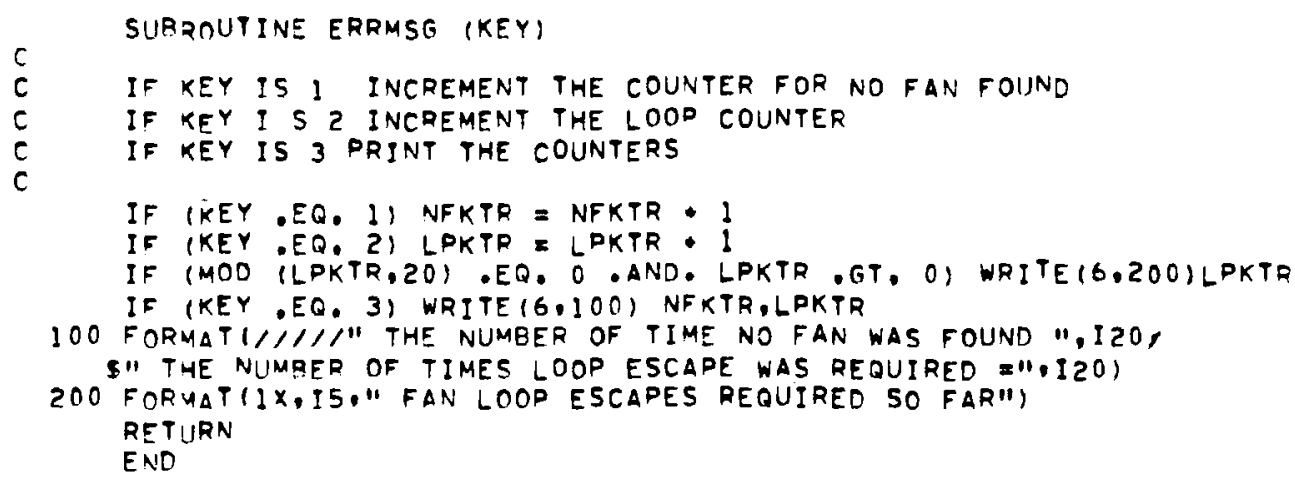

$\begin{array}{ll}\text { ERRMSG } & 2 \\ \text { ERRMSG } & 3 \\ \text { ERRMSG } & 4 \\ \text { ERRMSG } & 5 \\ \text { ERRMSE } & 6 \\ \text { ERRMSG } & 7 \\ \text { ERRMSG } & 8 \\ \text { ERRMSG } & 9 \\ \text { ERRMSG } & 10 \\ \text { ERRMSG } & 11 \\ \text { ERRMSG } & 12 \\ \text { ERRMSG } & 13 \\ \text { ERRMSG } & 14 \\ \text { ERRMSG } & 15 \\ \text { ERRMSG } & 16\end{array}$


$74 / 74 \quad$ ODT $=1$

SUBROUTINE FAN SELECTS THE OPTIMUM FAN SYSTEM FOR PHE PARTICIILAR HEAT EXCHANGER GEOMETRY SELECTED. FOR THE PARTICULAR FAN SYSTEM THE FOLLOWING PARAMETER ARE SPECIFIED-

\section{BLADE DIAMETER}

2. HUB DIAMETER

3. NUMBER OF BLADES DER FAN

4. BLADE ANGLE

KNOWING THE NUMAER OF FANS AND THE PACKING FACTOR OF THE FANS, THE CIRCUIALR TOWEG DIAMETER CAN EASILY BE DETERMINED. THE COST OF THE FAN SYSTEM IS THEN FOUND. THE FAN SYSTEN RESULTING IN THE MINIMUM COST IS SPECIFIED AS THE OPT IMUM,

IN THIS SUAROUTINE THERE ARE TWO ARRAYS WHICH CONTAIN DATA

FOR THE FOLLOWING FAN DIAMETERS $(24,26.2 B, 30.40 .60)$. FOR

EACH FAN OIAMETER THERE IS PERFORMANCE DATA FOR FANS WITH

DIFFERENT NUMBER OF BLADES ANO BLADE ANGLES. THE DATA

ARRAYS FANDAP CONTAINS THE FOLLOWING INFORMATIONFANDAD (I.J)

$J=1.5$ - COEFFICIENTS FOR THIRD ORDER CURVE FIT OF VOLUMETRIC FLOW RATE VERSUS TOTAL PRESSURE DROP ACROSS THE FAN

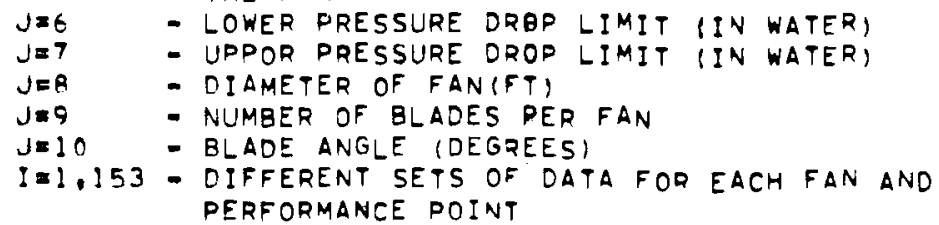




$$
74 / 74 \quad \text { OPT }=1 \quad \text { FTN } 4.5+414 \quad 06 / 05 / 78 \quad 07.59 .59
$$

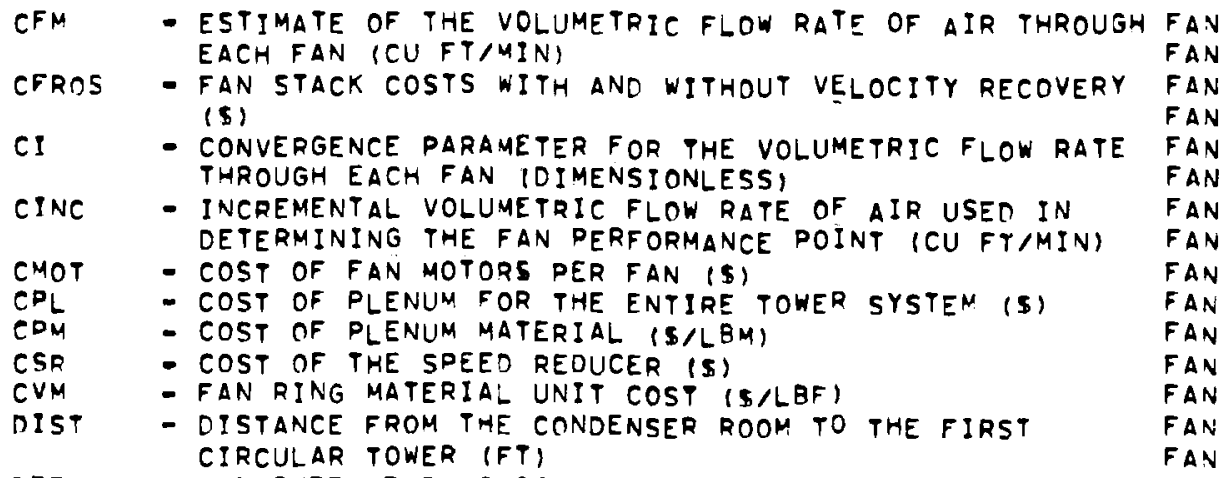

\begin{tabular}{ll} 
FAN & 59 \\
FAN & 60 \\
FAN & 61 \\
FAN & 62 \\
FAN & 63 \\
FAN & 64 \\
FAN & 65 \\
FAN & 66 \\
FAN & 67 \\
FAN & 68 \\
FAN & 69 \\
FAN & 70 \\
FAN & 71 \\
FAN & 72 \\
FAN & 73 \\
FAN & 74 \\
FAN & 75 \\
FAN & 76 \\
FAN & 77 \\
FAN & 78 \\
FAN & 79 \\
FAN & 80 \\
FAN & 81 \\
FAN & 82 \\
FAN & 83 \\
FAN & 84 \\
FAN & 85 \\
FAN & 86 \\
FAN & 87 \\
FAN & 88 \\
FAN & 89 \\
FAN & 90 \\
FAN & 91 \\
FAN & 92 \\
FAN & 93 \\
FAN & 94 \\
FAN & 95 \\
FAN & 96 \\
FAN & 97 \\
FAN & 96 \\
FAN & 99 \\
FAN & 100 \\
FAN & 101 \\
FAN & 102 \\
FAN & 103 \\
FAN & 104 \\
FAN & 105 \\
FAN & 106 \\
FAN & 107 \\
FAN & 108 \\
FAN & 109 \\
FAN & 110 \\
FAN & 111 \\
FAN & 112 \\
FAN & 113 \\
FAN & 114 \\
FAN & 115 \\
\hline &
\end{tabular}


$74 / 74 \quad O D T=1 \quad F T N 4.5+414 \quad 06 / 05 / 79 \quad 07.59 .59$

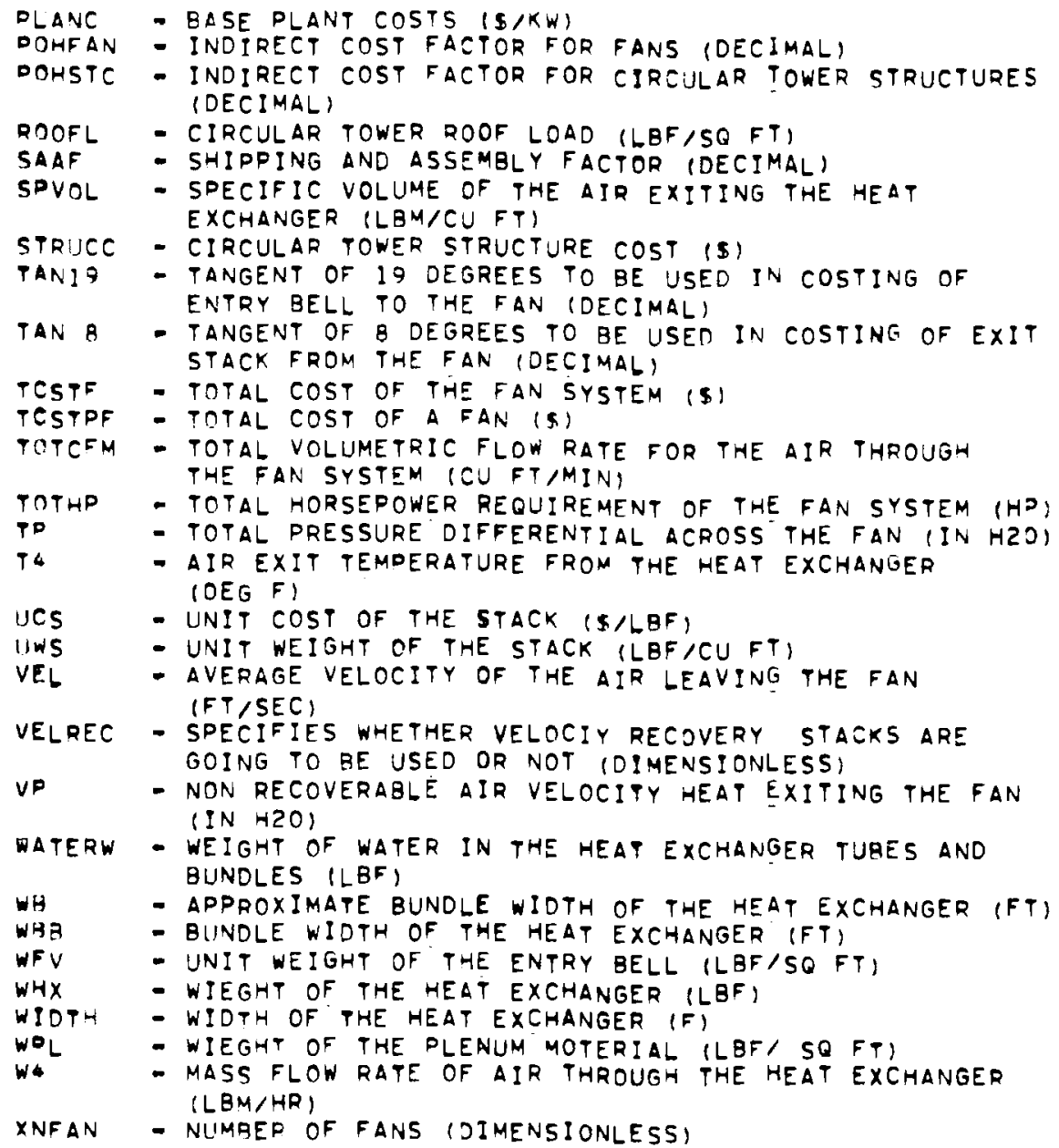

REAL NAF

LOGICAL LOODER

COMMON/FAN/ FANDAP $(153,10)$, FANDAH $(153,10)$,FMCPF, FBCPF,PZ,R, S.

S VELDEC, AFC, ELEV. EHR, LOOPER, CTMAX

LOGICAL FIRST, FRST, FXTEMP,FXTVAR,FXTTTD, FXTLNG COMMON

5 $\triangle F T R, A L P H A, A N G(3)$

- BDLjM(16)

S CADF, CSSPKW, CONF, CONL, COSTL, CAPCHG, CONMAT, CONMAZ, CAF

FAN $\quad 116$

FAN 118

FAN 110

FAN $\quad 120$

FAN 121

FAN 122

FAN 123

FAN 12

FAN 125

FAN 126

FAN 127

FAN 125

FAN 129

FAN 130

FAN 13:

FAN 132

133

134

135

136

137

138

139

140

141

142

FAN

150

FAN 15:

FAN $\quad 152$

FAN 153

FAN 150

FAN 155

FAN 156

FAN 157

FANDEK

FANDEK 3

FANDEK

BIGCOM

BIGCOM 3

BIGCOM

BIGCOM

BIGCOM

BIGCOM

BIGCOM

BIGCON

$5, D F I N, D E E D$
5 DEF, EFS

\$ :FCR,FIRST,FIXL,FCOS, FFHX,FXTEMP, FACTOR,FRST, FACTORD, F SHOT, FDELUG

5 , FIXV,FIXTTD,FXTVAR,FXTTTC,FXTING

5 , GAEFF

F. HXNP,HDCST.HCD

BIECOM

RI GCOM

BIGCOM

BISCOM 
5. ITMAX, ITMIN

- JCONS

, KCONV, KALEXT

5. MXEXT

5 .NPA,NSIDES, NBUNHI COMMON

S OOR

- PSIZE, PER, pHCOS, PLANC, PFACT, POMDPL

5 POHBAF, POHFAN, POHLEC, POHCIR, POHCND, POHSTC, POHSCL

5 , RBP,REDUCE, REDUCV, ROOFL, RHOUT

S QREJ, QREDUCE, QREDUCV

S. SJGMAG, SAAF, SEGL

S:TO, PPO (4), TEFF, TLIM, THFIN, TLPRA, TFIX

$5, T C D, T W$

5 .UCS. UWS

5 WEV. WATCONA, WATCOST

S.VAS (5)

5. XDEDA, $X W, X \cap$

COMMON /SUPPLY, VAL (5), CPLN, CPIP, CCOS, W3, COSM, EFF, 5 OIN, CLAND, REAIR, Z,HRFACZ, W: U, DELPW: PPOW:

5 FPOW, DELFC, WIDTH, ELENG, VAIR, VWAT, HI, HO, T4, 5 REWAT, NW, NT, NP,PLANCZ,PSIZI2, SPBP, 5W4, SW3. 5 SPC,PTOTAL,PLANC3,SAFRON, AAIR, YFP, SPCD,PSIZ13, WTZ. 5 BPZ, NWZ, TCOS, DELPA,DELPWT, DELPWC, CONBAS, XNTSX, VELDX

5. TLAX, UCON, PMCST, FMCST, FBACC, EFFC, CH, CA, CS

- CLTC, CFC, CPCC, FITCO, CSC,CEPREP,DELPIP, AST,OLDCST

\$. CAPCST, XNFAN, BLDANG, CPLEN, DPFEL, WHX, WTTUB, ADIA, ABLN

S, WTHOR, WTFRM, STRUCC,CFPERF, HDPERF, TOTCF, TPC, EFFIN, ANTU

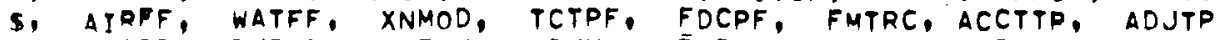

5, $\triangle C T B P D, \triangle D J B P D, \quad A C T V H, A D J V H, \triangle D D J P P$, THPAIR, TCTF, $\triangle F C S T$

\$, CSRD, WATERW, WSTRCT, CFOUND, HUBDIA, TOWLEN, NUMTOW, CTOWD

5. SQR J,SORJS, ATUBC, CBA, TTDI, TTOZ, DELPS, CSTLVR,PIPCSTD

5 , CSTHS, CYLRNG, CFANEL, PSTACS, PUFITC, PMPCST, RPTPL, SEPCST

5. DWD (16), WATCWD (16), DEPER (16), DEPERO (16)

S.WBR,HTOW,NTOWDE, CSTIF, CSJ,CVF, CDP,W5

COMMON /SCALER/HRFACI, ATUB, CPERA, SSCD,PLANCI,CASSSI.

S GAIR, FAIR, HPAIR, HPWAT, WLRTP, PLANCTI

COMMON /HEADEX, CHH,CN,CHM, CMW,CRJ,CWJ,CST,CMO,CSR, CSM,PITCHF, CPM, 1 PRESS - HEDMAT, HEDTYP, NPASS, WB, TSP, CSH, CSHP

COMMON /FNLOOP, LFB.LFE

COMMON /PLCOST/ WPL

COMMON /INDIS/ DIST

COMMON /PME/ PMELEC

COMMON/TOTAL/RECVEL,A6O,EA6O, VPCOEF, STATP

DATA (FANDAPI $1 . J), J=1,10)$,

$\$ .7961 \quad 0.6520 \quad 0.316$

DATA (FANDAPi $2, J), J=i, 240$;,

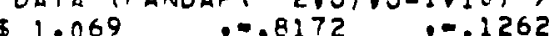

SDATA (FANDAPi $3, j), J=i, 10)$,

01.635 .

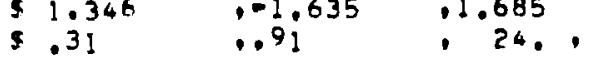

$, 11.944, .08 .252$

DATA (FANDAP( $4, \mathrm{~J}), J=1,10)$ )

$\$ .5643 \quad .4 .692 \quad .014 .336 \quad .15 .960,0.6062$.

s.365 $1.94 \quad 24.16 ., 12.1$

DATA (FANDAP, $5, J), J=1,10)$,

6.090

$$
. .2533 \quad 0.02331
$$

6. 8.1

.-1.666

. .5886 


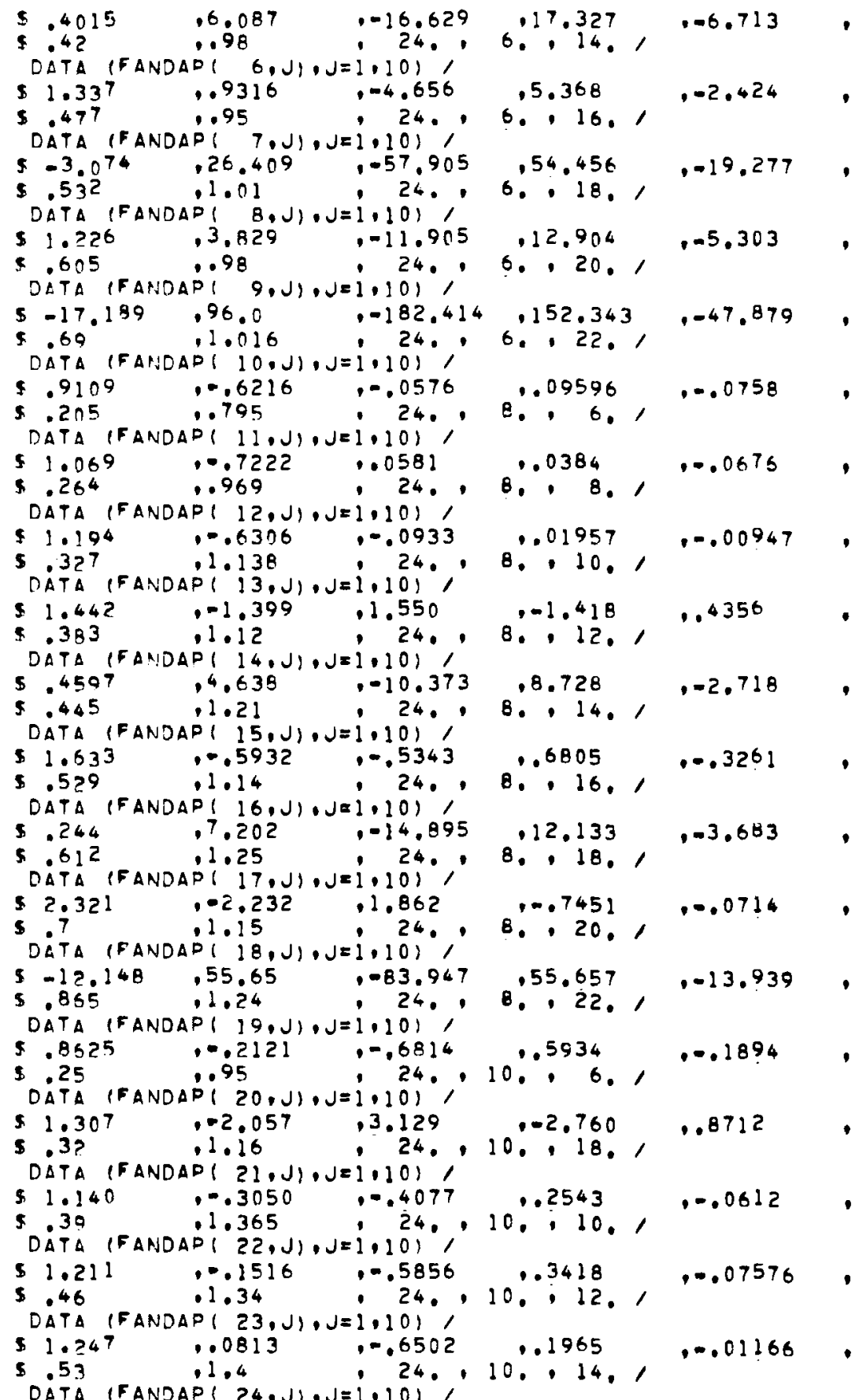

\begin{tabular}{|c|c|}
\hline $\begin{array}{l}\text { FAN } \\
\text { FAN }\end{array}$ & $\begin{array}{l}182 \\
183\end{array}$ \\
\hline $\begin{array}{l}F A N \\
F A N\end{array}$ & $\begin{array}{l}186 \\
185\end{array}$ \\
\hline $\begin{array}{l}F \Delta N \\
F A N \\
F \Delta N\end{array}$ & $\begin{array}{l}186 \\
187 \\
188\end{array}$ \\
\hline FAN & 189 \\
\hline $\begin{array}{l}\text { FAN } \\
\text { FAN }\end{array}$ & $\begin{array}{l}190 \\
191\end{array}$ \\
\hline$F A V$ & 192 \\
\hline$F A N$ & 193 \\
\hline$F \Delta N$ & 196 \\
\hline$F \Delta N$ & 195 \\
\hline FAN & 196 \\
\hline FAN & 197 \\
\hline FAN & 198 \\
\hline FAN & 199 \\
\hline FAV & 200 \\
\hline FAN & 201 \\
\hline FA:V & 202 \\
\hline FAN & 203 \\
\hline FAN & 204 \\
\hline FAN & 205 \\
\hline FAN & 206 \\
\hline FAN & 207 \\
\hline FAN & 208 \\
\hline FAN & 209 \\
\hline FAN & 210 \\
\hline $\begin{array}{l}F A N \\
F A N\end{array}$ & $\begin{array}{l}211 \\
212\end{array}$ \\
\hline FAN & 213 \\
\hline $\begin{array}{l}F A N \\
F A N\end{array}$ & $\begin{array}{l}214 \\
215\end{array}$ \\
\hline FAN & 216 \\
\hline FAN & 217 \\
\hline$F \triangle N$ & 218 \\
\hline FAN & 219 \\
\hline FAN & 220 \\
\hline $\begin{array}{l}F A N \\
F A N\end{array}$ & $\begin{array}{l}221 \\
222\end{array}$ \\
\hline FAV & 223 \\
\hline FAN & 224 \\
\hline$F A N$ & 225 \\
\hline FAN & 226 \\
\hline FAN & 227 \\
\hline FAN & 228 \\
\hline FAN & 229 \\
\hline FAN & 230 \\
\hline $\begin{array}{l}\text { FAN } \\
\text { FAN }\end{array}$ & $\begin{array}{l}231 \\
232\end{array}$ \\
\hline FAN & 233 \\
\hline FAN & 234 \\
\hline $\begin{array}{l}F A N \\
F A N\end{array}$ & $\begin{array}{l}235 \\
236\end{array}$ \\
\hline FAN & 237 \\
\hline FAN & 238 \\
\hline
\end{tabular}




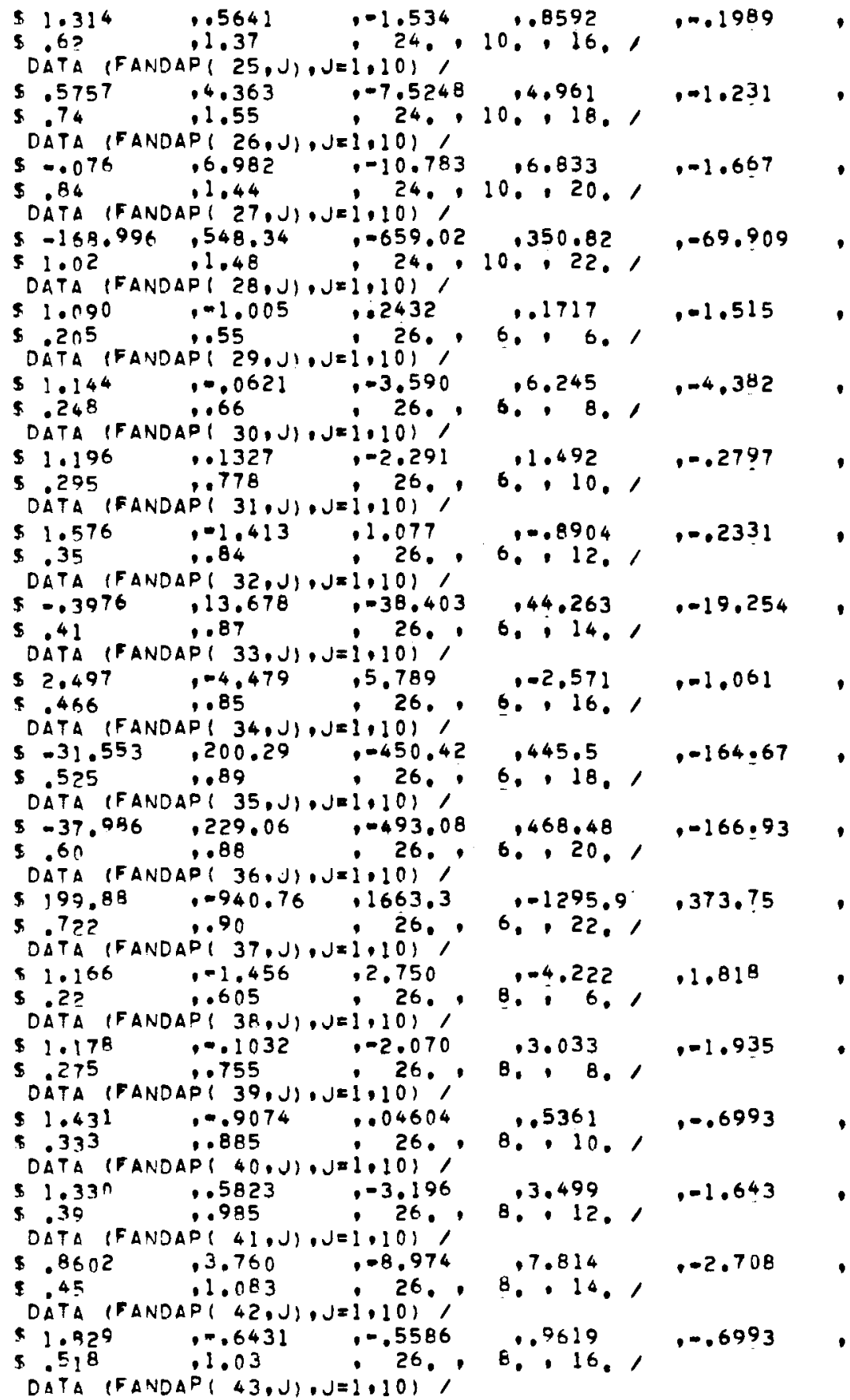

\begin{tabular}{ll} 
FAN & 239 \\
FAN & 240 \\
FAN & 241 \\
FAN & 242 \\
FAN & 243 \\
FAN & 244 \\
FAN & 245 \\
FAN & 246 \\
FAN & 247 \\
FAN & 248 \\
FAN & 249 \\
FAN & 250 \\
FAN & 251 \\
FAN & 252 \\
FAN & 253 \\
FAN & 254 \\
FAN & 255 \\
FAN & 256 \\
FAN & 257 \\
FAN & 258 \\
FAN & 259 \\
FAN & 260 \\
FAN & 261 \\
FAN & 262 \\
FAN & 263 \\
FAN & 264 \\
FAN & 265 \\
FAN & 266 \\
FAN & 267 \\
FAN & 268 \\
FAN & 269 \\
FAN & 270 \\
FAN & 271 \\
FAN & 272 \\
FAN & 273 \\
FAN & 274 \\
FAN & 275 \\
FAN & 276 \\
FAN & 277 \\
FAN & 278 \\
FAN & 279 \\
FAN & 280 \\
FAN & 281 \\
FAN & 282 \\
FAN & 283 \\
FAN & 284 \\
FAN & 285 \\
FAN & 286 \\
FAN & 287 \\
FAN & 288 \\
FAN & 289 \\
FAN & 290 \\
FAN & 291 \\
FAN & 292 \\
FAN & 293 \\
FAN & 294 \\
FAN & 295 \\
& \\
\hline
\end{tabular}




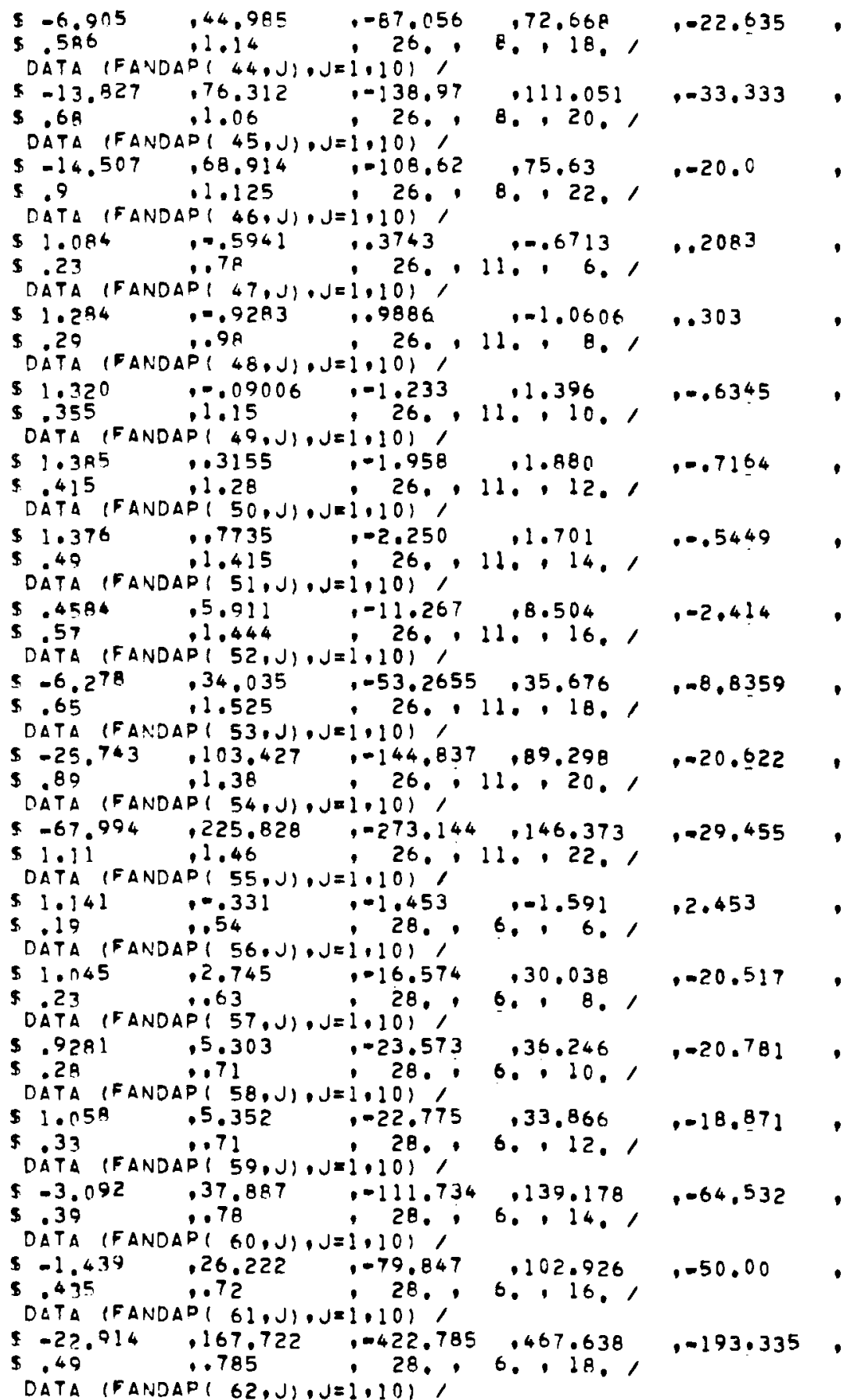

\begin{tabular}{|c|c|}
\hline$F A N$ & 276 \\
\hline$F A N$ & 297 \\
\hline FAN & 298 \\
\hline FAN & 299 \\
\hline FAN & 300 \\
\hline $\begin{array}{l}\text { FAV } \\
\text { FAN }\end{array}$ & $\begin{array}{l}301 \\
302\end{array}$ \\
\hline FAV & 303 \\
\hline FAN & 304 \\
\hline$F A V$ & 305 \\
\hline$F A N$ & 306 \\
\hline$F A N$ & 307 \\
\hline FAV & 308 \\
\hline$F A N$ & 309 \\
\hline F $\triangle N$ & 310 \\
\hline$F \triangle N$ & 311 \\
\hline FAV & 312 \\
\hline FAV & 313 \\
\hline FAN & 314 \\
\hline FAN & 315 \\
\hline FAN & 316 \\
\hline FAN & 317 \\
\hline FAN & 318 \\
\hline FAN & 319 \\
\hline FAN & 320 \\
\hline FAN & 321 \\
\hline FAN & 322 \\
\hline FAN & 323 \\
\hline FAN & 324 \\
\hline FAV & 325 \\
\hline$F \Delta N$ & 326 \\
\hline FAN & 327 \\
\hline FAN & 328 \\
\hline FAN & 329 \\
\hline FAN & 330 \\
\hline FAN & 331 \\
\hline FAN & 332 \\
\hline$F A V$ & 333 \\
\hline FAN & 334 \\
\hline$F \Delta N$ & 335 \\
\hline FAN & 336 \\
\hline FAN & \\
\hline FAN & 338 \\
\hline FAN & 339 \\
\hline FAN & \\
\hline$F A N$ & 341 \\
\hline FAN & 342 \\
\hline FAN & 343 \\
\hline FAN & 344 \\
\hline FAN & 345 \\
\hline FAN & 346 \\
\hline FAN & 347 \\
\hline FAN & 348 \\
\hline FAN & 349 \\
\hline FAN & 350 \\
\hline FAN & 35 \\
\hline FAN & 352 \\
\hline
\end{tabular}




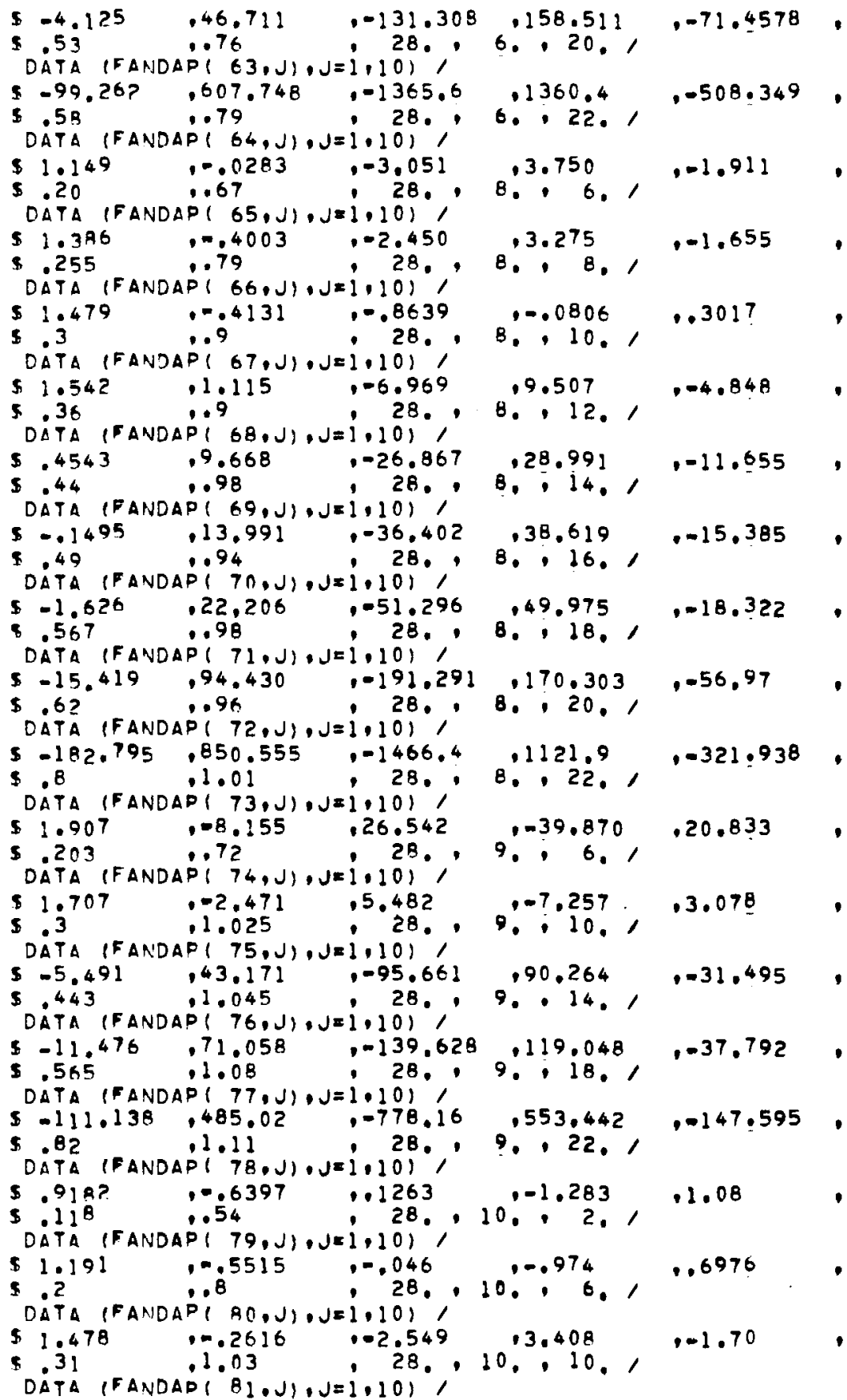

\begin{tabular}{|c|c|}
\hline FAN & 353 \\
\hline $\begin{array}{l}F A N \\
F A N\end{array}$ & $\begin{array}{l}354 \\
355\end{array}$ \\
\hline FAN & 356 \\
\hline FAN & 357 \\
\hline$F A N$ & 358 \\
\hline FAN & 359 \\
\hline FAN & 360 \\
\hline FAN & 361 \\
\hline FAN & 362 \\
\hline FAN & 363 \\
\hline$F A N$ & 364 \\
\hline FAN & 365 \\
\hline FAN & 366 \\
\hline$F \Delta N$ & 367 \\
\hline FAN & 368 \\
\hline FAN & 369 \\
\hline FAN & 370 \\
\hline FAN & 371 \\
\hline FAN & 372 \\
\hline FAN & 373 \\
\hline FAN & 374 \\
\hline FAN & 375 \\
\hline FAN & 376 \\
\hline$F A N$ & 377 \\
\hline FAN & 378 \\
\hline FAN & 379 \\
\hline FAN & 380 \\
\hline FAN & 381 \\
\hline FAN & 382 \\
\hline FAN & 383 \\
\hline FAN & 384 \\
\hline FAN & 385 \\
\hline FAN & 386 \\
\hline FAN & 387 \\
\hline FAN & 388 \\
\hline FAN & 389 \\
\hline FAN & 390 \\
\hline FAN & 391 \\
\hline FAN & 392 \\
\hline FAN & 393 \\
\hline FAN & 394 \\
\hline FAN & 395 \\
\hline FAN & 396 \\
\hline $\begin{array}{l}\text { FAN } \\
\text { FAN }\end{array}$ & $\begin{array}{l}397 \\
398\end{array}$ \\
\hline FAN & 399 \\
\hline FAN & 400 \\
\hline FAN & 401 \\
\hline$F A N$ & 402 \\
\hline FAN & 403 \\
\hline FAN & 404 \\
\hline FAN & 405 \\
\hline FAN & 406 \\
\hline FAN & 407 \\
\hline FAN & 406 \\
\hline$F A_{N}$ & 409 \\
\hline
\end{tabular}




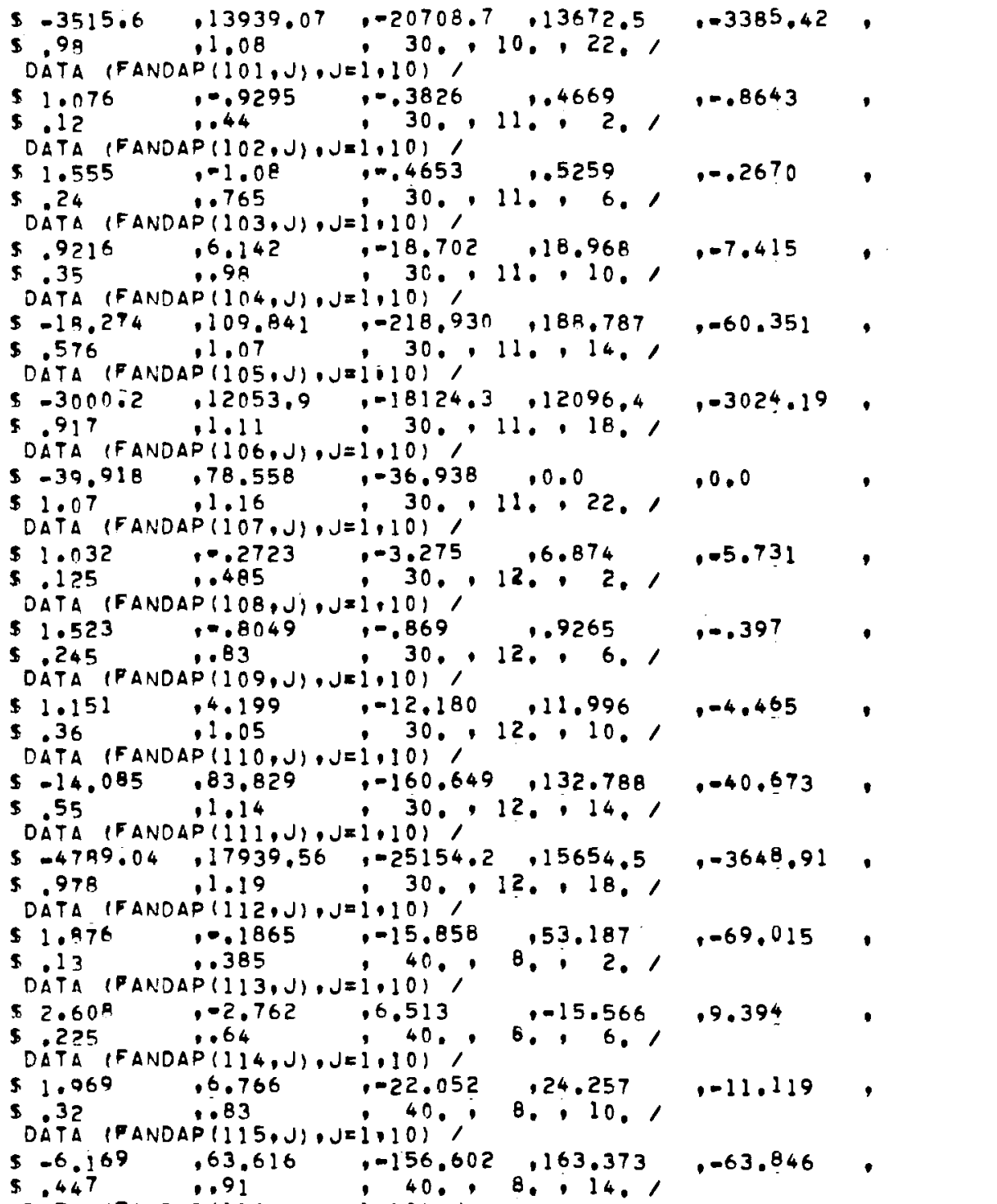




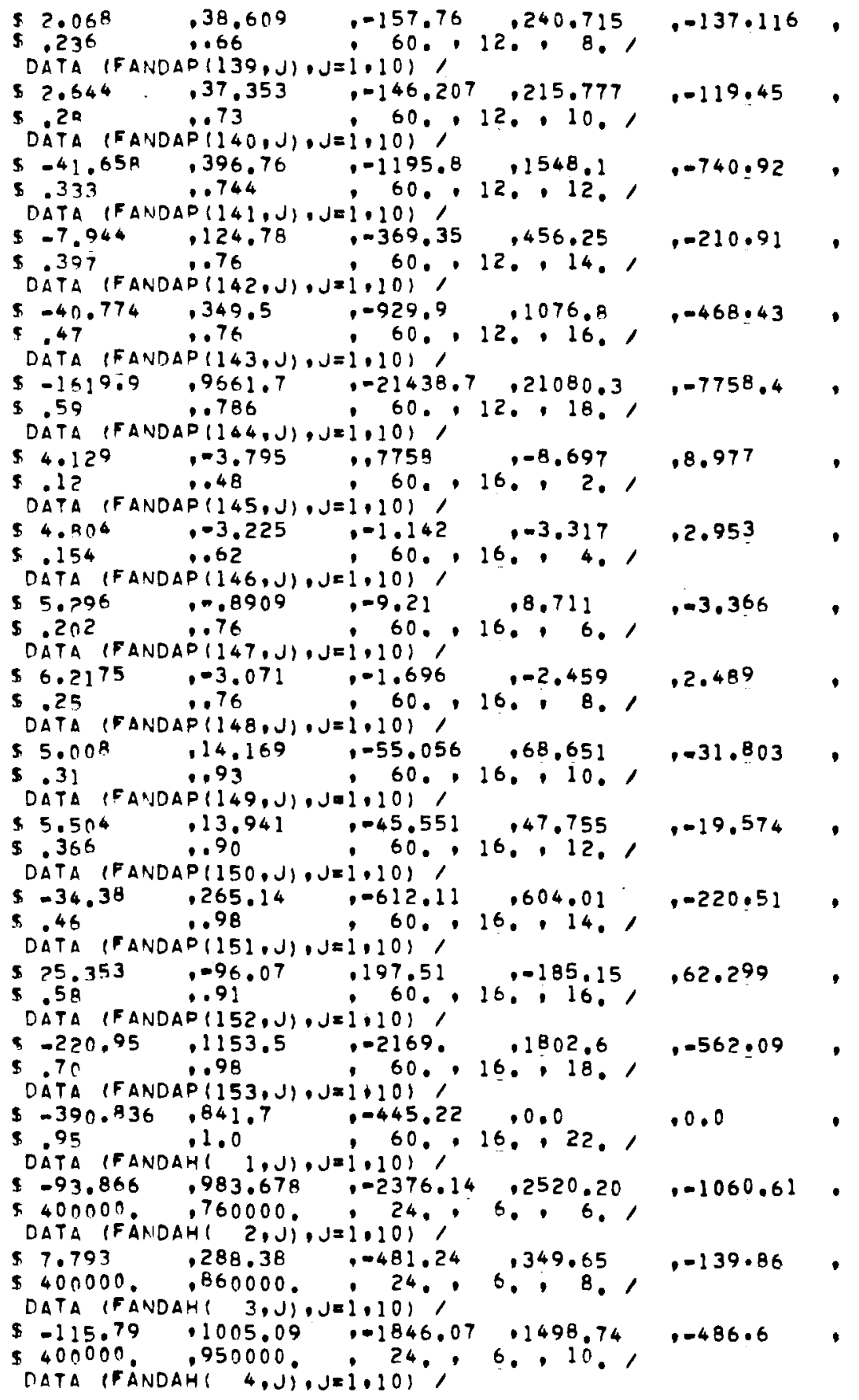

\begin{tabular}{ll} 
& \\
FAN & 581 \\
FAN & 582 \\
FAN & 583 \\
FAN & 584 \\
FAN & 585 \\
FAN & 586 \\
FAN & 587 \\
FAN & 588 \\
FAN & 589 \\
FAN & 590 \\
FAN & 591 \\
FAN & 592 \\
FAN & 593 \\
FAN & 594 \\
FAN & 595 \\
FAN & 596 \\
FAN & 597 \\
FAN & 598 \\
FAN & 599 \\
FAN & 600 \\
FAN & 601 \\
FAN & 602 \\
FAN & 603 \\
FAN & 604 \\
FAN & 605 \\
FAN & 606 \\
FAN & 607 \\
FAN & 608 \\
FAN & 609 \\
FAN & 610 \\
FAN & 611 \\
FAN & 612 \\
FAN & 613 \\
FAN & 614 \\
FAN & 615 \\
FAN & 616 \\
FAN & 617 \\
FAN & 618 \\
FAN & 619 \\
FAN & 620 \\
FAN & 621 \\
FAN & 622 \\
FAN & 623 \\
FAN & 624 \\
FAN & 625 \\
FAN & 626 \\
FAN & 627 \\
FAN & 628 \\
FAN & 629 \\
FAN & 630 \\
FAN & 631 \\
FAN & 632 \\
FAN & 633 \\
FAN & 634 \\
FAN & 635 \\
FAN & 636 \\
FAN & 637 \\
& \\
\hline
\end{tabular}




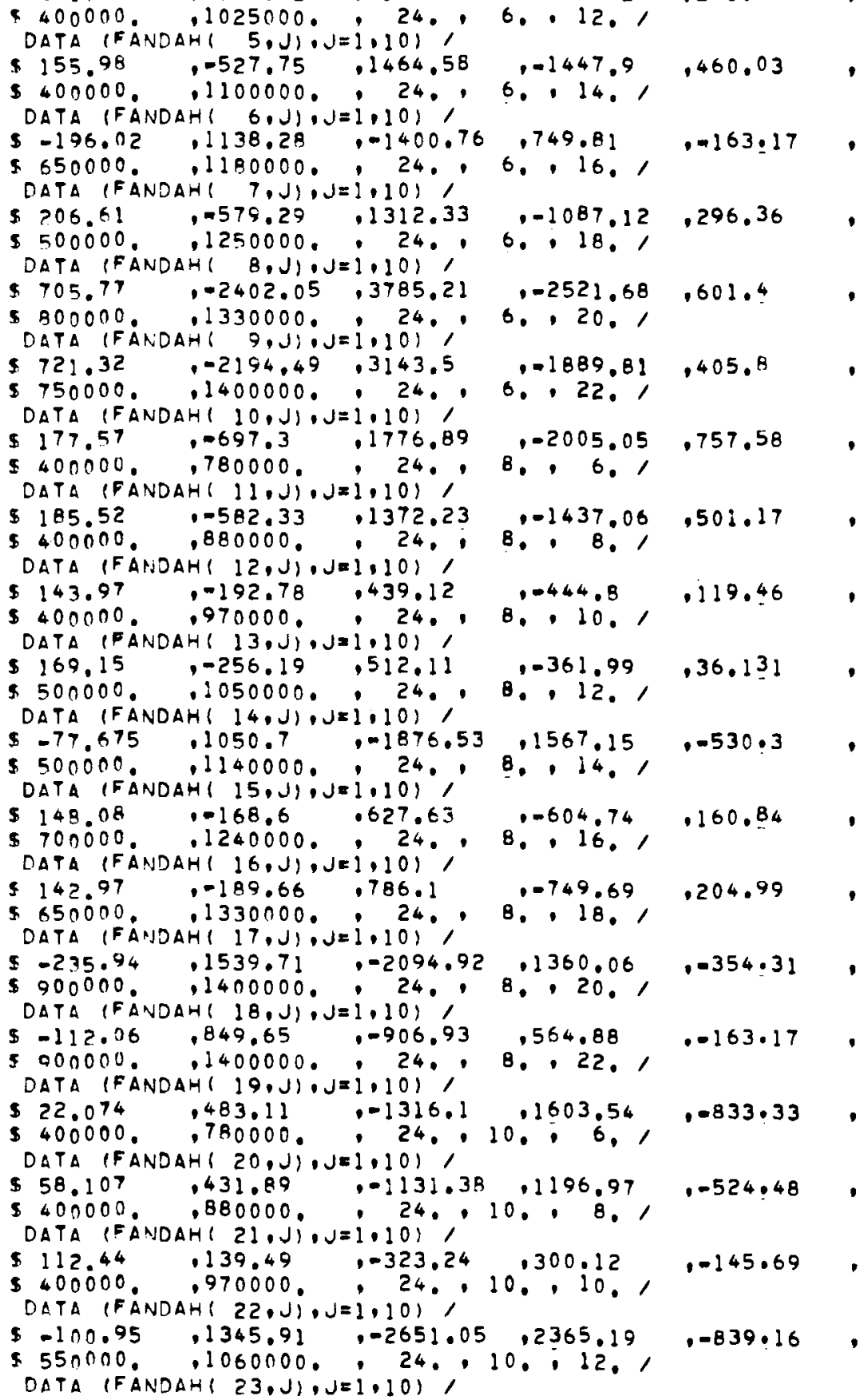

$.0848 .42 .281 .78 \quad$ FAN

FAV

FAN

FAN

FAN

FAN

FAN

FAN

FAN

FAN

FAV

FAV

$F \triangle N$

FAN

FAN

$F A N$

$F A N$

FAN

FAN

FAN

FAN

FAN

FAN

FAN

$F A N$

FAN

FAN

FAV

FAN

FAN

FAV

$F A N$

$F \triangle N$

$F A N$

FAN

FAN

FAN

$F \Delta V$

FAN

FAN

FAN

FAN

$F A N$

$F A N$

FAN

FAN

FAN

FAN

FAN

FAV

FAV

FAV

FAN

FAN

FAN

FAN

FAN 


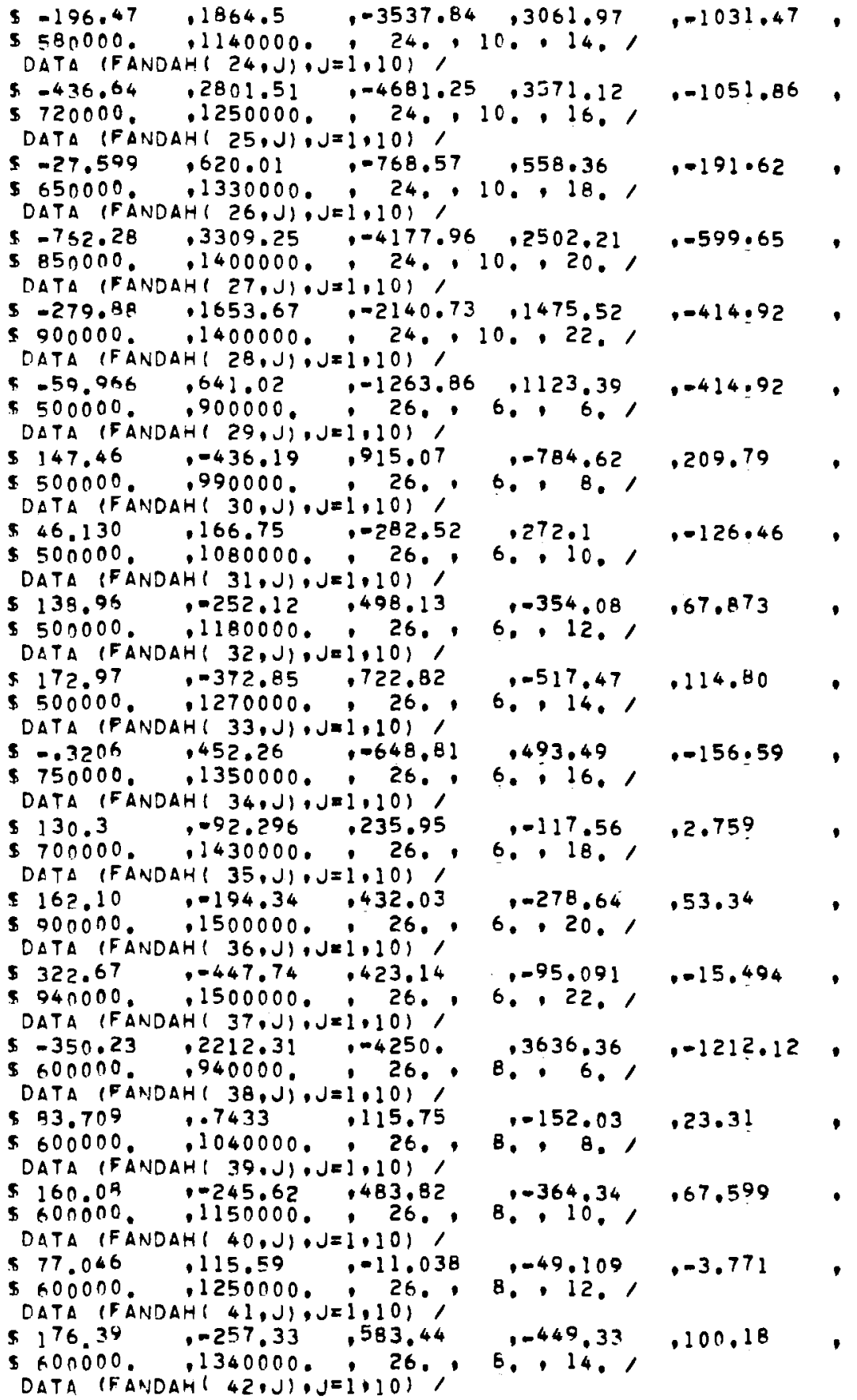




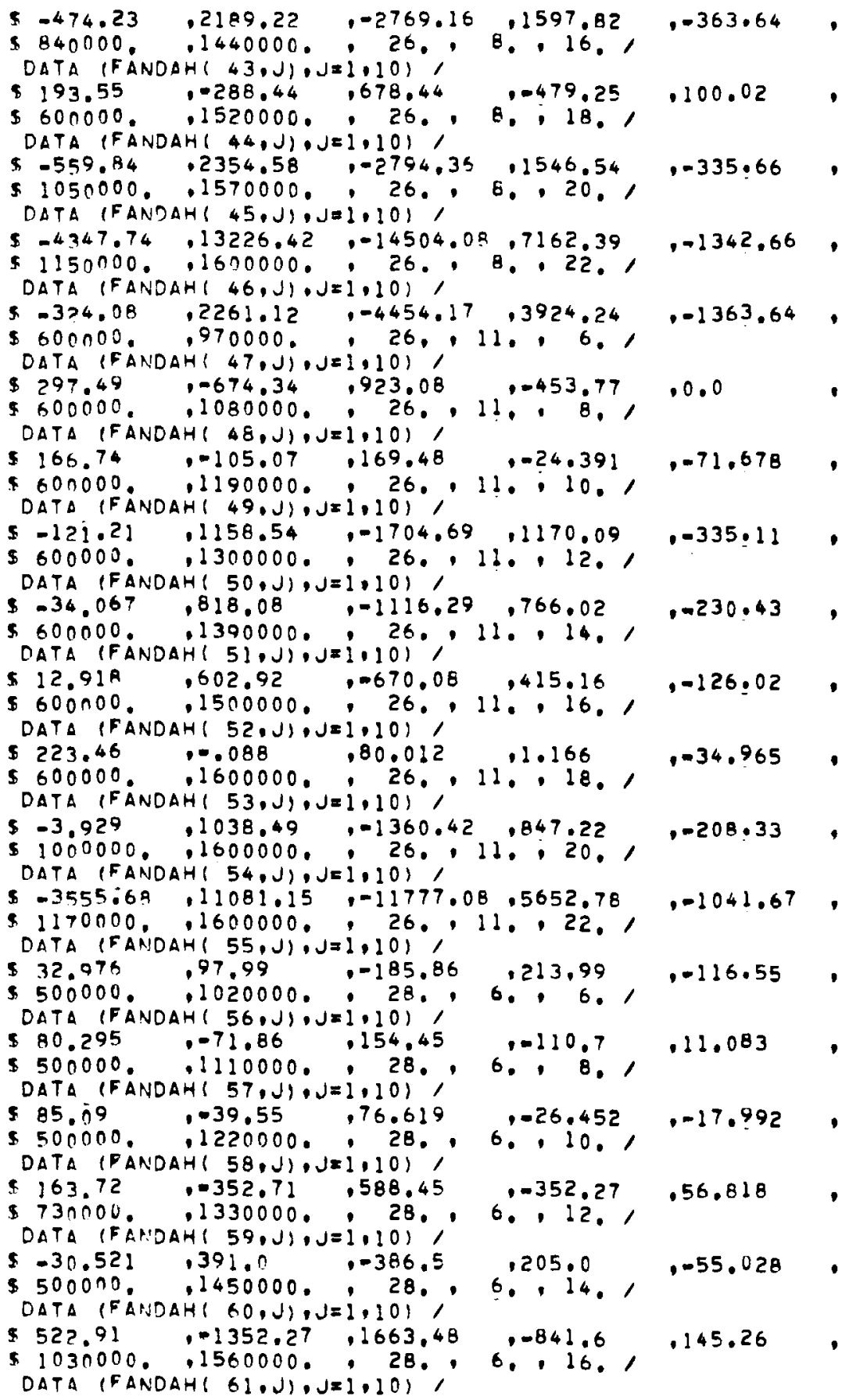

\begin{tabular}{ll} 
FAN & 752 \\
FAN & 753 \\
FAN & 754 \\
FAN & 755 \\
FAN & 756 \\
FAN & 757 \\
FAN & 758 \\
FAN & 759 \\
FAN & 760 \\
FAN & 761 \\
FAN & 752 \\
FAN & 763 \\
FAN & 764 \\
FAN & 765 \\
FAN & 766 \\
FAN & 767 \\
FAN & 768 \\
FAN & 769 \\
FAN & 770 \\
FAN & 771 \\
FAN & 772 \\
FAN & 773 \\
FAN & 774 \\
FAN & 775 \\
FAN & 776 \\
FAN & 777 \\
FAN & 778 \\
FAN & 779 \\
FAN & 780 \\
FAN & 781 \\
FAN & 782 \\
FAN & 783 \\
FAN & 786 \\
FAN & 785 \\
FAN & 786 \\
FAN & 787 \\
FAN & 788 \\
FAN & 789 \\
FAN & 790 \\
FAN & 791 \\
FAN & 792 \\
FAN & 793 \\
FAN & 794 \\
FAN & 795 \\
FAN & 796 \\
FAN & 797 \\
FAN & 798 \\
FAN & 799 \\
FAN & 800 \\
FAN & 801 \\
FAN & 802 \\
FAN & 803 \\
FAN & 804 \\
FAN & 805 \\
FAN & 806 \\
FAN & 807 \\
FAN & 808 \\
& \\
\hline
\end{tabular}




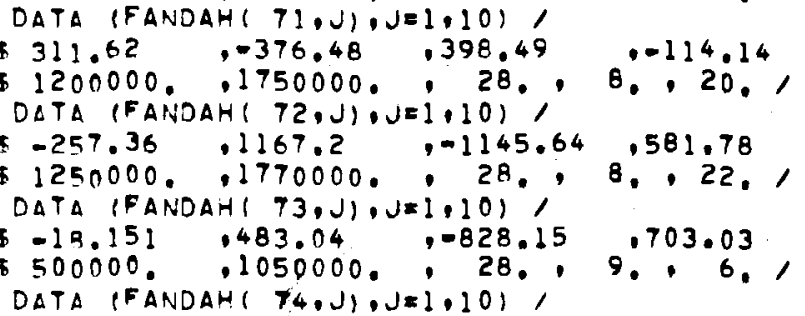




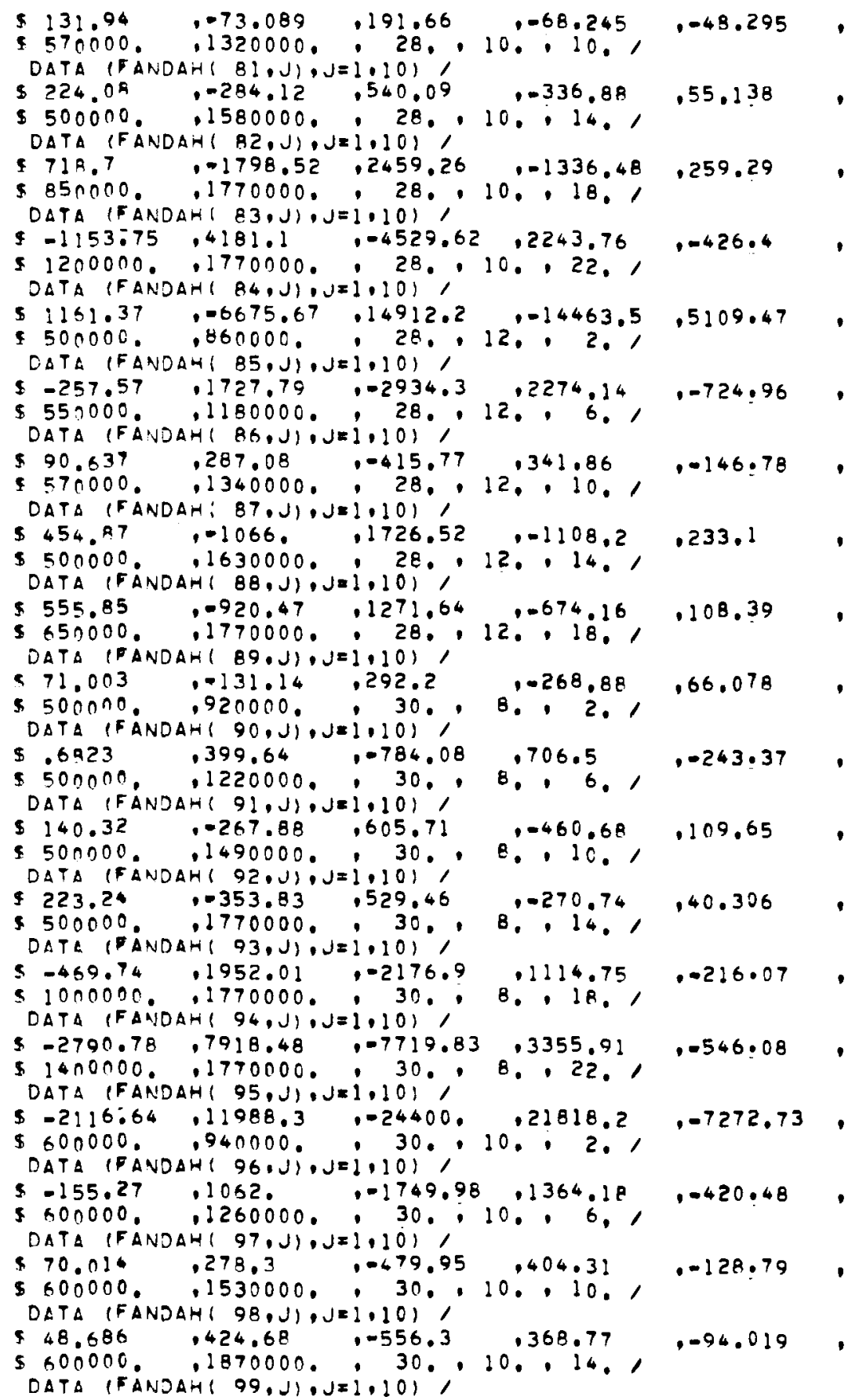


$\$ 1839.82 \quad .-4736.59 \quad .5146 .6 \quad .-2386.56$ $\$ 1110000,01870000,130 ., 10 ., 18,1$ DATA (FANDAH $(100, \mathrm{~J}), \mathrm{J}=1,10) /$

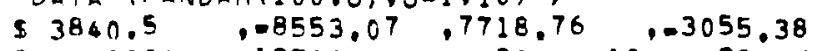

.400 .67

FAN

FAN

.449 .55

$1440000.1870000,30 ., 10 ., 22,1$

DATA (FANDAH $(101, J) \cdot J=1.10)$ )

$5573.86 \quad,-2682.76, .5269 .32$

$\$ 600000,950000 . ; 30 .: 11: ; 2,1$

DATA (FANDAH(102,J),J $=1,10) /$

$\begin{array}{lll}\$ 104.31 & 077.393 & 160.7\end{array}$

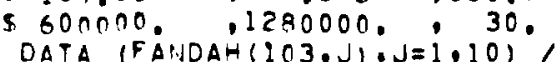

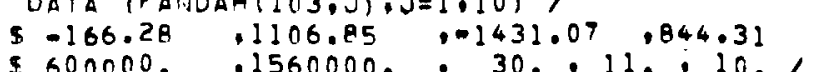

DATA (FANDAH(104,J),J $=1,10)$,

$\$ 105.35 \quad .142 .38 \quad .-19.254$

$5700000,1870000,30,11.14 .095$

DATA FFANDAH (105,J):Jel, 10$) 1$

s $1262.38 \quad, 03070.33 \quad .3409 .94 \quad,-1571.34$

$\$ 1040000,1870000,30,11.18 .1$

DATA (FANDAH $(106, \mathrm{~J}), \mathrm{J}=1,10)$,

\$ $6428.32 \quad,=14968.6 \quad .13695 . \quad, 05504.54$

$51450000,1870000,130,11 ., 22.1$

DATA (FANDAH $(107, \mathrm{~J}), \mathrm{J}=1,10)$,

$\$-58.06 \quad, 308.6 \quad .102 .27$

$5600000,950000, \quad 30,12 ., 2.1$

DATA IFANDAH $(108, J\}, J E 1,10) /$

$\begin{array}{llllll}-272.65 & .1420 .3 & 1-1840.91 & .1030 .3 & .-227.27\end{array}$

. 600000, $, 1290000,130,12,16.1$

DATA (FANDAH $(109 . J) \cdot J=1.10)$,

$\$-107.44 \quad .844 .15 \quad .0980 .16 \quad .527 .0 .119 .46$

$\$ 600000,1580000,130 ., 12 ., 10.1$

DATA (FANDAH $(110, J), J=1,10)$,

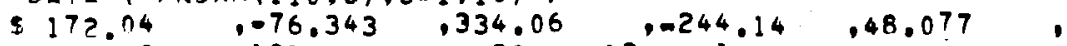

F 700000.,1870000, 30.,12.,14.,

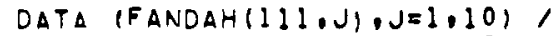

1050000 . $1854.72 \quad .2097 .31 \quad, .952 .54 \quad .148 .6$

DATA (FANDAH(112,J):J=i,10)'

S. $442.59 \quad .1667 .56,1862.69$

5 1000000 .1620000, 40. DATA (FANDAH $(113, \mathrm{~J}), \mathrm{J}=1,10)$,

$\$-234.8 \quad, 936.78 \quad .837 .06$

5. $1000000,2160000,40$.

DATA (FANDAH $(114, \mathrm{~J}), \mathrm{J}=1,10)$,

$559.02 \quad 0212.2 \quad .-81.972 \quad 014.603 \quad 0-2.878$.

$\$ 1000000, .2570000,, 40$.,

DATA (FANDAH $(115, J), J=1,10)$,

$5420.1 \quad, 0458.47 \quad, 450.55$

$51200000,03040000,, 40$. DATA (FANDAH $(116 . J) \cdot \mathrm{J}=1.10)$,

$\$ 502.0 \quad, 0434.25,0352.16$

DATA (FANDAH(1) $17, \mathrm{~J}), \mathrm{J}=1,10)$,

$5-266.24 \quad .1014 .48,0557.16,0149.48,015.597$,

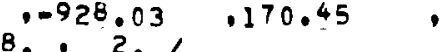




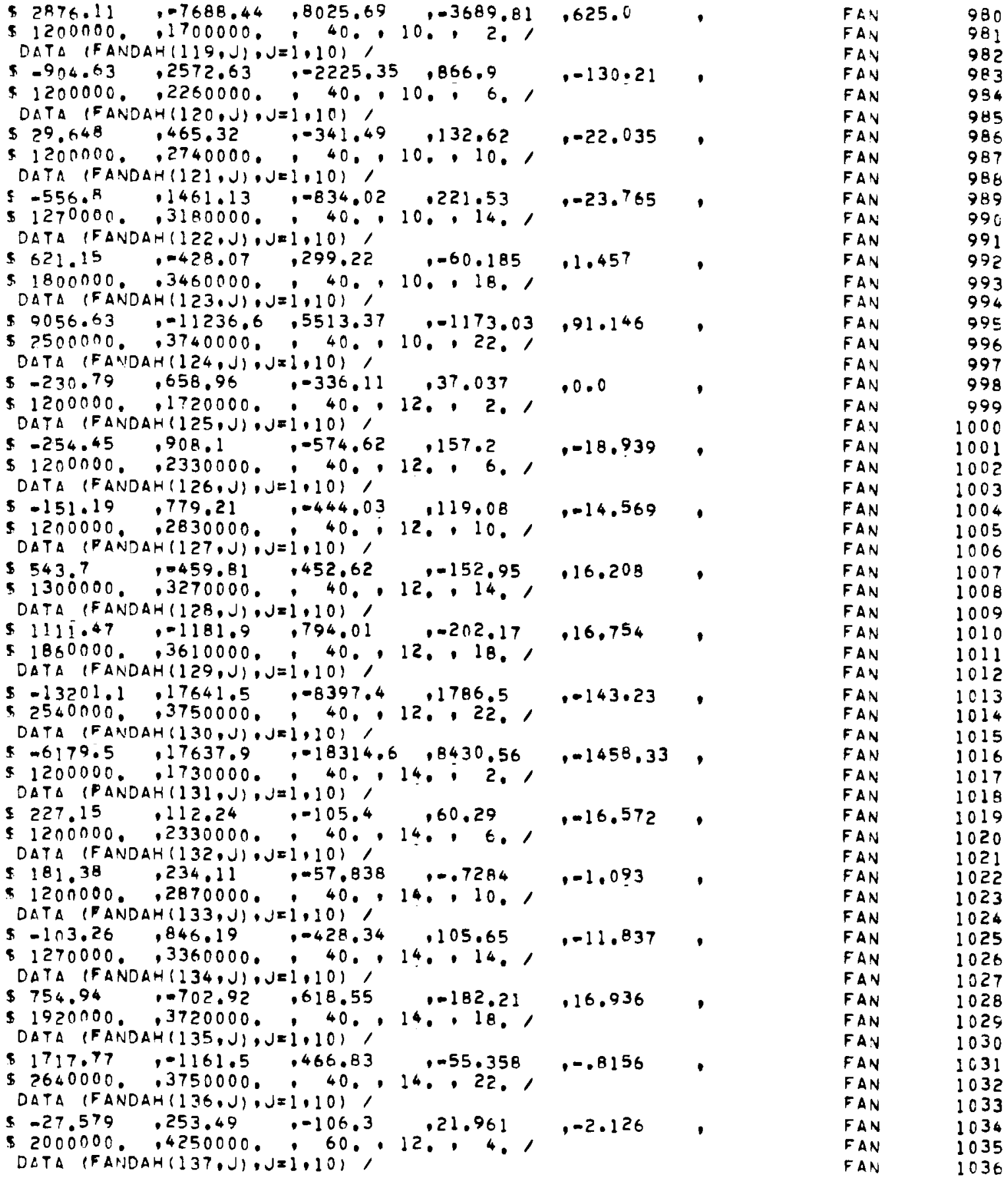




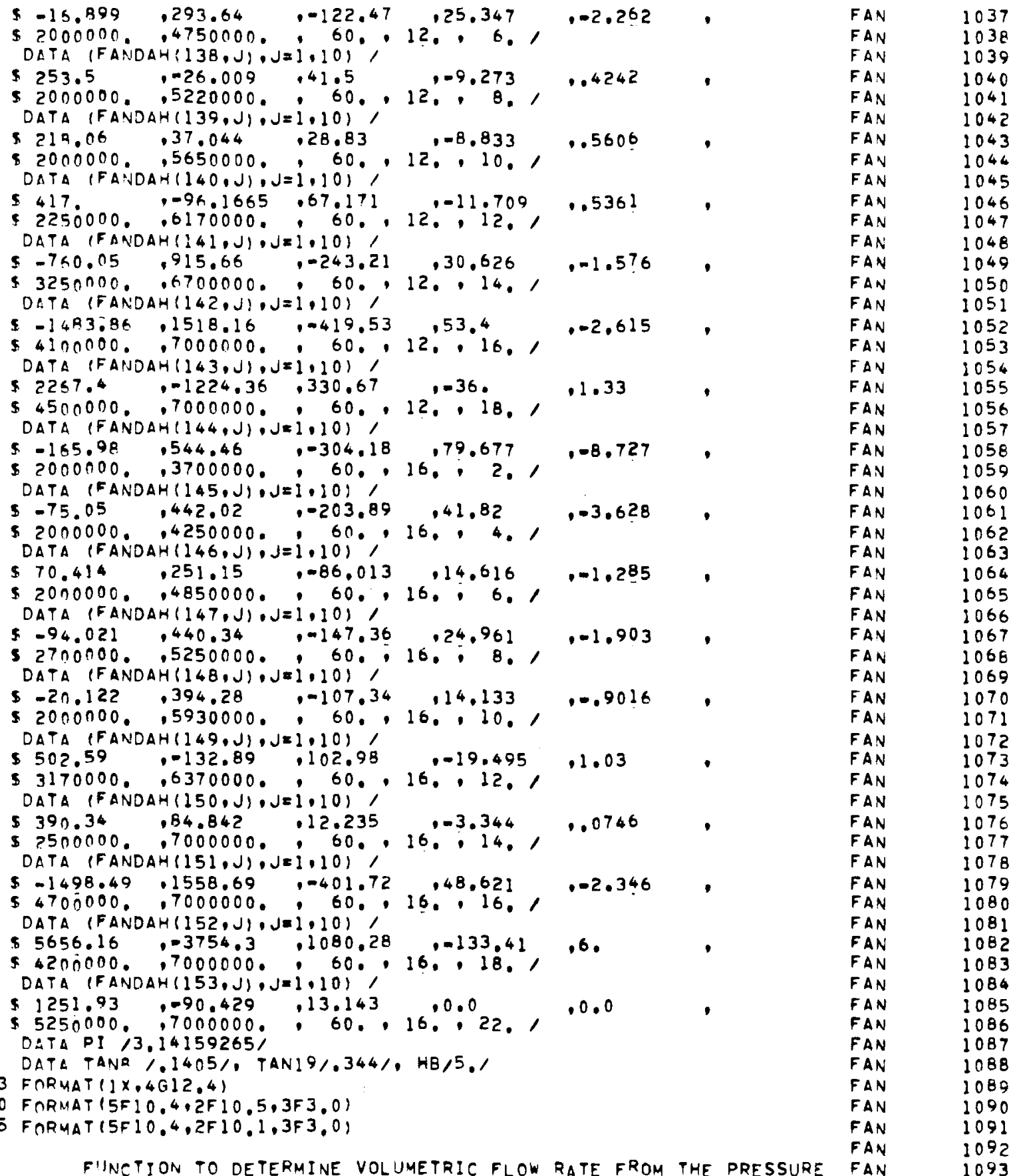


FAN

FAN

FAN

$B A R L N=3.39853-3.81835 E-5 * E L E V$

$B A R=E X P$ (AARLN)

SPVOL $=(T 4+459.67) / 1.325 / B A R$

TOTCFM $=W 4$ SPVOL 160 .

$A F C=5 \cdot E 08$

$A C=0$.

$A H P=0$.

$\triangle D I A=0$

$\triangle B L N=0$

$A B L A=0$ :

$A N F=n$.

$A V E L=0$ :

EXIT STACK FACTOR USED TO COMPUTE STACK EXIT DIAMETER WHEN VELOCITY RECOVERY IS USED. VELOCITY RECOVERY IS BASED ON 13 FOOT HIGH STACKS FOR 28 FOOT DIAMETER FANS. THE DIFFUSER ANGLE IS \& DEGREES. ESF $\approx 2 . T A N(B D E G) * 13,12 B$.

$E S F=0.1305$

VELOCITY PRESSURE COEFFICIENT USED TO COMPUTE VELDCITY PRESSURE IN INCHES OF WATER. VPCOEF =0.07495*29.92*13.5951/(2.*32.174*144.*14.696)

VDCOEF $=2.239 E-04$

STATP $0.07495 *$ SPVOL 0 DPTINW

RECVFL IVELREC

IF (LFE,LT.]) LFE $=153$

IF (LFB:LT.I) LFB =1

START OF ITERATIVE LOOP FOR DETERMINING THE OPTIMUM FAN SYSTEM

DC 400 I.LFB.LFE

IF $(1 . E 0.27)$ GO TO 400

IF (I.EO.IO3) GO TO 400

IF (I.EO.122) GO TO 400

0
$c$
$c$

INITIALIZATION OF FAN OPTIMIZATION DARAMETERS

$T P=0$.

PCF MPF $=0$.

FAN

AN

FAN

FAN

FAN

FAN

FAN

FAN

FAN

FAN

FAN

FAN

FAN

FAN

FAN

FAN

FAN

FAN

FAN

FAN

FAN

FAN

FAN

FAN

FAN

FAN

FAN

FAN

FAN

FAN

FAN

FAN

FAN

FAN

FAN

FAN

FAN

FAN

FAN

FAN

FAN

FAV

FAN

FAN

FAN

FAN

FAV 
CFMPFEO

$\mathrm{NOF}=\mathrm{O}$

HDPF $=0$ :

TOTHPEO

FCST $=0$

CMOT $=0$ :

$C S R=0$.

$C F D=0$.

CEC $=0$.

$C D L=0$.

TCSTPF $=0$.

TCSTFEO.

$V E L=0$.

CINCE $=90000$.

$C F M=400000$ :

$F L O V D=0$.

$T D=0$.

$D F=0$.

$C I=0$.

IF (I.LE.27) $A=429.08$

IF (I.GP. . 35) $A=2680.4$

FANDEFANDAD $(I, B)$

EXITD $=(1,+E S F)$ FAND

EXITA $=0.7854 * E X I T D * E X I T D$

EA6001:/160.EXXITA)

$A G 0=1.1(60 . * A)$

CROSS-SECTIONAL FLOW AREA FOR THE FAN DEPENDING ON THE FAN DIAMETEF

IF (I.GT.27. AND.I.LE.54) $A=495.96$

IF (I.GT.54.AND.I.LE.AB) $A=584.5$

IF (I.GT.B8.AND.I.LE. III) $A=658.37$

IF (I.GT.111.AND.I.LE.135) A=1123.91

DETERMINE THE NUMBER FANS, THE VOLUMETRIC FLOW RATE THROUGH

FAN

FAN

FAN

FAN

FAN

FAN

FAN

FAN

FAN

FAN

FAN

FAN

FAN

FAN

FAN

FAN

FAN

FAN

FAN

FAN

FAN

FAN

FAN

FAN

FAN

FAN

FAN

FAN

FAN

FAN

FAN

FAN

FAN

FAN

FAN

FAN

FAN

FAN

FAN

EACH FAN, AND THE TOTAL PRESSURE DROP ACROSS EACH FAN

FAN

C

THIS ITERATION SCHEME DETERMINES THE OPERATING POINT FOR

FAN

FAN "I". THE OPERATING POINT IS THE INTERSECTION OF THE FAV

DRESSURE CURVE AND THE TOTAL DRESSURE CURVE. THE ITERATION

USES THE FAN DATA AS CFM VERSUS TOTAL DRESSURE, PLOTTED AS

FAN

FAN

$Y$ VERSUS $X$. THE DOINTS (XD. PP) REFER TO THE POINTS ON THE

FAN

FAN PRESSURE CURVE. THE POINTS (XT,YT) REFER TO THE POINTS

IN THE TOTAL PRESSURE CURVE. THE LIMITING VALUES OF CFM AND

AND FAN PRESSURE FROM THE FAN DATA ARE USED TO GET THE

FAN

FAN

LIMITING POINTS OF THE FAN'PRESSURE CURVE AND THE TOTAL

FAN

FAN

FAN

PRESSURE CURVE. THESE POINTS ARE USED TO DETERMINE TWO

FAN

STRAIGHT LINES: YPEAP XP PBP AND YTEAT $X T+B T$. THE
INTERSECTION OF THESE LINES IS THE NEW FLOW RATE FOR THE NEXT FAN

STERATION, THE ITERATIONS CONTINUE UNTIL CONSECUTIVE FAN

INTERSECTIONS ARE NEARLY IJENTICAL.

FAN

FAN

$K Y=0$

FAN

115 !

152

1153

1154

1155

1156

1157

1158

1159

1160

1161

1262

1163

1164

1165

1166

1167

1166

1159

1170

1171

1172

1173

1174

1175

1176

1177

1179

1179

1180

118 ?

1182

1183

1184

1185

1186

1187

1188

1189

1190

1191

1192

1193

1194

1195

1196

1197

1198

1199

1200

1201

1202

1203

1204

1205

1206

1207 
IUBROUTINE FAN TAN FTN $4.5+414 \quad 0 P T=1 \quad 0 \quad 06 / 05 / 78 \quad 07.59 .59$

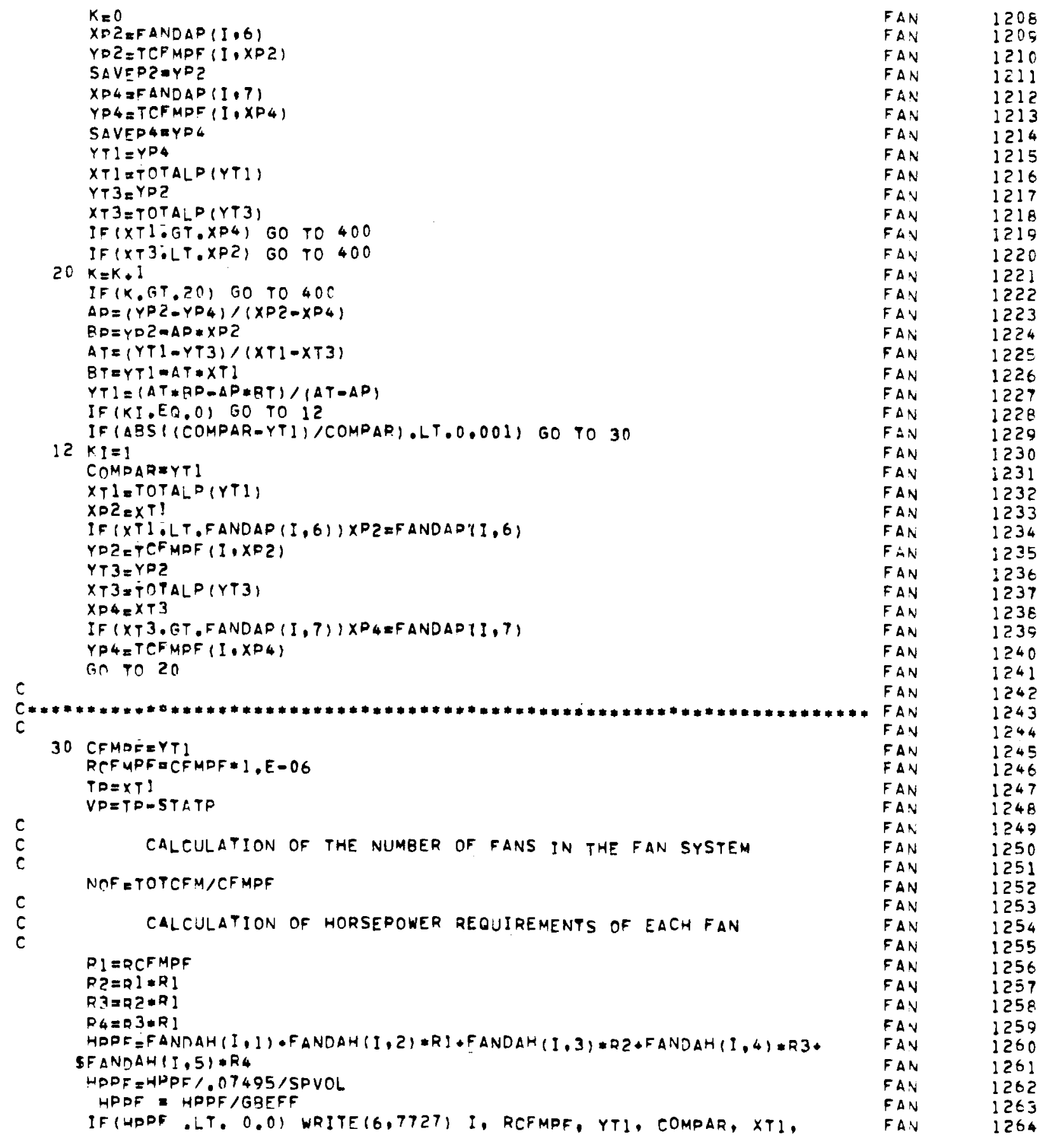


SURROUTINE FAN $\quad 74 / 74 \quad O P T=1 \quad$ FTN $4.5+414 \quad 06 / 05 / 78 \quad 07.59 .59$

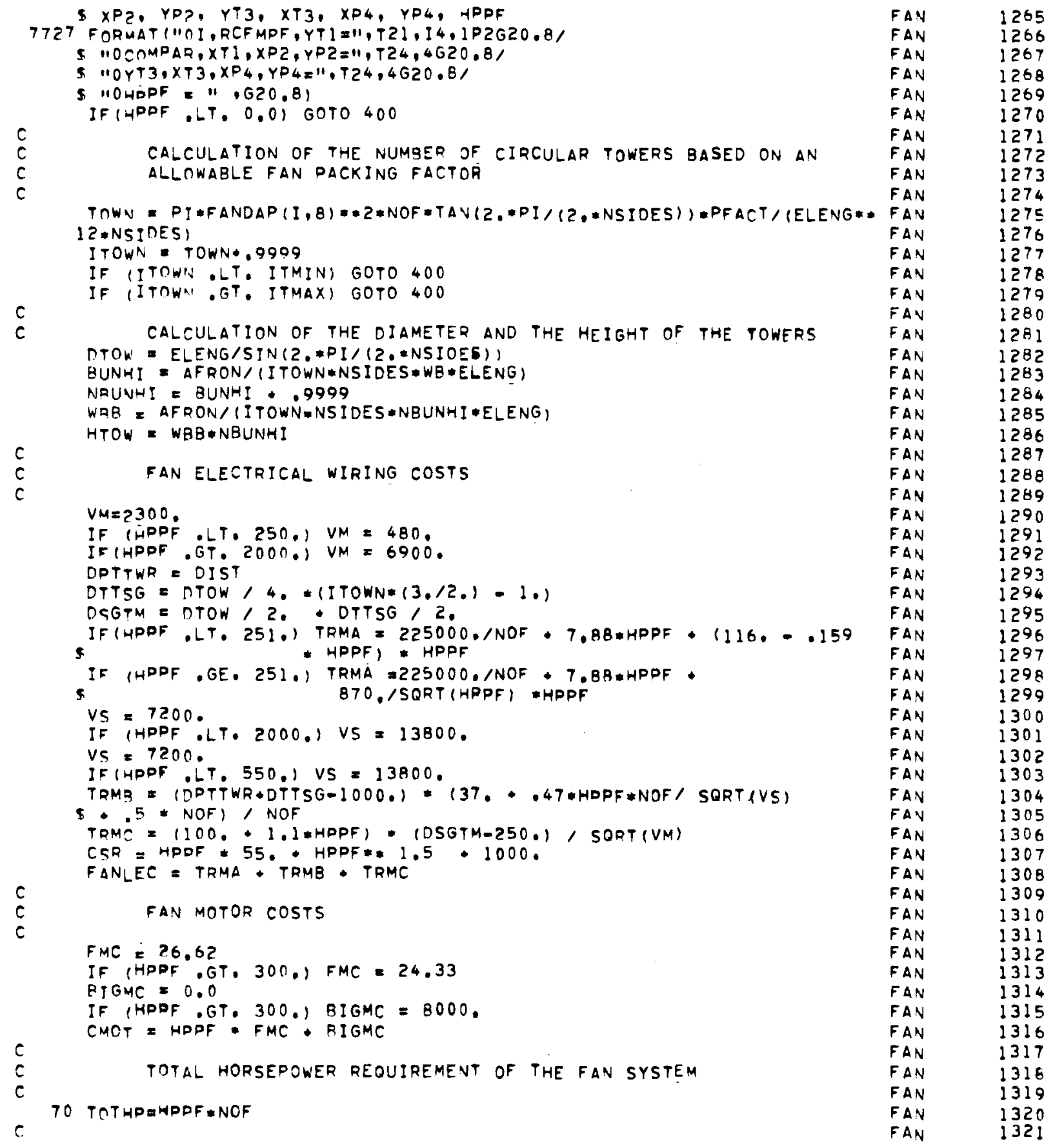




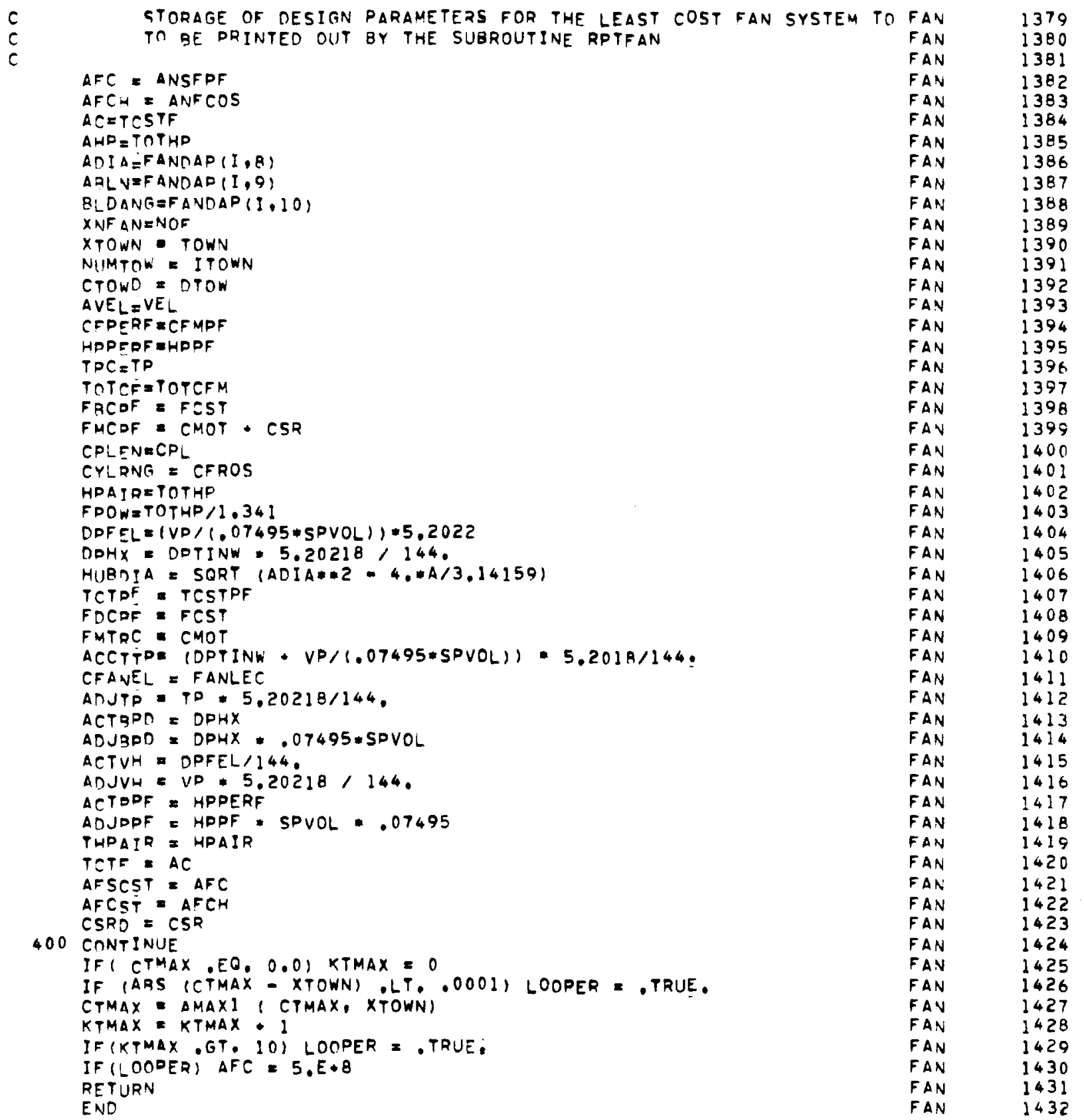


07.59 .59

SURROLTINE FINEFF CALCULATEG THE FIN EFFECTIVENESS OF AN EXTENDED FIN USING DUSINBERRE"S RELATIONSHIP AND A CUPVE FIT OF THE DIFFERENCE BETWEEN DUSINBEPREIS AND THE BESSEL FIINCTION SOLUTION OF THE FIN EFFECTIVENESS SECOND ORDER. DIFFERENTIAL EQUATION WITH CONSTANT FIN THICKNESS.

LOGICAL FIRST,FRST,FXTEMP,FXTVAF,FXTTTO,FXTLNG COMMON

\& AFTR, ALPHA, ANG (3)

* Bolim(16)

- CaDF, cSSPKw, conf, conl, costl, capchg, conmat, conmaz, caf

5. CaJ, CTURB, CDANG, CLUVR, CHAILS, CVM

\$. DEIN,DEEPL, DESVEL, DESVELV, DESVELD, DENSIF

\$ EEFD, EFS

-FOR,FIPST,FIXL,FCOS,FFHX,FXTEMP, FAETOR,FRST, FACTORD, FSHOT,FDELUG

\$.FIXV,FIXTTO,FXTVAR,FXTTTD,FXTLAG

I GREFF

- HXND.HDCST.HCD

S ITMAX, ITMIN

5 . JCONS

S. KCONV, KALEXT

S. $M \times E \times T$

S.NTA, NSIDES, NBUNHI COMMON

$S \mathrm{ODQ}$

\$ PSIZE, PER, PWCOS, PLANC, PFAET, POMDPL

5 POHAAF, POHFAN, POHLEC, POHCIR, POHCND, POHSTC, POHSEL

S RRP, REDUCE, REDUCV, ROOFL. RHOUT

$\$$ - DREJ, OREDUCE, OREDUCV

- SIGMAG, SAAF. SEGL

\$.TD, TPO(4). TEFF, TLIM, THFIN, TLPRA, TFIX

S, TED. TW

$\$$,UES, UWS

S. WFV, WATCONA, WATCOST

5 VAS (5)

S.XOEPA, XW, XD

LOGICAL PATH

DATA DATH 1 .TRUE.

DATA A, R, $0.06313766,0.00745032$,

$c$
$c$
$c$

IF (NOT, DATH) GO TO 10

RE $=$ DFIN $/ 12.0,2.0$

$R A=D O R / 12.0 / 2.0$

$R C=P E=R A$

$Y A=$ THFIN/I2.0/2.0

CST1 = RE/RB

$A W=R C \# R C$ SORT (CSTI)/CONF/YB/3.0

PATH $=$.FALSE.

10 CONTINIJE

$E F F O=1.0 / 11.0+H O=A W)$

DUSINAERRE"S AND BESSEL FUNETION SOLUTION DIFFERENCE

FINEFF

FINEFF

FINEFF

FINEFF

FINEFF

FINEFF

FINEFF

FINEFF

BI GCOM

BISCDM

BISCOM

BISCOM

BIGCOM

SIGCOM

3I GCOM

BI GCOM

BIGCOM

EIGCON.

BI GCOM

BI GCOM

BI GこOM

BIGCOM

BIGCOM

BI SCOM

BISCON

BI SCOM

BI SCON

BIGCOM

BIGCOM

BI GCOM

EIGCON

BIGCOM

BIGCOM

BI GCOM

BISCOM

BI GCON

BI GCOM

BIGCON

FINEFF

FINEFF

FINEFF

FINEFF

FI VEFF

FI NEFF

$F$ INEFF

FI NEFF

$F$ I NEFF

FI NEFF

FINEFF

FINEFF

FINEFF

FINEFF

FINEFF

FINEFF

FINEFF

FINEFF

FI NEFF 
SUBROUTINE FINEFF T4/74. OPTEI

FTN $4.5+414$

$06 / 05 / 78 \quad 07.59 .59$

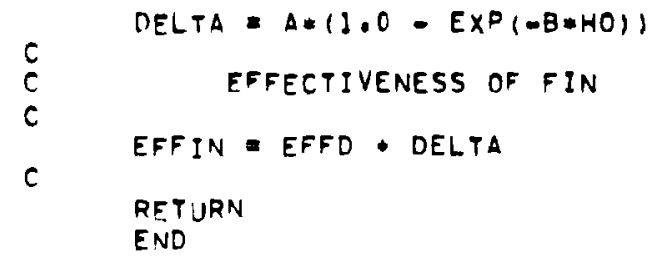

F I NEFF

FINEFF

FINEFF

FINEFF

FINEFF

FINEFF

FINEFF

FINEFF

30

32

34

35

36

37 
FUNCTION FLUID (NPROP, NFLUID, TEMP.ALTITD.LOCAT)

FLUID

DIMENSION LOCAT (2)

DIMENSION COLLAJ $(12,8,2)$

DIMENSION FLU(?), PRCP $(12,3)$

LOGICAL WARN

IFLAG $=1$

FLUID

FLUID

FLJID

FLUID

FLUID

FLUID

SURPOLITINE FLUID DETERMINES THE THERMODYNAMIC AND PHYSCIAL

FLU:S

PROPERTIES OF SATURATED AMMONIA AND AIR. THE VALUES ARE

FLUIJ

DETERMINED BY CURVE FITS OF THE PROPERTIES VERSES TEMPERATURE FLUID THE LIOUID PROPERITIES OF AMMONIA ARE FOR SATURATED AMMONIA. TME THERMODYNAMIC PROPERTIES FOR AMMONIA GAS ARE FOF ATMOSPHERIC PRESSURE WHILE THE PHPSICAL PROPERTIES ARE FOR SATURATED AMMONIA GAS.

$$
\text { COLLAJINFLUID, NDRQP, ICLA) }
$$

NFLUID - FLUIC INDEX

$$
=1-\triangle M M O N I A
$$

$=2 \cdot \operatorname{AIR}$

NOROP - PROPERTY INDEX

$=1$ - SPECIFIC HEAT (LIQUID) (BTU/LRM-DEG F)

- 2 - THEOMAL CONDUCTIVITY (LIQUID (RTU/HR-FT-DEG F)

$=3$ - DENSITY (LIOUID) (LBMICUFT)

$=4$ - VISCOSITY (LIOUID) (LBM/FT-HR)

$=5$ - SPECIFIC HEAT (GAS) (BTU/LBM=DEG F)

$=6$ - THERMAL CONDUCTIVITY (GAS) (BTU/HR-FT-DEG F)

= 7 - DENSITY (GAS) (LBM/CU FT)

$=8$ - VISCOSITY (GAS) (LBM/FT-HR)

= 9 - HEAT OF VAPORIZATION (BTULLBM)

$=10$ - PRESSURE AS A FUNCTION OF TEMPERATURE (PSI)

$=11$ - TEMPERATURE AS A FUNCTION OF PRESSURE (DEG F)

$=12$ - SURFACE TENSION (LGF/FT)

ICLA - COEFFICIENTS, LIMITS AND ADJUSTMENT FACTORS

- 1 - FIRST COEFFICIENT

- 2 - SECONO COEFFICIENT

= 3 - THIRD COEFFICIENT

= 4 - FOURTH COEFFICIENT

= 5 - LOWER TEMPERATURE LIMIT

$=6$ - UPDER PEMDERATURE LIMIT

$=7$. FIRST ADJUSTMENT FACTOR, ADDED TO TEMPERATURE

- 8 - SECONO ADJUSTMENT FACTOR, DIVIDEO INTO TEMPERATURE

DATA VALUES FOR AMMONIA

DATA( (COLLAJ II,J,1), J:1,8), I: 1,9$)$,

FLUID

FLUID

FLUIO

FLUID

FLUID

FLUI?

FLUID

FIUID

F-UID

F-UID

FLUID

FLUID

FLUID

FLUID

FLUID

FLUID

FLUIO

FLUID

FLUIJ

FLUIJ

FLUID

FEUID

FLUID

FLUID

FLUIO

FLUID

FLUID

FLUID

FLUIO

FLUID

$c$ 


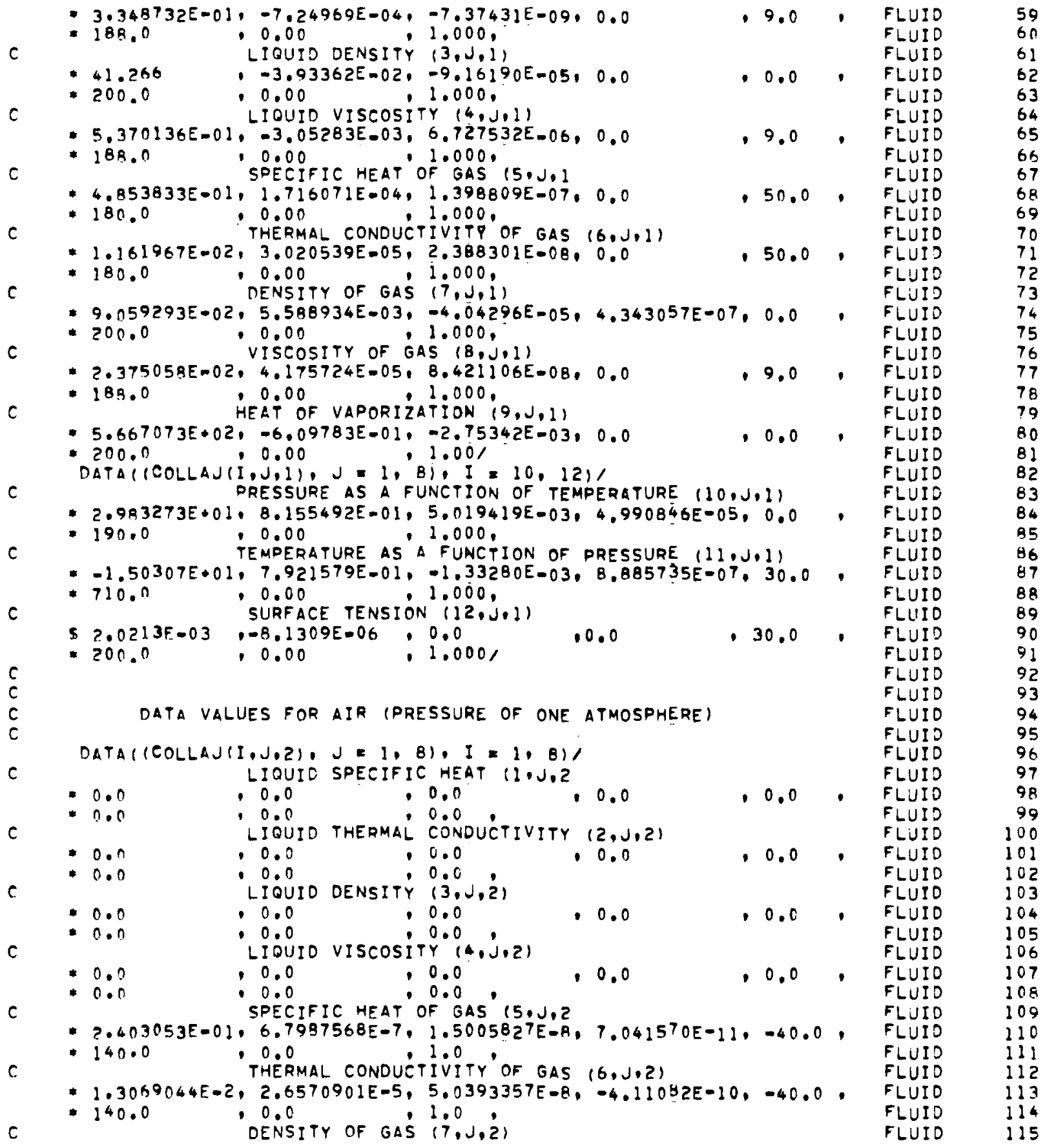


$\begin{array}{lllll}\text { FUNCTION FLUID OPT:I } & 74 / 74 & \text { FTN } 4.5 .4 ! 4 & 06 / 05 / 78 & 07.59 .59\end{array}$

$c$

$* 9.6353904 E-z,-1.874752 E-4,3.7316434 E-7 .=4.66929 E-10,-40.0$,

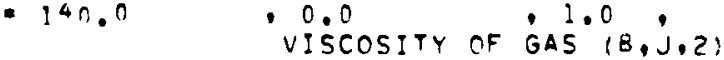

$.3 .9387711 E-\bar{z}, 6.8722417 F-5,-3.483392 E-8,2.4524095-11,-40.0$,

$+140.0$

- 0.0

OATA IFLU(I). I $=1.21 /$ "WATER", "AIR",

DATA FOF PRINTING DF ERRDR MESSAGES

OATA (PROP(I.J), JE 1,3$), I=1,121)$

* "SOECIFIC h" "EAT of LiQ", "UIO

- "THERMal con" "NDUCTIVITY"." of liouid".

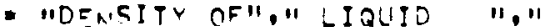

- "Viscnsity " "of liquio "."

* "SDECIFIC H", "EAT of Gas","

- "thermal co", "nouctivity"." of gas

- "DEnsitr OF"." gas

* "viscosity ". "of gas

11,11

- "heat if va", "PORIzation"."

- "paEssure "i" a a functi", "On of -

- "TEmperatup", "E as a fun" "CTion of pu"

" "SuRface te","nsion as a"," functiont",

c

c

c

IF (NFLUID, ST, 2) RETURN

NF $=$ NFLUID

ND $=$ NDROD $c$

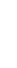

C

c

AIR PROPERTIES NOT AVAILABLE

IF (NF :EO. 2 . AND. NO.LE. 4 ) GO TO 50

WARNE OFALSE.

PROPERTIES ASKED FOR OUTSIDE THE RANGE OF TEMPERATURES

IF (TEMD LLT. COLLAJINP,5.NF)) WARN = .TRUE.

IF (TEMP. GT. COLLAJINP.G.NF)) WARN = TRUE.

$c$

FLIID PROPERTY SALCULATION

$T=$ (TEMP * COLLAJ (NP,T,NF))/COLLAJ (NP, B, NF)

FLUITO EOLLAJ(NP,I,NF) + COLLAJINP,Z,NF)*T * COLLAJ(NP,3,NF)*T**Z

* cOllaj(NP,4,NF)*T*3

ALTITUDE CORRECTION FOR DENSITY OF AIR

IF (NF $-E O .2$. AND. ND.EG.7) FLUIDE

5 $1.325 * E X P(3.39853-3.81835 E-5 *$ ALTITD), (TEMP.459.67)

DRIUTING OF ERRORS

IF (. NOY. WARN) RETURN

IF (IFLAG3 EQ. O) RETURN $\triangle L L O W E D$ FOR
FLUID

FLUID

FLUID

FLUIO

FLUIO

FLUID

FLUID

FLUID

FLUID

FLUID

FLUID

FLUID

FLUIO

FLUIO

FLUID

FLUID

FLJIJ

FLUID

FLUIO

FLUID

FLUID

FLUID

FLUID

FLUIO

FLUID

FLUID

FLUIO

FLUID

FLUID

FLUID

FLUID

FLUID

FLUID

FLUID

FLUIO

FLUID

FLUID

FLUID

FLUID

FLUIS

FLUID

FLUID

FLUID

FLUID

FLUID

FLUID

FLUID

FLUID

FLUID

FLUID

FLUID

FLUID

FLUID

FLUID

FLUID

FLUID

FLUID
116

117

118

119

120

121

122

$: 23$

124

125

126

127

128

129

130

131

132

133

134

135

136

137

$13 R$

139

140

141

142

143

144

145

146

147

148

149

150

351

152

153

154

155

156

157

158

159

160

161

162

163

164

165

166

167

168

169

170

171 
FLUID

FLUID

FLUID

FLUID

FLUID

FLUID

FLUID

FLUID

FLUID

FLUID

FLUID

FLUID

FLUID

FLUID

FLUID

FLUID

FLUID

FLUID
RETURN

END 
SUBROUTINE FRICT FOR GIVEN VALUES OF INSIDE OIAMETER (IN), MASS FLUX (LBM/SQ FT-SEC), ANO VISCOSITY (LBM/FT-HR), OETERMINES THE FRICTION FACTOR OF DRAWN TUBING (IDEI), STEEL FPICT PIDE (ID=2), OR CAST IRON PIPE (IDE3 USING MOODY FRICTION FRICT FACTOR CHART EQUATIONS

CALCULATE RECIPPOCAL OF RELATIVE ROUGHNESS AND RECIPROCAL OF SOUARE ROOT OF FRICTION FACTOR FOR FULLY TURBULENT FLOW

101 DFEDIAIEPIID:

$F F=4 . * A L D G) O(R F)+2.28$

$c$
$c$

CHECK TO DETERMINE IF TURBULENT FLOW IS FULLY DEVELOPED

$C F=D F * F F / R E$

IFICF $=0.01) 104.103,103$

DETERMINE RECIPROCAL OF SQUARE ROOT OF FRICTION FACTOR FOR

TRANIS!TION FLOW INITIALLY ASSUMING IT TO BE THE SAME AS FOR

FRICT

FPICT

FRICT

FRICT

FRICT

FRICT

FRICT

FRICT

FRICT

FRICT

FRICT

FRICT

FRICT

FRICT

FRICT

FRICT

FRICT

FEICT

FRICT

FRICT

FRICT

FRICT

FRICT

FRICT

FRICT

FRICT

FRICT

FRICT

FRICT

FRICT

FRICT

FULLY TURBULENT FLOW

FRICT

FRICT

FRICT

103 TRFEFF

107 TRECEFF=4.*LLOG $10(1 .+4.67 * R F * T R F / R E)$

FRICT

FRICT

IF CALCULATEO AND ASSUMED VALUES OF RECIPROCAL OF SQUARE ROJT FRICT

OF TRANSITION FLOW FRICTION FACTOR DO NOT AGREE WIYHIN

FRICT

SDECIFIED LIMITS, ASSIJME NEW VALUE EQUAL TO CALCULATED VALUE ANO REPEAT CALCULATION

FRICT

FRICT

FRICT

IF( (ABS (TRFC-TRF) /TRF) 0.001$) 105,105,106$

106 TRF =TRFC

GO TO 107

FRICT

FRIET

FRICT

FRICT

CALCULATE FULLY DEVELOPED TURGULENT FLOW OR TRANSIYION FLOW

FRICT

FRICT 


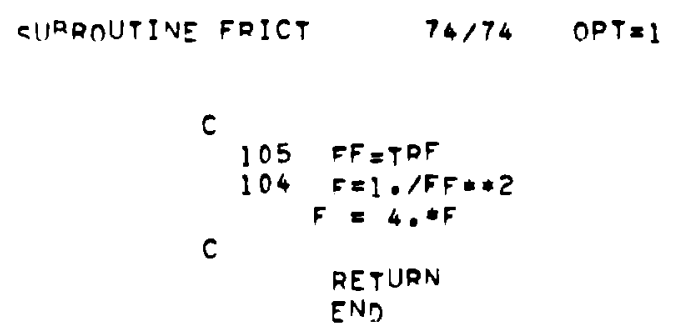

FTN $4.5 \cdot 414$

$06 / 05 / 78$

07.59 .59

FRIC

FRICT

FRICT

FRICT

FRICT

FRICT

FRICT

59

60

61

62

63

64 
$74 / 74 \quad$ OPT $=1$

FTN $4.5+414$

$06 / 05 / 79$

07.59 .59

SUBROUTINE HEADERIOOTM,DOTBR, DESVEL,RHO,RED, J,NN,DIAJ,DIL,TCST, SRCST, UPCST, ELCOST, FLGCOST, EJCOST)

THIS SUBROUTINE DETERMINES DESIGN AND COST INFORMATION FOR THE MAIN CIRCULATION PIPING AND THE QUADRANT PIPING.

DEFINITION OF VARIABLES

APIPCST - ABOVE GROUND PIPE COST (\$IOOOIFT)

DESVEL - DESTGN VELOCITY (FT/SEC)

OIA - PIPE COMPONENT JIAMETER (INCHES)

DOTRR - MASS FLOW RATE TO BRANCH, I.E. TO CIRCULAR TOWER OR TO TUBE BUNDLE (LBM/HR)

DOTM - MASS FLOW RATE IN GIVEN HEADER SECTION (LAM/HR)

EJCOST - EXPANSION JOINT EOST (\$1000)

ELCOST - 90 DEGREE ELBOW COST $1 \$ 1000$

FLGCOST - FLANGE COST (\$1000)

NKOS - NUMBER OF POSITIOHS IN UNIY COST ARRAYS

NN $\quad$ IF $=0$ THEN MAIN CIRCULATION HEAOER IS DESIGNED IFEI THEN QUADRANT HEADEP IS DESIGNED

DIPCST - BELOW GROUND PIPE COST (S1000/FT)

RED - ALLOWABLE REDUCTION IN DIAMETER BETWEEN ADJACENT HEADER SECTIONS (INCHES). IF RED=O THE PROGRAM CALCULATES THE REDUCTION

REDCOST - REDUCER COST (\$1000)

REDUET - REDUETION IN DIAMETER BETWEEN AOJACENT HEADER SECTIONS (INCHES). ONLY USED WHEN REDEO

RHO

- DENSITY (LBM/CUFT)

TEECOST - TEE COST $(\$ 1000)$

IIPCST - UNIT PIPE COST (S1000/FT)

VACSY - VALVE COST ISI0001, VALVES aRE MOTOR OPERATED BUTTERFLY VALVES

DIMENSION DIAM(24), FLGCST (24), EJCST (24), REDCST(24), ELCST(24) \$. VACSY $(24)$, TEECST $(24)$, PIPCST $(24)$. APIPCST $(24)$

DATA DIAM,

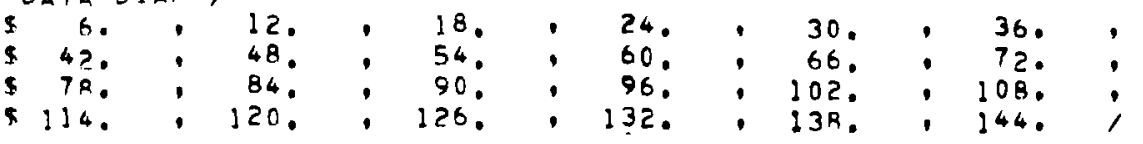

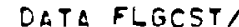

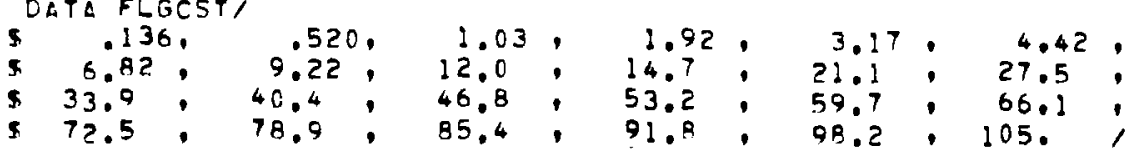

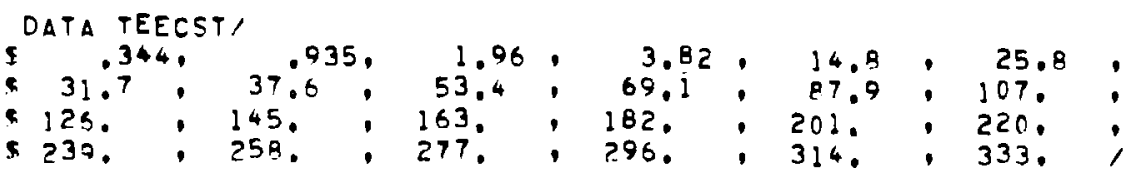

C

DATA PIPCST/

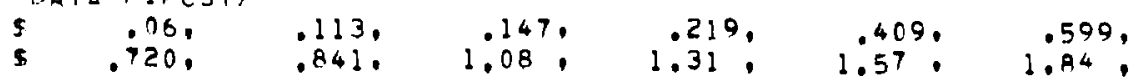

HE ADER

HE ADER

HE ADER

HE ADER

HE $A D E R$

HE ADER

HE $\triangle D E R$

HE ADER

HE $\triangle D E R$

HE ADER

HE ADER

HEADER

HE ADER

HE ADER

HEADER

HEADER

HEADER

HE ADER

HE ADER

HE ADER

HEADER

HE ADER

HEADER

HE ADER

HE $\triangle D E R$

HEADER

HE ADER

HEADER

HEADER

HE ADER

HEADER

HE ADER

HE ADER

HEADEF

HEADER

HEADER

HE ADER

HE $\triangle D E R$

HE $\triangle D E R$

HE $\triangle D E R$

HE ADER

HE ADER

HEADER

HE ADER

HE ADER

HE ADER

HEADER

HEADER

HEADER

HEADER

HEADER

HE ADER

HEADER

HEADER

HEAOER

HE ADER

HEADER 
$c$

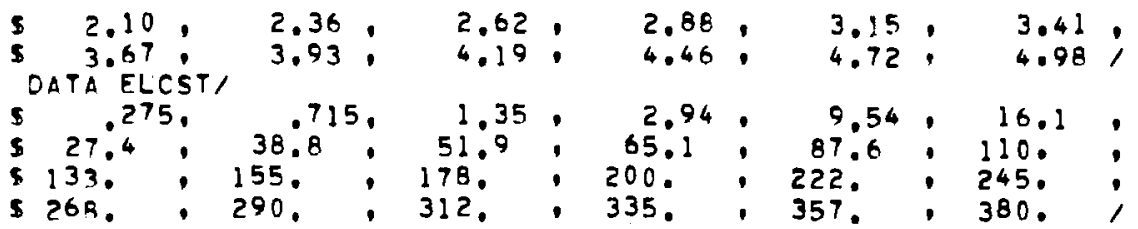

C

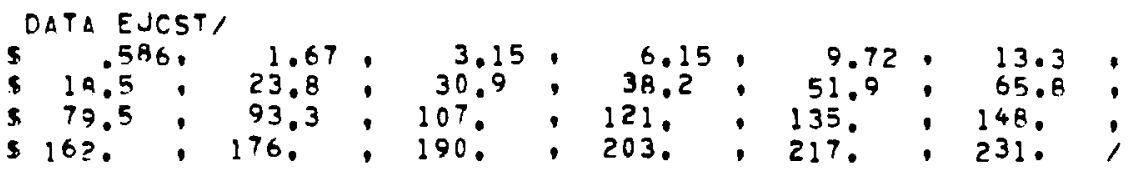

$C$

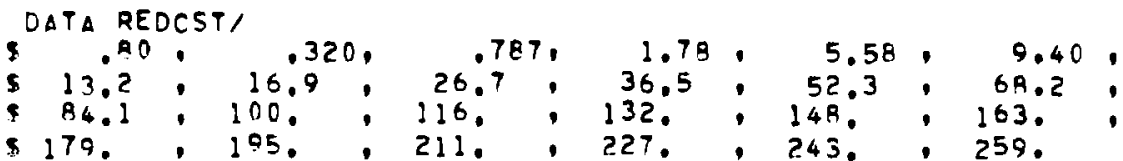

DATA APIDCSTI

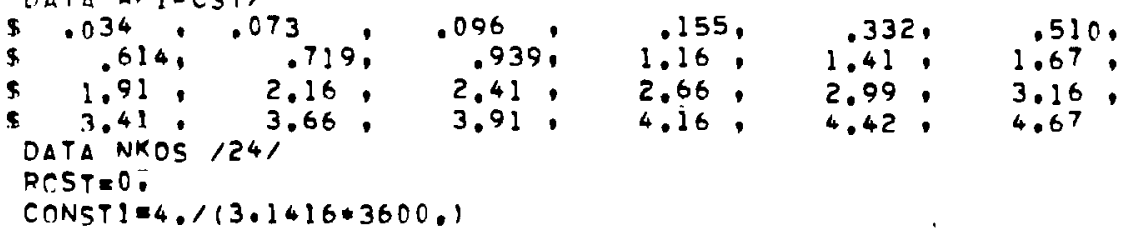

CALCULATE THE REQUIRED DIAMETER BASED ON MASS FLOW RATE AND DESIGN VELOCITY. THE DIAMETER ASSIGNED TO THIS HEADER SECTION IS THE NEXT LARGEST PIPE IN 6 INCH INCREMENTS FROM THE ARRAY DIAM.

DIA =SORT (DOTM*CONST I/(RHO*DESVEL)) *12. DO IO II $=1$.NKOS

$I=I I$

IF (DIA DIAM(I) ) 11,11,10

10 CONTINUE

1) DIAEDIAM (I)

THE MAIN CIRCULATION HEADER HAS AN EXPANSION JOINT AND APPROPRIATE FLANGES AT THE CONDENSER INLET AND OUTLET.

IF (J.EQ.1.AND. NN.EQ.1)FLGCOST $=3, * F L G C S T$ (I)

IF (J.EO.).AND.NN,EO.I)EJCOST=EJCST (I)

IN THE STATEMENTS THROUGH STATEMENT 12 THE REDUCTION IN DI AMETER BETWEEN ADJACENT HEADER SECTIONS IS SDECIFIED. THE REDUCER COST IS DETERMINED. THE INDEXES USED TO DEFINE THE ARRAY POSITIONS FOR OTHER COMPONENT COSTS ARE DETERMINES.

FOR JEI THIS ROUTINE IS BYAPASSED BECAUSE THE FIRST HEADER WILL NOT HAVE A REDUCER PRECEDING IT.

IF (J.ER.1) GO TO 12

HEADER $\quad 59$

HEADER 60

HEADER 61

HEADER 62

HEADER 63

HEADER 64

HEADER 65

HEADER 66

HEADER 67

HEADER $\quad 68$

HEADER $\quad 69$

HEADER 70

HEADER 71

HEADER T2

HEADER 73

HEADER 74

HEADER 75

HEADER $\quad 76$

1 HEADER 77

HEADER 78

HE $\triangle D E R \quad T 0$

HEADER 80

HEADER RI

HEADER B?

HEADER

HEADER B4

HEADE? 85

HEADER 86

HEADER 87

HEADER 88

HEADER $\quad 89$

HEADEQ 90

HEADER 91

HEADER 92

MEADER 93

HEADER 94

HEADER 95

HEADER 96

HEADER 97

HEADER 98

HEADER 99

HEADER 100

HEADER 101

HEADER 102

HEADER IOJ

HEADER 104

HEADER 105

HEADER 106

HEADER 107

HEADER 108

HEADER 109

HEADER 110

HEADER 111

HEADER IIZ

HEADER 113

HEADER 114 
THE PROGRAM MAY HAVE A SPECIFIED VALUE OF RED WHICH FIXES THE MIIST BE A MULTIPLE OF 6 . IF RED=0 THE ROUTINE CALCULATES A HEADER REDIJCTION. FOR EXAMPLE, HEADER SECTION J-I HAS BEEN ASSIGNED HEADER A DIAMETER OF 96 INCHES, IT IS DETERMINED THAT HEADER SECTIDN HEADER $J$ SHOULD BE 84 INCHES. THE REQUIRED REDUCTION IS I INCHES. IF DEDEC THEN A 96 INCH REDUCER IS COSTED AND HEADER SECTION . WILL BE $\triangle$ SSIGNED $\triangle$ DIAMETER OF 78 INCHES. IF RED $=6$ INCHES $\triangle 96$ JNCH REDUCER IS COSTED BUT DIAMETER J WOULO BE ASSIGNED A VALUE OF 90 INCHES. IF RED 12 INCHES THEN A 96 INCH REDUCER IS COSTED AND DIAMETER J IS ASSIGNED A VALUE OF 84 INCHES. IF REDEIB INCHES OR MORE THEN NO REDUCER IS SPECIFIED AND DIAMETER J IS ASSIGNED A VALUE OF 96 INCHES, LARGE VALIJES OF RED RESULT IN PIDE SIZES LARGER THAN REQUIRED BUT FEWER REDUCERS ARE REQUIRED.

CALCULATION OF REDUCTION, RED NOT SPECIFIED.

IF (RED.NE, 0.1GO TO 13

IF (DIA:EO.DIAJ) GO TO 12

REDISCT DDIAJ-DIA

$M=R E D U C T / 5, \ldots 0$ !

$I J K=I$

GO TO 14

RED SPECIFIED, POSSIBLE REASSIGNMENT OF DIAMETER J.

HEADER

HEADER

HEADER

HEADER

HEADER

HEADER

HEADER

HEADER

HEADER

HEADER

HEADER

HEADER

HEADER

HEADER

HEADER

HEADER

HE ADER

HEADER

HEADER

HEADER

HEADER

HEADER

3 IF(DIA:GT.OIAJ-RED)GO TO 15 $D$ IA $=D I A J=R E D$

$M=R E D / 6,+.01$

I.JKE OIIA-DIAM(I) /6,+.0I

$14 K=0$

$J J=M$

HEADER

HEADER

HEADER

HEADER

HEADER

HEADER

HEADER

HEADER

DETERMINE REOUCER COST. IT HAS BEEN ASSUMED FROM THE REDUCER COST DATA THAT. FOR EXAMPLE, A OS INCH REDUCEP WILL COST THE SAME WHETHER THE REDUCTION IS TO 90,84, OR 78 INCHES. IF THE TOTAL REDUETION BETWEEN ADJACENT HEADER

HEADER

HEADER

HEADER

SECTIONS EXCEEDS IB INCHES THEN AN ADDITIONAL REDUCER MUST BE HEADER COSTED, IN THE EXAMPLE, THIS WOULD BE A 78 INCH REDUCER, IF HEADER TME TOTAL REDUCTION EXCEEDS 36 INCHES, SAY 96 TO 54 , THEN A HEADER 96. 78. AND 60 INCH REDUCER WOULD BE COSTED. AND SO ON.

16 CONTINUE

IF (IJK•JJ.GT.NKOS) STOP"IN HEADER IJK +JJ EXCEEDS NKOS"

RCST = RCST * REDCST (IJK•JJ)

$K=K+1$

$J J=M-3 m K$

IF (JJ.LE, O) GO TO 12

GO TO 16

15 DIA $=D I A J$

$12 M=(D I A-D I A M(I)) / 6 \ldots+01$

IF $(I+M$. GT. NKOS) STOP MIN HEAJER I +M EXCEEOS NKOS

IF THE LAST HEADER SECTION IS BEING DESIGNED USE AN ELBOW RATHER THAN A TEE.

HEADER

MEAOER

HEADER

HEADER

HEADER

HEADER

HEADER

HEADER

HEADER

HEADER

HEADER

HEADER

HEADER

HEADER

HEADER

HEADER

116

117

118

119

120

121

122

123

124

125

126

127

128

129

130

131

132

133

134

135

136

137

$13 \mathrm{~A}$

139

140

141

142

143

144

145

146

147

148

149

150

151

152

153

154

155

156

157

158

159

160

161

162

163

164

165

166

167

168

169

170

171

172 
SUBROUTINE HEADER $\quad 74 / 74$ OPT=

FTN $4.5+414$

$06 / 05 / 78 \quad 07.59 .59$

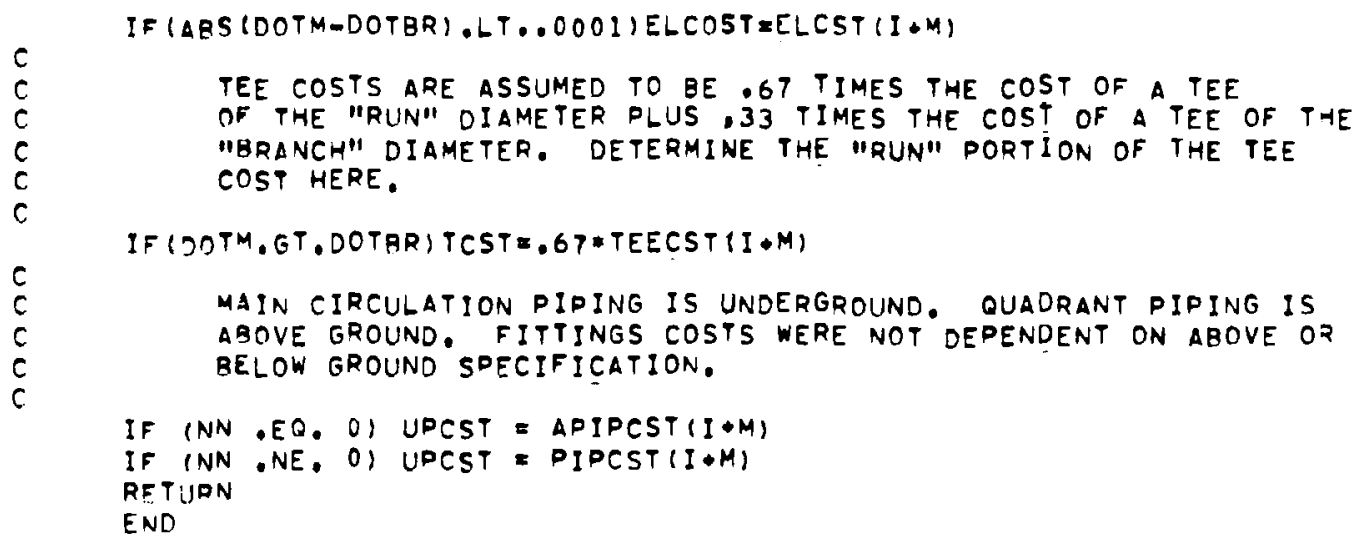

END

HEADER 173

HEADER 174

HEADER $\quad 175$

HEADER $\quad 176$

HEADER 177

HEADER $\quad 178$

HEADER $\quad 179$

HEADER 180

HEADER 181

HEADER 182

HEADER 183

HEADER 184

HEADER 185

HEADER 186

HEADER 187

HEADER 188

HE $\triangle D E R$ 


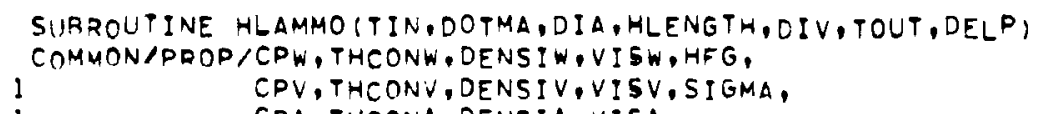



SUBROUTINE HLAMMO
$74 / 74 \quad O P T=1$
FTN $4.5 \cdot 414$
$06 / 05 / 73 \quad 07.59 .59$

$c$

PIPE

$T I=F L U I O(11,1, P 2, E L E V \cdot " C A L C$ IPH)

DELTI $=.001$

$20 F T I=-P 2$. FLUID $10.1 . T I, E L E V, " C A L C 2 ")$

FTIDEL = - P2 - FLUID $(10,1$,TI DELTI,ELEV, "CALC 3")

$T I P I=T I$ - DELTI*FTI/(FTIDEL - FTI)

IF(ABSITIPI - TII.LT..0001) GO TO 30

$T I=T I P I$

Go To 20

30 TOUT $=$ TI

$c$
$c$
$c$

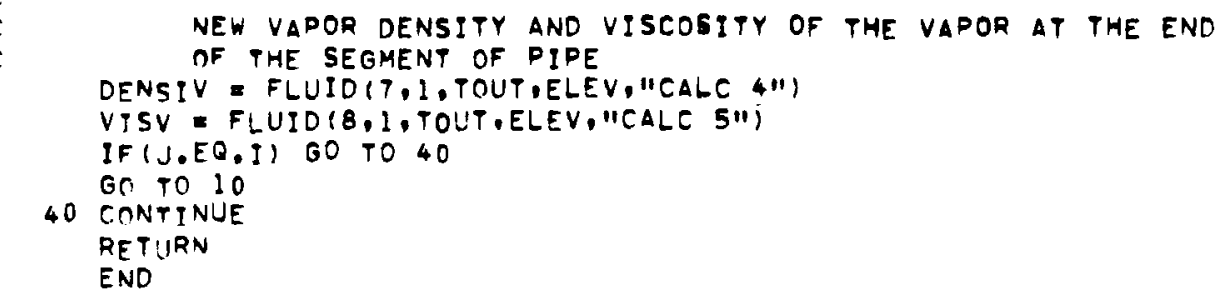


$74 / 74 \quad O P T=1$

FTN $4.5 \cdot 414$

$06 / 05 / 78$

07.59 .59

SURROUTINE HOTERV (FAIR,HO,SIGMAG,ALPHA, XD,GAIR)

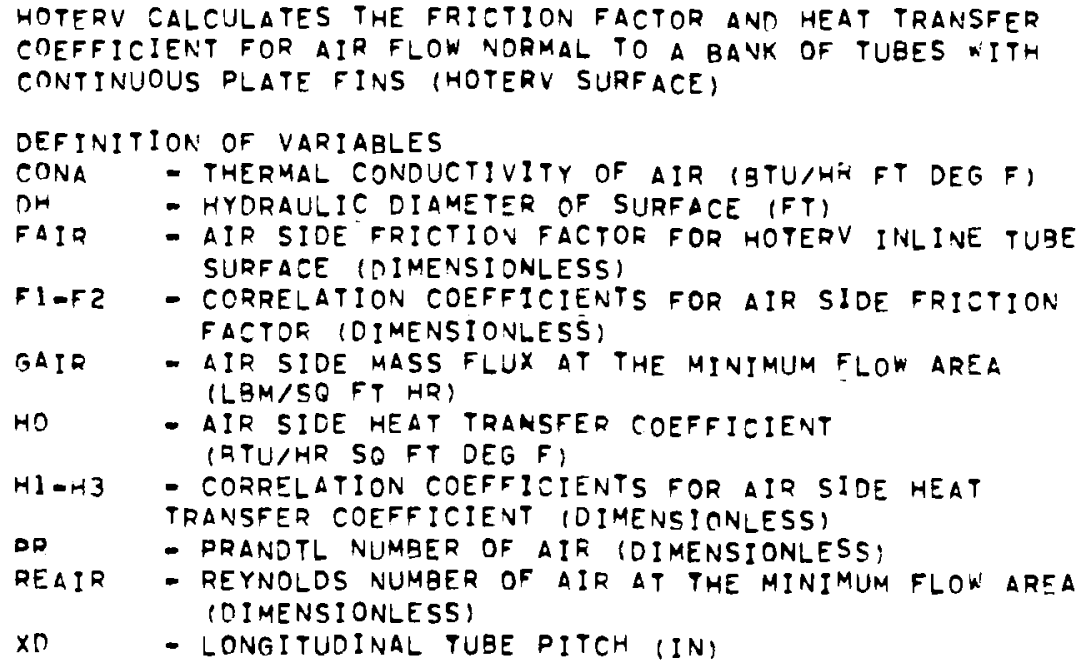


SUBROUTINE HTCLOIDTI, DL, ANG,GT, XI, XZ,PRL, DENL, DENV, VISL, VISV, TKL, ICDL,HTL,HTC,HTE,HTN,HTK,RETI

DIMENSION ANG (3)

SUBROUTINE HTCLO CALCULATES THE TUBESIDE TWO-PHASE LIQUID
FILM HEAT TRANSFER COEFFICIENT FOR THE CONDENSATION OF a FlUID ON THE WALLS OF a TUBE

DEFINITION OF VARIABLES

HTCLO

HTCLO

HTCLO

HTCLO

HTCLO

HTCLO

HTCLO

HTCLO

HTCLO

ARG (1) - ANGLE THE TUBE MAKES FROM THE hORIZONTAL (RAD)

ARG (2) - SINE OF THE ANGLE THE TUBE MAKES FROM THE

HTCLO

HTCLO

HTCLO HORIZONTAL (DIMENSIONLESS)

DENL - DENSITY OF THE LIQUID (LBM/CU FT)

DENV - DENSITY OF THE VAPOR (LBM/CUFT)

OL - LENGTH OF THE LIOUID AND VAPOR TUBE (IN)

HTCLO

HTCLO

HTCLO

HTCLO

DR - RATIO OF THE DENSITIES (DIMENSIONLESSI

DTI - INSIDE DIAMTER OF THE TUBE (IN)

GT - MASS FLUX OF THE FLUID THROUGH THE TUBE (LBM/

HTCLO

HTCLO HR $-S Q$ FT)

HTCLO

HTCLO

HPB - HEAT TRANSFER COEFFICIENT ACCORDING TO THE CORRELA- HTCLO TION BY BOYKO AND KRUZHILIN (BTU/HR-SO FT-DEG F) HTCLO

HTC - HEAT TRANSFER COEFFICIENT ACCORDING TO THE CORRELATION BY CHATO (BTU/HR-SO FT-DEG F)

HTK - HEAT TRANSFER COEFFICIENT ACCORDING TO THE CORRELATION BY KIRKBRIDE AND BADGER (BTU/HR-SQ FTDEG F)

HTN - HEAT TRANSFER COEFFICIENT ACCORDING TO THE CORRELATION BASED ON THE NUSSELT TYPE ANALYSIS (BTU/HR-SQ FT-DEG F)

PRL - PRANDTL NUMBER OF THE LIOUID (DIMENSIONLESS)

RET - REYNOLDS NUMBER (DIMENSIONLESS)

TKL - THERMAL CONDUCTIVITY OF THE LIOUID(BTU/HR-FT-DEG F)

VISL - VISCOSITY OF THE LIQUID (CENTIPOSE)

XD - CHANGE IN VAPOR QUALITY FROM THE INLET TO THE EXIT OF THE TUBE IDIMENSIONLESS

$X I$ - INLET QUALITY (DIMENSIONLESS)

XD - EXIT QUALITY (DIMENSIONLESS)

$D R=D E N L / D E N V$

RET $=D T I * G T /$ IVISL*29.)

$X 0=4 M I N I(X 2, .999)$

$c$
$c$

BRAVITY CONTROLLED STRATIFIED CONDENSATION (CHATO)

$\triangle N G L E$ OF THE CONDENSATE - IZO DEGREES

$X_{D}=\triangle M A X I(X I-X 0 . .001)$

HTC = 9?4."TKL*(DENL"(DENL-DENV) $D$ DLANG $(3)$

(VISL*DTI*Z*GT*XD))*.33

c

PRESSURE GRADIENT CONTROLLED ANNULAR CONDENSATION (BOYKOKRUTHILIN)

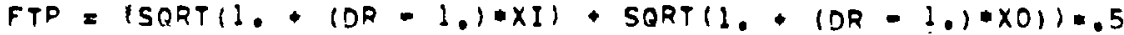

HTB $=.024 * T K L * R E T * .8 * P R L * .43 * F T P / O T I * 12$.

$K \cap O L=0$

IF (ANG (1) -0.017$) \quad 30.30 .50$

30 IF (HTCAHTB) $100,100,40$

$40 \mathrm{KOOL}=1$

HTCLO

HTCLO

HT CLO

HTCLO

HTCLO

HTCLO

HTCLO

HTCLO

HTCLO

HTCLO

HTELO

HTCLO

HTCLE

HTCLO

HTCLO

HTCLO

HTCLO

HTCLO

HTCLO

HTCLO

HTCLO

HTCLO

HTCLO

HTCLO

HTCLO

HTCLO

HTCLO

HTCLO

HTELO

HTCLO

HTCLO

HTCLO

HTCLO

HTCLO

HTCLO 


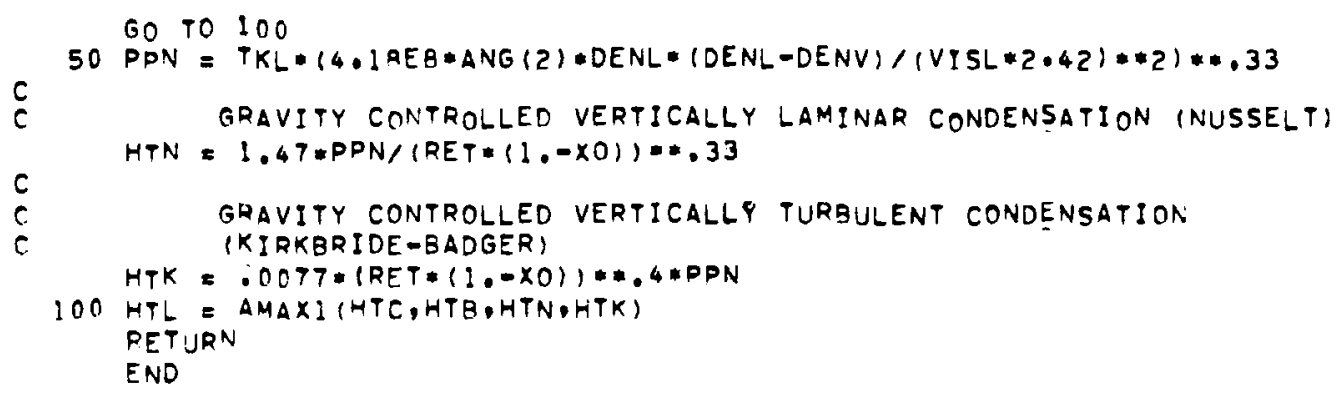

$c$

c

HTCLO

HTCLO

HTCLO

HTCLO

HTCLO

HTCLO

HTELO

HTCLO

HTCLO

HTCLO

HTCLO

HTCLO

59

60

61

63

64

65

66

67

68

69

70 
SUBROUTINE HYOLOS $\quad 74 / 74 \quad$ OPT $=1$

FTN $4,5+414$

$06 / 05 / 78 \quad 07.59 .59$

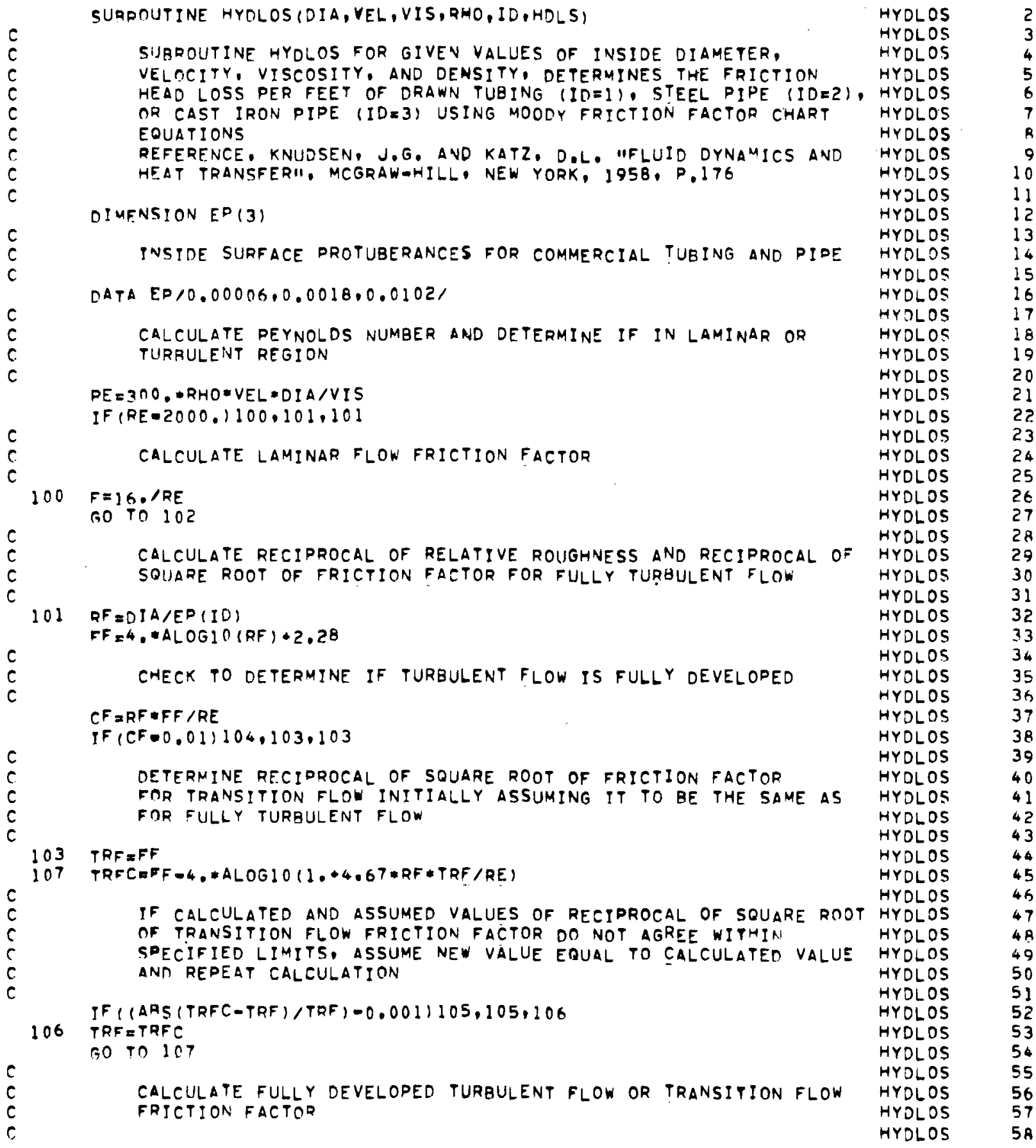




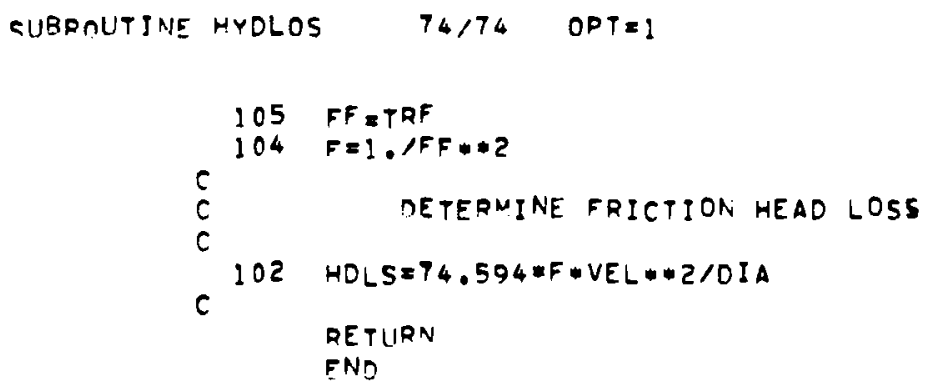

$\begin{array}{ll}\text { HYDLOS } & 59 \\ \text { HYOLOS } & 60 \\ \text { HYDLOS } & 6 ! \\ \text { HYDLOS } & 62 \\ \text { HYDLOS } & 63 \\ \text { HYOLOS } & 64 \\ \text { HYDLOS } & 65 \\ \text { HYDLOS } & 66 \\ \text { HYOLOS } & 67\end{array}$


220 FORMAT (1H+,TBO,"TURBINE OUTLET TEMPERATURE, TI",F10.3," F")

INPSUM

INPSUN

INPSUM

226 F ORMAT $11 \%+, 774, " L E N G T H$ OF HEAT EXCHANGER TUBES ELENG",F10,1,

INPSUM I" FEFT")

230 FORMAT (T4. "EXCHANGER LENGTH FIXED AT ".F5.0." FEET")

INPSUM

233 F RRMÄT IT4."TERMINAL TEMPERATURE DIFFERENCE CONSTRAINED TO $1, F 5,2$, J" FII)

234 FARYAT (T4, "TERMINAL TEMPERATURE DIFFERENCE UNCONSTRAINED")

235 FORUAT (1H+.T74."TERMINAL TEMPERATURE OIFFERENCE TTDI",F10.3," F")

240 FORMAT (T4, "RUN W'ITH VELOCITY RE COVERY")

245 FORHAT IT4, "RUN I'ITHOUT VELOCITY RECOVERYI

255 FORMAT (T4,"NTU CALCULATION IS BY ",2AIO)

260 FORMATIT4,"IFRONTAL AIR VELOCITY CONSTRAINEO TO ",F5.2," FT/SECI":

261 FORMAT (TA."IFRONTAL AIR VELOCITY UNCONSTRAINED")

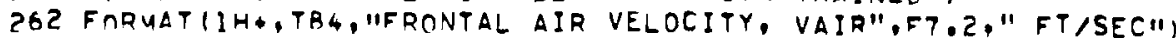

300 FORMAT1//T51,"UNTT COSTS AND COSTING FACTORS"/T51,30(1-1)/11

305 FORMATITE." IFUEL ",F7.3," CENTS/MMBTU"

\$,T3ก, "POLER PLANT CONSTRUCTION",FI1.3," $\$ / K W "$

S.TIOL."FIXED CHARGE RATE",F9.3)

310 FORMATIT2,"LANO ".F7.3," \$/SO FT"

S.T30."REPLACEMENT POWER", 7X,F $11,3, " M I L L S / K W H "$

I.TI IO, "CAPACITY FACTOR",F9.3)

315 FORMATITZ,"MOTORS",F7.3," S / HDI

S,T30,"STEAM SUPPLY",T54,FII.3," \$/KW TURBINE OUTPUT"

\$.T9B,"BASE THERMAL EFFICIENCY",F9.31

320 FARMATIT30, "CADACITY CHARGE", T54,F11,3," S/MW"

S. T93, "CONSTRUCTION COST MULTIPLIER",F9.3!

325 F RRMATIT95, "MAINT COSTS /CAPITAL COSTS",FO,31

WDITE (6.202)

WRITE $(6, ? 04)$

WRI TE $(6, \geq 06)$

W'RITE $(6,204)$

WRITE $(6.200)$

WDITE $(0,208)$ TLIM

$c$

WRITE $(6,207)$ FFHX

$c$

I (FXTEMP) WRITE $(6,218)$

IF (. NOT. FXTEMP) WRITE (6.210)

C

WRITE $(6.220)$ VAS (1)

IF (FXTVAP) WRITE $(6,260)$

IF (.NOT, FXTVAR) WRITE $(6,261)$

c

WRITE $(6.262)$ VAS(2)

IF (FXTTTD) WRITE $(6,233)$

IF (.NOT. FXTTTD) WRITE $(6.234)$

c

WEITE $(0,235)$ VAS (3)

IF (FXTLNG) WRITE $(6,230)$ FIXL

IF (.NAT,FXT:NG) WRITE $(6.225)$

C

WDITE $(0,2.26)$ VAS $(4)$

INPSJM

INDSUM

INPSUM

INPSUM

INPSUM

INPSUM

INDSJM

INPSUM

INDSUM

INDSUM

INDSUM

INPSUM

INOSUM

INPSUM

INPSUM

INPSUM

INPSUM

INDSUM

INPSUM

INPSUM

INPSUM

INPSUM

INDSUM

INPSUM

INPSUM

INPSUM

INPSUM

INPSUM

INDSUM

INPSUM

INPSIM

INDSUM

INPSJM.

INPSUM

INDSUM

INDSUM

INDSUM

INPSUM

INDSUM

INPSUM

INPSUM

INPSUM

INPSUM

INDSUM

INPSUM

INPSUM

INPSUY

IF (DEEPL.GT.0.00) WRITE $(6,215)$ DEEPL

IF (TEEPL,EQ. $) .00)$ WRITE $(6,210)$

INPSIJM

INPSUM

INDSUM WDITE $(6,211)$ VAS (5)

INDSUM 
QUBROUTINE INPSUM T4,74 OPTEI

FTN 4.5 .414

$06 / 05 / 78$

07.59 .59

IF (VELREC.EQ.0.00) WRITE $(6,245)$

IF (VELQEC.NE.0.00) WRITE $(6,240)$

$c$

c

WRITE $(6,126)$

WRITE $(6,127)$

WDITE $(6,128)$ (TPO(I),I $=1,4)$

WRITE $(6,129)$ RAP

c

KRITE $(6,130)$ ELEV

WRITE $(6.300)$

WRITE $(6,305)$ FCOS, PLANC,FCR

WRITE 16.310$)$ COSTL,PWCOS,CAPF

WPITE $(6,3)$ HPCST,CSSDKW, TEFF

WRITE $(6.320)$ CAPCHG.CCM

WDITE $(6,325)$ PER

RETIIRN

ENO

$\begin{array}{ll}\text { INPSUM } & 85 \\ \text { INPSUM } & 86 \\ \text { INPSUM } & 87 \\ \text { INPSUM } & 88 \\ \text { INDSUM } & 89 \\ \text { INPSUM } & 90 \\ \text { INPSUM } & 91 \\ \text { INPSUM } & 92 \\ \text { INPSUM } & 93 \\ \text { INPSUM } & 93 \\ \text { INPSUM } & 94 \\ \text { INPSUM } & 95 \\ \text { INPSUM } & 96 \\ \text { INDSUM } & 97 \\ \text { INPSUM } & 96 \\ \text { INPSUM } & 99 \\ \text { INPSUM } & 100 \\ \text { INPSUM } & 101 \\ \text { INPSUM } & 102 \\ \text { INDSUM } & 103\end{array}$


SURROUITINE LIMIT (ATM, TMAX,RIMAX,TMIN,RIMIN)

LIM:T SUAROLITINE LIMIT CALCUIATES THE MAXIMUM AND MIN:MUM ALLOWASLE LINIT TEMFERATURES. THE NINIMUM TEMPERATURE TO TWO DEGREES F. THE LIMIT MAXIMUM TEMPERATURE IS ARBITRARILY SET EOUAL TO 2 DEG F. TIMIT F LESS THAN THE BOILING POINT ITHE ENTHALPY/LB OF TRY AIR HAS NO PHYSICAL MEANING FOR PARTIAL PRESSURES FRUAL TO OR EXCEEDING THE ATMOSPHERIC DQESSURE)

LIMIT

LIMIT WHICH CAN CAUSE INSTABILITIES IN SUBROUTINE TCALC.

LIMIT

LIMIT

LIMIT

FINACTIONS FD, FAH, FCS AND FRI COMPUTE THE PARTIAL DRESSURE

$L I M I T$ AF WATER (IN, HG.), AESOLUTE HUNIOITY ILE OF WATER,

LITIT LH OF DRY AIR). HEAT CAPAEITP (RTU/DEG. FELE OF DRY AIR) ANJ LIMIT

LIMIT ENTHALPY (BTU/LR OF DRY AIR), RESPECYIVELY.

$F P(D H, T)=R H * 29.92 *(0.000203 *(1 T-32) / 1.8) *$.

- - 0.0118 (T-32.1/1.8.1.095)

* Expl - 0.000426*1:T-32.1/1.8:*2

- 0.0828 (?-32.1/2.8-5.163)

$c$

FD (2H.T) ERH EXP $(17.268-9 ? 40.1(T+460.1)$

$F A H(D, A T M)=D * 18.02 /((A T M M 25.92-0) \cdot 28.97)$

$F C S(A H)=.24+.45 * A H$

FRI (CS,T,AH)ECS*T+1094, \#AH

$c$

$R_{H}=1$ THE RELATIVE HUMIDITY IS SET EQUAL TO 1.

C THE BOILING POINT IS COMPUTED.

$T=-46 C .-0240.1(A L O G(A T M+29.92 / R M)-17.168)$

$T M A X=T-2$.

DMAXEFP (DH,TMAX)

AHMAXEFAH (PMAX,ATM)

CSMAX $=F C S(\triangle H M A X)$

PIMAX JPRI (CSMAX, TMAX, AHMAX)

$c$
$C$
$C$

THE MINIMUM ALLOWEO TEMPERATURE IS ARBITRARILY SET EQUATO ? DEGREES F. AND THE CORRESPONOING VALUES OF PARTIAL DRESSURE, ABSOLUTE HUMIDITY. SPECIFIC HEAT AND ENTHALPY $\triangle D E$ COMPUTEN.

$T M I N=2$.

DMIN=FP(DH,TMIN)

AHMINDFAH (DMIN,ATM)

CSMINEFCS (AHMIN)

PIMYNEFRI (CSMIN,TMIN,AHMIN)

WDITE(6.:0) TMAX, TMIN,RIMAX, RIMIN

10 FORMATI"OIN SR LIMIT TMAX,TMIN, RIMAX,RIMIN=", IPAEI5,5) RETURN

END

LIVIT

LINIT

LIMIT

LIMIT

LIMIT

LIMIT

LIMIT

LIMIT

LIMIT

LIMIT

LIMIT

LIMIT

LIMIT

LIMIT

LIMIT

LIMIT

LIMIT

LIMIT

LIMIT

LIMIT

LIMIT

LIMIT

LIMIT

LIMIT

LIMIT

LINIT

LIMIT

LIMIT

LIMIT

LIMIT

LIMIT 
SUBROUTINE MTCH $\quad 74 / 74 \quad$ OPTEI FTN $4.50414 \quad 06 / 05 / 78 \quad C$

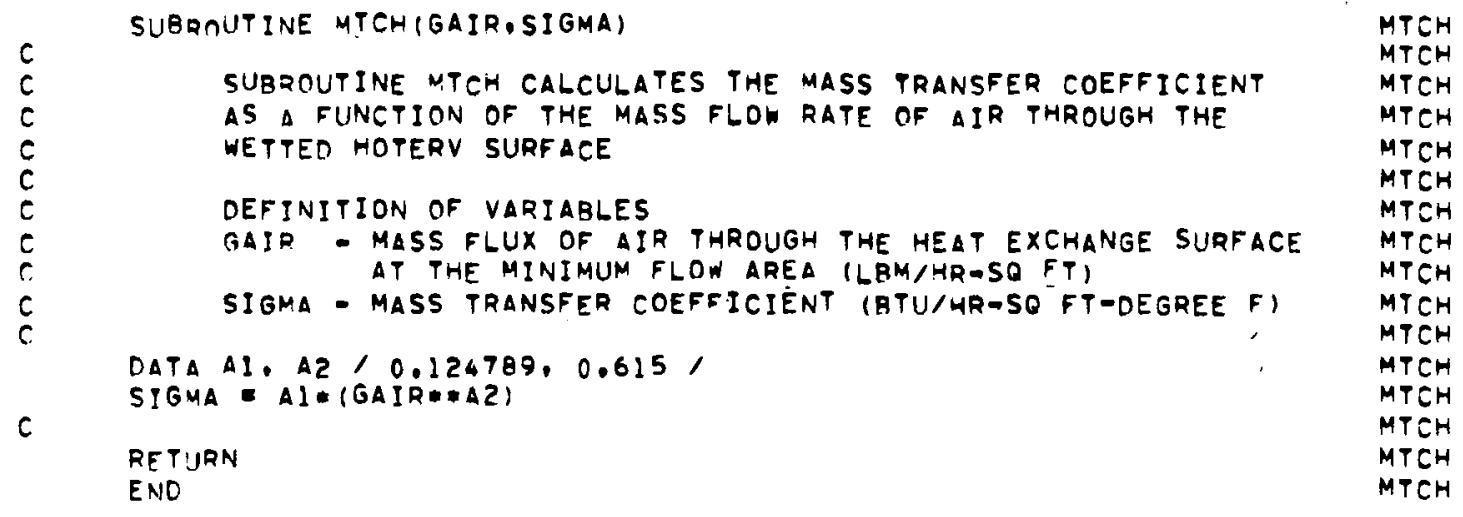


$74 / 74 \quad O P T=1$

SUBRDUITINE NOVART (VCST, RANGE:

SUBROUTINE NOVART DETERMINES THE PERFORMANCE OF A PLANT DESIGNED AT A SDECIFIC AMBIENT TEMPERATURE OVER A WHOLE YE.AD THE ROUTINE ALSO DETERMINES THE INCREMENTAL COST OF OPERATING THE POWER PLANT OVER THE ENTIRE YEAR

DEFINITION OF VARIABLES

CAPCHG - CAPACITY CHARGE (\$MEGAWATT)

CADCST - CAPITAL COST FOR AUXIIARY POWEP (S)

CAPF - CAPICITY FACTOR (OIMENSIONLESS)

CCOS - COST OF THE HEAT EXCHANGER (\$)

CLAND - COST OF THE LAND(\$)

CONGAS - COST OF THE CONDENSER/REBOILEA (\$)

COSM - MAINTAINENCE COST (MILLS/KWH)

CPID - COST OF THE PIPING (\$)

CSTCL - AUXILIARY POWER COST (MILLS/KWH)

DELFC - INCREASED FUEL COST FOR THE DLANT (MILLS/KWH)

FCOS - FUEL COST (STMM BTU)

FCR - FIXED CHARGE RATE (DIMENSIONLESS)

FPOW - FAN POWER (KWH)

HRFACI - HEAT RATE FACTOR LT RATING CONOITION(DIMENSIONLESS)

HPFACZ - HFAT RATE FACTOR AT DESIGN CONDITIONS (DIMENSIONLESS)

HRFAC4 - HEAT RATE FACTOR AT OFF DESIGN CONDITIONS (DIMENSI ONLESS)

NTA - NUMAER OF TEMPERATURE INCREMENTS IN THE TEMPERATURE

- RANGE OF THE SITE

DARCOS - INCREMENTAL POWER PRODUCTION COST AT THE AMBIENT TEMPERATURE (MILLS/KWH)

DREN - POWER GENERATING BP THE PLANT (MEGAWATTS)

DPOW - PUMP POWER (KWH)

PSIZE - PLANT SIZE (MEGAWATTS)

PSI713 - TOTAL POWER OUTDUT OF PLANT AT DESIGN CONDITIONS (MEGAWATTS)

DTOT - TOTAL POWER OUTPUT OF PLANT AT OFF DESIGN CONDITIONS (MEGAWATTS)

DNCOS - REDLACEMENT POWER COST (MILLS/KWH)

D22 - $A M M O N I A$ PRESSURE AT THE MEAT EXEHANGER (LBF/SQ ET)

DINI

- HFAT INPUT INTO THE POWER PLANT (ETU/HR)

DREJCTI - HEAT REJECTED AT THE DESIGN CONDITIONS OF THE PLAVT (RTU/HR)

RQEJCTZ - HEAT REJECTED AT THE OFF DESIGN CONDITIONS OF THE PLANT (BTU/HR)

SPCD - DIFFERENTIAL COST OF PLANT EXCLUDING THE COOLING SYSTEM (S)

TEFF

- BASE THERMAL EFFIEIENCY OF THE PLANT (DIMENSIONLESS)

TEFF

- Rated thermal efFicienCy of the plant (DIMENSIONLESS)

TEFF3 - DESIGN THERMAL EFFICIENCY OF THE PLANT (OIMENSIONLESS)

TEFF4 - OFF DESIGN THERMAL EFFICIENCY OF THE PLANT (OIMENSIONLESS:

TPER - FRACTION OF THE YEAR THAT THE CORRESPONDING AMBIENT TEMPERATJRE EXISTS (DIMENSIONLESS)
$06 / 05 / 75 \quad 07.59 .59$

NOVART

NOVART

NOVART

NOVART

HOVART

NOVART

NOVART

NOVART

NOVART

NOVART

NOVART

NOVART

NOVART

NOVART

NOVAPT

NOVART

NOVART

NOVART

NOVART

NOVART

NOVART

NOVART

NOVART

NOVART

NOVART

NOVART

NOVART

NOVART

NOVART

NOVART

NOVART

NOVART

NOVART

NOVART

NOVART

NOVART

NOVART

NOVART

NOVART

NOVART

NOVART

NOVART

NOVART

NOVART

NOVART

NOVART

NOVART

NOVART

NOVART

NOVART

NOVART

NOVART

NOVART

NOVART

NOVART

NOVART

NOVART 
$74 / 74 \quad$ OPT $=1$

VEST - INCREMENTAL INCREASE IN COST OF THE POWER PRODUCED BY THE OPTIMUM POWER PLANT DUE TO THE COOLING SYSTEM (MILLS/KWH)

YFP - PLANT SCALING FACTOR FOR FAN AND PUMP POWER LOSS (DIMENSIONLESS)

DEFINITION OF ARRAYS

DWD - WET/DRY BACK PRESGURE CURVE FOR THE AMBIENT TFMPERATURE RANGE (IN HGA)

TDO - ARRAY OF COEFFICIENTS FOR THE CUBIC CURVE FIT OF HEAT RATE FACTOR AS A FUNCTION OF TURBINE BACK PRESSURE (DIMENSIONLESS)

VTPER - FRACTION OF THE YEAR THAT THE AMBIENT AND WET BUL TEMPERATURE INCREMENTS EXIST (DIMEÑSIONLESS)

COMMON /VARVAR, VTPER(16); VTA(16), VTAWB(16)

COMMON / PROP/ CPW, THCONW, DENSIW, VISW. HFG.

-

* CPV, THCONV, DENSIV, VISV, SIGMA,

CPA, THCONA, DENSIA, VISA

COMMON

- aFTr, alPha, ang (3)

- Bolim(16)

S CAPF, CSSPKW, CONF, CONL, COSTL, CAPCHG, CONMAT, CONMAZ, CAF

5 , CRJ, CTURB, CDANG, CLUVR, CHAILS, CVM

\$. DFIN.DEEPL, DESVEL, DESVELV, DESVELD,DENSIF

5 EFFP, EFS

5 ,FCR,FIRST, FIXL, FCOS,FFHX, FXTEMP, FACTOR, FRST , FACTORD, FSHOT , FDELUE

5. FIXV,FIXTTD,FXTVAR,FXTTTD,FXTLNG

S. GBEFF

S. HXNP, HPCST,HCD

5 ITMAX, ITMIN

S JCONS

S. KCONV, KALEXT

S.MXEXT

S NTA,NSIDES, NBUNHI COMMON

5 $\mathrm{NOP}$

5. PSIZE, DER, PWCOS, PLANC, PFACT, POMDPL

5 POHBAF, POHFAN, POHLEC, POHCIR, POHCND, POHSTC, POHSCL

S RPP, REDUICE, REDUCV, ROOFL, RUOUT

$\$$, TREJ. QREDUCE, OREDUCV

S SIGMAG, SAAF, SEGL

S, TO, TPO(4), TEFF, TLIM, THFIN, TLPRA, TFIX

$5, T C D$. TW

S.UES, UWS

S.WFV. WATCONA, WATCOST

S. VAS (5)

S, XDEDA, XW, XO COMMON/SUPPLY/ VAL (5).

a OIN, CLAND. REAIR, FPOW, DELFC, WIOTH, ELENG, DAIR, VWAT, DEWAT. NW, NT, NP,PLANCZ,PSIZIZ, SPBP, SW4, SW3. 5DC,PTOTAL,PLANC3,SAFRON, AAIR, YFP, SPCD,PSIZ13, WTZ, RO2, NW2, TCOS, DELPA,DELPWT,DELPWC, CONBAS, XNTSX, VELDX

- TLAX, UCON, PMCST, FMCST, FEACC, EFFC, CH, CA, CS 5: CLTC, CFC, CPCC, FITCO, CSC,CEPREP,DELPIP, AST,OLDCST S.CADCST, XNFAN,BLDANG, CPLEN, DPFEL: WHX, WTTUG, ADIA, ABLN
$06 / 05 / 78$

07.59 .59

NOVART

NOVART

NOVART

NOVART

NOVART

NOVART

NOVART

NOVART

NOVART

NOVART

NOVART

NOVART

NOVART

NOVART

NOVART

NOVART

NOVART

NOVART

BIGCOM

BI GCOM

BI GCOM

BI GCOM

BIGCOM

BIGCOM

BIGCOM

BIGCOM

BIGCOM

BIGCOM

RIGCOM

BIGCOM

BI GCOM

BI GCOM

BIGCOM

BI GCOM

BI GCOM

BIGCOM

BI GCOM

BIGCOM

BIGCOM

BIGCOM

BIGCOM

BI GCOM

BI GCOM

BI GCOM

BIGCOM

BI GCOM

BIGCOM

BI GCOM

SUPPLY

SUPPLY

SUPPLY

SUPPLY

SUPPLY

SUPPLY

SUPPLY

SUPPLY

SUPPLY
59

60

6)

62

63

64

65

66

67

68

69

70

71

72

73

74

75

2 
5. WTHOF, WTFRM, STRUCC, CFPERF, HPPERF, TOTCF, TPC, EFFIN, ANTU 8. HIRFF, WATFF, XNMOD, TCTPF, FDCPF, FMTRC, $\triangle C C T T P$, ADJTP S. $\triangle E T E P C, \triangle D J B P D$, ACTVH, ADJVH, $\triangle D J P P F$, THOAIR, TCTF, AFCST 5. CSRI, WATERW, WSTRCT, CFOUND, HUBDIA, TOWLEN, NUMTOW, CTOWD S. SORJ.SQRJS, $A T U B C$, CRA, TTOI, TTDZ, DELPS, CSTLVF,PIPCSTD

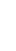

SUDOEY I:

SUPPLY I?

SUPDIY II

SUPDEY 14

SUPP_Y 15

SUPPLY 16

SUPPLY 17

SUPPLY 18

SCALER 2

SCALER 3

NOVART BC

NOVART BI

NOVART BE

NOVART 83

NOVART BL

NOVART 85

NOVART 86

NOVART RT

NOVART $8 E$

NOVART 89

NOVART 90

NOVART 91

NOVART 92

NOVART 93

NOVART 94

NOVART 95

NOVART GE

NOVART 97

NOVART 98

NOVART 99

NOVART 100

NOVART IOI

NOVART 102

NOVART 103

NOVART IOL

NOVART IO5

NOVART $10 G$

NOVART 107

NOVART $10 R$

NOVART 109

NOVART IIO

NOVART ?!!

NOVART IIE

NOVART 113

NOVART II4

NOVART 115

NOVART ILE

NOVART 117

NOVART IIR

NOVART IIO

NOVART 120

NOVART III

NOVART $12 E$

NOVART 123

NOVART 124

NOVART 125

NOVAR: $\$ 26$ 


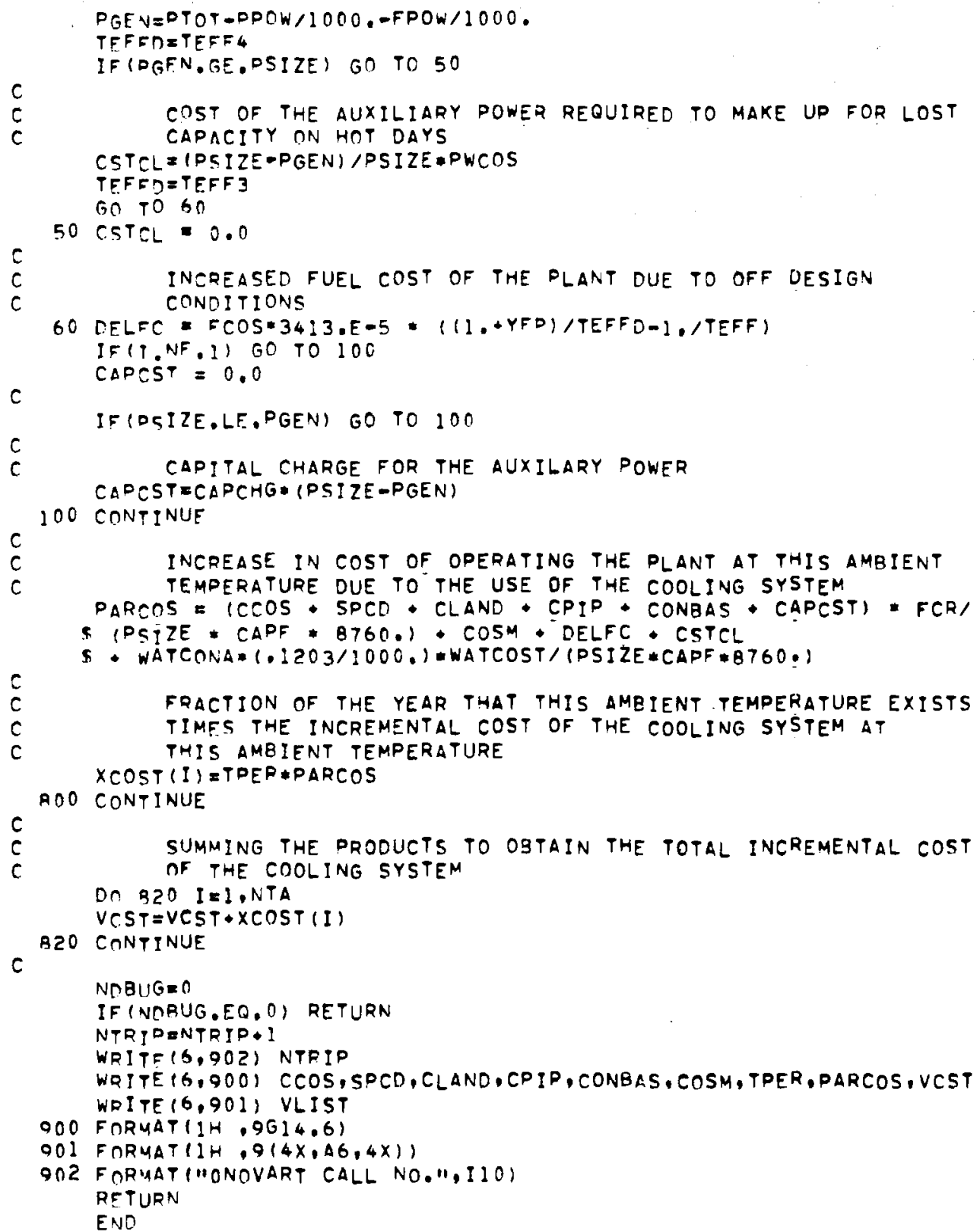

\begin{tabular}{|c|c|}
\hline $\begin{array}{l}\text { NOVART } \\
\text { NOVART } \\
\text { NOVART }\end{array}$ & $\begin{array}{l}127 \\
126 \\
129\end{array}$ \\
\hline NOVART & 130 \\
\hline $\begin{array}{l}\text { NOVART } \\
\text { NOVART }\end{array}$ & $\begin{array}{l}131 \\
132\end{array}$ \\
\hline NOVART & \\
\hline NOVART & \\
\hline NOVART & 135 \\
\hline NOVART & 136 \\
\hline NOVART & 137 \\
\hline NOVART & 138 \\
\hline NOVART & 139 \\
\hline NOVART & $1<0$ \\
\hline NOVART & $1<1$ \\
\hline NOVART & 142 \\
\hline NOVART & 143 \\
\hline NOVART & 144 \\
\hline NOVART & 145 \\
\hline NOVART & 146 \\
\hline NOVART & 147 \\
\hline NOVART & 148 \\
\hline NOVART & 149 \\
\hline NOVART & 150 \\
\hline NOVART & 15 \\
\hline NOVART & 52 \\
\hline $\begin{array}{l}\text { NOVART } \\
\text { NOVART }\end{array}$ & \\
\hline $\begin{array}{l}\text { NOVART } \\
\text { NOVART }\end{array}$ & \\
\hline $\begin{array}{l}\text { NOVART } \\
\text { NOVART }\end{array}$ & 56 \\
\hline NOVART & 57 \\
\hline NOVART & 58 \\
\hline NOVART & 150 \\
\hline NOVART & 160 \\
\hline NOVART & 161 \\
\hline NOVART & 162 \\
\hline NOVART & 163 \\
\hline NOVART & 164 \\
\hline NOVART & \\
\hline NOVART & 16 \\
\hline NOVART & 167 \\
\hline NOVART & 168 \\
\hline NOVART & \\
\hline NOVART & 11 \\
\hline NOVART & \\
\hline NOVART & \\
\hline NOVART & 17 \\
\hline NOVART & 17 \\
\hline NOVART & \\
\hline NOVART & \\
\hline NOVART & \\
\hline & \\
\hline
\end{tabular}


WHEN CALLED WITH KWHICH E I THIS ROUTINE REPORTS CONDITIONS AFTER OUT4 SCALING PLANT AND STEAMM SUPPLY BUTT RE- OUT4 FORE SCALING FOR FANS AND PUMPS OUT4

OUT4

WHEN CALLEO WITH KWHICH NOT I THE ROUTINE REPORTS CONDITIONS AFTER OUT 4 LOGICAL FIRST, FRST, FXTEMP, FXTVAR, FXTTTD, FXTLNG COMMON

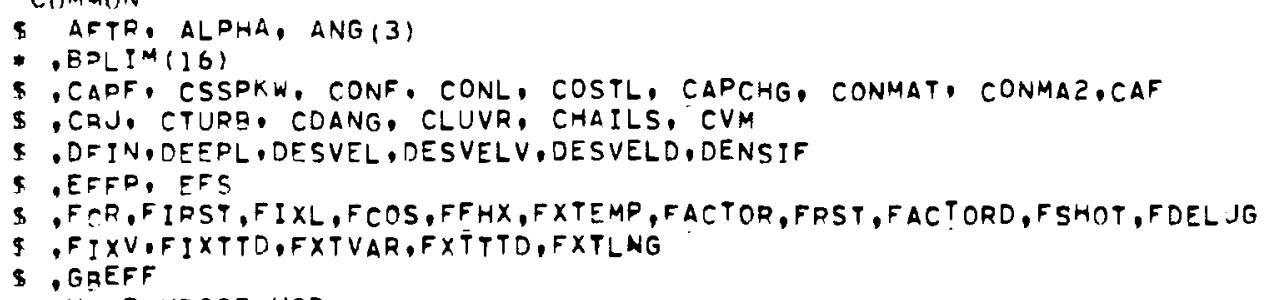


s GAIR, FAIF, HPAIR, HPWAT, WLRTP, PLANCTI COMMON /OJE/ DI, XOG,SF

901 FORMATI" CONOITIONS AFTER SCALING PLANT AND STEAM SUPPLY IBEFORE - SCALING FOR FANS AND PUMPS)", T38,"HRFAC2E", G.5.7)

906 FORMAT (IX,T88, "DSIZ12=",GI5.7," MW")

911 FORMAT (IX,TRR, "PLANCZ=",G15,7," $S / K W 11)$

916 FORMATIIX.TB8," SPBP $=11, G 15,9, "$ S")

QI7 FORMAT IIX.TBI, "BACK PRESSURE=",G15,7," IN, HEN)

918 FORMAT (1).T79."SAT TEMPERATURE=1", $15.9, "$ DEG F")

921 FORMAT IIX,TIZ,"AMMONIA FLOW RATE ",GI5.9," LS MASS , HRM)

$9 ? 3$ FORMATIIX,TI4," HEAT REJECTED ", G 15,9," BTU/HRU"?

926 FORMATIIX.T14,"A!R FLOW RATE ", G15.9, "LB MASS, HR")

Q31 FORMATIIX,TIM." PUMP POWER ", G15.9, "KILOWATTS")

936 F CRMATIIX,TI4," FAN POWER ", G15.9," KILOWATTS")

Q41 FORMAT (IX,TI4,"HX SURFACE AREA ", G15.9," SO FT")

946 FORMATIIX,TI4," FRONTAL AREA ", G15.9," "SO FTI)

951 FORMAT $11 \times$,T11,"WIDTH/LENGTH RATIO ", G15.9)

956 FORMATIX,T15," WIDTH ".111.T45," TUSES")

961 FORMAT (1X,T15," WIDTH ", G15.9." FEET")

962 F RRMATIIX,T9,"NO. CONDENSER TUBES "I, 15.9

963 FORMATIX,TR,"CONDENSER TUBE LENGTH ".G I5,9," FT.")

Q64 FORMAT (IX,TI3,"AMMONIA VELOCITY "G15.9,"FT/SEC")

965 FORMAT!IX,T7."CONDENSER HT TRFR AREA ", G15,9," SQ FT TOTAL")

1001 FORMATIIX,"SCALING FOR FAN AND PUMP DOWER REQUIREMENTS".

- T80."SCALing factor ", G15.9)

1005 FORMLT (IX,T85," PSIZE ",G15,9," MW")

IOOG FORMATIIX,T88,"PSIZI3 ", G15.9, "MW"I

1011 FORMATIIX,TQR, "PTOTAL ".G15,9," MW")

IOI6 FORMATIIX,T88, "PLANC3" G15.9," 5/KW")

In2l FCRUAT (IX.TRB," SPC ", G15.9." Si")

I 26 FORMAT (IX,T88," SPCD ",G15.9," \$1")

888Q FORMATIIHO,12("***** "1)/1)

WDITF $(6,8 B 8 B)$

WLPTD $=$ WOTI/ELENI

IF (KWHICH.NE,I) GOTO 2000

WDITE $(6,901)$ HRFAZS

WDITF $(6,906)$ ZSIZ12

WDITE $(6,011)$ ZPLNCZ

WRITE $(6,016)$ ZSPBP

WRITE $(6,917)$ BPZMIN

WDITE $(6,018)$ VAR (1)

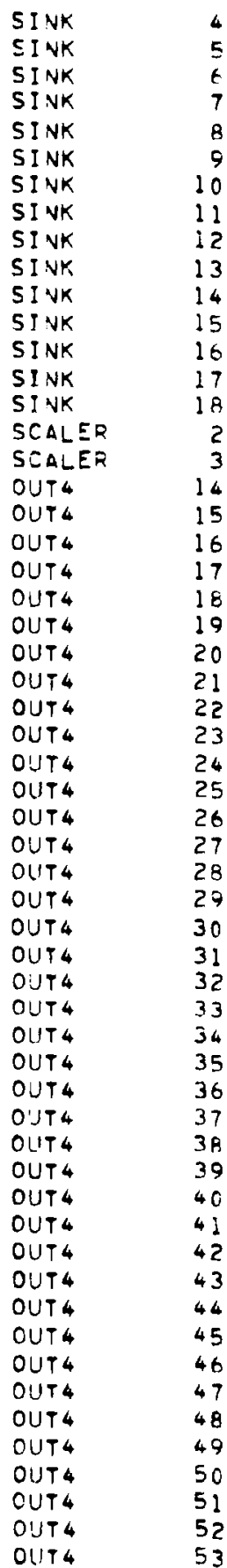




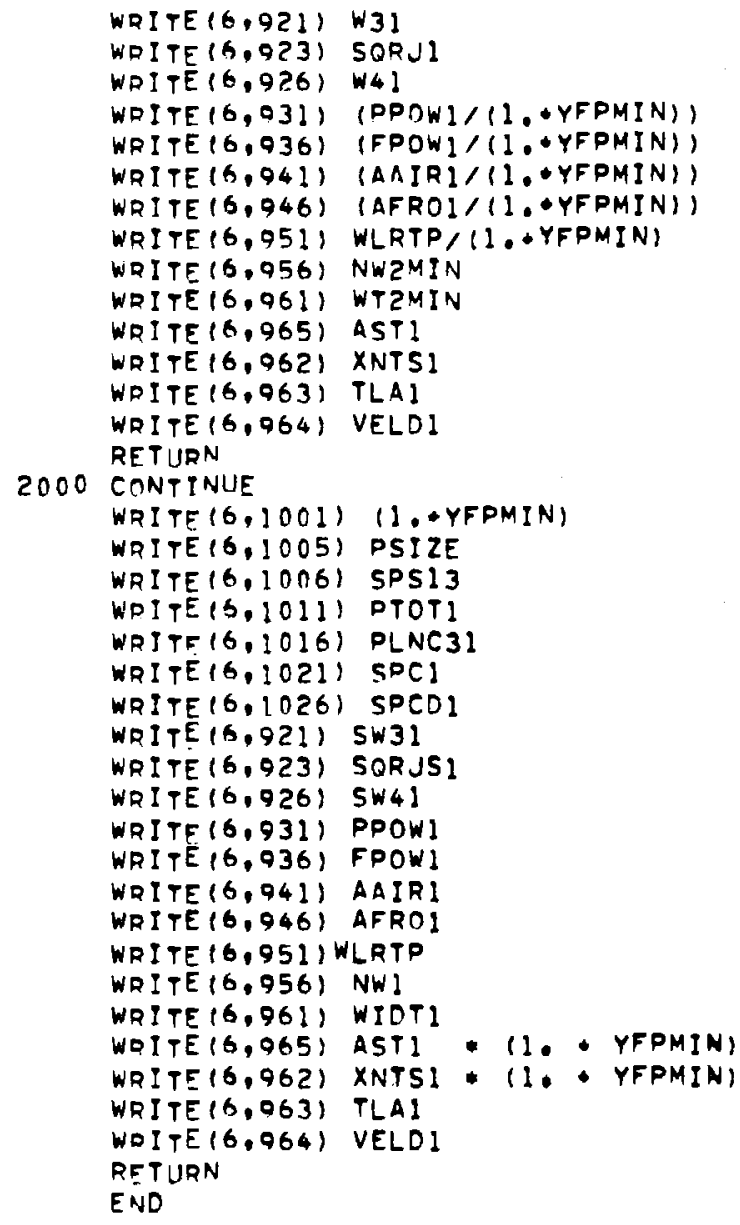

$\begin{array}{ll}\text { OUT4 } & 54 \\ \text { OUT4 } & 55 \\ \text { OUT4 } & 56 \\ \text { OUT4 } & 57 \\ \text { OUT4 } & 58 \\ \text { OUT4 } & 59 \\ \text { OUT4 } & 60 \\ \text { OUT4 } & 61 \\ \text { OUT4 } & 62 \\ \text { OUT4 } & 63 \\ \text { OUT4 } & 64 \\ \text { OUT4 } & 65 \\ \text { OUT4 } & 66 \\ \text { OUT4 } & 67 \\ \text { OUT4 } & 68 \\ \text { OUT4 } & 69 \\ \text { OUT4 } & 70 \\ \text { OUT4 } & 71 \\ \text { OUT4 } & 72 \\ \text { OUT4 } & 73 \\ \text { OUT4 } & 74 \\ \text { OUT4 } & 75 \\ \text { OUT4 } & 76 \\ \text { OUT4 } & 77 \\ \text { OUT4 } & 78 \\ \text { OUT4 } & 79 \\ \text { OUT4 } & 80 \\ \text { OUT4 } & 81 \\ \text { OUT4 } & 82 \\ \text { OUT4 } & 83 \\ \text { OUT4 } & 84 \\ \text { OUT4 } & 85 \\ \text { OUT4 } & 86 \\ \text { OUT4 } & 87 \\ \text { OUT4 } & 88 \\ \text { OUT4 } & 89 \\ \text { OUT4 } & 90 \\ \text { OUT4 } & 91 \\ \text { OUT4 } & 92 \\ & \end{array}$


C

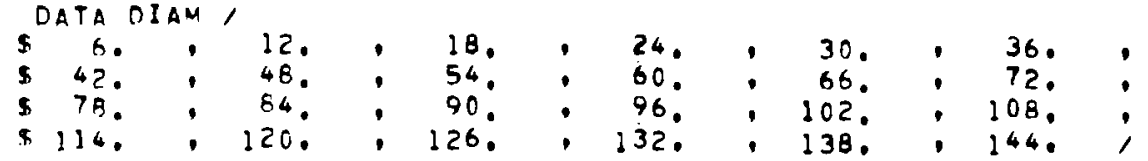

$c$

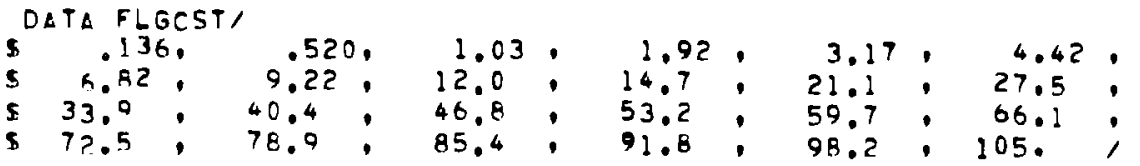

C

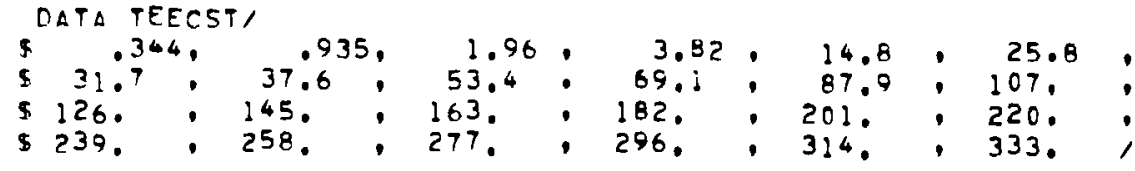

DATA PIDCSTI

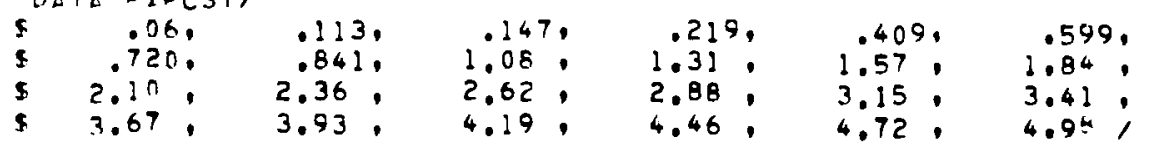

c

DATA VACST,

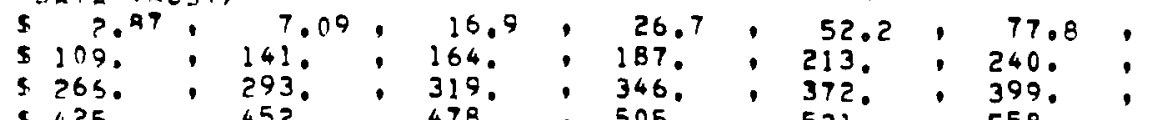

$c$

$\$ 425 ., 452,478$

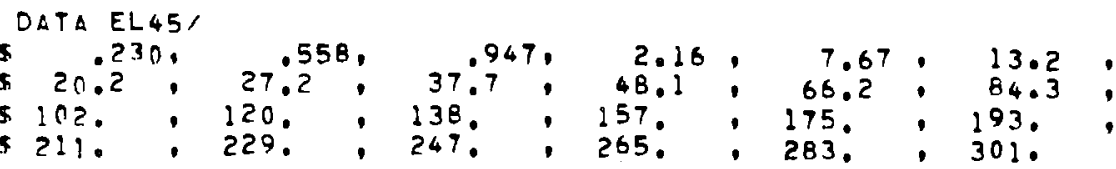

$c$

DAT A APIPCST/

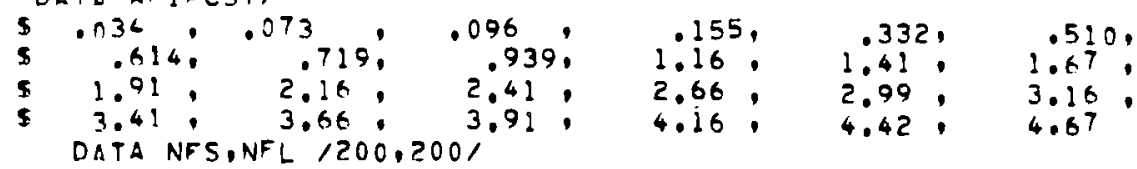

I CHORTANSIDES/4

IF IICHORD.EO.O) ICHORD = 2

ICHECK $=$ ICHORD $/ 2$

IF IS.ICHECK -ICHORD.LT.OI ICHORD = ICHORD I

IFIICHOPO.LE. OISTOP "IN PIPCLR ICHORO .LE. ZERO"

NRIINDAO =ICHORD N NBUNHI

INAUNENSUTIDQD

IF INAIINDOD.LE. OISTOP "IN PIPCLR NBUNPOD IS ZERC OR LESSN

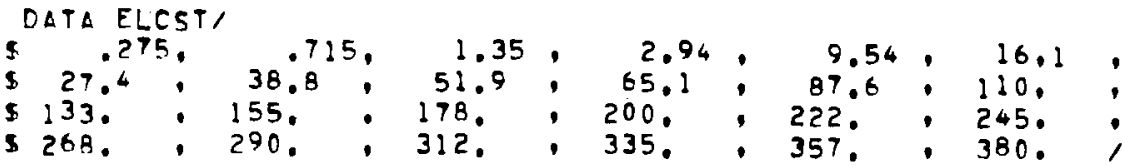

PIPCLR $\quad 173$

PIPCLR $\quad 174$

PIPCLR I75

PIPCLR I76

PIPCLR I77

DIPCLR I7A

PIPCLR $\quad 170$

PIPCLR IRO

PIPCLR IRI

PIPCLR IUD

PIPCLR 183

PIPCLR IB6

PIPCLR 185

PIDCLR 186

PIPCLR 187

PIPC-R 188

PIDCLR 189

PIPCIR 190

PIDCLR 19

PIPCIR I92

PIPCLR 193

PIPCLR 194

PIPCLF 195

PIPCLR 196

PIPCLR 197

PIPCLR 198

PIPCLR 190

PIPCLR 200

I PIDCLR 201

PIPCLR 202

PIPCLR 203

PIPCLR 204

PIPCLR 205

PIPCLF 206

PIPCLR 207

PIPCLR 208

PIDCLR 200

PIPCLR 210

PIPCLP $21 !$

PIDCLF 212

IPIDCLR 213

PIPCLR 214

PIPCLR 215

PIDCLR 216

PIPCLR 217

PIPCLD 218

PIPCLR 215

PIPCLR 220

PIPCLR 221

PIPCLR 222

DIPCLP 223

PIPCLE 224

PIPCLP 225

PIPCLR 226

PIPCLR 227

PIPCLR 228

PIDCLF 229 


$$
74 / 74 \quad \text { OPT }=\text { ? }
$$

$(\$ 1000)$

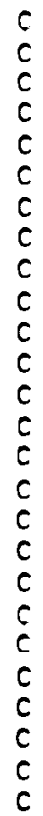

C

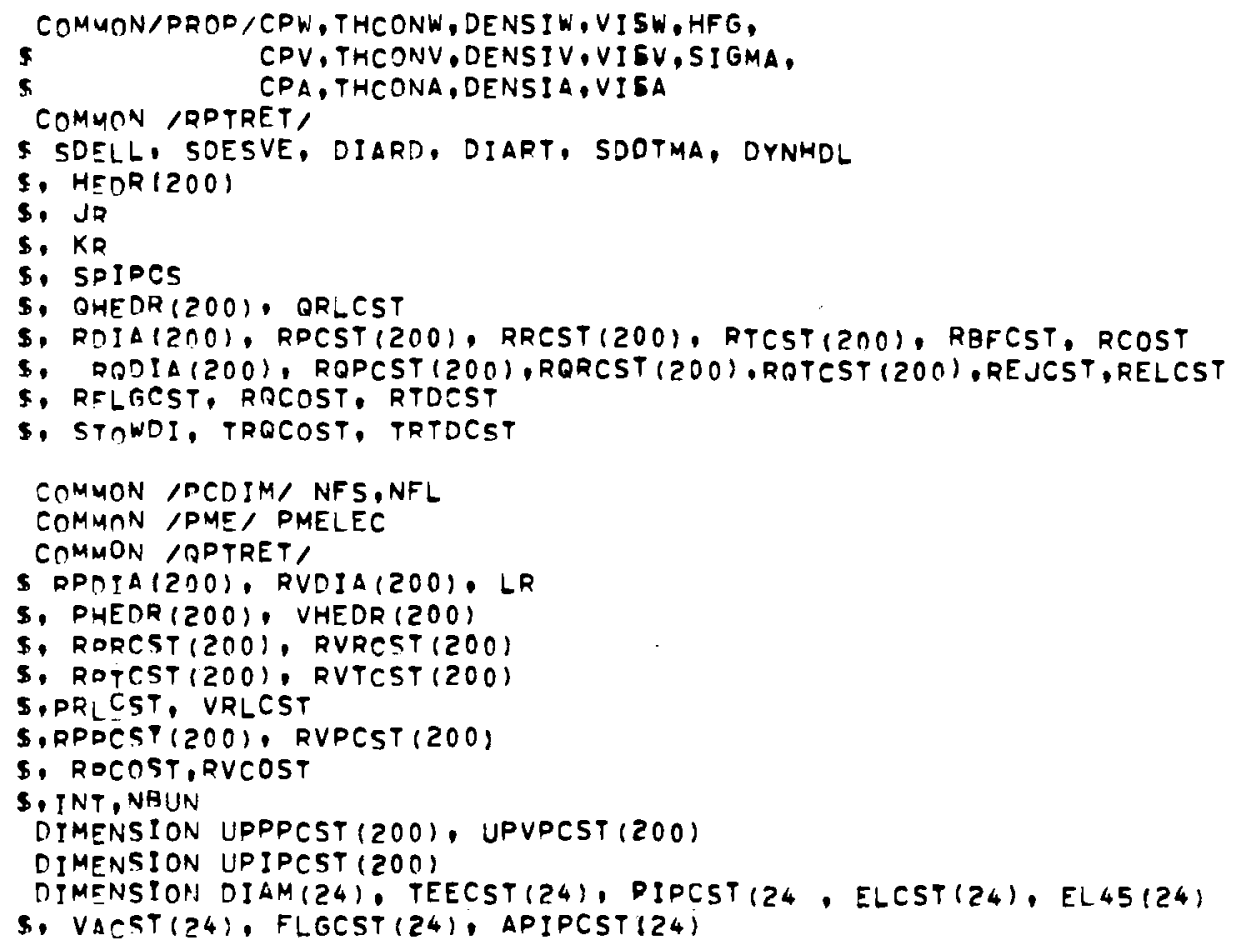

$06 / 05 / 78 \quad 07.59 .59$

\begin{tabular}{ll} 
PIPCLR & 116 \\
PIPCLR & 117 \\
PIPCLR & 118 \\
PIDCLR & 119 \\
PIPCLR & 120 \\
PIPCLR & 121 \\
PIPCLR & 122 \\
PIPCLR & 123 \\
PIPCLR & 124 \\
PIPCLR & 125 \\
PIPCLR & 126 \\
PIPCLR & 127 \\
PIPCLR & 128 \\
PIPCLR & 129 \\
PIPCLR & 130 \\
PIPCLR & 131 \\
PIPCLR & 132 \\
PIPCLR & 133 \\
PIPCLR & 134 \\
PIPCLR & 135 \\
PIPCLR & 136 \\
PIPCLR & 137 \\
PIPCLR & 138 \\
PIPCLR & 139 \\
PIPCLR & 130 \\
PIPCLR & 141 \\
PIPCLR & 142 \\
PIPCLR & 143 \\
PIPCLR & 144 \\
PIPCLR & 145 \\
PIPCLR & 146 \\
PIPCLR & 147 \\
PIPCLR & 148 \\
PIPCLR & 149 \\
PIPCLR & 156 \\
PIPCLR & 151 \\
PIPCLR & 152 \\
PIPCLR & 153 \\
PIPCLR & 154 \\
PIPCLR & 155 \\
PIPCLP & 156 \\
PIPCLR & 157 \\
PIPCCLR & 158 \\
PIPCCLR & 159 \\
PIPCCLR & 160 \\
PIPCLR & 161 \\
PIPCLR & 162 \\
PIPCLR & 163 \\
PIPCLR & 164 \\
PIPCLR & 165 \\
PIPCLR & 166 \\
PIPCLR & 167 \\
PIPCLR & 168 \\
PIPCLR & 169 \\
PIPCCR & 170 \\
PIPCLR & 171 \\
PIPCLR & 172 \\
\hline
\end{tabular}




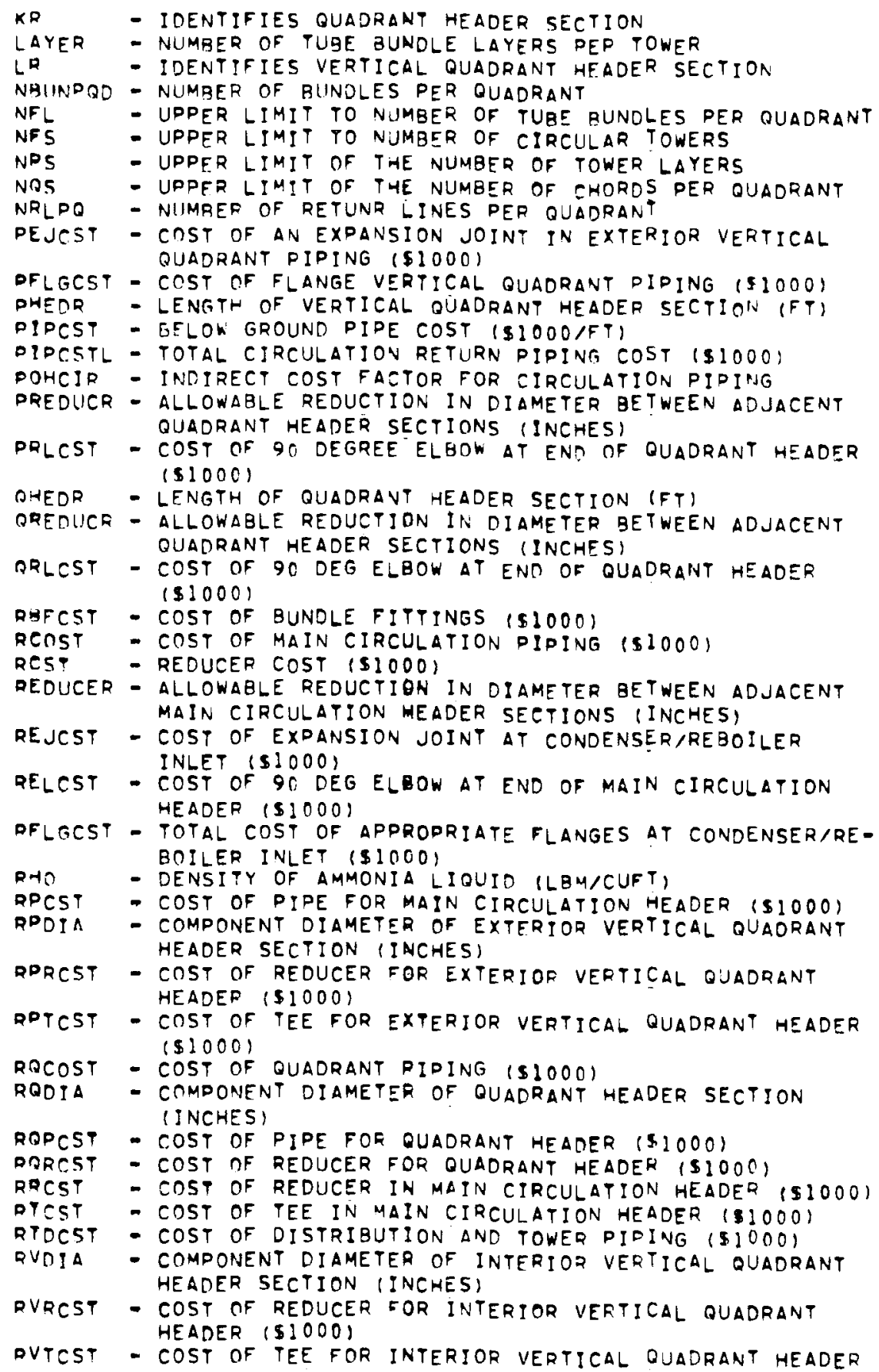

PIDCLR

PIPCLR

PIPCLR

PIPCLR

PIPCLF

PIPCLR

PIDCLR

PIDCLR

PIPCLR

PIDCLF

FIPCLP

PIPCLR

PIPCLR

PIPCLR

PIPCLR

PIPCLR

PIPCLR

PIDCLR

PI PCLP

PI CLL

PIDCLR

PIPCLR

PIPCLR

PIDCLR

PIPCLR

PIPCLR

PIDCLR

DIDCLR

PIDCLR

PIPCLR

FIPCLR

PI PCLF

PIPCLP

PIPCLR

PIPCLR

PIPCLR

PIPCLR

PIDCLR

PIPCLR

PIPCLF

PIPCLR

PIPCLR

PIPCLR

PIPCL

PIPCLR

PIPCLR

PIPCLR

PIPCLR

PI PCLR

PIPCLR

PIDCLR

PIDCLR

PIPCLD

PIPCLR

PIPCLR

PIPCLR

PI PCLR

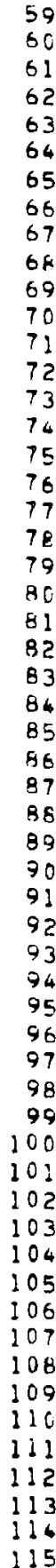


C

PIPCLR

PIPCLR

PIPCLR

PIPCLF

PIPCER

PIPCLR

PIPCLR

PIPCLR

PIPCLR

PI PCLP

PIPCLR

PIPCIR

PIDCLR

PIPCLR

PIDCIR

PIPCLR

PIPCLR

PIPCIR

PIPCLR

PIPCLR

PIDCLR

PIPCLR

PIDCLR

PIDCLR

PIPCLR

PIPCLR

PIDCLR

PIPCLR

PIPCLR

PIPCLR

PIPCLR

PIPCLR

PIPCLR

PIPCLR

PIPCLR

PIPCLR

PIPCLR

PIDCLR

PIPCLR

$P I D C L R$

PIPCLR

PIPCLR

PIDCLR

PIPCLR

PIDCLR

PIDCLR

PIDCLR

PI PCLR

PIPCLP

PIPCLR

PIPCLR

PIDCLR

PIPCLR

PIDCLR

PIPCLR

PIDCLR

PIDCLR 
43 CALL HEADER IDOTREM, DOTMAT, DESVELL, RHO,REDUCR, JR, I, DIAJ,

SDIAZ, TEST, RCST, UPCST, RELCST, RFLGCST, RE JCST)

THE DIAMETER OF HEADER SECTION JF (DIAR) IS REPLACED BY DIAJ IN ORDER TO COMPARE THE DIAMETER OF SECTION JR WITH SECTION JR - I TO DETERMINE REDUCER REQUIIREMENTS. RDIA IS THE STORED VALUE OF THE DIAMETER OF HEADER SECTION JR.

$C \backslash A J=\Pi \backslash A R$

$R \cap ! A(J R)=D I A R$

RTCST (JR) $=$ TCST

ASSIGN THE COST OF A REDUCER TO THE PRECEDING HEADER SECTION. THE COST OF A REDUCER IS BASED ON THE LARGEST DIAMETER OF TAE REDUCER.
PIPCLR 230

PIPCLR 231

PIPCLR 232

PIPCLR 233

PIPCLR 234

PIPCLR 235

PIDCLR 236

PIPCLR 237

PIDCLR 238

PIPCLR 239

PIPCLR 240

PIPCLR 241

PIPCLP 242

PIPCLR 243

PIPCLR 244

PIPCLR 245

PIPCLR 240

PIPCLR 247

PIPCLR 248

PIPCLR 249

PIPCLR 250

PIPCLR 251

PIDCLR $255^{\circ}$

PIPCLR 253

PIPCLF. 254

PIDCLR 255

PIPCLP 256

PIDCLR 257

PIPCLR 258

PIPCLR 259

DIPCLR 260

PIPCLR 261

PIPCLR 262

PIPCLR 263

PIPCLR 264

PIPCLR 265

PIPCLR 266

PIPCLR 267

PIPCLR 268

PIPCLR 269

PIPCLP 270

PIPCLR 27 I

PIPCLR 272

PIPCLR 273

PIPCLR 274

PIPCLR 275

PIPCLR 276

PIPCLR 277

PIPCLR 278

PIDCLR 279

PIPCLR 280

PIOCLR 281

PIPCLR 282

PIPCLF 283

284

PIDELR 285 
SUEROUTINE PIPCLR $\quad \mathbf{7 4 / 7 4}$ OPTEI

FTN $4.5+414$

$06 / 05 / 78$

07.59 .59

c

IF $(J R \cdot B T, J)$ RRCST (JR-1)=RCST

PIPCLR 287

UPIDCST $(J R)$ EUPCST

PIPCLR

PIPCLR

PIPCLR

$c$
$c$
$c$
$c$
$c$
$c$
$c$
$c$

DETERMINE LENGTH OF HEADER GECTION, THE FIRST HEADER SECTION IS DESIGNATED HEADER SECTION 10 ALL EVEN NUMBERED HEADER SECTIONS ARE EFFECTIVELY ZERO LENGTH BECAUSE THE CIRCULAR TOWERS ARE PAIRED ON OPPOSITE SIDES OF THE MAIN CIRCULATION HEADER. THERE IS ONE HEADER SECTION DESIGNATED FOR EACH PIPCLR PIPCLR PIPCLR PIPCLR CIRCULAR TOWER.

IF (JR.EQ.1) GO TO 10

IF $(M O D(J R, 2), E Q, O)$ HEDR $(J R)=0$.

IF $(M O D(J R, 2), N E, O)$ HEDR $(J R)=1,5 *$ TOWOIA

GO TO 11

10 HEDR (JA) $=$ DIST $+.5 *$ TOWDIA

11 CONTINUE

DETERMINE THE HEAD LOSS AND PRESSURE DROP IN HEADER SECTION JR. THE EOUIVALENT LENGTH INCLUDES AN LID OF 20 FOR THE TEE "RUN".

EQLEN=HEDR (JR) +20. 0 DIAR/ 12 .

VELO $=D O T R E M+C O N S T 2 /$ (RHO $=$ DIAR $* D I A R$ )

CALL HYDLOS (DIAR, VELO,VIS,RHO; 2, READ)

$D E L R H=D E L R H * H E A D * R H O * E Q L E N / I O O$.

SUBTRACT OUT THE FLOW TO ONE CIRCULAR TOWER TO GET THE FLDW IN HEADER SECTION JR * 1 .

DOTREMEDOTREM-DOTMAT

If THE REMAINING fLOW IS ZERO THEN THE LAST HEADER SECTION HAS BEEN DESIGNED.

IF CDOTREM.LE.0.) GO TO 42

$J R=J R \cdot 1$

IF( JR .GT. NFS) PIPCSTL = 1.E०40

IF( JA .GT. NFS) RETURN

GO TO 43

c

C

DETERMINE THE DIAMETER OF THE DISTRIBUTION PIPING.

42 BRCHDIAESQRT (DOTMAT:CONST)/(RHO:DESVELL) ) *12.

IF (BRCMDIA.LT.DIAM(I)) BRCHDIAZDIAM(I)

Do $44 \quad I=1.23$

$I I=I$

IF (

44 CONTINUE

45 RRCHOIAEDIAM(II)

THE COST OF A TEE IS ASSUMEO TO BE .67 TIMES THE COST DF A TEE OF THE "RUN" DIAMETER PLUS . 33 TIMES THE COST OF A TEE nF THE "BRANCH" DIAMETER. THE "RUN" PORTION WAS DETERMINED IN SUBROUTINE "HEADER". THE "BRANCH" PORTION IS DETERMINED HERE .

PIPCLR

PIPCLR

PIPCLR

PIDCLR

PIPCLR

PIPCLR

PIDCLR

PIPCLR

PIPCLR

PIPCLR

PIPC!R

PIPCLR

PIPCLR

PIPCLR

PIPCLR

PIPCLR

PIPCLR

PIPCLR

PIPCLR

PIPCLR

PIPCLR

PIPCLR

PIPCLR

PIPCLR

PIPCLR

PIPCLR

PIPCLR

PIPCLR

PIPCLR

PIPCLR

PIPCLR

PIPCLR

PIPCLR

PIPCLR

PIPCLR

PIPCLR

PIPCLR

PIPCLR

PIPCLR

PIPCLR

PIPCLR

PIPCLR

PIPCLR

PIPCLR

PIPCLR

PIPCLR

PIPCLR

PIPCLR 
RRCSTE.33*TEECST (II)

DO $46 \quad I=1 . J R$

FTCST (I) =RTCST(I) +BRCST

46 RDCST (I) =UFIPCST (I) *HEDR(I)

RTCST (JR) $=0$.

Dก $47 I=1 . J R$

IF (I.LT.JR)RCOST =RCOST \&RDCST (I) +RRCST (I) +RTCST II)

IF (I .EO.JR) RCOST =PCOST +RPCST (I) +RRCST II) +RELCST

47 CONTINLIE

IN THE STATEMENTS THROUGH 5J * 4 THE DISTRIRUTION PIPING IS NESIGNEO. INDEXES FOR UNIT COST ARRAYS ARE DEFINED. PRESSURE LOSS IS EVALUATED.

DIARDESORT (DOTMAT*CONST I/IRHOWDESVELL) I*12.

IF (SIARD,LT,DIAM()) DIARD=DIAM(I)

DO $50 \quad 1=1,23$

$I D=I$

IF (OIARD-DIAM(ID) $51,51,50$

50 CONTINIJE

$5 I$ DIARDEDIAM(ID)

THE EQUIVALENT LENGTH INCLUDES THE LENGTH OF PIPE CONNECTING THE MAIN CIRCULATION HEADER TO THE TOWER DISTRIBUTION HEADER. THIS LENGTH IS ASSUMED TO BE .2 TIMES THE TOWER DIAMETER.

EOLENE:Z-TOWDIA+200. DIARD/IZ.

VELOEDCTMAT*CONST2/IRHO\#DIARD*DIARD)

CALL HYDLOS(DIARD, VELO, VIS,RHO, 2 , HEAD)

$D E L D D=H E A D=R H O=E Q L E N / 100$.

IN THE STATEMENTS THROUGH 53 * 4 THE TOWER PIPING IS DESIGNED. INDEXES FOR UNIT COST ARRAYS ARE DEFINED. PRESSURE LOSS IS EVALUATED.

DIART = SQRT (DOTMAQ CONST I/(RHOWOESVELL) ) 12 .

IF (OIART.LT.DIAM(I))DIART=DIAM II)

D० $52 \quad I=1.23$

$I T=$ !

IF (DI ART-OIAM (IT) $53,53,52$

52 CONTINIJE

53 DIARTEDIAM (IT)

EDLENETOWDIA*60.DIART/12.

VELOADOTMAO*CONST2/(RHO DIART*DIART)

CALL HYDLOS (DIART, VELO,VIS,RHO, Z, HEAD)

DELDT IHEAD $=R H O=E Q L E N / I O O$.

DELTD * DELPT + DELPD

SUM THE PRESSURE DROPS IN THE TOWER AND DISTRIBUTION PIPING.

COMPUTE THE COST OF THE DISTRIBUTION AND TOWER PIPJNG. PIPE LENGTHS INCLUDE THE LENGTH OF PIPE CONNECTING THE MAIN CIRCULATIDN HFADER TO THE TOWED DISTRIBUTION HEADER (.2"TOWDIA)
PIPCLR 344

PIPCLR 345

PIPCLR 346

PIPCLR 347

PIDCLD 348

PIPCLR 349

PIPCLR 350

PIPCLR 351

PIPCLR 352

PIPCLR 353

PIPCLR 354

PIPCLR 355

PIPCLP 356

PIPCLR 357

PIPCLE 35R

PIPCLR 359

PIPCLR 360

PIPCLR 361

PIPCLR 362

PIPCLR 363

PIPCLR 364

PIPCLR 365

PIPCLR 366

PIPCLR 367

367

369

370

371

372

373

374

375

376

377

378

379

380

381

385

383

384

385

386

387

388

399

390

391

392

393

394

395

396

397

398

399

400 
SUBROUTINE PIPCLR T4/74 OPTEI FTN $4.5+414 \quad 0405 / 78 \quad 07.59 .59$

AND THE TOTAL LENGTH OF PIPE CONNECTING THE TOWER DISTRIBUTION HEADER TO THE FOUR QUADRANT HEADERS $13.25 *$ TOWDIA). THESE LENGTHS ARE GENERAL APPROXIMATIONS. THE TOWER DISTRIBUTION HEADER IS ASSUMED TO APPROXIMATE THE COST OF FOUR TEES. INDEX "ID" REFERS TO THE DISTRIBUTION DIPING, "IT" TO THE TOWER PIPING.

RTDCST*.2*TOWDIA*PIPCST $(I D) * 4 . *(.67 * T E E C S T(I D)+.33 *$ TEECST $(I T)$ $S+3.25 * T O W D I A * P I P C S T(I T) \cdot 10 . * E L C S T(I T)+2 . * E L 45(I T)$

S*4, *VACST (IT) \& * *FLGCST (IT) TRTDCST $=$ RTDCST *CTOW

$c$
$c$
$c$
$c$
$c$
$c$
$c$
$c$
$c$
$c$
$c$
$c$
$c$
$c$
$c$
$c$
$c$
$c$
$c$
$c$
$c$
$c$
$c$
$c$
$c$
$c$

IN THE STATEMENTS THROUGH 67 + 11 THE QUADRANT PIPING IS DESIGNED AND COSTED. THE PRESSURE LOSS IN THE QUADRANT HEADER AND BUNDLE FITTINGS IS DETERMINED.

THE QUADRANT PIPING CAN BE SEPARATED INTO TWO GROUPS- THE DORTION THAT RUNS ALONG THE BASE OF THE QUADRANT AND OISTRIBUTES NH3 TO THE CHORDS (HORIZONTAL) AND THE

PIPING THAT OISTRIBUTES NH3 TO THE TUBE BUNDLES (VERTICAL)

EACH QUADRANT MUST HAVE INDEPENDENT PIPING SO THAT PARTIAL SHUTDOWN IS AVAILABLE. THE TUBE BUNOLES ARE INSTALLED WITH A SLIGHT SLOPE TO ACCOMODATE THE FLOW OF NH3 LIOUID. TO FACI!ITATE DESIGN EACH OUADRANT MUST HAVE AN EVEN NUMBER OF CHORDS.

TO DESIGN THE VERTICAL RETURN PIPING TWO SITUATIONS MUST BE CONSIDERED-THE PIPING AT THE ENDS OF THE QUADRANT (EXTERIOR) WHICH SUPPLIES ONLY ONE CHOBD AND THE PIPING INBETMEEN THE EXTERIOR PIPING IINTERIOR) WHICH SUPPLIES TWO CHORDS.

DOTMAE ANO DOTMAI ARE THE FLOWS TO THE EXTERIOR AND INTEPIOR VERTICAL PIPING, RESPECTIVELY.

DESIGN AND COST THE HORIZONTAL OUADRANT PIPING

NRLDQ ICHORD $/ 2+1$

IF(NRLPO.GT.200) STOP "IN PIPCLR NRLPO EXCEEDS $200 "$ DO 60 I $=1$, NRLPO

60 RQRCST III $=0$.

$K R=$ I

DOTREMEDCTMAG

DOTMAEADOTMAG/ICHORD

DOT MAT 2 2. DOTMAE

63 DOTMAHDDOTMAI

IF (KR,ED, I, OR,KR,EO,NRLPG) DOTMAHEDOTMAE

CALL MEADER IDOTREM, DOTMAH, DESVELL, RHO, QREOUCR, KR, O,DIAJ, DIAR,TCST, SRCST, UPCST, QRLCST, QFLFCST, QEJCSTI

THE DIAMETER OF HEADER SECTION KR (DIAR) IS REPLACED BY DIAJ IN ORDER TO COMPARE THE DIAMETER OF SECTION KR WITH KR I TO DETERMINE REDUCER REQUIREMENTS. RQDIA IS THE STORED VALUE OF THE DIAMETER OF HEADER SECTION KR.

$D I A J \cong D I A R$

$R Q D I A(K R)=D I A R$

PIPCLR

PIPCLR

PIPCLR

PIPCLR

PIPCLR

PIPCLR

PIPCLR

PIDCLR

PIPCLR

PIPCLR

PIPCLR

PIPCLR

PIPCLR

PIPCLR

PIPC:R

PIPCLR

PIPCLR

PIPCLR

PIPCLR

PIPCLR

PIPCLR

PIPCLR

PIPCLR

PIPCLR

PIOCLR

PIPCLR

PIPCLR

PIPCLR

PIPCLR

PIPCLR

PIPCLR

PIPCLR

PIPCLR

PIPCLR

PIPCLR

PIPCLR

PIPCLR

PIDCLR

PIPCLR

PIPCLR

PIPCLR

PIPCLR

PIPCLR

PIPCLR

PIPCLR

PIPCLR

PIPCLA

PIPCLR

PIPCLR

PIPCLR

PIPCLR

PIPCLR

PIPCLP

PIPCLR

PIPCLR

PIPCLR

PIPCLR
401

402

403

404

405

406

407

408

409

410

411

412

413

414

415

416

417

418

419

420

421

422

423

426

425

426

427

428

429

430

431

432

433

434

435

436

437

438

439

440

441

442

443

444

445

446

447

448

449

450

451

452

453

454

455

456

457 
RPTCST (KR) $=$ TCST

ASSIGN THE COST OF \& REDUCER TO THE PRECEDING HEADER SECTION. THE COST OF A REDUCER IS BASED OF THE LARGEST DIAMETER OF THE REDUCER.

IF (KR.GT.1) RQRCST $(K R-1)=R C S T$

UPIDCST $(K R)=U P C S T$

THE FIRST QUADRANT HEADER IS ASSUMED TO HAVE ZERO LENGTH. THE PIPE LENGTH FDR THE REMAINING SECTIONS IS THE CHORD LENGTH (TUBEL).

IF (KR.EQ. I) QHEDR (KR) =0. IF (KR.OT. I) OHEDR (KR) =2. *TUBEL

DETERMINE THE HEAD LOSS AND PRESSURE OROP IN THE MORIZONTAL OUADRANT HEADER SECTION.

$E R L E N=Q H E D R(K R)+20, * D I A R / 12$

VELO=DOTREM *CONST 1/ (RHO DIAR *DIAR)

CALL HYOLOS (DIAR, VELO,VIS,RHO, 2, HEAD)

$D E L R Q=D E L R O=H E A D=R H O * E Q L E N / 100$.

SUBRTACT OUT THE FLOW TO ONE TUBE BUNDLE. IF THE FLOW REMAINING IS ZERO THEN THE LAST HORIZONTAL QUADRANT HEADER SECTION HAS BEEN DESIGNED.

DOTREMEDOTREM-DOTMAH

IF (DOTREM.LE.O) GO TO 62

$K P=K P+1$

IF (KR, OT, NQS) PIPCSTLEI,E +40

IF (KR.GT.NQS) RETURN

GO TO 63

$c$
$c$
$c$

62 CONTINUE

NOW DESIGN THE VERTICAL QUADRANT HEADER PIPING. THE

EXTERIOR PIPING WILL BE DESIGNED FIRST.

IF (NBUNHI.GT.200) STOP "IN PIPCLR LAYER EXCEEDS 2000 DO $70 I=1, N B U N H I$

70 RPREST II) =0

LRE!

DOTREMDDOTMAE

73 CALL HEADERIDOTREM, DOTMAB, DESVELL,RHO,PREDUCR,LR, O, DIAJ,DIAR,TCST, SRCST, IJPCST, PRLCST, PFLGCST,PEJCST)

THE DIAMETER OF HEADER SECTION LR (OIAR) IS REPLACED BY DIAJ IN ORDER TO COMPARE THE DIAMETER OF SECTION LR WITH LR I TO NETERMINE REDUCER REOUIREMENTS. RPDIA IS THE STORED VALUE DF THE DIAMETEP OF HEADER SECTION LR.

OJAJEDIAR

$R D D I A(L R)=D ! \angle R$

RDT SST (LQ) =TCST

PIDCLR

PIPCLR

PIPCLR

PIPCLR

PIPCLR

PIPCLR

PIPCLR

PIPCLR

PIPCLR

PIPCLR

PIPCLR

PIPCLR

PIPCLR

PIPCLR

PIPCLR

PIPCLR

PI CLR

PIPCLR

PIPCLR

PIPCLR

PIPCLR

PIPCLR

PIPCLR

PIPCLR

PIPCLR

PIPCIR

PIDCLR

PIPCLR

PIPCLR

PIPCLR

PIPCLF

PIPCLR

PIPCLR

PIPCLR

PIPCLR

PIPCLR

PIPCLR

PIPCLR

PIPCLR

PIPCLR

PIPCLR

PIPCLR

PIPCLR

PIPCLR

PIPCLR

PIPCLR

PIPCLR

PIPCLR

PIPCLR

PIPCLR

PIDCLR

PIDCLR

PIDCER

PIDCLR

FIPCLR

PIPCLR

ASSIGN THE COST OF A REDUCEA TO THE PRECEDING HEADER SECTION.

PIPCLL 
SURROUTINE PIPCLR $\quad 74 / 74 \quad$ OPTE1 FTN $4.5+414 \quad 06 / 05 / 78 \quad 07.59 .59$

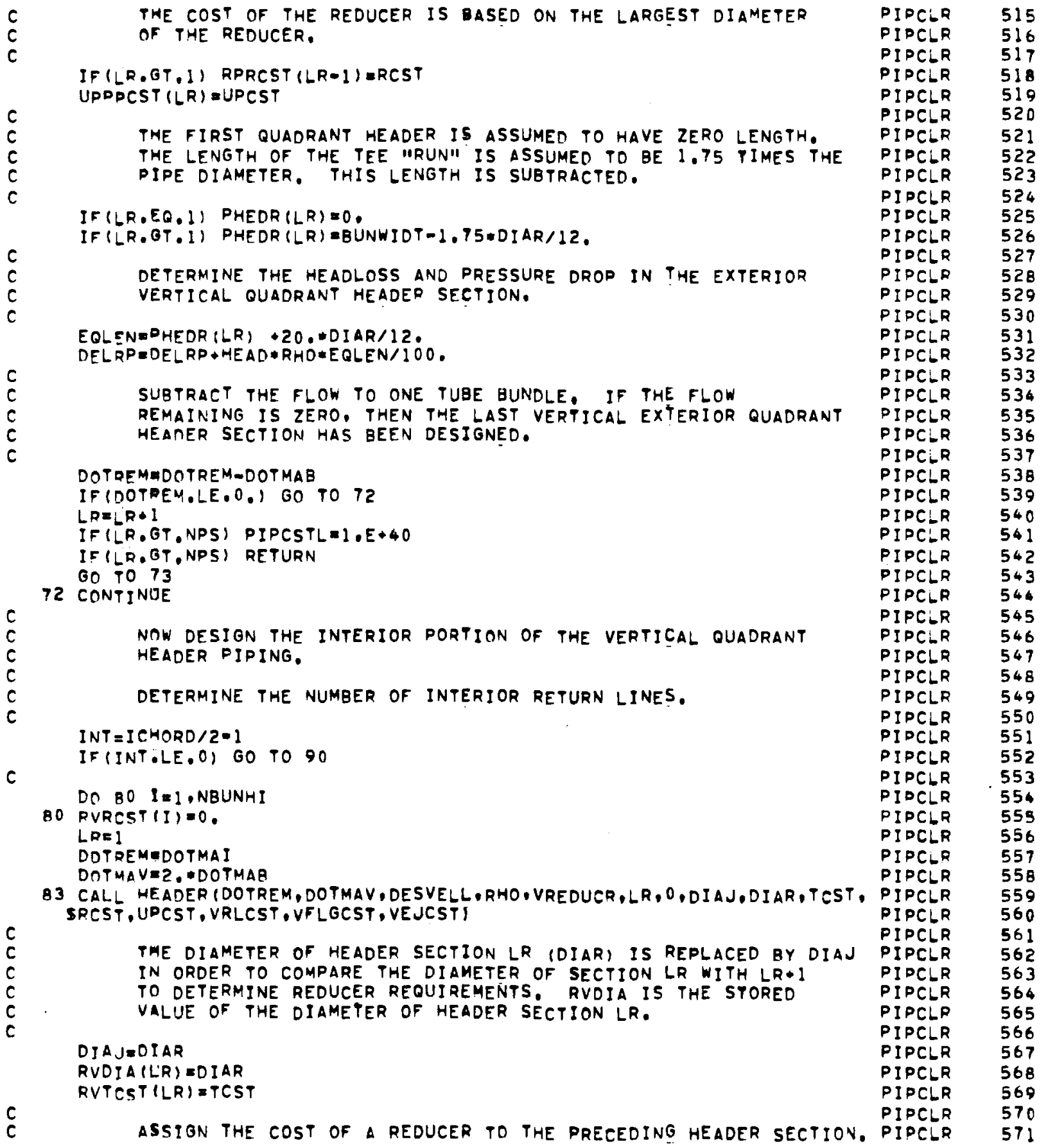


PHE COST IS BASED OF THE LABgeSt DIAMETER OF THE REDUCER.

PIPCLR

THE FIRST OUADRANT HEADER IS ASSUMED TO HAVE ZERO LENGTH. THE LENGTH OF THE TEE "RUN" IS ASSUMED TO BE 1.75 TIMES

THE DIPE DIAMETER. THIS LENGTH IS SUBTRACTED.

IF (IR,EQ. I) VHEQR (LR) $=0$.

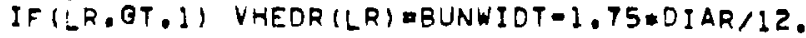

DETERMINE THE HEADLOSS ANO PRESSURE DROD IN THE INTEPIOR VERTICAL QUADRANT HEADER SECTION.

EOLEN=VHEDR (LR) *20.:DIAR/I2.

VELO=DOTREM CONSTI \{RHO DIAR DIAR\}

CAL! HYDLOS (DIAR, VELO,VIS,RHO, 2, HEAD)

DELRVEDELRV *HEAD $=R H O=E Q L E N / 100$.

SUETRACT THE FLOW TO TWO TUBE BUNDLES, IF THE FLOW

REMAINING IS ZERO, THEN THE LAST VERTICAL INTERIOR QUADRANT HEADER SECTION HAS BEEN DESIGNED.

DOTREMEDOTREM-DOTMAV

IF (JOTREM.LE,O.) GO TO 90

$L R=L \dot{D} \cdot 1$

IF (IF.BT.NPS) PIPCSTLEI,E*40

IF (LP.OT,NPS) RETURN

GO TO $^{\mathrm{B}} \mathrm{3}$

COMPUTE THE DIAMETER OF THE BUNOLE FITTINGS

90 BRCHDIASSORT (DOTMAS*CONSTI/(RHOEDESVELL)) $* 12$.

IF (BPCHDIA.LT.DIAM(I)) BRCHDIAIDIAM(I)

DO $911=1.23$

$I I \times I$

IF (BRCHOIA-OIAMIII) 92.92 .91

SI CONTINUE

92 BRCHDIA=DIAM(II)

$c$
$c$
$c$
$c$
$c$
$c$
$c$
$c$
$c$
$c$
$c$
$c$
$c$

DETERMINE THE COST OF THE SUNDLE FITTINGS

THE PIPE CONNECTING THE QUADRANT HEADER

TO THE TUBE BUNDLE HEADER

RBFCSTE3,*APIPCSTIII)+ELCST III)+2,*FLGCSTIII)

THE BRANCH PORTION OF THE TEE COST IS ASSLIMED TO BE. .33

TIMES THE GRANCH DIAMETER.

BRCSTE.33*TEECST (II)

evaluate the cost of the ojadrant header

FIRST DETERMINE THE COST OF THE HORIZONTAL SECTION.

DO $931=1, K R$

PIPCLR 573

$P I P C$ R $\quad 574$

PIPCLR 575

PIPCLR $\quad 576$

PIPCLR 577

PIPCLR 578

PIPCLP $\quad 579$

PIPCLR 580

PIPCLR 58 I

PIPCLR 582

PIPCLR 583

PIPCLR 564

PIPCLR 585

PIPCLR 586

PIPCLR 587

PIPCLR $\quad 588$

PIPCLR 569

PIPCLR 590

PIPC-R 591

PIPCLR 592

PIPCLR 593

PIPCLR 59.

PIPCLR 595

PIPCLR 596

PIPCLR 597

PIPCLR 598

PIPCLR 590

PIPCLR 600

FIPCLR 601

PIPCLR 602

PIPCLR 603

PIPCLP 604

PIPCLR 605

PIPCLR 606

PIDCLE 607

PIPCLR 608

PIPCLR 609

PIOCLR 610

PIPCLR 6II

PIDELR 612

DIPCLR 613

PIPCLR 614

PIPCLR 615

PIPCLR 616

PIPCLR 617

PIPCLR 618

PIPCLR SIG

PIPCLR 620

PIPCLR 62I

PIPCLR 622

PIPCLR 623

PIPCLR 624

PIPCLR 625

PIPCLR 626

PIPCLR $\quad 627$

PIPCLR $\quad 628$ 
93 RAPCSTII) =UPIPCST (I) *QHEDR (I)

PIPCLR

PIPCLR

629

POTCST (KR) $=0$.

DO $94 I=1, K R$

IF (I.LT,KR) ROCOST=ROCOST RROPCST (I) RRQTCST (I) \&RORCST (I)

IF (T.EQ.KR) ROCOST =ROCOST \&RQPCSTII) RQRCST (I) •QRLCST

94 CONTINUE

SECOND EVALUATE THE COST OF THE VERTICAL EXTERIOR QUADRANT

HEADER PIPING

DO $951=1 . L R$

RPTOSTIIIERDTCST (I) +BRCST

95 RPPCSTII) $=U P P P C S T(I) \triangle P H E D R(I)$

RDTCST (LRIEO O.

DO 96 I $=1 \cdot L R$

IF (I.LT.LR) RPCOSTIRPCOST RPPCST (I) +RPTCST (I) RPRCST (I)

IF (I,EQ.LR) RPCOST =RPCOST+RPPCSTII) +RPRCST (I) +PRLCST

$c$

96 CONTINUE

$c$
$c$
$c$

THIRD EVALUATE THE COST OF THE VERTICAL INTERIOR OUADRANT HEAOER PIPING

IF (INTILE.O) GO TO 99

DO 97 III,LR

RVTCST (I) aRVTCST (I)+2 *BRCST

97 RVPCSTII) =UPVPCST (I) VHEDR II)

DO $98 \quad I=I, L R$

IF (I.LT.LR) RVCOSTaRVCOST•RVPCSTII) +RVTCST (I) +RVRCST (I)

IF (I,EQ.LR) RVCOST $=R V C O S T+R V P C S T I I)+R V R C S T(I)+R V T C S T(I)$

98 CONTINUE

c

99 CONTINUE

DETERMINE THE TOTAL COST OF THE RETURN QUADRANT PIPING

$R Q C O S T=R O C O S T+2, * R P C O S T+I N T * R V C O S T+R B F C S T * N B U N P Q D$

TRQCOST $=$ RQCOST $*$ CTOW $* 4$.

$D E L R Q=D E L R Q+D E L R P+D E L R V$

c DETERMINE HEAD LOSS AND PRESSURE DROP IN THE BUNDLE FITTINGS.

EOLEN - 150. BRCHDIA/12.

VELO $=D O T M A B \approx C O N S T 2 /(R H O * B R C H D I A * B R C H D I A)$

CALL HYDLOS (BRCHDIA,VELO,VIS,RHO,Z, HEAD)

$c$
$c$
$c$
$c$

$D E L P B A=H E A D * R H O * E Q L E N / I O O$.

DETERMINE TOTAL COST AND PRESSURE DROP IN RETURN PIPING SYSTEM.

PIPASTL'ERCOST +TRTDCST +TRQCOST

PIPCSTL = PIPCSTL * POHCIR

$D E L L=D E L P H \bullet D E L T D \circ D E L R Q \bullet D E L P B R$

DYNHDL $=D E L L / R H O$

STORE VALUES IN COMMON BLOCK /RPTRET/ FOR LATER USE IN OUTPUT.

SDESVE = DESVELL

SOOTMA = DOTMA

PIPCLR

PIPCLR 633

PIPCLR $\quad 634$

PIPCLR 635

PIPCLR 636

PIPCLR 637

PIPCLR 638

PIPCLR 630

PIPCLR 640

PIDCLR 64]

PIPCLR 642

PIPCLR 643

PIPCLR 644

PIPCLR 645

PIPCLR $\quad 646$

PIPCLR 647

PIPCLR $\quad 648$

PIPCLR 640

PIPCLR 650

PIPCLR 65I

PIPCLR 652

PIPCLR 653

PIPCLR 654

PIPCLR 655

PIPCLR 656

PIPCLR 657

PIPCLR 658

PIPCLR 659

PIPCLR 660

PIPCIR 66I

PIOCLR 662

PIOCLR 663

PIPCLR

PIPCLR 665

665
666

$\begin{array}{ll}\text { PIPCLR } & 667 \\ \text { PIPCLR } & 668\end{array}$

PIPCLR $\quad 668$

PIPCLR 670

PIPCLR 671

PIPCLR 672

PIPCLR 673

PIPCLR 676

PIPCLR 675

PIPCLR 676

PIDCLR 677

PIPCLR 678

PIPCLR 670

PIPCLR 680

PIPCLR 68 I

PIPCLR 682

PIPCLR 683

PIPCLR 684

PIPCLR 685 


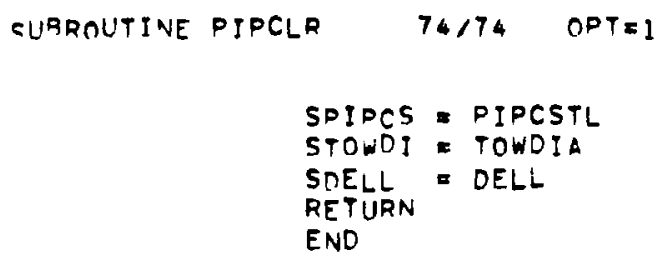

FTN $4.5+414$

$06 / 05 / 78$

07.59 .59

PIPCLR

PIPCLP

PIPCLR

PIPCLR

PIPC-R

686

687

688

689

690 
SUBROUTINE PIPCLSICTOW, TOWDIA, DOTMA,TCONOUT, DIST, DESVELV, BUNWIDT, S REDUCS, QREDUCS, DOHCIR, NSIDES, HTOW, NBUNHI, TUSEL, DELV. PIPCSTV, STHXINI

THIS SUBROUTINE DESIGNS AND COSTS THE SUPPLY PIPING SYSTEM WHICH TRANSPORTS THE VAPOR AMMONIA. THIS INCLUDES ALL THE PIPE, VALVES. AND FITTINGS BETWEEN THE HEAT ÉXCHANGER AND THE CONDENSER/REBOILER. PIPE DIAMETER IS DETERMINED FROM THE AMMONIA FLOW RATE AND THE VAPOR DESIGN VELOCITY. VALVE AND FITTING SIZES CORRESPOND TO THE PIPE SIZES WITH THE SAME FLOW RATE.

AMMONIA PROPERTIES ARE FIRST EVALUATED AT THE CONDENSER/ DEBOILER OUTLET TEMPERATURE. CONDITIONS ARE ASSUMED TO BE SATURATED, AFTER DESIGNING EACH HEADER OR PIPE SECTION THE DRESSURE DROP IS DETERMINEO IN THAT SECTION BY CALLING HLAMMO. THIS PROVIDES THE OUTLET TEMPERATURE OF THAT SECTION WHICH BECOMES THE INLET TEMPERATURE FOR THE NEXT SECTION.

\section{DEFINITION OF VARIABLES}

APIPCST * ABOVE GROUND PIPE COST ( $\$ 1000 / F T$ )

QRCHDIA - DIAMETER OF DISTRIBUTION PIPING OR BUNDLE FITTINGS (INCHES), IS USED TO GET "BRANCH" PORTION OF THE TEE COSTS

BRCST - COST OF UBRANCHII PORTION OF TEE COST (\$1000)

BSDYNHD - DYNAMIC HEAD LOSS IN BUNDLE FITTINGS (FT OF AMMONIA)

BUNWIDT - WIDTH OF TUBE BUNDLE (FT)

CDANG - ANGLE OF DELTA HEAT EXCHANGER (DEGREES)

CDV - SPECIFIC HEAT OF AMMONIA VAPOR (BTU/LBM DEG F)

CPOW - NUMBER OF CIRCULAR TOWERS

DOYNHD - DYNAMIC HEAD LOSS IN DISTRIBUTION PIPING IFT OF AMMONIA)

DELP - PRESSURE DROP IN MEADER SECTION (PSF)

DELPBS - PRESSURE DROP IN BUNDLE FITTINGS (PSF)

DELPD, - PRESSURE DROP IN DISTRIBUTION PIPING, TOWER PIPING (T) , (PSF)

DELSH,Q - PRESSURE DROD IN MAIN CIRCULATION HEADER, QUADRANT HEADER (Q), (PSF)

DELSP - PRESSURE DROP IN VERTICAL QUADRANT HEADER (PSF)

DELV - PRESSURE DROP IN SUPPLY CIRCULATION PIPING FOR VAPOR AMMONIA (PSF)

DENS - DENSIV

DENSIV - DENSITY OF AMMONIA VAPOR (LBM/CU FT)

DESVELV - AMMONIA VAPOR DESIGN VELOCITY (FT/SEC)

PIPCLS

PIPCLS

PIPCLS

PIPCLS

PIPCLS

PIPCLS

PIPCLS

PIPCLS

PIPCLS

PIPCLS

PIPCLS

PIPCLS

PIPCLS

PIPCLS

PIPCLS

PIPCLS

PIPCLS

PIPCLS

PIPCLS

PIPCLS

PIPCLS

PIPCLS

PIPCLS

PIPCLS

PIPCLS

PIPCLS

PIPCLS

PIPCLS

PIPCLS

PIPCLS

PIPCLS

PIPCLS

PIPCLS

PIPCLS

PIPCLS

PIPCLS

PIPCLS

PIPCLS

PIPCLS

PIPCLS

PIPCLS

PIPCLS

PIPCLS

PIPCLS

PIPCLS

PIPCLS

DIAS - DIAMETER OF MAIN CIRCULATION SUPPLY HEADEP (INCHES)

DIASD - DIAMETER OF DISTAIBUTION PIPING (INCHES)

PIPCLS

DIAST - DIAMETER OF TOWER PIPING (INCHES)

DIST - DISTANCE FROM CONDENSER/REBOILER TO BOUNDARY OF TOWERS

DOTMA - TOTAL MASS FLOW RATE (LBM/HR)

DOTMAB,Q.T - MASS FLOW RATE TO TUBE BUNDLE, QUADRANT $(0)$, TOWER (T), (LBM/HR)

DOTMAH - MASS FLOW RATE TO VERTICAL SECTION OF OUADRANT PIPING (LAM/HR)

PIPCLS

PIPCLS

-IPCLS

PIPCLS

PIPCLS

PIPCLS

PIPCLS

PIPCLS

PIPCLS

PIPCLS 


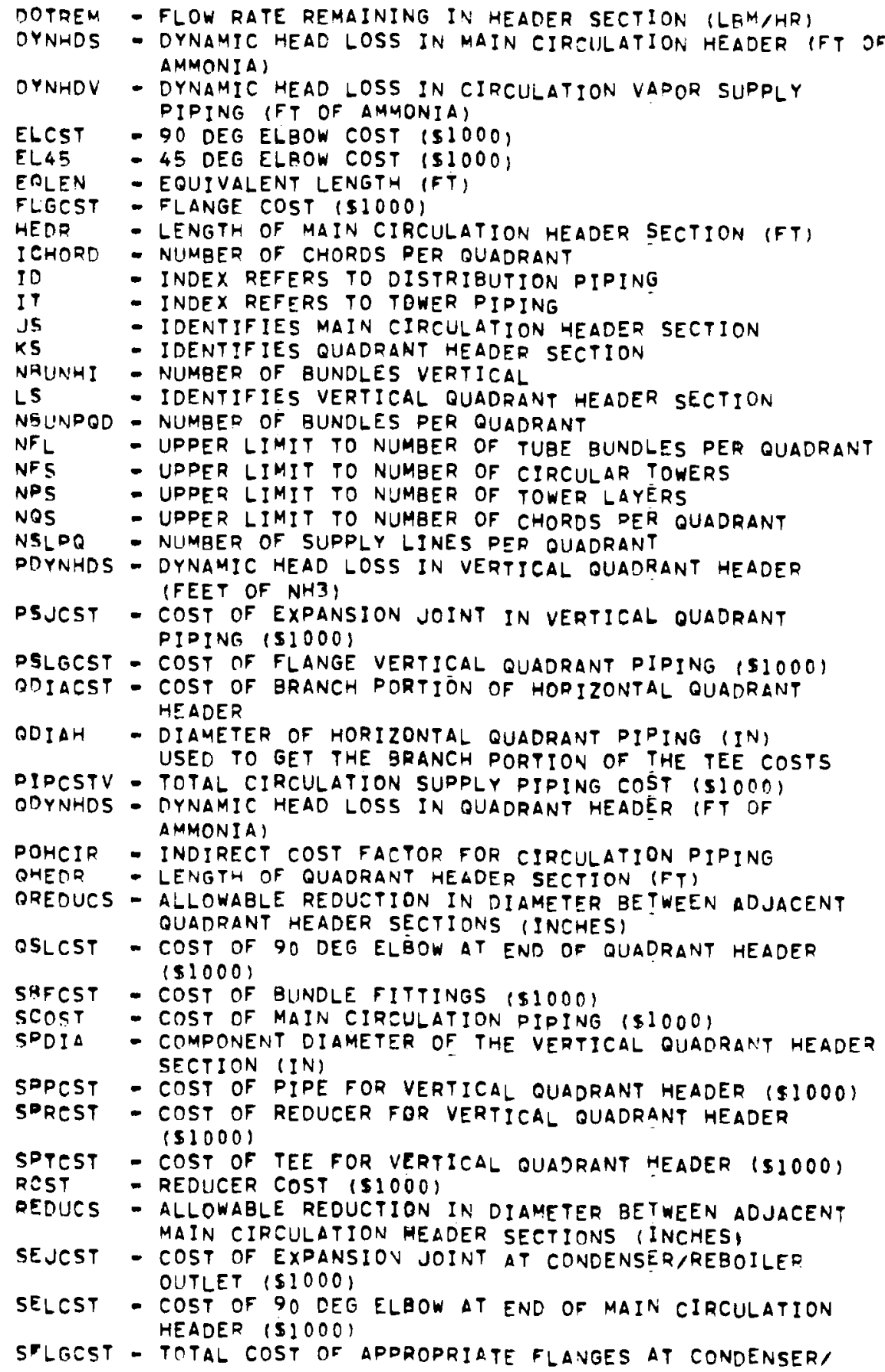




$$
14 / 74 \quad \text { OPT }=1
$$

REBOILER OUTLET $(\$ 1000)$

$c$
$c$
$c$
$c$
$c$
$c$
$c$
$c$
$c$
$c$
$c$
$c$
$c$
$c$
$c$
$c$
$c$
$c$
$c$
$c$
$c$
$c$
$c$
$c$
$c$
$c$
$c$
$c$
$c$
$c$
$c$
$c$
$c$

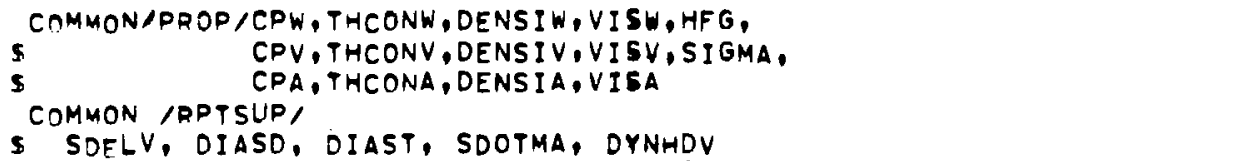

S. HEDR $(200)$

S. JS

S. $K S$

S. SOCSTV

\$. QSLEST, QHEDR (200)

S, SQFCST, SCOST, SEJCST, SELCST, SFLGCST, SOCOST, STDCST

5. SDIA $(200)$, SPCST $(200)$, SQDIA $(200)$, SOPCST $(200)$, SQRCST $(200)$

S. SOTCST (200), SRCST $(200)$, STCST $(200)$

5. STCOND, STHXIN, STOWDI. TSQCOST, TSTDCST COMMON /PCDIM/ NFS, NFL COMYON IOPTSUP,

SSPDIA $(200)$. LS, PHEDR $(200)$, SPPCST $(200)$, SPRC5T $(200)$, SPTCST $(200)$ 5, SOCOST, PSLCST, PSLGCST, PSJCST, UPPPCST $(200)$

S, DF, NRUN

DIMENSION UPIPCST $(200)$

DIMENSION DIAM(24), TEECST (24), PIPCST (24, ELCST (24), EL45(24)

$c$
$06 / 05 / 78$

07.59 .59

PIPCLS

PIPCLS 118

PIPCLS 119

PIPCLS 120

PIPCLS 121

PIPCLS 122

PIPCLS 123

PIPCL5 124

PIPCLS 125

PIPCLS 126

PIPCLS 127

PIPCLS 128

PIPCLS 129

PIPCLS 130

PIPCLS 13?

PIPCLS 132

PIPC-S 133

PIPCLS 134

PIPCLS 135

PIPCL5 136

PIPCLS 137

PIPCLS 136

PIPCLS 139

PIPCLS 140

PIPCLS 141

PIPCLS 142

PIPCLS 143

PIPCLS 144

PIPCLS 145

PIPCLS 146

PIPCLS 147

PIDC-S 148

PIPCLS 149

PIPCLS 150

PIPCLS 151

PIPCL5 152

PIPCLS 153

PIPCLS 154

PIPCL5 155

PIPCLS 156

PIPCLS 157

PIPCLS 158

PIPCLS 159

PIPCLS 160

PIPCIS 161

PIPCLS 162

PIPCLS 163

PIPCLS 160

PIPCLS 165

PIPCLS 166

PIPCLS 167

PIPCLS 168

PIPCLS 160

PIPCLS 170

PIPCLS 171

PIPCLS 172 
$c$

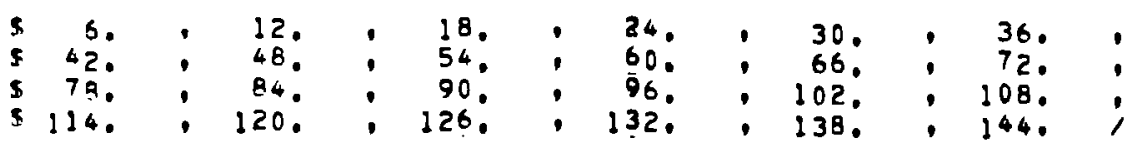

$$
\text { DATA FLGCST, }
$$

$$
\begin{aligned}
& 1.03 \\
& 12.0 \\
& 46.8 ; \\
& 85.4 ;
\end{aligned}
$$

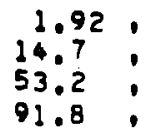

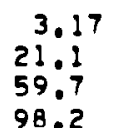

98.2

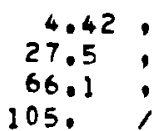

105.;

c

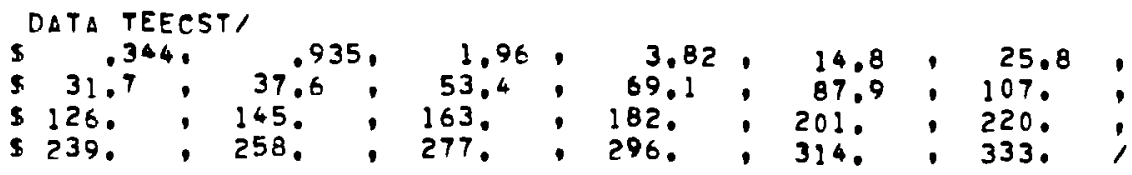

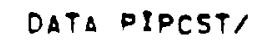

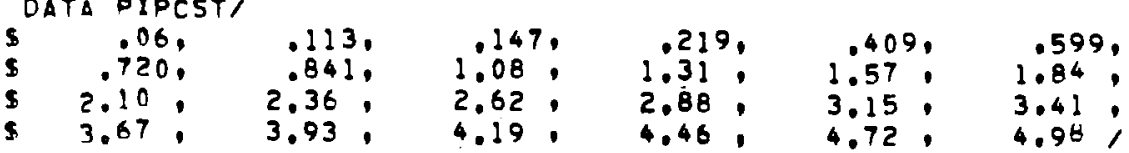

c

$$
\text { DATA VACST, }
$$

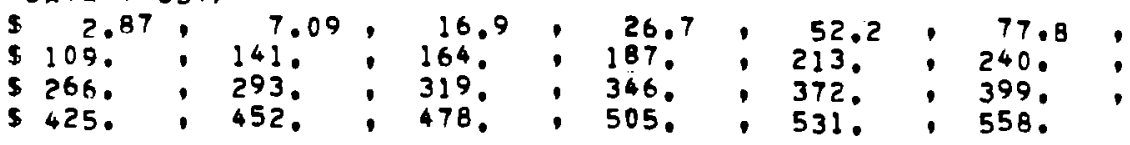

$c$

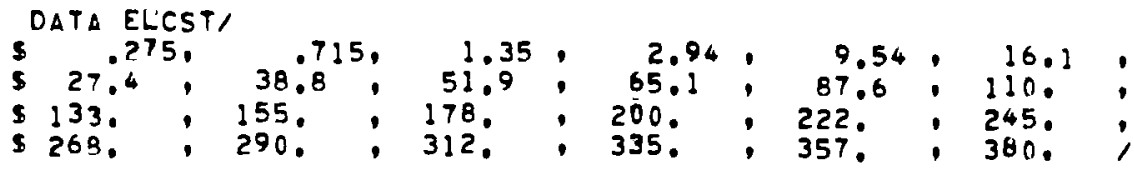

C

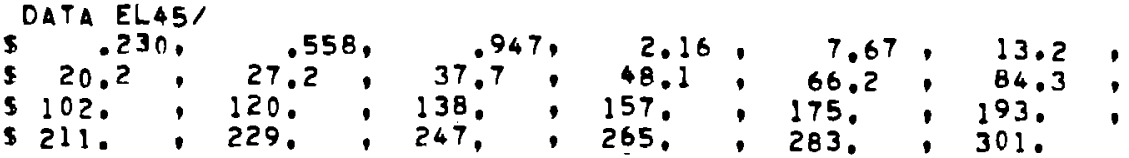

$c$

DATA APIPCST/

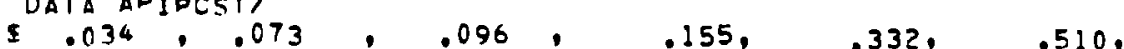

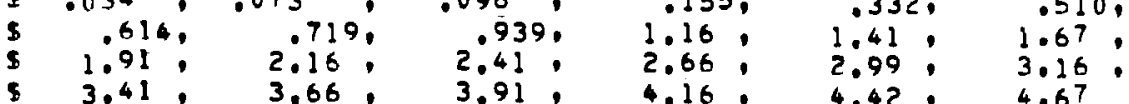

$$
\begin{aligned}
& \text { C } \\
& 100 \text { FRRMAT (BFI0.2) } \\
& P F=D O H C I R \\
& \text { NPS }=100 \\
& \text { NOS }=100 \\
& I C H O R D=N S I D E S / 4 \\
& \text { IF (ICHORD,EO.O) ICHORD =2 } \\
& \text { ICHECKEICHORD } / 2 \\
& \text { IF (?. ICHECK -ICHORD.LT.O) ICHORD = ICHORD I } \\
& \text { IF IICHORD. LE,O) STOD "IN PIPCLS ICHORD .LE.ZERO" } \\
& \text { NBUNPDO }=\text { I CHORD } * \text { NBUNHI }
\end{aligned}
$$

PIPCL5 173

PIPC-S 174

PIPCLS $\quad 175$

PIPCLS 176

PIPCL5 $\quad 177$

PIPCLS $\quad 178$

PIPCLS $\quad 179$

PIPCLS 180

PIPCLS 181

PIDCLS IB2

PIPCLS 183

PIPCLS 184

PIDCLS 185

PIPCLS 186

PIPCLS IBT

PIPCLS $18 B$

DIPCLS 189

PIPCLS 190

PIPCL5 191

PIPCLS 192

PIPCLS 193

PIPCLS 194

PIPCIS 195

PIPCLS 196

PIPCLS 197

PIPCLS 198

PIPCLS 199

1 PIPCLS 200

PIPCLS 201

PIPCLS 202

PIPCL5 203

PIPCLS 204

PIPCLS 205

PIPCSS 206

PIPCLS 207

PIPCLS 208

PIDCLS 209

PIPCLS 210

PIDCLS 211

1 PIPCLS 212

PIPCLS 213

PIPCLS 214

PIDCLS 215

PIPCLS 216

PIPCLS 217

1 PIPCLS 218

PIDCLS 219

PIPCIS 220

PIPCLS 221

PIPCLS 222

PIPCLS 223

PIPCLS 224

PIPC-5 225

PIPCLS 226

PIPCLS 227

PIPCLS 228

PIPCLS 229 
$c$
$c$
$c$
$c$
$c$
$c$
$c$
$c$
$c$

NBUN=NAUNPQD

DOTMATEDOTMA/CTOW

DOTMAQDDOTMAT $/ 4$.

DOTMAREDOTMAQ/NBUNPQD

TINETCONOUT

CONSTI $=4 . /(3.1416 * 3600$.

DELSH=O.

DYNHDSEO.

SCOST=0.

DIACSTOO.

DIAHEO :

PDYNHOSEO.

DEL SPEO

SPCOSTRO.

DO $1 I=I$.NFS

1 SACST (I) 0 .

DO 2 I I. NFL

2 SQRCSTII) $=0$.

DELSQEO.

QOYNHDS 0 .

SOCOSTOO.

SELCST:O.

SFLGCST:0.

SEJCSTEO.

DOTREMEDOTMA

$J S=1$

IN THE STATEMENTS THROUGH 17 + I THE MAIN CIRCULATION PIPING IS DESIGNED AND COSTED. THE DYNAMIC HEAD LOSS AND PRESSURE RROP ARE DETERMINED FOR PUMPING REQUIREMENTS.

CALL HEADER TO DESIGN AND COST THE COMPONENTS OF HEADER

SECTION JS. ALL HEADER SECTION COMPONENTS ARE DETERMINED

BETWEEN STATEMENTS 13 AND 12 - 1 .

13 CALL HEADER IDOTREM,DOTMAT, DESVELV, DENSIV,REDUCS,JS, I DIAJ, SOIAS, TCST, RCST, UPCST, SELCST, SFLGCST, SEJCST)

THE DIAMETER OF HEADER SECTION JS (DIAR) IS REPLACEO BY DIAJ IN ORDER TO COMPARE THE DIAMETER OF SECTION JS WITH SECTION JS * I TO DETERMINE REDUCER REQUIREMENTS. SDIA IS THE STORED VALUE OF THE DIAMETER OF HEADER SECTION JS.

PIPCLS 230

PIPCLS 231

PIPCLS 232

PIPCLS 233

PIPCLS 234

PIPCLS 235

PIPCLS 236

PIDCLS 237

PIPCLS 238

PIPCLS 239

PIPCLS 240

PIPCLS 24 !

PIPCLS 242

PIDCLS 243

PIDCLS 244

PIPCLS 245

PIPCLS 246

PIPCLS 247

PIPCLS 248

PIPCLS 249

PIPCLS 250

PIPCLS 251

PIPCLS 252

PIPCLS 253

PIPCLS 254

PIPCLS 255

PIDCLS 256

PIPCLS 257

PIDCLS 258

PIPCLS 259

PIPCLS 260

PIPCLS 261

PIPCLS 262

PIPCLS 263

PIPCLS 264

PIPCLS 265

PIPCLS 266

PIPCLS 267

PIPCLS 268

PIPCLS 269

PIPCLS 270

PIPCLS 271

PIPCLS 272

DIAJEDIAS

SDIA (JS) EDIAS

STCST(JS) $=$ TCST

PIPCLS

PIPCLS

PIPCLS

PIPCLS

ASSIGN THE COST OF a REDUCER TO THE PRECEDING HEADER SECTION. THE COST OF A REDUCER IS BASED ON THE LARGEST DIAMETER OF

THE REDUCER.

IF (JS.GT . I) SRCST (JS-I) =RCST

UPIDCST (JS) =UPCST

PIPCLS

PIPCLS

PIPCLS

PIPCLS

PIPCLS

PIPCLS

DETERMINE LENGTH OF HEADER SECTION. THE FIRST HEAOER SECTION PIPCLS IS DESIGNATED HEADER SECTION 1. ALL EVEN NUMBERED HEADER SECTIONS ARE EFFECTIVELY ZERO LENGTH BECAUSE THE CIRCULAR 
TOWERS ARE PAIRED ON OPPOSITE SIDES OF THE MAIN CIRCULATION HEADER. THERE IS ONE HEADER SECTION DESIGNATED FOR EACH CIRCULAR TOWER.

IF (JS,EQ.1) 1 GO TO 10

IF (MOD (JS, Z) ,EO,O) HEDR (JS) $=0$.

IF $(M \cap D(J S, 2), N E, 0)$ HEDR (JS) $=1.5$ * TOWDI A

GO TO 11

10 HEDQ $(J 5)=D I S T \cdot .5 *$ TOWDIA

DETERMINE THE OUTLET TEMPERATURE AND DYNAMIC HEAD LOSS FOR HEADER SECTION JS. THE EOUIVALENT LENGTH INCLUDES AN LID OF 20 FOR THE TEE "RUN". THE OUTLET TEMPERATURE BECOMES THE INLET TEMPERATURE FOR THE NEXT HEADER SECTIOÑ. SAYE THE VAPOR DENSITY TO COMPUTE DYNAMIC HEAD LOSS. HLAMMO PROVIDES A NEW VAPOR DENSITY (THROUGH COMMON) FOR THE NEXT HEADER SECTION.

II EQLEN $=$ HEOR (JS) +20. ०DIAS/12.

SURTRACT OUT THE FLOW TO ONE CIRCULAR TOWER TO GET THE FLOW IN HEADER SECTION JS * 1 .

DENSEDENSIV

CALL HLAMMO(TIN,DOTREM,DIAS,EQLEN, I, ,TOUT,DELP)

$D E L S H=D E L S H+D E L P$

DYNHOSMDYNHDS D DELP/DENS

TIN $=$ TOUT

$c$
$c$
$c$
$c$

DOTREMADOTREM-DOTMAT

IF THE REMAINING FLOW IS ZERO THEN THE LAST HEADER SECTION HAS BEEN DESIGNED.

IF IDOTREM.LE. O.,1GO TO 12

$\mathrm{J} 2=\mathrm{JP}+1$

IF ( JS .GT. NFSI PIPCSTV $=1 \cdot E * 40$

IF ( JS, GT. NFS) RETURN

GO TO 13

DETERMINE THE DIAMETER OF TAE DISTRIBUTION PIPING.

12 BRCHOIAESORT (DOTMAT \#CONSTI/ (DENSIV DESVELV)) * I2. IF (BRCHDIA.LT.DIAM (I)) BRCHDIA =DIAMI)

DO $14 I=1 \cdot 24$

II $=$ I

IF (IRCHDI A-DIAM(II) ) 15,15,14

14 CONTINUE

15 BRCHDIA $=$ DIAM(II)

$c$
$c$
$c$
$c$
$c$
$c$

THE COST OF A TEE IS ASSUMED TO BE .67 TIMES THE COST OF A

TEE OF THE "RUN" DIAMETER PLUS . 33 TIMES THF COST OF a TEE OF THE "BRANCH" DIAMETER. THE "RUN" PORTION WAS DETERMINED IN SURROUTINE "HEADER". THE "BRANCH" PORTION IS DETERMINED HERE. 
SUBROUTINE PIPCLS $\quad 74 / 74 \quad$ OPTEI $\quad$ FTN $4.50414 \quad 06105 / 78 \quad 07.59 .59$

STCST (I) $=S T C S T(I)+B R C S T$

16 SPCST (I) $=H E D R(I): U P I P C S T$ (I)

STCST (JS) 0 .

Dก $17 \quad I=1$, JS

IF (I.LT. JS) SCOSTESCOST +SPCST II) +SRCST (I) + STCST (I)

IF (I.EQ.JS) SCOSTESCOST +SPCST (I) + ERCST II) +SELCST

17 CONTINUE

SCOST $=$ SCOST + SFLGCST+SEJEST

IN THE STATEMENTS THROUGH 21 - THE DISTRIBUTION PIPING IS DESIGNED. INDEXES FOR UNIT COST ARRAYS ARE DEFINED. PRESSURE LOSS AND DYNAMIC HEAO LOSS ARE EVALUATED.

DIASD = SART (DOTMAT - CONSTI/ (DENSIVEDESVELV) 12 . DO $20 \quad I=1.24$

IDEI

IFIDIASD-DIAM(ID)) 2], 2I, 20

20 CONTINUE

21 DIASDEDIAM(ID)

EQLENE.2*TOWDIA+200.*DIASD/12.

THE EQUIVALENT LENGTH INCLUDES THE LENGTH OF PIPE CONNECTING TME MAIN CIRCULATION HEADER TO THE TOWER OISTRIBUTION HEADER, THIS LENGTH IS ASSUMED TO BE .2 TIMES THE TOWER DIAMETER.

DENSEDENSIV

CALL HLAMMO (TIN, DOTMAT, DIASD,EQLEN, I, , TOUT, DELPD)

DOY NHDADELPD/DENS

THE TEMPERATURE OUT OF THE DISTRIBUTION PIPING IS THE TEMPERATURE INTO THE TOWER PIPING.

TINETOUT

IN THE STATEMENTS THROUGH 23 - 4 THE TOWER PIPING IS DESTGNED. INDEXES FOR UNIT COST ARRAYS ARE DEFINED. PRESSURE LOSS AND DYNAMIC HETA LOSS ARE EVALUATED.

DIASTISQRT (DOTMAO*CONSTI/ (DENSIVHDESVELV) *12.

DO $22 \quad I=1.24$

$I T=!$

IF (DIAST-DIAM(IT) $23,23,22$

22 CONTINUE

23 DIASTEDIAM(IT)

EQLENETOWDIA+BO, DIAST/12. DENS $=D E N S I V$

CALL HLAMMO (TIN, DOTMAQ, DIAST, EQLEN, \& , TOUT, DELPT)

TOYNHOEDELPT/DENS

THE TEMPERATURE OUT OF THE TOWER PIPING IS THE TEMPERATURE

INTO THE QUADRANT PIPING.

$T I N=T O U T$

COMOUTE THE COST OF THE DISTRIBUTION AND TOWER PIPING. PIPE LENGTHS INCLUDE THE LENGTH OF PIPE CONVECTING THE MAIN CIRCLILATION HEADER TO THE TOWER DISTRIRUTION HEADER (.2 ETOWDIA)

$\begin{array}{ll}\text { PIPCLS } & 344 \\ \text { PIPCLS } & 345 \\ \text { PIPCLS } & 346 \\ \text { PIPCLS } & 347 \\ \text { PIPCLS } & 348 \\ \text { PIPCLS } & 349 \\ \text { PIPCLS } & 350 \\ \text { PIPCLS } & 351 \\ \text { PIPCLS } & 352 \\ \text { PIPCLS } & 353 \\ \text { PIPCLS } & 354 \\ \text { PIPCLS } & 35 \\ \text { PIPCLS } & 356 \\ \text { PIPCLS } & 357 \\ \text { PIPCLS } & 358 \\ \text { PIPCLS } & 359 \\ \text { PIPCLS } & 360 \\ \text { PIPCLS } & 361 \\ \text { PIPCLS } & 367 \\ \text { PIPCLS } & 363 \\ \text { PIPCLS } & 364 \\ \text { PIPCLS } & 365 \\ \text { PIPCLS } & 366 \\ \text { PIPCLS } & 367 \\ \text { PIPCLS } & 368 \\ \text { PIPCLS } & 369 \\ \text { PIPCLS } & 370 \\ \text { PIPCLS } & 371 \\ \text { PIPCLS } & 372 \\ \text { PIPCLS } & 373 \\ \text { PIPCLS } & 374 \\ \text { PIPCLS } & 375 \\ \text { PIPCLS } & 376 \\ \text { PIPCLS } & 377 \\ \text { PIPCLS } & 376 \\ \text { PIPCLS } & 379 \\ \text { PIPCLS } & 380 \\ \text { PIPCLS } & 381 \\ \text { PIPCLS } & 382 \\ \text { PIPCLS } & 333 \\ \text { PIPCLS } & 384 \\ \text { PIPCLS } & 385 \\ \text { PIPCLS } & 366 \\ \text { PIPCLS } & 387 \\ \text { PIPCLS } & 388 \\ \text { PIPCLS } & 389 \\ \text { PIPCLS } & 390 \\ \text { PIPCLS } & 391 \\ \text { PIPCLS } & 397 \\ \text { PIPCLS } & 393 \\ \text { PIPCLS } & 394 \\ \text { PIPCLS } & 395 \\ \text { PIPCLS } & 396 \\ \text { PIPCLS } & 397 \\ \text { PIPCLS } & 398 \\ \text { PIPCLS } & 399 \\ \text { PIPCLS } & 400\end{array}$


AND THE TOTAL LENGTH OF PIPE CONNEETING THE TOWER

PIPCLS DISTRIBUTION HEADER TO THE FOUR QUADRANT HEADERS 13.25 . TOWOIA). THESE LENGTHS ARE GENERAL APPROXIMATIONS. THE TOWER DISTRIBUTION HEADER IS ASSUMED TO APPROXIMATE THE COST OF FOUR TEES. INDEX "ID" REFERS TO THE OISTRIBUTION PIJCis PIPCSS PIPCLS PIPING, "IT" TO THE TOWER PIPING.

PIPCLS

PIPCLS

PIPCLS

PIPCLS

STOCSTะ, 2*TOWDIA*PIPCST (ID)*4,*1.67*TEECST (ID)*.33*TEECST (IT)) S+ (3.25*TOWDIA) *PIPCST (IT) +14.*ELCST!IT) +2, *EL 65 (IT) $5+4$ * VACST (I)+B.*FLGCST (IT)

TSTDCST $=5$ TOCST *CTOW

PIPCLS

PIPCLS

PIPCLS

IN THE STATEMENTS THROUGM 35 + 11 THE OUADRANT PIPING IS DE SIGNED AND COSTED. THE DYNAMIC HEAD LOSS AND PRESSURE DROP PIPCLS

PIPCIS

PIPCLS IN THE OUADRANT HEADER AND BUNOLE FITTINGS ARE DETERMINED.

THE QUADRANT PIPING CAN BE SEPARATED INTO TWO GROUPS - THE PORTION THAT RUNS ALONG THE TOP OF THE OUADRANT AND DISTRIBUTES NH3 TO THE CHORDS (HORIZONTAL) AND THE PIPING THAT DISTRIBUTES NH3 TO THE TUBE BUNDLES (VERTICAL)

ipCle

PIPCLS

PIPCLS

PIPCLS

PIPC:S

PIPCLS

PIPCLS

PIPCLS

EACH QUADRANT MUST HAVE INOEPENDENT PIPING SO THAT

PARTIAL SHUT DOWN IS AVAILABLE. TO FACILITATE DESIGN

PIPCIS

DIPCLS

PIPCLS

EACH OUADRANT MUST HAVE AN EVEN NUMBER OF CHOROS.

PIPCLS

DESIGN AND COST THE HORIZONTAL PIPING,

NSLDREICHORD/Z

IF (NSLPQ.GT.2OO) STOP "IN PIPCLS NSLPO EXCEEDS $200 "$

DO $30 \quad 1=1 . N S L P 0$

30 SORCSTITIEO.

KS $=1$

DOTREMEDOTMAQ

DOTMAHE2 - DOTMAO/ICHORO

31 CALL HEADER IDOTREM, DOTMAH, DESVELV, DENSIV, OREDUCS,KS,O,DIAJ,DIAS,TC SST.RCST.UPCST, OSLCST, SFLGCST, SEJCSTI

PIPCLS

PIPCLS

PIPCLS

PIPCLS

PIPCLS

PIPCLS

PIPCLS

PIPCLS

PIPCLS

PIPCLS

401

402

403

404

405

406

407

408

405

410

412

412

413

414

415

416

417

418

419

420

421

42 ?

423

424

425

426

427

428

429

430

431

432

433

434

435

436

437

438

439

440

441

442

443

444

445

446

447

448

449

450

451

452

453

454

455

456

457

THE FIRST OUADRANT HEADER IS ASSUMED TO HAVE ZERO LENGTH. PIPCLS LENGTH OF THE REMAINING SECTIONS IS THE CHORD LENGTH (TUBEL). PIPCLS 


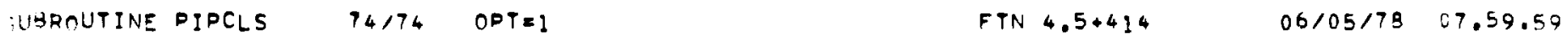

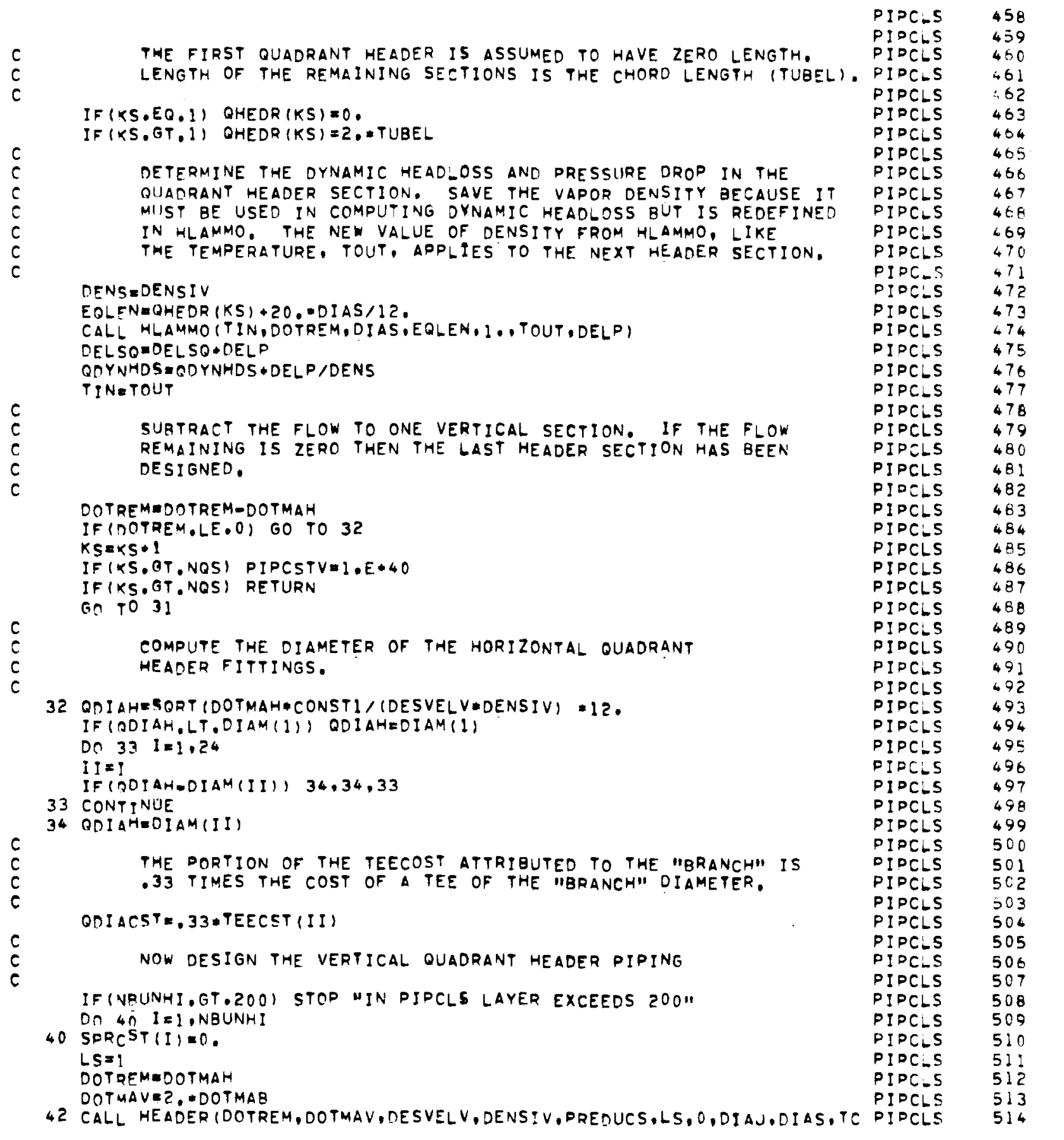




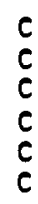

$c$
$c$
$c$
$c$

$c$
$c$
$c$
$c$

$c$
$c$
$c$
$c$
$c$
SST , RCST, UPCST, PSLCST, PSLGCST,PSJCST)

PIPCLS

PIPCLS

THE DIAMETER OF HEADER SECTION LS (DIAS) IS REPLACED BY DIAJ

IN ORDER TO COMPARE THE DIAMETER OF SECTION KS WITH KS+I

TO DETERMINE REOUCER REQUIREMENTS. SPDIA IS THE STORED

VALUE OF THE DIAMETER OF HEADER SECTION LS.

DIAJEDLAS

SPDIA (L'S) $=D I A S$

SPTCST (LS) ETCST

ASSIGN THE COST OF A REDUCER TO THE PRECEDING HEADER SECTION. THE COST OF A REDUCER IS BASED ON THE LARGEST DIAMETER OF THE REDUCER.

IF (LS.OT. I) SPRCST (LS-1) =RCST

UPPPCST $(L S)=U P C S T$

THE FIRST QUADRANT HEADER IS ASSUMED TO HAVE ZERO LENGTH. THE LENGTH OF THE TEE "RUN" IS ASSUMED TO BE 1.75 TIMES

THE PIPE DIAMETER. THIS LENGTH IS SUBTRACTED.

IF (LS.EO.1) PHEDR (LS) $=0$.

IF (LS.6T.1) PHEDR (LS) $=B U N W I D T-1, T 5+D I A S / 12$.

DETERMINE THE OYNAMIC HEADLOSS AND PRESSURE DROP IN THE OUADRANT HEADER SECTION. SAVE THE VAPOR DENSITY BECAUSE IT MUST BE USED IN COMPUTING DYNAMIC HEADLOSS BÜT IS REDEFINED IN HLAMMO. THE NEW VALUE OF OENSITY FROM HLAMMO. LIKE THE TEMPERATURE, TOUT, APPLIES TO THE NEXT HEADER SECTION.

DENSEDENSIV

EOLENEPHEDR (LS) +20, DIAS/12.

CALL HLAMMO (TIN, DOTREM, DIAS,EQLEN, I . TOUT, DELP)

DELSPEDELSP DDELP

PDYNHOSEFOYNHDS +DELP/DENS

TINETOUT

c

41 BRCHDIA=SQRT (DOTMAB * CONST I/ (DESVELV*DENSIV) ) 12 ,

IF (BRCHDIA.LT.DIAM(I)) BRCHDIAEDIAM(I)

DO $43 I=1.24$

I I $=$ I

IF (BRCHDIA-DIAM(II)) $44,44.43$

43 CONTINIJE
SUBTRACT THE FLOW TO TWO TUBE BUNDLES. IF THE FLOH REMAINING IS ZERO THEN THE LAST HEADER SECTION HAS BEEN

DOTREMEDOTREM-DOTMAB:2.

IF (DOTREM.LE.0) GO TO 41

$L S=L S+I$

IF (LS.GT.NPS) PIPCSTV $=1, E \$ 40$

IF(L.OT.NPS) RETURN

GO TO 2

COMPUTE THE DIAMETER OF THE BUNDLE FITTINGS.
PIPCLS

PIPCLS

PIPCLS

PIPCLS

PIPCLS

PIPCLS

PIPCLS

PIPCLS

PIPCLS

PIPCLS

PIPCLS

PIPCLS

PIPCLS

PIPCLS

PIPCLS

PI PCLS

PIPCLS

PIPCLS

PIPCLS

PIPCLS

PIPCLS

PIPCLS

PI PCLS

PIPCLS

PIPCLS

PIPCLS

PI PCLS

PI PCLS

PIPCLS

PIPCLS

PIPCLS

PIPCLS

PIPCLS

PI PCLS

PIPCLS

PIPCLS

PIPCLS

PIPCLS

PIPCLS

PI PCLS

PIPCLS

PIPCLS

PIPCLS

PIPCLS

PIPCLS

PIPCLS

PIPCLS

PIPCLS

PIPCLS

PIPCLS

PIPCLS

PIPCLS

PIPCLS

PIPCLS

PIPCLS
515

516

517

518

519

520

521

522

523

524

525

526

527

528

529

530

531

532

533

534

535

536

537

538

539

540

541

542

543

544

545

546

547

548

549

550

551

552

553

554

555

556

557

558

559

560

561

562

563

564

565

566

567

568

569

570

571 
THE PORTION OF THE TEECOST ATTRIBUTED TO THE "BRANCM" IS

.33 TIMES THE COST OF A TEE OF THE "BRANCH" DIAMETER.

BRCSTE:33*TEECSTIII)

TME PIDE CONNECTING THE OUADRANT HEADER DETERMINE THE COST OF THE BUNDLE FITTINGS TO THE TUBE BUNDLE HEADER

SPFESTE2,*APIPCST (II) *2**FLGCST (II)

EVALUATE THE COST OF THE QUADRANT HEADER.

FIRST DETERMINE THE COST OF THE HORIZONTAL SECTION.

DO $50 \quad I=1, K S$

SOTEST (I) = SQTCST (I) QODIACST

50 SOPCST II) EQHEDR (I) \#UPIPCST (I)

SQTCST $(K S)=0$.

DO $51 I=1, K S$

IF (I.LT.KS) SQCOST ESQCOST+SQPCST II) +SQRCST (I) +SQTCST (I)

IF (I.EQ.KS) SQCOST=SQCOST +SQPCST II) +SQRCST (I)+QSLCST

C

51 CONTINUE

SECONO EVALUATE THE COST OF THE VERTICAL QUADRANT hEADER PIPJNG.

DO 52 InI.LS

SPT-ST (I) aSPTCST (I) +BRCST*2.

52 SPPCSTII) $\approx$ UPPPCST (I) *PHEDR (I)

SDTCST (LS) $=0$.

Do $53 \quad I=1,1.5$

SPCDST =SPCOST + SPPCST (I) +SPRCST (I) + SPTCST (I)

53 CONTINUE

DETERMINE THE TOTAL COST OF THE SUPPLY QUADRANT PIDING.

SOCOSTESQCOST +NSLPQ SPCOST * NBUNDQD*SBFCST

TSQCOST $=$ SOCOST *CTOW*4.

DETERMINE THE PRESSURE DROP AND DYNAMIC HEADLOSS IN THE QUADRANT PIPING.

DELSADDELSQ+DELSD

QOYNHOS $=$ QDYNHDS \$POYNHDS

COMPUTE DYNAMIC HEAO LOSS IN BUNDLE FITTINGS.

DENS=DENSIV

EQLFN = I2ก. * BRCHDIA/12.

CALL HLAMMO\{TIN, DOTMAB, BRCHDIA, EQLEN, 1,. TOUT, DELPBS )

BSDYNHDEOELPES/DENS

C

THE VAPOR TEMPERATURE AT THE HEAT EXCHANGER INLET HAS BEEN

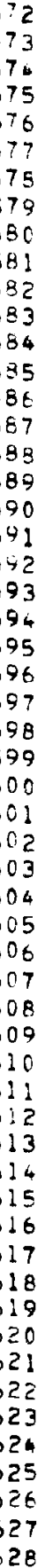


FUNCTION PNLIM TH/74 OPTEI FTN $4.5+414$

$06 / 05 / 78 \quad 07.59 .59$

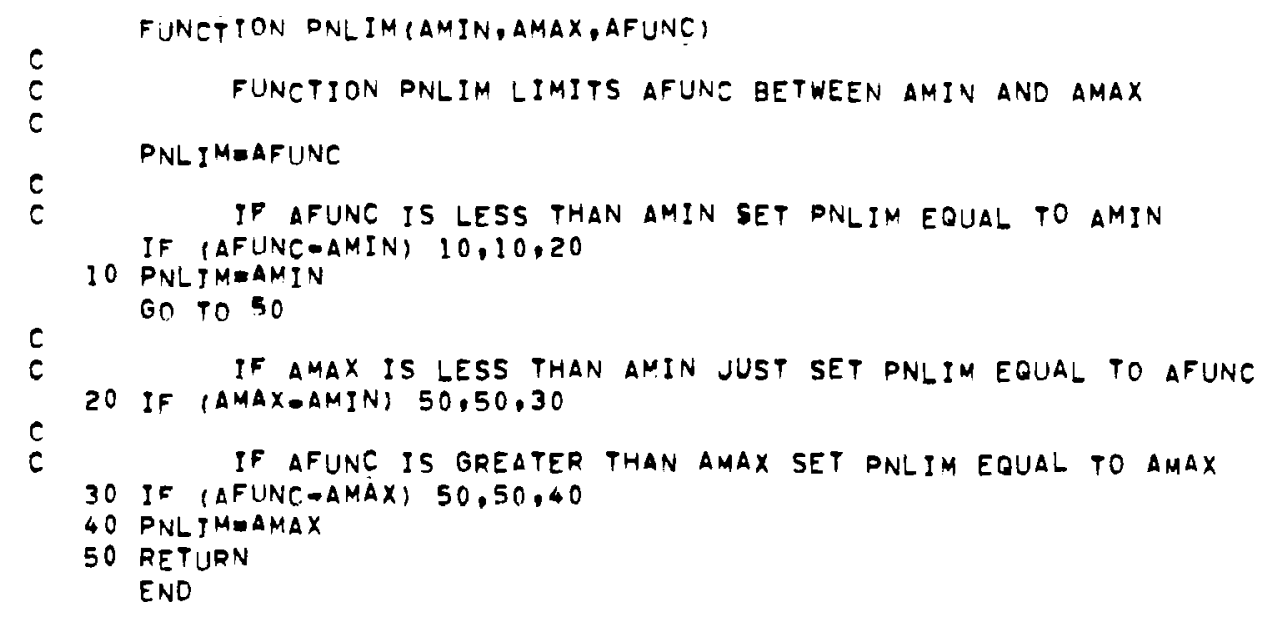

$\begin{array}{ll}\text { PNLIM } & 2 \\ \text { PNLIM } & 3 \\ \text { PNIIM } & 4 \\ \text { PNLIM } & 5 \\ \text { PNLIM } & 6 \\ \text { PNLIM } & 7 \\ \text { PNLIM } & 8 \\ \text { PNLIM } & 9 \\ \text { PNLIM } & 10 \\ \text { PNLIM } & 11 \\ \text { PNLIM } & 12 \\ \text { PNIIM } & 13 \\ \text { PNLIM } & 14 \\ \text { PNLIM } & 15 \\ \text { PNIIM } & 16 \\ \text { PNLIM } & 17 \\ \text { PNLIM } & 18 \\ \text { PNLIM } & 19 \\ \text { PNLIM } & 20\end{array}$


$74 / 74 \quad$ OPT $=1$

FTN $4.5+414$

$06 / 05 / 78$

07.59 .59

SUBROUTINE PRPERT (TWATAV,TAIRAV,ALTITD)

PRPERT

SUBROUTINE PRPERT DETERMINES THE THERMOOYNAMIC AND PHYSICAL DROPERTIES OF AIR AND SATURATED AMMONIA

DEFINITION OF VARIABLES

CDA - HEAT CAPACITY OF AIR IBTU/LBM-DEG F)

CPU - HEAT CAPACITY OF AMMONIA VAPOR (BTU/LBM-DEG F)

CDW

DENSIA

- heAT capacity of amMONia liQuid (BTU/LBMADEg F

DENSIV

DENSIW

HF G

- DENSITY OF AIR (LBM/CU FT)

- DENSITY of amMONIA VAPOR (LBM/CU FT)

SIGMA

TAIRAV

THCONA

- DENSITY OF AMMONIA LIOUID (LBM/CU FTI

- hEAT OF VAPORIZATION OF AMMONIA (B TUULBM)

- SURFACE TENSION OF amMONIA (LGF/FT)

- aVERAGe air temperature (DEG F)

THCONV

- THERMAL CONDUCTIVITY OF AIR (BTU/HR-FT-DEg F)

- THERMAL CONDUCTIVITY OF AMMONIA VAPOR IBTU/HR-FTDEG FI

THCONW - THERMAL CONDUCTIVITY OF AMMONIA LIQUID IBTU/HR-FTDEG F)

TWATAV - AMMONIA TEMPERATURE IDEG F

VISA - VISCOSITY OF THE AIR (LBM/HR-FT)

VISV - VISCOSITY OF THE AMMONIA VAPOR (LBM/FT-HR)

VISW - VISCOSITY OF THE AMMONIA LIQUID (LBM/FT-HR)

COMYON / PROP, CPW, THCONW, DENSIW, VISW, HFG.

- CPV, THCONV, DENSIV, VISV, SIGMA,

$+$

CPA, THCONA, DENSIA, VISA

AMMONIA PROPERTIES

CPW = FLUID (1, 1, TWATAV,ALTITD, "SUBROUTINE PRPERT I")

THCONW I FLUID $(2,1$, TWATAV,ALTITD, "SUBROUTINE PRPERT 2")

DENSIW FLUID 13,1 , TWATAV,ALTITD,"SUBROUTINE PRPERT 3")

VISW = FLUIO (4,1, TWATAV,ALTITD, "SUBROUTINE PRPERT 4")

CPV = FLUID\{5,1,TWATAV,ALTITO, "SUBROUTINE PRPERT 511)

THCONV = FLUID $(6,1$, TWATAV,ALTITD, "SUBROUTINE PRPERT $6 " 1$

DENSIV = FLUID 17, I, TWATAV,ALTITD, "SUBROUTINE PRPERT 7")

VISV = FLUIO $(8,1$, TWATAVOALTITD, "SUBROUTINE PRPERT 8")

HFG = FLUID (9,1, TWATAV,ALTITD, "SUBROUTINE PRPERTB.5")

c

SIGUA "FLUID112,1, TWATAV,ALTITD."SUBROUTINE PRPERTB.7",

AIR PROPERTIES

CPA = FLUID (5,2.TAIRAV,ALTITD,"SUBROUTINE PRPERT 9")

THCONA = FLUID 16,2 , TAIRAV, ALTITD,"SUBROUTINE PRPERT IO"4

DENSIA FLUID 17,2,TAIRAV,ALTITD,"SUBROUTINE PRPERT II")

c

VISA = FLUID $(8,2$,TAIRAV,ALTITD, "EUBROUTINE PRPERT I2")

RETURN

END

PRPERT

PRPERT

PRPERT

PRPERT

PRPERT

PRPERT

PRPERT

PRPERT

PRPERT

PRPERT

PRPERT

PRPERT

PRPERT

PRDERT

PRDERT

PRPERT

PRPERT

PRPERT

PRPERT

PRPERT

PRPERT

PRPERT

PRDERT

PRPERT

PRPERT

PRPERT

PRPERT

PRPERT

PRPERT

PRPERT

PRPERT

PRPERT

PRPERT

PRPERT

PRPERT

PRPERT

PRPERT

PRPERT

PRPERT

PRPERT

PRPERT

PRPERT

PRPERT

PRPERT

PRDERT

PRPERT

PRPERT

PRPERT

PRPERT 
SUBROUTINE PUMLEC T4/74 OPTa1 FTN $4.5+414 \quad 06105 / 78 \quad 07.59 .59$

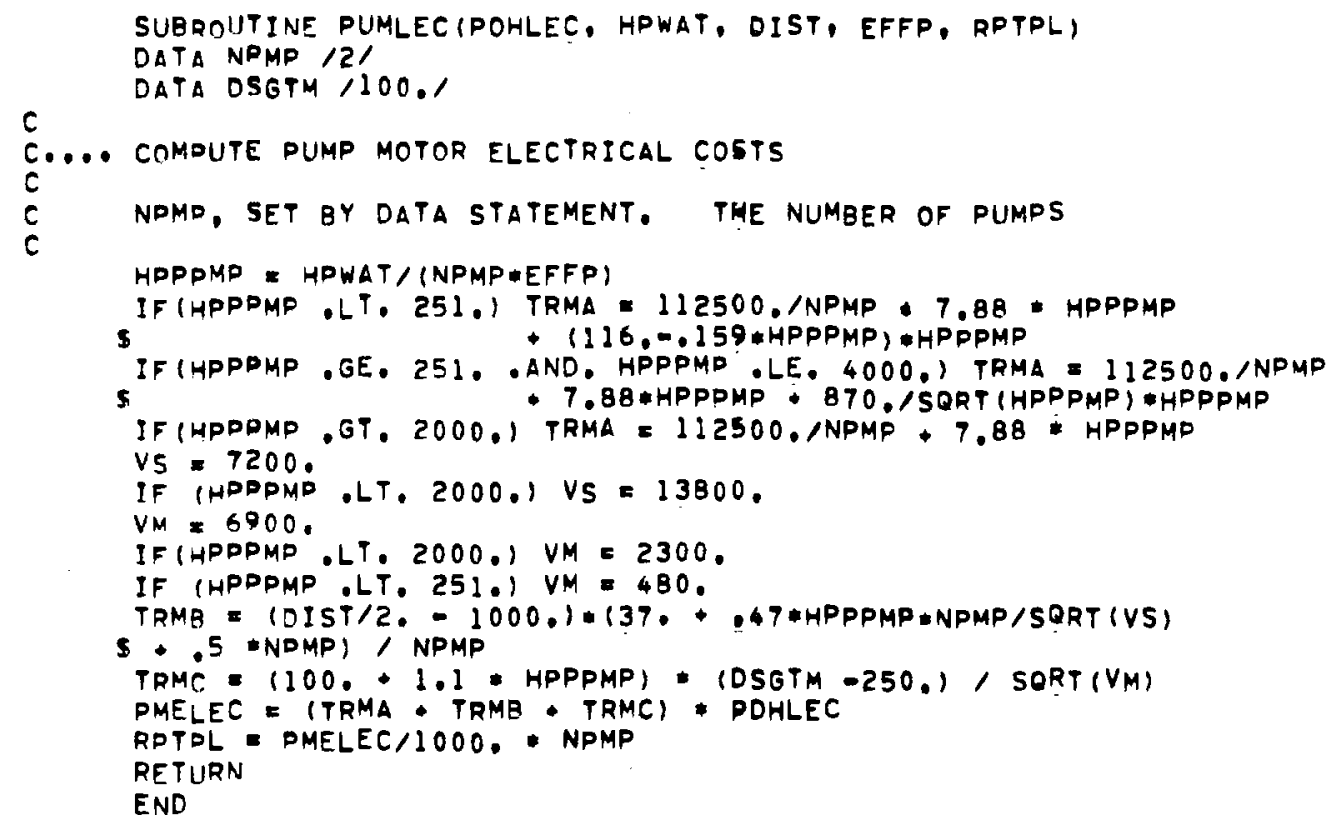

PUMLEC

PUMLEC

PUMLEC

PUMLEC

PUMLEC

PUMLEC

PUMLEC

PUMLEC

PUMLEC

PUMLEC

PUMLEC

PUMLEC

PUMLEC

PUMLEC

PUMLEC

PUMLEC

PUMLEC

PUMLEC

PUMLEC

PUMLEC

PUMLEC

PUMLEC

PUMLEC

PUMLEC

PUMLEC

PUMLEC 
SUBROUTINE PUMPCST,W3, DELPS, DELPR, DELPWT, DELDWC, $X$, DESVEL,

PMPCST S DMPCST, PUFITCI SUBROUTINE PUMPCST DETERMINES THE COST OF THE PUMPS AND
THE FITTINGS FOR THE AMMONIA LOOP

\section{DEFINITION OF VARIABLES}

CPA - HEAT CAPACITY OF AIR (BTU/LBM-DEG F)

CPV - HEAT CAPACITY OF AMMONIA VAPOR (BTU/LBM-DEG F)

CPA - HEAT CAPACITY OF AMMDNIA LIOUID (BTU/LBM-DEG F)

DELPR - PRESSURE DROP IN THE RETURN PIPING (LBF/SOFT)

DELPS - PRESSURE DROP IN THE SUPPLY PIPING (LBF/SO FT)

DELPWC - PRESSURE OROP IN THE CONDENSER/REBOILER (LBF/SOFT)

DELPWT - PRESSURE DROP IN THE HEAT EXCHANGER (LBF/SQ FT)

DENSIW - DENSITY OF AMMONIA LIQUID (LBM/CU FT)

DESVEL - DESIGN LIOUID VELOCITY (FT/SEC)

DIA - DIAMETER OF THE VALVES ANO FLANGES (IN)

GDMI - GALLONS PER MINUTE OF AMMONIA IN THE RETURN PIPING (GPM)

GPMZ - GALLONS PER MINUTE OF AMMONIA ENTERING THE CONOENSER/REBOILER (GPM)

PIPCSPI - FLANGE AND VALVE COST FOR PUMPING IN THE RETURN PIPING (KS)

PIPCSPZ - FLANGE AND VALVE COST FOR PUMPING IN THE LOOP TO THE CONDENSER/REBÖILER IKS

PMPCST - TOTAL COST OF PUMPING SYSTEM (KS)

PUMPCSZ - COST OF PUMP FDR LOOP TO THE CONDENSER/ REBOILER (KS)

TOHI - DYNAMIC HEAD TO gE MADE UP BY THE RETURN PUMP (FT)

TDH2 - DYNAMIC HEAD TO BE MADE UP BY THE PUMP IN THE CONDENSER/REBOILER LDOP (FT)

W3 - MASS FLOW OF AMMDNIA (LBM/HR)

$X$ - EXIT QUALITY OF FLUID EXITING THE CONDENSER/ REBOILER (DIMENSIONLESS)

DIMENSION DIAM(24), FLGCST (24), VACST (24)

COMMON/PROP/CPW, THCONW, DENSIW, VISW, HFG,

1

CPV, THCONV, DENSIV, VISV, SIGMA.

DATA DIAM,

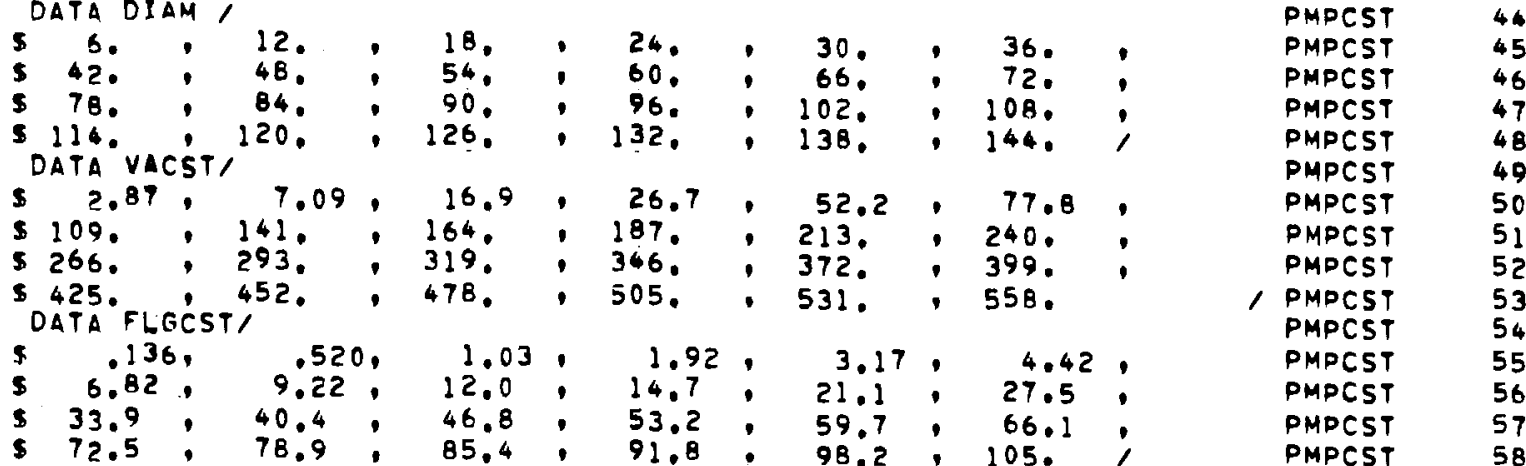

PMPCST

PMPCST

PMPCST

PMPCST

PMPCST

PMPCST

PMDCST

PMPCST

PMPCST

PMPCST

PMPCST

PMPCST

PMPCST

PMPCST

PMPCST

PMPCST

PMPCST

PMPCST

PMPCST

PMPCST

PMPCST

PMPCST

PMPCST

PMPCST

PMPCST

PMPCST

PMPCST

PMPCST

PMPCST

PMPCST

PMPCST

PMPCST

PMPCST

PMPCST

PMPCST

PMPCST

PMPCST

PMPCST

PMPCST

PMPCST

PMPCST

PMPCST

PMPCST

PMPCST

PMPCST

PMPCST

PMPCST 


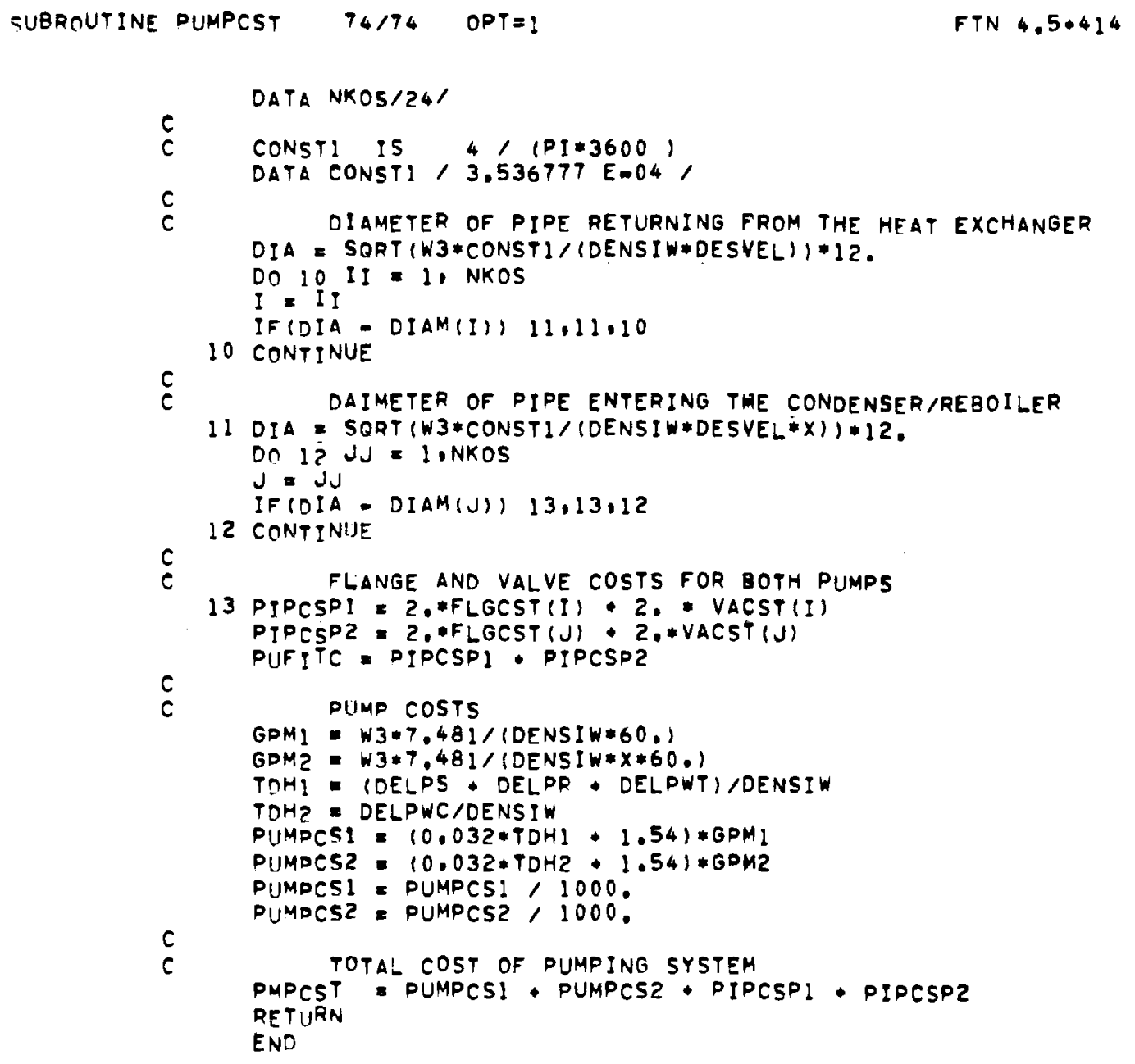


L T14,"AMMONIA TEMPERATURE",F6,1, "F"

R,T6?, "STEAM HEAT TRANSFER COEFFIEIENT",FB,2, "BTU/HR SO FT DEG FII) 6120 FORMATI

$L$ TII."INLET AMMONIA VELOCITY",FB.2,"FT/SEC"

R.T60,"AMMONIA HEAT TRANSFER COEFFICIENT",FB,2,"BTU/HR SO FT DEG F" R,

6125 FORMATI

$L$ TIO,"OUTLET AMMONIA VELOCITY",FB,2, "FT/SEC"

R.T63,"WALL HEAT TRANSFER COEFFICIENT",FB,2,"BTU/HR SQ FT DEG F"I)

6I28 FORMATI

$L$ TO5,"PRESSURE DROP THRU CONDENSER",FB, ILB/SO FT"

$R, T 64, " F O R C E D$ CONVECTION COEFFICIENT",FB, 2 " "BTU/HR SO FT DEG F"', 6130 FORMATI

$L / T O G, \| \cdots$ CONDENSER DESCRIPTION $\rightarrow-"$

R, T79,"1-O CONDENSER COSTS -.-11/1/

6135 FORMATI

L T17,"NUMBER OF SHELLS",16.

R T7S,"TUBING MATERIAL $\$ 1, F 14,2,2 \times 1 "(1,2 A 10,11)$

6140 FORMATI

L T05,"HEAT TRANSFER AREA PER SHELL".611.5." S0 FT"

R T85,"SHELLS SH,F\{4,2\}

6145 FORMATI

L TOT, "NUMBER OF PASSES PER SHELL", I6.

R T77,"IFIELD ERECTION \$11,F14,21

0150 FORMATI

L T22,"PUAE LENGTH", I6,T45,"FEET"I

6155 FORMATI

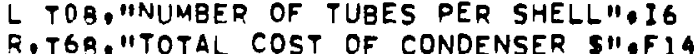

C

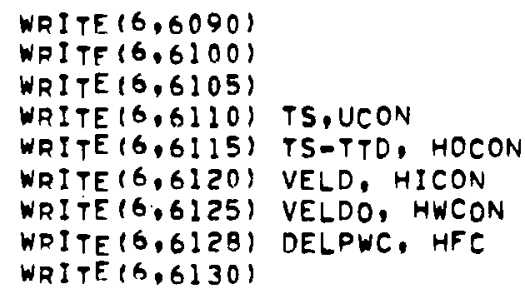

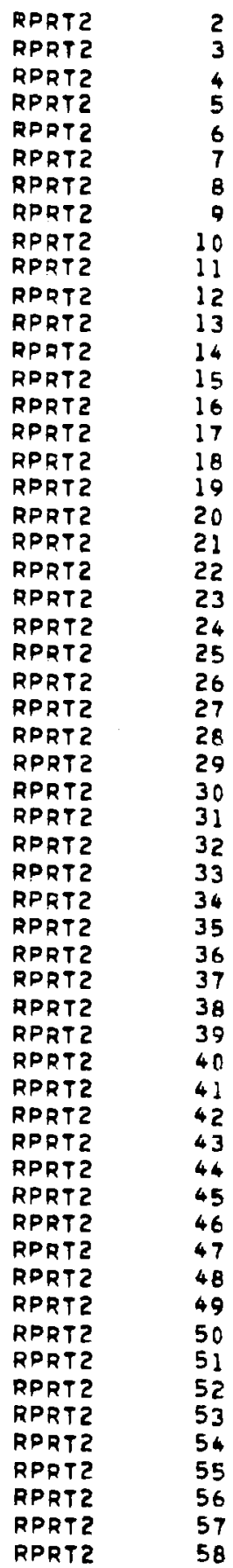




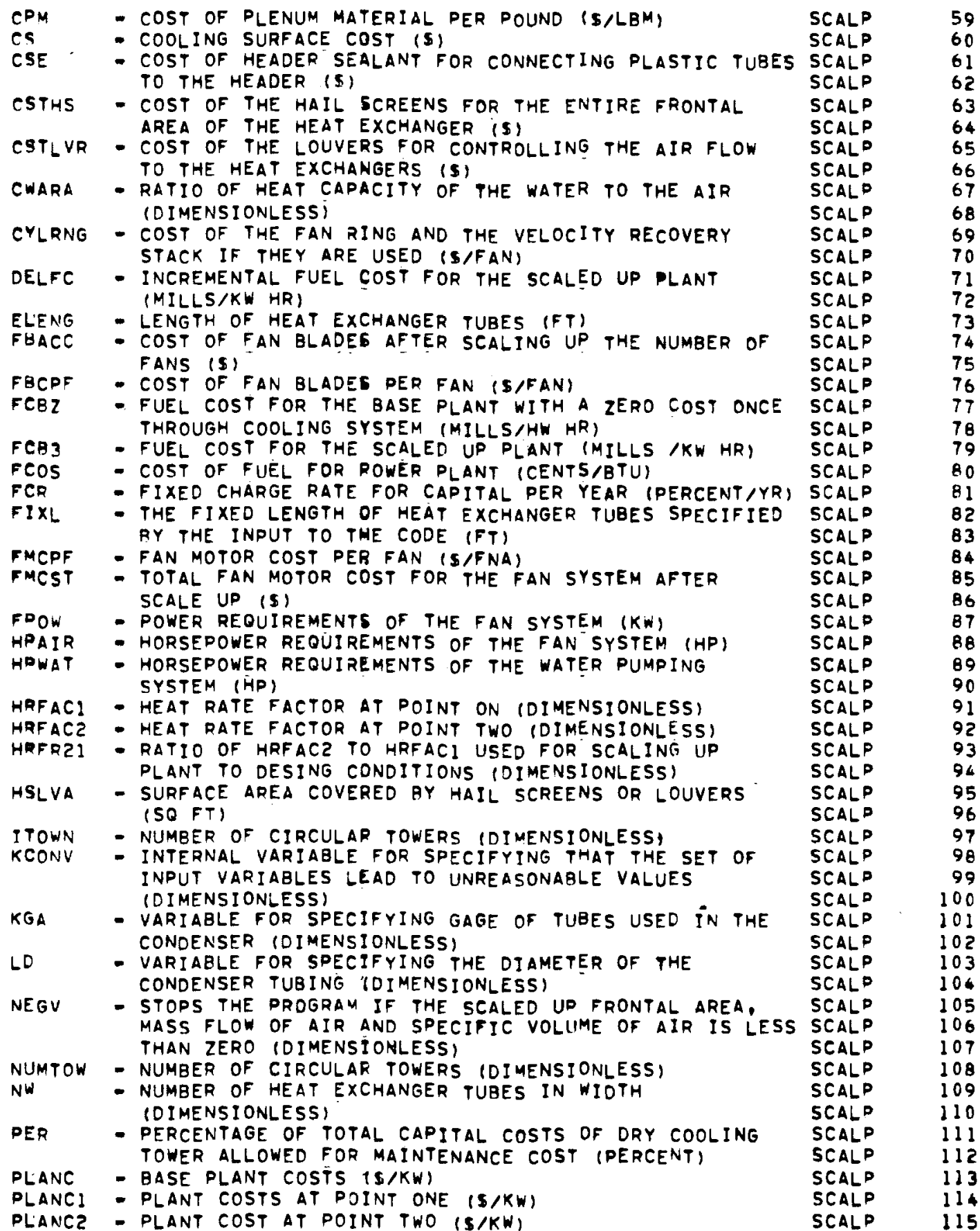


$74 / 74$ OPTE1 FTN $4.5+414 \quad 06 / 05 / 78 \quad 07.59 .59$

SUBROUTINE SCALPITI,RANGE, AFRON, ULRAT,TCWFCS,ITOWN)

SCALP $\quad 2$

SUBROUTINE SEALP SEALES THE PLANT AND DRY COOLING TOWER DESIGNS AND COSTS LINEARLY UPWARD OR DOWNWARD TO ACCOUNT FOP THE FOLLOWING FACTORS-

1. THE OESIGN TEMPERATURE OF THE POWER PLANT DOES NOT CORRESPOND TO THE RATED BACK PRESSURE OF THE STEAM TURAINE.

2. PART OF THE TOTAL POWER OUTPUT OF THE PLANT IS USED IN SUPPLYING POWER TO THE FAN AND PUMPING SYSTEMS,

SCALP

SCALD

SCALP

SCALD

SCALP

SCALD

SCALD

SCALP

SCALD

SCALD

THE FOLLOWING ARE POINTS ON THE HEAT RATE FACTOR CURVE WHICH SCALD

$\triangle R E$ USED IN DESERIBING THE SCALING OF THE PLANT AND DRY COOL- SCALD ING TOWERS. AT EACH OF THEGE POINTS THE HEAT RATE FACTOR, SCALP

PLANT SIZE ANO PLANT COSTS ARE DETERMINED.

PIINT O - CONVENTIONAL TURBINE OPERATING AT RATED BACK PRESSURE

DOINTT 1 - STEAM TURBINE OPERATING AT RATEO BACK PRESSURE

POINT 2 - STEAM TURBINE OPERATING AT SYSTEM DESIGN EXIT TEMPERATURE WITH OUTPUT OF TURBINE GENERATOR EQUAL TO THE DESIGN POWER OUTPUT

DOINT 3 - STEAM TURBINE OPERATING AT SYSTEM DESIGN EXIT TEMPERATURE WITH THE POWER OUTPUT OF PLANT EQUAL TO THE DESIGN POWER OUTPUT

SCALP

SCALP

SCALP

SCALD

SCALD

SCALP

SCALD

SCALD

SCALP

SCALP

SCALD

THE DIFFERENCE BETUEEN POINT TWO ANO THREE IS THE PLANT HaS

SCALP

REEN SCALED UD TO ACCOUNT FOR THE POWER CONSUMPTION OF THE FANS AND PUMPS, BETWEEN POINT ONE AND TWO THE POWER PLANT HAS BEEN SCALED UP OR DOWN TO ACCOUNT FOR AN INCREASE OR DECREASE IN THE HEAT RATE FACTOR.

SCALD

SCALP

SCALP

DEFINITION OF VARIABLES

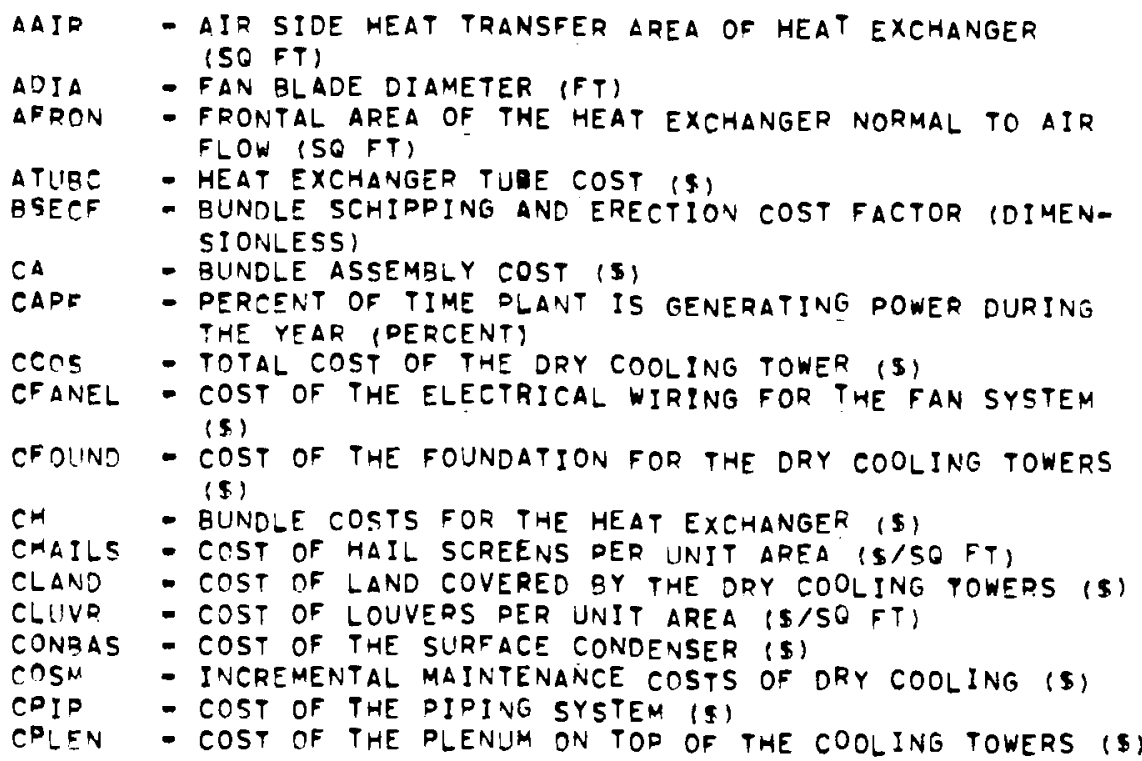

SCALP

SCALP

SCALD

SCALP

SCALP

SCAL?

SCALD

SCALD

SCALP

SCALP

SCALD

SCALD

SCALP

SCAL:

SCALD

SCALP

SCALP

SCALP

SCALD

SCALP

SCALD

SCALP

SCALP

SCALP

SCALP

SCALD

CPLEN

- COST OF THE PLENUM ON TOP OF the cOOLING TOWERS 


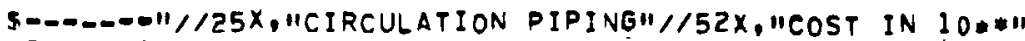

" RPTSUP $\$ 3$ DOLLARS FOR"/40X,"1-.END

RPTSUP

107 
FTN $4.5+414$

$06 / 05 / 78$

07.59 .59

$c$
$c$
$c$
$c$
$c$

SUBROUTINE RPTSUP IDESVELV, NUMTOW, CTOWD I, WBB I, HTOW I, NTOWDE I)

RPTSUP

RPTSUP

RPTSUP

RPTSUP

RPTSUP

RDTSUP

COMMON /KPTSUP/

S DELVI, DIASDI, DIASTI, DOTMAI, DYNHDI

5. HEORI $(200)$

S. JSSAVE

S, KSSAVE

S. PIPCSI

s, OSLCS), OHEDRI (200)

S. SRFCSI, SCOSTI, SEJCSI, SELCST, SFLGCI, SOCOSI, STDCSI

S. SDIAI $(200)$, SPCST) (200), SQDIAI (200), SOPCSI(200), SORCS1 (200)

S. SOTCSI $(200)$, SRCSTI $(200)$, STCSTI $(200)$

5. TCONOI. THXINI, TOWDII. TSOCOI, TSTDEI COMMON /KKSUP/

S SPDIAI (200), LSSAVE, PHEDSI (200), SPPCST1(200), SPRCSTI $(200)$

S. SOTCSTI(200), SPCOSTI,PSLCSTI,PSLGCS1, PSJCSTI, UPPPCSI (200)

$S, D F, N A I J N$

LSELSSAVE

JS E JSSAVE

c

KS: KSSAVE

203 FORMAT (1H $114 \times, 12,1 \times, 7 F 10.1)$

204 FORMATI//140X, "TOTAL COST OF CIRCULATION SUPPLY PIPING $\$ 1, F 5.0$, S" $10 * 3 n / 1 / 11$

205 FORMATI/25X, "HORIZONTAL QUADRANT SUPPLY PIPING"

RDTSJP

RPTSUP

RPTSUP

RPTSUP

RPTSUP

RPTSUP

RPT SUP

RPTSUP

RPTSUP

RPTSUP

RPTSUP

RPTSUP

RPTSUP

RDTSUO

RPTSUP

RPTSJD

RPTSUD

RPTSUP

RPTSUP

RPTSUP

RPTSUP

RPTSUP

RPTSUP

$1 / 42 x_{*}$ "COST IN $10 * 3$ DOLLARS FOR" $/ 40 x$ $5, " 1-0.011 / 12 X$, "HEADER DIAMETER LENGTH $\$$ PIPE TEES REDUCERS"/2!X,"(INCHES) (FEET)"1/)

208 FORMATI///42X,"TOTAL COST OF QUADRANT SUPPLY HEADERS S",F5.O,

\$1" $10 . * 311 / 1 / 1$

209 FORMAT1/25X, "TOWER SUPPLY PIPING"

S IIIX,"NUMBER OF TOWERS", $21 x_{1}$ IS

$511 n x$, "NUMREF OF TOWERS WITH DELUGE PIPING", $2 x, I 5$

S /10X."TOWER DIAMETER",23X,F5,0," FEET"/

S 10X,"TOWER HEIGHT",25X,F5,1," FEET"/

S lox, "HUNDLE WIDTH",25X,F5.1," FEET"/

$510 x$,

"DIAMETER OF DIPE FROM CIRCULATION RPTSUD

S"110X,"HEADER TO TOWER DISTRIBUTION HEADER",57.0," INCHES"/IOX, "DI RPTSUP

SAMETER OF TOWER DISTRIBUTION PIPE",F 7.0." INCHES"//1OX, "COST OF DI RPTSUP

SSTRIRIITION PIPING PER TOWER S",F5.0," $10 * 3 " / 1 / 40 X, " T O T A L$ COST O RPTSUP

SF TOWER DISTRIBUTION PIPING S",F5,0," *10*3"1/1/1

211 FORMATIIOX, "VAPOR DESIGN VELOCITY",F5.0,"FPSH/

S IOX."MASS FLOW RATE", IPE10.3," LBM/HR"/

- InX."CONDENSER OUTLET TEMPERATURE",OPF7.2," DEg. F",

$510 \times$ "HEAT EXCHANGER INLET TEMPERATURE",F7,2," DË. F"/

S $1 \cap X, " S U P P L Y$ PIPING PRESSURE DRDP",F $7,1, "$ PSF"/

5 InX."SUPPLY DIPING DYNAMIC HEAD",F7.1," FEET"1/1/1

\$ $47 X$ "ITTAL SUPPLY PIPING SYSTEM COST S",F6.0," $10 * 31111$

213 FORMAT (IKI)

214 FORMATIIH, $13 \times, 12,1 \times, 7 F 10,1)$

215 FORMATI/10X,"COST OF SUPPLY HEADERS PER QUADRANT S",F5.1, क" *1 $0 * 311$

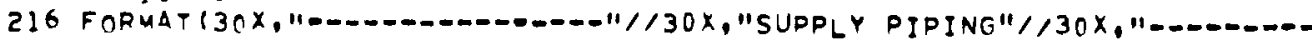

RPTSUP

RPTSUP

RPTSUP

RPTSUD

RPTSUP

RPTSJO

RPTSUP

RPTSJP

RPTSJP

RPTSUP

RPTSUP

RPTSUP

RDTSUP 


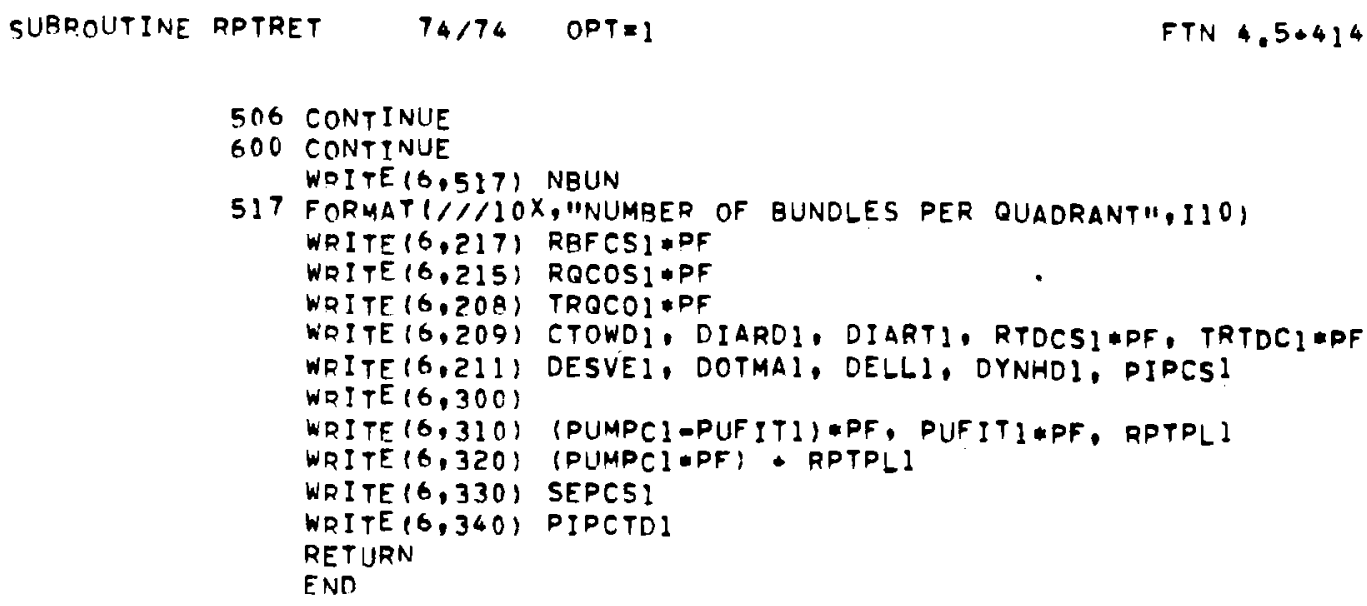

$\begin{array}{ll}\text { RPTRET } & 116 \\ \text { RPTRET } & 117 \\ \text { RPTRET } & 118 \\ \text { RPTRET } & 119 \\ \text { RPTRET } & 120 \\ \text { RPTRET } & 121 \\ \text { RPTRET } & 122 \\ \text { RPTRET } & 123 \\ \text { RPTRET } & 124 \\ \text { RPTRET } & 125 \\ \text { RPTRET } & 126 \\ \text { RPTRET } & 127 \\ \text { RPTRET } & 128 \\ \text { RPTRET } & 129 \\ \text { RPTRET } & 130 \\ \text { RPTRET } & 131\end{array}$


c

217 FORMATI/110X,"FITTINGS COST PER BUNOLE \$1,F7.3." $10 \cdots 311$

RPTRET

RPTRET

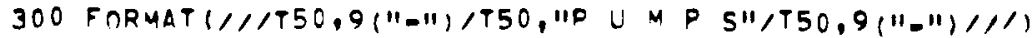

310 FORMATIT10, "PUMP COST",T30,"\$",FE,0,1110*3"

\$ TI0, "PUMP FITTINGS",T30,"\$",FB.0,"*10**3"

5 ITIO,"PUMP ELECTRICAL", T30,"\$",FB,0,110.10:311)

320 FORMATI/T6O."OUMP STATION", TBO, "\$", FB.0,110.103",

330 FORMATI/1TIO."SEPARATOR COST".T30."\$",FII.0.1110*3")

c

340 FORMATI//TIO,"DELUGE PIPING COST",T30,"S",F11.0,"1.10\%3")

\section{JRIJRSAVE \\ KREKRSAVE \\ LRILPSAVE}

WRITE $(6,213)$

WRITE (6.2 16$)$

DQ $501 \quad I=1, J R$

IF (I.GT.I) GO TO 202

WRITE (A:203) I, ROIAI (I), HEDRI (I), RPCSTI II) APF, RTCSTI (I) APF

S RRCSTI II)*PF, REJCSI*PF, RFLGCI*PF GO TO 201

202 IF (I.EO.JR) GO TO 210

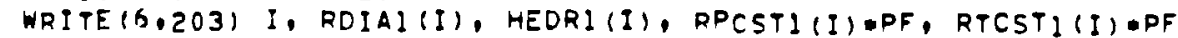

S.RRCSTIII) APF

GO TO 201

210 WRITE(6.203) I, RDIAI (I), HEDRI I), RPCSTIII) PPF, RELCSIAPF

F.RRCSTI I I $\rightarrow$ PF

201 CONTINUE

WRITE $(6.204)$ RCOSTI APF

WRITE $(6.205)$

DO $206 \quad I=1, K R$

IF (I.EQ,KR)GO TO 207

WRITE $(6,214)$ I, RQDIAI (I), QHEDRI (I), ROPCSI (I) *PF, ROTESI II) MPF

S ,RORCSI (I) $\triangle P F$

GO TO 206

207 WRITE (S.214) I, RODIAI II), OHEDRI (I), ROPCSI II) UDF, QRLESI HPF

S, RORCSI (I) DPF

206 CONTINUE

WDITE $(0.405)$

405 FORUAT $1 / 25 X$, "EXTERIOR VERTICAL OUADRANT RETURN PIPING"/142X,"COST

SIN $10 * 3$ DOLLARS FOR $1 / 40 x, 30(11-1) / 12 x$, "HEADER DIAMETER LENGTH

5 SIPE TEES REDUCERS"/2IX,"(INCHES: (FEET)"/1)
THOR DO $406 I: I, L R$

IF (I.EO.LR) GO TO 407

WRITE (6,214) I,RPDIAI (I),PHEDRI I), RPPCSTI I) $\triangle P F, R P T C S T I(I) \backsim P F$, SRDRESTI (I) $D F$

GO TD 406

407 WRITE $(6,214)$ I,RPDIAI (I), PHEDR II),RPPCSTI I) $\$$ PF, PRLCST \&PF,

SRDRCSTI (I) PF

406 CONTINUE

IF (INT.LE.O) GO TO 600

WDITE $(6.505)$

505 FORMATI/25X."INTERIOR VERTICAL QUADRANT RETURN PIPINGI//42X,"COST

\$IN 10*3 DOLLARS FOR "/40X,30 "1-")/12X,"HEADER DIAMETER LENGTH

5 SIN IOA.3 DO

TEES REDUCERS"/2IX,"IINCHES

(FEET) 11 )

DO $505 I=1$,LR

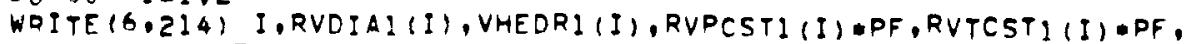
SPVRCSTI (I) $*$ PF

RPTRE

RPTRET

RPTRET

RPTRET

RPTRET

RPTRET

RPTRET

RPTRET

RPTRET

RPTRET

RPTRET

RPTRET

RPTRET

RPTRET

RPTRET

RPTRET

RPTRET

RPTRET

RPTRET

RDTRET

RPTRET

RPTRET

RPTRET

RDTRET

RPTRET

RPTRET

RPTRET

RPTRET

RPTRET

RPTRET

RPTRET

RDTRET

RPTRET

RPTRET

RPTRET

RPTRET

RPTRET

RPTRET

RPTRET

RPTRET

RPTRET

RPTRET

RPTRET

RPTRET

RPTRET

RPTRET

RPTRET

RPTRET

RPTRE i

RPTRET

RPTRET

RDTRET

ROTRET

RPTRET
59

60

61

62

63

64

65

66

6?

$-8$

60

7

71

72

73

76

79

78

70

80

81

82

83

84

85

86

87

A8

80

t:

91 
SURROIITINE RPTRET(CTOWD1, PF, PSTACI, PUFITI, PUMPCI, RPTPLI,

RPTRET S SEPCSI,DIPCTDI)

S. HEDRI $(200)$

S. JRSAVE

S. KRSAVE

S. PIPCSI

S. QHEDRI (200), QRLCSI

S. RDIAI (200), RPCSTI (200), RRCSTI (200), RTCSTI (200), RBFCSI, RCOSTI

5. RADIAI (200), RQPCS) (200), RORCS 1 (200), RQTCSI (200), REJCS)

5, RELCSI, RFLGCI, RQCOSI, RTDCSI

S. TOWDII. TROCOI, TRTDCI

COMMON /KKRET/

S RPOIAI $(200)$, RVDIAI $(200)$, LRSAVE

S.PHEORI $(200)$, VHEDRI $(200)$

S.RPRCSTI(200), RVRCSTI(200)

S.RPTCSTI (200), RVTCSTI (200)

S.DRLCSTI, VRLCSTI

S.PPDCSTI (200), RVPCSTI(200)

S.RPCOSTI, RVCOSTI

C

S INT • NBUN

203 FORMATIIH $, 14 \times, 12,1 \times, 7 F 10,11)$

204 FORMATI///40X,"TOTAL COST OF CIRCULATION RETURN PIPING \$",F5.0. S" $10 \cdots 31 / 1 / 11$

205 FORMATI/Z5X, "HORIZONTAL QUADRANT RETURN PIPING"

$S$

$1 / 42 x_{0}$ "COST IN 10**3

S."1-0.-

F PIPE TEES REDUCERS"/21X,"(INCMES) (FEET)" $/ 1)$

RPTRET

RPTRET

RPTRET

RPTRET

RPTRET

RPTRET

RPTRET

RPTRET

RPTRET

RPTRET

RPTRET

RPTRET

RPTRET

RPTRET

RPTRET

RPTRET

RPTRET

RPTRET

RPTRET

RPTRET

RPTRET

RPTRET

RPTRET

RPTRET

RPTRET

RPTRET

RPTRET

RPTRET

RPTRET

RPTRET

RPTRET

RPTRET

RPTRET

RPTRET

S"1 $10 * 311 / 1 / 1$

209 FORMATI/25X, "TOWER RETURN PIPING"

5

$510 x$

//10X, HTOWER DIAMETERH, 23X,F5, O, FEET"/ RPTRET "DIAMETER OF PIPE FROM CIRCULATION RPTRET

S"/10X,"HEADER TO TOWER DISTRIBUTION HEADER",F7.0," INCHES"/IOX, "DI RPTRET

SAMETER OF TOWER DISTRIBUTION PIPE",F $.0, "$ INEHES"1/10X,"COST OF JI RPTRET SSTRIRIITION PIPING PER TOWER \$",F5,0," $10 * 31 / 1 / 40 x \cdot "$ "TOTAL COST O RPTRET SF TOWER DISTRIBUTION PIPING S",F5,0," *10*311/111

211 FORMATIIOX, "LIQUID DESIGN VELOCITY",F5,0," FPS"/

I IOX, "MASS FLOW RATE", IPEIO.3," LBM/HR"/,

\$ IOX."RETURN PIPING PRESSURE DROP", OPFT, I," PSF"/

\$ IOX, "RETURN PIPING DYNAMIC HEAD",FT.1, "FEET"1/11,

$547 \times$ "TOTAL RETURN PIPING SYSTEM COST S"F, F.0," $10 * 311111$

213 FORMAT (IHI)

2 I4 FORMATIIH, I3 $\left., 12,1 x_{1}, 7 F 10,1\right)$

215 FORMATI/IOX, "COST OF RETURN HEADERS PER QUADRANT SHOF5.1, 5" * $10 * 311$

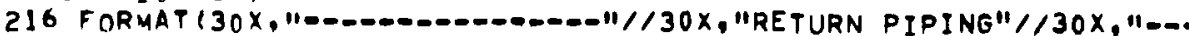
S- $\ldots+\infty / / 25 x$, "CIRCULATION PIPING"//52 $x$, "COST IN $10 \ldots * 11$ SOLLARS FOR"140X,"1-D.-D SRS EXPANSION FLANGES"/2IX."(INCMES) S11/11 PIDE TEES REDUCE RPTRET

RPTRET

RPTRET

RPTRET

RPTRET

RPTRET

RPTRET

RPTRET

RPTRET

RPTRET

RPTRET

(FEET)", 33X, "JOINT (1)

(3 RPTRET

RPTRET
2 


\section{c}

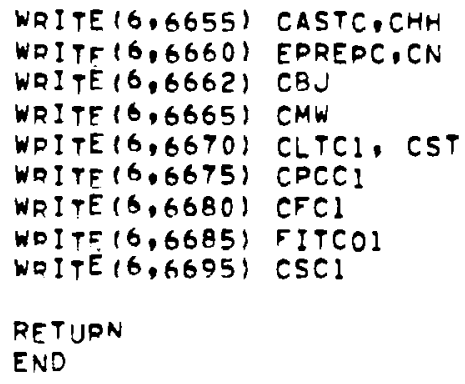

$\begin{array}{ll}\text { RPTHXD } & 112 \\ \text { RPTHXD } & 113 \\ \text { RPTHXD } & 114 \\ \text { RPTHXD } & 115 \\ \text { RPTHXD } & 116 \\ \text { RPTHXD } & 117 \\ \text { RPTHXD } & 118 \\ \text { RPTHXD } & 119 \\ \text { RPTHXD } & 120 \\ \text { RPTHXD } & 121 \\ \text { RPTHXD } & 122 \\ \text { RPTHXD } & 123\end{array}$




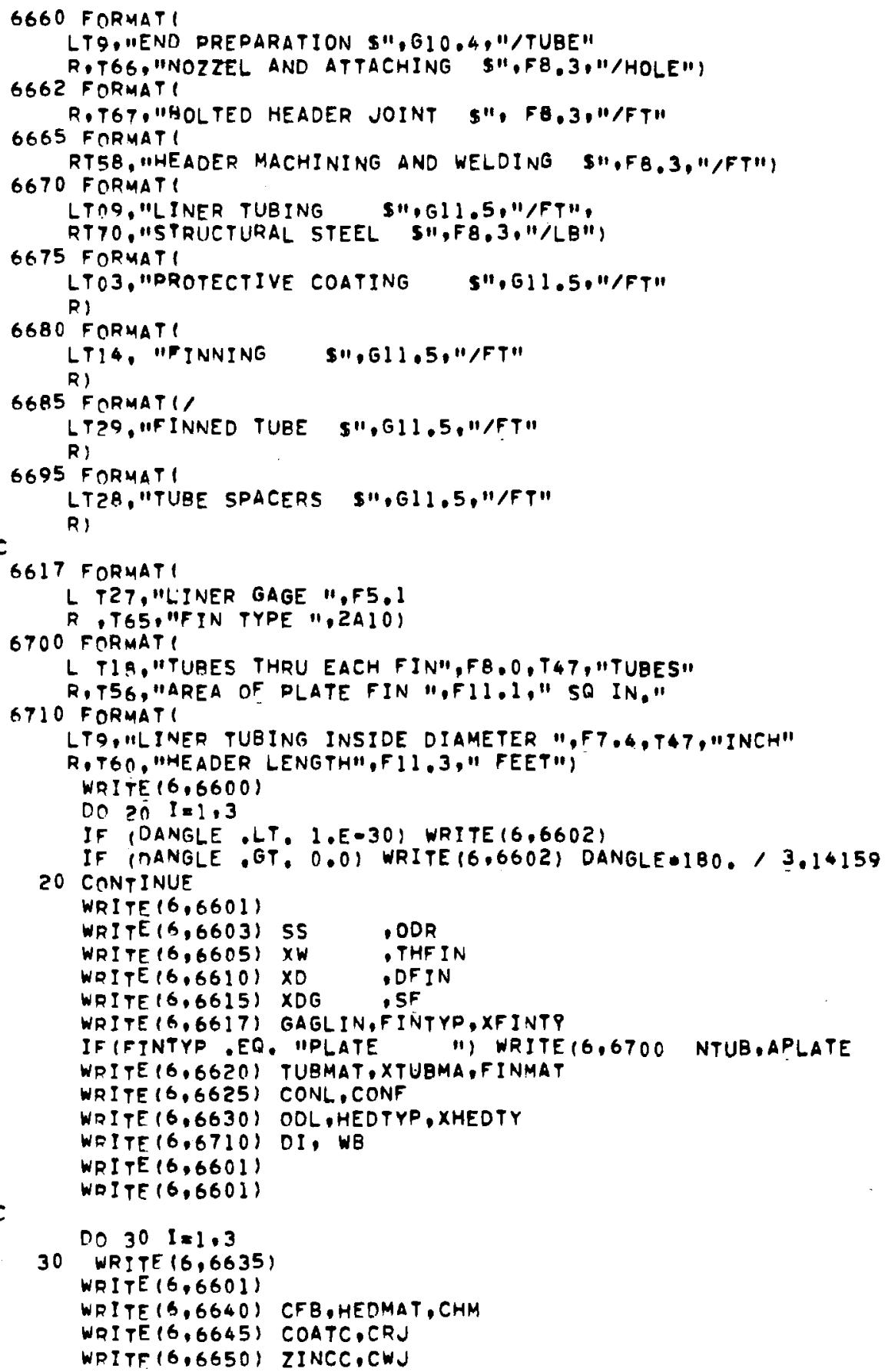


RDTHXD

SUPPLY

SUPPLY

SUPPLY

SUDPLY

SUDPLY

SUPP:Y

SUPPLY

SUPPIYY

SUPPLY

SUPPLY

SUPPLY

SUPPLY

SUPPLY

SUPPLY

SUPPLY

SUPPLY

SUPP:Y

RPTHXD

RPTHXD

RPTHXD

RPYHXD

RPTHXD

RPTHXD

RPTHXD

RPTHXD

RPTHXD

RPTHXD

RPTHXD

RPTHXD

RPTHXD

RPTHXD

RPTHXD

RPTHXO

RPTHXD

RPTHXD

RPTHXD

RPTHXD

RPTHXD

RPTHXD

RPTHXD

RDTHXD

RPTHXD

RPTHXC

RPTHXD

RDTHXD

RPTHXJ

RPTHXD

RPTHXD

RPTHXD

RPTHXD

RPTHXO

RPTHXD

RPTHXD

RPTHXJ

RPTHXD

RDTHXO 
THIS ROUTINE REPORTS HEAT EXCHANGER DESCRIPTION AND UNIT COST DATA RPTHXD

RPTHXD

LOGICAL' FIRST, FRST, FXTEMP,FXTVAR,FXTTTD,FXTLNG

BIGCOM COMMON

s AFTR, ALPHA, ANG (3)

* Bdim(16)

5. CAPF, CSSPKW, CONF, CONL, COSTL, CAPCHG, CONMAT, CONMAZ, CAF

BIGCOM

BIGCOM

BIGCOM

BIGCOM

, CBJ. CTURB, CDANG, CLUVR, CHAILS, CVM

5 DFIN, DEEPL , DESVEL, DESVELV, DESVELD, DENSIF

S.EFFP. EFS

I IFR, FIRST,FIXL, FCOS,FFHX, FXTEMR, FACTOR, FRST, FACTORD, FSHOT, FDELUG

S ,FIXV.FIXTTD,FXTVAR,FXTTTD,FXTLNG

S.GQEFD

5. HXNP, HPCST, HCD

5 ITMAX, ITMIN

S. JCONS

SEONV, KALEXT

5 , MXEXT

S ,NTA,NSIDES, NBUNHI

COMMON

$S O D R$

S.PSIZE, PER, PWCOS. PLANC, PFACT, POMDPL

5 , POHBAF, POHFAN, POHLEC, POHCIR, POHCND, POHSTC, POHSCL

5. RBP,REDUCE, REDUCV, ROOFL, RHOUT

S DREJ, QREDUCE, QREDUCV

S SIGMAG, SAAF, SEGL

S.TD, TPO(4), TEFF, TLIM, THFIN, TLPRA, TFIX

S.TCD. TW

S.UCS, UWS

5 . WFV. WATCONA, WATCOST

S , VAS (5)

S. XDEPA, XW, XD

COMMON/HEADEX/ CHH, CN, CHM, CMW, CRJ, CWJ, CST 5 CMO, CSR, CSM, PITCMF, CPM, PRESS, HEDMAT, HEDTYP, NPASS S WB, TSP, CSH,CSHP

COMMON /SINK/ VAR(5), CPLN1, CPIPI, CCOS1, W31, COSMI, EFFI, QINI, CLANI, REAII, ZI, HRFAZS, WHI, UI, DEPWI, PPOWI. FPOWI, DEFCI, WIOTI, ELENI, VAIRI, VWATI, HII, HOI, T4I: REWA1, NWI, NTI, NPI,ZPLNC2,ZSIZ12, ZSPBP, SWA1, SW31. SPC1, PTOT1,PLNC31, AFROI, AAIRI, YFPMIN, SPCDI, SPSI3,WTZMIN,

BDZMIN,NWZMIN, TCOS1, DEPAI, DPWT1, DPWCI,CONBAL, XNTSI, VELDI - TLAl, UCON1, PMCSI, FMCSI, FBACl, EFFCl, CKI, CAI: CSI S. CADCSI, XNFANI, BLDANI, CPLEN1,DPFELI, WKI, WTTUI, ADI I, ABLI S, WTHDI, WTFRI, STRUCl, CFMPFI, HPPFI, TOTCFI, PPI,EFFINI, ANTUI

S. AIRFF1, WATFFI, XNMODI, TCTPF 1; FDCPFI, FMTRCI, ACTTPI, ADJTP I

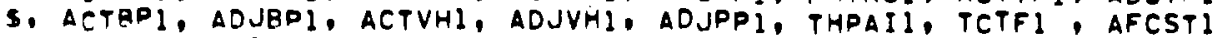

5, CSRI, WATERI, WSTRCI, CFOUNI, HUBDI1, TOWLEI, NUMTOI, CTOWDI

F, SORJI, SORJSI, ATUBCI , CBAI, TTDII, TTD2I, DELPSI, CSTLVI , PIPCTDI

5 .CSTHSI, CYLRNI, CFANEI, PSTACI, PUFITI, PMPCSI, RPTPLI, SEPCSI

S.PWD 1 (16), WATCWO1 (16), DEPER 1 (26), DEPERQ1 (16)

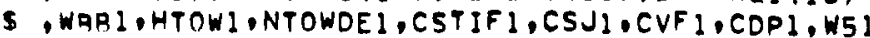

COMMON /TINE/ XNS, TLA, XNTS, ARPS, TTOZOE, LINOR, XNP, XQUALY

COMMON /SURFEX, ODL,GAGLIN, NTUB, TUBMAT, FINTYP,FINMAT, CFG

5. APLATE, COATC, ZINCC, CASTC, SS,EPREPC, XTUBMA, XHEDTY, XFINTY

BIGCOM

BIGCOM

BIGCOM

BIGCOM

BIGCOM

BI GCOM

BIGCOM

BIGCOM

BI GCOM

BI GCOM

BI GCOM

BIGCOM

BIGCOM

BI GCOM

BI GCOM

BIGCOM

BIGCOM

BISCOM

BI GCOM

BIGCOM

BIGCOM

BIGCOM

BIGCOM

BI GCOM

BIGCOM

RPTHXD

RPTHXD

RPTHXD

SINK

SINK

SINK

SINK

SINK

SINK

SINK

SINK

SINK

SINK

SINK

SINK

SINK

SINK

SINK

SINK

SINK

RPTHXD

RPTHXD

RPTHXD 


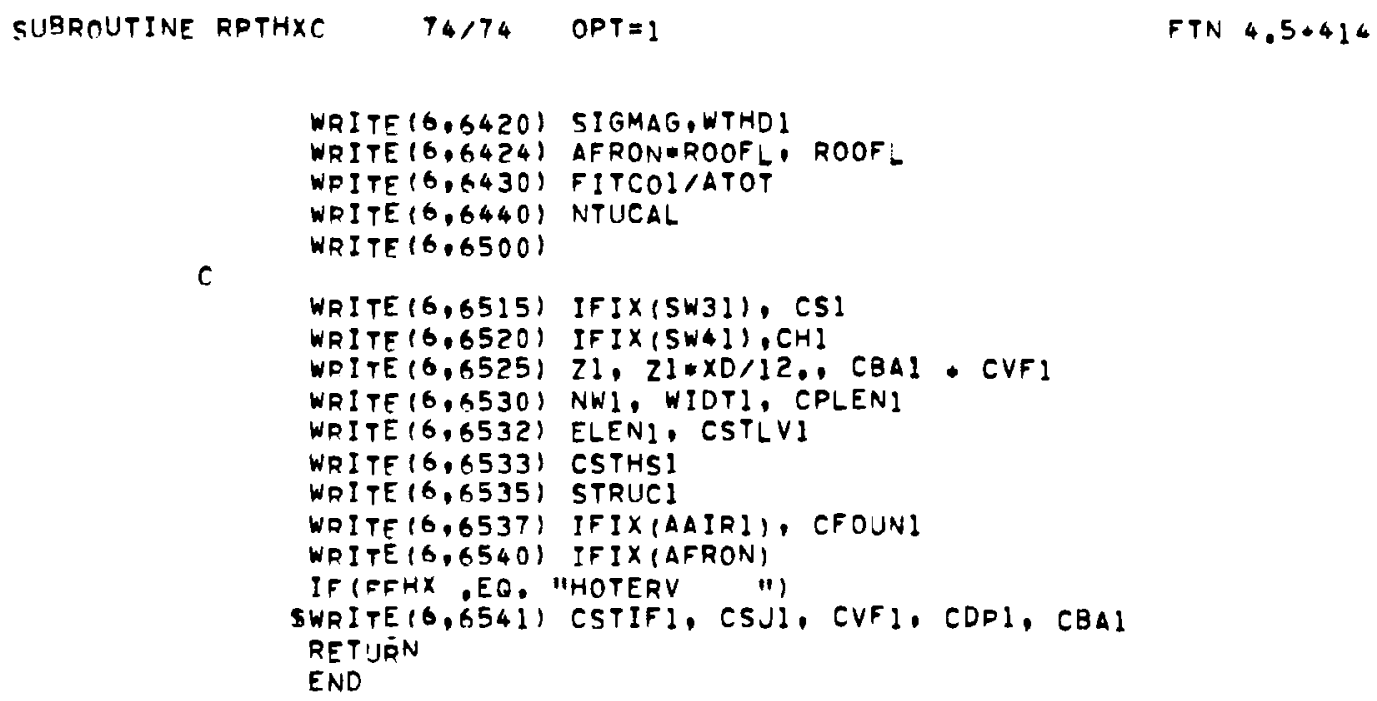




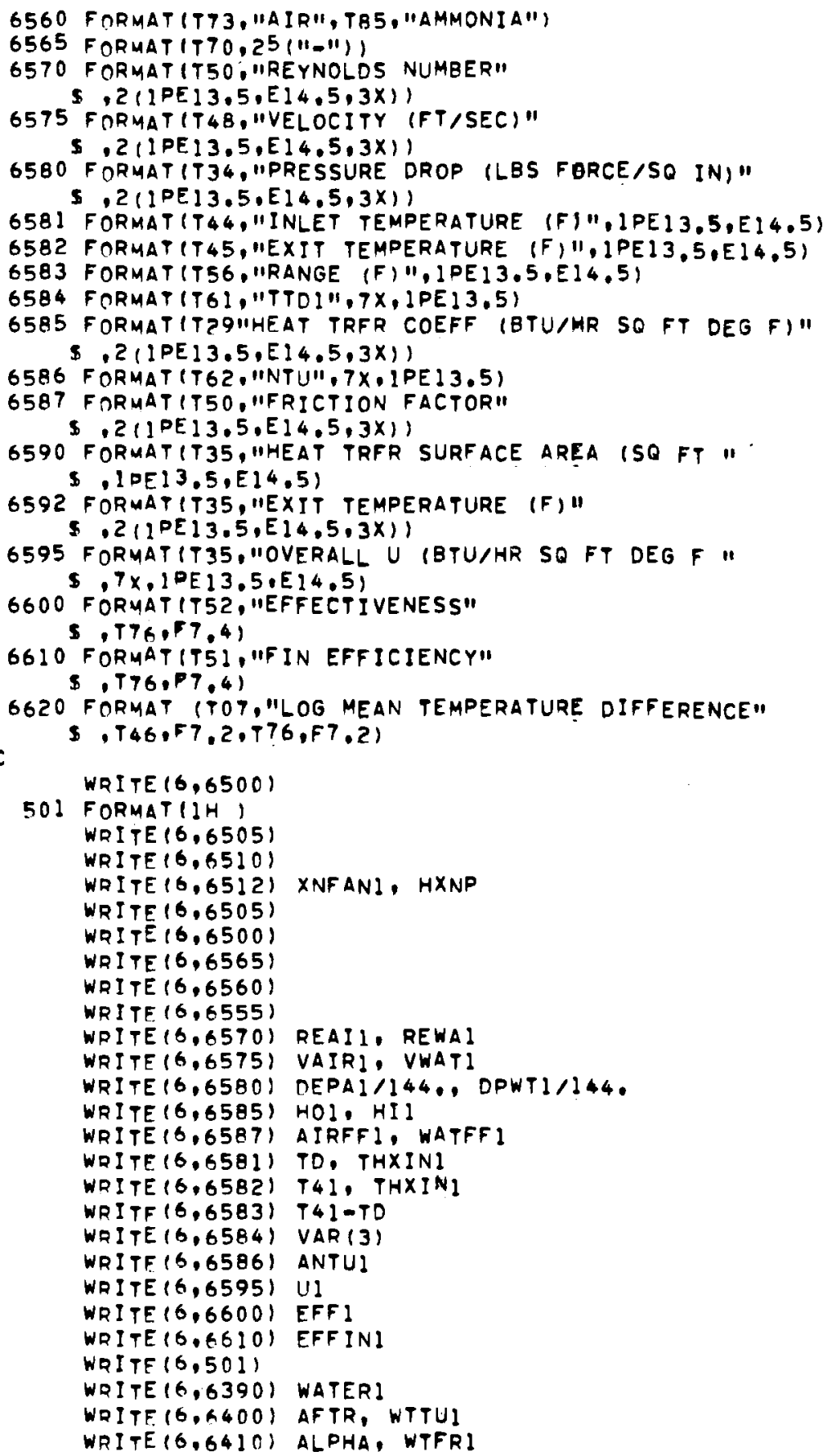

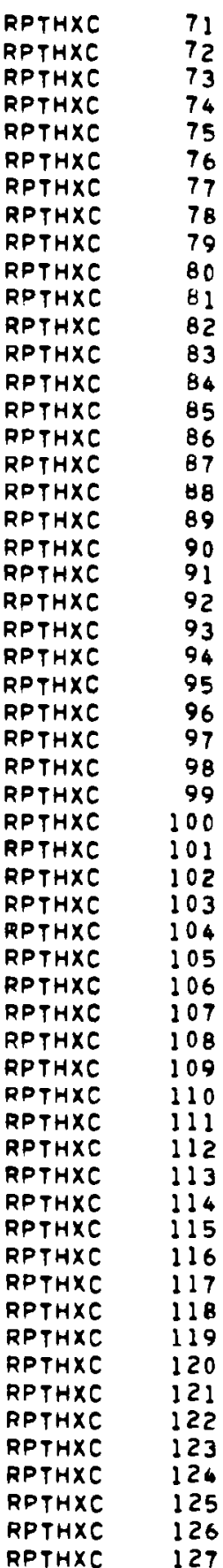




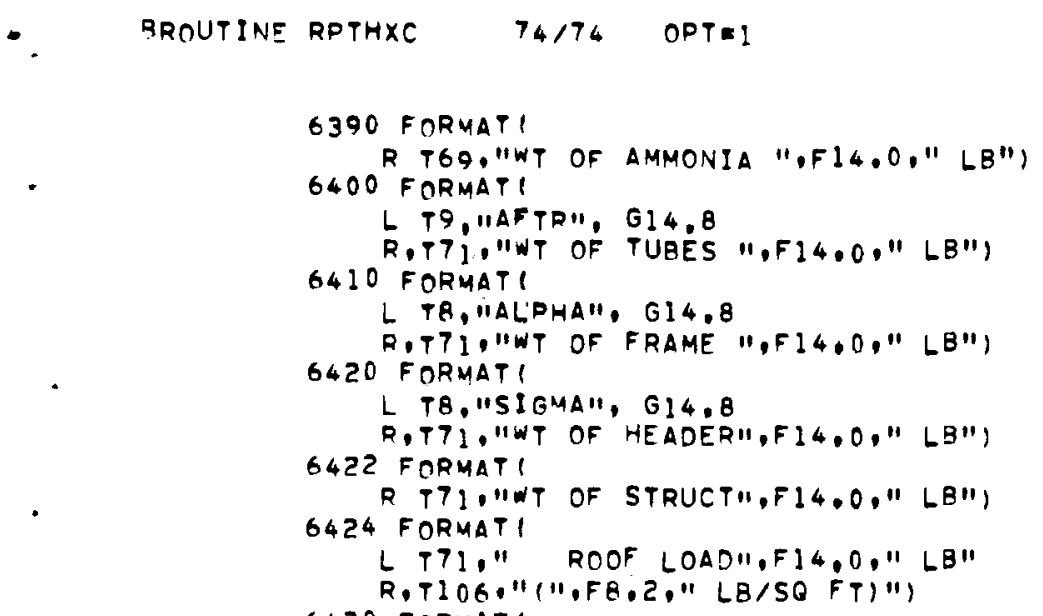


COMMON /TINE/ XNS, TLA, XNTS, ARPS, TTOZQE, LINOR, XNP, XQUALY COMMON /HXD/ AI, ATOT,SFA,RFA,ACS COMMON /ESCAL/ BSECF

BIGCOM

BI GCOM

BI GCOM

BI GCOM

BI GCOM

BI GCOM

BIGCOM

BI GCOM

BI GCOM

SI NK

SINK

SINK

SINK

SINK

SINK

SINK

SINK

SINK

SINK

SI NK

SINK

SINK

SINK

SINK

SINK

RPTHXC

RPTHXC

RPTHXC

RDTHXC

RPTHXC 


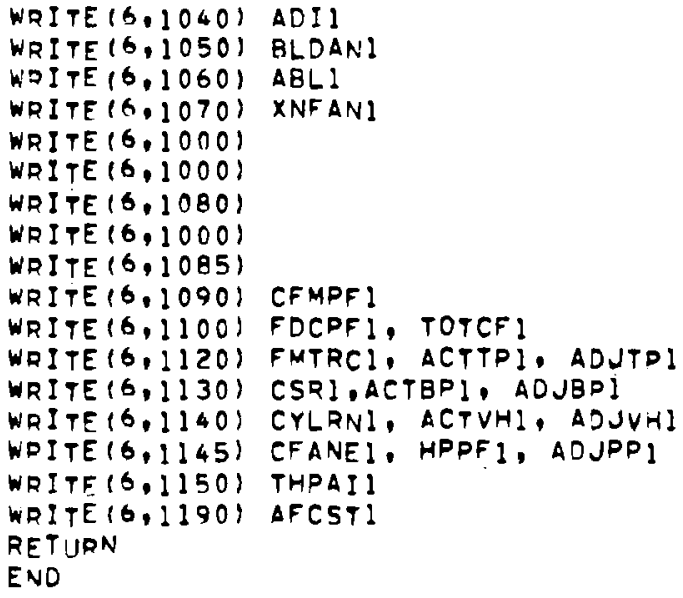

FTN $4.5+414$

$06 / 05 / 78 \quad 07.59 .59$ 


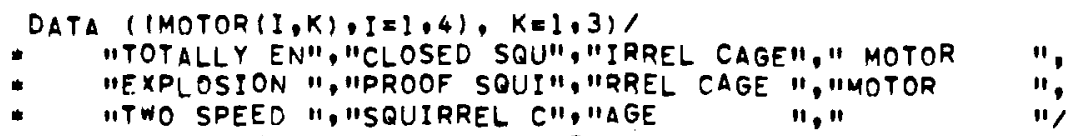


S VELDEC, AFC. ELEV, EHR, LOOPER, GTMAX LOGICAL FIRST, FRST, FXTEMP, FXTVAR OFXTTTD, FXTLNG COMMON

5 AFTR, ALPHA, ANG (3)

- Boljm(j6)

S CAPF, CSSDKW, CONF, CONL, COSTL, CAPCHG, CONMAT, CONMAZ,CAF

\$ CRJ, CTURB, CDANG, CLUVR, CHAILS, CVM

\$. DFIN . OEEPL, DESVEL DESVELV, DESVELD, DENSIF

S EFFD. EFS

S FCR, FIRST, FIXL, FCOS,FFHX, FXTEMP, FACTOR, FRST , FACTORD, F SHOT , FDEL JG

S. FIXV,FIXTTD,FXTVAR,FXTTTD,FXTLNG

$S$, GAEFF

5 , HXND,HPCST, HCD

S ITMAX, ITM!N

5 iJCONS

S, KCONV, KALEXT

S. MXEXT

S ,NTA,NSIDES, NBUNHI COMMON

$5 O D R$

\$.PSIZE, DER, PWCOS, PLANC, PFACT, POMDPL

S. POHBAF, POHFAN, POHLEC, POHCIR, POHCND, POHSTC, POHSCL

5 - RSP.REDUCE, REDUCV, ROOFL, RHOUT

\$ PREJ, OREDUCE, OREDUCV

\$. SIGMAG, SAAF, SEGL

? TD. TPO(4), TEFF, TLIM, THFIN, TLPRA, TFIX

S.TCD, TW

S. UES, UWS

S ,WFV, WATCONA, WATCOST

5 . VAS (5)

c

S.XEPA, XW, XO

COMMON /SINK/ VAR(5), CPLNI, CPIPI, CCOSI, W31, COSMI, EFFI, 5 OINI, CLANI, REAII, ZI,HRFAZS, WAI, UI, DEPWI, PPOWI. S FPAWI, OEFCI, WIDTI, ELENI, VAIRI, VWATI, HII, HOI, T4l, 5 REWAI, NWI, NTI, NPI,ZPLNC2,ZSIZ12, ZSPAP, SWA1, 5W31.

5 SPCI, PTOTI,PLNC31, $\triangle F R O 1, \triangle A I R I, Y F P M I N$, SPCDI, SPSI3,WT2MIN, 5 BDIMIN.NWZMIN. TCOSI, DEPAI, DPWTI, DPWCI,CONBAI, XNTSI, VELDI S, TLA1, UCON1, PMCS1, FMCS1, FBACl, EFFCl, CHI, CAI, CSI 5 - CLTCI, CFCI, CPCCI,FITCOI, CSCI,CEPREI,DEPIPI, ASTI,OLDCSI S. CADC51, XNFANI, BLDANI, CPLENI,DPFELI, WH! WTUI: ADI1, ABLI

s, WTHO1, WTFRI, STRUCI,CFMPF1, HPPFI, TOTCFI, TPI,EFFINI, ANTU1

5, $\triangle I P F F$ 1. WATFF1, XNMODI, TCTPF1, FOCPF1, FMTRC1, ACTTPI, ADJTDI

5. $A C T R P 1, \triangle D J B P 1, A C T V H 1, \triangle D J V H 1, \triangle D J P P I, T H P A I 1, T C T F 1, A F C S T 1$

5. CSRI, WATERI, WSTRCI, CFOUNl. HUBDII, TOWLEl. NUMTOI, CTOWDI

S , SPRJ1, SORJS1, ATUBCI, CBAI, TTOI1, TTO21, DELPSI, CSTLVI, PIPCTDI

S CSTHS1, CYLRN1, CFANE1, PSTAC1, PUFIT1, PMDCS1, RPTPL1, SEPCSI

5. PWDI (16), WATCWD \& (16), DEPERI (16), DEPERQ (16)

S. WRBI, HTOWI,NTOWDE , CSTIFI,CSJI,CVFI,CDPI, W51

RPTFAN

RDTFAN

RPTFAN

RPTFAN

FANDEK

FANDEK

FANDEK

BI GCOM

BIGCOM

BI GCOM

BIOCOM

BI GCOM

BIGCON

BI GCON

BI GCOM

BIGCOM

BIGCOM

BI GCOM

BI GCOM

BIGCOM

BI GCOM

BI GCOM

BIGCOM

BI GCOM

BIGCOM

BIGCOM

BI GCOM

BI GCOM

BIGCOM

BI GCOM

BI GCOM

BI GCOM

BIGCOM

BI GCOM

BI GCOM

BI GCOM

BI GCOM

RPTFAN

SINK

SINK

SINK

SINK

SINK

SINK

SINK

SINK

SINK

SINK

SINK

SINK

SINK

SINK

SINK

SINK

SINK

RPTFAN

RPTFAN 
DO $401: 1 . N N N$

$\left.N_{1}=1 ! \cdot 1\right\} * 42 \cdot 22$

$N 2=N 1+9$

WRITE (6.1075) (SPRT4 (IJ), IJ = NI,N2)

40 CONTINUE

WRITE $(6,1000)$

WQITE $(6,1045)$

DO $501=1$. NNN

$N_{1}=1 I \cdot 11 \cdot 42 \cdot 32$

$N 2=N I: 9$

WRITE(6.1075) (SPRT4(IJ). IJ $=N 1, N 2$ )

50 CONTINUE

WRITE $(6.1000)$

WRITE $(6,1046)$

DO $55 I=1$. NNN

$N 1=(1-1) \cdot 42 \cdot 42$

$N 2=N 1$

WPITE (6.1075) (SPRT4 (IJ), IJ = NI,N2)

55 CONTINUE

Go TO 100

10

WRITE $(6,1050)$

DO 6 I I I. NNN

$N 1=1 ! \cdot 1\} * 26 \cdot 1$

$\mathrm{N} 2=\mathrm{N} 1+9$

WRITE $(6,1075)$ (SPRT4 (IJ),IJ=N1,N2)

GO CONTTNIJE

WRITE $(6,1000)$

WRITE (6.1055)

DO $701=1, N N N$

N1 $=11-1) \div 26 \cdot 11$

$N 2: N 1 \cdot 2$

WRITE $(6,1075)$ (SPRT4 (IJ), I JNNI,N2)

70 CONTINUE

WRITF $(6,1000)$

WRITE $(6,1025)$

WRITE $(6,1000)$

WRITE $(0,1060)$

DO OO I= 1 , NNN

$\left.N^{\prime}=\mid I \cdot 1\right) \cdot 26 \cdot 14$

$N 2=N 1.9$

WRITE (6,1075) (SPRT4 (IJ), I JENI, N2)

BO CONTTNUE

WRI TE $(6,1000)$

WRITE (6,1065)

DO $001 \div 1$. NNN

$N_{1}=(1 \cdot 1) * 26 \cdot 24$

N2 $=N 1 * 2$

WRITE $(6,1075) \quad($ SPRT $4(I J), I J=N 1, N 2)$

90 CONTINUE

100 CONTINUE

RETURN

END

$\begin{array}{ll}\text { RPTDEL } & 87 \\ \text { RPTDEL } & 88 \\ \text { RPTDEL } & 89 \\ \text { RPTDEL } & 90 \\ \text { RPTDEL } & 91 \\ \text { RPTDEL } & 92 \\ \text { RPTDEL } & 93 \\ \text { RPTDEL } & 94 \\ \text { RPTDEL } & 95 \\ \text { RPTDEL } & 96 \\ \text { RPTDEL } & 97 \\ \text { RPTDEL } & 98 \\ \text { RPTDEL } & 99 \\ \text { RPTDEL } & 100 \\ \text { RPTDEL } & 101 \\ \text { RPTDEL } & 102 \\ \text { RPTDEL } & 103 \\ \text { RPTDEL } & 104 \\ \text { RPTDEL } & 105 \\ \text { RPTDEL } & 106 \\ \text { RPTDEL } & 107 \\ \text { RPTDEL } & 108 \\ \text { RPTDEL } & 109 \\ \text { RPTDEL } & 110 \\ \text { RPTDEL } & 111 \\ \text { RPTDEL } & 112 \\ \text { RPTDEL } & 113 \\ \text { RPTDEL } & 114 \\ \text { RPTDEL } & 115 \\ \text { RPTDEL } & 116 \\ \text { RPTDEL } & 117 \\ \text { RPTDEL } & 118 \\ \text { RPTDEL } & 119 \\ \text { RPTDEL } & 120 \\ \text { RPTDEL } & 121 \\ \text { RPTDEL } & 122 \\ \text { RPTDEL } & 123 \\ \text { RPTDEL } & 124 \\ \text { RPTDEL } & 125 \\ \text { RPTDEL } & 126 \\ \text { RPTDEL } & 127 \\ \text { RPTDEL } & 128 \\ \text { RPTDEL } & 129 \\ \text { RPTDEL } & 130 \\ \text { RPTDEL } & 131 \\ \text { RPTDEL } & 132 \\ \text { RPTDEL } & 133 \\ \text { RPTDEL } & 134 \\ \text { RPTDEL } & 135 \\ \text { RPTDEL } & 136 \\ \text { RPTDEL } & 137 \\ \text { RPTDEL } & 138 \\ \text { RP } & \end{array}$




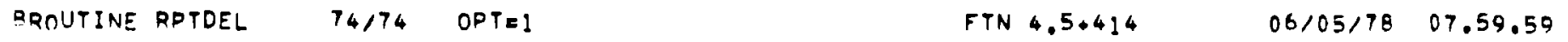

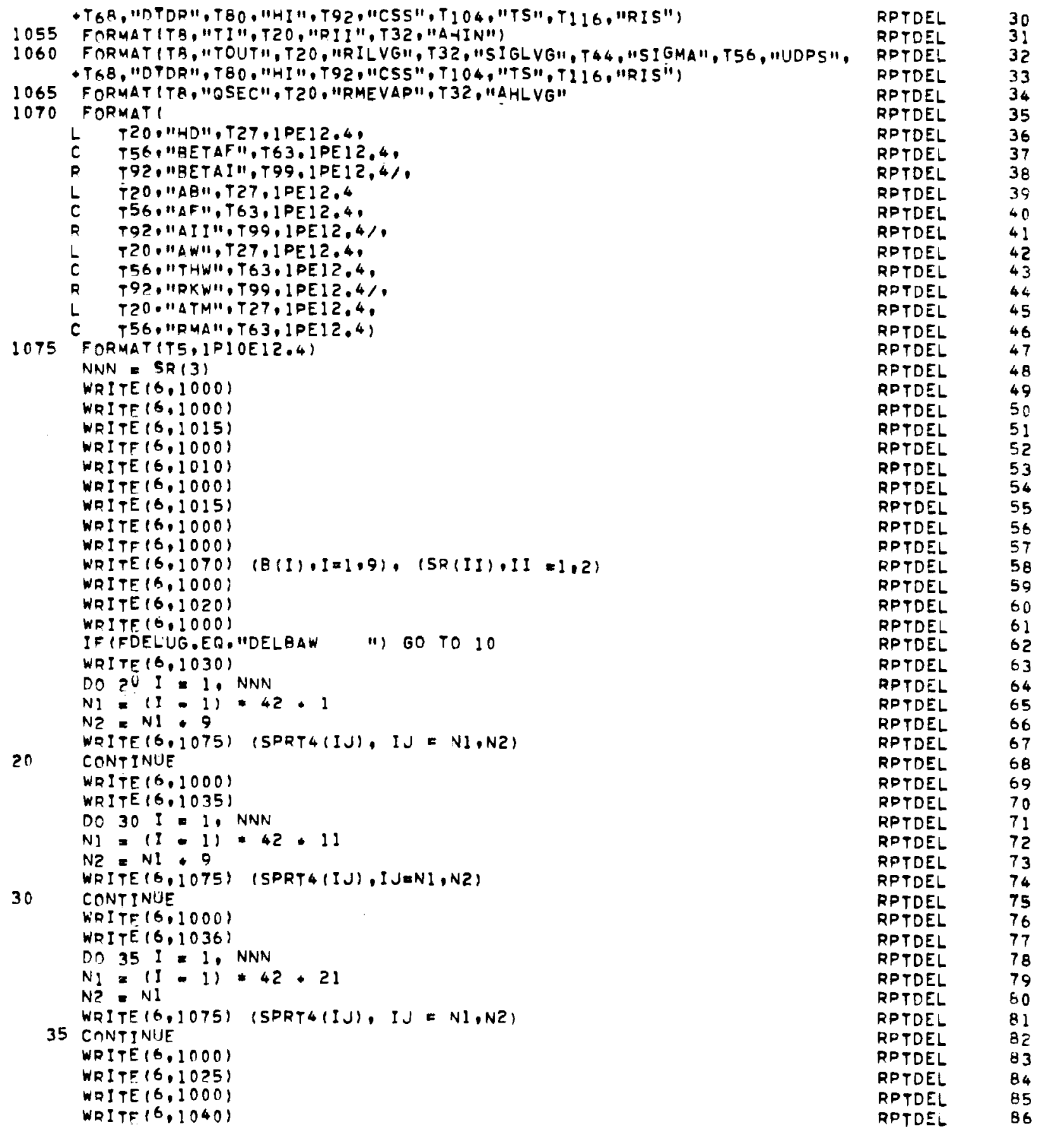


SUBROUTINE RPTDEL

THIS ROUTINE REPORTS THE OUTPUT FROM THE DELUGING ROUTINES (DELUG, UDEL OR DELBAW, SIGBAW)

RPTOEL

HPTDEL

RPTOEL

RPTOEL

RPTOEL

RPTDEL

LOGICAL FIRST,FRST, FXTEMP,FXTVAR,FXTTTO,FXYLNG

COMMON

5 AFTR, ALPHA, ANG (3)

* BDLIM(16)

5 CAPF, CSSPKW, CONF, CONL, COSTl, CAPCHg, CONMAT, CONMAz, CaF

\$ CBJ. CTURB, CDANG, CLUVR, CHAILS, CVM

\$ DFIN. DEEPL, DESVEL, DESVELV, DESVELD, DENSIF

S. EFFP, EFS

\$ IFCR, FIRST, FIXL , FCOS ,FFHX, FXTEMP, FACTOR, FRST ,FACTORD,F SHOT , FDELUG

S ,FIXV,FIXTTD,FXTVAR,FXTTTD, FXTLNG

$\$$.GSEFF

S. HXNDIHDCST, HCD

S, ITMAX, ITMIN

$\$$. JCONS

\$. KCONV, KALEXT

5 . MXEXT

S NTA.NSIDES,NBUNHI

COMMON

$S O D R$

5 .PSIZE, PER, PWCOS, PLANC, PFACT, POMDPL

5 P POHBAF, POHFAN, POHLEC, POHCIR, POHCND, POHSTC, POHSCL

S RRD,REDUCE, REDUCV, ROOFL, RHOUT

S QREJ, QREDUCE, QREDUCV

S. SIGMAG, SAAF, SEGL

\$, TO, TPO (4), TEFF, TLIM, THFIN, TLPRA, TFIX

S,TCD. TW

S.UCS, UWS

\$ WFV, WATCONA, WATCOST

S.VAS(5)

5 ,XOEPA, XW, XO

COMYON/KPRT4/SPRT 4 (6T2),SR (3)

COMMON /HXD/A $(5)$

C

COMMON /CONS/ $B(9)$

BI GCOM

BIGCOM

BIGCOM

BI GCOM

BIGCOM

BIGCOM

BIGCOM

BIGCOM

BIGCOM

BI GCOM

BIGCOM

BI GCOM

BIGCOM

BIGCOM

BIGCOM

BIGCOM

BIGCOM

BIGCOM

BIGCOM

BIGCOM

BIGCOM

BIGCOM

BI GCOM

BI GCOM

BIGCOM

BI GCOM

BIGCOM

BIGCOM

BI GCOM

BIGCOM

RPTDEL

RPTOEL

RPTDEL

RPTDEL

RPTOEL

1000 FORMATIIH,

1005 FORMAT (IHI)

1010 FORMATITSI, "DELUGEO HEAT EXCHANGER DATAMI)

1015 FORMAT (T51.29("-11)

1020 FORMATIT50, "LEADING EDGE OF HEAT EXCHANGER")

1025 FORMAT (T50."TRAILING EDGE OF HEAT EXCHANGER")

1030 FORMATITA,"TAIN", T20, "RIIN", T32, "UDIN", T44,"UDRS",T56, "HOD", T6B, - "DTORIR", TBO,"UDRA", T92,"UDPP", TI 104 , "EFFIN", T116,"HI")

1035 F ORMATITB,"CSR", T20, "DTDR",T32,"TR", T44, "RIR", T56,"TS", T68, "RIS", "TRO, "TFAV", T92, "RIFAV",TI04,"TI",TI16,"RII"I

1036 FORMATITB, "AHIN"I

1040 FORMATIT8,"TOUT",T20, "RILVG",T32,"UDLVG",T44,"UDRS", T56, "HOD",T68, " "DTDRIR", TBO, "UDRA", T92, "UDPP", T I O4, "EFFIN", T116,"HI"I

1045 FORMATITE,"CSR",T20,"DTDR", T32,"TR",T44, "RIR",T56,"TS", T68, "RIS", -TAO, "TFAV",T92, "RIFAV",TI 4 , "OSEC", TI16, "RMEVAP")

1046 FORMATITE, "AHLVG"I)

1050 FORMATITB,"TAIN", T20,"RIIN",T32,"SIGIN", T44,"SIGMA", T56, "UDPS",

RPTDEL
RPTDEL

RPTDEL

RPTDEL

RPTDEL

RPTDEL

RPTDEL

RPTDEL

RPTDEL

RPTOEL

RPTDEL

RPTDEL

RPTDEL

RPTDEL

RPTDEL

RPTDEL 


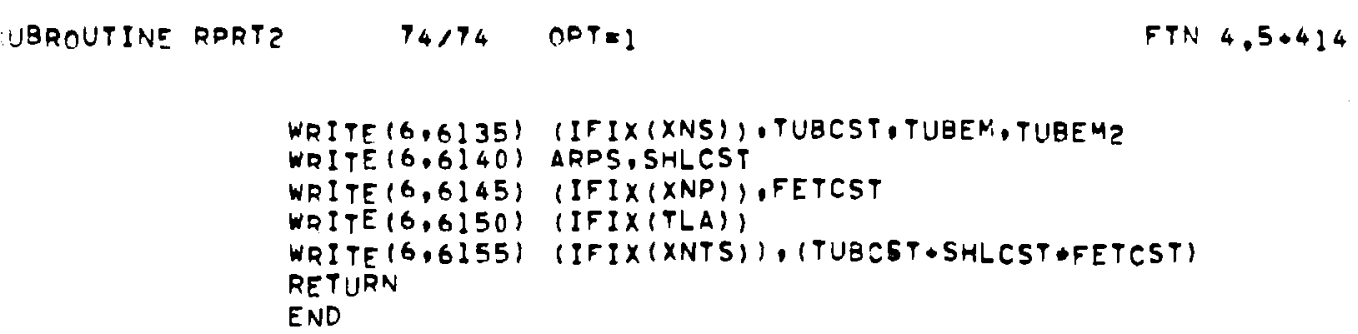

$\begin{array}{ll}\text { RPRTZ } & 59 \\ \text { RDRT2 } & 60 \\ \text { RPRT2 } & 61 \\ \text { RPRT2 } & 62 \\ \text { RPPT2 } & 63 \\ \text { RPRT2 } & 64 \\ \text { RPRT2 } & 65\end{array}$


SUBROUTINE RPTDEL

THIS ROUTINE REPORTS THE OUTPUT FROM THE DELUGING ROUTINES (DELUG, UDEL OR DELBAW, SIGBAW)

RPTDEL

RPTDEL

RPTDEL

RPTDEL

RPTDEL

RPTDEL

LOGICAL' FIRST ,FRST, FXTEMP, FXTVAR,FXTTTD,FXTLNG COMMON

5 AFTR, ALPHA, ANG (3)

* BDLIM(I6)

5 CAPF, CSSPKW, CONF, CONL, COSTl, capchg, conMat, conmaz, caF

5 . CBJ, CTURB, CDANG, CLUVR, CHAILS, CVM

$S$. DFIN, DEEPL, DESVEL, DESVELV, DESVELD, DENSIF

IEFFP, EFS

I FCR, FIRST, FIXL,FCOS, FFHX,FXTEMP, FACTOR, FRST, FACTORD, FSHOT , FDELUG

S ,FIXV,FIXTTD,FXTVAR, FXTTTD, FXTLNG

S.GBEFF

S. HXNPIHPCST,HCD

S. ITMAX, ITMIN

5 , JCONS

S.KEONV, KALEXT

S. MXEXT

5 ,NTA.NSIDES, NBUNHI

COMMON

5 ODR

5 PSIZE, PER, PWCOS, PLANC, PFACT, POMDPL

5 POHBAF, POHFAN, POHLEC, POHCIR, POHCND, POHSTC, POHSCL

\$ RRP,REDUCE, REDUCV, ROOFL, RHOUT

\$ QREJ, QREDUCE, OREDUCV

S SIGMAG, SAAF, SEGL

S TD, PPO $(4)$, TEFF, TLIM, THFIN, TLPRA, TFIX

S, TCD. TW

S.UCS, UWS

5. WFV, WATCONA, WATCOST

S. VAS (5)

5 .XDEPA, XW. XD

COMMON/KPRT4/SPRT4 (6T2), SR (3)

COMMON /HXD/ $(5)$

C COMMON /CONS/ B $(9)$

1000 FORMATIIH,

1005 FORMAT IIHI)

1010 FORMATIT5!. "DELUGED HEAT EXCHANGER DATAII)

1015 FORMAT1T51.29(11-11))

1020 FORMAT (T50, "LEADING EDGE OF HEAT EXCHANGER")

1025 FORMAT IT50, "TRAILING EDGE OF HEAT EXCHANGER"I

1030 FORMAT TT8, "TAIN",T20, "RIIN", T32, "UDIN", T44,"UDRS", T56, "HOD", T6B, + "DTDRIR", T80, "UDRA", T92, "UDPP", TI O4, "EFFIN", TI16, "HI")

1035 FORMATITB,"CSR",T20, "DTDR",T32,"TR",T44, "RIR",T56,"TS", T68, "RIS", -TAO, "TFAV", T92, "RIFAV", TI04, "TI", TI16,"RII"I

1036 FORMAT (TB, "AHIN")

1040 FORMATITB," TOUT", T20, "RILVG",T32,"UDLVG", T44,"UDRS", T56,"HOD", T68, +"DTORIR", T80, "UDRA", T92, "UDPP", T 104 , "EFFIN", T 116, "HI")

1045 FORMATPTE, "CSR",TZ0, "DTDR", T32, "TR", T44, "RIR", T56, "TS", T68, "RIS", +TBO, "TFAV", T92, "RIFAV", T104,"OSEC", T116, "RMEVAPH)

1046 FORMAT (TB, "AHLVG")

1050 FORMATITB,"TAIN", T20, "RIIN", T32, "SIGIN", T44,"SIGMA", T56, "UDPS",

BI GCOM

BI GCOM

BIGCOM

BI GCOM

BI GCOM

BIGCOM

BI GCOM

BI GCOM

BIGCOM

BI GCOM

BI GCOM

BIGCOM

BISCOM

BI GCOM

BI GCOM

BI GCOM

BI GCOM

BIGCOM

BI GCOM

BIGCOM

BI GCOM

BI GCOM

BI GCDM

BIGCOM

BIGCOM

BI GCOM

BI GCOM

BI GCOM

BIGCOM

BI GCOM

RPTDEL

RPTDEL

RPTDEL

RPTDEL

RPTDEL

RPTDEL

RPTDEL

RPTDEL

RPTDEL

RPTDEL

RPTDEL

RPTDEL

RPTDEL

RPTDEL

RPTDEL

RPTDEL

RPTDEL

RPTDEL

RPTDEL

RPTDEL

RPTOEL 
RROUTINE RPTDEL T4/74 OPTEI FTN $4.5+414 \quad 06105 / 78 \quad 07.59 .59$

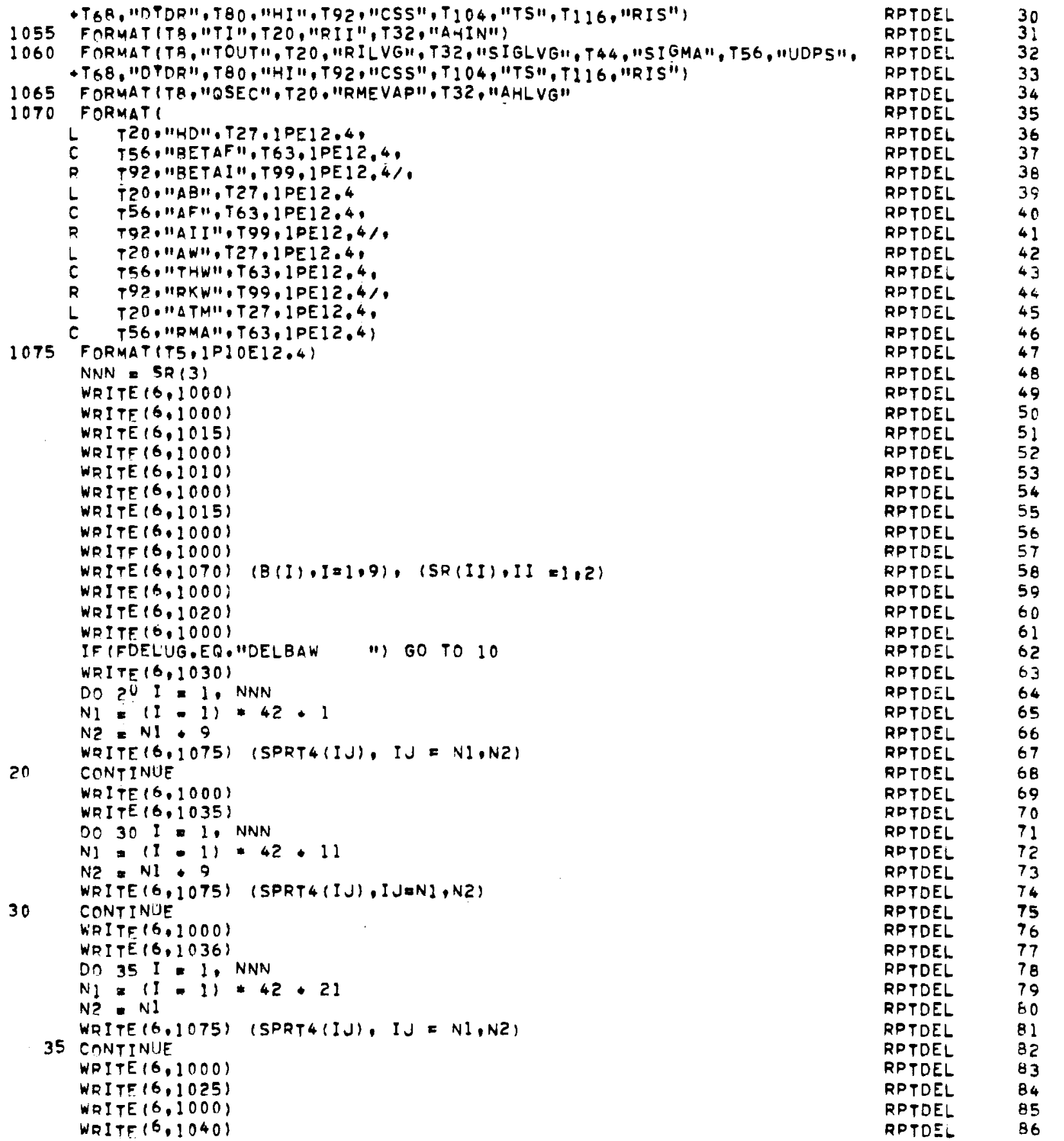


DO $401 \div 1$. NNN

$N_{1}=(1 \cdot 1) \cdot 42 \cdot 22$

$N 2=N !+Q$

WRITE (6,1075) (SPRT4 IJ), IJ = NI,N2)

40 CONTINUE

WRITE $(6,1000)$

WRIYE (6.1045)

DO $501=1$. NNN

$N !=(1-1) \cdot 42+32$

$N 2=N 1 * 9$

WRITF (6,1075) (SPRT4(IJ), IJ $=N$ I,N2)

50 CONTINUE

WRI TE $(6.1000)$

WRITE $(6,1046)$

DO $551=1$. NNN

$\left.N_{1}=\mid 1 \cdot 1\right\} \cdot 42 \cdot 42$

$N 2=N 1$

WRITE (6,1075) (SPRY4 (IJ), IJ = NI,N2)

55 CONTINUE

GO TO 100

10

WRITE 16,$\rfloor 050)$

DO 6 ก I $=1$. NNN

$N]=(1 \cdot 1)=26 \cdot 1$

$N 2=N ! * 9$

WDITE (6,1075) (SPRT4 (IJ),IJEN1,N2)

60 CONTFNIJE

WRITE $(6,1000)$

WRITE $(6,1055)$

DO $701=1$, NNN

NI $=11 \cdot 1) * 26 \cdot 11$

N2 $=N 1 \cdot 2$

WRITE $(6,1075)$ (SPRT (I J), I JaNI, N2)

70 CONTINUE

WRITE $(6,1000)$

WRITE $(6,1025)$

WRITE $(6,1000)$

WRITE (6,1060)

DO QO $1: 1$, NNN

$N 1=11 \cdot 11 \cdot 26+14$

$N 2=N 1 * Q$

WRITE (6,1075) (SPRT 4 (IJ),IJ=NI,N2)

BO CONTTNUE

WRITE $(6.1000)$

WRITF $(6,1065)$

$00001=1$. NNN

$N 1=(1 \cdot 1) \cdot 26 \cdot 24$

$N 2 \div N 1 \cdot 2$

WRITE (6,1075) (SPRT $4(I J), ~ I J=N I, N 2)$

90 CONTINUE

100 CONTINUE

RETURN

END

$\begin{array}{ll}\text { RPTDEL } & 87 \\ \text { RPTDEL } & 88 \\ \text { RPTDEL } & 89 \\ \text { RPTDEL } & 90 \\ \text { RPTDEL } & 91 \\ \text { RPTDEL } & 92 \\ \text { RPTDEL } & 93 \\ \text { RPTDEL } & 94 \\ \text { RPTDEL } & 95 \\ \text { RPTDEL } & 96 \\ \text { RPTDEL } & 97 \\ \text { RPTDEL } & 98 \\ \text { RPTDEL } & 99 \\ \text { RPTDEL } & 100 \\ \text { RPTDEL } & 101 \\ \text { RPTDEL } & 102 \\ \text { RPTDEL } & 103 \\ \text { RPTDEL } & 104 \\ \text { RPTDEL } & 105 \\ \text { RPTDEL } & 106 \\ \text { RPTDEL } & 107 \\ \text { RPTDEL } & 108 \\ \text { RPTDEL } & 109 \\ \text { RPTDEL } & 110 \\ \text { RPTDEL } & 111 \\ \text { RPTDEL } & 112 \\ \text { RPTDEL } & 113 \\ \text { RPTDEL } & 114 \\ \text { RPTDEL } & 115 \\ \text { RPTDEL } & 116 \\ \text { RPTDEL } & 117 \\ \text { RPTDEL } & 118 \\ \text { RPTDEL } & 119 \\ \text { RPTDEL } & 120 \\ \text { RPTDEL } & 121 \\ \text { RPTDEL } & 122 \\ \text { RPTDEL } & 123 \\ \text { RPTDEL } & 124 \\ \text { RPTDEL } & 125 \\ \text { RPTDEL } & 126 \\ \text { RPTDEL } & 127 \\ \text { RPTDEL } & 128 \\ \text { RPTDEL } & 129 \\ \text { RPTDEL } & 130 \\ \text { RPTDEL } & 131 \\ \text { RPTDEL } & 132 \\ \text { RPTDEL } & 133 \\ \text { RPTDEL } & 134 \\ \text { RPTDEL } & 135 \\ \text { RPTDEL } & 136 \\ \text { RPTDEL } & 137 \\ \text { RPTDEL } & 138 \\ \text { RDTD } & \end{array}$




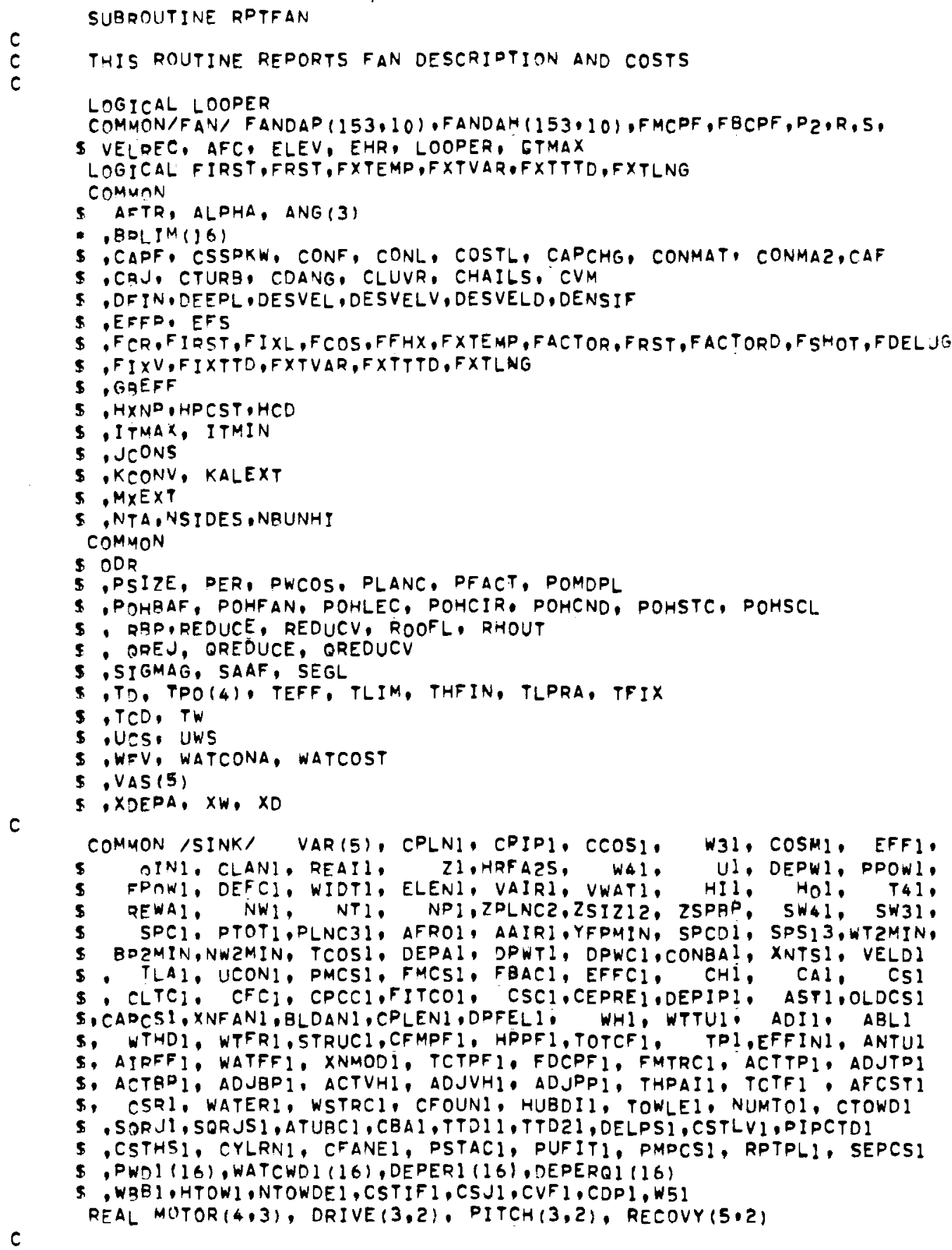

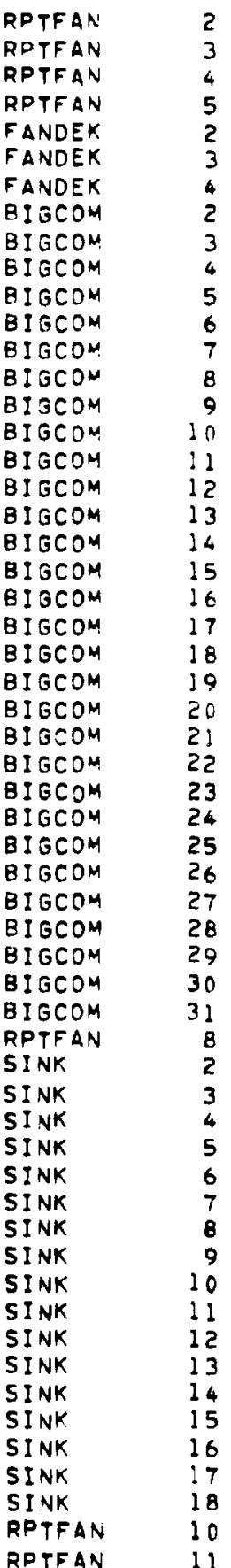


c

DATA (IMOTOR $(I, K), I=1,4), K=1,3)$ )

" "TOTALLY EN", "CLOSED SQU", "I RREL CAGE"," MOTOR

- "EXPLOSION ", "PROOF SOUI", "RREL CAGE ", "MOTOR

* "TWO SPEED "."SQuiRREL C", "AGE

","

"1

DATA (IDRIVE $(I, K), I=1,3), K=1,2)$ )

" "SPIRAL BeV", "El gear or","IVe ",

" "V-BELT DRI","VE "," "/

DATA (IPITCH(I,K),I=1,3),K=1,2),

",

" "manual Dit", "Ch blades ","

- "auto-varia", "ble pitch ","Blades ",

DATA (IRECOVY (I,K), I $=1,5), K=1,2\}$ )

" "FANS NOT E", "QUIPPED WI","TH VELOCIT","Y RECOVERY",

- "DIFFUSERS",

" "fans equipu,"ped with V","Elocity re", "covery dif",

$$
\text { * "fusers "/ }
$$

1000 FORMATIIH )

1005 FORMAT (1HI)

1010 FORMAT (T52,"F A N S")

1020 FORMAT (T51,"10........"I)

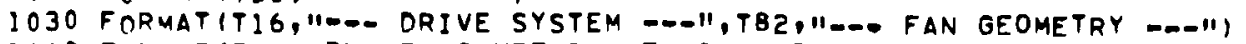

1040 FORMAT ITE4,"BLADE DIAMETER ",F6.2," FEET"

1050 FORMAT 1T87."BLADE ANGLE ",F6.2," DEGREES"

1060 F RRMAT (T7B,"NUMBER OF BLADES/FAN ", F6.2)

1070 FORMAT (TB4,"NUMBER OF FANS ",F6.01

c

1080 FORMATITI7,"-.. FAN COSTS -..", TTB,"-. DESIGN CONDITIONS -."

1085 FORMAT (TI00."(ACTUAL)",T122,"(ADJUSTED)"

1 O90 FORMAT TT73."VOLUMETRIC FLOW RATE/FAN ",FI2.0." CU FT/MIN")

1100 FORMATITI6."BLADE AND HUB COST/FAN S1, F10,0,T71.

* "total volumetric flow rate ",Fl2.0." CU FT/MIN"I)

1120 FORMAT TT24."IMOTOR COST/FAN \$", F10.0.T70.

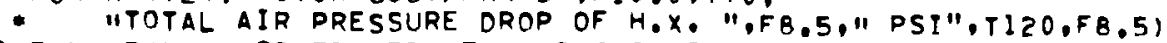

1130 FARMATITIO, "SPEED REDUCER DRIVE COST/FAN \$1, F10.0.T69,

* "PRESSURE DROP ACROSS H.X. BUNDLE ",FB.5," DSI", TI20,FB.5I

1140 FORMATIT24."ISTACK COSTIFAN S",F10,0.T72,

* "Non-REcoverable VELOCITY HEAD ",FB.5," PSI", I120,FB.51

1145 FORMATTT19."ELECTRICAL COST/FAN S",F 10.0

\$.TR3."HORSEPOWER/FAN",F $13,0 . "$ HP",T $116, F 12.01$

1150 FORMATITT9,

* "FAN POWER REQUIREMENTS ",FB,0," hP")

1:90 FORMATIT2,"*** ANNUAL OPERATIONAL COSTS $\$ 1, F 10.01$

1200 FORMAT $(T 37,5 A 10)$

1210 FORMATI

R T86,"HUB OIAMETER ",F6.2," FEET")

WRITE $(5,1000)$

WRITE $(6,1000)$

WRITE $(6,1020)$

WRITE $(6,1010)$

WQITE $(h, 1020)$

WR.ITE $(6,1000)$

IF (VELAEC.EO.0) WRITE $(6,1200)$ (RECOVY $(I, 1), I=1,5)$

IF (VELREC.EQ.1) WRITE $(6,1200)$ (RECOVY $(I, 2), I=1,5)$

WDITE $(6,1000)$

WRITE $(6,1030)$

WRITE $(6,1000)$

WPITE $(6.1210)$ HUBDII

$\begin{array}{ll}\text { RPTFAN } & 12 \\ \text { RPTFAN } & 13 \\ \text { RPTFAN } & 14 \\ \text { RPTFAN } & 15 \\ \text { RPTFAN } & 16 \\ \text { RPTFAN } & 17 \\ \text { RPTFAN } & 18 \\ \text { RPTFAN } & 19 \\ \text { RPTFAN } & 20 \\ \text { RPTFAN } & 21 \\ \text { RPTFAN } & 22 \\ \text { RPTFAN } & 23 \\ \text { RPTFAN } & 24 \\ \text { RPTFAN } & 25 \\ \text { RPTFAN } & 26 \\ \text { RPTFAN } & 27 \\ \text { RPTFAN } & 28 \\ \text { RPTFAN } & 29 \\ \text { RPTFAN } & 30 \\ \text { RPTFAN } & 31 \\ \text { RPTFAN } & 32 \\ \text { RPTFAN } & 33 \\ \text { RPTFAN } & 34 \\ \text { RPTFAN } & 35 \\ \text { RPTFAN } & 36 \\ \text { RPTFAN } & 37 \\ \text { RPTFAN } & 38 \\ \text { RPTFAN } & 39 \\ \text { RPTFAN } & 40 \\ \text { RPTFAN } & 41 \\ \text { RPTFAN } & 42 \\ \text { RPTFAN } & 43 \\ \text { RPTFAN } & 44 \\ \text { RPTFAN } & 45 \\ \text { RPTFAN } & 46 \\ \text { RPTFAN } & 47 \\ \text { RPTFAN } & 48 \\ \text { RPTFAN } & 49 \\ \text { RPTFAN } & 50 \\ \text { RPTFAN } & 51 \\ \text { RPTFAN } & 52 \\ \text { RPTFAN } & 53 \\ \text { RPTFAN } & 54 \\ \text { RPTFAN } & 55 \\ \text { RPTFAN } & 56 \\ \text { RPTFAN } & 57 \\ \text { RPTFAN } & 58 \\ \text { RPTFAN } & 59 \\ \text { RPTFAN } & 60 \\ \text { RPTFAN } & 61 \\ \text { RPTFAN } & 62 \\ \text { RPTFAN } & 63 \\ \text { RPTFAN } & 64 \\ \text { RPTFAN } & 65 \\ \text { RPTFAN } & 66 \\ \text { RPTFAN } & 67 \\ \text { RPTFAN } & 68 \\ \text { RAAAAAAAA } & \end{array}$




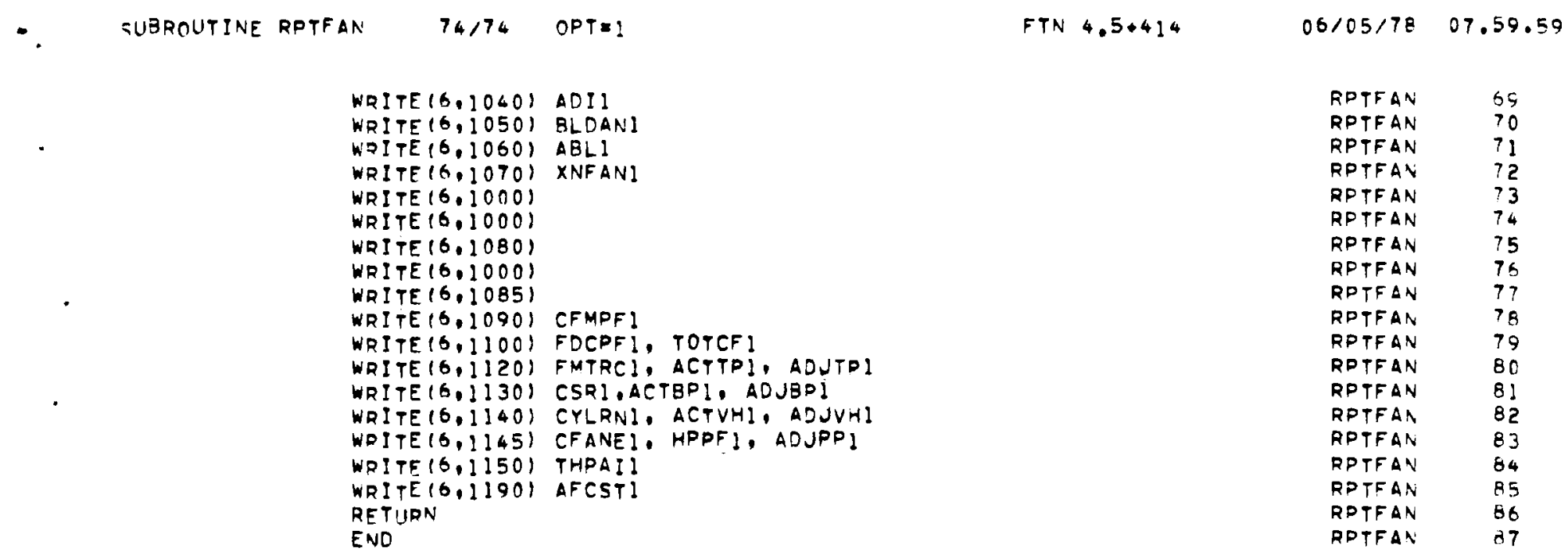


THIS ROUTINE REPORTS HEAT EXCHANGER DESIGN, OPERATING CONDITIONS AND COST

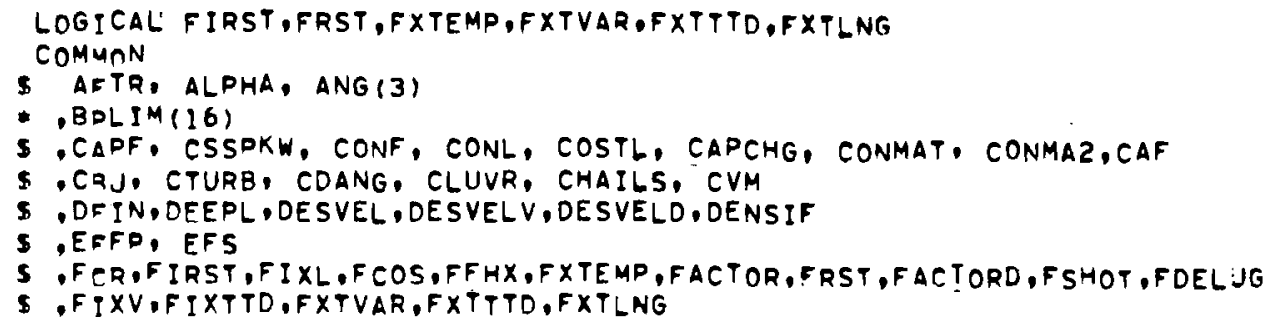

COMMON /TINE/ XNS, TLA, XNTS, ARPS, TTDZOE, LINOR, XNP, XQUALY COMMON /HXO/ AI, ATOT, SFA,RFA,ACS

COMMON /ESCAL/ BSECF

COMMON /KPTSUP, DUM(2017), THXINI, DUMZ (3)

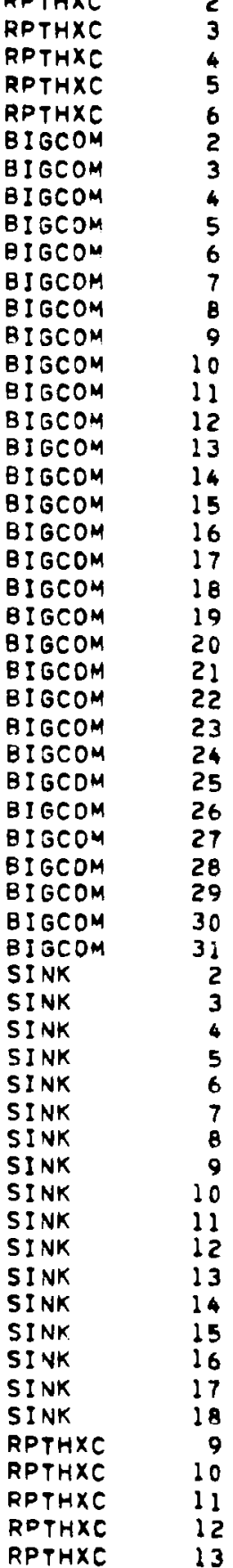




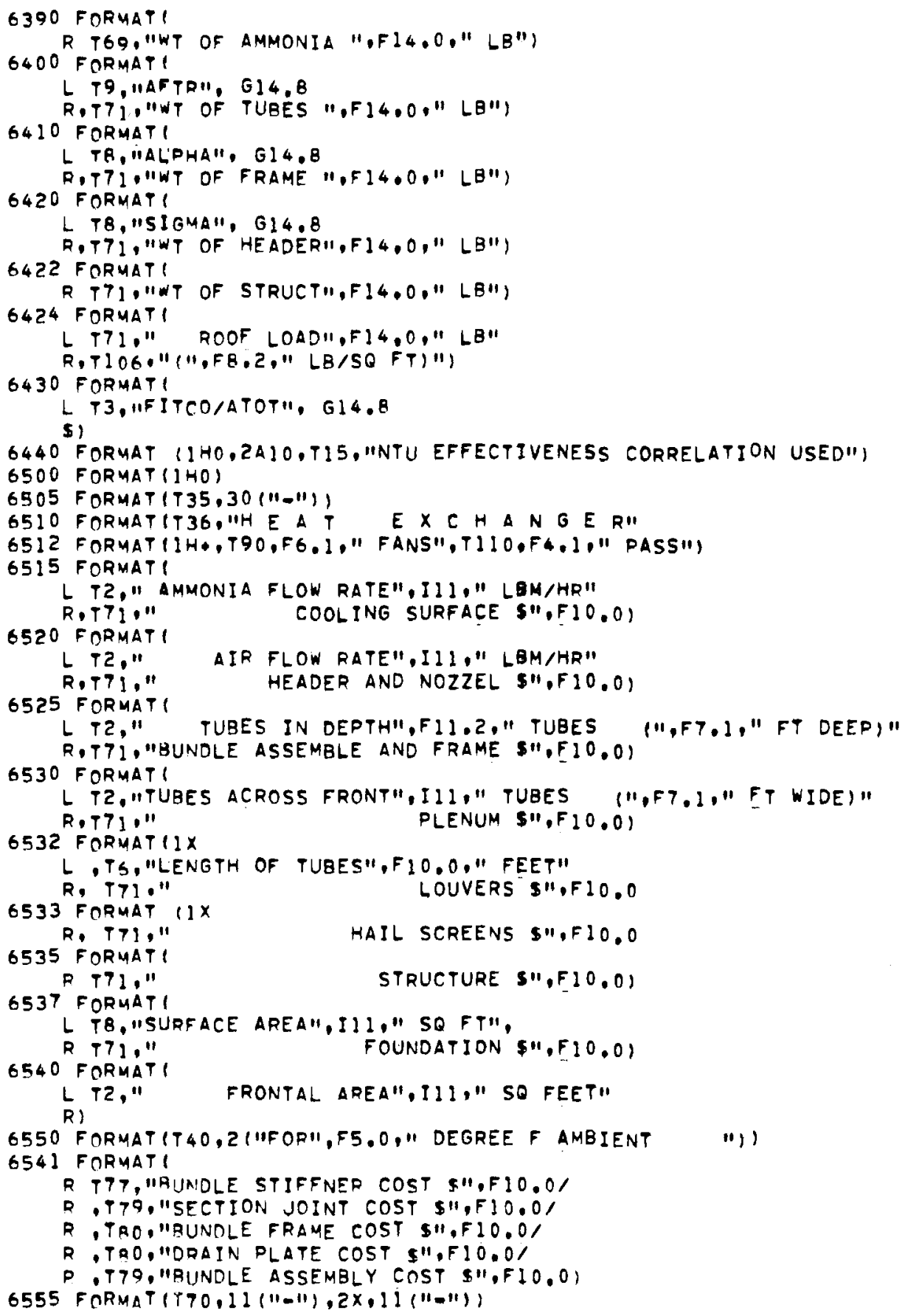




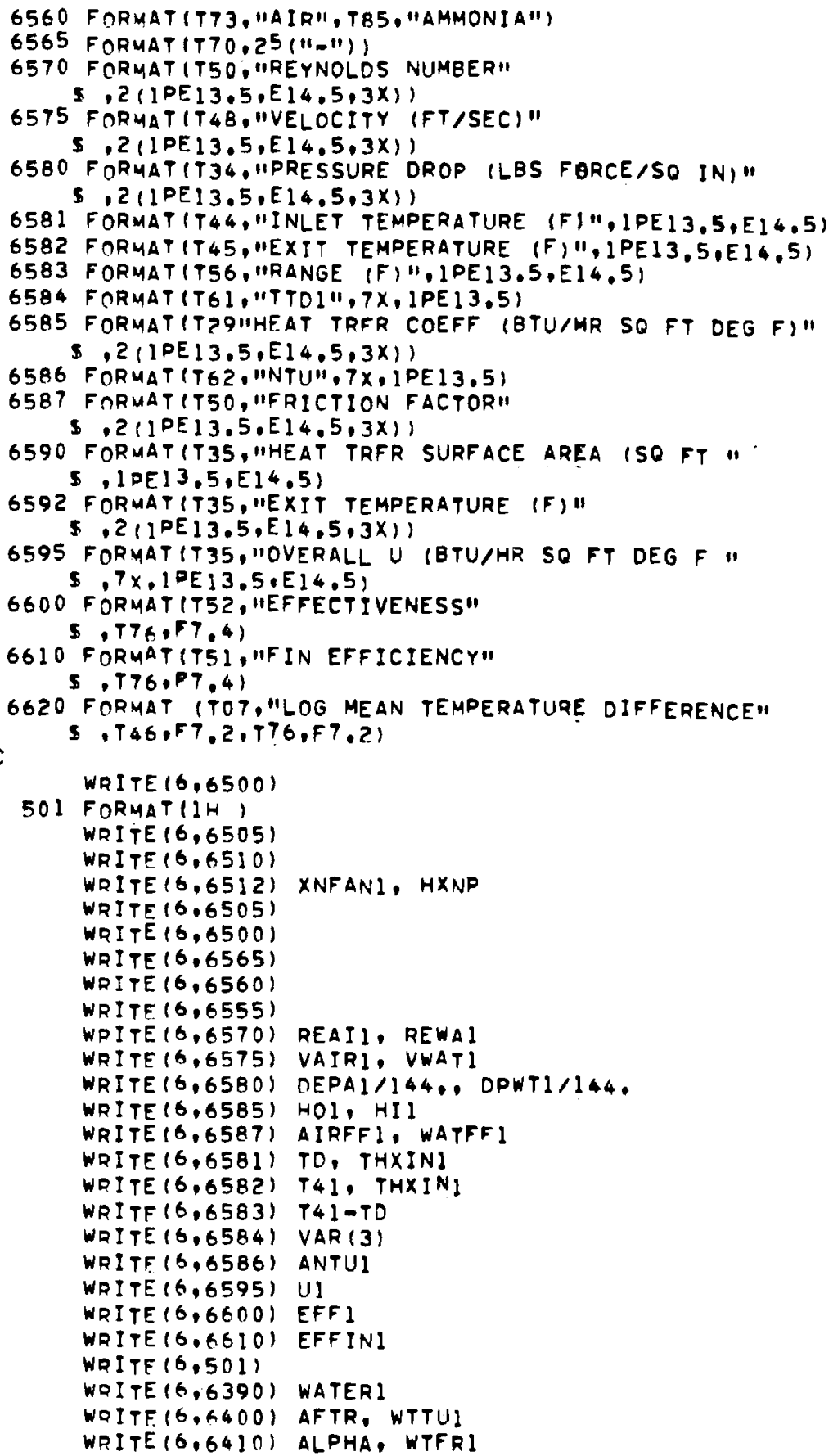

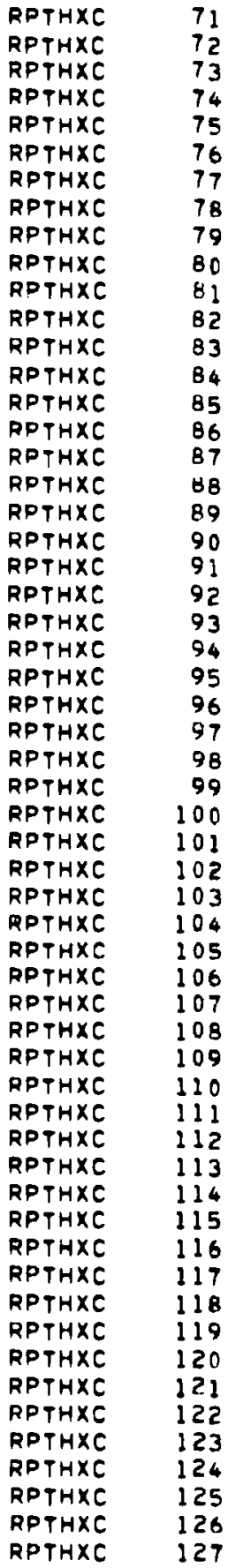




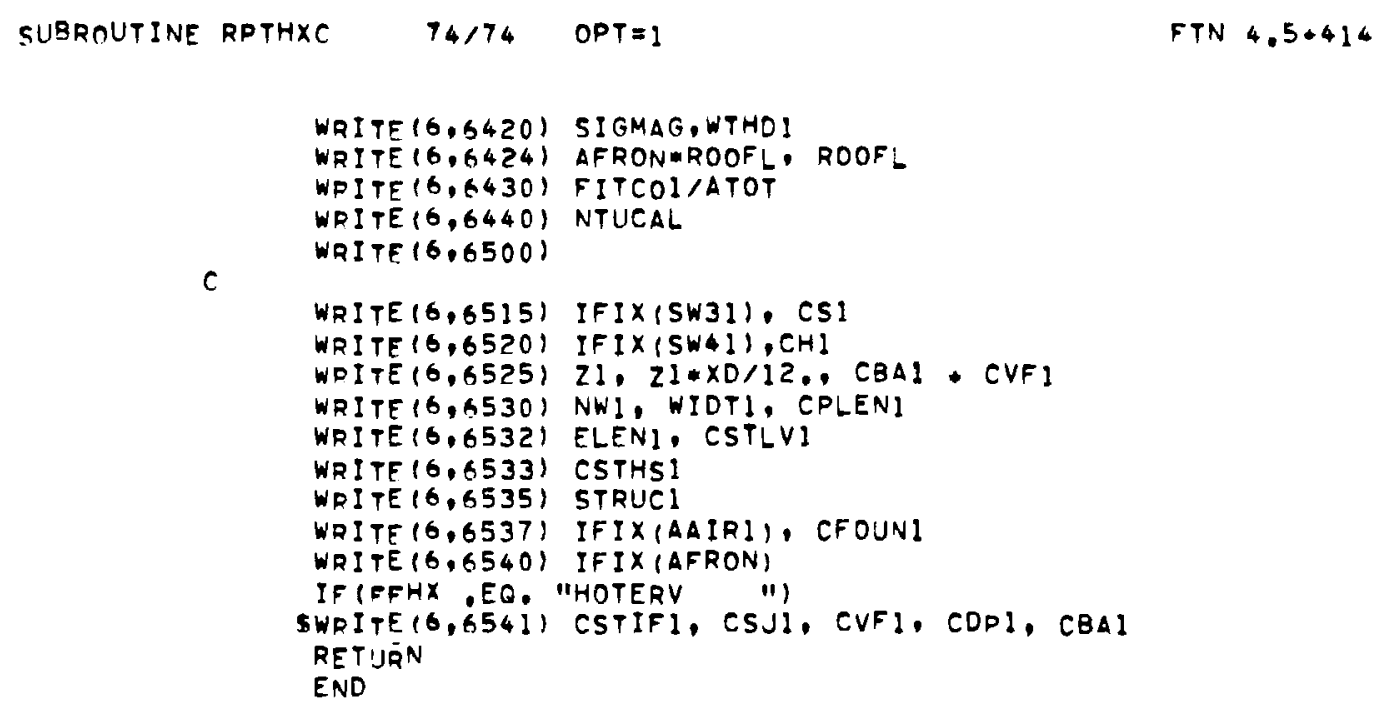

$\begin{array}{ll}\text { RPTHXC } & 128 \\ \text { RPTHXC } & 129 \\ \text { RPTHXC } & 130 \\ \text { RPTHXC } & 131 \\ \text { RPTHXC } & 132 \\ \text { RPTHXC } & 133 \\ \text { RPTHXC } & 134 \\ \text { RPTHXC } & 135 \\ \text { RPTHXC } & 136 \\ \text { RPTHXC } & 137 \\ \text { RPTHXC } & 138 \\ \text { RPTHXC } & 139 \\ \text { RPTHXC } & 140 \\ \text { RPTHXC } & 141 \\ \text { RPTHXC } & 142 \\ \text { RPTHXC } & 143 \\ \text { RPTHXC } & 144 \\ \text { RPTHXC } & 145 \\ \text { RPTHXC } & 146\end{array}$




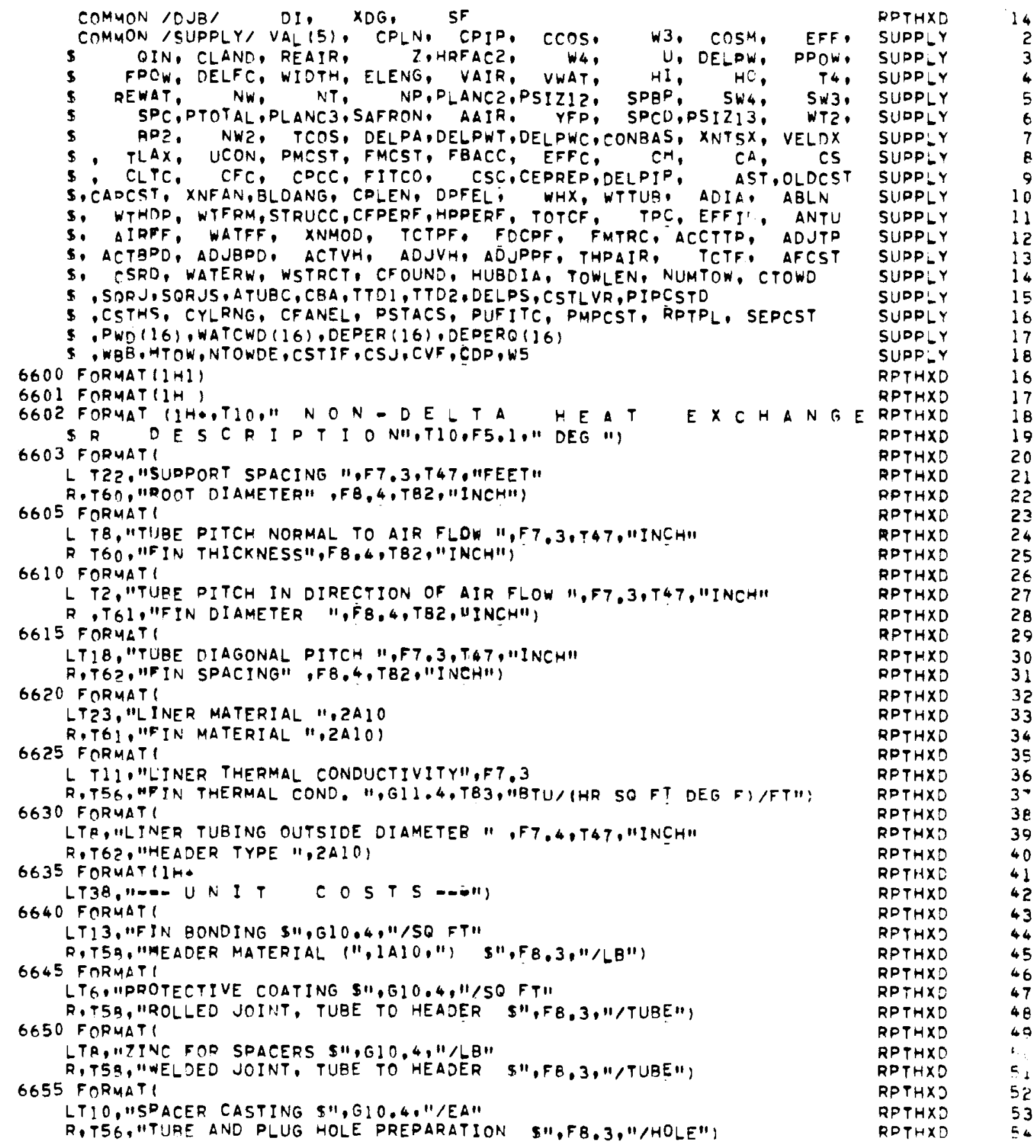




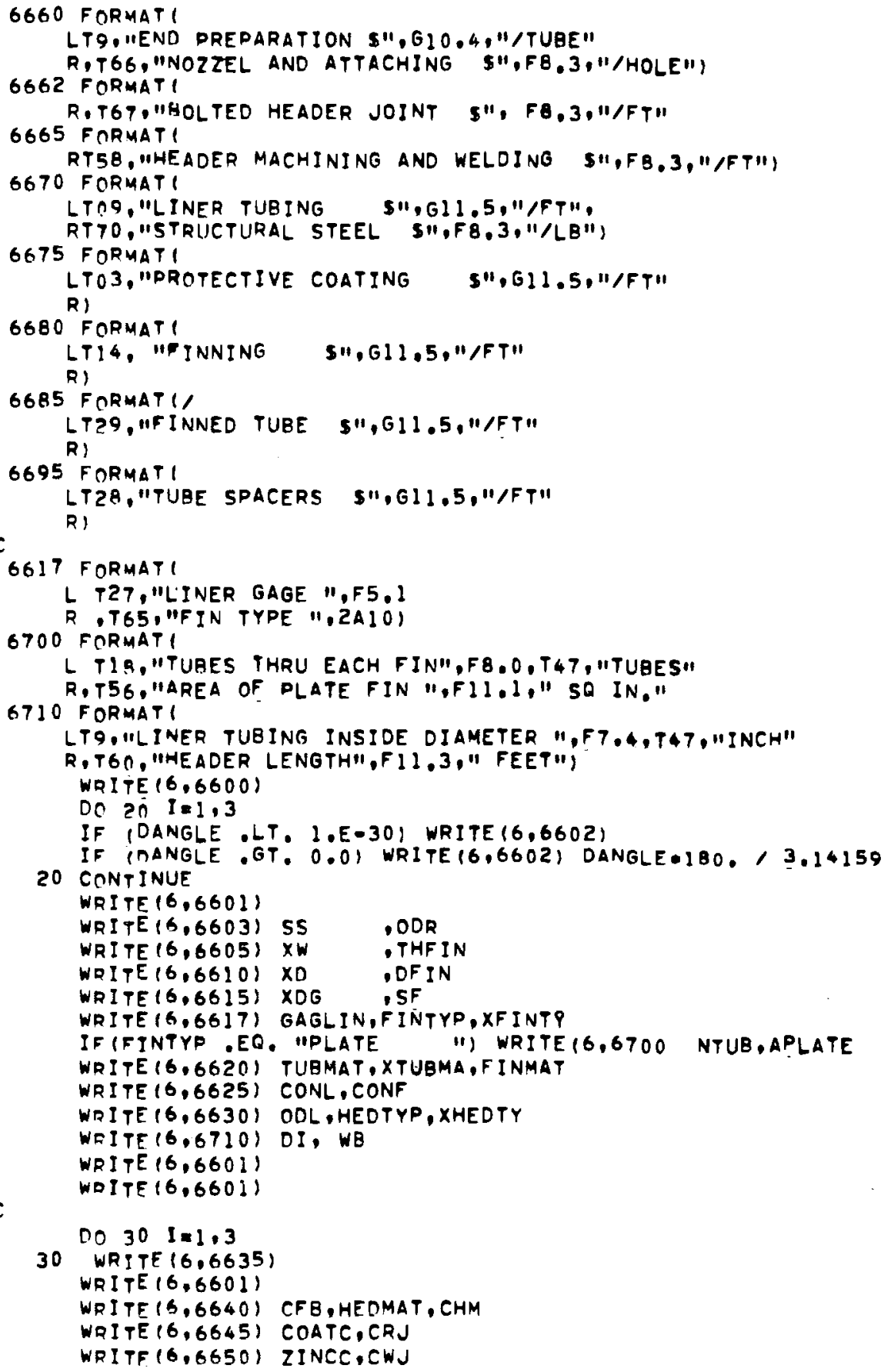

\begin{tabular}{ll} 
RPTHXD & 55 \\
RPTHXD & 56 \\
RPTHXD & 57 \\
RPTHXD & 58 \\
RPTHXD & 59 \\
RPTHXD & 60 \\
RPTHXD & 61 \\
RPTHXD & 62 \\
RPTHXD & 63 \\
RPTHXD & 64 \\
RPTHXD & 65 \\
RPTHXD & 66 \\
RPTHXD & 67 \\
RPTHXD & 68 \\
RPTHXD & 69 \\
RPTHXD & 70 \\
RPTHXD & 71 \\
RPTHXD & 72 \\
RPTHXD & 73 \\
RPTHXD & 74 \\
RPTHXD & 75 \\
RPTHXD & 76 \\
RPTHXD & 77 \\
RPTHXD & 78 \\
RPTHXD & 79 \\
RPTHXD & 80 \\
RPTHXD & 81 \\
RPTHXD & 82 \\
RPTHXD & 83 \\
RPTHXD & 84 \\
RPTHXD & 85 \\
RPTHXD & 86 \\
RPTHXD & 87 \\
RPTHXD & 88 \\
RPTHXD & 89 \\
RPTHXD & 90 \\
RPTHXD & 91 \\
RPTHXD & 92 \\
RPTHXD & 93 \\
RPTHXD & 94 \\
RPTHXD & 95 \\
RPTHXD & 96 \\
RPTHXD & 97 \\
RPTHXD & 98 \\
RPTHXD & 99 \\
RPTHXD & 100 \\
RPTHXD & 109 \\
RPTHXD & 102 \\
RPTHXD & 103 \\
RPTHXD & 104 \\
RPTHXD & 105 \\
RPTHXD & 106 \\
RPTHXD & 107 \\
RPTHXD & 108 \\
RPTHXD & 109 \\
RPTHXD & 110 \\
RPTHXD & 111 \\
& \\
RP & 100 \\
\hline
\end{tabular}


SUSROUTINE RDTHXE $74 / 74 \quad$ OPTEI

WRITE $(6.6655)$ CASTC,CHH

WDITE $(6,6660)$ EPREPC,CN

WRITE $(6.6662)$ CBJ

WRITE $(6,6665)$ CMW

WPITE $(0,6670)$ CLTCI, CST

WRITE $(6.6675)$ CPCCI

WRITE $(6,6680)$ CFEI

WDITE (6.6685) FITCOI

C

WRITE $(6.6695)$ CSCI

RETURN

END
FTN $4.5+414$

$06 / 05 / 78$

07.59 .59

$\begin{array}{ll}\text { RPTHXD } & 112 \\ \text { RPTHXD } & 113 \\ \text { RPTHXD } & 114 \\ \text { RPTHXD } & 115 \\ \text { RPTHXD } & 116 \\ \text { RPTHXD } & 117 \\ \text { RPTHXD } & 118 \\ \text { RPTHXD } & 119 \\ \text { RPTHXD } & 120 \\ \text { RDTHXD } & 121 \\ \text { RPTHXD } & 122 \\ \text { RPTHXD } & 123\end{array}$


C

SUBROHTINE RPTRETICTOWDI, PF, PSTACI, PUFITI, PUMPCI, RPTPLI. S SEPC̄SL,DIPCTDII

5 DELLI, DESVEI, DIARDI, DIARTI, DOTMAI, DYNHDI

S. HEDRI $(200)$

s. Jasave

S. KRSAVE

S. PIPCSI

5. QHEDRI 200$)$. QRLCS

S. RDIAI (200), RPCSTI (200), RRCSTI (200), RTCSTI $(200), R B F C S I, R C O S T I$

5. RRDIAI (200), RQPCSI (200), RORCSI (200), ROTCSI $(200)$, REJCSI

S, RELCS1, RFLGCI, RQCOSI, RTDCSI

5. TOWDII. TRQCOI. TRTDCI

COMHON /KKRET/

S RPDIAI (200), RVDIAL $(200)$, LRSAVE

S.PHEORI $(200)$. VHEDRI $(200)$

S.RPRCSTI (200), RVRCSTI (200)

S.RPTCSTI (200). RVTCSTI (200)

S.PRLCSTI, VRLCSTI

S.PPDCSTI (200), RVPCSTI (200)

S.RPCOSTI, RVCOSTI

S.INT.NBUN

203 FORMATIIH $, 14 x, I 2,1 X, 7 F 10.1)$

204 FORMATI//140X,"TOTAL COST OF CIRCULATION RETURN PIPING SH,F5.O. S" $10 * 311 / 1 / 11$

205 FORMAT $/ / 25 \times$, "HORIZONTAL OUADRANT RETURN PIPING" 5

$1 / 42 X, " C O S T$ IN IO

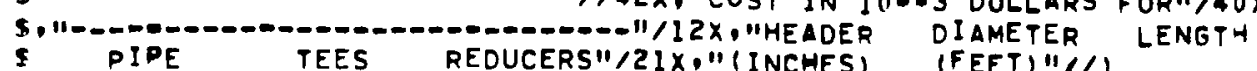

208 FORMAT $/ / / / 42 X$, "TOTAL COST OF QUADRANT RETURN HEADERS SW.F5.0, S"1 $10 * 311 / 1 / 1)$

209 FORMATI/25X, "TOWER RETURN PIPING"
5

$\sin x$

PIPING"

RPTRET "DIAMETER OF PIPE FROM CIRCULATION RPTRE
RPTRET

RPTRET

RPTRET

RPTRET

RPTRET

RPTRET

RPTRET

RPTRET

RPTRET

RPTRET

RPTRET

RPTRET

RPTRET

RPTRET

RPTRET

RPTRET

RPTRET

RPTRET

RPTRET

RPTRET

RPTRET

RPTRET

RP TRET

RPTRET

RPTRET

RPTRET

RPTRET

RPTRET

RPTRET

RPTRET

RPTRET

RPTRET

RPTRET

RPTRET

RPTRET

S"/1OX."HEADER TO TOWER DISTRIBUTION HEADER",FT.0," INCHES"/IOX, "DI RPTRET SAMETER OF TOWER DISTRIBUTION PIPE",F7.0, "INEHESN/IIOX, MOST OF JI RPTRET SSTRIRIITION PIPING PER TOWER \$",F5.0," $10 * 31 / 1 / 40 \times, " 10 T A L$ COST O RPTRET SF TOWER DISTRIBUTION PIPING \$11,F5.0.11 $10=311 / 1 / 1$

211 FORMAT $110 X$, "LIQUID DESIGN VELOCITY",F5,0," FOS"/

5 IOX, "MASS FLOW RATE", IPE 10.3," LBM/HR",

$\$$ I $10 \times$ "RETURN PIPING PRESSURE DRQP",OPF 7. $1, "$ PSF"/

$\$$ IOX. "RETURN PIPING DYNAMIC HEAD",F7.1, "FEET"1/1/1

5 47."TOTAL RETURN PIPING SYSTEM COST $\$ 11.66 .0 .1110 * 311111$ 213 FORMAT (IHI)

214 FORMAT (1H, $13 X, I 2,1 \times, 7 F 10,1)$

215 FORMAT! $/ 10 X$, "COST OF RETURN HEADERS PER OUADRANT SH,F5.1. SII $10 * 311)$

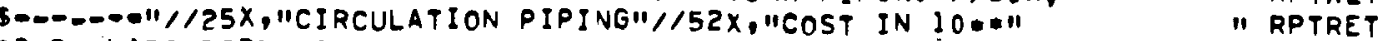

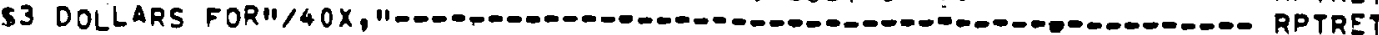
\$- - " / / $2 X, " H E A D E R$ DIAMETER LENGTH PIDE TEES REDUCE RPTRET SRS EXPANSION FLANGES"/ZIX,"(INCHES) (FEET) ",33X, "JOINT (1) (3 RPTRET S) $11 / 1$
RPTRET

RPTRET

RPTRET

RPTRET

RPTRET

RPTRET

RPTRET

RPTRET

RPTRET$$
\text { Rt }
$$

RPTRET

2

3

4 
c

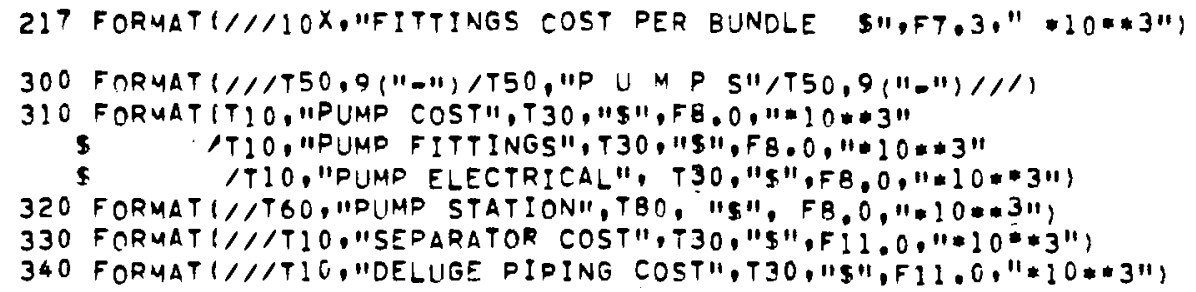

RPTRET

RPTRET

RPTRET

RPTRET

RPTRET

RPTRET

RPTRET

RPTRET

RPTRET

RPTRET

RPTRET

RPTRET

RPTRET

RPTRET

RPTRET

RPTRET

RPTRET

RPTRET

RPTRET

RPTRET

RPTRET

RPTRET

RPTRET

RPTRET

RPTRET

RPTRET

RDTRET

RPTRET

RPTRET

RPTRET

RPTRET

RPTRET

RPTRET

RPTRET

RDTRET

RPTRET

RPTRET

RPTRET

RPTRET

RPTRET

RPTRET

PPTRET

RPTRET

RPTRET

RPTRET

RPTRET

RPTRET

RPTRET

RPTRET

RPTRET

RPTRET

RPTRET

RPTRET

RPTRET

RPTRET

RPTRET

RPTRET 
506 CONTINUE

600 CONTINUE

WDITE $(6,537)$ NBUN

517 FORMAT $/ / 110 X$, "NUMBER OF BUNOLES PER QUADRANT", IIO)

WRITE $(6,217)$ RBFCSI*DF

WRITE $(6,215)$ RQCOSI*PF

WRITE (6,208) TROCOI HPF

WRITE $(6,209)$ CTOWDI, DIARDI, DIARTI, RTDCSI $\#$ PF, TRTDCI $A P F$

WRITE $\{6,211)$ DESVE], DOTMAI, DELLI, DYNHDI, PIPCS I

WRITE $(6,300)$

WRITE (6,310) (PUMPCI-PUFITI) $*$ PF, PUFITI $\#$ PF, RPTPLI

WRITE $(6,320)$ (PUMPCI $\# P F$ ) RPTPLI

WRITE $(6,330)$ SEPCSI

WRITE $(6,340)$ PIPCTD

RETURN

END

$\begin{array}{ll}\text { RPTRET } & 116 \\ \text { RPTRET } & 117 \\ \text { RPTRET } & 118 \\ \text { RPTRET } & 119 \\ \text { RPTRET } & 120 \\ \text { RPTRET } & 121 \\ \text { RPTRET } & 122 \\ \text { RPTRET } & 123 \\ \text { RPTRET } & 124 \\ \text { RPTRET } & 125 \\ \text { RPTRET } & 126 \\ \text { RPTRET } & 127 \\ \text { RPTRET } & 128 \\ \text { RPTRET } & 129 \\ \text { RPTRET } & 130 \\ \text { RPTRET } & 131\end{array}$


SUBROUTINE RPTSUP $\quad 74 / 74 \quad$ OPT=1 FTN $4.5 * 414$

$06 / 05 / 78 \quad 07.59 .59$

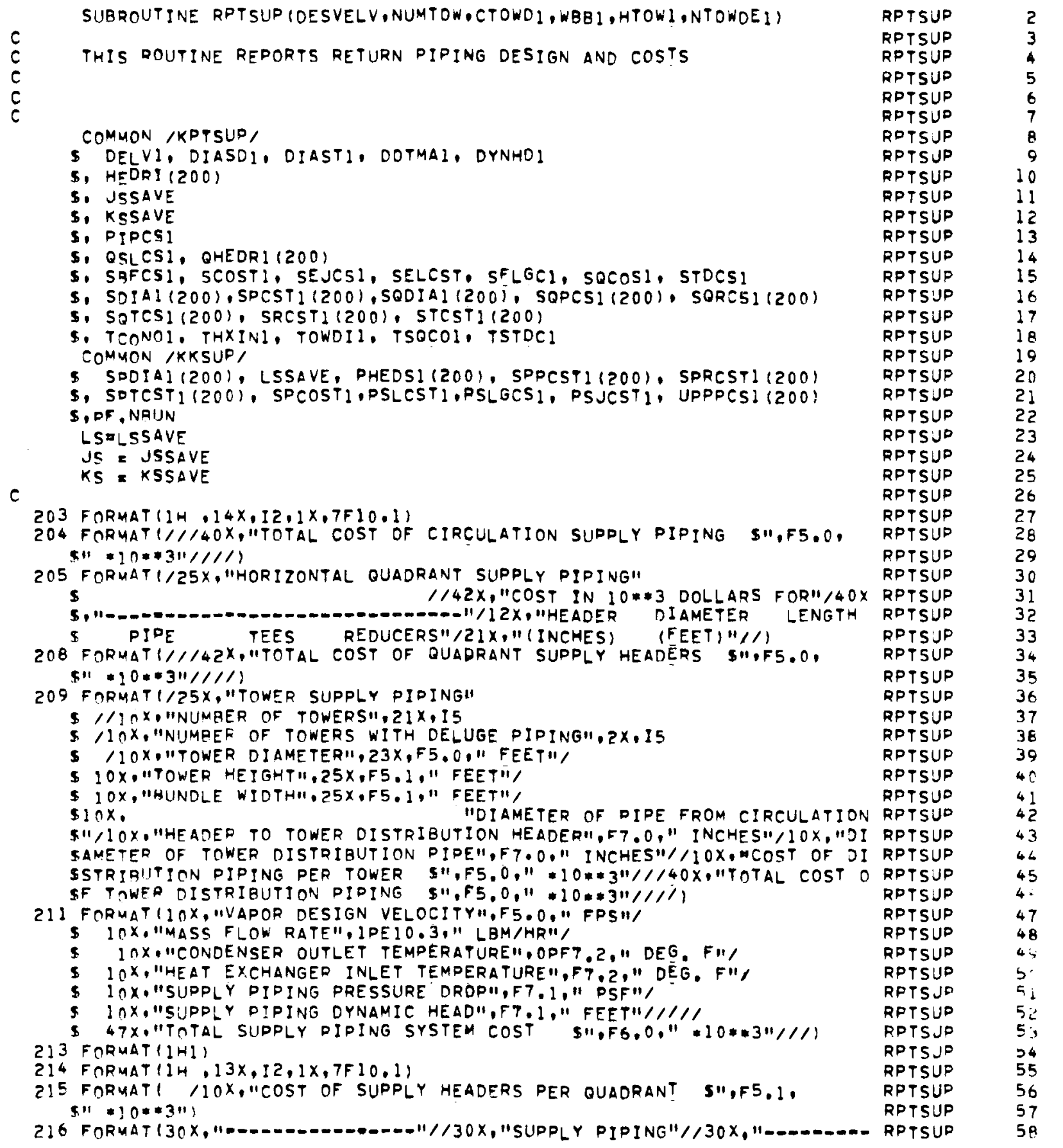




\section{C}

$\$=-\infty=1 / / 25 x, " C I R C U L A T I O N$ PIPING"//52X,"COST IN $10 *\|\|$

" RPTSUP

$\$ 3$ DOLLARS FOR"/4OX,"I-OTMETER

DIAMETER LENGTH

PIOE

TEES

REDUEE RPTSUP

SRS EXPANSION FLANGES"/2IX,"(INCHES) (FEET)",33X, "JOINT(I) (3 RPTSUP 5) "I/I

217 FORMATI//110X,"FITTINGS COST PER BUNDLE S1".55.3." *10*311,

RPTSUP

RPTSUP

RPTSUP

RPTSUP

WRITE $(6,213)$

WRITE $(6,216)$

DO $201 \quad I=1 . \mathrm{JS}$

IF (I.GT.1) GO TO 202

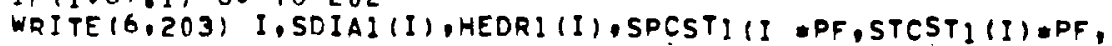

SSPCST I I )*PF, SEJCSI *PF, SFLGCI*PE

GO TO 201

202 IF(I,EQ.JS) GO TO 210

WRITE (6.203) I,SDIAI (I) OHEDRI (I) ISPCSTI (I \#PF,STCSTI(I) \#PF,

SSRCSTI (I) $\%$ PF

GO TO 201

210 WRITE (6.203) I,SDIAI II) HEDRI (I).SPCSTI II *PF, SELCSTHPF,

SSRCSTI (I) \#PF

201 CONTINUE

WRITE $(6,204)$ SCOSTI DF

WRITE $(6,205)$

DO $206 \quad I=1 . K 5$

IF (I.ER,KSIGO TO 20 T

WRITE $(6,2$ I 4 ) I,SQDIAI (I), QHEDRI (I),SQPCSI (I) PF,SQTCSI (I) *PF,

RPTSUP

RPTSUP

RPTSUP

RPTSUP

RPTSUP

RPTSUP

RPTSUD

RPTSUD

RPTSUP

RPTSUP

RPTSUP

RPTSUP

RPTSUP

RPT SUP

RPTSUP

RPTSUP

RPTSUP

RPTSUP

SSORCSI (I) *PF

GO TO 206

207 WRITE $(6,214)$ I,SODIAI (I), OHEORI (I),SOPCSI II) APF, OSLCS1,

SSORCSI (I) $P F$

206 CONTINUE

WRI TE $(6,305)$

305 FORMAT (/25X, "VERTICAL QUADRANT SUPPLY PIPING

SIN 10:3 DOLLARS FOR "/40X,30("-"1)/12X, "HEADER

5 PIPE TEES

REDUCERS" $/ 2$ IX," (INCHES)

"/ $/ 42 X, " C O S T$
LENGT

RPTSUP

RPTSUD

RPTSUP

RPTSUP

RPTSUP

RPTSUP

RPTSUP

DO $306 \quad I=1, L S$

(FEET) $/ 11$

RPTSUP

RPTSUP

RPTSUP

WRITE $(6.214)$ I.SPDIAI (I),PHEDSI (I),SPPCSTI II),SPTCSTI II),SPRCSTIII 5)

306 CONTINUE

WRITE $(6,517)$ NBUN

517 FORMAT (//10X,"NUMBER OF BUNDLES PER QUADRANT".110)

WRITE $(6,217)$ SBFCSI*PF

WRITE $(6.215)$ SOCOS $\#$ DF

WRITE $(6,20 B)$ TSOCO) $\#$ PF

RPTSUP

RPTSUP

RPTSUP

RPTSUP

RPTSUP

RPTSUP

RPTSUP

RPTSUP

WRITE 16,209 , NUMTOW,NTOWDEI,CTOWDI, HTOWI,WBBI, DIASDI,DIASTI,STOCSI RPTSUP

$1 * D F, T S T D C L \rightleftharpoons P F$

WRITE (6,211) DESVELV, DOTMAI, TCONOI, THXINI, DELVI,DYNHDI, PIPCSI RETURN

RPTSUP END

RPTSUP

RPTSUP

61

62

63

64

65

66

67

68

69

70

71

72

73

74

76

77

78

79

80

81

82

83

84

85

86

87

88

$B 9$

90

91

92

93

94

95

96

97

98

99

100

101

102

103

104

105

106 
$74 / 74$ OPTE1 FTN $4.5 .414 \quad 06 / 05 / 75 \quad 07.59 .59$

SUBROLITINE SCALP(TI, RANGE, AFRON, WLRAT, TCWFCS, ITOWN)

SUBROUTINE SCALP SCALES THE PLANT AND DRY COOLING TOWER DESIGNS AND COSTS LINEARLY UPUARD OR DOWNWARD TO ACCOUNT FOP THE FOLLOWING FACTORS-

1. THE DESIGN TEMPERATURE OF THE POWER PLANT DOES NOT CORRESPOND TO THE RATED BACK PRESSURE OF THE STEAM TURRINE.

2. PART OF THE TOTAL POWER OUTPUT OF THE PLANT IS USED IN SUPPLYING POWER TO THE FAN AND PUMPING SYSTEMS,

SCALD

SCALP

SCALP

SCALP

SCALP

SCALP

SCALD

SCALP

SCALP

SCALP

SCALP

THE FOLLOWING ARE POINTS ON THE HEAT RATE FACTOR CURVE WHICH ARE USED IN DESCRIBING THE SCALING OF THE PLÁNT AND DRY COOLING TOWERS. AT EACH OF THESE POINTS THE HEAT RATE FACTOR, DLANT SIZE aNO PLANT COSTS ARE DETERMINED.

PIINT O - CONVENTIONAL TURBINE OPERATING AT RATED BACK PRESSURE

DOINIT I - STEAM TURBINE OPERATING AT RATEO BACK PRESSURE

DOINT 2 - STEAM TUREINE OPERATING AT SYSTEM DESIGN EXIT TEMPERATURE WITH OUTPUT OF TURBINE GENERATOR EQUAL TO THE DESIGN POWER OUTPUT

DOINT 3 - STEAM TURBINE OPERATING AT SYSTEM DESIGN EXIT TEMPERATURE WITH THE POWER OUTPUT OF PLANT EQUAL TO THE DESIGN POWER OUTPUT

THE DIFFERENCE BETWEEN POINT TWO AND THREE IS THE PLANT HAS REEN SCALED UD TO ACCOUNT FOR THE POWER CONSUMPTION OF THE FANS AND PUMPS, BETWEEN POINT ONE AND TWO THE POWER PLANT HAS BEEN SCALED UP OR DOWN TO ACCOUNT FOR AN INCREASE OR DECREASE IN THE HEAT RATE FACTOR.

DEFINITION OF VARIABLES

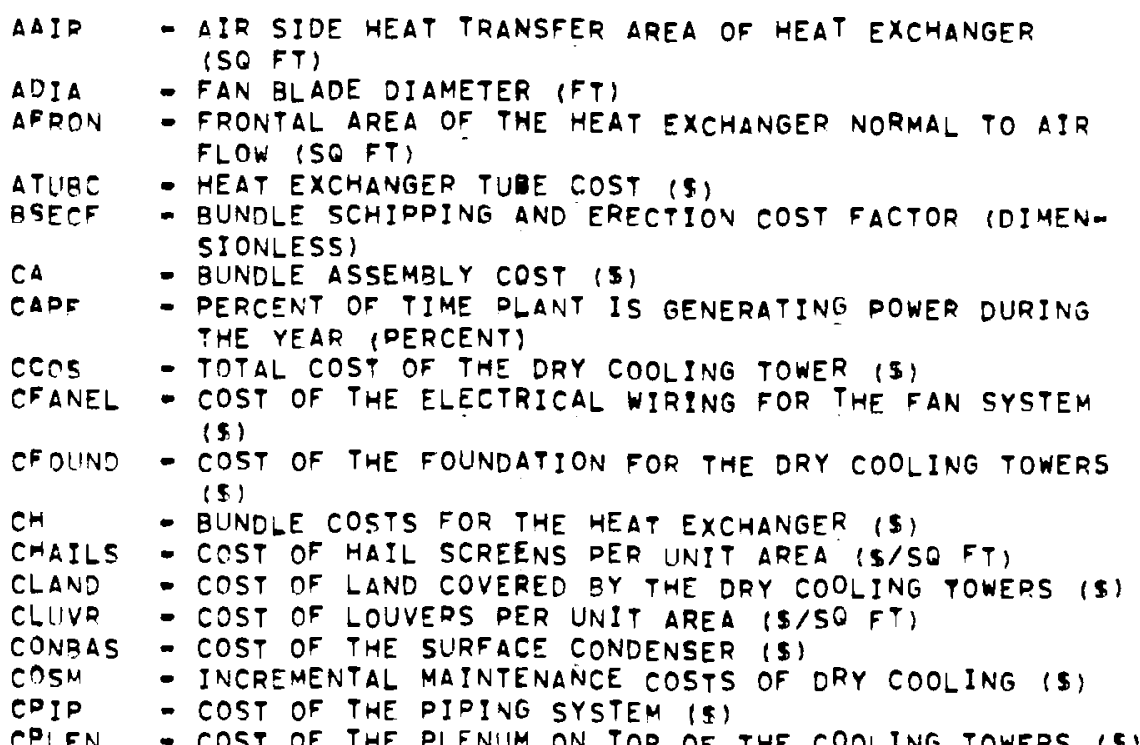

SCALD

SCALD

SCALP

SCALP

SCALD

SCALD

SCALD

SCALP

SCALP

SEALP

SCALP

SCALP

SCALD

SCALP

SCAL?

SCALP

SCALP

SCALP

SCALP

SCALP

SCALP

SCALP

SCALD

SCALP

SCAL.

SCALP

SCALP

SCALP

SCALP

SCALD

SCALP

SCALD

SCALD

SCALD

SCALP

SCALP

SCALP

SCALP

SCALD

SCALE

SCALD

SCALP

SCALP

SCALD

CPLEN

- cost of the plenum on top of the coOling towers

(s) SCALP 


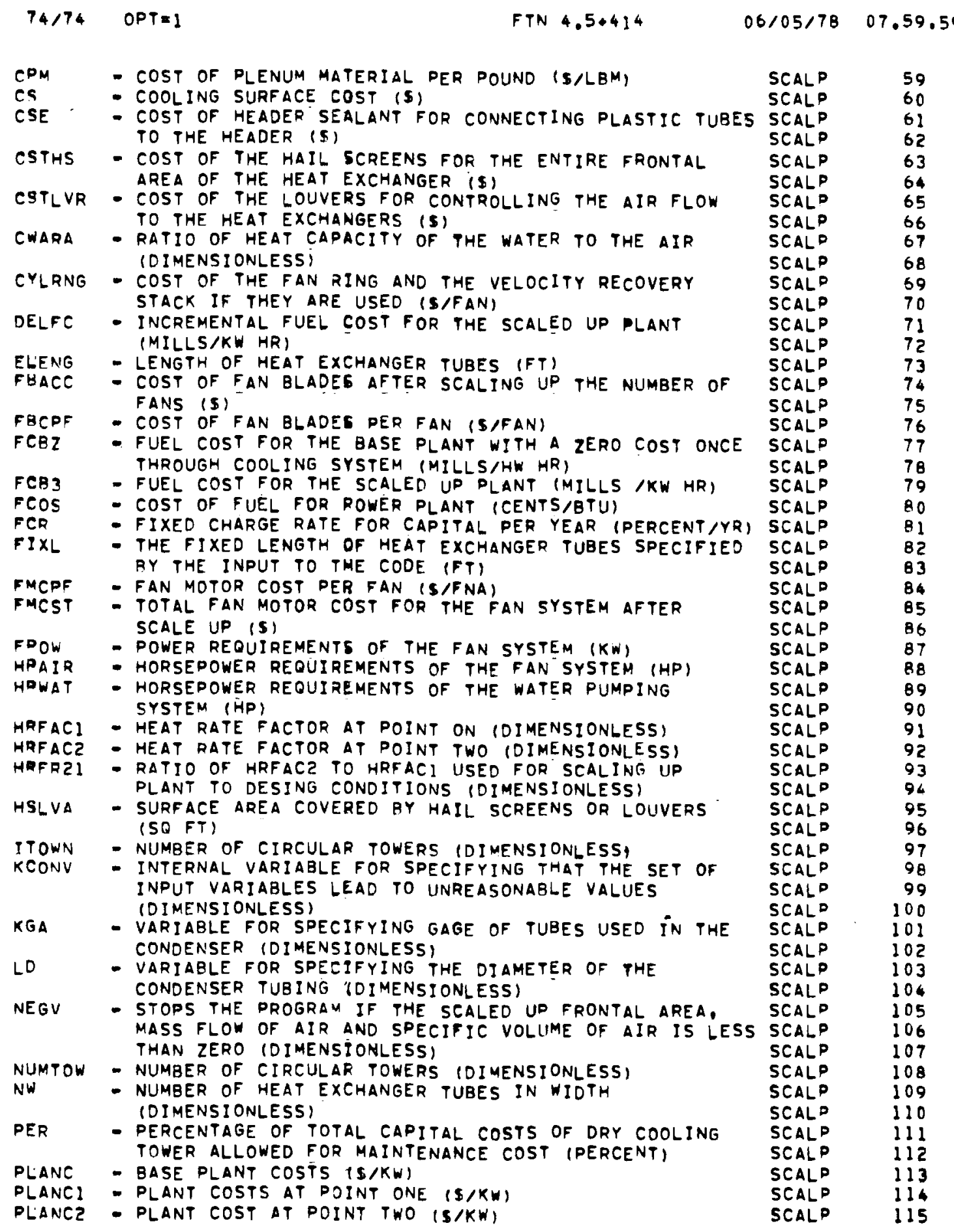




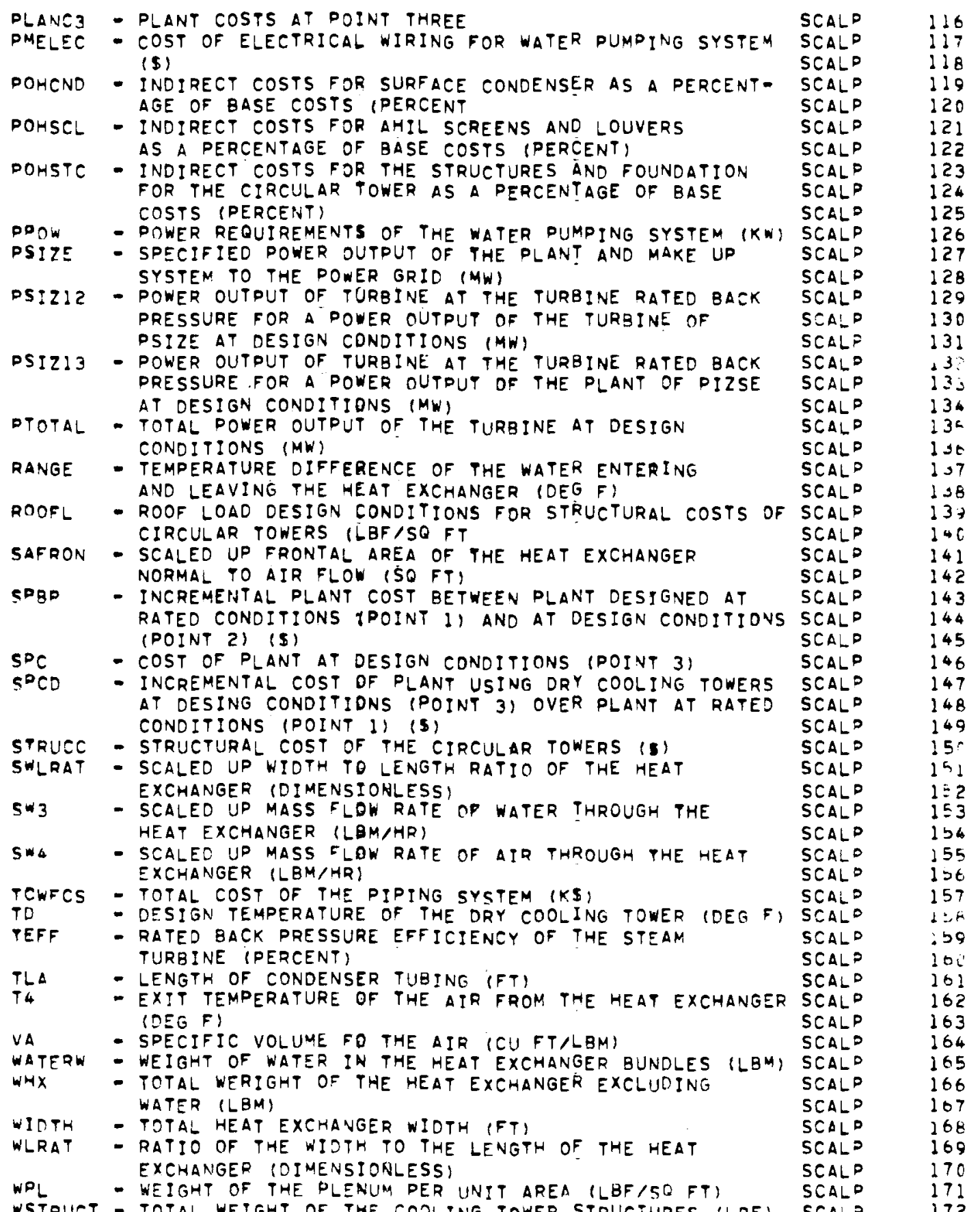


$$
74 / 74 \quad \text { OPT }=1
$$

FTN $4,5+414$

$06 / 05 / 78$

07.59 .59

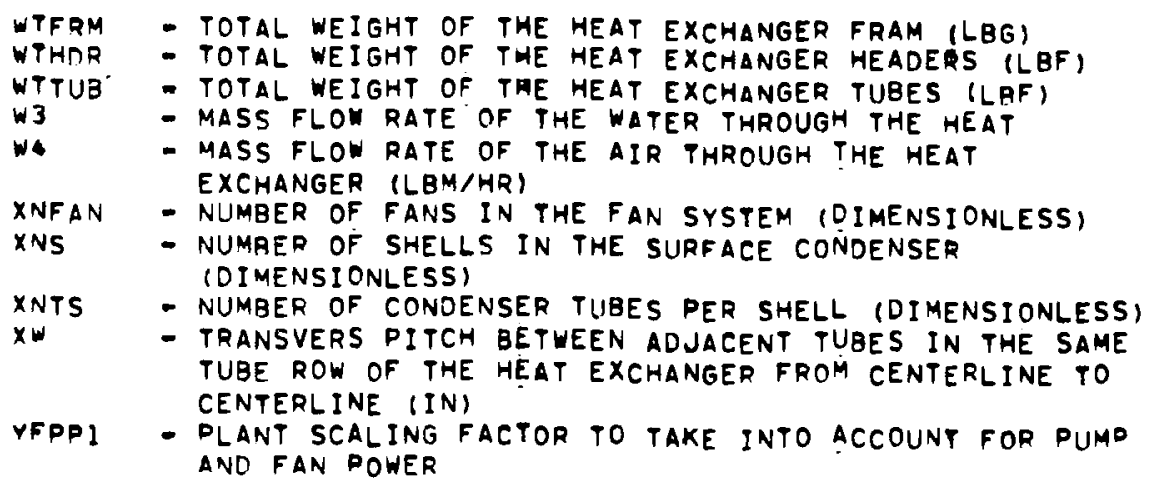

LOGICAL NEGV

LOGICAL FIRST,FRST, FXTEMP, FXTVAR, FXTTTD,FXTLNG COMMON

S AFTR, ALPHA, $A N G(3)$

- BDLIM(16)

S, CAPF, CSSPKW, CONF, CONL, COSTL, CAPCHG, CONMAT, CONMAZ,CAF

\$. CRJ, CTURB, CDANG, CLUVR, CMAILS, CVM

$\$$.DFIN.DEEPL, DESVEL, DESVELV,DESVELD, DENSIF

$S, E E F P, E F S$

S :FCR, PIRST, FIXL, FCOS,FFHX, FXTEMP, FACTOR, FRST, FACTORD, FSHOT, FDELUG

S ,FIXV,FIXTTD,FXTVAR,FXTTTD,FXTLNG

5 , GBEFF

S, HXNP,HDCST,HCD

F. ITMAX, ITMIN

5 , JCONS

S KCONV, KALEXT

S.MXEXT

S, NTA, NSIOES, NRUNHI

COMMON

$S O D P$

5 ,PSIZE, PER, PWCOS, PLANC, PFAET, POMDPL

- POHBAF, pOHFAN, POHLEC, POHCIR, POHCND, POHSTC, POHSCl

S RBP.REDUCE, REDUCV, ROOFL, RHOUT

S QREJ, QREDUCE, QREDUCV

\$ SIGMAG, SAAF, SEGL

\$, TD, TPO(4), TEFF, TLIM, THFIN, TLPRA, TFIX

$\$, T C D, T W$

TUCS. UWS

\$. WFV, WATCONA, WATCOST

S. VAS (5)

$5, X D E P A, X W, X D$

COMMON /SUPPLY, VAL (5), CPLN, CPIP, CCOS, W3, COSM, EFF, $\$$ OIN, CLAND, REAIR, Z,HRFACZ, WA, U, DELPW, PPOW: FPOW, DELFC, WIDTH, ELENG, VAIR, VWAT, REWAT, NW, NT, NP,PLANCZ,PSIZ12, SAIR, YFP, SPCD,PSIZI3, WTZ,

AP2, NW2, TCOS, DELPA,DELPWT, OELPWC, CONBAS, XNTSX, VELDX

5 , TLAX, UCON, PMCST, FMCST, FBACC, EFFC, CM, CA, CS

S, CLTC, CFC, CPCC, FITCO, CSC,CEPREP,DELPIP, AST, OLDCST

\$. CAPCST, XNFAN, BLDANG, CPLEN, DDFEL, WHX, WTTUB, ADIA, ABLN

5. WTHDR, WTFRM, STRUCC,CFPERF, HPDERF, TOTCF, TPC, EFFIN, ANTU

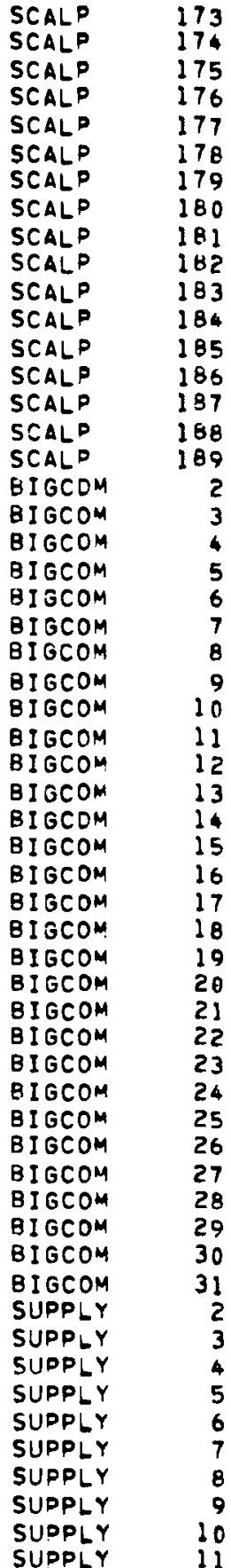


SUBROUTINE SCALP $\quad 74 / 74 \quad$ OPT $=1 \quad$ FTN $4.5 .414 \quad 09 / 05 / 78 \quad 09.59 .59$

5. AIRFF, WATFF, XNMOD, TCTPF, FDCPF, FMTRC, ACCTTP, ADJTP

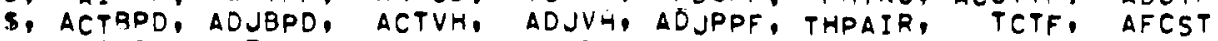
8. CSRD, WATERW, WSTRCT, CFOUND, HUBDIA, TOWLEN, NUMTOW, CTOWD 5. SORJ,SORJS,ATUBC, CBA, TTDI, TTOZ, DELPS, CSTLVR,PIPCSTD

5 , CSTHS, CYLRNG, CFANEL, PSTACS, DUFITC, PMPCST, RPTPL, SEPCST

\$. PWD (16), WATCWD (16),DEPER (16), DEPERO (16)

5. WBE,HTOW,NTOWDE, CSTIF, CSJ,CVF, CDP,W5

COMMON / SINK/ VAR(5), CPLN1, CPIPI, CCOS1, W31, COSM1, EFFI, OINI, CLANI, REAIl, ZI,HRFAZS, W4I, UI, DEPWI, PPOWI, FOOWI, DEFCI, WIOTI, ELENI, VAIRI, VWATI, HII, HOI, T4I, REWAI, NWI, NTI, NPI,ZPLNC2,ZSIZI2, ZSPBP, SWAI, SW3I. SPCI, PTOTI,PLNC3I, $\triangle F P O I, ~ \triangle A I R I, Y F P M I N$, SPCDI, SPSI3,WTZMIN, BPZMIN, NWZMIN, TCOSI, DEPAI, DPWTI, DPWCI,CONBAI, XNTS1, VELDI

5 - TLAI, UCONI, PMCSI, FMCSI, FBACl, EFFCI, CHI, CAI, CSI 3: CLTCI, CFCl, CPCCI,FITCOI, CSCl,CEPREI,OEPIPI, ASTI,OLDCSI

S.CADCS1, XNFANI, BLDANI,CPLENI,DPFELI, WHI, WTTUI, AOII, ABLI

5. WTHOI, WTFRI, STRUCI,CFMPFI, HPPFI,TOTCFI, TPI,EFFINI, ANTUI

5, AIRFFl, WATFFl, XNMODI, TCTPF1, FDCPF I, FUTRCl, ACTTPI, ADJTPI

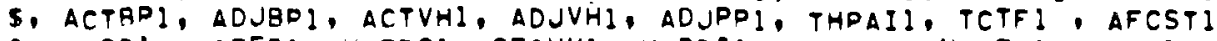

s, CSRI, WATERI. WSTRCI, CFOUNI. HUBDIl, TOWLEI, NUMTOI, CTOWDI

5 ,SORJI,SORJSI, ATUBCI, CBAI, TTDII, TTD2 I, DELPSI, CSTLVI,PIPCTOI

5 .CSTHSI, CYLRNI, CFANEI, PSTACI, PUFITI, PMPCSI, RPTPLI, SEPCSI

5 .PWD 1 (16), WATCWD1 (16),DEPERI (16), DEPERQ1(16)

S WBE I. HTOW I, NTOWDE I, CSTIF I, CSJI, CVF I, CDP I, W5I

COMMON /SCALER/HRFACI. ATUB, CPERA, SSCD,PLANCI, CASSSI.

I GAIR, FAIR, HPAIR, HPWAT, WLRTP, PLANCTI

COMMON /LACALL, SRN

COMMON /ESCAL/ BSECF

COMMON ITINE/ XNS. TLA, XNTS, ARPS, TTDZOE, LINOR, XNP, XQUALY

COMMON/TUBE/KGA,LD, TID $(T, 6)$

COMMON /PLCOST, WPL

COMMON /DME/ PMELEC

COMMON IHEADEX, CHH,CN,CHM, CMW, CRJ,CWJ,CST, CMO,CSR,CSM,RITCHF, CPM,

1 DRESS, HEDMAT, HEDTYP, NPASS, WB, TSP, CSH, CSHP

LOGJCAL LOOPER

COMMON/FAN/ FANDAP $(153,10)$, FANDAM $(153,10), F M C P F, F B C P F, P 2, R, S$,

I VELREC, $\triangle F C$, ELEV, EHR, LOOPER, ETMAX

DATA NEGV/,FALSE,

901 FORMATIIX, AI0,"TI, RANGE, CWARA, AFRON, WLRAT",5G 14.4 )

NAMELIST $109 N L /$ FLOSS, AFRON, VA,WG, THITE, TO, TH, CWARA, RANGE

$X$ TOW $=$ ITOWN

SCALING FACTOR FROM POINT I TO DOINT 2 TO ACCOUNT FOR CHANGE IN HEAT RATE FACTOR

HRFR2I $=H R F A C 2 / H R F A C$ I

DOWER OUTPUT OF TURBINE AT RATED BACK PRESSURE (POINT 1$)$ WHICH GIVES REQUIREO POWER OUTPUT PSIZE AT THE DESIGN BACK PRESSURE (POINT 2)

PSIZIZOPSIZE:HRFRZI

- DLANT COSTS aT DESIGN CONDITIONS (POINT 2 ) WhICh INCLUDE STEAM SUPPLY AND BACK PRESSURE SCALING TO POINT 2

Plancz-PLANCl * hrFRzi

SUPPLY $\quad 12$

SUPDLY

SUPDIY

SUPD:Y

SUPP_Y

SUPPEY

SUPD:Y

SINK

SINK

SINK

SINK

SINK

SINK

SINK

$\operatorname{SINK}$

SINK

SINK

SINK

SINK

SINK

SINK

SINK

SINK

SI NK

SCALER

SCALER

SCALP

SCALP

SCAL?

SCALP

SCALP

SCALP

SCALP

SCALP

FANDEK

FANDEK

FANDEK

SCALP

SCALD

SCALD

SCALD

SCALP

SCALP

SCALP

SCALP

SCALP

SCALP

SCALP

SCALP

SCAL?

SCALD

SCALD

SCALP

SCALP

SCAL?

SCALP

SCALD
2

14

15

26 
SCALP 223

SCALP 224

SCALP 225

SCALP 226

SCALP 227

SCALP 228

SCALD 520

SCALP 230

SCALP 231

SCALP 232

SCALP 233

SCALD 234

SCALD 235

SCALD 236 
SUBROUTINE SCALP $\quad 74 / 74$ ODTE

FTN $4.5+414$

$06 / 05 / 78$

07.59 .59

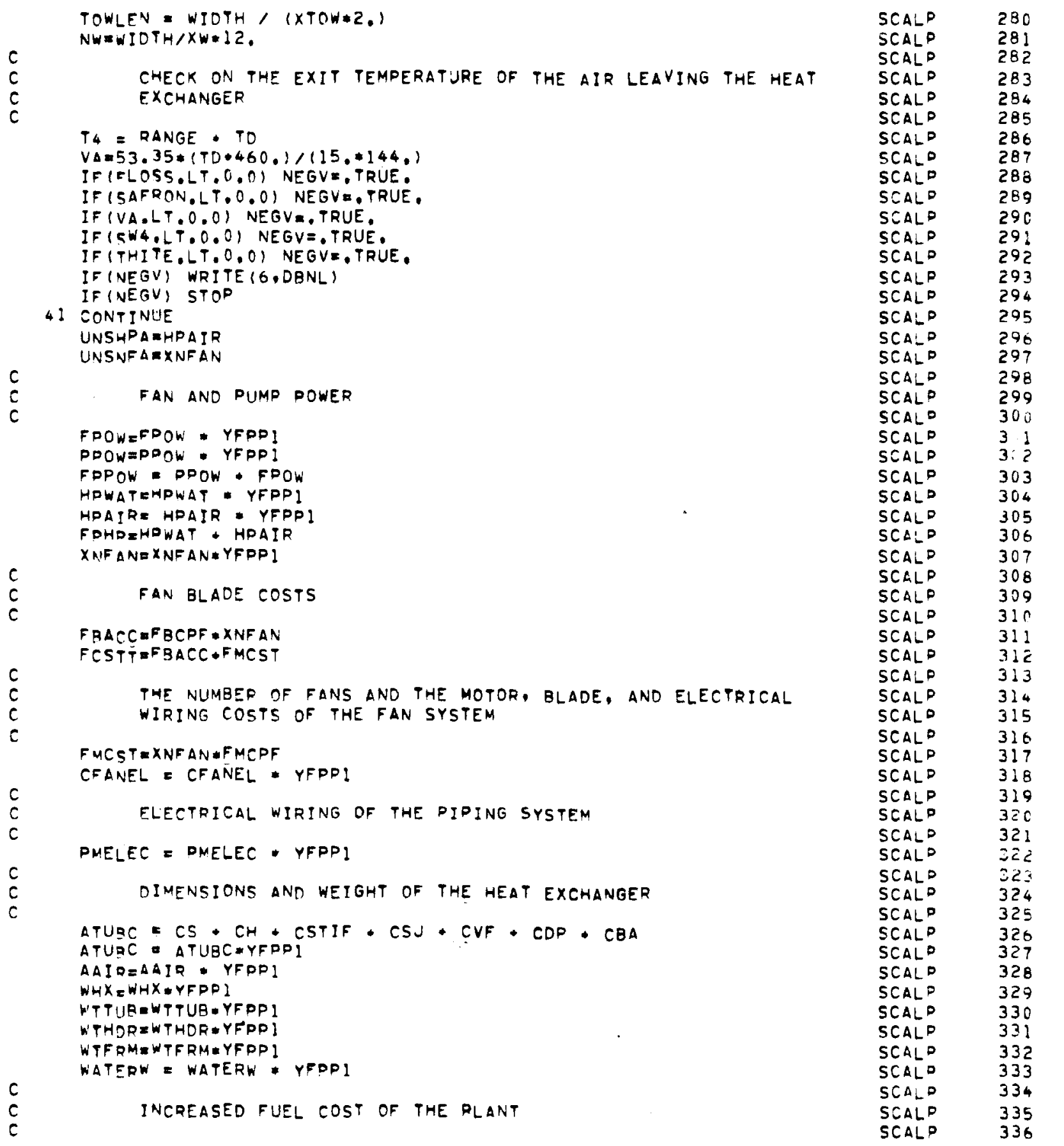




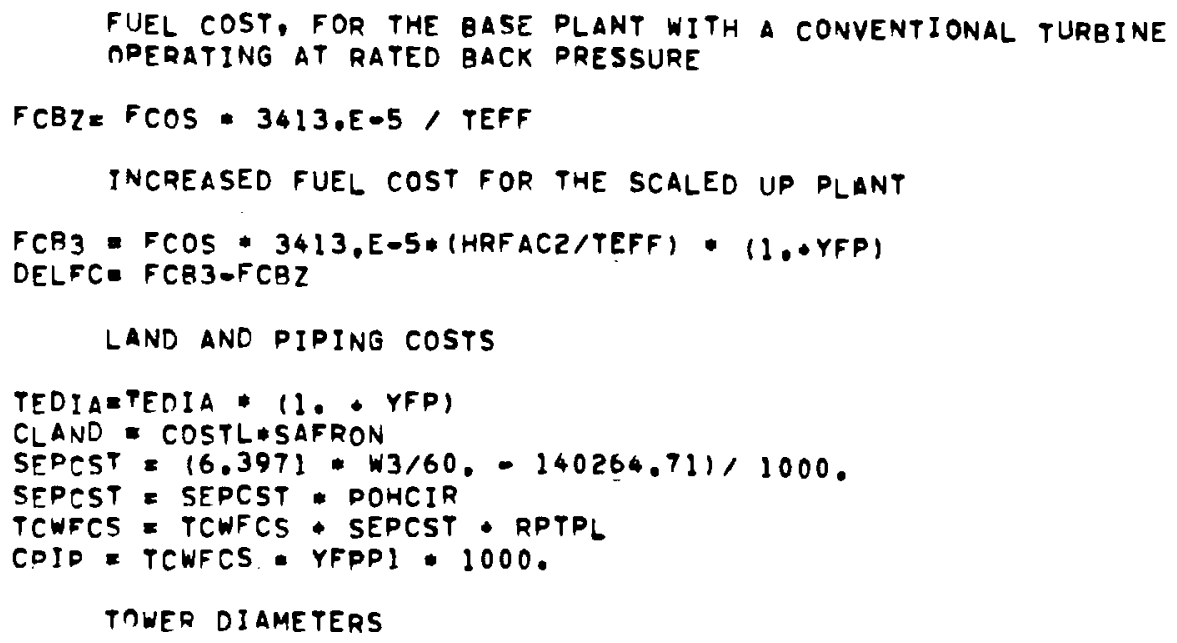

CALL STRUET IITOWN, NSIDES, HTOW, ELENG, SEGL, NBUNHI, WHX, WATERW, ADIA, IXNFAN,POOFL, STRUCC, CFOUND) 
SURROUTINE SCALP $\quad 74 / 74$ OPTEI

FTN $4.5+414$

$06 / 05 / 78$

07.59 .59

$\because$

C APTIMIZATION)

SCALP 394

OLDEST* (CCOS+SPCD+CLAND+CPIP+CONBAS) *FCR/(DSIZE*CAPF *8760.) 5 \& COSM+ DELFC RETIJRN

SCALP $\quad 395$

SCALP $\quad 396$

SCALP 397

SCALD $\quad 398$

END

SCALD 399 


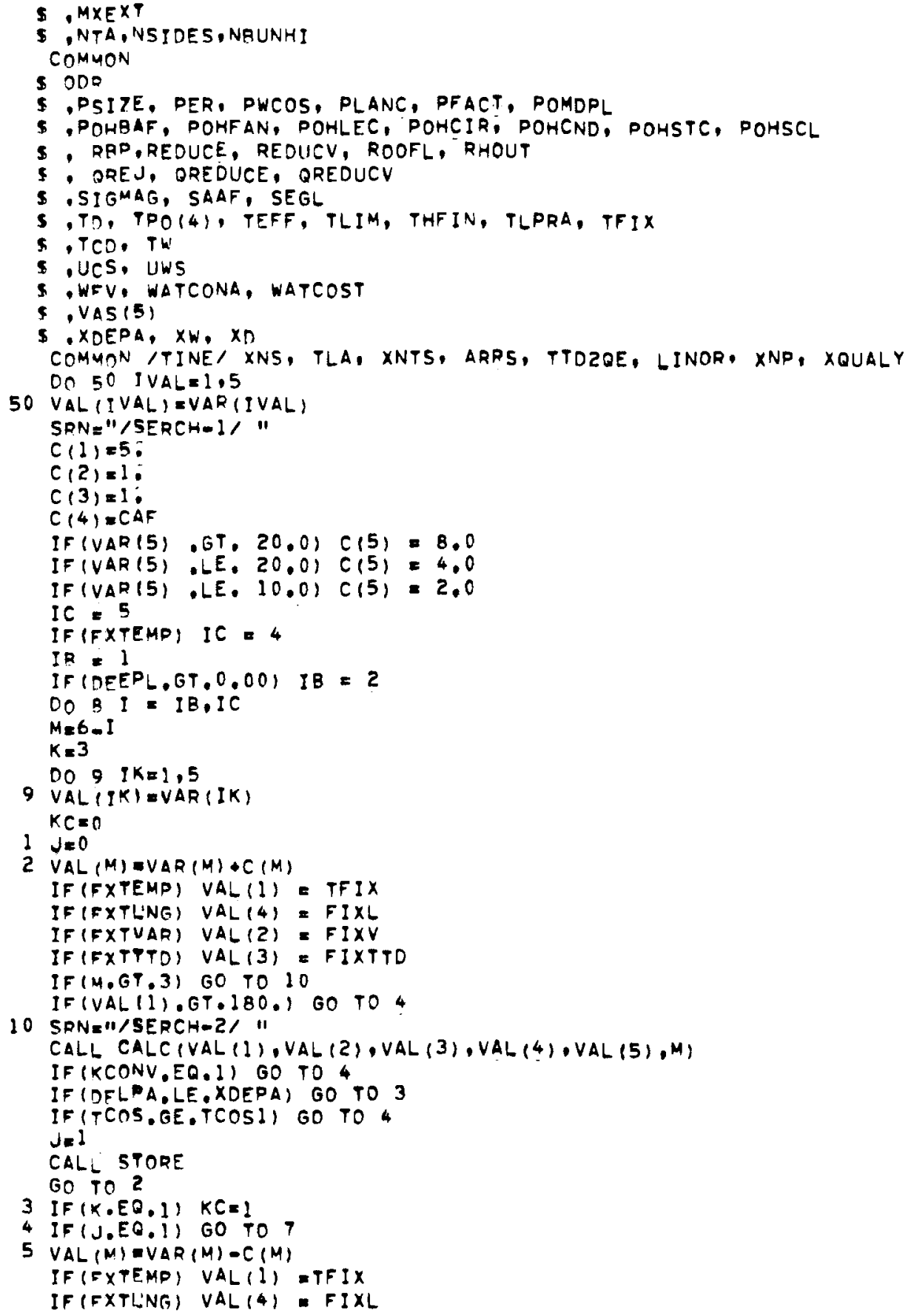

BIGCOM 27

BIGCOM 18

BIGCOM 19

BISCOM 20

BIGCOM 2]

BI GCOM 22

BIGCOM 23

BIGCOM 20

BIGCOM 25

BIGCOM 26

BIGCOM 27

BIGCOM 28

BIGCOM 20

BIGCOM 30

PI GCOM 3I

SERCH II

SERCH I

SERCH 14

SERCH 15

SERCH 16

SERCH 17

SERCH 18

SERCH IQ

SERCH 2

SERCH 21

SERCH 22

SERCH 23

SERCH 24

SERCH 25

SERCH 26

SERCH 27

SERCH 28

SERCH 29

SERCH 30

SERCH 3

SERCH 32

SERCH 33

SERCH 3 W

SERCH $3=$

SERCH 36

SERCH

SERCH 3

SERCH IS

SERCH 40

SERCH 4

SERCH 42

SERCH 43

SERCH

SERCH 45

SERCH 46

SERCA 47

SERCH

SERCA 40

SERCA 50

SERCA 51

SERCH 5 S 
IF (FXTVAR) VAL (Z) $=F I X V$

IF (FXTYTD) VAL ( 3 ) FIXTTD

IF (VAL (M) :LE, 0.0$)$ GO TO 7

SRNE" /SERCH=3/"

CALL CALC (VAL (1), VAL (2), VAL (3), VAL (4), VAL (5), M)

IF (KCONV.EQ.1) GO TO 7

IF (DELPA.LE,XDEPA) GO TO 6

IF (TCOS.GE,TCOSI) GO TO $T$

CALL STORE

$K C=0$

Go in 5

6 IF (K,ER. I) KCEI

7 IF (KC.EQ.1) CALL CHNGE (M)

$K=K-1$

IF $(K \cdot E Q, O)$ GO TO $B$

IF (M,EQ. 5 . AND. $C(M), E Q, 1.0)$ GO TO $B$

IF $(M, E Q, 5) C(M)=C(M) * 5.0 / 2.0$

$C(M)=C(M) / 5$.

Go TO 1

8 CONTINUE

RETURN

END

$\begin{array}{ll}\text { SERCH } & 54 \\ \text { SERCH } & 55 \\ \text { SERCH } & 56 \\ \text { SERCH } & 57 \\ \text { SERCH } & 58 \\ \text { SERCH } & 59 \\ \text { SERCH } & 60 \\ \text { SERCH } & 61 \\ \text { SERCH } & 62 \\ \text { SERCH } & 63 \\ \text { SERCH } & 64 \\ \text { SERCH } & 65 \\ \text { SERCH } & 66 \\ \text { SERCH } & 67 \\ \text { SERCH } & 68 \\ \text { SERCH } & 69 \\ \text { SERCH } & 70 \\ \text { SERCH } & 71 \\ \text { SERCH } & 72 \\ \text { SERCH } & 73 \\ \text { SERCH } & 74 \\ \text { SERCH } & 75\end{array}$


$14 / 74 \quad$ OPT $=1$

FTN $4.5+414$

$06 / 05 / 78$

07.59 .59

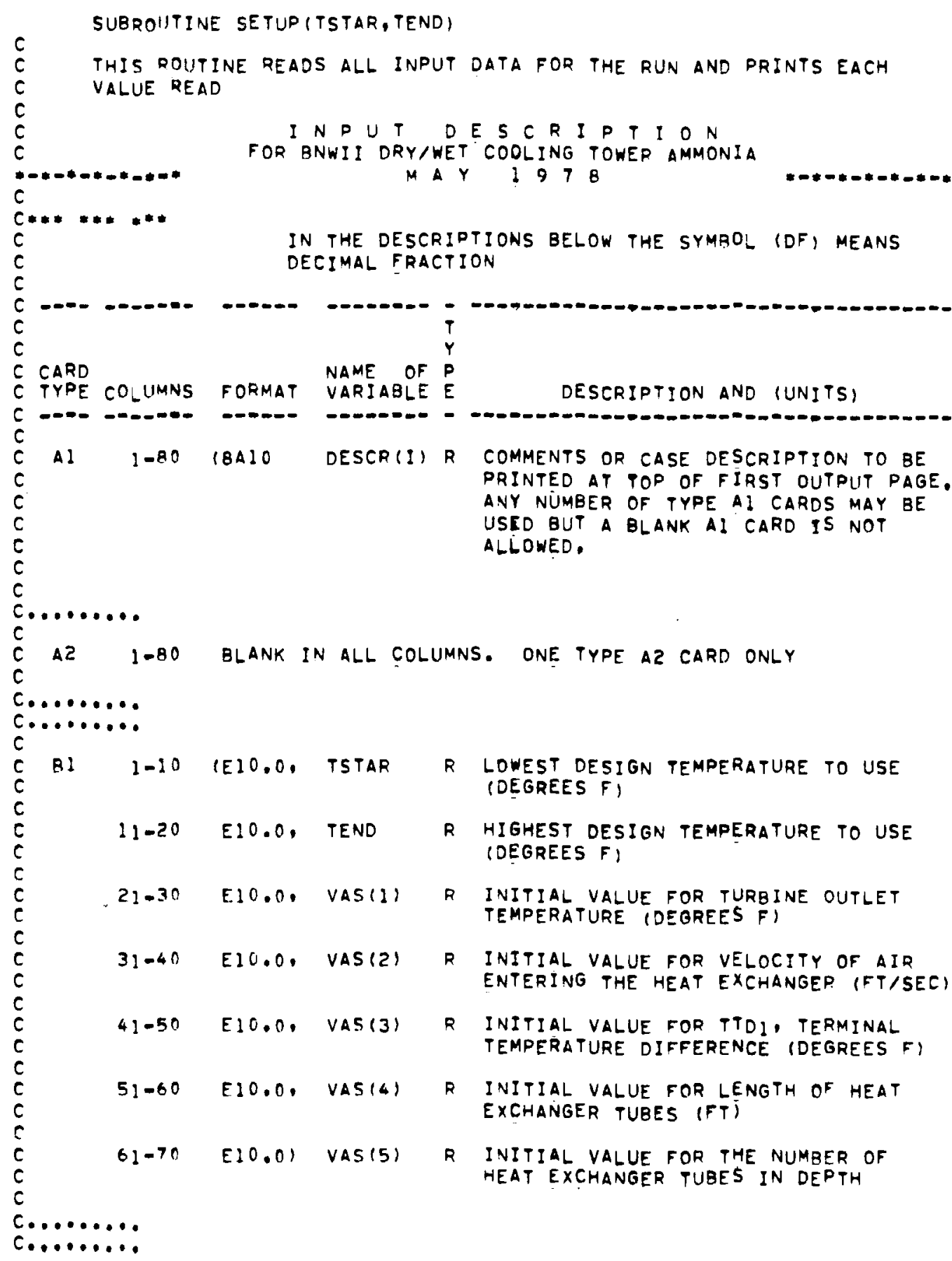

SE TUP

SETUP

SETUP

SETUP

SETUP

SETUP

SETUP

SETUP

SETUP

SETUP

SETUP

SETUP

SETUP

SETUP

SETUP

SETUP

SETUP

SETUP

SETUP

SETUP

SETUP

PRINTED AT TOP OF FIRST OUTPUT PAGE SETUP

SETUP

SETUP

SETUP

SETUP

SETUP

SETUD

SETUP

SETUP

SETUP

SETUP

SETUP

SETUP

SETUP

SETUP

SETUD

SETUP

SETUP

SETUP

SETUP

SETUP

SETUP

SETUD

SETUD

SETUP

SETUP

SETUP

SETUP

SETUP

SETUP

SETUD

SETUD

SETUD

SETUD

SETUP

SETUP 
$74 / 74 \quad$ OPT $=1$

FTN $4.5+414$

$06 / 05 / 78$

07.59 .59

c

$\mathrm{CC}$

\begin{tabular}{|c|c|c|c|c|c|}
\hline B2 & $1-10$ & KE10.0. & $T F I X$ & R & $\begin{array}{l}\text { FIXED TUBINE OUTLET TEMPERATURE (F) } \\
\text { ENTRY IS IGNORED IF BLANK OR ZERO }\end{array}$ \\
\hline & $11-20$ & $E 10.0$, & $F I X V$ & $\mathbf{R}$ & $\begin{array}{l}\text { FIXED FACE VELOCITY OF THE AIR } \\
\text { ENTERING THE HEAT EXCHANGER (FT/SEC) } \\
\text { ENTRY IS IGNORED IF BLANK OR ZERO }\end{array}$ \\
\hline
\end{tabular}

SETUP

SETUP

SETUP

SETUP

SETUP

SETUP

SETUP

SETUP

SETUP

21.30 E10.0, FIXTTO R FIXED TERMINAL TEMPERATURE DIFFERENCE OF THE SURFACE CONDENSER IFI ENTRY IS IGNORED IF BLANK OR ZERO

SETUP

SETUP

SETUP

SETUP

SETUP

$31=40 \quad E 10.0, \quad F I \times L$

R

FIXED LENGTH OF HEAT EXCHANGER (FT) IGNORED IF SET EQUAL TO ZERO

ENTRY IS IGNORED IF BLANK OR ZERO

SETUP

SETUP

SETUP

IF THE ABSOLUTE VALUE OF FIXL $=0$. THEN THE PROGRAM WILL CALCULATE

THE LENGTH.

41.50 E10.0) DEEPL R FIXED NUMBER OF TUBES IN DEPTH

ENTRY IS IGNORED IF BLANK OR ZERO

SETUP

SETUP

SETUP

SETUP

SETUP

SETUP

SETUP

SETUP

SETUP

SETUP

SETUP

SETUP

$\mathrm{C} \cdot \cdots \cdots$

Cl $1-40 \quad(8 F 5.0, T A(I)$

TEMPERATURES REPRESENTATIVE OF

SETUP

TPER (I) FRACTION OF YEAR (DEGREES F) SETUP

SETUP

41-80 BF5.0) TPER(I) R FRACTION OF YEAR OVER WHICH TEMPERATURE TA(I) IS TYPICAL. (DF)

C A UT I ON - HIGHEST TA

SETUP

SETUP

SETUO

SETUP

SETUP

SETUP

SETUP

C2 1.40 (BF5.0, TA(I) $R$ CONTINUATION OF TAII) FOR IE 9 TO 16

SETUP

AI-AO BF5.0) TPER(I) R CONTINUATION OF TPER(I)

SETUP

SETUP

NOTE - A TYPE C2 CARD IS REQUIRED EVEN IF BLANK.

SETUP

SETUP

SETUP

SETUP

SETUP

SETUP

c.

C

ClA

1040 (8F5.0, TAHB(I) R

WET BULB AIR TEMPERATURE REPRESENTATIVE OF TPER (I) FRACTIION OF YEAR (DEGREES $F$ )

SETUD

SETUP

SETUP

SETUP

SETUP

41-80 AF5.0) BPLIMII) R BACK PRESSURE LIMIT ON THE STEAM TURBINE REPRESENTATIVE OF TPER(I)

SETUP FRACTION OF YEAR (IN, HG.)

SETUP

SETUP 
$74 / 74 \quad$ OPT $=1$

FTN $4.5+414$

$06 / 05 / 73$

07.59 .50

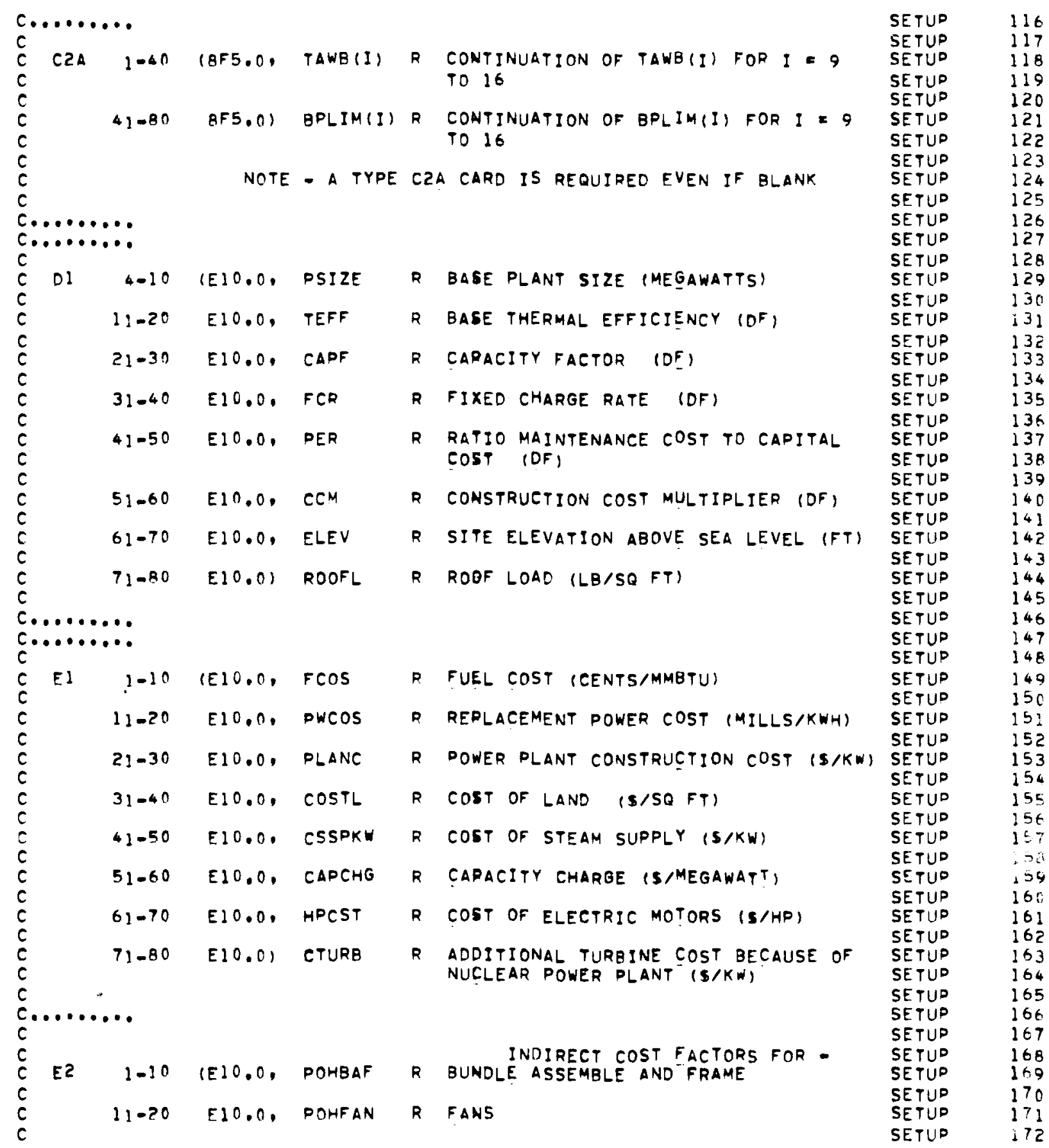




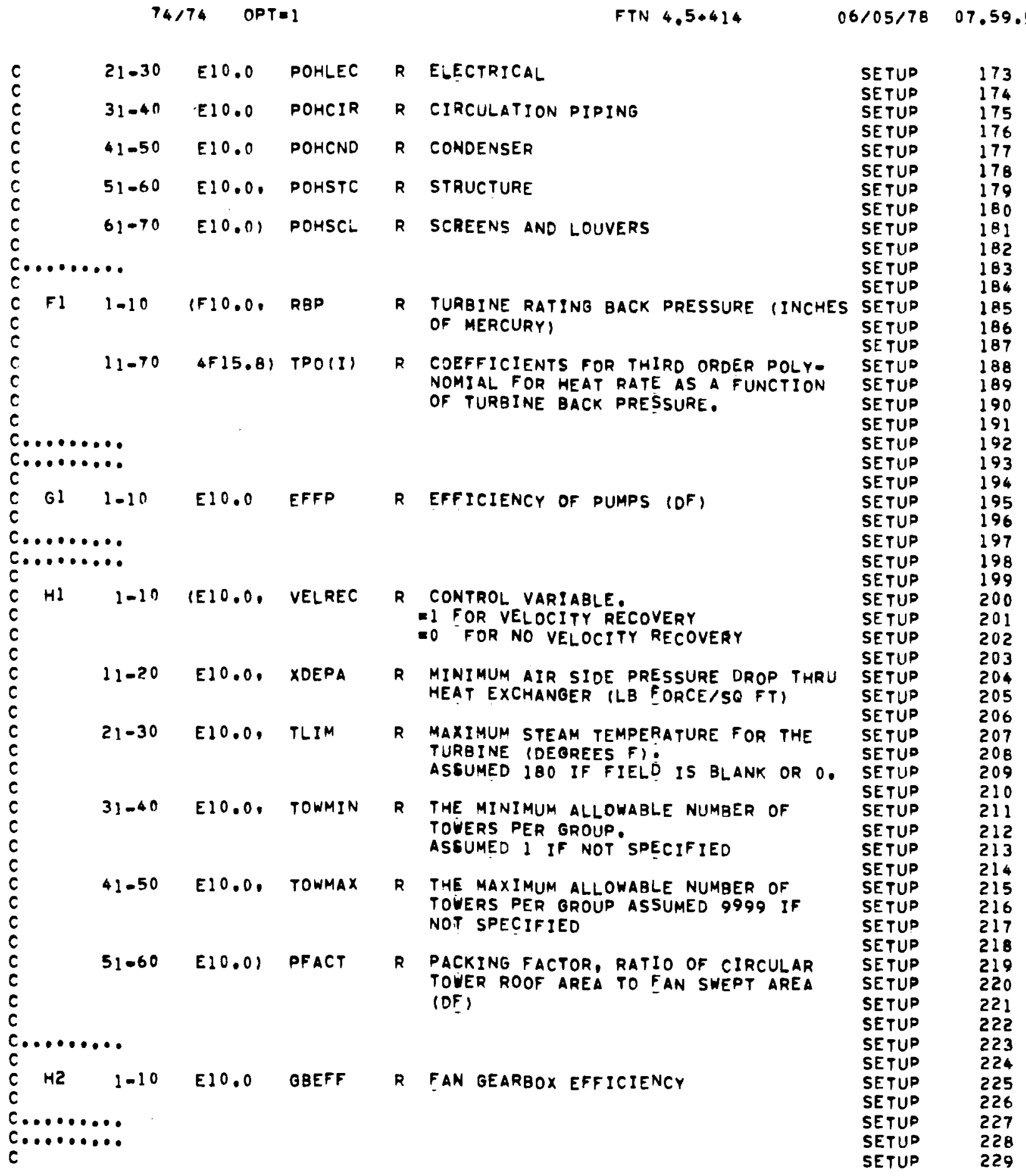




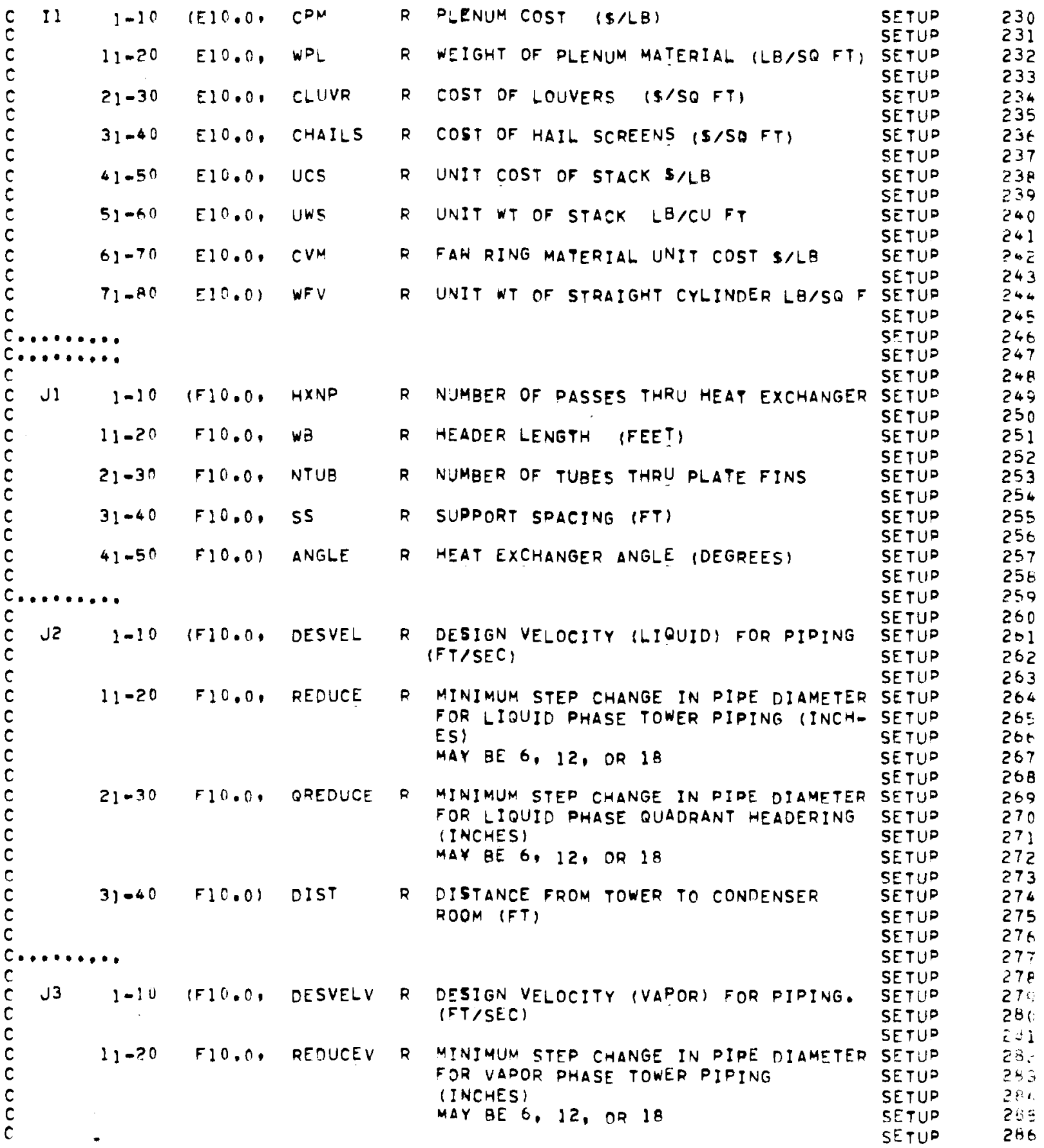


$74 / 74 \quad$ OPT 1

FTN 4.5 .414

$06 / 05 / 78$

07.59 .59

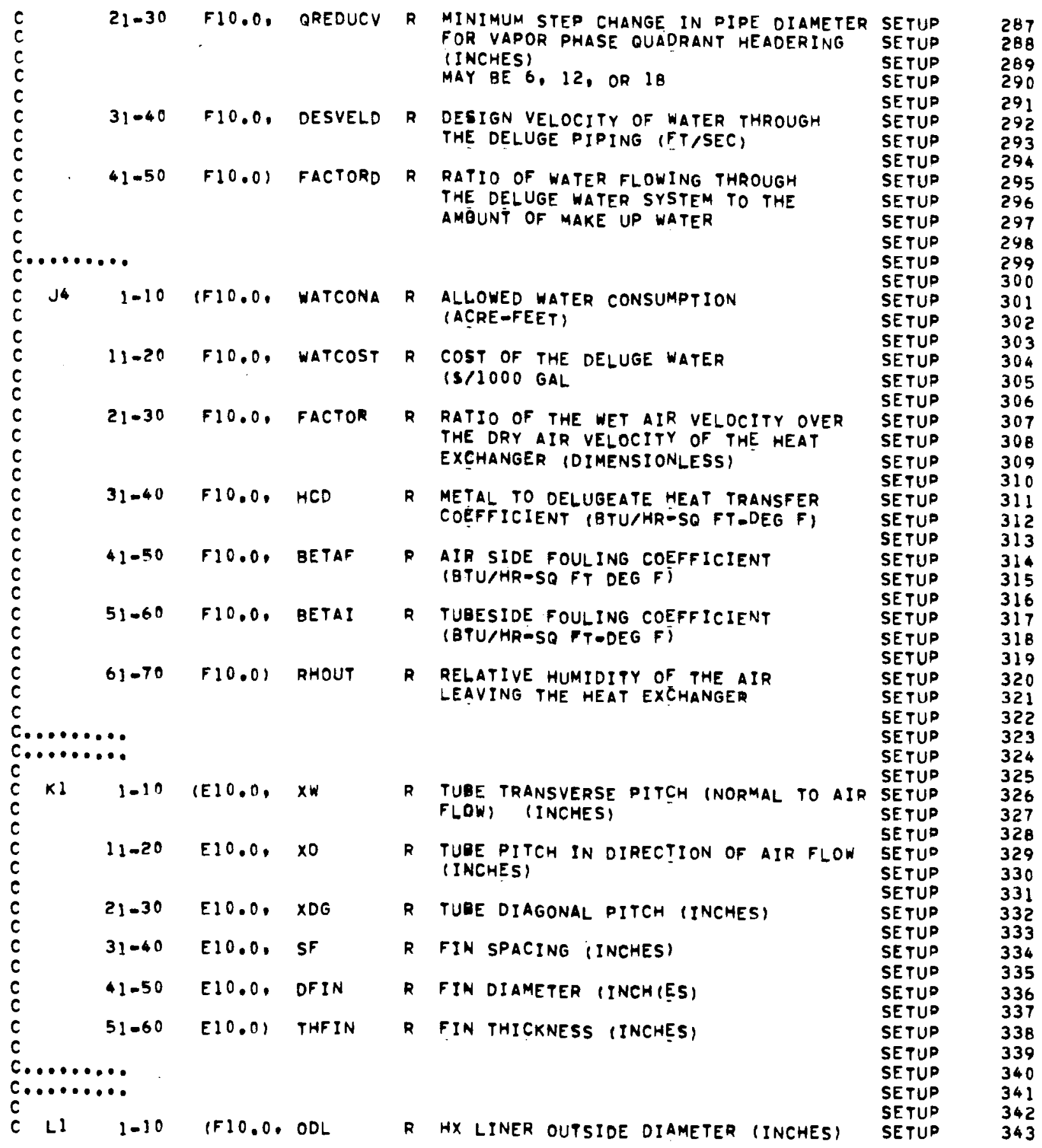




$$
74 / 74 \text { OPT } 1
$$

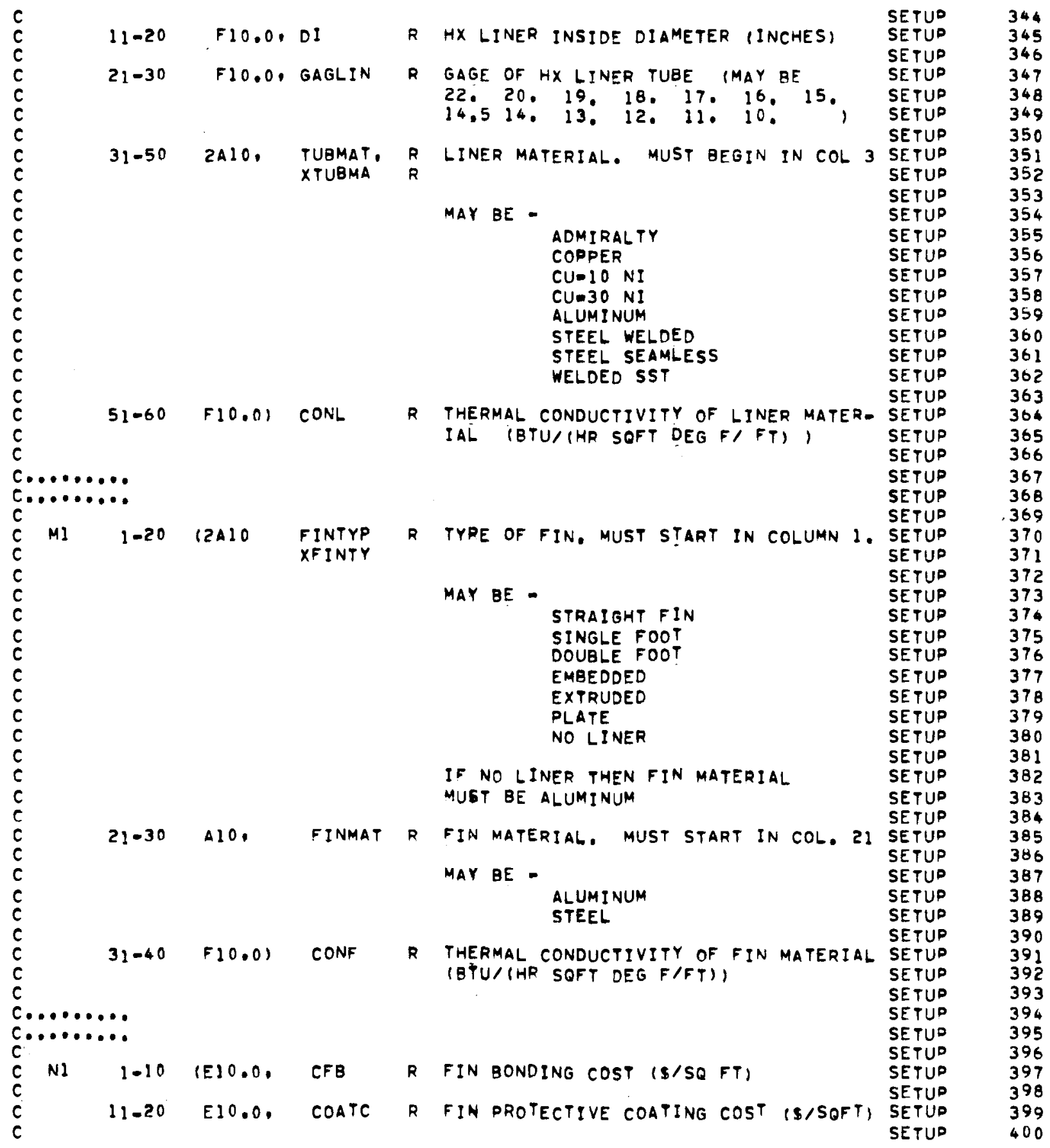




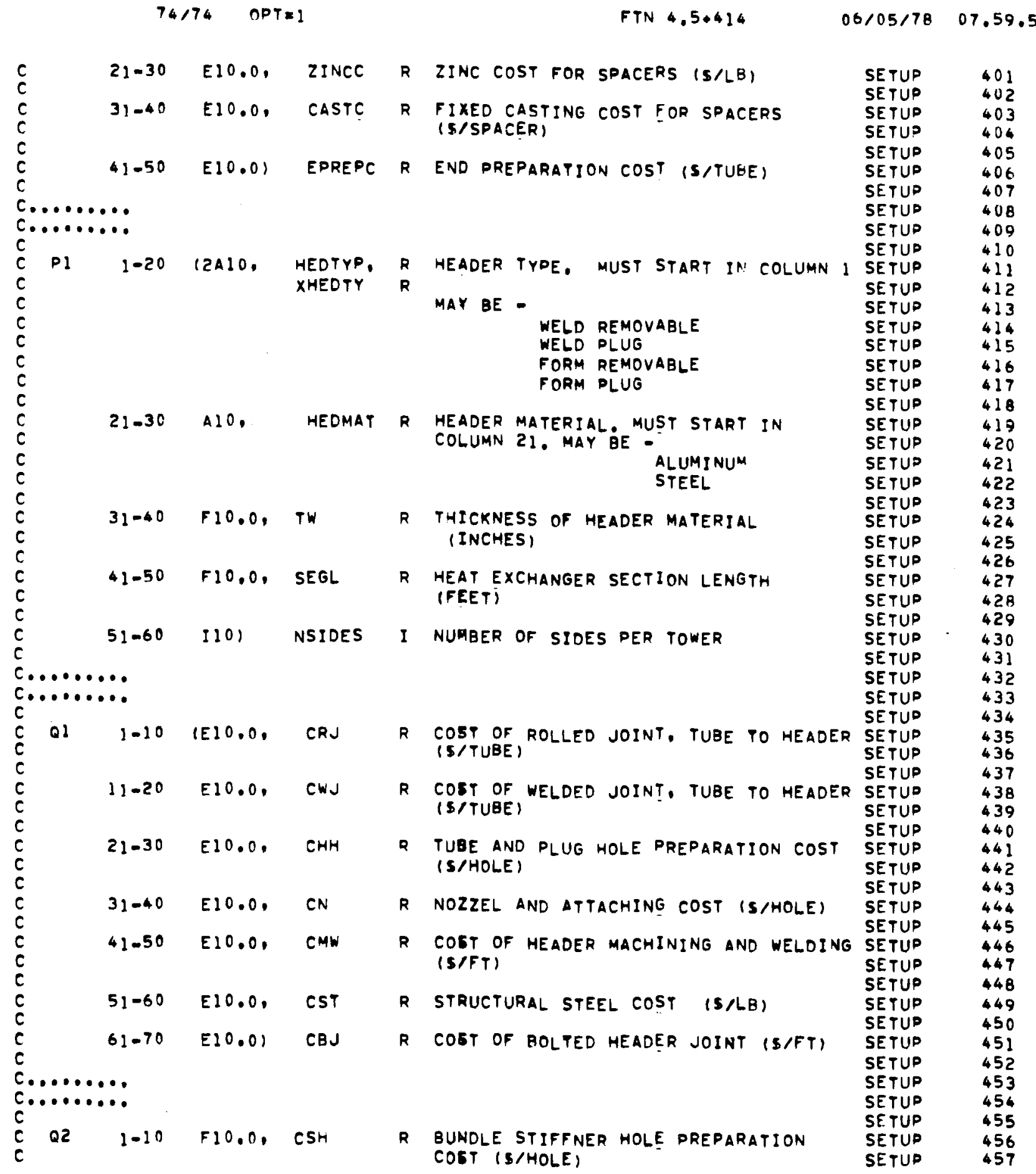


74/74 OPT:

FTN $4.5+414$

$06 / 05 / 78$

07.59 .59

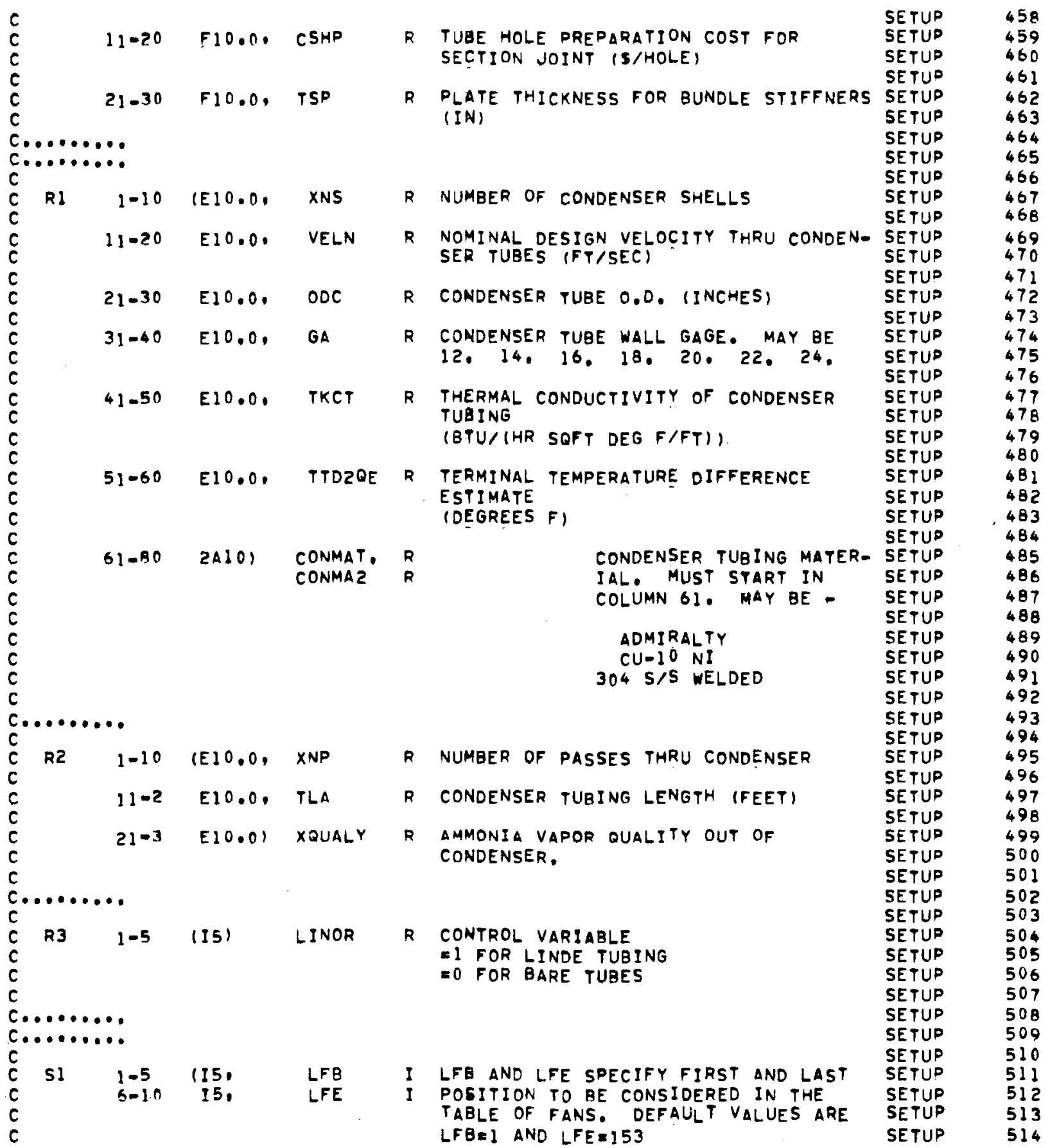


$74 / 74 \quad$ OPT $=1$$$
\begin{aligned}
& c \\
& c \\
& c \\
& c \\
& c \\
& c \\
& c \\
& c \\
& c \\
& c \\
& c \\
& c \\
& c \\
& c \\
& c \\
& c \\
& c \\
& c \\
& c \\
& c \\
& c \\
& c \\
& c \\
& c \\
& c \\
& c \\
& c \\
& c \\
& c \\
& c \\
& c \\
& c \\
& c \\
& c \\
& c \\
& c \\
& c \\
& c \\
& c \\
& c \\
& c \\
& c \\
& c
\end{aligned}
$$

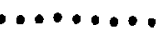

c.........

$T 1$

$1-10 \quad$ A10
FTN $4.5+414$

$06 / 05 / 78$

07.59 .59

\begin{tabular}{ccc} 
& & \\
\hline DIAMETER & IFAN & LFAN \\
\hline 24. & 1 & 27 \\
26. & 28 & 54 \\
28, & 55 & 88 \\
30. & 89 & 111 \\
40. & 112 & 135 \\
60. & 136 & 153
\end{tabular}

11-15 (5) MXEXT I THE MAXIMUM ALLOWABLE NUMBER OF CALLS TO SUBROUTINE XTEND FOR ANY ONE DESIGN TEMPERATURE.

DEFAULT VALUE IS 10

FFHX

R

CONTROL VARIABLE FOR SPECIFYING METHOD TO USE IN COMPUTING FRICTION FACTOR. MUST START IN COLUMN 1 . MAY BE -

$$
\text { HOTERV }
$$

$11-20$ AIO FSHO

R

CONTROL VARIABLE FOR SPECIFYING TYPE OF SEARCH ROUTINE TO BE USED IN SEARCH FOR OPTIMUM MAY BE -

\section{SKIP SHOT} CONSTANT

$21-30 \quad A 10 \quad$ FDELUG $R$

CONTROL VARIABLE FOR SELECTING TYPE OF METHOD TO CALCULATE EVAPORATIVE HEAT TRANSFER MAY BE -

DELUG

DELBAW

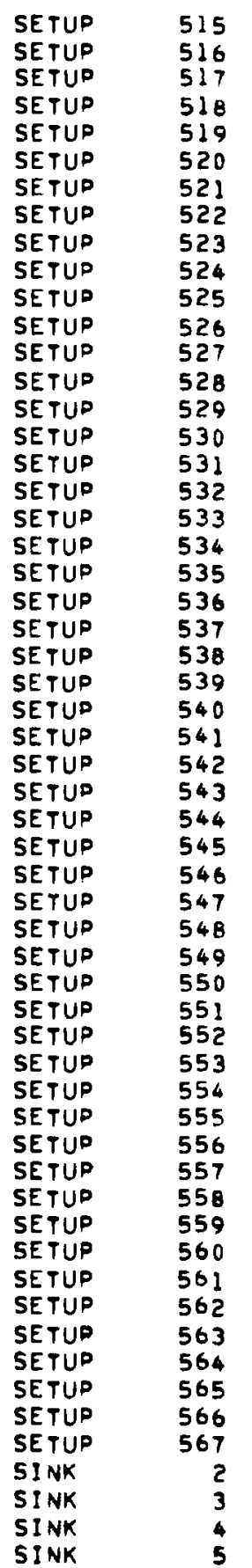

REAL NTUB

DIMENSION TOD (6), TGA (7)

COMMON/OJB/DI, XOG,SF

COMMON /INOIS/ DIST

COMMON /HEADEX/ CHH,CN,CHM,CMW,CRJ,CWJ,CST,CMO,CSR,CSM,PITCHF,CDU, -PRESS, HEDMAT, HEDTYP, NPASS, WB, TSP, CSH, CSHP

COMMON /SURFEX, ODL, GAGLIN, NTUB, TUBMAT, FINTYP, FINMAT, CFB, APLATE, SCOATC, ZINCC, CASTC, SS,EPREPC, XTUBMA, XHEDTY, XFINTY

COMMON /TINE/ XNS, TLA, XNTS, ARPS, TTOZQE, LINOR, XNP, XQUALY COMMON/TINEZ/CCM

COMMON/TUBE/KGA,LD,TID (7,6)

COMMON $O M T C / V E L N, O D C, T K C T$ COMMON /SINK' VAR(5), CPLNI, CPIPI, CCOSI,
OINI, CLANI, REAII, ZI, HQFAZS, WHI. FPOWI, DEFCI, WIOTI,

W31. COSMI. EFFl,

UI, DEPWI, PPOWl. ELENI, VAIRI, VWATI, HII, HOI, T4l, NPI, ZPLNCZ,ZSIZ12, ZSPBP, SW41, SW3!
NWI. NTI.
REWAI.
NPIOZONCZ, ZSIZIZ

\begin{abstract}
ZSPBP. SW4l, SW3I.
\end{abstract}


5 CLTC, CFC, CPCC, FIYCO, CSC, CEPREP,DELPIP, AST,OLDCST S, CADCST, XNFAN, BLDANG, CPLEN, DPFEL, WHX, WTTUB, ADIA, ABLN

1. WTHDR, WTFRM, STRUCC,CFPERF,HPPERF, TOTCF, TPC, EFFIN, ANTU

5. $A I D F F$, WATFF, XNMOD, TCTPF, FDCPF, FMTRC, ACCTTP, ADJTP

5, $\triangle C T B P D, A D J B P D, A C T V H, A D J V H, A D J P P F, T H D A I R$, TCTF, AFCST

5. CSRD, WATERW, WSTRCT, CFOUND, HUBDIA, TOWLEN, NUMTOW, CTOWD

5 , SQRJ.SORJS,ATUBC, CBA, TTDI, TTDZ, DELOS, CSTLVR, PIDCSTD

- CSTHS, CYLRNG, CFANEL, PSTACS, PUFITC, PMPCST, RPTPL, SEPCST

\$.PWO (16), WATCWD (16), DEPER (16) , DEPERO (16)

\$ WBB. HTOW, NTOWDE, CSTIF, CSJ, CVF, CDP,W5

COMMON /VARVAR/ TPER (16), TA(16): TAWB(16

COMMON/FAN/ FANDAP $(153,10)$, FANDAH $(153,10)$, FMCPF, FBCPF, P2,R, 5.

SVELREC,AFC, ELEV, EHR

COMMON/CONS/HD, BETAF, BETAI, $X X(6)$

DATA BLANK/" "I

C SURFACE CONDENSER TUBE OD ANO TUBE gage arRaYS

DATA TOD/0.625,0.750,0.875,1.000.1.125.1.250/

DATA TGA/12.114.116.118..20.122.124.1

$c$
$c$
$c$
$c$

CONDENSER TUBE ID AND GPM PER FPS VELOCITY THROUGH TUBE AS A FUNCTION OF TUBE GAGE AND TUBE OD.

6000 FORMATIIHI///TIO. "BNWII DRY/WET COOLING TOWER COST MODEL -

S." AMMONIA CIRCULAR TOWER VERSION"

S, T85, "THIS RUN MADE",A10,5X,A10)

5001 FNRMAT 184101

6001 FORMAT (T21.8A10)

5002 FORMAT (BEI0.0)

5012 FORMAT (6EI0.0.110)

6002 FORMAT $/ 1 \times .8615,8$ )

6012 FORMAT $(1) \times, 6 G 15, B, 115)$

5003 FORMAT (16F5.0)

6003 FORMAT $(1 / 1 \times 8 F 8,1, A F B .4)$

5004 FORMAT (F $10.0 .4 F 15.8)$

6004 FORMAT $(/ 1 X, F 10.2 .4 G 15 . B)$

5005 FORMAT $(6 F 10.0)$

6005 FORMAT (/1X.6615.8)

5006 FORMAT $13 F 10.0,2 A 10, F 10.01$

6006 FORMAT $(/ 1 X, 3 F\{0.5,1 X, 2 A 10, F \perp 0.5)$

5007 FORMAT $(3 A 10.5 F 10.0)$

6007 FORMAT $(11 X, 2 A 10.1 X, A 10.5615 .8)$

5008 FORMAT (BI5)

6008 FORMAT $(/ 1 \times, B I 6)$

5009 FORMAT $16 E 10.0 .2 A 10)$

6009 FORMAT $(/ 1 \times, 6615,6.5 \times .2 A 10)$

5010 FORMAT $(3 A 10,2 F 10.0,110)$

6010 FORMAT1/1X,2A10, IX,A10,2G10,2,1101

5011 FORMAT (TEI0.0.2F5.0)

6011 FORMATI/1X,BG15.8,G10.41

SUPPLY 9

SUPPLY 11

SUPPLY IO

SUPPLY 13

SUPPLY 14

SUPPLY 15

SUPPLY 16

SUPPLY 17

SUPPLY 18

SETUP 577

SETUP 578

SETUD $\quad 579$

SETUP 580

SETUP 5BI

SETUP 582

SETUP 583

SETUP 584

SETUP 585

SETUP 586

SETUP $\quad 587$

SETUP 588

SETUP 589

SETUP 590

SETUD 591

SETUP 592

SETUP 593

SETUP 596

SETUP 595

SETUP 596

SETUD 597

SETUD 598

SETUP 599

SETUP 600

SETUP 601

SETUP 602

SETUP 603

SETUP 604

SETUP 605

SETUP 606

SETUD $\quad 607$

SETUP 608

SETUP 609

SETUP 610

SETUP 611

SETUP 612

SETUP 613

SETUP 614

SETUP 615

SETUP 616

SETUP 617

SETUP 618

SETUP 619

SETUP 620

SETUP 621

SETUP 622

SETUP 623 
5014 FORMAT (4AIO)

6OI FORMAT $(/ 1 X, 4 A 10)$

7060 FORYAT"TG,"TSTAR", T21, "TEND",T36,"VAS(1)",T5), "VAS (2)", Y66, S"VAS (3)". TB! "VAS (4)", T96."VAS (5)")

706 I FRMAT (T6, "TFIX", T2 I, "F IXV", T36, "F IXTTD", T5I, "F IXL", T66, "DEEPL")

7090 FORMATI \&X, "TA(1)".3X"TA(2)", 3X"TA(3)", 3X"TA (4)", 3X"TA(5)"

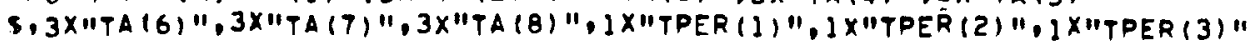

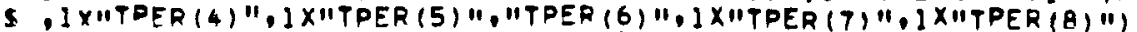

7120 FORMAT1 4X"TA $(9) ", 2 X " T A(10) ", 2 X " T A(1)$ ", $2 X " T A(12)$ ", XXTA(13)" S2X"TA(14)", $2 \times 1 T A(15) ", 2 \times 1 T A(16) " \cdot 1 \times " T P E R(9)$ TPER(10)TPER(1) TPER(1 S2TPER (13) TPER (14) TPER (15) TPER (16)")

7130 FORMAT (4X,"TWB(1) TWB(2) TWB(3) TWB(4) TWB(5) TWB(6) TWB(T) SETUP

I TWB(8)",IX,"BPLM(1) BPLM(2) BPLM(3) BPLM(4) BPLM(5) BPLM(6) BPLM) SETUP 27) $Q P L M(B) 11)$

SETUP

SETUP

SETUP

SETUP

SETUP

SETUP

SETUP

SETUP

7140 FORMAT (4X, $T W B(9)$ TWB(10) THB(11) TWB(12) TWB(13). TWB(14) TWB(15) SETUP ITWB(16)".1X,"BPLM(9) BPLM(10) BPLM(11)BPLM(12) BPLM(13)BPLM(14)BDL4) SETUP $215) \mathrm{BP}(M(16) 11)$

7150 FORMAT IT5,"PSIZE",T23,"TEFF", T38, "CAPF", T54, "FCR", T69, "PER" 5. TA4,"CCM", T96, "ELEV", T108, "ROOFL")

7180 FORMAT IT5, "FCOS",T23, "PWCOS",T3B,"PLANC", T52, "COSTL",T67, "CSSPKW" S ,TAZ, "CADCHG",T97,"HPCST",T112, "CTURB")

7185 FORMAT 1T5,"POHBAF", T20, "POHFAN",T35, "POHLEC", T50, "POHCIR", S T65, "POHCND", T80, "POHSTC", T95, "POHSCL")

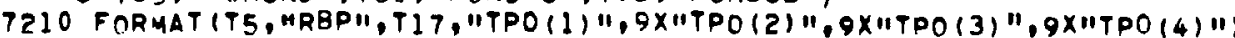

7240 FORMAT (T5. "EFFPU)

7270 FORMAT (T4, "VELREC", T20, "XDEPA", T35, "TLIM", T50, "TOWMIN", T65, S "TOWMAX", T90, "PFACT")

T2AO FORMAT (5X, "IGBEFF")

7300 FORMAT IT5, "CPM", T20, "WPL", T35, "CLUVR", T50, "CHAILS", T65, "UCS", TBO S." "UWS", T95, "CVM", T110, "WFV")

7330 FORMAT (T5, "HXNP", T24, "WB", T44, "NTUB", T58,"SS", T75, "ANGLE")

7335 FORMAT (T5, "DESVEL", T21, "REDUCE", T35, "OREDUCE", T50, "OIST 1 )

7340 FORMAT (T5, "DESVELV", T21, "REDUCV", T35, "OREDUCV", T50, "DESVELD",T65, I"FACTORD"I

7345 FORMAT 1T5, "WATCONA", T20, "WATCOSTH, T36, "FACTOR", T50, "HCDH, T65, J"RETAF", TBO, "BETAI", T95, "RHOUT"!

7360 FORMAT 15X,"XW", 13X,"XD", 13X,"XDG", 12X,"SF", 13X,"DFIN", I IX, S"THFIN")

7390 FORMAT (5X"ODL", I 2X"DI", 03X"GAGLIN", 9X"TUBMA", I 3X"CONL")

7420 FORMAT ( 3 X"F INTYD", 14 X"F INMAT", 4 X "CONF")

7450 FORMAT 15X"CFB", 12X"COATC",10X"ZINCC", 10X"CASTC", 10X, "EPREPC")

7480 FORMATIT4, "HEDTYDH, T24, "HEDMAT",T36, "TW", T50, "SEGL", T65, "NSIDES")

7490 FORMAT(T5,"CSH", $12 X$, "CSHP", $12 X, " T S P ")$

7510 FORMAT 15X,"CRJ", 12X,"CWJ", 12X,"CHH", 2 2X,"CN", $12 x_{\text {, }}$ "CMW", $12 x_{,}$"C5T", S $12 x \cdot$ "CBJ")

7540 FORMAT I5X"XNS", 1 2 X"VELN", 11 X"ODC", 12X"GA", $10 X " T K C T ", 10 X$, "TTOZOE" 5 . $15 \times$ "CONMAT")

7550 FORMAT (5X,"XNP", I OX, "TLA", 1 OX,"XQUALY")

7555 FORMAT ( $3 X_{0}$, "LINOR")

7570 FORMAT (TA, "LFB", TII, "LFE", TI5, "MXEXT")

7580 FORMAT (5X,"FFHX",6X,"FSHOT",5X, "FDELUG")

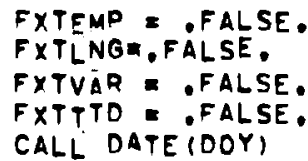

SETUP

SETUP

SETUP

SETUP

SETUP

SETUP

SETUP

SETUP

SETUP

SETUP

SETUP

SETUP

SETUP

SETUP

SETUP

SETUP

SETUP

SETUP

SETUP

SETUP

SETUP

SETUP

SETUP

SETUD

SETUP

SETUO

SETUP

SETUP

SETUP

SETUP

SETUP

SETUD

SETUP

SETUP

SETUP

SETUP

SETUP

SETUP

SETUP

SETUP

SETUP

624

625

626

627

628

629

630

631

632

633

634

635

636

637

638

639

640

641

642

643

644

645

646

647

648

649

650

651

652

653

654

655

656

657

658

659

660

661

662

663

664

665

666

667

668

669

670

671

672

673

674

675

676

677

678

679 


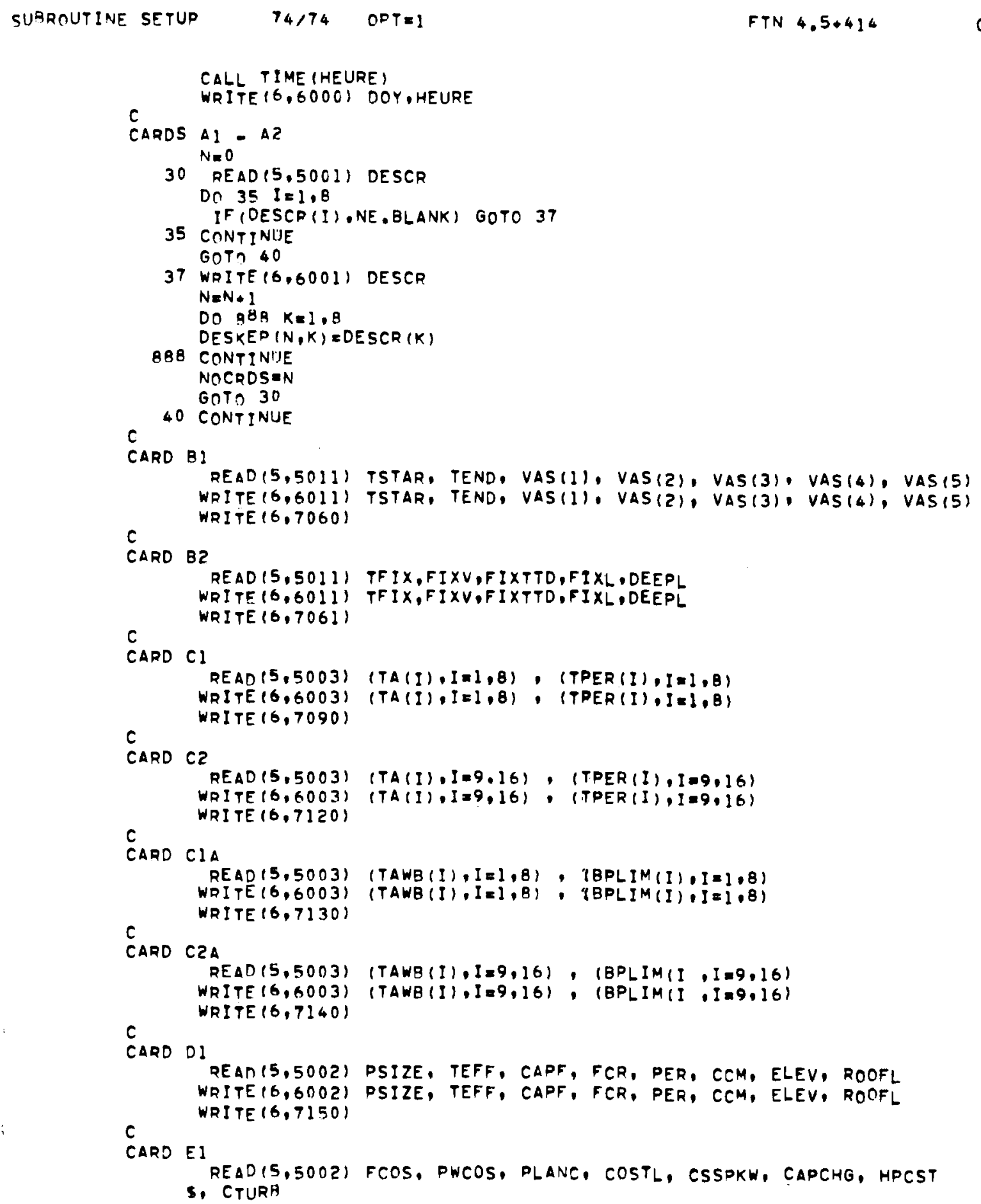

$\begin{array}{ll}\text { SETUP } & 681 \\ \text { SETUP } & 682 \\ \text { SETUP } & 683 \\ \text { SETUP } & 684 \\ \text { SETUP } & 685 \\ \text { SETUP } & 686 \\ \text { SETUP } & 687 \\ \text { SETUP } & 688 \\ \text { SETUP } & 689 \\ \text { SETUP } & 690 \\ \text { SETUP } & 691 \\ \text { SETUP } & 692 \\ \text { SETUP } & 693 \\ \text { SETUP } & 694 \\ \text { SETUP } & 695 \\ \text { SETUP } & 696 \\ \text { SETUP } & 697 \\ \text { SETUP } & 698 \\ \text { SETUP } & 699 \\ \text { SETUP } & 700 \\ \text { SETUP } & 701 \\ \text { SETUP } & 702 \\ \text { SETUP } & 703 \\ \text { SETUP } & 704 \\ \text { SETUP } & 705 \\ \text { SETUP } & 706 \\ \text { SETUP } & 707 \\ \text { SETUP } & 708 \\ \text { SETUP } & 709 \\ \text { SETUP } & 710 \\ \text { SETUP } & 711 \\ \text { SETUP } & 712 \\ \text { SETUP } & 713 \\ \text { SETUP } & 716 \\ \text { SETUP } & 715 \\ \text { SETUD } & 716 \\ \text { SETUP } & 717 \\ \text { SETUP } & 718 \\ \text { SETUP } & 719 \\ \text { SETUP } & 720 \\ \text { SETUP } & 721 \\ \text { SETUP } & 722 \\ \text { SETUP } & 723 \\ \text { SETUP } & 724 \\ \text { SETUP } & 725 \\ \text { SETUP } & 726 \\ \text { SETUP } & 727 \\ \text { SETUP } & 728 \\ \text { SETUP } & 729 \\ \text { SETUP } & 730 \\ \text { SETUP } & 731 \\ \text { SETUP } & 732 \\ \text { SETUP } & 733 \\ \text { SETUP } & 734 \\ \text { SETUP } & 735 \\ \text { SETUP } & 736 \\ \text { SETUP } & 737 \\ \text { STU } & \end{array}$




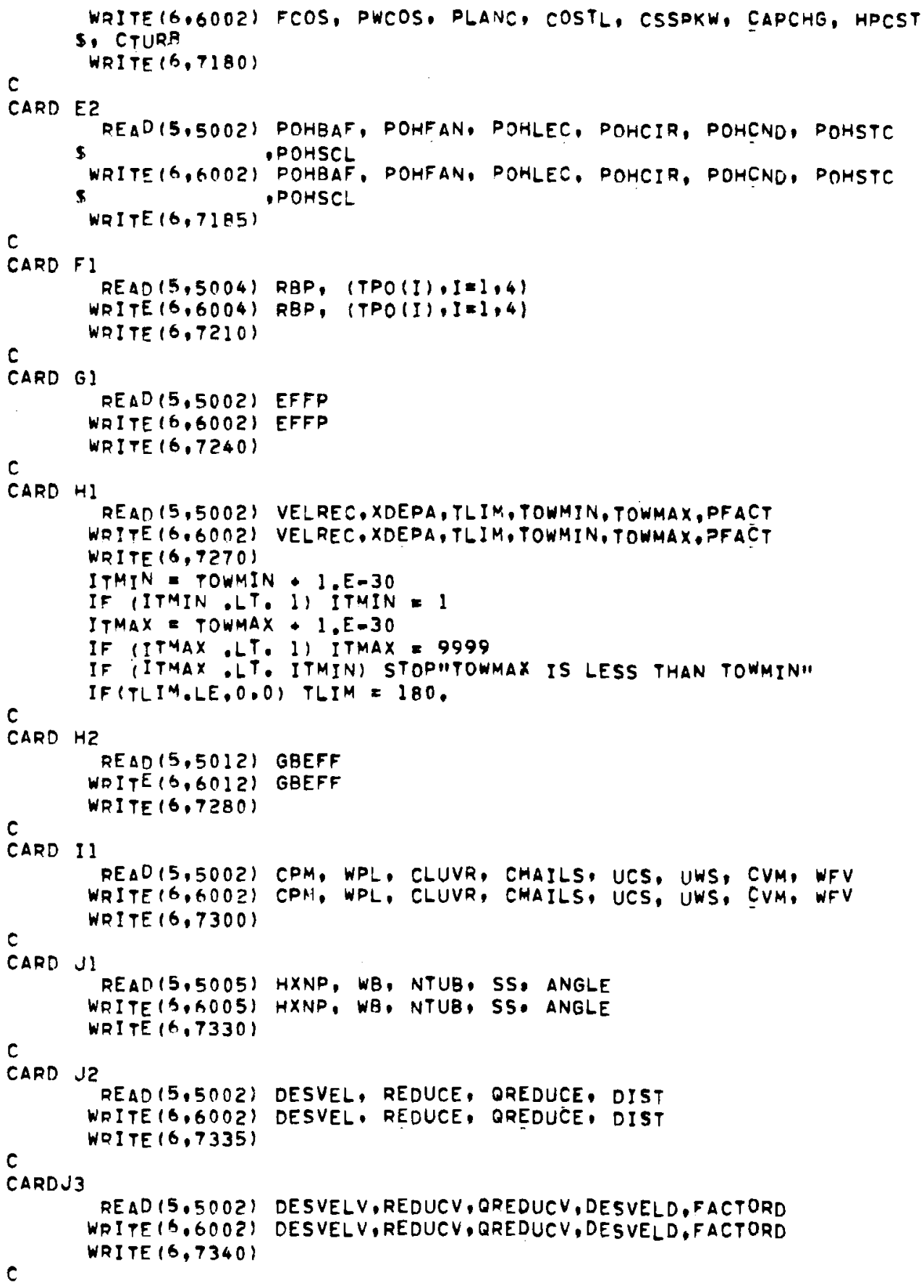

$\begin{array}{ll}\text { SETUP } & 738 \\ \text { SETUP } & 739 \\ \text { SETUP } & 740 \\ \text { SETUP } & 741 \\ \text { SETUP } & 742 \\ \text { SETUP } & 743 \\ \text { SETUP } & 744 \\ \text { SETUP } & 745 \\ \text { SETUP } & 746 \\ \text { SETUP } & 747 \\ \text { SETUP } & 748 \\ \text { SETUP } & 749 \\ \text { SETUP } & 750 \\ \text { SETUP } & 751 \\ \text { SETUP } & 752 \\ \text { SETUP } & 753 \\ \text { SETUP } & 754 \\ \text { SETUP } & 755 \\ \text { SETUP } & 756 \\ \text { SETUP } & 757 \\ \text { SETUP } & 758 \\ \text { SETUP } & 759 \\ \text { SETUP } & 760 \\ \text { SETUP } & 761 \\ \text { SETUP } & 762 \\ \text { SETUP } & 763 \\ \text { SETUP } & 764 \\ \text { SETUP } & 765 \\ \text { SETUP } & 766 \\ \text { SETUP } & 767 \\ \text { SETUP } & 768 \\ \text { SETUP } & 769 \\ \text { SETUP } & 770 \\ \text { SETUP } & 771 \\ \text { SETUP } & 772 \\ \text { SETUP } & 773 \\ \text { SETUP } & 774 \\ \text { SETUP } & 775 \\ \text { SETUP } & 776 \\ \text { SETUP } & 777 \\ \text { SETUP } & 778 \\ \text { SETUP } & 779 \\ \text { SETUP } & 780 \\ \text { SETUP } & 761 \\ \text { SETUP } & 782 \\ \text { SETUP } & 783 \\ \text { SETUP } & 786 \\ \text { SETUP } & 785 \\ \text { SETUP } & 786 \\ \text { SETUP } & 787 \\ \text { SETUP } & 788 \\ \text { SETUP } & 789 \\ \text { SETUP } & 790 \\ \text { SETUP } & 791 \\ \text { SETUP } & 792 \\ \text { SETUP } & 793 \\ \text { SETUP } & 796 \\ & \end{array}$




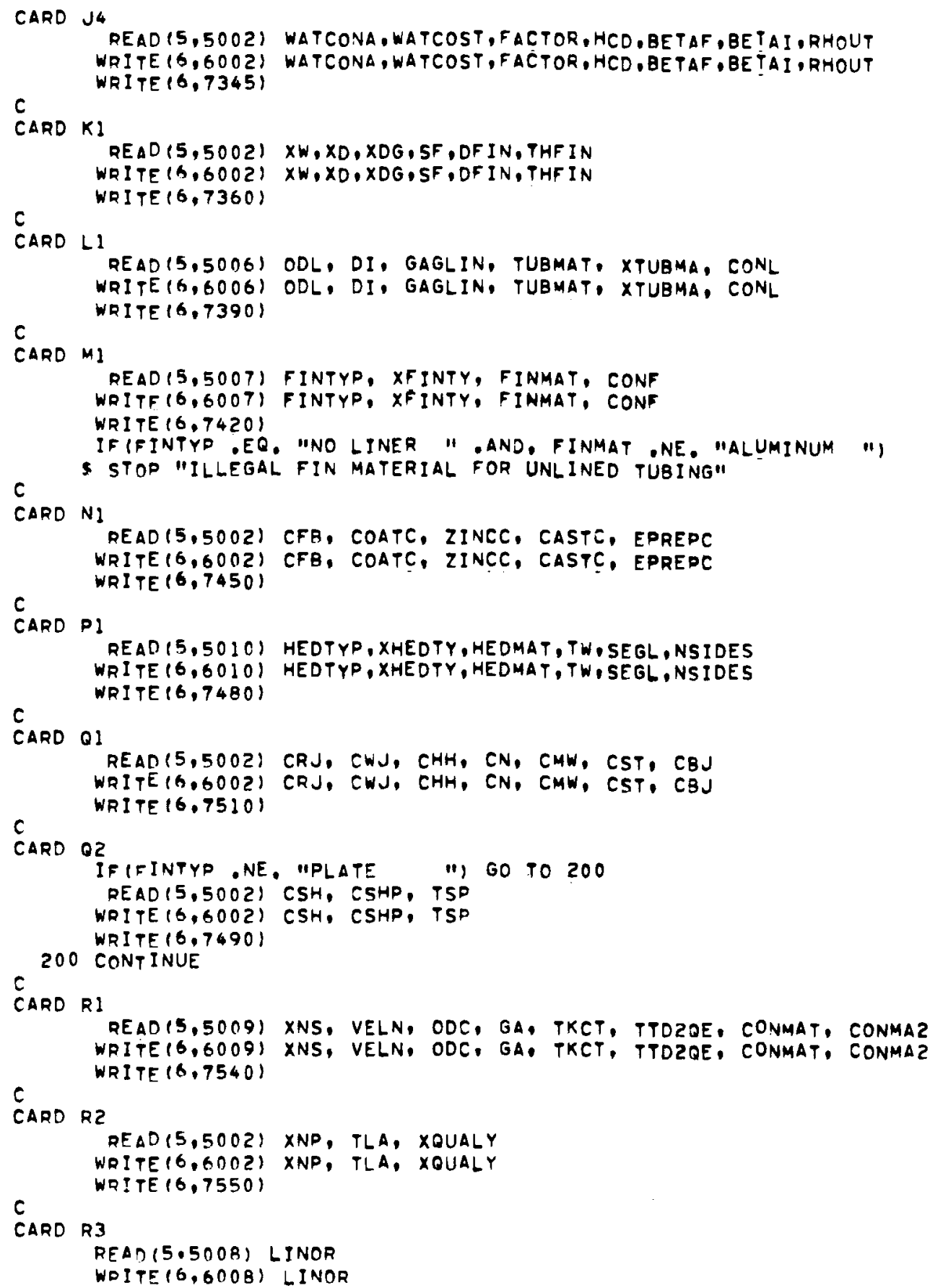

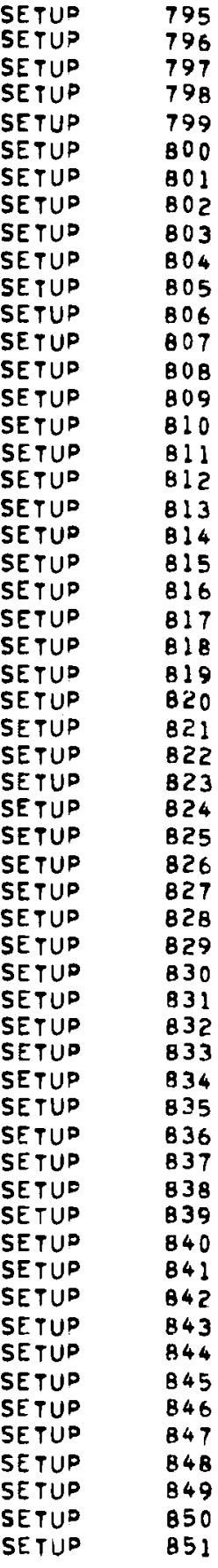


$C$ CARD SI

REAT $(5,5008)$ LFB, LFE, MXEXT

WRITE $(6,6008)$ LFB, LFE, MXEXT

WRITE (6.7570)

IF (MXEXT.LT. 1) MXEXT $=10$

C

IF ILFE .LT. LFB) STOP "LFB EXCEEDS LFE ON INPUT CARD"

CARD TI

READ $(5,5014)$ FFHX,FSHOT,FDELUG

WRITE $(6,6014)$ FFHX,FSHOT,FDELUG

WRI TE $(6,7590)$

IFIFFHX. NE. "PFR " AND. FFHX .NE, "FRICHO "

\$. ANO. FFHX. NE. "HOTERV ")

c

5 STOP "*a* ** UNRECOGNIZEO FFHX ON CARD TI"

$E H R=3414.43 /$ TEFF

IF (FIXL',GT,0.0) FXTLNGE. YRUE.

IF (FIXV .GT. 0.00 ) FXTVAR = .TRUE.

IFIFIXTTS .GT. 0.00) FXTYTD: .TRUE.

IF (TFIX,GT. 0.00$)$ FXTEMP = .TRUE.

DO $45 \mathrm{~J}=1,16$

IF(TPER (J).LT.1,E-20) GOYO 46

NTAZJ

45 CONTINUE

46 CONTINUE SMPER $=0.0$

DO $20 \quad I=1.16$

20 SMPER=SMPER. TPEA(I)

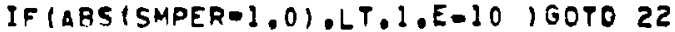

WRITE $(6,89)$

;

$$
\text { STOD }
$$

c

22 CONTINUE

C

)

C

DETERMINE POSITION OF TUBE O.D. AND GAGE IN RESPECTIVE ARRAYS

DO $90 \quad I=1.6$

$\angle D=I$

IF( ABS IODC-TOD(LD), .LT. .001) GOTO 1011

j

99 CONTINUE

C

STOD "ILLEgal VAlUe fop condenser tUBe od"

1011 DO $98 \quad 1=1,7$

$K G A=I$

IF( ABS(GA-TGA(KGA)).LT. .0O1) GOTO 100

)

98 CONTINUE

STOD "ILlegal VALUe fOR CONDENSER tube gage"

C

100 CONTINUE

WATCONA = WATCONA*2719015.

j

ANG (1) = ANGLE*3.141592654/180.

ANG $(2)=$ SIN(ANG(2))

ANG (3) $=\operatorname{COS}(A N G(1))$

c

IF (ARS(ANG (3)).LT,.0001) ANG(3)=0.0

SETUP

SETUD

SETUP

SETUP

SETUP

SETUP

SETUD

SETUP

SETUP

SETUP

SETUP

SETUD

SE TUP

SETUP

SETUP

SETUP

SETUP

SETUP

SETUP

SETUP

SETUP

SETUP

SETUD

SETUP

SETUP

SETUP

SETUP

SETUP

SETUP

SETUP

SETUP

SE TUP

SETUP

SETUP

SETUP

SETUP

SETUP

SETUP

SE TUP

SETUP

SETUP

SETUD

SETUP

SETUP

SETUP

SETUD

SETUP

SETUP

SETUP

SETUD

SETUP

SETUP

SETUP

SETUP

SETUD

SETUP

SETUP
852

853

854

855

856

857

858

B5o

$B 60$

86 !

86 .

86 ;

864

8: 5

806

8.7

$B 68$

869

870

871

B72

873

874

B75

876

B.7

878

879

B

B: 1

BF

BA 2

$B B_{4}$

885

886

B87

$B$ B

889

890

ค9)

892

893

894

$B 95$

B. 6

897

B 38

990

900

901

912

963

9.

9.5

906

907 


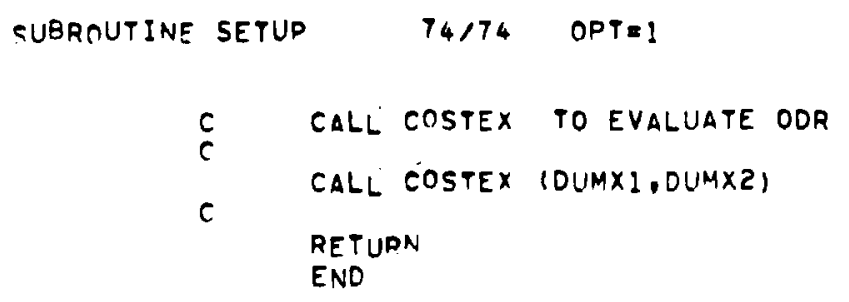

FTN $4.5 \cdot 414$

$06 / 05 / 78 \quad 07.59 .59$

$\begin{array}{ll}\text { SETUD } & 910 \\ \text { SETUP } & 910 \\ \text { SETUD } & 911 \\ \text { SETUP } & 912 \\ \text { SETUP } & 913 \\ \text { SETUD } & 914\end{array}$


DIMENSION T(3), CWAR (4), RANG (5), AFRO (5), WLRA (4), VAM(5) LOGICAL FIRST, FRST, FXTEMP, FXTVAR, FXTTTD,FXTLNG COMMON

S AFTR, ALPHA, ANG (3)

- BDLIM(16)

S CADF, CSSPKW, CONF, CONL, COSTL, CAPCHG, CONMAT, CONMAZ, CAF

SHOT

SHOT

BIGCOM

BIGCOM

BI GCOM

HIGCOM

BI GCOM

CAJ, CTURB, CDANG, CLUVR, CHAILS, CVM

$\$$.DFIN.DEEPL, DESVEL, DESVELV, DESVELD, DENSIF

S. EFFP: EFS

BI GCOM

BIGCOM

5 ,FCR,FIRST, FIXL,FCOS, FFHX, FXTEMP, FACTOR, FRST, FACTORD, FSHOT, FDELUG

S ,FIXV,FIXTTD,FXTVAR, FXTTTD,FXTLNG

F. GQEFF

S. HXND,HPCST, HCD

S. ITMAX, ITMIN

2 , JCONS

. KCONV, KALEXT

5 , MXEXT

S NTA, NSIDES, NBUNHI

COMMON

$\$$ ODR

* PSIZE, PER, PWCOS, PLANC, PFACT, POMDPL

S. POHBAF, POHFAN, POHLEC, POHCIR, POHCND, POHSTC, POHSCL

S RRP, REDUCE, REDUCV, ROOFL, RHOUT

? QREJ, QREDUCE, OREDUCV

S SIGMAG, SAAF, SEGL

S, TD, TPO(4), TEFF, TLIM, THFIN, TLPRA, TFIX

S. TCD. TW

S.UES, UWS

S WFV. WATCONA, WATCOST

$\$$ VAS (5)

\$, XDEPA, XW, XO

COMMON /LACALL/ SRN

COMMON /SUPPLY VAL 51 I,

BIGCOM

BIGCOM

BIGCOM

BI GCOM

BI GCOH

BI GCOM

BI GCOM

BI GCOM

BI GCOM

BI GCOM

BI GCOM

BI SCOM

BI GCOM

BI GCOM

BIGCOM

BI GCOM

BI GCOM

BI GCOM

BI GCOM

BIGCOM

BI GCOM

BI GCOM

SHOT

SUPPLY

SUPPLY

SUPPLY

SUPPLY

SUPP:Y

SUPPLY

SUPPLY

SUPPLY

SUPPLY

SUPPLY

SUPPLY

SUPPLY

SUPPLY

SUPPLY

SUPPLY

SUPPLY

SUPPLY

SI NK

UI. DEPWI, PPOWI: SINK
HII, HOI, T4I, SINK 
5 REWAI, NWI, NTI, NPI, ZPLNCZ,ZSIZIZ, ZSPBP, SW4I, SW3I,
5 SPCI, PTOTI,PLNC3I, AFROI, AAIRI, YFPMIN, SPCDI, SPSI3,WTZMIN, 5 BPZMIN,NWZMIN, TCOSI, DEPAI, DPWTI, DPWCI,CONBAI, XNTSI, VELDI 5 - TLAI, UCONI, PMCSI. FMCSI. FBACI. EFFCI, CHI, CAI, CSI 5. CLTCl. CFCl. CPCCI.FITCOI. CSCl,CEPREI.DEPIPI. ASTI.OLDCSI S. CADCSI, XNFANI, BLDANI, CPLENI, DPFELI, WHI, WTTUI, ADII, ABLI 5. WTHDI. WTFRI,STRUCI,CFMPFI, HAPFI, TOTCFI, TPI,EFFINI, ANTUI 5. AIRFFI, WATFFI. XNMODI, TCTPFI, FDCPFI, FMTRCI, ACTTPI, ADJTPI

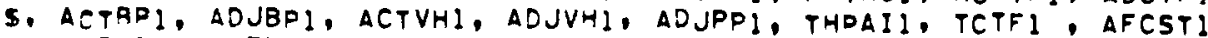
5. CSRl. WATERI, WSTRCI, CFOUNI, HUBDII, TOWIEl. NUMTOI, CTOWDI

5 , SORJI. SORJSI. ATUBCI, CBAI, TTDII, TTD2I. DELPSI, CSTLVI, PIPCTOI

5 .CFTHSI, CYLRNI, CFANEI, PSTACI, PUFIT!, PMPCSI, RPTPLI, SEPCSI

5.PWDI (16),WATCWDI (16), DEPER 1 (16),DEPERQ1 (16)

5. WBPI. HTOWI NTOWDEI, CSTIFI.CSJI.CVF I . CDPI, W5 I

COMHON /SCALER/HRFACI, ATUB, CPERA, SSCD,PLANEI,CASSSI.

5 GAID, FAIR, HPAIR, HPWAT, WLPTP, PLANCTI

COMMON /TINE/ XNS, TLA, XNTS, ARPS, TTDZQE, LINOR, XNP, XQUALY

9500 FORMATI"OIN SHOT AT CHECKPOINT A: VAR $(4)=11$, IPEI2,4,6X, UVAM(4) $=11$, SE 12,4,6X, "OAFRO =" E I2.41

9501 FORMAT "OIN SHOT AT CHECKPOINT B. IE"II, 6X, "DAFROE",1PEI2,4,6X, $\$$ "VAR (4) =",EI2,4,6X,"AFRO(I) =",EI2,4)

9502 FORMATI"OIN SHOT AT CHECKPOINT C. I $C=11,6 X, 11 V A R(4)=1,1 P E I 2.4$, $56 \times, " F A F=11, E \backslash 2,41$

9503 FORMATI"OIN SHOT AFRO WILL BE BAD BECAUSE VAS(4) ". IPEI2.4) $I D B=0$ IF (L.EQ.2) GO TO 3$)$

DO $1, T=1,5$

$1 \operatorname{VAM}(I S)=0.0$

TCOsian. $T \cos 2=0$

$\operatorname{TT}=3$

IF (FXTEMD) ITT $=$ I

DC $20 I=1 . I T T$

$T(I)=V A S(1)+(I-2) * 5$.

IF $(F X T E M D) T(I)=T F I X$

IF (TII) .GT. 180.$) T(I)=180$.

20 IF (TII) .LE, 0.0$) T(I)=10.0$

IF (.NOT. FXTTTD) GO TO 200

$L C=1$

CWAR(I) FIXTTD

Go TO 210

200 CONTINIIE

DO $71 \quad I=1.4$

CWAR II =VAS (3) + (I-2) $=2$.

21 IF (CWAR (I).LE.0.) CWARII)=.5

$L C=4$

210 CONTINUE

IF (DEEPL.GT.0.00) GO TO 220

$L W=4$

DO $22 I=1, L W$

WLRA (I) = VAS(5) * II - 2)

22 IF (WLRA II) .LE. 0.1 WLRAII) = 1.0

Go TO 221

$220 \mathrm{LW}=1$

WLRA 11 × DEEPL

22 I CONTINUE

IF (.NOT. FXTLNG) GO TO 23

SINK

SINK

SINK

SINK

SINK

SINK

SINK

SINK

SINK

SINK

SINK

SINK

SINK

SINK

SCALER

SCALER

SHOT

SHOT

SHOT

SHOT

SHOT

SHOT

SHOT

SHOT

SHOT

SHOT

SHOT

SHOT

SHOT

SHOT

SHOT

SHOT

SHOT

SHOT

SHOT

SHOT

SHOT

SHOT

SHOT

SHOT

SHOT

SHOT

SHOT

SHOT

SHOT

SHOT

SHOT

SHOT

SHOT

SHOT

SHOT

SHOT

SHOT

SHOT

SHOT

SHOT

SHOT 


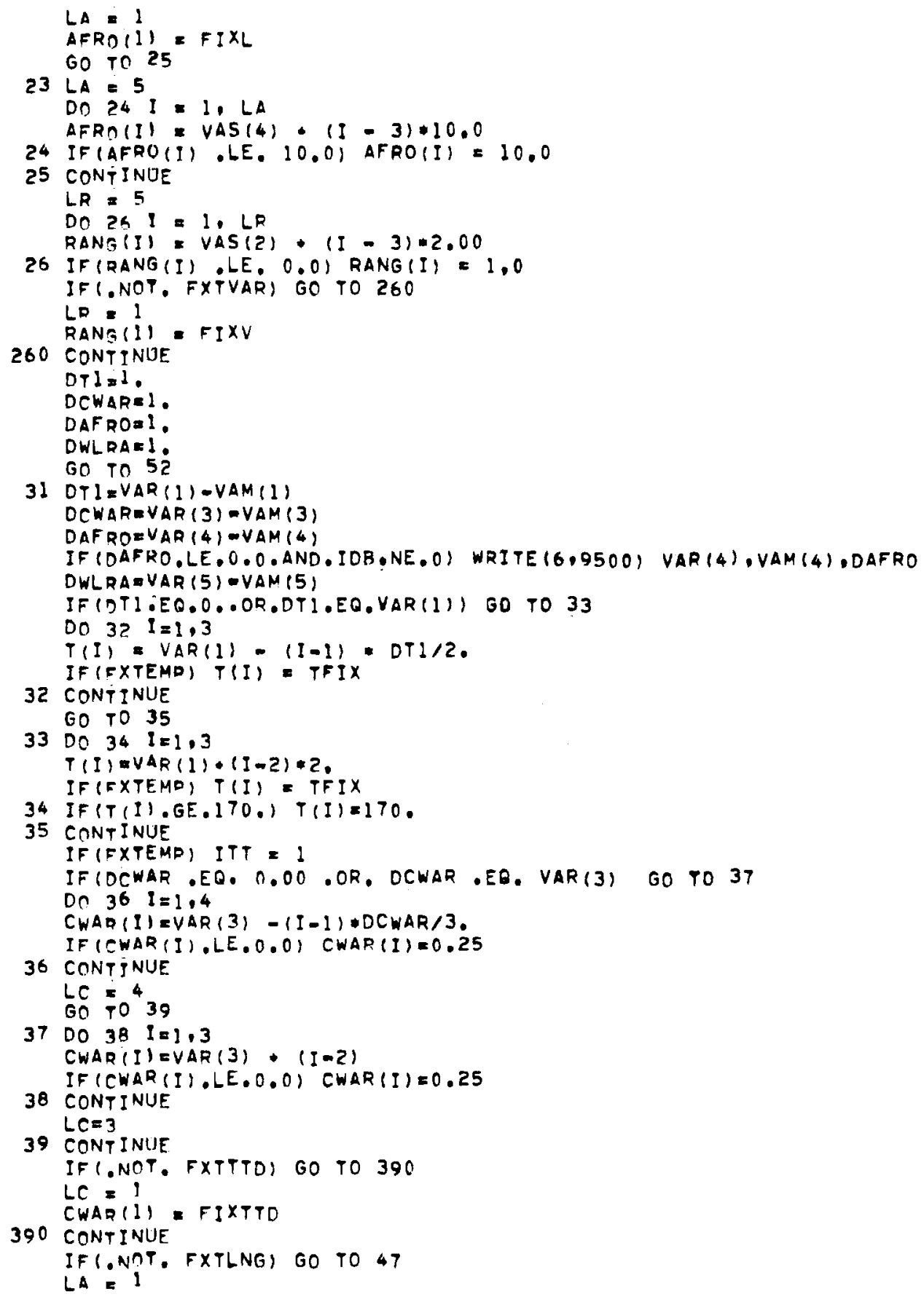

SHOT

SHOT

SHOT

SHOT

SHOT

SHOT

SHOT

SHOT

SHOT

SHOT

SHOT

SHOT

SHOT

SHOT

SHOT

SHOT

SHOT

SHOT

SHOT

SHOT

SHOT

SHOT

SHOT

SHOT

SHOT

SHOT

SHOT

SHOT

SHOT

SHOT

SHOT

SHOT

SHOT

SHOT

SHOT

SHOT

SHOT

SHOT

SHOT

SHOT

SHOT

SHOT

SHOT

SHOT

SHOT

SHOT

SHOT

SHOT

SHOT

SHOT

SHOT

SHOT

SHOT

SHOT

SHOT

SHOT

SHOT

54 


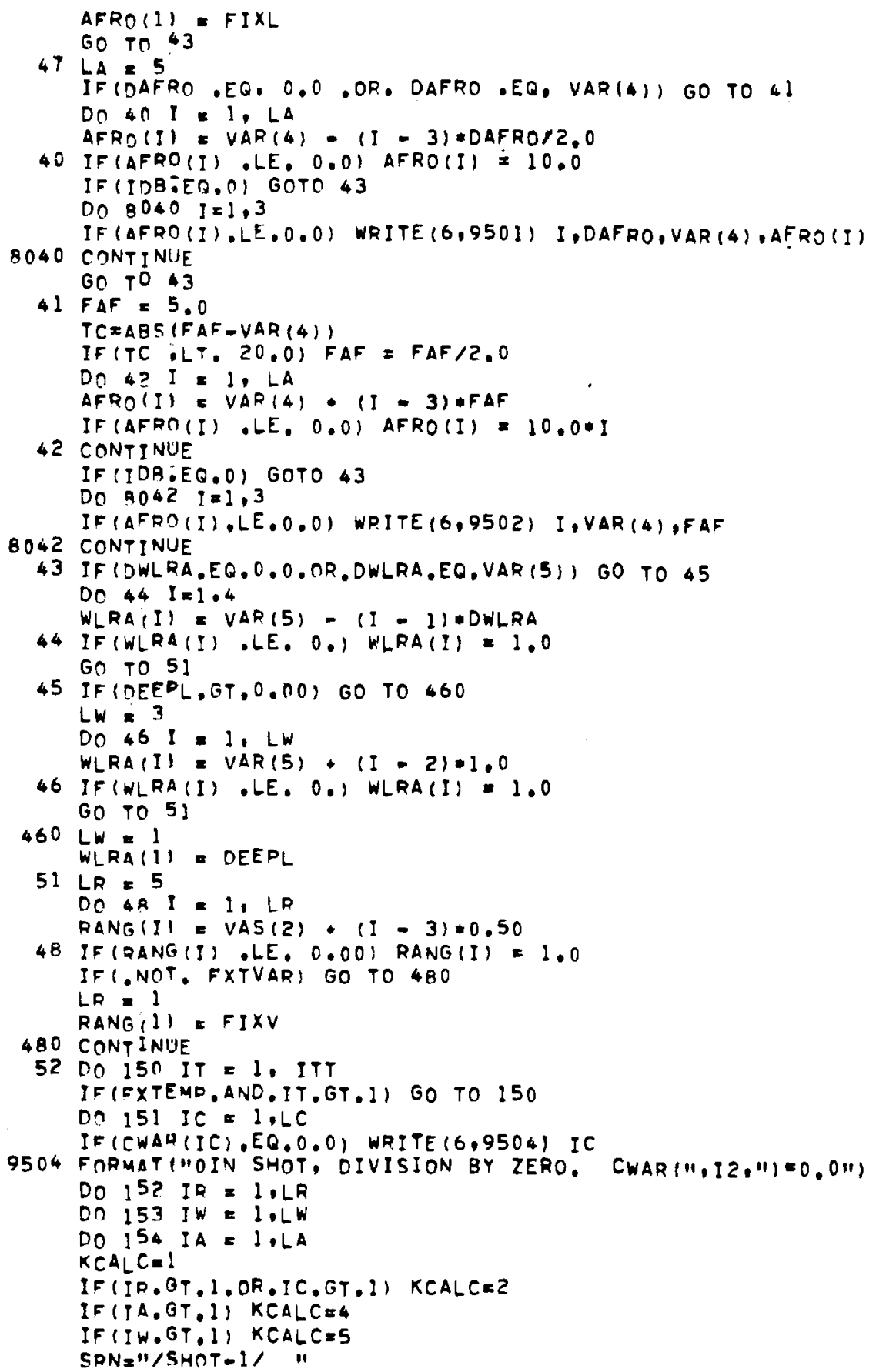

$\begin{array}{ll}\text { SHOT } & 111 \\ \text { SHOT } & 112 \\ \text { SHOT } & 113 \\ \text { SHOT } & 116 \\ \text { SHOT } & 115 \\ \text { SHOT } & 116 \\ \text { SHOT } & 117 \\ \text { SHOT } & 119 \\ \text { SHOT } & 119 \\ \text { SHOT } & 120 \\ \text { SHOT } & 121 \\ \text { SHOT } & 122 \\ \text { SHOT } & 123 \\ \text { SHOT } & 124 \\ \text { SHOT } & 125 \\ \text { SHOT } & 126 \\ \text { SHOT } & 127 \\ \text { SHOT } & 128 \\ \text { SHOT } & 129 \\ \text { SHOT } & 130 \\ \text { SHOT } & 131 \\ \text { SHOT } & 132 \\ \text { SHOT } & 133 \\ \text { SHOT } & 134 \\ \text { SHOT } & 135 \\ \text { SHOT } & 136 \\ \text { SHOT } & 137 \\ \text { SHOT } & 138 \\ \text { SHOT } & 139 \\ \text { SHOT } & 140 \\ \text { SHOT } & 141 \\ \text { SHOT } & 142 \\ \text { SHOT } & 143 \\ \text { SHOT } & 144 \\ \text { SHOT } & 145 \\ \text { SHOT } & 146 \\ \text { SHOT } & 147 \\ \text { SHOT } & 148 \\ \text { SHOT } & 149 \\ \text { SHOT } & 150 \\ \text { SHOT } & 151 \\ \text { SHOT } & 152 \\ \text { SHOT } & 153 \\ \text { SHOT } & 154 \\ \text { SHOT } & 155 \\ \text { SHOT } & 156 \\ \text { SHOT } & 157 \\ \text { SHOT } & 158 \\ \text { SHOT } & 159 \\ \text { SHOT } & 160 \\ \text { SHOT } & 161 \\ \text { SHOT } & 162 \\ \text { SHOT } & 163 \\ \text { SHOT } & 164 \\ \text { SHOT } & 165 \\ \text { SHOT } & 166 \\ \text { SHOT } & 167 \\ & \end{array}$


$\because$

CALL CALCIT(IT),RANG (IR), CWARIIC),AFRO (IA ,WLRA IIW), KCALC)

IF (KCNNV.EO.1) GO TO 154

IF (DELPA.LE,XDEPA) GO TO 16

IF (TCOSI.EQ.0.) GO TO 14

IF (TCOS.GE.TCOS1) GO TO 16

IF (TCOS2, EQ, 0.) GO TO 18

18 TCOSExTCOSI

DO 19 II $=1,5$

19 VAM II I $=V A R$ (I I)

$14 T \cos 1=T \cos$

$\operatorname{VAR}($ I) $=T$ (IT)

$\operatorname{VAR}(2)=R A N G(I R)$

$\operatorname{VAR}(3)=C W A R$ (IC)

$\operatorname{VAR}(4)=A F R O(1 A)$

$\operatorname{VAR}(5) \cup W L R A(I W)$

CAF $\triangle A F P O(1) *$.

Go in 154

16 IF (L.EQ.2) GO TO 154

IF(TCOS2.EQ.0.) 60 TO 17

IF (TCOS.GE.TCOS2) GO TO 154

17 TCOSZl- $\cos$

$\operatorname{VAM}(1) \backsim T(I T)$

$\operatorname{VAM}(3)=C W A R(I C)$

VAM (4) $\triangle A F R O(I A)$

$\operatorname{VAM}(5) \cup W L R A(I W)$

154 CONTINUE

153 CONTINUE

152 CONTINUE

151 CONTINUE

150 CONTINUE

IF (IÖB:ER.0) RETURN

WRITE $(6,553)$ TCOS 1, VAR (1), VAR (2), VAR (3), VAR (4), VAR (5)

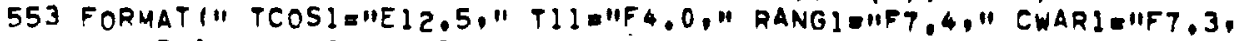
I" $A F R O I=1 " E\lfloor 0.3, " W L R A I=11 F 4.01$

RETURN

END

$\begin{array}{ll}\text { SHOT } & 168 \\ \text { SHOT } & 169 \\ \text { SHOT } & 170 \\ \text { SHOT } & 171 \\ \text { SHOT } & 172 \\ \text { SHOT } & 173 \\ \text { SHOT } & 174 \\ \text { SHOT } & 175 \\ \text { SHOT } & 176 \\ \text { SHOT } & 177 \\ \text { SHOT } & 178 \\ \text { SHOT } & 179 \\ \text { SHOT } & 180 \\ \text { SHOT } & 181 \\ \text { SHOT } & 182 \\ \text { SHOT } & 183 \\ \text { SHOT } & 184 \\ \text { SHOT } & 185 \\ \text { SHOT } & 186 \\ \text { SHOT } & 187 \\ \text { SHOT } & 188 \\ \text { SHOT } & 189 \\ \text { SHOT } & 190 \\ \text { SHOT } & 191 \\ \text { SHOT } & 192 \\ \text { SHOT } & 193 \\ \text { SHOT } & 194 \\ \text { SHOT } & 195 \\ \text { SHOT } & 196 \\ \text { SHOT } & 197 \\ \text { SHOT } & 198 \\ \text { SHOT } & 199 \\ \text { SHOT } & 200 \\ \text { SHOT } & 201 \\ \text { SHOT } & 202 \\ \text { SHOT } & 203 \\ \text { SHOT } & 204 \\ & \end{array}$


SUBROUTINE SIGBAW CALCULATES THE DVERALL MASS TRANSFER SI GBAW SIGBAW COEFFICIENT BETWEEN THE TUBESIDE FLUID AND THE AIR, THIS

SIGBAW MASS TRANSFER COEFFICIENT USES ENTHALPY AS THE DRIVING FORCE SIGBAW (ENTHALPY DIFFERENCE IS ANALOGOUS TO TEMPERATURE DIFFERENCE). SIGBAW

SIGBAW

A NOMENCLATURE OF SOME OF THE MORE FREQUENTLY USED TERMS FOLLOWS.

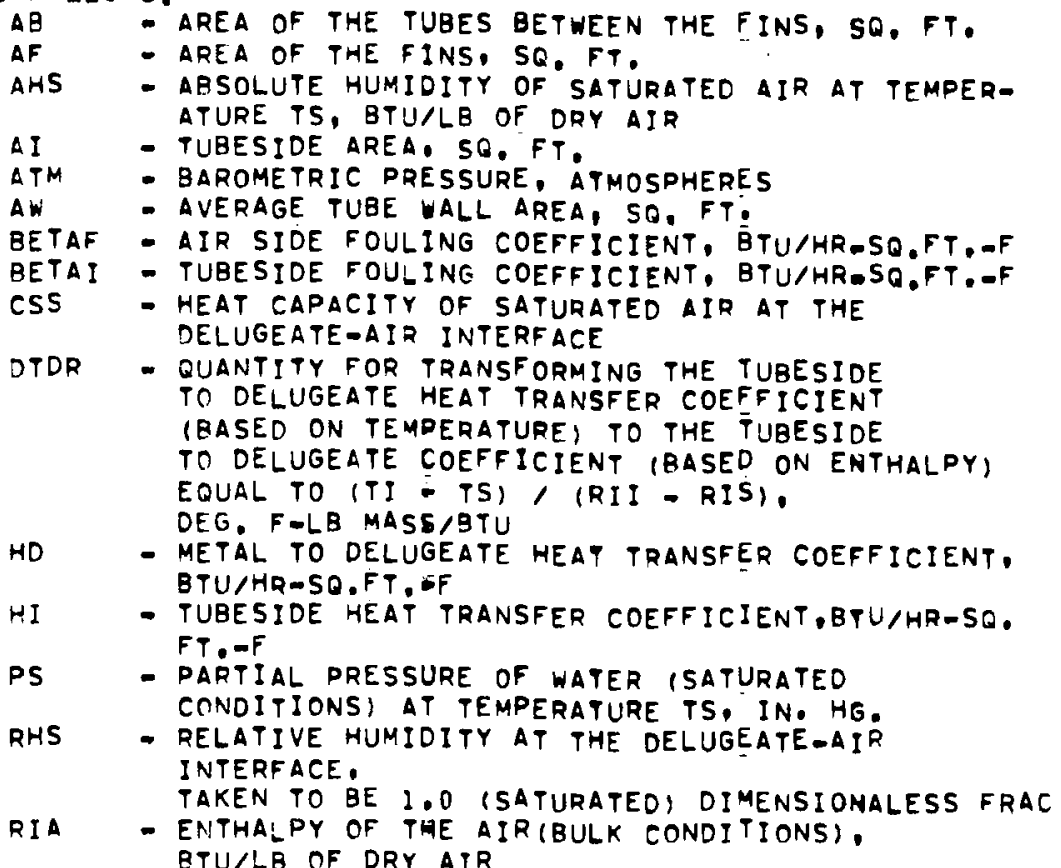

SIGBAW

SIGBAW

SIGBAW

SIGBAW

SI GBAW

SI GBAW

SIGBAW

SI GBAW

SI GBAW

SIGBAW

SIGBAW

SIGBAW

SIGBAW

SIGBAW

SIGBAW

SI GBAW

SIGBAW

SIGBAW

SI GBA

SI GBAW

SI GBAW

SI GBAW

SIGBAW

SI GBAW

SIGBAW

SIGBAW

SIGBAW

SIGBAW

SIGBAW

SI GBAW BTU/LB OF DRY AIR

RII - ENTHALPY OF SATURATED AIR AT THE TUBESIDE

SI GBAW

SI GBAW TEMPERATURE, BTU/LB OF DRY AIR

RIMAX - ENTHALPY OF SATURATED AIR AT TMAX. BTU/ LB OF DRY AIR

RIMIN - ENTHALPY DF SATURATED AIR AT TMIN, BTU/ LB OF DRY AIR

RIS - ENTHALPY OF SATURATED AIR AT THE TEMPERATURE OF THE DELUGEATE-AIR INTEQFACE, TS, BTU/LB OF DRY AIR

RKW - THERMAL CONDUETIVITY OF THE TUBE WALL. BTU/HRSO.FT -F/FT

SIG - TUBESIDE TO AIR MASS TRANSFER COEFFICIENT. LB OF DRY AIR/SO.FT, -HR.

SIGMA - DELUGEATE TO AIR MASS TRANSFER COEFFICIENT, LR OF DRY AIR/SO,FT, -HR, LB MASS/SQ,FT. WHR.

TA - BULK TEMPERATURE OF AIR, DEG, F.

TAIN - TEMPERATURE OF THE AIR EVTERING THE HEAT 


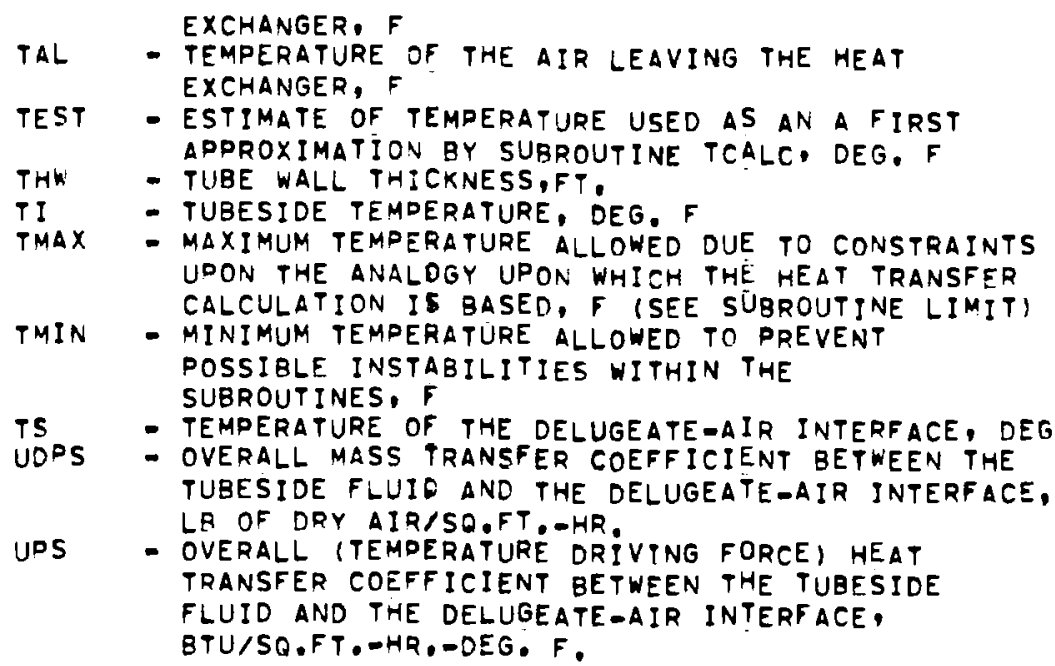

LOGICAL' DATH. FRST 3

COMMON /WETDRY/ NCYCLE, TMAX, RIMAX, TMIN, RIMIN

SIGBAW BI

COMMON/COUNT/NSLOPE, FRST3

COMMON/RPRT $4 / R 4$ (6T2), RR (3)

DATA MAX/20/

$F D(R H, T)=R H * 29.92 * 10.000203 *((T-32) / 1 . B) *$.

- $0.0118 *(T-32.1 / 1.8+1.095)$

- EXP( $-0.000426(1 T-32) / 1.8.)=2$

$+0.0928 *(T-32.1 / 1.8-5.163)$

$F D(R H, T)=R H * E X D(17.168-9240.1(T+460)$.

$F A H(P . A T M)=P * 18.02 /((A T M+29.92-0) \cdot 2 B .97)$

FCS $(A H)=.24 * .45 * A H$

$F R I(C S, T, A H)=C S * T+1094, * A H$

THE QUANTITIES INPUTED TO SIGBAW ARE PRINTED OUT.

SIGBAW B2

SIGBAW 83

SIGBAW B4

SIGBAW 85

SIGBAW 86

SIGBAW B7

SIGBAW 88

SIGBAW 89

SIGBAW 90

5IGBAW 91

SIGBAW 92

SIGBAW 93

SIGBAW 94

SIGBAW 95

SIGBAW 96

SI GBAW 97

SIGBAW 9 B

NDBUG5 $=0$

NDRIJG51 = 0

IF (NDBUGS EQ. I) WRITE $(6,75)$

75 FORMAT (IHO,"TA,RIA, ATM, HD, BETAF, AB, AF, AI", / IX,

S"AW, HI, BETAI, THW,RKW, TI,RII, SIGMA,SIG,NTREST",/)

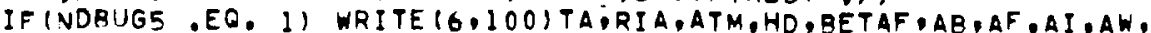
+HI P RETAI , THW, RKW, TI,RII SIGMA,SIG,NTREST

100 FORMAT IX, "FROM SIGBAW",BEI2,5/IX, BEI2,5,10X,E12,5,I5,1/1)

$\mathrm{N}=\mathrm{O}$

AN INITIAL ESTIMATE OF TS IS MADE WHEN NTREST IS NOT EQUAL TO ONE AND PATH IS TRUE

SIGBAW

SIGBAW

SIGBA.

SIGBAW

SIOBAW

SIGBAW

SI GBAW

SI GBAW

SIGBAW

SIGBAW

SI GBAW

SIGBAW

SIGBAW

SIGBAW

SIGBAW

SI GRAW

IFI.NOT. DATH. AND. NTREST, EO. 1) GO TO 1200

IF (NSLOPE .LE. Z) GO TO 1010

SI GBAW 


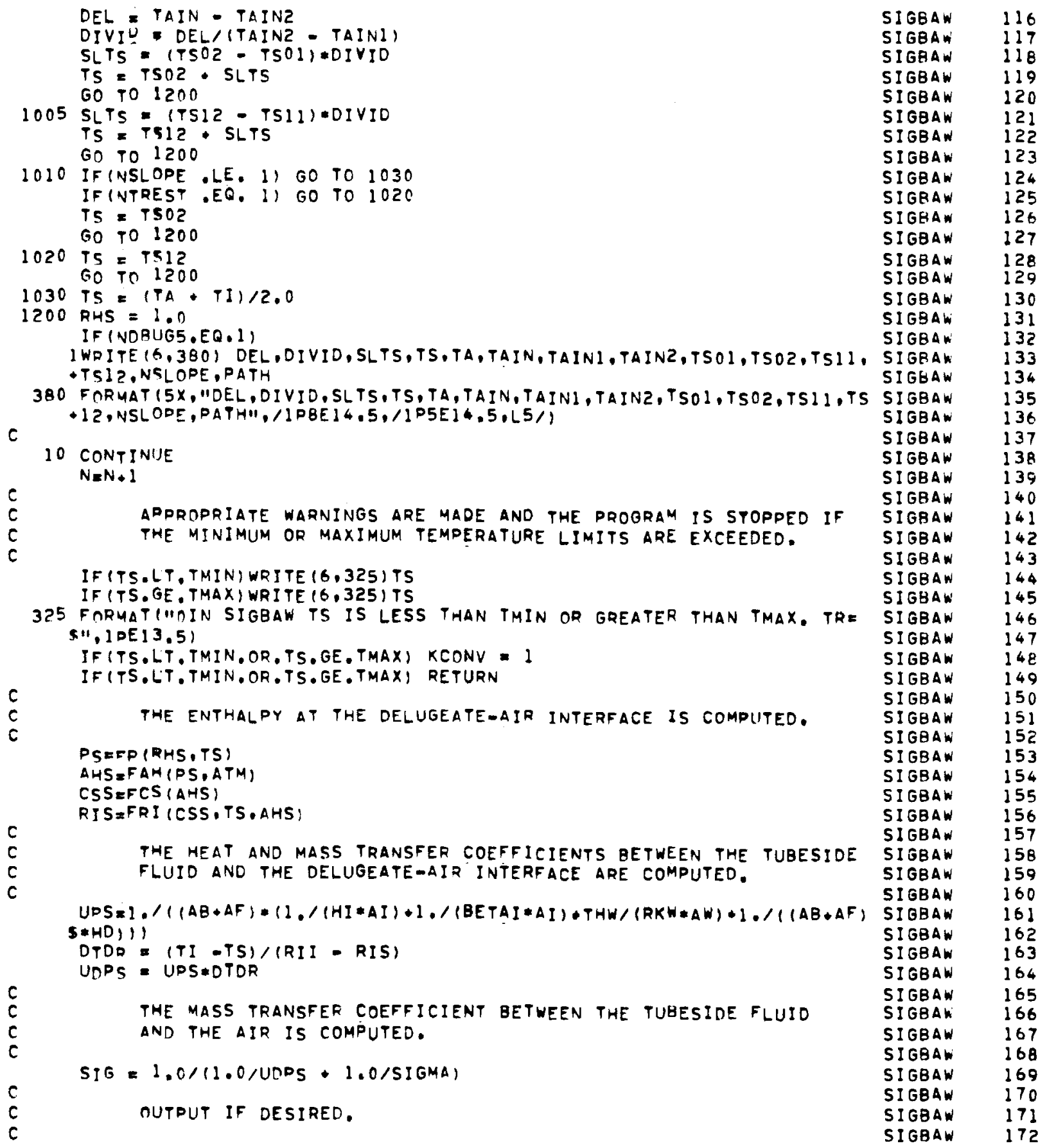


SURROUTINE SIGBAW $\quad 74 / 74 \quad$ OPTEI

FTN $4.5+414$

$06 / 05 / 78$

07.59 .59

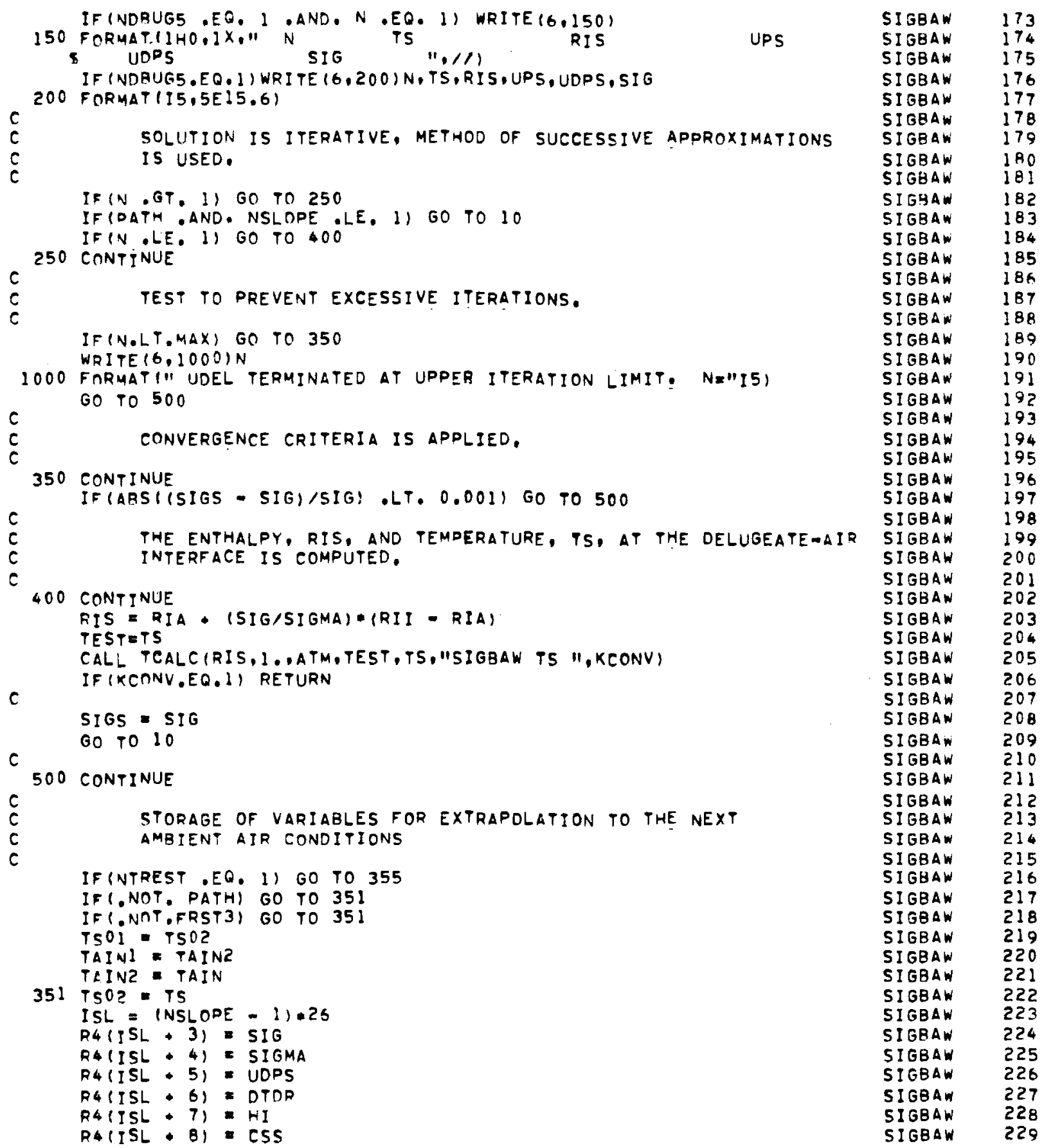


R4 (ISL+9)=TS

RAIISL+1O) = RIS

$R 4(I S L+11)=T I$

$R 4(I S L+12)=R I I$

$S I G S=5 I G$

GO TO 370

355 IF (.NAT. PATH) GO TO 356

IF(.NOT.FRST3) GO TO 356

TSII $=T S 12$

356 TSI? = TS

ISL $=$ (NSLNPE -11.26

$R 4(I S L+16)=S I G$

R4 (ISL -17$)=$ SIGMA

R4 (ISL + 18) = UDPS

$R 4(1 S L \cdot 19)=D T D R$

$24(I S L+20)=H I$

R4 (ISL * 2I) = CSS

$R 4(I S L \cdot 22)=T S$

$R 4(I S L+23)=R I S$

SIGS $=$ SIG

370 CONTINUE

IF (NDAUG5,EQ.1)

IWRITE (6,390) TAINI,TAINZ,TAIN,TS,TSO),T502,TS11, TS1Z,PATH

390 FORMAT 15X,"TAINI, TAIN2, TAIN,TS,TSOL,TSOZ,TSII,TSIZ, PATHM,

. IPAE 14.5.L5/I

c

PATH $=$.FALSE.

C OPTION TO OUTPUT QUANTITIES OF INTEREST.

IF (NDAUG51.NE.1) RETURN

RETURN

END

$\begin{array}{ll}\text { SIGBAW } & 230 \\ \text { SIGBAW } & 231 \\ \text { SIGBAW } & 232 \\ \text { SIGBAW } & 233 \\ \text { SIGBAW } & 234 \\ \text { SIGBAW } & 235 \\ \text { SIGBAW } & 236 \\ \text { SIGBAW } & 237 \\ \text { SIGBAW } & 238 \\ \text { SIGBAW } & 239 \\ \text { SIGBAW } & 240 \\ \text { SIGBAW } & 241 \\ \text { SIGBAW } & 242 \\ \text { SIGBAW } & 243 \\ \text { SIGBAW } & 244 \\ \text { SIGBAW } & 245 \\ \text { SIGBAW } & 246 \\ \text { SIGBAW } & 247 \\ \text { SIGBAW } & 248 \\ \text { SIGBAW } & 249 \\ \text { SIGBAW } & 250 \\ \text { SIGBAW } & 251 \\ \text { SIGBAW } & 252 \\ \text { SIGBAW } & 253 \\ \text { SIGGAW } & 254 \\ \text { SIGBAW } & 255 \\ \text { SIGBAW } & 256 \\ \text { SIGBAW } & 257 \\ \text { SIGBAW } & 258 \\ \text { SIGBAW } & 259 \\ \text { SIGBAW } & 260 \\ \text { SIGBAW } & 261\end{array}$

SIGBAW 


$$
74 / 74 \quad \text { OPT }=1
$$

FTN $4.5+414$

$06 / 05 / 78$

07.59 .59

SUBROUTINE SPDESTTS,TTD,XNS,XNP, CHLD,TLA,X,VELD, VELDO, ARPS, XNTS, IDELP,LINOR)

SUBROUTINE SPDES DESIGNS A CONDENSER/REBOILER ON THE BASIS OF THE TEMPERATURE DIFFERENCE BETWEEN THE SATURATED STEAM AND THE SATURATED AMMONIA. THE AMOUNT OF HEAT TO BE TRANSFERED, THE TEMPERATURE OF THE STEAM, THE LENGTH OF THE TUBES, AND THE EXIT OUALITY OF THE AMMONIA LEAVING THE TUBES THE CONDENSER/REBOILER CAN BE DESIGNED WITH TWO TYPES OF TUBES (1) BARE TUBES, AND (2) ENHANCED TIJBES

FOR BARE TUQES THE OUTSIDE COEFFICIENT IS PROVIDED BY NUSSULT AND THE INSIDE COEFFICIENT BY ROHSENOW FOR THE ENHANCED TUBES THE OUTSIOE AND INSIDE COEFFICIENTS ARE PROVIDED BY A REPORT FROM LINDE CORDORATION

\section{DEFINITION OF VARIABLES}

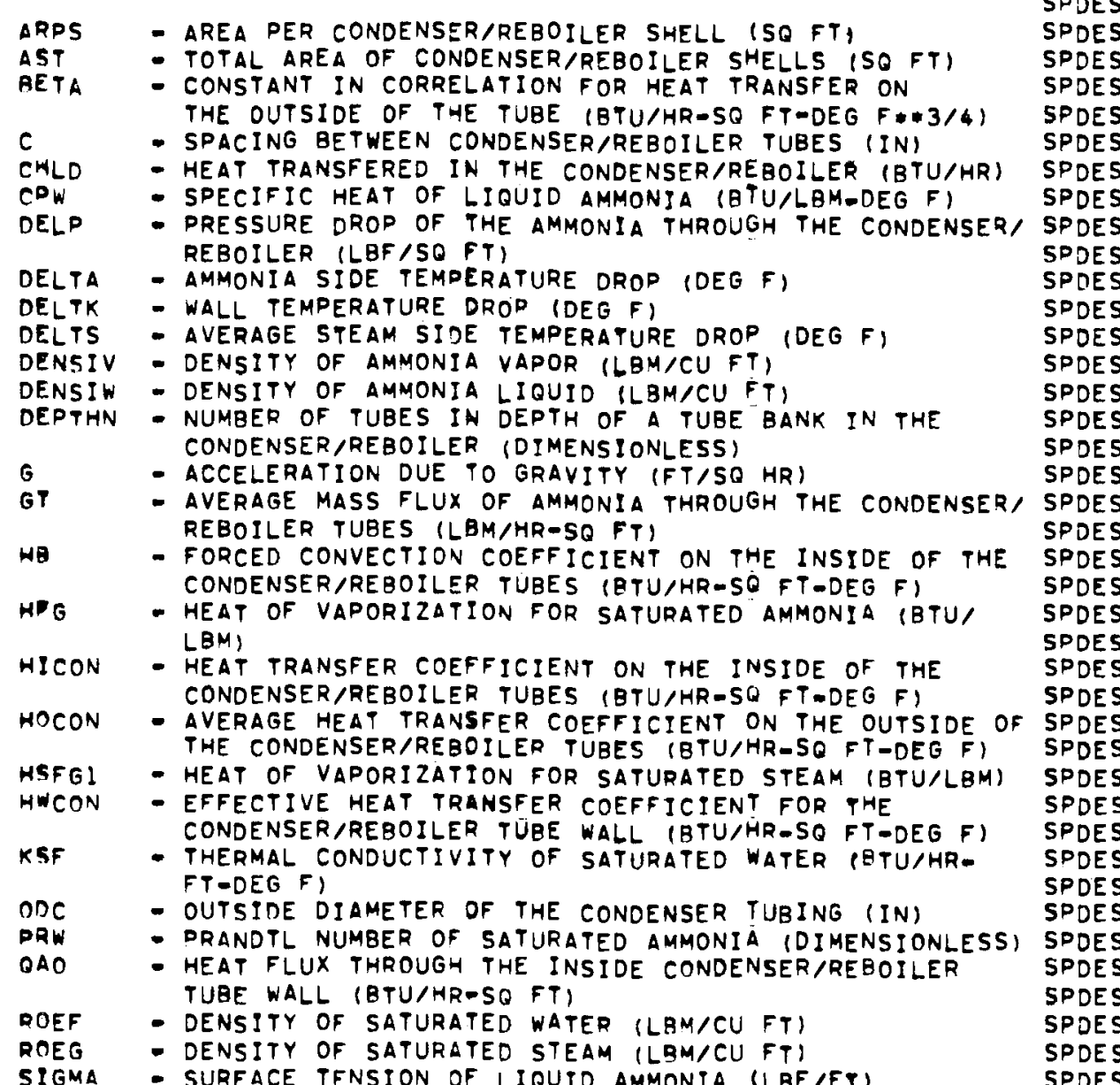

SPDES $\quad 2$

SPDES

SPDES

SPDES

SPDES

SPDES

SPDES

SPOES

SPDES

SPDES

SPDES

SPDES

SPDES

SPDES

SPDE 5

SPDES

SPDES

SPDES

SPDES

SPDES

SPDES

SPDES

SPDES

SPDES

SPDES

PDES

SPDES

SPDES

SPDES

SPDES

SPDES

SPDES

SPDES

SPDES

SPDES

SPDES

SPDE 5

SPDES

SPDES

SPDES

SPDES

SPDES

SPDES 
$74 / 74 \quad$ OPTE1

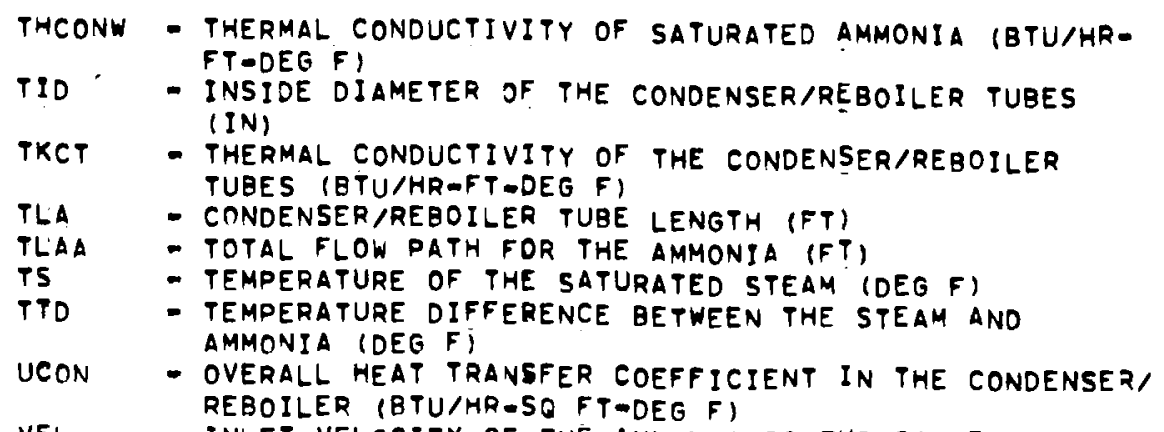

VEL

- INLET VELOCITY OF THE AMMONIA TO THE CONDENSER/ REBOILER TUBES (FT/SEC)

VELO - INLET VELOCITY OF THE AMMONIA TO THE CONDENSER/ REBOILER TUBES (FT/SEC)

VELDO - EXIT VELOCITY OF THE AMMONIA LEAVING THE CONDENSER/ REBOILER TUBES (FT/SEC)

VISS - VISCOSITY OF SATURATED WATER (LBM/FT-HR)

VISV - VISCOSITY OF SATURATED AMMONIA VAPOR (LBM/FT-HR)

VISW - VISCOSITY OF SATURATED AMMONIA LIOUID (LBM/FT-HR)

VSF) - SPECIFIC VOLUMN OF. SATURATED WATER (CU FT/LBM)

VSGI - SPECIFIC VOLUMN OF SATURATED STEAM (CU FT/LBM)

$x$

- EXIT OUALITY OF THE AMMONIA LEAVING THE CONDENSER/ REBOILER TUBES (DIMENSIONLESS)

XNP - NUMBER OF PASSES OF THE AMMONIA THROUGH PHE CONDENSER (DIMENSIONLESS)

XNS - NUMBER OF SHELLS IN THE CONDENSER/REBOILER (DIMENSIONLESS)

XNTS - NUMBER OF TUBES PER SHELL (DIMENSIONLESS)

REAL' KSF

DIMENSION TSAT (64), VSG (64), VSF (64), HSFG (64), ANG (3)

COMMON/PROP/CPW, THCONW, DENSIW, VISW, HFG,

2

COMMON $O A T C / V E L N, O D C$ TKCT

CPV, THCONV, DENSIV, VI SV, SIGMA

COMMON/TUBE/KGA,LD,TID $(7,6)$

COMMON IRPRTZ/UCONN, HOCON, HICON, HWCON, HFC, VELDT, VELDO7, TYDT, TST, I XNPT, DELPW T, ARPST, TLA 7, XNT57, XNST

COMMON /SUPPLY, VAL (5), CPLN, CPIP, CCOS, W3, CO5M, EFF,

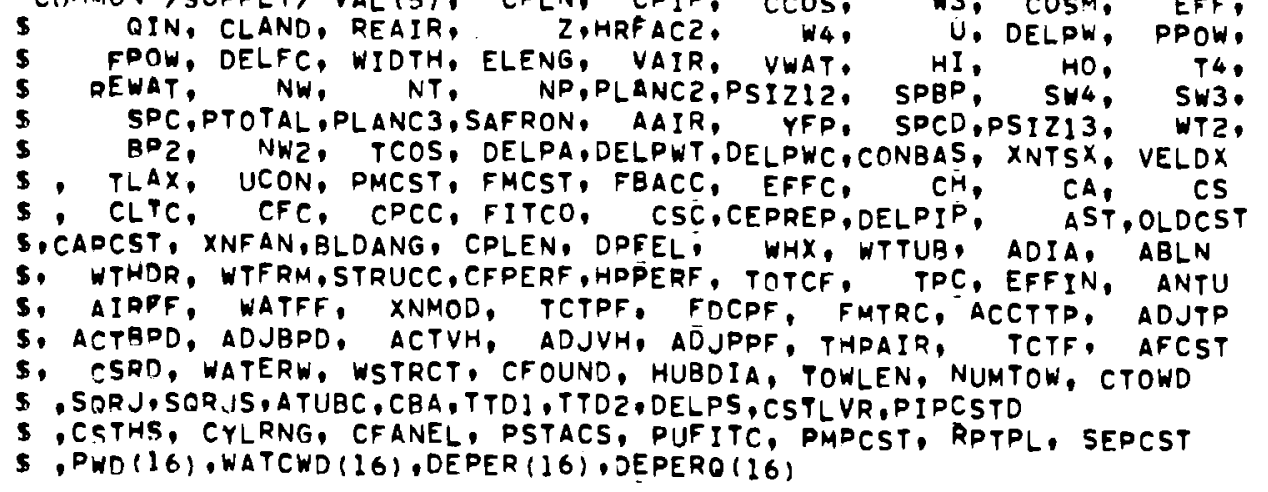

SPDES

SPOES

SPDES

SPDES

SPDES

SPDES

SPDES

SPDES

SPDES

SPDES

SPDES

SPDES

SPDES

SPDES

SPDES

SPDES

SPDES

SPDES

SPDES

SPDES

SPDES

SPDES

SPDES

SPDES

SPDES

SPDES

SPDES

SPDES

SPDES

SPDES

SPDES

SPDES

SPDES

SPDES

SPDES

SPDE 5

SPDES

SPDES

SPDES

SUPPLY

59

60

62

63

64

65

66

67

68

69

70

71

72

73

74

76

77

78

79

80

81

82

83

84

85

86

87

88

89

90

91

92

94
95

96

97

98

99

SUPPLY

SUPPLY

SUPPLY

SUPPLY

SUPPLY

SUPPLY

SUPPLY

SUPPLY

SUPPLY

SUPPLY

SUPPLY

SUPPLY

SUPPLY

SUPPLY

SUPPLY 
$c$

c

$c$

c
S WBB,HTOW, NTOWDE, CSTIF, CSJ, CVF, CDP,W5

PRODERTIES OF STEAM VERSUS TEMPERATURE

DATA TSAT/ $40.24,44.98 .49,07,52.67,55,90$,$$
\text { , }
$$$$
58,82,61,5,63,97,66,27,68,42,70
$$
$177.49,79,04,81.96,84,66,87,17,89,52,91,72,93,8,95,78,97,65,99,43$, $2101.14,102,77,104.34,105,85,107,3,108,7,110.06,111.36,112.63,113.8$ $36.115 .06,1 i 6.22,1 i 7.34,1 i 8,44,119,51,120,56,125,42,129,78,133.75$, $4137.4,140,77,143,91,146,85,149.62,152.24,154,72,157,08,159.33$. $5161.48,165,53,169,28,172,77,176,05,179,13,182.05,184,81,187,45$, $6189.96 .192 .37 \%$

DATA VSF $/ .016019, .016020,00160220.016025, .016028$, $.016032, .016035, .016039, .016043, .016047, .016051, .016055$

$1.016058, .016062, .016066, .016070,0016077, .016084, .016091, .016097$, $2.016104 .016110,0.016116, .016122, .016128, .016134 \% .016139, .016145$, $3.0161500 .016155,00161601.016165,0016170,0016175 \ldots 016180,016184$, $4.016189 .016194, .016198, .016202,016207, .016227 \% 016246,0016264$. $5.016281, .016297, .016312, .016327, .016341, .016354, .016367,0.016380$, $6.016392, .016403, .016426, .016447, .016467, .016486, .016505, .016522$. $7.016539,0.016556, .016572 .016587$,

DATA VSG/2423.96.2038.97,1761.71,1552.30,1388.42, $1 \quad 1256.58,1148,17,1057,42,980,30,913,94,856,22,805,54$. $1760.68,720.69,684,80,652.41,596.25,549.23,509,27,474.87,444.95$, $2418,60^{3} 395,42,374,68,356,06,339,26,324,02,310,13,297,41,285,73$, $3274,95,264.99,255,74,247,13,239,11,231,6,224,56,217,95,211,74$, $4205,87,200,34,176,7,158,19,143,28,131,01,120,73,112,1104.47,97,92$, $592.17,87,08,82,53,78,46,74,77,68,38,63,03,58,48,54,55,51,14,48,143$ $6,45,496,43,116,40.989,39.0671$

DATA HSFG/1070,8,1068.1,1065.8,1063.8,1062.0. $1060,3,1058,8,1057,4,1056,1,1054,9,1053,8,1052,7,1051.7$. $11050,7,1049,8,1048,9,1047,3,1045,8,1044,3,1043,1041,8,1040,6$. $21039.5,1038,4,1037,4,1036,4,1035,5,1034,6,1033,7,2032,9,1032,1$. $31031.3,1030,6,1029,9,1029,1,1029,5,1027,8,1027,1,1026,5,1025,9$, $41025.3 .1022,5,1020.11017,7,1015.5,1013.6,1011.7,1010,1008.4$, $51006, R_{1} 1005.4,1004,11002.6,1001.3,998.9,996.7,994.6,992.6,990.7$. $6988,9,987,3,985,6,984.1,982.61$

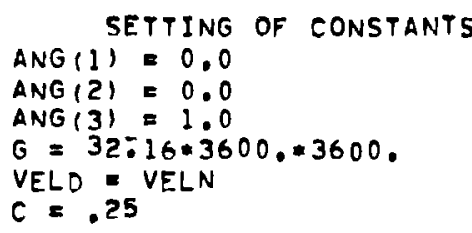

SUPPLY

SPDES

SPDES

SPDES

SPDES

SPDES

SPDES

SPDES

SPDES

SPDES

SPDES

SPDES

SPDES

SPDES

SPDES

SPDES

SPDES

SPDES

SPDES

SPDES

SPDES

SPDES

SPDES

SPDES

SPDES

SPDES

SPDES

SPDES

SPDES

SPDES

SPDES

SPDES

SPDES

SPDES

SPOES

SPDES

SPDES

SPDES

SPDES

SPDES

SPDES

SPDES

SPDES

SPDES

SPDES

SPDES

SPDES

SPDES

SPDES

SPDES

SPDES

SPDES

SPDES

SPDES

SPDES

SPDES

SPDES
18

101

10 ?

103

104

105

106

107

108

109

110

111

112

113

114

115

116

117

$11 \mathrm{~B}$

119

120

121

122

123

124

125

126

127

128

129

130

131

132

133

134

135

136

137

138

139

140

141

142

143

144

145

146

147

148

149

150

151

152

153

154

155

156 


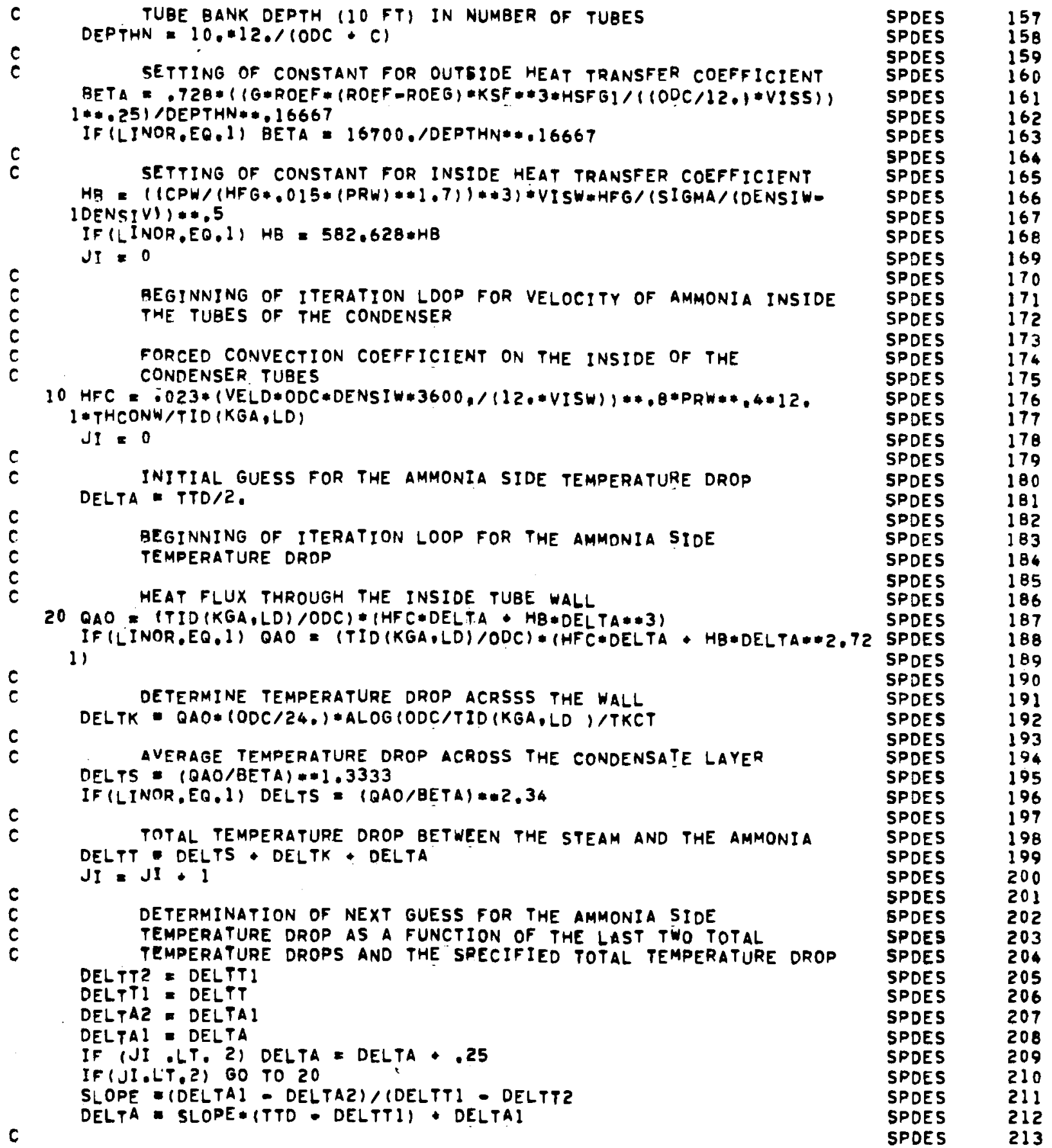


CONVERGENCE CRITERIA FOR THE AMMONIA SIDE TEMPERATURE DROP IF (ABS (IDELTAODELTAI) /DELTA) .LT. .000I) BOTO 30 GO TO 20

c

$c$
$c$

VELOCITY OF AMMONIA BASED ON THE INSIDE WALL HEAT FLUX AND THE EXIT QUALITY OF THE AMMONIA

30 VEL $=$ DDC *OAO*TLA*XNP/(75, *X*HFG*TID(KGA,LD)*\#2*DENSIW)

CONVERGENCE CRITERIA FOR THE VELOCITY OF AMMONIA THRU THE TUBES IF (ABS ( IVELD-VEL)/VEL) .LT. .0OI) GOTO 40 $V E L \cap=V E L$

$c$
$c$ GO TO 10

C NIJMBER OF TUBES AND SURFACE AREA PER SHELL and TOTAL SURFACE AREA

40 ARPS $=$ CHLD/ (XNS*QAO)

XNTS = AINT (ARPS/ $(3.14159 *(00 C / 12) * T L A.) \cdot 0.5)$

XNTSX $=$ XNTS*XNS

c

AST $=$ ARPS $* X N S$

C OVERALL HEAT TRANSFER COEFFICIENT

c

UCON $=$ QAO/TTD

C OUTSIDE HEAT TRANSFER COEFFICIENT

HOCON = QAO/DELTS

C

c

INSIDE HEAT TRANSFER COEFFICIENT

HICON = (QAO/DELTA) * ODC/TIOIKGA,LD)

C EFFECTIVE WALL HEAT TRANSFER COEFFICIENT HWCON = QAO/DELTK

c

EXIT VELOCITY OF THE AMMONIA LEAVING THE CONDENSER TUBES VELDO * VELD*DENSIW*(1, - X) /DENSIW * X/DENSIV $)$

c

PRESSURE DROP THROUGH THE CONDENSER/REBOILER TUBING GT: VELD*DENSIW*3600.

TLAA $=$ TLA\#12. \#NP

CALL DPCONIGOWS,TID (KGA,LD), TLAA,ANG,GT,O, ,X, DENSIW,DENSIV, IVISW*.41338,VISV\#.41338,DELP,DPF,DPG,DPM,XLM,PHIL,PHILZ,VF,FL,RL. ZRVI

$c$
$c$
$c$

DELP = DELP 144.

STORAGE OF VARIABLES

TLAX = TLA

VELOX V VELD

EFFC $=1.0$

UCONN - UCON

TTDT $=$ TTD

VELDT - VELD

VELDOT = VELDO

DELPW7 = DELP

ARPST - ARPS

TLAT = TLA

XNTST $=$ XNTS

SPDES

SPDES

SPDES

SPDES

SPDES

SPDES

SPDES

SPDES

SPOES

SPDES

SPDES

SPDE 5

SPDES

SPDES

SPDES

SPDES

SPDES

SPDES

SPDES

SPDES

SPDES

SPDES

SPDES

SPDES

SPDE 5

SPDES

SPDES

SPDES

SPDES

SPDES

SPDES

SPDES

SPDES

SPDES

SPDES

SPDES

SPDES

SPDES

SPDES

SPDES

SPDES

SPDES

SPDES

SPDES

SPDES

SPDES

SPDES

SPDES

SPDES

SPDES

SPDE 5

SPDES

SPDES

SPDES

SPDES

SPDES

SPDES
214

215

216

217

218

219

220

221

222

223

224

225

226

227

228

229

230

231

232

233

234

235

236

237

238

239

240

241

242

243

244

245

246

247

248

249

250

251

252

253

254

255

256

257

258

259

260

261

262

263

264

265

266

267

268

269

270 
$06 / 05 / 78 \quad 07.59 .59$

XNST $=$ XNS

XNPT $=X N P$.

TST $=$ TS

RETURN

END

SPDES

SPDES

SPDES

SPDES

SPDES

271

272

273

274

275 
$74 / 74 \quad$ OPT $=1$

FTN $4.5 \cdot 414$

$06 / 05 / 78$

07.59 .59

$c$
$c$
$c$
$c$
$c$
$c$
$c$
$c$
$c$
$c$
$c$
$c$
$c$
$c$
$c$
$c$
$c$
$c$
$c$
$c$
$c$
$c$
$c$

SUBROUTINE SSCALE

SSCALE

SSCALE

SUBROUTINE SSCALES SCALES THE STEAM SUPPLY SYSTEM UP TO

SSCALE

OBTAIN THE SPECIFIED POWER OUTPUT OF THE PLANT (PSIZE) aT THE RATED BACK PRESSURE OF THE STEAM TURBINE. THE INCREMENTAL COST OVER THAT OF THE SYSTEM USING A CONVENTIONAL TURBINE IS CALCULATED at THE RATED BACK PRESSURE.

DEFINITION OF VARIABLES

CASSSI - INCREMENTAL COST OF STEAM SUPPLY FOR MAKE UP OF POWER OUTPUT DUE TO INCREASED HEAT RATE AT RATED BACK PRESSURE (5)

CSSPKW - COST OF STEAM SUPPLY SYSTEM PER KILOWATT (S/KW)

HRFACI - HEAT RATE FACTOR OF STEAM TURBINE AT RATED BACK

- PRESSURE (DIMENSIONLESS)

PlIANC - COST OF POWER PLANT PER KILOWATT (S/KW)

PSIZE - PLANT SIZE (MW)

RBP - STEAM TURBINE RATED BACK PRESSURE (IN HGA)

SSCD - INCREMENTAL PLANT COST DUE TO INCREASED HEAT RATE AT RATED BACK PRESSURE OF STEAM TURBINE (\$)

TPO - COEFFICIENTS OF THIRO ORDER HEAT RATE CURVE FIT FOR STEAM TURBINE (DIMENSIONLESS)

LOGICAL FIRST,FRST, FXTEMP,FXTVAR,FXTTTD,FXTLNG
COMMON

9 AFTR, ALPHA, ANG (3)

- BDlim (16)

5 , CAPF, CSSPKW, CONF, CONL, COSTL, CAPCHG, CONMAT, CONMAZ, CAF

\$. CBJ. CTURB. CDANG, CLUVR, CHAILS, CVM

$\$$ DFIN, DEEPL, DESVEL, DESVELV, DESVELD, DENSIF

5 .EFFP, EFS

S FCR, FIRST,FIXL, FCOS, FFHX,FXTEMP, FACTOR, FRST FACTORD, FSHOT, FDELUG

S.FIXV,FIXTTD,FXTVAR,FXTTTD,FXTLNG

S. GBEFF

S. HXNP, HPCST,HCD

S. ITMAX, ITMIN

5 . JCONS

S. KCONV, KALEXT

5. MXEXT

S NTA,NSIDES, NBUNHI COMMON

5 ODR

5.PSIZE, PER, PWCOS, PLANC, PFACT, POMDPL

5 POHBAF, POHFAN, POHLEC, POHCIR; POHCND, POHSTC, POHSCL

$S$, RBPIREOUCE, REOUCV, ROOFL, RHDUT

\$ OREJ. QREDUCE. QREDUCV

S. SIGMAG, SAAF, SEGL

5 TD. TPO (4), TEFF, TLIM, THFIN, TLPRA, TFIX

S.TCD, TW

- UCS. UWS

5 WFV. WATCONA. WATCOST

S. VAS (5)

S:XDEPA, XW, XD

COMMON /SUPPLY/ VAL (5), CPLN, CPIP, CCOS $\$$ OIN, CLAND, REAIR, Z,HRFACZ, W4,

5. FPOW, DELFC, WIDTH, ELENG, VAIR, VWAT,

W3. COSM,
U1, DELPW:
HO:

SSCALE

SSCALE

SSCALE

SSCALE

SSCALE

SSCALE

SSCALE

SSCALE

SSCALE

SSCALE

SSCALE

SSCALE

SSCALE

SSCALE

SSCALE

SSCALE

SSCALE

SSCALE

SSCALE

SSCALE

BI GCOM

BI GCOM

BI GCOM

BI GCOM

BI GCOM

BI GCOM

BI GCOM

BI GCOM

BIGCOM

BI GCOM

BI GCOM

BI GCOM

BIGCOM

BIGCOM

BI GCOM

BI GCOM

BI GCOM

BI GCOM

BI GCOM

BI GCOM

BI GCOM

BI GCOM

BI GCOM

BIGCOM

BI GCOM

BI GCOM

BI GCOM

BI GCOM

BI GCOM

BIGCOM

SUPPLY

EFF,

T4, SUPPLY 
SUBROUTINE SSCALE $\quad 74 / 74$ OPYEI FTN 4.5 .414

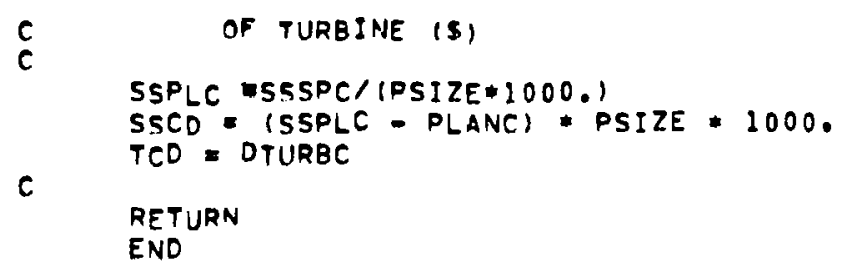

$06 / 05 / 78$

07.59 .59

SSCALE

SSCALE

SSCALE

SSCALE

SSCALE

SSCALE

SSCALE

SSCALE
54

55

56

57 


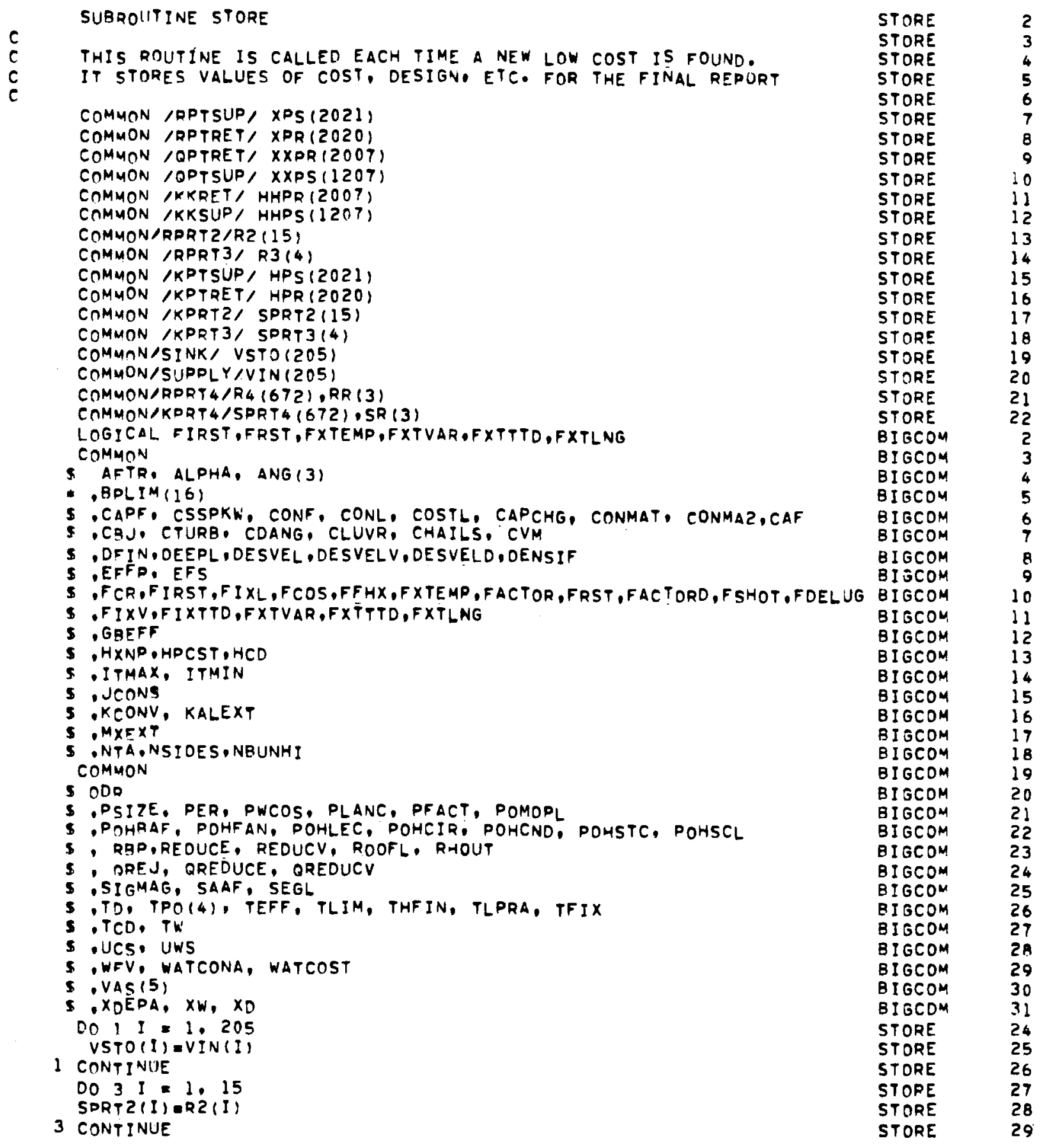


SUBROUTINE STORE $\quad 74 / 74 \quad$ OPTEI

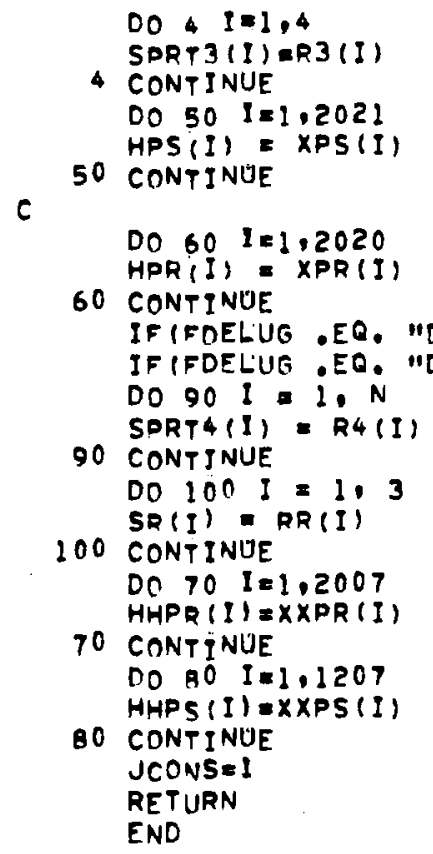

07.59 .59

$\begin{array}{ll}\text { STORE } & 30 \\ \text { STORE } & 31 \\ \text { STORE } & 32 \\ \text { STORE } & 33 \\ \text { STORE } & 34 \\ \text { STORE } & 35 \\ \text { STORE } & 36 \\ \text { STORE } & 37 \\ \text { STORE } & 38 \\ \text { STORE } & 39 \\ \text { STORE } & 40 \\ \text { STORE } & 41 \\ \text { STORE } & 42 \\ \text { STORE } & 43 \\ \text { STORE } & 44 \\ \text { STORE } & 45 \\ \text { STORE } & 46 \\ \text { STORE } & 47 \\ \text { STORE } & 48 \\ \text { STORE } & 49 \\ \text { STORE } & 50 \\ \text { STORE } & 51 \\ \text { STORE } & 52 \\ \text { STORE } & 53 \\ \text { STORE } & 54 \\ \text { STORE } & 55 \\ \text { STORE } & 56\end{array}$


SUBROUTINE STRUCTINTOW, NSIDES, HTOW, BUNL, SEGL, NBUNHI, WHX, WATERW, IDFAN, NOF, ROOFL, CSTRUC, CFNDI

THIS SUBROUTINE CALCULATES THE STRUCTURAL AND FOUNDATION

COSTS FOR A SYSTEM OF ROUND COOLING TOWERS USING THE

DELUGEO HOTERV SURFACE

DEFINE VARIABLES

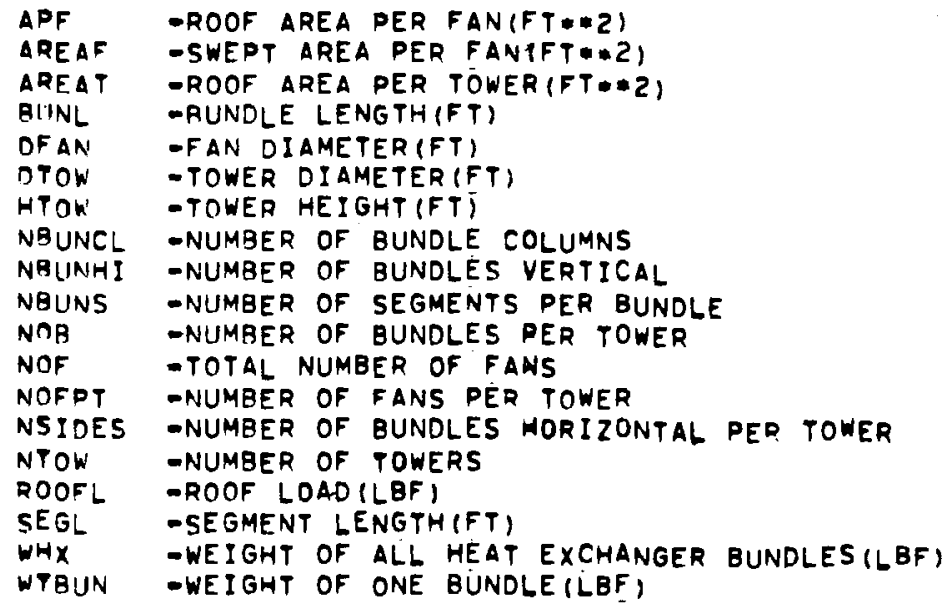


SUBROUTINE STRUCT $\quad 74 / 74 \quad$ OPT $=1$

$C F C=A *(1 B \cdot C) / 129340 . * .4)$

$c$

$A=13.8 B * N B U N C L * H T O W$

BENQUNHI *WTBUN/NBUNS

CEROOFL*AREAT/NBUNCL

$c$

$C B C=A *((B+C) / 54 B \perp 0 .+4$.

$A=0.0682 *$ AREAT $*$ SRAPF

$B=17.0$ F ANDS/APF +ROOFL

c

$C F D F=A \cdot(B / 45.5 * .5)$

C

ILEVEL $=$ HTOW $/ 25.0$

$A=16.74 *$ NOFPT

$B E S Q R T$ (HTOW HTOW+APF)

c

$C \times B=A * B$

c

$C D=1.631 *(A R E A T-A R E A F)$

C

C

c
FTN 4.5 .414

$06 / 05 / 7 B$

07.59 .59

STRUCT

STRUCT

STRUCT

STRUCT

STRUCT

STRUCT

STRUCT

STRUCT

STRUCT

STRUCT

STRUCT

STRUCT

STRUCT

STRUCT

STRUCT

STRUCT

STRUCT

STRUCT

STRUCT

STRUCT

STRUCT

STRUCT

STRUCT

STRUCT

STRUCT

STRUCT

STRUCT

STRUCT

STRUCT
59

60

61

62

63

64

65

66

67

68

69

70

71

72

73

75

76

77

78

79

80

81

82

83

84

85

86

RETURN

END 


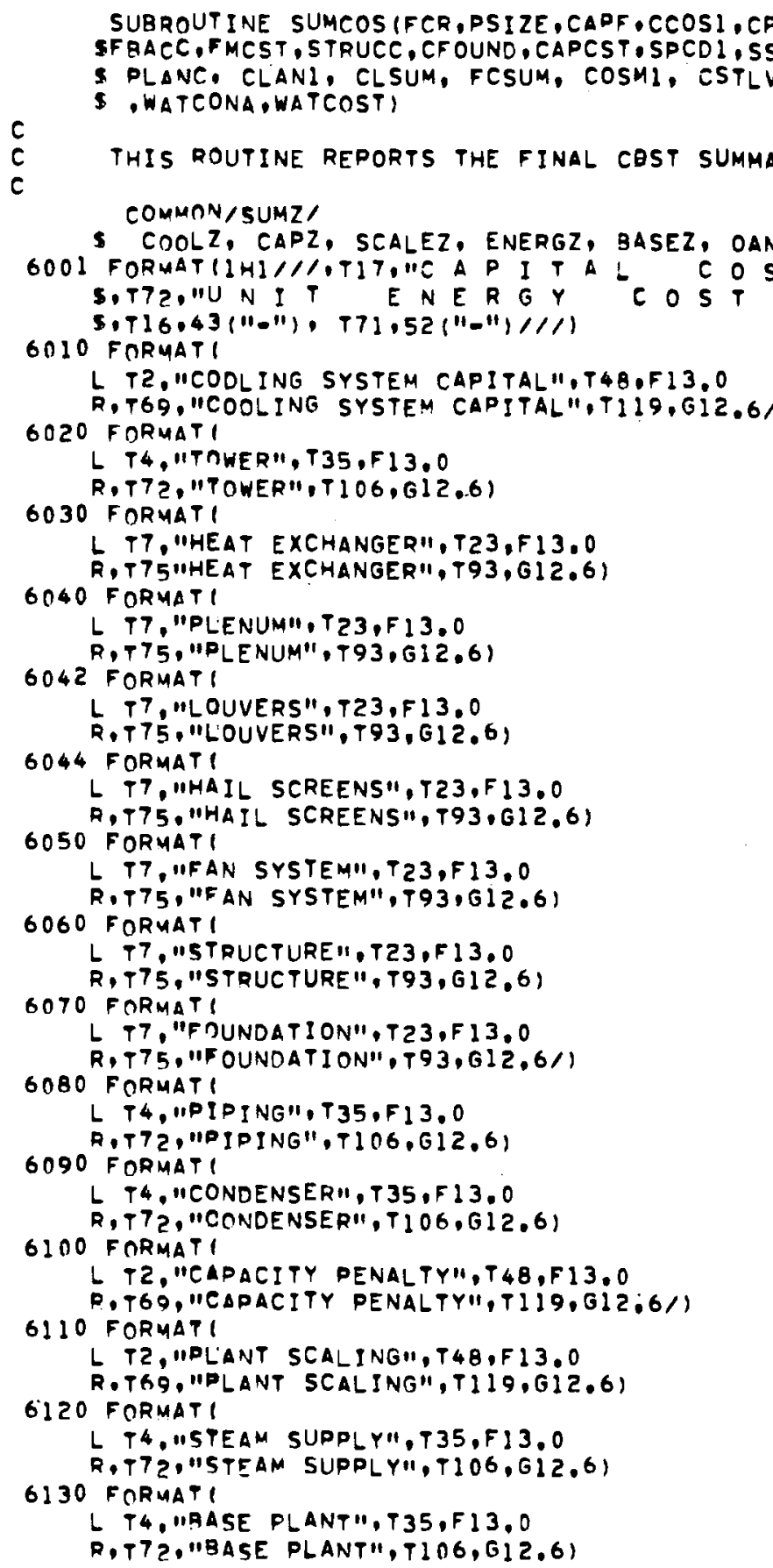

SUMCOS

SUMCOS

SUMCOS

SUMCOS

SUMCOS

SUMCOS

SUMCOS

SUMCOS

SUMCOS

SUMCOS

SUMCOS

SUMCOS

SUMCOS

SUMCOS

SUMCOS

SUMCOS

SUMCOS

SUMCOS

SUMCOS

SUMCOS

SUMCOS

SUMCOS

SUMCOS

SUMCOS

SUMCOS

SUMCOS

SUMCOS

SUMCOS

SUMCOS

SUMCOS

SUMCOS

SUMCOS

SUMCOS

SUMCOS

SUMCOS

SUMCOS

SUMCOS

SUMCOS

SUMCOS

SUMCOS

SUMCOS

SUMCOS

SUMCOS

SUMCOS

SUMCOS

SUMCOS

SUMCOS

SUMCOS

SUMCOS

SUMCOS

SUMCOS

SUMCOS

SUMCOS

SUMCOS

SUMCOS

SUMCOS

SUMCOS 
L T7,"HEAT RATE",T38,F13,0

R,T75, "HEAT RATE", T108,G12,6)

6134 FORMATI

LTT,"FANS AND PUMPS", T38,F13.0

R.T75, "FANS AND PUMPS", TIOB,G12.6/1

6136 FORMATI

L T2, "WATER",T4B,F13,0

R,T69,"WATER",T119,G12.6/

6140 FORMATI

L T2, "LAND", T48,F13,0/1

6150 FORMATI

$6160^{\circ}$ FORMATI

L TH.13(11-11)/1)

L T2,"TOTAL CAPITAL COST",T46,"SM,T48,F13,0

R,T69,"TOTAL CAPITAL COST".TI19.612.6/)

6170 FORMATI

R T69,"ENERGY PENALTYH,T119,G12.6/1

6180 FORMATI

R T69,"ADDITIONAL BASE PLANT FUEL",T119,012.6/1

6190 FORMATI

R T69."OPERATION AND MAINTENANCE",TI19.612.6/1

6200 FORMATI

$R, T 72$, "TOTAL",T119,612,6)

YFPOI = YFP 1 .

FACTOR FCR/(PSIZE-CAPF \#B760)

WRITE $\{6,6001)$

WRITE $(6,6010) \quad$ (CCOSI +CPIPI+CONBA1), ( (CCOSI +CPIPI +CONBAI) *FACTOR)

WRITE $(6,6020)$ CCOS1, CCOSI*FACTOR

WRITE $(6,6030)$ ATUBC.ATUBC FACTOR

WRITE $(6,6040)$ CPLEN.CPLEN WACTDR

WRITE $(6,6042)$ CSTLVI, CSTLVI \#FACTOR

WRITE $(6,6044)$ CSTHSI, CSTHSI WFACTOR

WRITE $(6,6050)$ (FBACC + FMCST: CYLRNI - CFANEI)

- (FBACC - FMCST - CPLRNI + CFANEII * FACTOR

WRITE $(6,6060)$ STRUCC,STRUCC \&FACTOR

WRITE $(6,6070)$ CFOUND,CFOUND FACTOR

WRITE $(6,6080)$ CPIPI,CPIPI FACTOR

WRITE $(6,6090)$ CONBAI, CONBAI FACTOR

WRITE $(6,6100)$ CAPCST.CAPCST FACTOR

WRITE $(6,6110)$ SPCD SPCD $*$ FACTOR

WRITE $(6,6120)$ SSCD HRFAC2/HRFAC1 *YFPPI, SSCO HRFAC2/HRFACI YYPPI*

SFACTOR

WRITE (6,6130) PLANC \{HRFAC2/HRFAC I*YFPPI-1.1*10,**3* P5IZE,

5 PLANC* IHRFACZ/HRFACI *YFPPI-1.1:10*3*PSIZE *FACTOR

WRITE $(6,6132)$ PLANC* (HRFACZ/HRFACI-1.1*10,*3*PSIZE, PLANC*

1 (HRFACZ/HRFACI - $1.1 * 10, * 3$ *PSIZE \#FACTOR

IHRFAC2 /HRFACI * (YFPPI - 1,1*10,*3*PSIZE *FACTOR

1*10**3*PSIZE,PLANC*

WRITE $(6,6136)$ WATCONA* $1.1203 / 1000.1$ *WATCOST, WATCONA*1, $1203 / 1000.1$

1 -WATCOST/(PSIZE $\approx C A P F=8760$.

WRITE $(6,6140)$ CLANI

WRITE $(6,6150)$

WRITE $(6,6160) \quad$ CLANI + SPCD + CAPCST +CCOSI \& CPIPI + CONBAI

S, (CL $\triangle N 1$ \&PCD I +CAPCST+CCOS I CPIP I CONBAI) *FACTOR

WRITE (6.6170) CLSUM

WRITE 16,6180$)$ FCSUM

SUMCOS

SUMCOS

SUMCOS

SUMCOS

SUMCOS

SUMCOS

SUMCOS

SUMCOS

SUMCOS

SUMCOS

SUMCOS

SUMCOS

SUMCOS

SUMCOS

SUMCOS

SUMCOS

SUMCOS

SUMCOS

SUMCOS

SUMCOS

SUMCOS

SUMCOS

SUMCOS

SUMCOS

SUMCOS

SUMCOS

SUMCOS

SUMCOS

SUMCOS

SUMCOS

SUMCOS

SUMCOS

SUMCOS

SUMCOS

SUMCOS

SUMCOS

SUMCOS

sumcos

SUMCOS

SUMCOS

SUMCOS

SUMCDS

SUMCOS

SUMCOS

SUMCOS

SUMCOS

SUMCOS

SUMCOS

SUMCOS

sumcos

SUMCOS

SUMCDS

SUMCOS

SUMCOS

SUMCOS

SUMCOS

\section{9}

60

62

63

64

65

66

67

68

70

71

72

73

74

75

76

77

78

79

80

81

82

84

86

89

0

91

93

95

96

97

98

99

100

101

102

103

104

105

106

107

108

100

110

111

112

113

114

115 
WDITE $(6.6190)$ COSM]

SUMCOS

SUMCOS

116

TOTAL = (CLAN] + SPCOI + CAPCST + CCOSI + CPIPI + CONBAI) *

SUMCOS

117

S FACTOR - CLSUM - FCSUM + COSMI

S*WATCONA* $1.1203 / 1000.) * W A T C O S T /(D S I Z E * C A P F * B 760$.

WRITE $(6,6200)$ TOTAL

COOLZ $=(C C O S 1+C P I P 1 \cdot \operatorname{CONBA} 1)$ FACTOR

CAPT $=$ CAPCST F ACTOR

SCALEZ SPCDI HFACTOR

ENEQGZ =CLSUM

BASEZTF CSUM

SUMCOS

118

SUMCOS

SUMCOS

SUMCOS

SUMCOS

SUMCOS

121

122

OANDMZ $=$ COSM]

SUMCOS

SUMCOS

124

END

SUMCOS

126

127 
SUBROUTINE SUMMARY

LOGICAL FIRST ,FRST, FXTEMP, FXTVAR, FXTTTD,FXTLNG

COMMON

- artr. alpha, ang (3)

* Bolim(16)

5 CAPF, CSSPKW, CONF, CONL, COSTh, CaPCHg, CONMAT, cONMAZ, CAF

5 , CBU, CTURB, CDANG, CLUVR, CHAILS, CVM

\$. DFIN, DEEPL, DESVEL, DESVELV, DESVELD, DENSIF

5 . ESFP, EFS

S ,FCR,FIRST, FIXL, FCOS, FFHX, FXTEMP, FACTOR, FRST , FACTORD, FSHOT , FDELUG

S, FIXV,FIXTTD,FXTVAR,FXTTTTD,FXTLNG

S.GBEFF

5 , HXNP,HPEST,HCD

5 ITMAX. ITMIN

5 . JCONS

S, KCONV, KALEXT

$5, M \times E X T$

5 ,NTA,NSIDES, NBUNHI

COMMON

$S O D R$

5 PSITE, PER, PWCOS, PLANC, PFACT, POMDPL

5 , POHAAF, POHFAN, POHLEC, POHCIR, POHCND, POHSTC, POHSCL

S RRP,REDUCE, REDUCV, ROOFL, RHQUT

S QREJ, QREDUCE, QREDUCV

\$. SIGMAG, SAAF, SEGL

S, TD, TPO (4), TEFF, TLIM, THFIN, TLPRA, TFIX

5. TCD, TW

5 ,UCS, UWS

5 WEV. WATCONA, WATCOST

S. VAS (5)

S. XDEPA, XW, XD

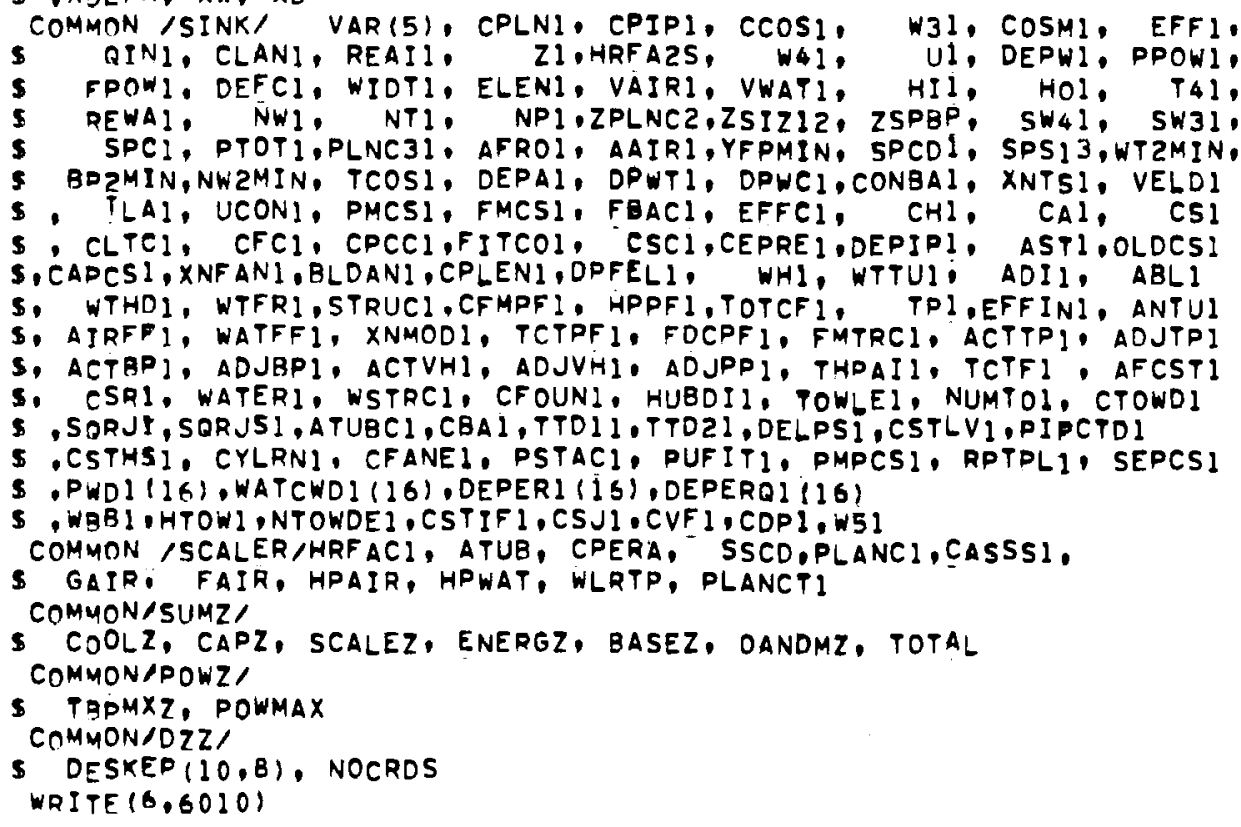

COMMON/SINK/ VAR(5), CPLNI, CPIPI, CCOSI, FPOWI, OEFCI, WIDTI, ELENI, VAIRI, VWATI, HII, HOI, TAI, SINK SPC1, PTOT1,PLNC31, AFROI, AAIRI,YFPMIN, SPCDI, SPSI3,WTZMIN, SINK ZMIN, NW2MIN, TCOS1, DEPAI, DPWT1, DPWC1, CONBAI, XNTSI, VELDI

, CLTCl, CFCl, CPCCI,FITCOI, CSCI, CEPREI,DEPIPI, ASTI,OLDCSI

S,CAPCSI, XNFANI,BLDANI, CPLENI,DPFELI, WHI, WTTUI, ADII, ABLI

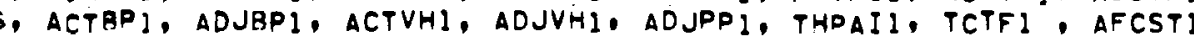

S, CSRl, WATERI, WSTRCl, CFOUNI, HUBDIl, TOWLEl, NUMTOl, CTOWDI

\$,SORJI, SORJ51, ATUBCI, CBAI, TTDII,TTOZI, DELPSI, CSTLVI,PIPCTDI

5.PWDI (16). WATCWDI (16),DEPERI (15),DEPERQ1 (16)

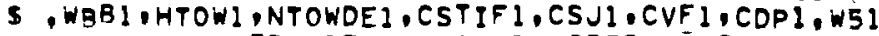

COMMON /SCALER/HRFACl, ATUB, CPERA, SSCD,PLANCI, CASSSI.

GAIP. FAIR HPAIR, HPWAT WLRTP, PLANCTI

SUMZ

COMMONAPOWZI

TAPHXZ, POWMAX

COMMONIDZZ/

WRITE $(6.6010)$

SUMMARY

BIGCOM

BIGCOM

BI GCOM

BIGCOM

BI GCOM

BIGCOM

BIGCOM

BIGCOM

BIGCOM

BI GCOM

BI GCOM

BI GCOM

BI GCOM

BI GCOM

BI GCOM

BI GCOM

BI GCOM

BI GCOM

BI GCOM

BI GCOM

BI GCOM

BIGCOM

BIGCOM

BI GCOM

BI GCOM

BIGCOM

BIGCOM

BIGCOM

BI GCOM

BI GCOM

SINK

SINK 


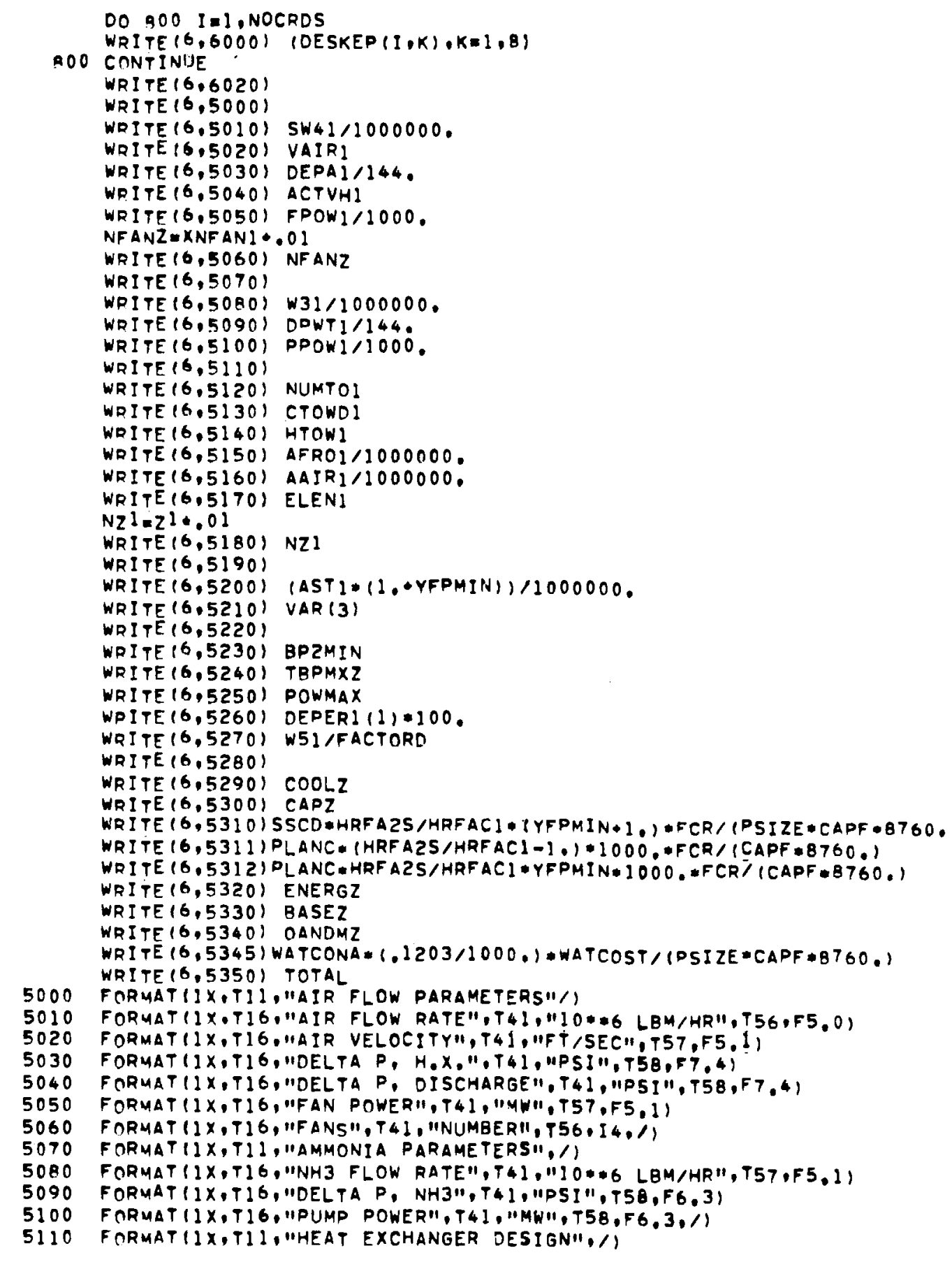

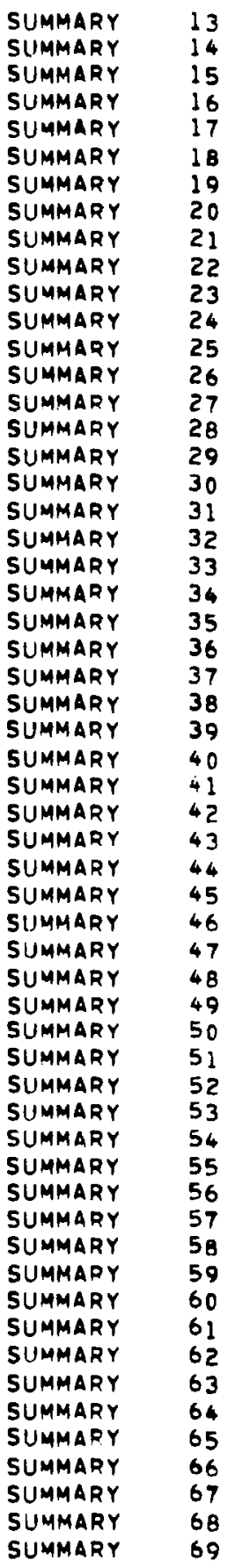


SUPROUTINE SUMMARY $74 / 74 \quad$ OPTEI FTN $4.5 * 414$

$06 / 05 / 78$

07.59 .59

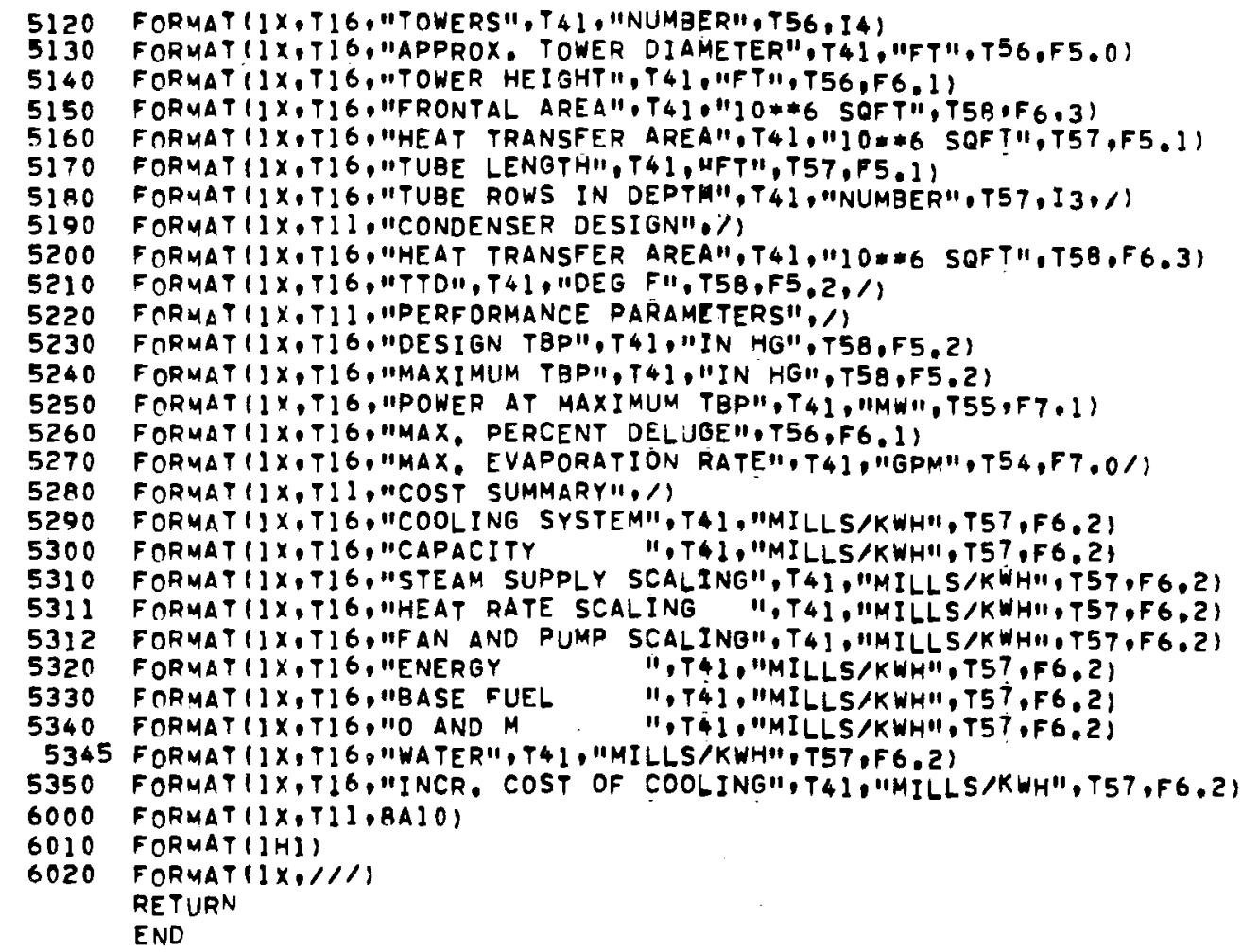

SUMMARY

SUMMARY

SUMMARY

SUMMARY

SUMMARY

SUMMARY

SUMMARY

SUMMARY

SUMMARY

SUMMARY

SUMMARY

SUMMARY

SUMMARY

SUMMARY

SUMMARY

SUMMARY

SUMMARY

SUMMARY

SUMMARY

SUMMARY

SUMMARY

SUMMARY

SUMMARY

SUMMARY

SUMMARY

SUMMARY

SUMMARY

SUMMARY

SUMMARY

SUMMARY

SUMMARY

SUMMARY 
SUBROUTINE SURCON IISUR, KGA, LD, TLA, XNTS, ARPS, XNS, CONCST

SURCON - CONMAT, POHCND)

PROGRAM SURCON DETERMINES THE CAPITAL COST OF A SINGLE PRESSURE OR SERIES-CONNECTED SURFACE CONDENSËR. REFERENCE

SURCON

SURCON

SURCON

SURCON "SURFACE CONDENSERS FOR LAND INSTALLATION", FORM PLIJ12, WESTINGHOUSE ELECTRIC CORPORATION, 26 NOVEMBER 1968 UPDATED ON FEBRUARY 12,1976 TO CURRENT PRICES

SURCON

SURCON

SURCON

DEFINITION OF VARIABLES

SURCON

SURCON

SURCON

ACCF - $\triangle C C E S S O R Y$ COST CORRECTION FACTOR FOR THE CONDENSE?

SURCON

AODER - INCREASES COST OF TUBING MATERIAL

ARPS - AREA PER SHELL OF THE CONDENSER (SQ FT)

$\triangle U X C S T$ - AUXILIARY COST (S)

CFAC - LENGTH CORRECTION FACTOR (OIMENSIONLESS)

CONCST - CONDENSER COST (\$)

CONMAT - VARIABLE FOR CONDENSER MATERIAL (DIMENSIONLESS)

OSCT - QUANTITY DISCOUNT FOR TUBES (OIMENSIONLESS)

ERTCST - ERECTION COST (S/SO FT)

- TUBING LENGTH PENALTY (DIMENSIONLESS)

FETCST - FIELD ERECTION COST (S/SO FT)

FOD - TUBE SIZE FACTOR (DIMENSIONLESS)

POHCND - INDIRECT COST FACTOR FOR THE TUBE, SHELL, AND FIELD ERECTION COSTS (DIMENSIONLESS)

SHLCST - SHELL COST FOR THE CONDENSER (S)

TESTPF - TOTAL COST OF TUBING MATERIAL IS/FT,

WT - WEIGHT OF TOTAL AMOUNT OF TUBES IN THE CONDENSER (LBF)

XNS - NUMBER OF SHELLS IN THE CONDENSER (DIMENSIONLESS)

SURCON

SURCON

SURCON

SURCON

SURCON

SURCON

SURCON

SURCON

SURCON

SURCON

SURCON

SURCON

SURCON

SURCON

SURCON

SURCON

SURCON

SURCON

SURCON

SURCON

SURCON

SURCON

SURCON

SURCON

SURCON

CL TUBE FIXED COSTS ISI

CL'M - TUBE MATERIAL COSTS $13 / L B M$

ROEL - DENSITY OF TUBE MATERIALS (LBM/CU IN)

GAGLI - TUBE GAGE (DIMENSIONLESS)

THKLIN - THICKNESS OF TUBE (IN)

OOLINE - COMMON DIAMETERS OF TUBES (IN)

SURCON

SURCON

SURCON

SURCON

SURCON

SURCON

SURCON

LOGICAL FIRST

DIMENSION XLCST $(6,2), \operatorname{SCC} 1(6), \operatorname{SCC}(6)$

DIMENSION TUBMA $(13), C L(10)$, CLMI10), ROEL $(10)$, GAGLI $(7)$,

SURCON

SURCON

SURCON

SURCON

COMMON /RPRT3/ TUBCST,SHLCST, FETCST,CONBAT

$c$
$C$

TUBING MATERIALS, COSTS. AND DENSITY

DATA CL, $0.00,0.00,0.00,0.08,0,04,0.38,0.12,0.20,0.15$,

- 0.10,

DATA CL'M / 1.30,1.52,1.83,1.40,1.36,0.44,0.32,1.63,7.60,

SURCON

SIJRCON

SURCON

SURCON

SURCON

SURCON

SURCON

7.00 )

SURCON

$.0 .163,0.163$,

SURCON 


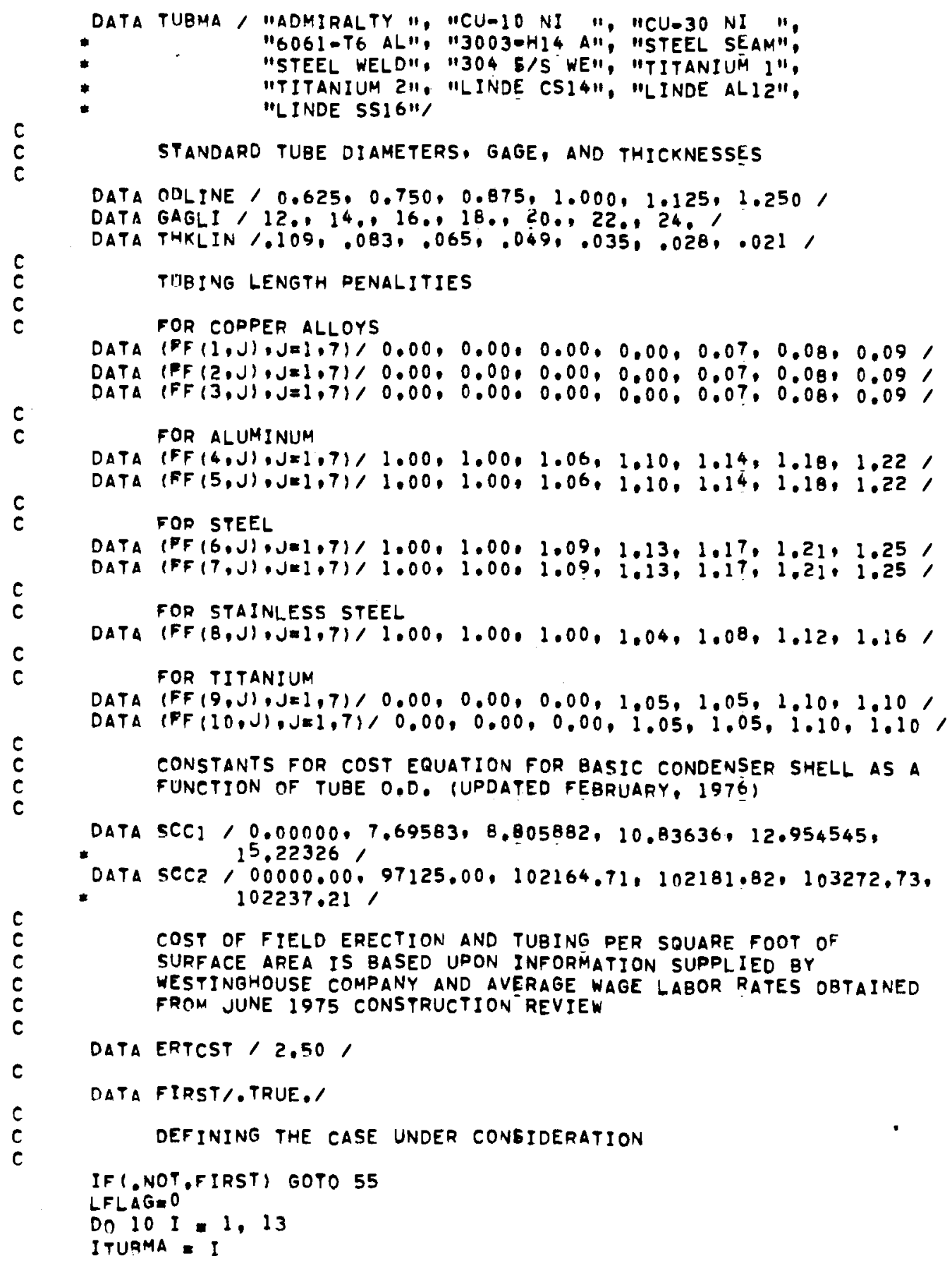

SURCON

SURCON

SURCON

SURCON

SURCON

SURCON

SURCON

SURCON

SURCON

SURCON

SURCON

SURCON

SURCON

SURCON

SURCON

SURCDN

SURCON

SURCON

SURCON

SURCON

SURCON

SURCON

SURCON

SURCON

SURCON

SURCON

SURCON

SURCON

SURCON

SURCON

SURCON

SURCON

SURCON

SURCON

SURCON

SURCON

SURCON

SURCON

SURCON

SURCON

SURCON

SURCON

SURCON

SURCON

SURCON

SURCON

SURCON

SURCON

SURCON

SURCON

SURCON

SURCON

SURCON

SURCON

SURCON

SURCON

SURCON

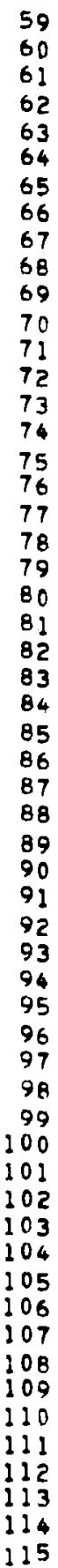




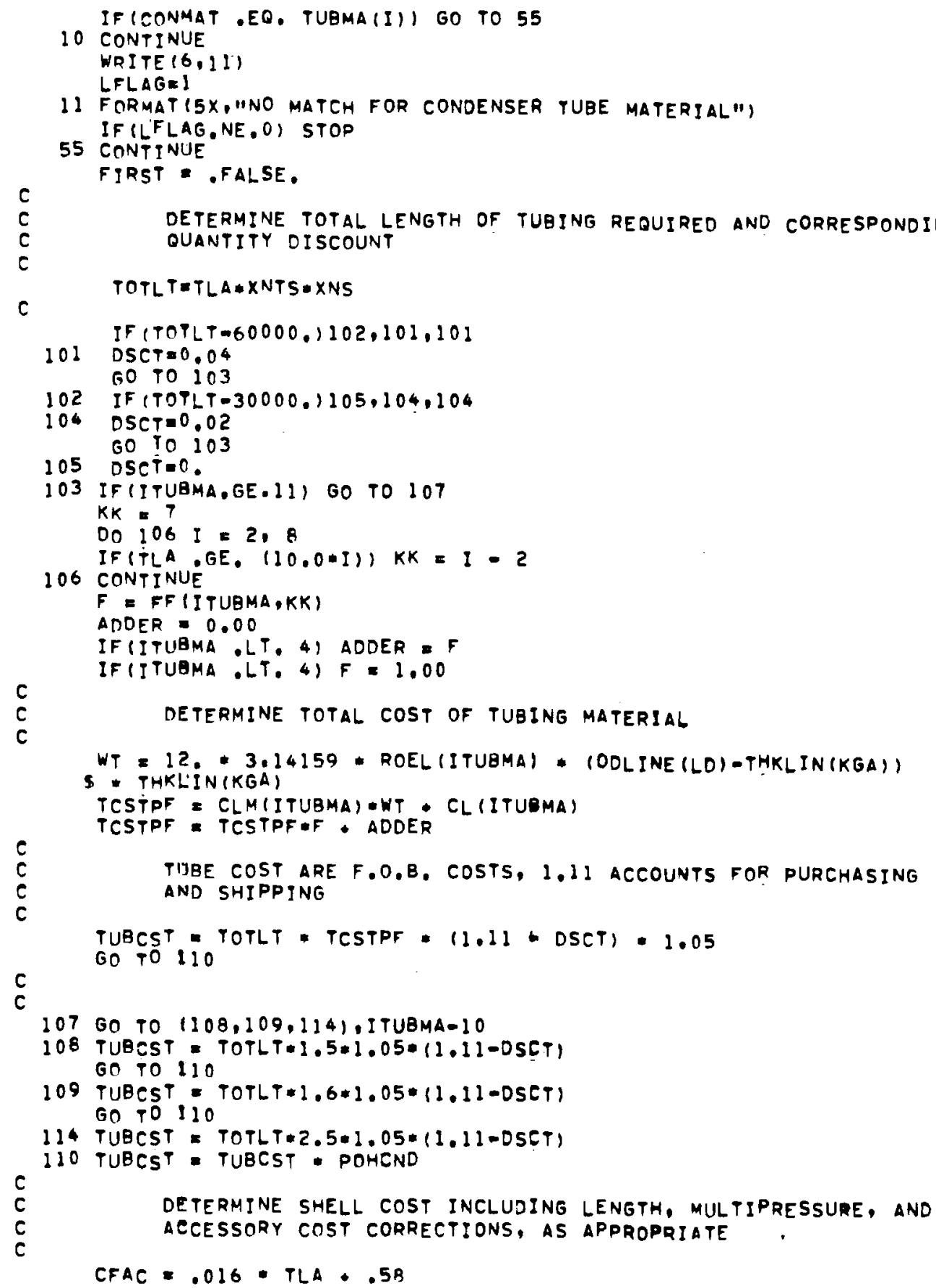

SURCON

SURCON

SURCON

SURCON

SURCON

SURCON

SURCON

SURCON

SURCON

SURCON

SURCON

SURCON

SURCON

SURCON

SURCON

SURCON

SURCON

SURCON

SURCON

SURCON

SURCON

SURCON

SURCON

SURCON

SURCON

SURCON

SURCON

SURCON

SURCON

SURCON

SURCON

SURCON

SURCON

SURCON

SURCON

SURCON

SURCON

SURCON

SURCON

SURCON

SURCON

SURCON

SURCON

SURCON

SURCON

SURCON

SURCON

SURCON

SURCON

SURCON

SURCON

SURCON

SURCON

SURCON

SURCON

SURCON

SURCON
116

117

118

119

120

121

122

123

124

125

126

127

128

129

130

131

132

133

134

135

136

137

138

139

140

141

142

143

144

145

146

147

148

149

150

151

152

153

154

155

156

157

158

159

160

161

162

163

164

165

166

167

168

169

170

171

172 
SUBROUTINE SURCON T4/74 OPTEI

GO TO1100,111,112,113), ISUR

111 CFACECFAE.O.0 GO in 200

$j 75$

180

$\lceil 85$

i95

200

205

112 CFACACFAC+0.16

GO TO 100

c

$113 C F A C=C F A C+0.03+X N S+0.02$

IF (LD :LT, 4) FOD $=0.95$

IF ILD :EO. 6) FOD = 1.05

$c$
$c$
$c$

90

c

$c$

AUXCST $=0.45 * A R P S=X N S$

SHLCST = SHLCST 1.05

SHLCST - SHLCST - POHCND

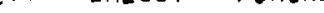

FETCST =ERTCST *ARDS*XNS

FETCST = FETCST : 1.05

FETCST = FETCST. POHCNO

CONCSTETUACST + SHLEST + FETCST

CONBATACONCST
$100 F 00 \div 1000$

ACCE = $(0.116 * A L O G(X N T S)-0.485) * F O D$

IF(ITU1BMA. EO. 2$)$ ACCF = $10,239 * A L O G(X N T S=1.416) * F O D$

THE CONDENSER SHELL COST
1973 PRICE LISTING 1312

BSHCST = (SCC) (LD) XXNTS - SCCZ (LO))

SHLCST = BSHCST * (CFAC+ACCF) *XNS * AUXCST

FTN $4.5+414$

$06 / 05 / 78$

07.59 .59

OETERMINE COST OF FIELD ERECTION AND TUBING

DETERMINE TOTAL COST OF CONDENSER

RETURN

END

$\begin{array}{ll}\text { SURCON } & 173 \\ \text { SURCON } & 174 \\ \text { SURCON } & 175 \\ \text { SURCON } & 176 \\ \text { SURCON } & 177 \\ \text { SURCON } & 178 \\ \text { SURCON } & 179 \\ \text { SURCON } & 190 \\ \text { SURCON } & 191 \\ \text { SURCON } & 182 \\ \text { SURCDN } & 183 \\ \text { SURCON } & 184 \\ \text { SURCON } & 185 \\ \text { SURCON } & 186 \\ \text { SURCON } & 187 \\ \text { SURCON } & 188 \\ \text { SURCON } & 189 \\ \text { SURCON } & 190 \\ \text { SURCON } & 191 \\ \text { SURCON } & 192 \\ \text { SURCON } & 193 \\ \text { SURCON } & 194 \\ \text { SURCON } & 195 \\ \text { SURCON } & 196 \\ \text { SURCON } & 197 \\ \text { SURCON } & 198 \\ \text { SURCON } & 199 \\ \text { SURCON } & 200 \\ \text { SURCON } & 201 \\ \text { SURCON } & 202 \\ \text { SURCON } & 203 \\ \text { SURCON } & 204 \\ \text { SURCON } & 205 \\ \text { SURCON } & 206 \\ \text { SURCON } & 207 \\ & \end{array}$

CARD NR. SEVERITY DETAILS DIAGNOSIS OF PROBLEM

$160 \quad 1$

AN IF STATEMENT MAY BE MORE EFFICIENT THAN A 2 OR 3 BRANCH COMPUTEO GD TO STATEMENT. 
SUBROUTINE TBPMAX IBPL,PSATMAX,TSATMAX,DPER, OPERO, WATCON, WATCONT, IPMAXDRY, TMAXDRY,POELUG, TDELUG,WATDE PLICK)

SUBROUTINE TBPMAX DETERMINES THE OPERATION OF THE HEAT TRANSFER SYSTEM OF THE POWER PLANT WHEN THE BACK PRESSURE CURVE OF THE TURBINE IS SPECIFIED OVER THE WHOLE AMBIENT TEMPERATURE RANGE. IT ALSO DETERMINES THE BACK PRESSURE OF THE TURBINE OVER THE AMBIENT TEMPERATURE RANGE WHEN THE HEAT EXCHANGER IS COMPLETELY ORY.

DEFINITION OF VARIABLES

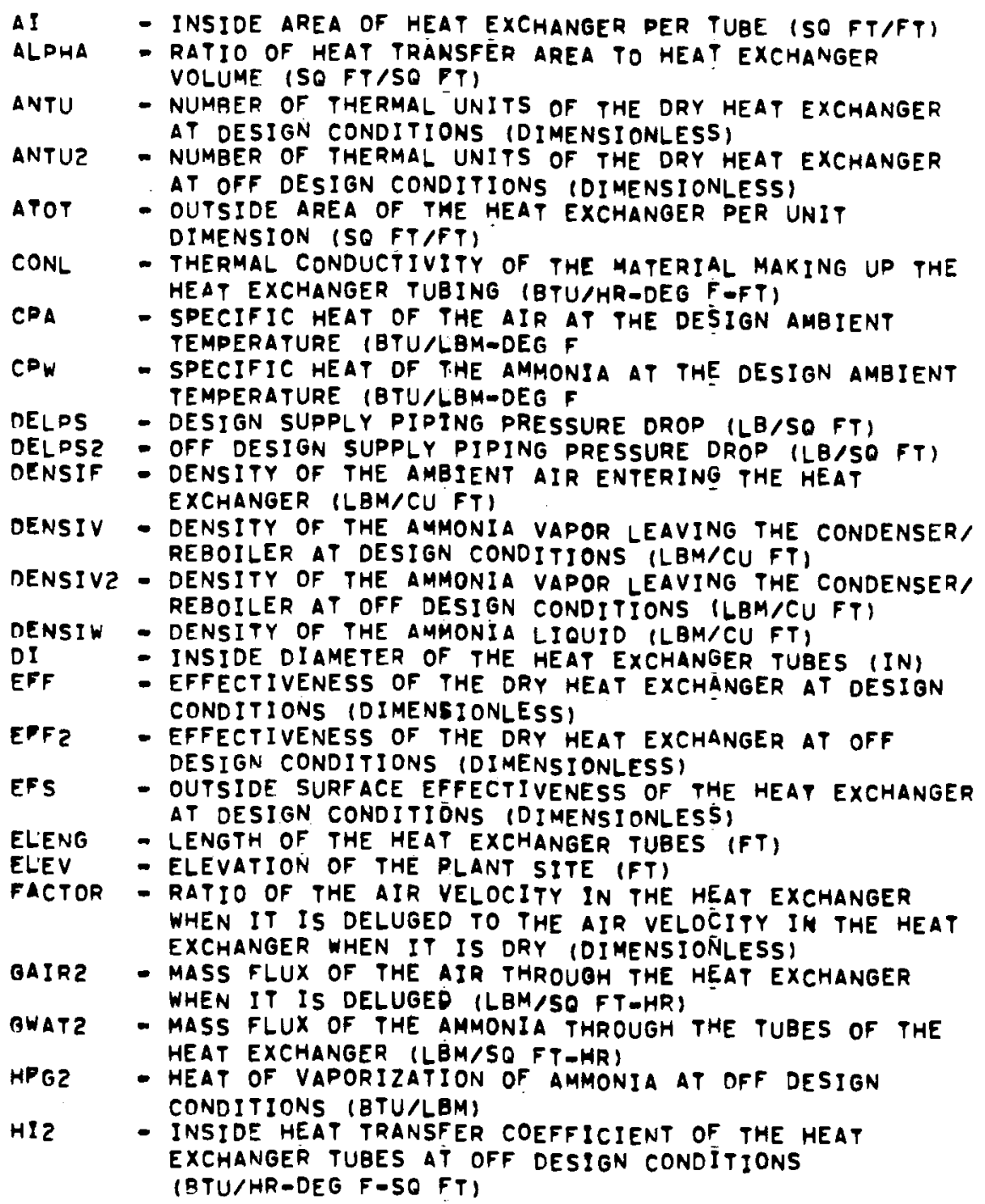

TBPMAX

TBPMAX

TBPMAX

TBPMAX

TBPMAX

TBPMAX

TBPMAX

TBPMAX

TBPMAX

TBPMAX

TBPMAX

TRDMAX

TBPMAX

TBPMAX

TBPMAX

TBPMAX

TBPMAX

TBPMAX

TBPMAX

TBPMAX

TBPMAX

TBPMAX

TBPMAX

TBPMAX

TBPMAX

TBPMAX

TBPMAX

TBPMAX

TBPMAX

TBPMAX

TBPMAX

TBPMAX

TBPMAX

TBPMAX

TBPMAX

TBPMAX

TBPMAX

TBPMAX

TBPMAX

TBPMAX

TBPMAX

TBPMAX

TBPMAX

TBPMAX

TBPMAX

TBPMAX

TBPMAX

TBPMAX

TBPMAX

TBPMAX

TBPMAX

TBPMAX

TBPMAX

TBPMAX

TBPMAX

TBPMAX 
$74 / 74$ OPTE1 FTN $4.5+414 \quad 06 / 05 / 78 \quad 07.59 .59$

HO - AIR SIDE HEAT TRANSFER COEFFICIENT OF THE HEAT TBPMAX 50 EXCHANGER AT DESIGN CONDITIONS (BTU/HR=DEG F-SQ FT) TBPMAX

HO2 - AIR SIDE HEAT TRANSFER COEFFICIENT OF THE HEAT TBPMAX EXCHANGER AT OFF DESIGN CONDITIONS (BTU/HR-DEG F-SO FT)

HRFACI - HEAT RATE FACTOR AT RATING CONDITION (DIMENSIONLESS)

HRFAC2 - HEAT RATE FACTOR AT DESIGN CONDITIONS (DIMENSIONLESS)

HRFAC4 - HEAT RATE FACTOR AT OFF DESIGN CONDITIONS (DIMENSIONLESS)

NTA - NUMBER OF TEMPERATURE INCREMENTS IN THE AMPIENT TEMPERATURE RANGE OF THE PLANT SITE (DIMENSIONLESS)

NW - NUMRER OF TUBES IN THE WIDTH OF THE HEAT EXCHANGER (OIMENSIONLESS)

OOL - OUTSIDE DIAMETER OF THE HEAT EXCHANGER TUBING (IN)

D - BACK DRESSURE OF THE TURBINE (IN HGA)

PATM - ATMOSPHERIC PRESSURE AT THE PLANT İITE (ATMOSPHERES)

PERCENT - FRACTION OF THE HEAT EXCHANGER DELUGED (DIMENSIONLESS

PERCENI - STORAGE VARIABLE FOR THE FRACTION OF THE HEAT EXCHANGER DELUGED (DIMENSIONLESS)

DRW - PRANDTL NUMBER OF THE AMMONIA LIQUID AT THE DESIGV CONOITIONS (DIMENSIONLESS)

PSIZ13 - TOTAL POWER OUTPUT OF THE PLANT AT DESIGN CONDITIONS (MEGAWATTS)

P12 - AMMONIA PRESSURE AT THE CONDENSER/REBOILER (LBF/SO FT)

PZ2 - AMMONIA PRESSURE AT THE HEAT EXCHANGER (LBF/SQ FT)

DDRY - AMOUNT OF HEAT REJECTED BY THE DRY PART OF THE HEAT EXCHANGER (BTU/HR)

QINI - HEAT INPUT INTO THE POWER PLANT (BTU/HR)

DREJTW - AMOUNT OF HEAT REJECTED BY THE WET PART OF THE HEAT TBDMAX EXCHANGER (BTU/HR)

DQEJTI - HEAT REJECTED AT THE DESIGN CONDITIONS OF THE PLANT (BTU/HR)

OREJT2 - HEAT REJECTED AT THE OFF DESIGN CONDITIONS OF THE PLANT (BTU/HR)

QSEC - AMOUNT OF HEAT REJECTED BY THE WET PART OF THE HEAT

RANGE - TEMPANGER PER UNIT DIMENSION (BTU/HR)

TBPMAX

TBPMAX

TBPMAX

TBPMAX

TBPMAX

TBPMAX

TBDMAX

TBPMAX

TBPMAX

TBPMAX

TBPMAX

TBDMAX

TBDMAX

TBPMAX

TBPMAX

TBOMAX

TBPMAX

TBPMAX

TBPMAX

TBPMAX

TBPMAX

TBPMAX

TBPMAX

TBPMAX

TBPMAX

TBPMAX

TBPMAX

TBPMAX

TBPMAX

TBPMAX

TBDMAX

TBPMAX

TBDMAX

TBPMAX

TBPMAX OF THE HEAT EXCHANGER AT DESIGN CONDITIONS (DEG F)

RANGE2 - TEMPERATURE RANGE OF THE AIR THROUGH THE DRY PART OF THE HEAT EXCHANGER AT OFF DESIGN CONDITIONS (DEG F)

RANGEZ1 - TEMPERATURE RANGE OF THE AIR THROUGH THE DRY PART OF THE HEAT EXCHANGER AT OFF DESIGN CONDITIONS (DEG F)

QMA - MASS FLOW RATE OF AIR FLOWING THROUGH EACH UNIT DIMENSION OF THE HEAT EXCHANGER (LBM/HR)

RMEV - AMOUNT OF WATER EVAPORATED PER UNIT DIMENSION OF THE HEAT EXCHANGER ILBM/HR AND THE TOTAL HEAT

TBPMAX

TBPMAX

TBDMAX

TBPMAX

TBPMAX

TBPMAX

TBPMAX

TBPMAX

TBPMAX

TBPMAX

TBPMAX

TBPMAX

TBPMAX EXCHANGER WHEN IT IS DELUGED (LBM/HR)

SIGMAG - FREE FLOW AREA TO FRONTAL AREA OF THE HEAT

TBPMAX

TBPMAX EXCHANGER (SO FT/SO FT)

TBOMAX 


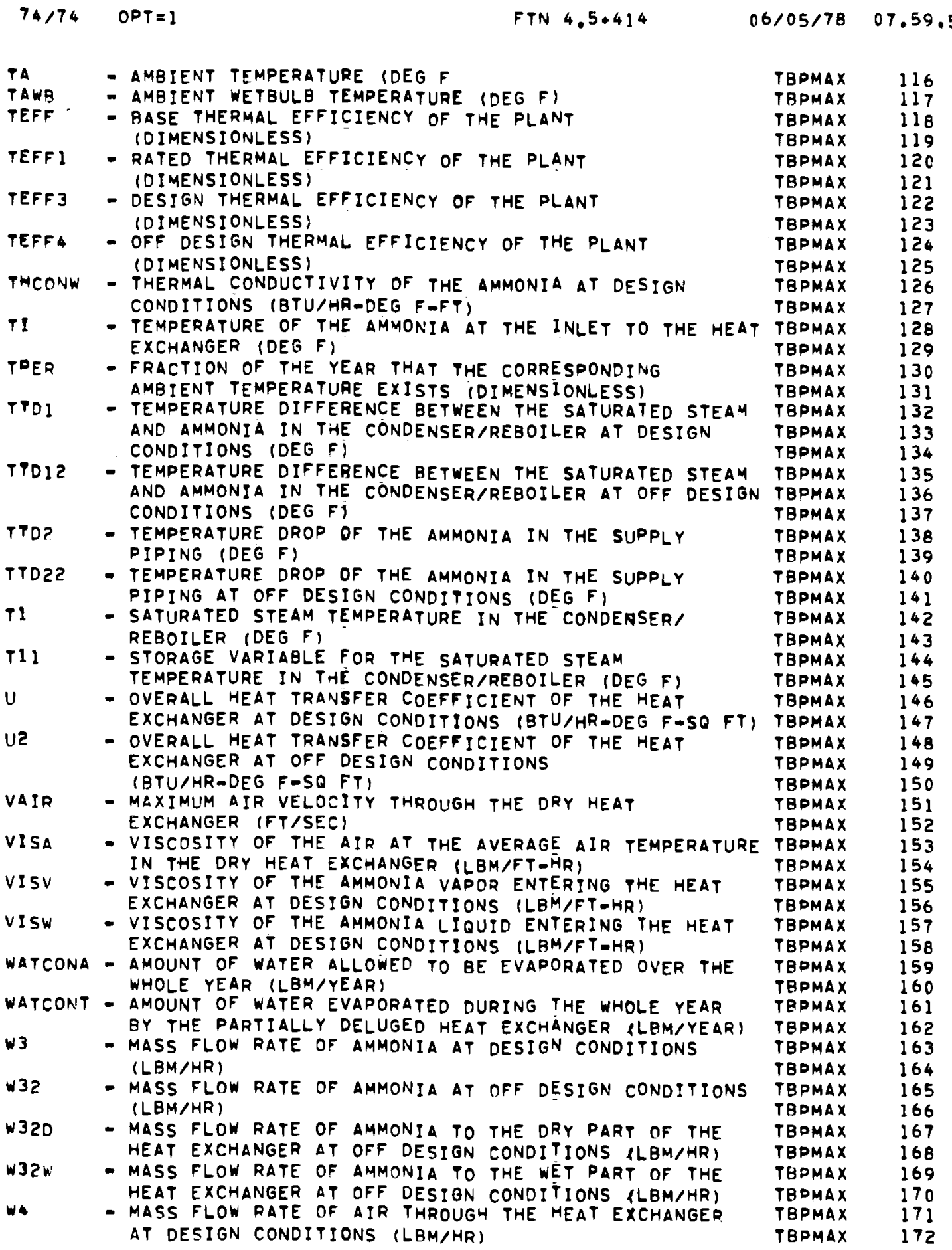


$74 / 74 \quad$ OPT $=1$

YFP - PLANT SCALING FACTOR FOR FAN AND PUMP POWER LOSS

XD - LONGITUDINAL DITEM OF THE TUBES IN THE HEAT EXCHANGER (IN)

YFP - PLANT SCALING FACTOR FOR FAN ANO PUMP POWER LOSS (DIMENSIONLESS)

$Z$ - HEAT EXCHANGER DEPTH IN NUMBER OF TUBES (DIMENSIONLESS)

20

- heAT EXCHANGER OEPTH IN NUMBER OF TUBES (DIMENSIONLESS)

DEFINITION OF ARRAYS

ANG

DPE

- the angle of the heat exchanger tubes from the HORIZONTAL AND THE SINE AND COSINE OF THAT ANGLE

- BACK PRESSURE LIMIT CURVE (IN HGA)

- FRACTION OF THE HEAT EXCHANGER THAT IS DELUGED FO? EACH AMBIENT TEMPERATURE INCREMENT (DIMENSIONLESS

DPERQ - FRACTION OF THE HEAT LOAD THAT IS CARRIED OFF BY THE DELUGED PART OF THE HEAT EXCHAÑGER FOR EACH AMBIENT TEMPERATURE INCREMENT (DIMENSIONLESS)

PDELUG - BACK PRESSURE CURVE WHEN THE HEAT EXCHANGER IS DELUGED COMPLETELY IIN HGA

PMAXDRY - ALL DRY BACK PRESSURE CURVE (IN HGA)

PSATMAX - BACK PRESSURE CURVE WHICH CAN INCLUDE ALL DRY OR ALL WET BACK PRESSURES (IN HGA)

TDELUG - TURBINE STEAM TEMPERATURE CURVE WHEN THE HEAT EXCHANGER IS DELUGED COMPLETELY (DEG F)

TMAXDRY - ALL DRY TURBINE STEAM TEMPERATURE CURVE (DEG F)

TPO - ARRAY OF COEFFICIENTS FOR THE CUBIC CURVE FIT OF HEAT RATE FACTOR AS A FUNCTION OF TURBINE BACK PRESSURE (DIMENSIONLESS)

TSATMAX - TURBINE STEAM TEMPERATURE CURVE WHICH CAN INCLUDE ALL DRY OR ALL WET TURBINE STEAM TEMPERATURES (DEG F)

VTA

VTAWB

VTPER

- AMBIENT TEMPERATURE CURVE (DEg F)

- AMBIENT WET BULB TEMPERATURE CURVE (DEg F)

- FRACTION OF THE year that THE aMBIENT AND WET BULB TEMPERATURE INCREMENTS EXIST (DIMENSIONLESS)

WATCON - WATER CONSUMPTION OF THE HEAT EXCHANGER OVER THE WHOLE YEAR FOR EACH AMBIENT TEMPERATURE INCREMENT (LBM/YEAR)

WATDE - WATER CONSUMPTION DF THE HEAT EXCHANGER WHEN IT IS COMPLETELY DELUGED OVER THE WHOLE YEAR FOR EACH AMBIENT TEMPERATURE INCREMENT (LBM/YEAR)

\section{LOGICAL FRST 3}

LOGICAL' FIRST, FRST, FXTEMP, FXTVAR,FXTTTD,FXTLNG COMMON

5 AFTQ. ALPHA, ANG(3)

* Bplim(16)

S CAPF; CSSPKW, CONF, CONL, COSTL, CAPCHG, CONMAT, CONMAZ, CAF

S COJ. CPURB, CDANG, CLUVR, CHAILS, CVM

$\$$, DFIN,DEEPL, DESVEL, DESVELV,DESVELD,DENSIF

$\$$,EFFP, EFS

S ,FCR,FIRST, FIXL,FCOS, FFHX, FXTEMP, FACTOR, FRST, FACTORD, FSHOT, FDELUG

S. FIXVIFIXTTD,FXTVAR,FXTTTD,FXYLNG

$\$$.GBEFF
$06 / 05 / 78$

07.59 .59

TBPMAX

TBPMAX

TBPMAX

TBPMAX

TBPMAX

TBPMAX

TBPMAX

TBDMAX

TBPMAX

TBPMAX

TBPMAX

TBPMAX

TBPMAX

TBPMAX

TBPMAX

TBPMAX

TBPMAX

TBPMAX

TBDMAX

TBPMAX

TBPMAX

TBPMAX

TBDMAX

TBPMAX

TBPMAX

TBPMAX

TBPMAX

TBPMAX

TBPMAX

TBPMAX

TBPMAX

TBPMAX

TBPMAX

TBPMAX

TBPMAX

TBPMAX

TBPMAX

TBPMAX

TBPMAX

TBPMAX

TBPMAX

TBPMAX

TBPMAX

TBPMAX

TBPMAX

TBPMAX

BIGCOM

BI GCOM

BIGCOM

BI GCOM

BIGCOM

BI GCOM

BIGCOM

BIGCOM

BIGCOM

BI GCOM

BIGCO4
173

174

175

176

177

178

179

180

181

182

183

184

185

186

137

188

189

190

191

192

193

194

195

196

197

198

199

200

201

202

203

204

205

206

207

$20 \mathrm{~B}$

209

210

211

212

213

214

215

216

217

218 


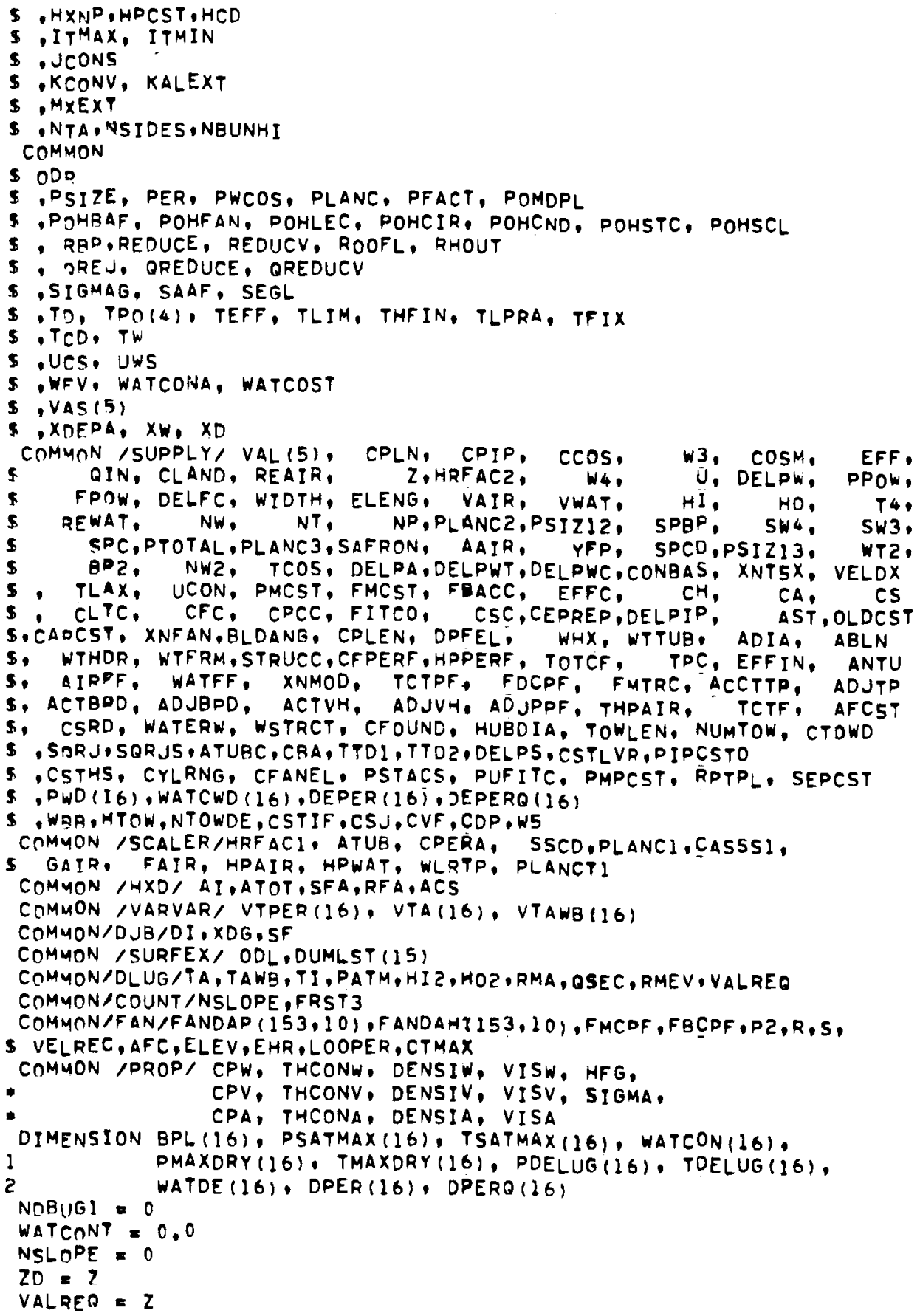

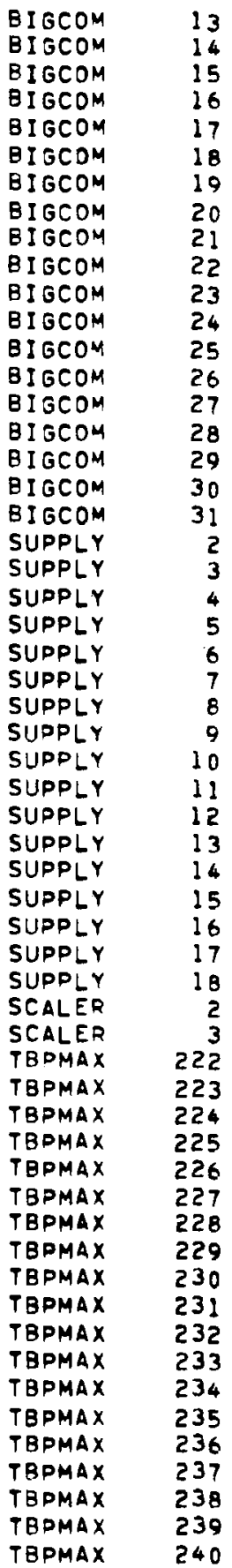


C DESIGN THERMAL EFFECTIVENESS OF THE POWER PLANT TEFF3 - TEFF/HRFAC2

c

C TEFF1 = TEFF/HRFAC 1

C HEAT INPUT INTO THE POWER PLANT

QIN1 $=3413, E \cdot 3 *$ PSIZ13/TEFFI

$c$
$c$
$c$
$c$
$c$
$c$
$c$
$c$
$c$

HEAT REJECTED AT THE OESIGN THERMAL EFFECTIVENESS OF THE DOWER PLANT OREJTI = QINI*11. - TEFF31

RANGE OF THE AIR AT THE DESIGN AMBIENT TEMPERATURE RANGE $\approx$ QREJT $1 /(W 4 *(1$, + YFP $)+C P A)$

IF (LICK.GT,O) GO TO 110

LDOP TO FIND THE BACK PRESSURE OF THE TURBINE OVER THE AMBIENT TEMPERATURE RANGE WITH AN ALL DRY HEÁT EXCHANGER

DO $100 \mathrm{~J}=1$, NTA

$I=N T A \cdot J \cdot I$

c

C

$T A=V T A(I)$

C DERCENT OF THE yEAR AT THE ABOVE AMBIENT TEMPERATURE TDED = VTPER (I)

$c$
$c$

c

C $10 P=E \times O(17.168-9240 . /(T) \cdot 460.1)$

INTIAL GUESS FOR THE ALL DRY TURBINE SATURATEO STEAN. TEMPERATURE

$T 1$ = TA * RANGE/EFF + TTDI + TTDZ

BACK PRESSURE OF THE TURBINE AT THE SATURATION TEMPERATURE

C HEAT RATE FACTOR AT THE ABOVE BACK PRESSURE

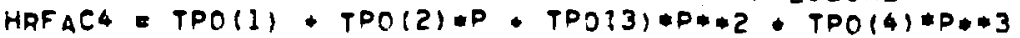

c

$c$ TEFF4 THERMAL EFFECT

HEAT REJECTED at THE abOVE THERMAL EFFECTIVENESS OF THE POWER PLANT

QREJTZ E QINI*11. - TEFF 4)

TEMPERATURE DIFFERENCE BETWEEN THE SATURATEO STEAM AND THE AMMONIA IN THE CONDENSER/REBOILER TTDI2 = (OREJT2/OREJT1) *TTDI

c

PRESSURE OF THE AMMONIA LEAVING THE CONDENSER/REROILER

$P, 2$ ELUID $130.1, T\}$ - TTDI2,ELEV."TBPMAXI")

HFG? = FLUID $19,1, T 1$ - TTDIZ,ELEV,"TBPMAXZ")

c DENSIV2 = FLUID(7,I,TI-TTDIZ,ELEV, "TEPMAX3")

MASS FLOW RATE OF AMMONIA THROUGH THE COOLING SYSTEM

TBPMAX

TBPMAX 242

TBDMAX 243

TBPMAX 244

TBPMAX 245

TBPMAX 246

TBPMAX 247

TBDMAX 248

TBPMAX 249

TBPMAX 250

TBPMAX 251

TBPMAX 252

TBDMAX 253

TBPMAX 254

TBPMAX 255

TBPMAX 256

TBPMAX 257

TBPMAX 258

TBPMAX 259

TBPMAX 260

TBPMAX 261

TBPMAX 262

TBPMAX 263

TBDMAX 264

TBPMAX 265

TBPMAX 266

TBDMAX 267

TBPMAX 268

TBPMAX 269

TBPMAX 270

TBPMAX 271

TBPMAX 272

TBPMAX 273

TBPMAX 274

TBPMAX 275

TBPMAX 276

TBPMAX 277

TBPMAX 278

TBPMAX 279

TBPMAX 280

TBPMAX 28 !

TBDMAX $2 E 2$

TBPMAX 283

TBPMAX 284

TBPMAX 285

TBPMAX 286

TBPMAX 287

TBPMAX 288

TBPMAX 289

TBPMAX 290

TBPMAX 291

TBPMAX 292

TBDMAX 293

TBDMAX 294

TBPMAX 295

TBDMAX 296

TBDMAX 297 


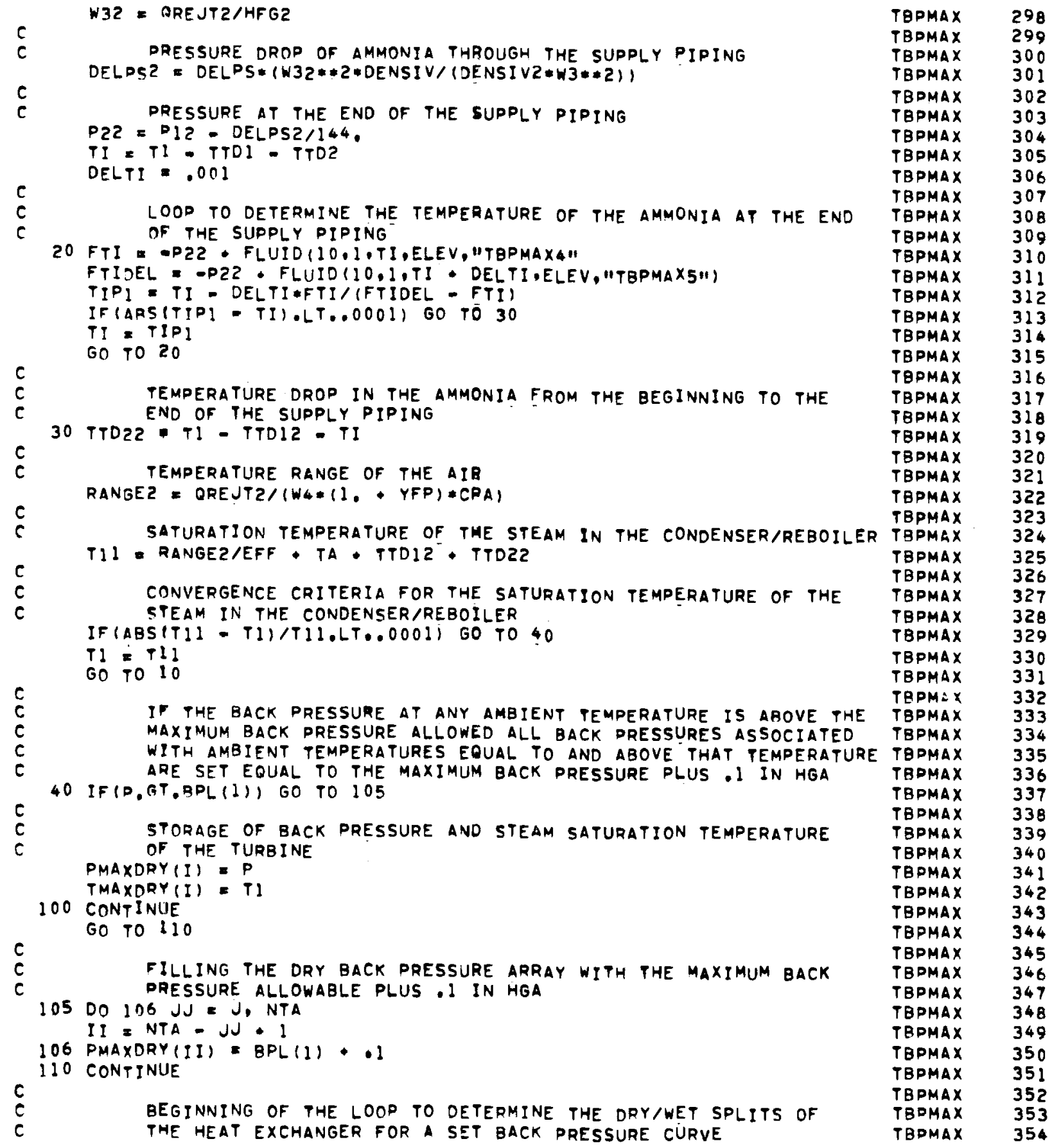




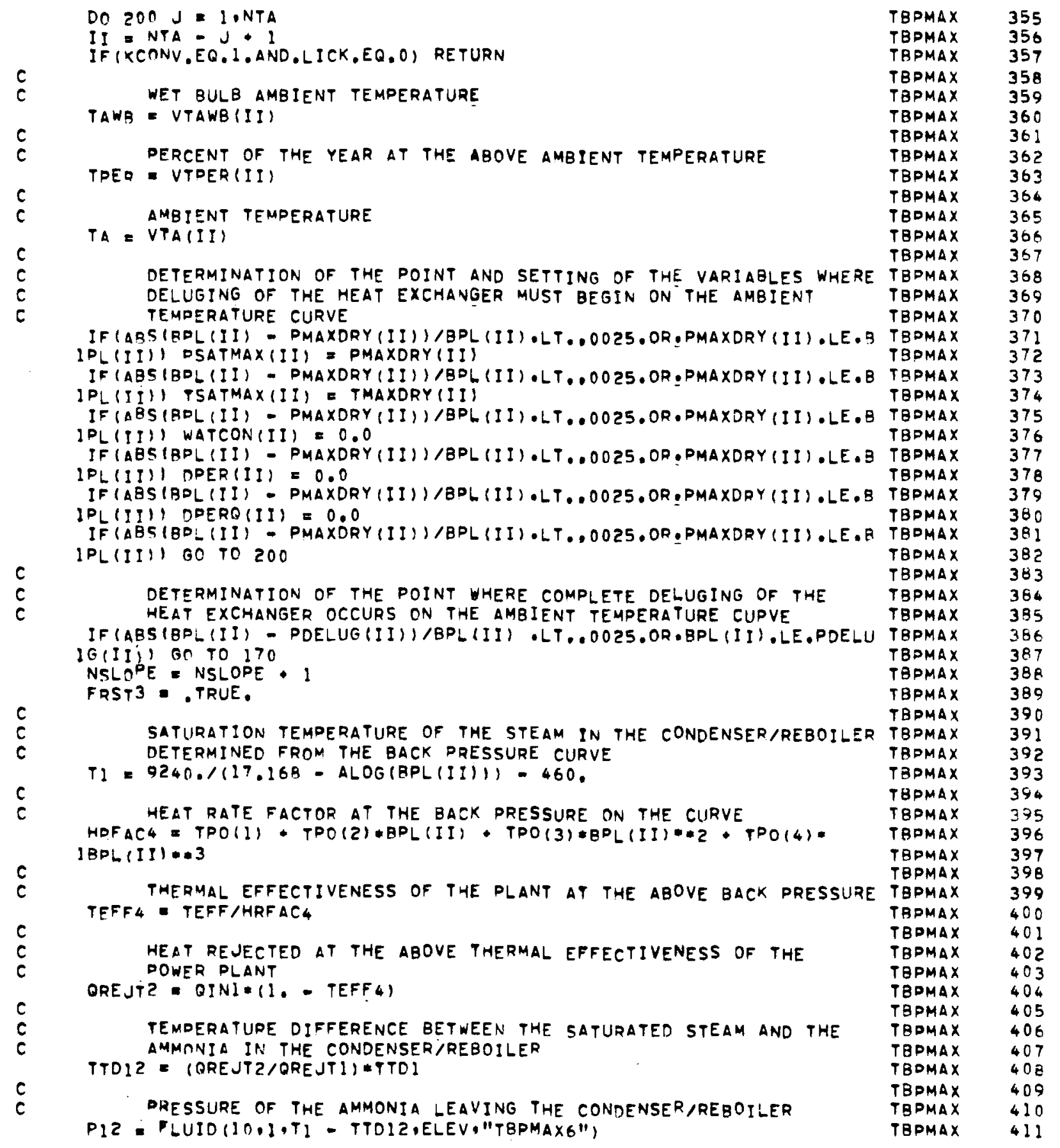




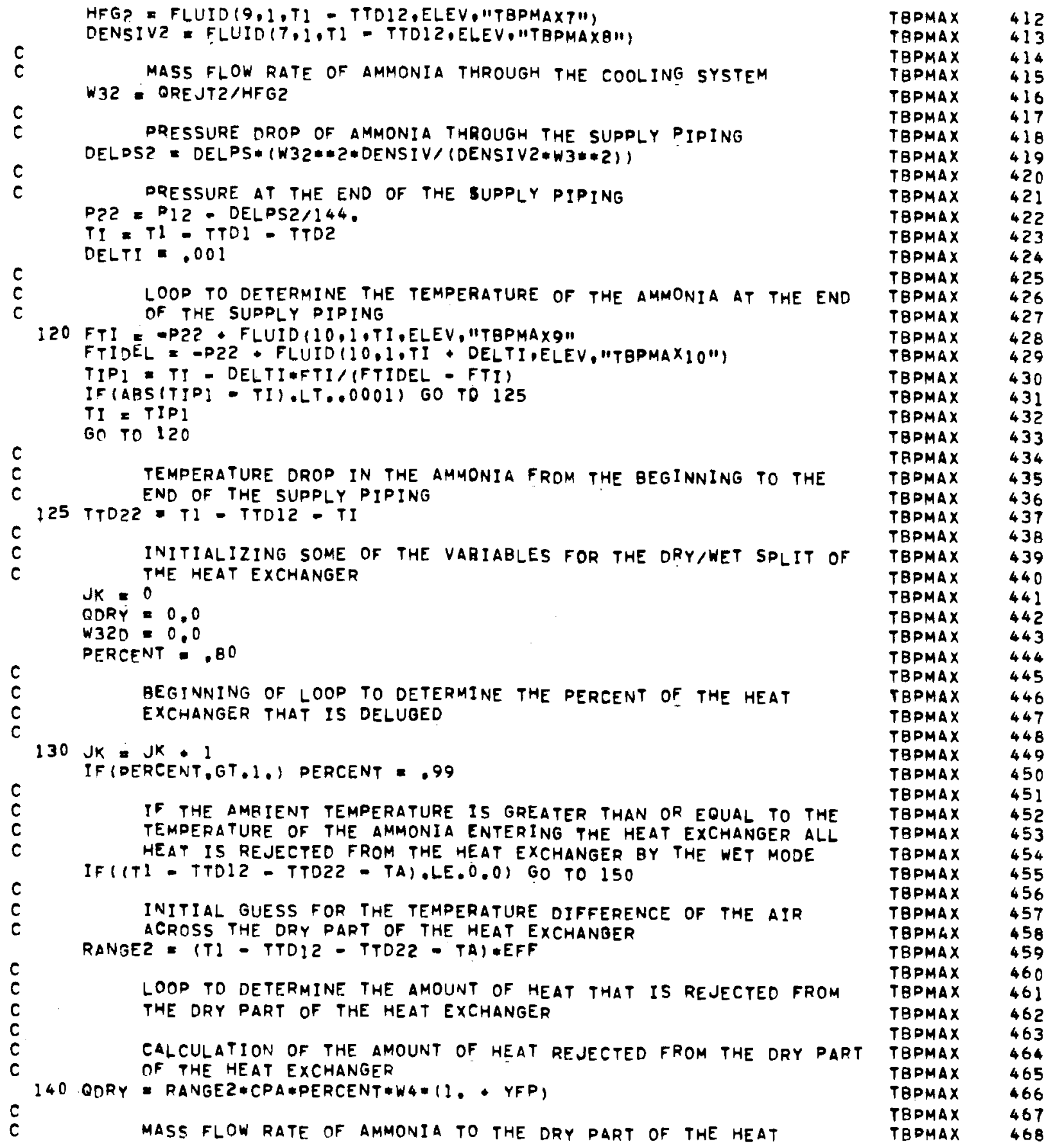


IGAIRZ,VISA)

IF IFDELUG,EQ. "DELBAW

IF (FDEL'UG:EQ, "DELUG

i) CALL MTEH(GAIRZ,HOZ)

IF (FDELUG,EQ, "DELBAW

I) CALL DELUG

IF (KCONV.EQ, I) RETURN

ii) CALL DELBAW

QREJTW = OSEC *NW*ELENG* (1 - PERCENT)

RMEV = NW*ELENG*(1. - PERCENT) *RMEV

IF (NOQUG I EQ.1) WRITE $(6,220)$ TI, GWATZ,HIZ,GAIRZ,HOZ,TA,TAWB,TI,

IPATM, RMA, QSEC, RMEV, PERCENT, QREJTW, QDRY, WA YCONT

c

SE OF THE CALCULATED HEAT REJECTION FROM THE TOTAL HEAT

EXCHANGER AND THE DESIRED HEAT REJECTION TO ESTIMATE THE

AMOUNT OF HEAT EXCHANGER THAT NEEDS TO BE DELUGED

IF (JK.LE. I) YI = OREJTW. ODRY

IF (JK.L'E. I) $X]$ I PERCENT

IF (JK.LE. I) PERCENT =.20

IF (JK.LE.1) GO TO 130

$Y Z=Y I$

$X_{2}=x_{1}$

$Y I=$ QREJTW - QORY

$X I=$ PERCENT

SLOPE $-(Y)=Y Z) /(X)-X Z)$

C

PERCEN1 = X1 * (QREJT2 = YI) /SLOPE

c

CONVERGENCE CRITERIA FOR THE PERCENT OF THE HEAT EXCHANGER THAT IS LEFT ORY

IF (ARS IPERCENI - PERCENT)/PERCENT.LT..001 GO TO 160

PERCFNT = PERCEN I

GO TO 130

C

STORAGE OF VARIABLES

160 WATCON(II) = TPER*8760.*RMEV

WATCONT - WATCONT - WATCON(II)

TSATMAX (II) = TI

PSATMAX (II) = BPL(II)

DPEQ (II) E 1 - - PERCENT

DPERR III) = QREJTW/OREJT2

$c$
$c$

GO TO 200

C SETTING OF VARIABLES WHEN THE HEAT EXCHANGER IS COMPLETELY DELUGED

170 WATCON III) = WATOE(II)

TSATMAX (II) = TDELUG (I I)

PSATMAX (II) = PDELUG III)

DPEQ II) $=1.0$

DPEROIII) $=1.0$

c

WATCONT = WATCONT - WATCONIII)

C IF THE WATER CONSUMEO OVER THE WHOLE YEAR AT THE MAXIMUM

IF THE WATER CONSUMEO OVER THE WHOLE YEAR AT THE MAXIMUM
BACK PRESSURE CURVE IS GREATER THAN THAT ALLOCATED THE DESIGN IS THROWN OUT

200 IF(WATCONT.GT.WATCONA.AND.LICK.EQ.0) KCONV =

$\begin{array}{ll}\text { TBPMAX } & 526 \\ \text { TBPMAX } & 527\end{array}$

TBPMAX 528

TBPMAX 529

TВPMAX 530

TBPMAX 531

TBDMAX 532

TBPMAX 533

TBPMAX 534

TBPMAX $\quad 535$

TBPMAX 536

TBPMAX 537

TBPMAX 538

TBPMAX 539

TBPMAX 540

TBPMAX 541

TBPMAX 542

TBPMAX 543

TBPMAX 544

TBPMAX 545

TBPMAX 546

TBPMAX 547

TBPMAX 548

TBPMAX 549

TBPMAX 550

TBPMAX 55

TBPMAX 552

TBPMAX 553

TBPMAX 554

TBPMAX $\quad 555$

TBPMAX 556

TBPMAX 557

TBPMAX 55B

TBPMAX 559

TBPMAX 560

TBPMAX 561

TBPMAX 562

TBPMAX 563

TBPMAX 564

TBPMAX 565

TBPMAX 566

TBPMAX 567

TBPMAX 568

TBPMAX 569

TBPMAX 570

TBPMAX 571

TBPMAX 572

TBPMAX 573

TBPMAX 574

TBPMAX 575

TBPMAX 576

TBPMAX 577 RETURN

TBPMAX

220 FORMAT $22 X$, ITI, GWATZ,HIZ, GAIRZ, HOZ,TA, TAWB,TI,PATM,RMA, OSEC,RMEV, PE TBPMAX IREENT , ORE JTW, QDRY,WATCONT $11,1,2 \times, 8 G 15.4,1.2 \times, 8615.411$

TBPMAX END 
SUBROUTINE TCALC COMPUTES THE TEMPERATURE, T, FROM A KNOWLEDGE OF

THE ENTHALPY (RI), RELATIVE HUMIDITY (RH), BAROMETRIC PRESSURE (ATM). TEST IS AN ESTIMATE FOR USE IN THE ITERATIVE CALCULATION. LIMITS ARE PLACED UPON THE RANGE OF RI WHICH WILL BE CONSIDERED IN ORDER TO PREVENT INSTABILITIES IN TCALC, IFOR EXAMPLE, RI APPROACHES INFINITY AS THE BOILING POINT IS REACHED--THUS, IF THE TEMPERATURE CORRESPONOING TO RI IS NEAR THE BOILING POINT, THE ITERATION WITHIN TCALC WILL BECOME UNSTABLE): THE UPPER LIMIT OF RI IS RIMAX. THE LOWER LIMIT IS RIMIN. SEE SUBROUTINE LIMIT FOR THE COMPUTATION OF RIMIN, RIMAX, TMIN AND TMAX.

COMMON /WETDRY, NCYCLE, TMAX,RIMAX, TMIN,RIMIN

COMMON/COUNT/NSLOPE, FRST 3

DIMENSION TNEW(20), RINEW(20)

DATA MAX/2O/

C

FUNCTION FP GIVES THE PARTIAL PRESSURE OF WATER, IN. HG.

$F D(R H, T)=R H * 29.92 *(0.000203 *(1 T-32) / 1.8) *$.

- $-0.0118=(T-32.111 .8+1.095)$

- ExP(-0.000426*(1T-32.)/1.8)*2

$+0.0828+(T-32,1 / 1.8-5.163)$

$F D(R H, T)=R H * E X P(17.168-9240.1(T+460)$.

TEST IS USED AS THE FIRST APPROXIMATION.

IF (N.EQ.1) TNEW (N) ETEST

IF EXTRAPOLATION GOES OUT OF TEMPERATURE BOUNDS. A NEW ESTIMATE IS MADE. NOTE THAT PMAX ANO TMIN ARE THE

TCALC

TCALC

TCALE

TCALC

TCALC

TCALC

TCALC

TCALC

TCALE

TCALC

TCALC

TCALC

TCALC

TCALC

TCALE

TCALC

TCALC

TCALC

TCALC

TCALC

TCALE

TCALC

TCALC

TCALC

TCALC

TCALC

TCALC

TCALC

TCALC

TCALC

TCALC

TCALE

TCALE

TCALC

TCALE

TCALC

TCALE

TCALE

TCALE

TCALC

TCALC

TCALE

TCALC

TCALC

TCALC

TCALE

TCALC

TCALC

TCALC

TCALC

TCALC

TCALE

TCALE

TCALE

TCALE 
TEMPERATURES CORRESDONDING TO RIMAX AND RIMIN, RE SPECTIVELY.

IF (TNEW (N) . GT - TMAX) TNEW (N) ETNEW(N-1) • (TMAX-TNEW $(N-1) 1 / 3$. IF (TNEW (N) .LT. TMIN) TNEW (N) ETMIN

$T=T N E W(N)$$$
\text { a VALUE OF ENTHALPY CORRESPONDING TO THE TNEW (N). }
$$$$
\text { RINEW(N), IS COMPUTED. }
$$

$P=F D(R H, T)$

$A H=F A H(P, A T M)$

CSETCS (AH)

RIDUMEFRI $(C S, T, A H)$

$c$
$c$
$c$
$c$
$c$
$c$
$c$
$c$
$c$

RINEW $(N)=R I D U M$

THE DEVIATION IN ENTHALPY IS COMPUTED.

$D R I=R I=R I N E W(N)$

C IF (NDBUG3.EQ.I) WRITE $(6,50) N$.TNEW (N) , RINEW (N), DRI

50 FORMAT (IX, "FROM TCALC 5ON,I5,3EIT,B)

A TEST FOR CONVERGENCE IS COMPUTED.

IF (ABSIDRI).LT.000I) GO TO 40

OIAGNOSTIC IF EXCESSIVE NUMBER OF ITERATIONS

IF (N,EQ.20) WRITE (6.170) FROM,N,RI,TEST,TNEW,RINEW

S, TMAX, TMIN, NCALC

170 FORMAT I/"ISUBSCRIPT LIMIT IN TCALC. CALLED FROM ".AIO," NE"

S. I5/1X, "RI $="$ " IPIEI3.5,3X, "TEST=", E 13.5/1X, "TNEW=",

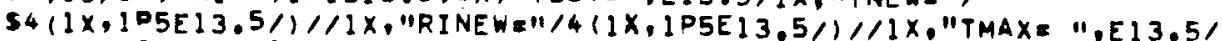

SIX, "TMINE ",EI3.5/IX,"NCALCE ". I5)

$c$
$c$
$c$
$c$
$c$
$c$
$c$ IF (N.EQ.20) RETURN

A NEW ESTIMATE FOR T IS OBTAINED BY USE OF THE DERIVATIVE OF T WITH RESPECT TO ENTHALPY FOR N EQUAL TO 1 LINEAR INTERPOLATION/EXTRAPOLATION TECHNIQUES ARE USED THEREAFTER.

IF (N, NE. I) GO TO 45

$T P=T+.0001$

$P P=F P(R H, T P)$

AHP $=F A H(P P, A T M)$

CSP IFCS (AHP)

$R I P=F R I$ (CSP, TP,AHP)

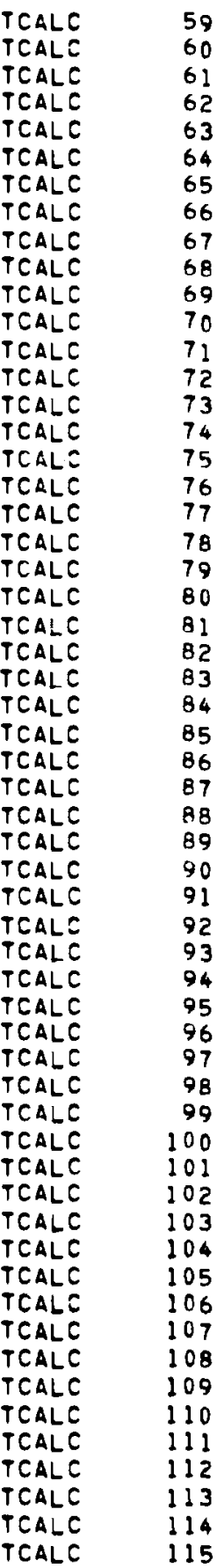


SUBROUTINE TCALC T4/74 OPTEI FTN $4.5 \times 414 \quad 06 / 05 / 78 \quad 07.59 .59$

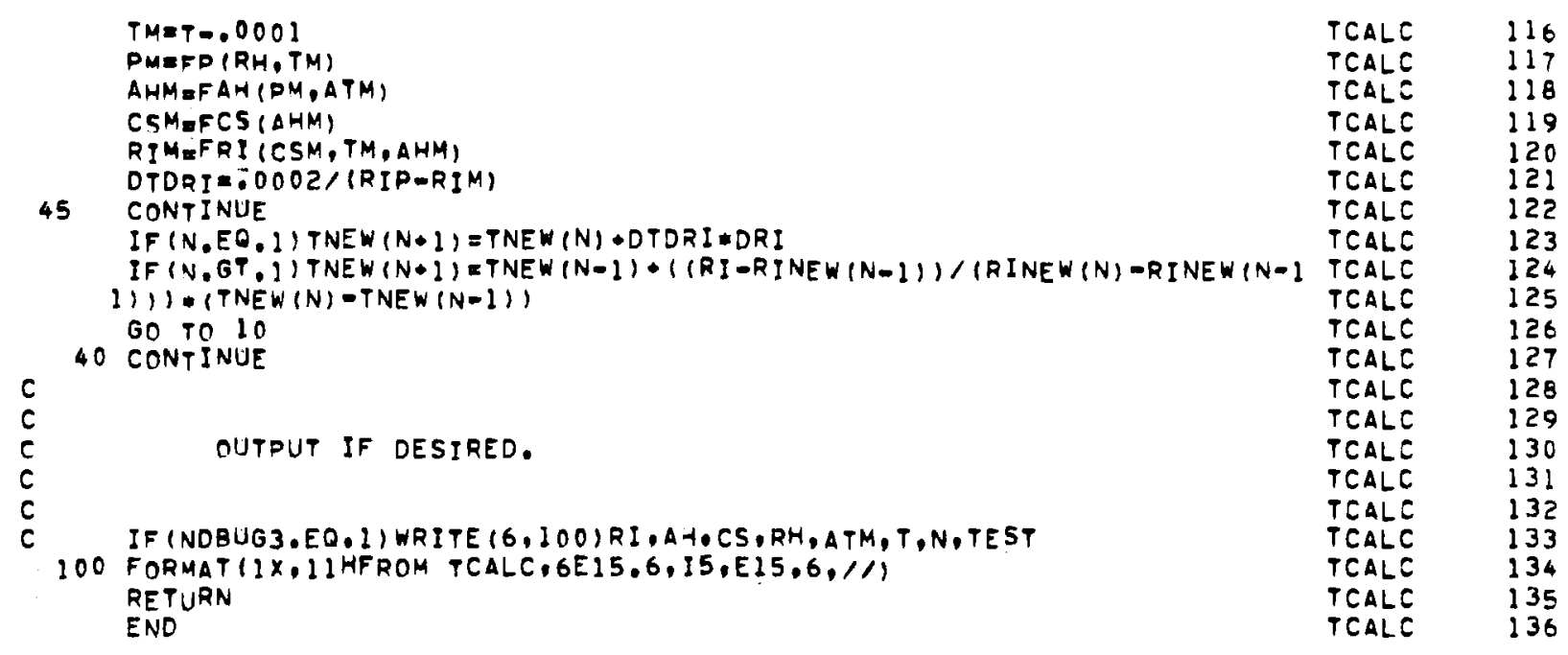




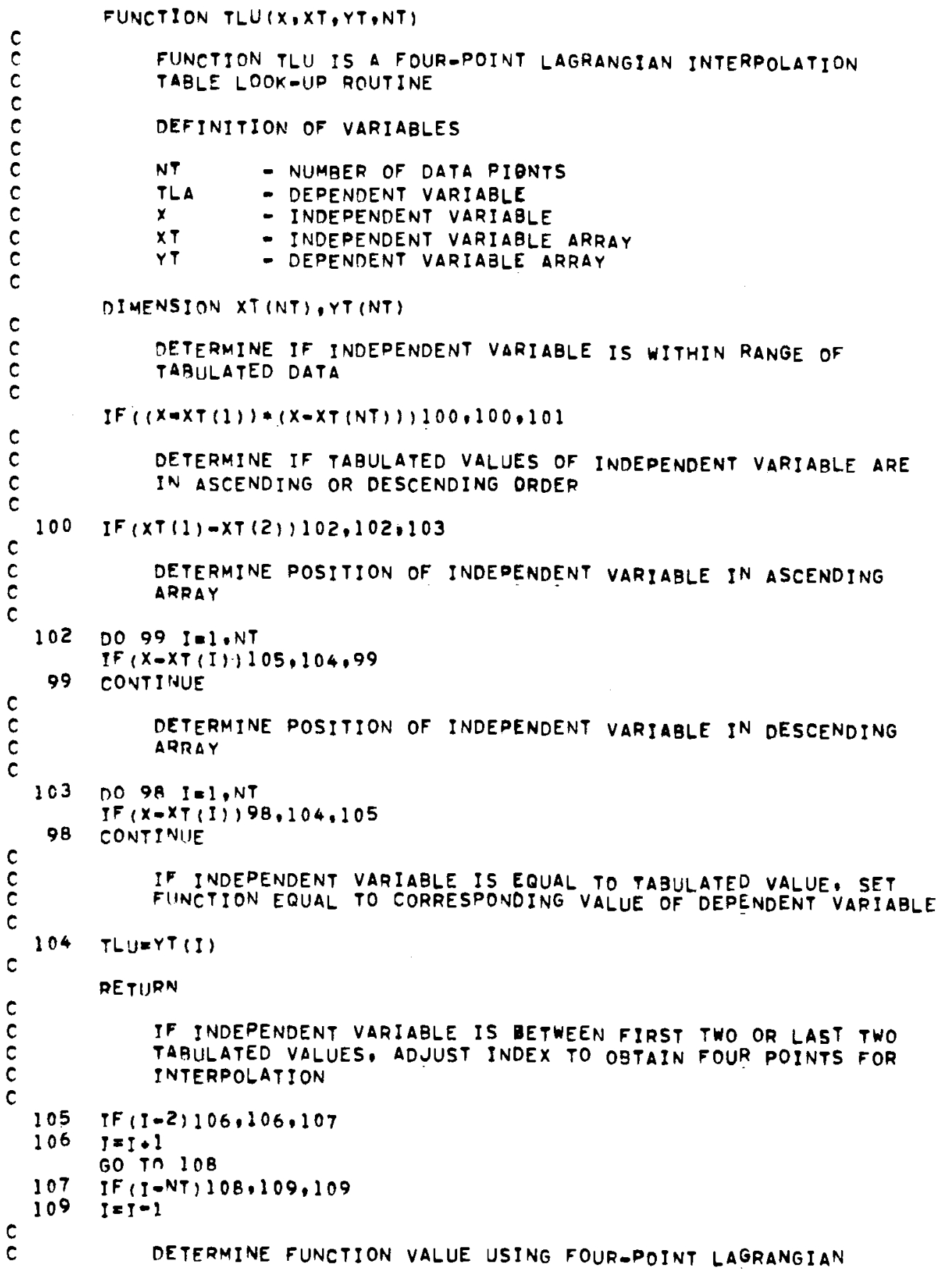




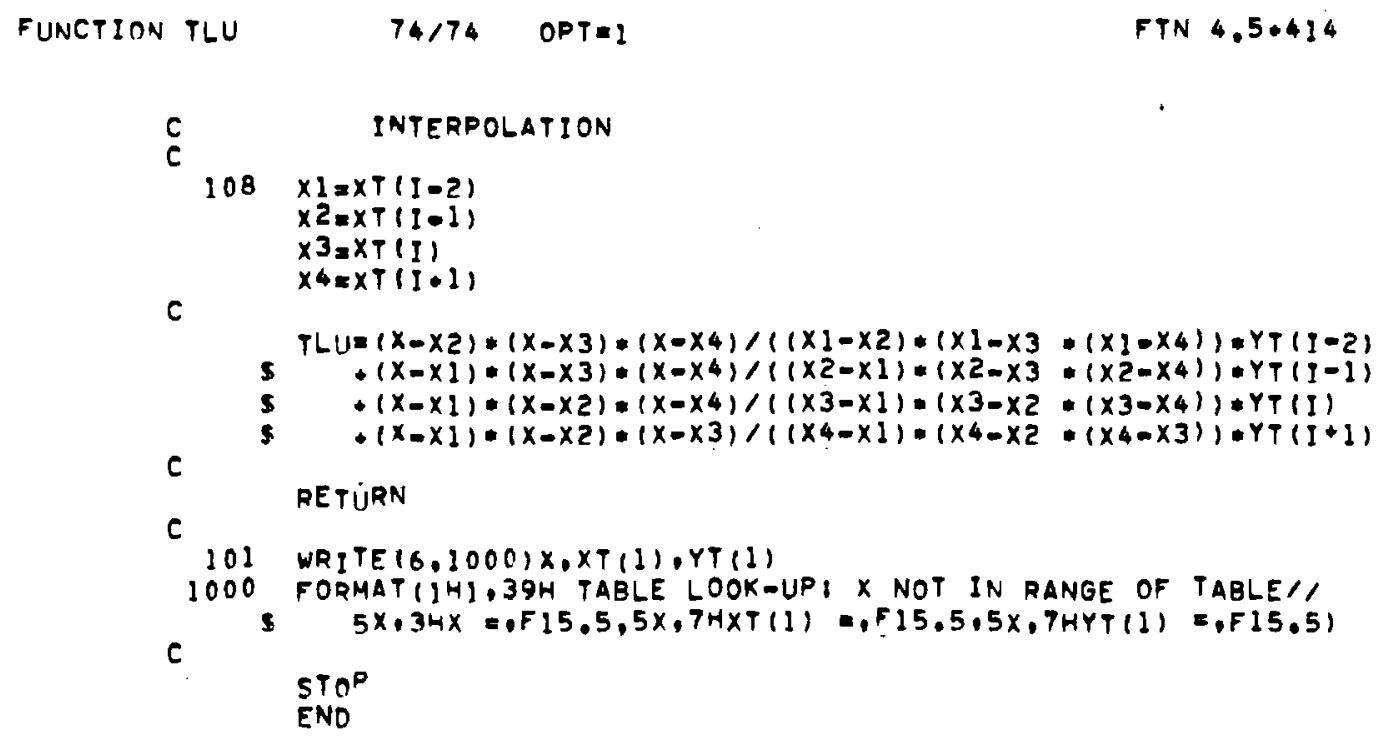




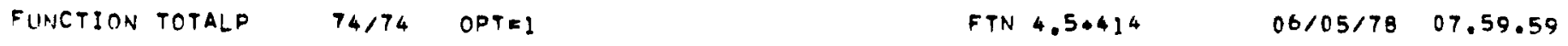

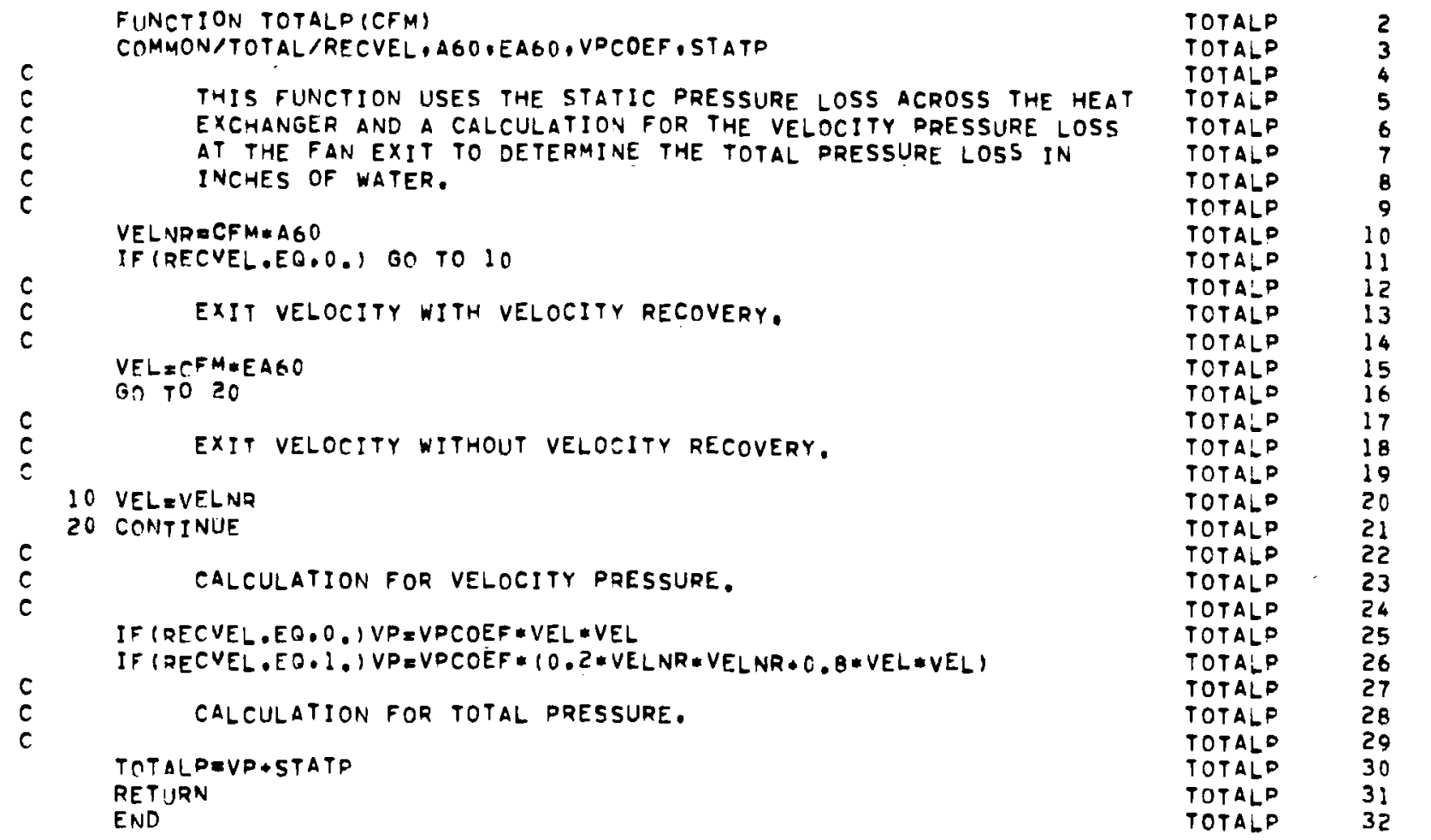


SUIBROUTINE UDEL ITA,RIA, ATM, HD, BETAF, AB, AF, AI, AW, HI, BETAI , THW, RKW, - TI,RII,HOD,UD,EFFIN,PATH,KCONV,NTRESTI SURROUTINE UDEL CALCULATES THE OVERALL TRANSFORMED HEAT TRAASFER COEFFICIENT BETWEEN THE TUBESIDE FLUID AND THE AIR. THIS TRANSFORMED HEAT TRANSFER COEFFICIENT USES ENTHALPY AS PHE DRIVING FORCE IENTHALPY DIFFERENCE DIVIDED BY HEAT CAPACITY OF THE AIR IS ANALDGOUS TO TEMPERATURE DIFFERENCE). THE OVERALL TRANSFORMED COEFFICIENT IS CALCULATED FROM

UDEL UDEL UDEL UDEL UDEL

UDEL

UDEL

UDEL TWO COEFFICIENTS. THAT BETWEEN THE TUBESIDE FLUID AND THE FIN UDEL ROOT, UDPR, AND THAT BETWEEN THE FIN ROOT AND THE AIR, UDRA, UDEL EACH OF THESE COEFFICIENTS ARE CALCULATED FROM LOWER ORDER UDEL COEFFICIENTS. THE RESULTS ARE DEPENDENT UPON THREE TEMPERA- UDEL TURES WHICH ARE UNKNOWN AT THE START OF THE CALCULATION, THE TEMPERATURE AT THE FIN ROOT. THE AVERAGE TEMPERATURE OF THE FIN AND THE TEMPERATURE OF THE DELUGEATE-AIR INTERFACE. THUS THE CALCULATION IS CARRIED OUT BY THE METHOD OF SUCCESSIVE APPRDXIMATIONS.

\section{A NOMENCLATURE OF SOME OF THE MORE FREQUENTLY USED} TERMS FOLLOWS.

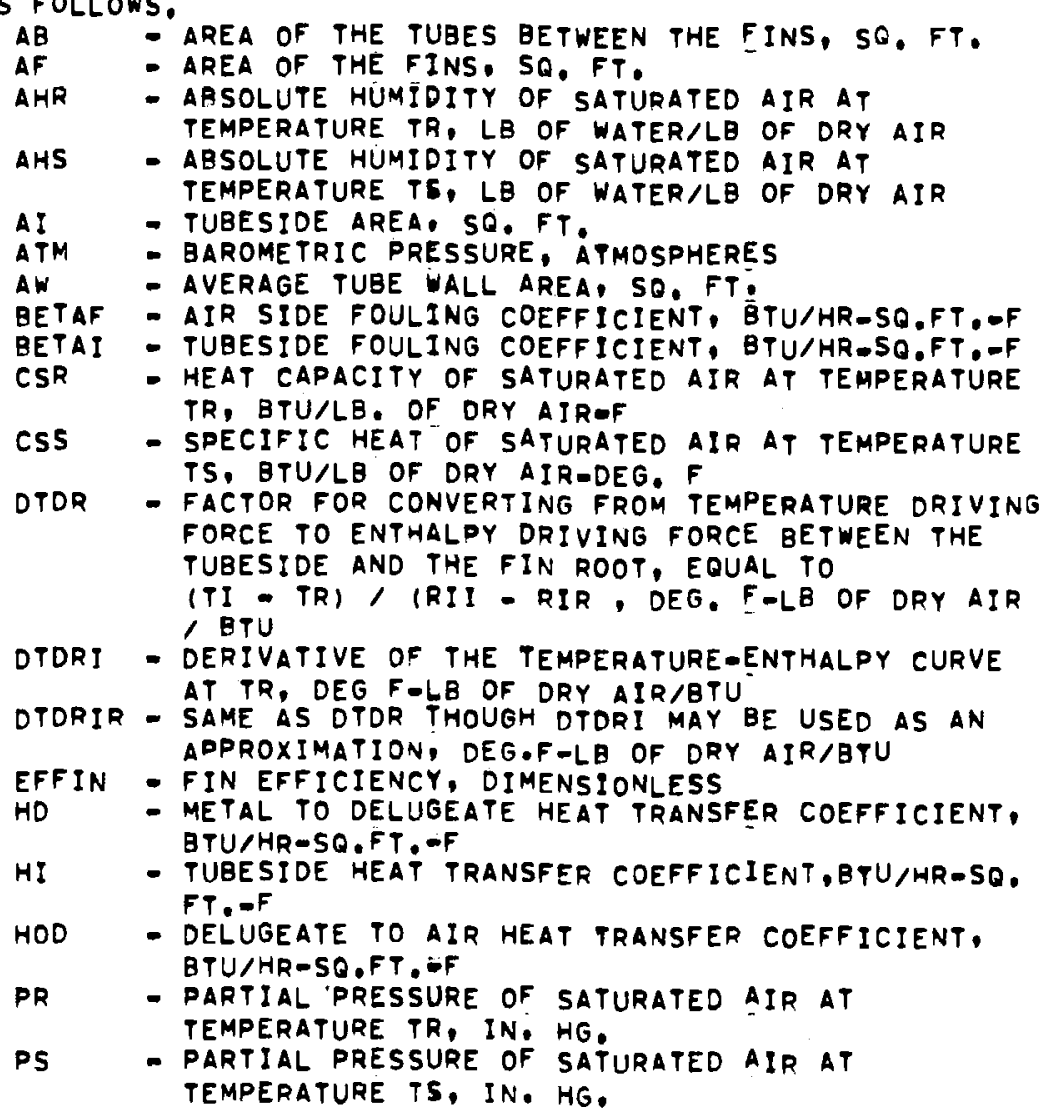

UDEL

UDEL

UDEL

UDEL

UDEL

UDEL

UDEL

UDEL

UDEL

UDEL

UDEL

UDEL

UDEL

UDEL

UDEL

UDEL

UDEL

UDEL

UDEL

UDEL

UDEL

UDEL

UDEL

UDEL

UDEL

UDEL

UDEL

UDEL

UDEL

UDEL

UDEL

UDEL

UDEL

UDEL

UDEL

UDEL

UDEL

UDEL

UDEL

UDEL

UDEL

UDEL

UDEL

UDEL 
$74 / 74 \quad$ OPT 1

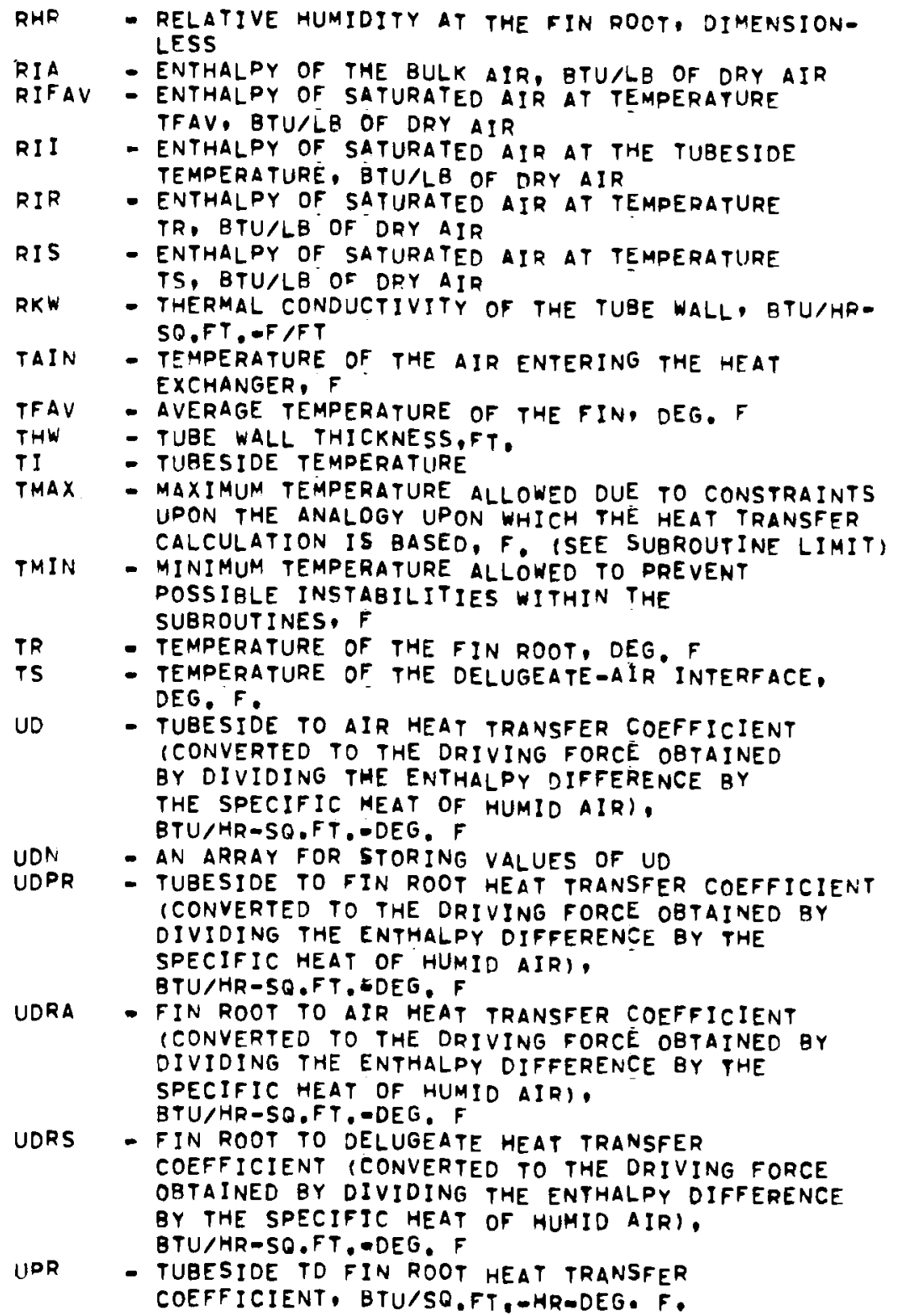

LOGICAL PATH. FRST3

COMMON /WETDRY, NCYCLE, TMAX,RIMAX, TMIN,RIMIN

COMMON/COUNT/NSLOPE,FRST 3

COMMON/RPRT 4/R4 (672), RR (3)

DIMENSION UDN(20), TRSAVE $(20)$, TSSAVE $(20)$, TFAVSA (20)

$\begin{array}{ll}\text { UDEL } & 59 \\ \text { UDEL } & 60 \\ \text { UDEL } & 61 \\ \text { UDEL } & 62 \\ \text { UDEL } & 63 \\ \text { UDEL } & 64 \\ \text { UDEL } & 65 \\ \text { UDEL } & 66 \\ \text { UDEL } & 67 \\ \text { UDEL } & 68 \\ \text { UDEL } & 69 \\ \text { UDEL } & 70 \\ \text { UDEL } & 71 \\ \text { UDEL } & 72 \\ \text { UDEL } & 73 \\ \text { UDEL } & 74 \\ \text { UDEL } & 75 \\ \text { UDEL } & 76 \\ \text { UDEL } & 77 \\ \text { UDEL } & 78 \\ \text { UDEL } & 79 \\ \text { UDEL } & 80 \\ \text { UDEL } & 81 \\ \text { UDEL } & 82 \\ \text { UDEL } & 83 \\ \text { UDEL } & 84 \\ \text { UDEL } & 85 \\ \text { UDEL } & 86 \\ \text { UDEL } & 87 \\ \text { UDEL } & 89 \\ \text { UDEL } & 89 \\ \text { UOEL } & 90 \\ \text { UDEL } & 91 \\ \text { UDEL } & 92 \\ \text { UDEL } & 93 \\ \text { UDEL } & 94 \\ \text { UDEL } & 95 \\ \text { UDEL } & 96 \\ \text { UDEL } & 97 \\ \text { UDEL } & 98 \\ \text { UDEL } & 99 \\ \text { UDEL } & 100 \\ \text { UDEL } & 101 \\ \text { UDEL } & 102 \\ \text { UDEL } & 103 \\ \text { UDEL } & 104 \\ \text { UDEL } & 105 \\ \text { UDEL } & 106 \\ \text { UDEL } & 107 \\ \text { UDEL } & 108 \\ \text { UDEL } & 109 \\ \text { UDEL } & 110 \\ \text { UDEL } & 111 \\ \text { UDEL } & 112 \\ \text { UDEL } & 113 \\ \text { UDE } & 114 \\ \text { UD } & 115 \\ \text { UD } & \end{array}$




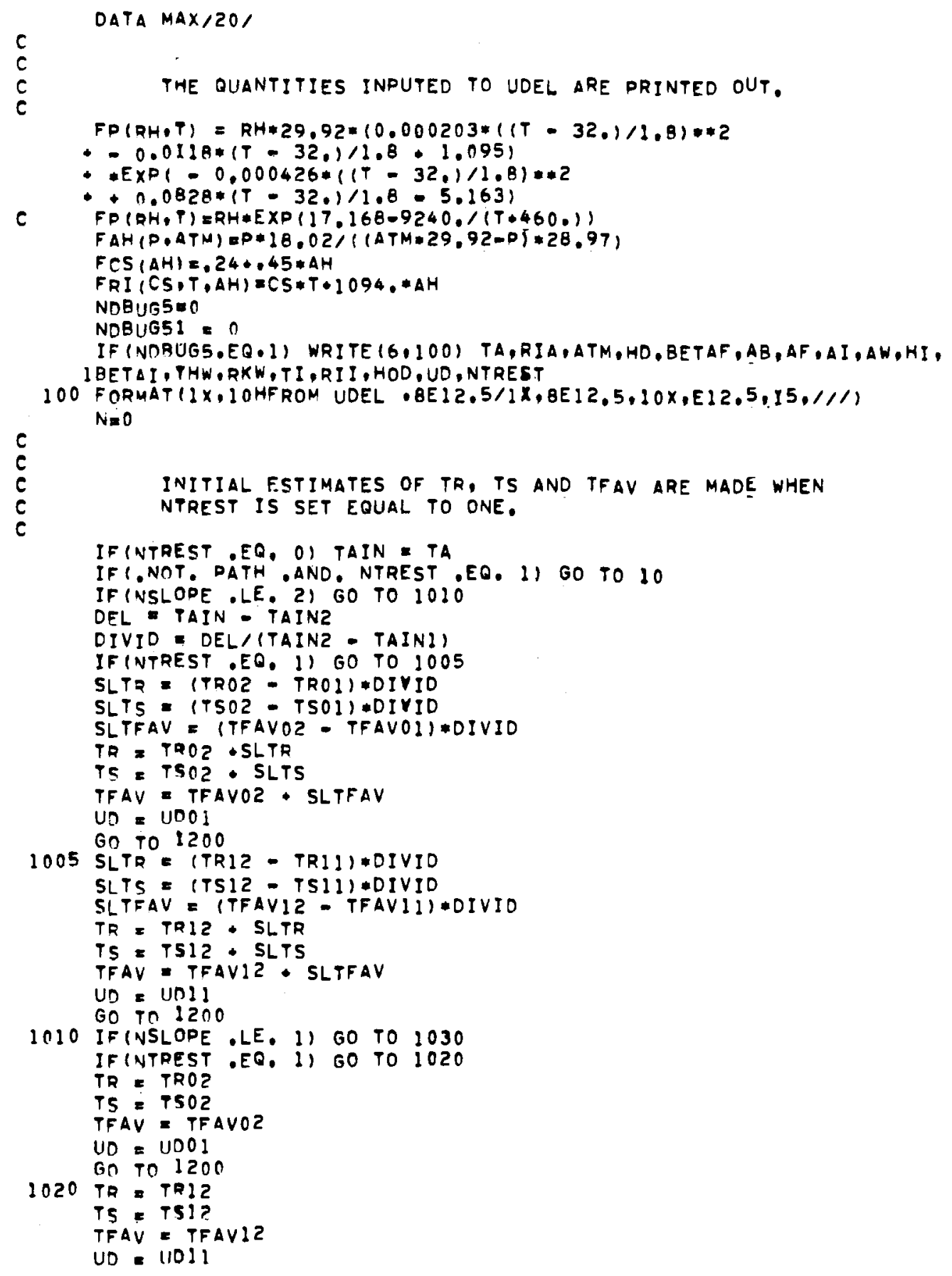

$\begin{array}{ll}\text { UDEL } & 116 \\ \text { UDEL } & 117 \\ \text { UDEL } & 118 \\ \text { UDEL } & 119 \\ \text { UDEL } & 120 \\ \text { UDEL } & 121 \\ \text { UDEL } & 122 \\ \text { UDEL } & 123 \\ \text { UDEL } & 124 \\ \text { UDEL } & 125 \\ \text { UDEL } & 126 \\ \text { UDEL } & 127 \\ \text { UDEL } & 128 \\ \text { UDEL } & 129 \\ \text { UDEL } & 130 \\ \text { UDEL } & 131 \\ \text { UDEL } & 132 \\ \text { UDEL } & 133 \\ \text { UDEL } & 134 \\ \text { UDEL } & 135 \\ \text { UDEL } & 136 \\ \text { UDEL } & 137 \\ \text { UDEL } & 138 \\ \text { UDEL } & 139 \\ \text { UDEL } & 140 \\ \text { UDEL } & 141 \\ \text { UDEL } & 142 \\ \text { UDEL } & 143 \\ \text { UDEL } & 144 \\ \text { UDEL } & 145 \\ \text { UDEL } & 146 \\ \text { UDEL } & 147 \\ \text { UDEL } & 148 \\ \text { UDEL } & 149 \\ \text { UDEL } & 150 \\ \text { UDEL } & 151 \\ \text { UDEL } & 152 \\ \text { UDEL } & 153 \\ \text { UDEL } & 154 \\ \text { UDEL } & 155 \\ \text { UDEL } & 156 \\ \text { UDEL } & 157 \\ \text { UDEL } & 158 \\ \text { UDEL } & 159 \\ \text { UDEL } & 160 \\ \text { UDEL } & 161 \\ \text { UDEL } & 162 \\ \text { UDEL } & 163 \\ \text { UDEL } & 164 \\ \text { UDEL } & 165 \\ \text { UDEL } & 166 \\ \text { UDEL } & 167 \\ \text { UDEL } & 168 \\ \text { UDEL } & 169 \\ \text { UDEL } & 170 \\ \text { UDEL } & 171 \\ \text { UDEL } & 172 \\ & \\ \text { UD } & 139\end{array}$


15 CONTINUE

$T P=T R+: 0001$

$P P=F P(R H A, T P)$

$\triangle H P=F A H$ (PD, $A T M$ )

CSP =FCS (AHP)

RIPEFRI (CSD, TP, AHP)

TMETRATOOOOI

$P M=F P(R H R, T M)$

$A H M=F A H$ (PM, ATM)

CSMEFCS (AHM)

RIMEFRI (CSM,TM,AHM)

DTDRIRE. $0002 /(R I P=R I M)$

\begin{tabular}{ll} 
UDEL & 173 \\
UDEL & 174 \\
UDEL & 175 \\
UDEL & 176 \\
UDEL & 177 \\
UDEL & 178 \\
UDEL & 179 \\
UDEL & 180 \\
UDEL & 181 \\
UDEL & 182 \\
UDEL & 183 \\
UDEL & 184 \\
UDEL & 185 \\
UDEL & 186 \\
UDEL & 187 \\
UDEL & 188 \\
UDEL & 189 \\
UDEL & 190 \\
UDEL & 191 \\
UDEL & 192 \\
UDEL & 193 \\
UDEL & 194 \\
UDEL & 195 \\
UDEL & 196 \\
UDEL & 197 \\
UDEL & 198 \\
UDEL & 199 \\
UDEL & 200 \\
UDEL & 201 \\
UDEL & 202 \\
UDEL & 203 \\
UDEL & 204 \\
UDEL & 205 \\
UDEL & 206 \\
UDEL & 207 \\
UDEL & 208 \\
UDEL & 209 \\
UDEL & 210 \\
UDEL & 211 \\
UDEL & 212 \\
UDEL & 213 \\
UDEL & 214 \\
UDEL & 215 \\
UDEL & 216 \\
UDEL & 217 \\
UDEL & 218 \\
UDEL & 219 \\
UDEL & 220 \\
UDEL & 221 \\
UDEL & 222 \\
UDEL & 223 \\
UDEL & 224 \\
UDEL & 225 \\
UDEL & 226 \\
UDEL & 227 \\
UDEL & 228 \\
UDEL & 229 \\
\hline
\end{tabular}




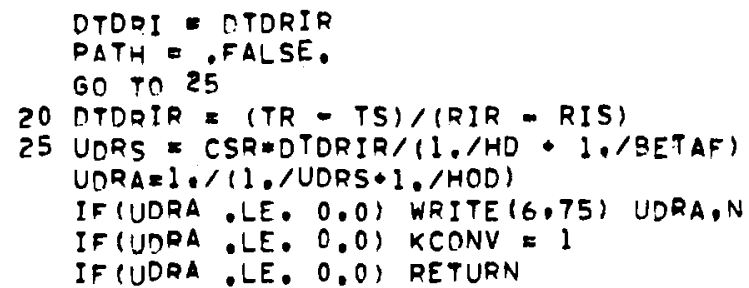

$\begin{array}{ll}\text { UDEL } & 230 \\ \text { UDEL } & 231 \\ \text { UDEL } & 232 \\ \text { UDEL } & 233 \\ \text { UDEL } & 234 \\ \text { UDEL } & 235 \\ \text { UDEL } & 236 \\ \text { UDEL } & 237 \\ \text { UDEL } & 238 \\ \text { UDEL } & 239 \\ \text { UDEL } & 240 \\ \text { UDEL } & 241 \\ \text { UDEL } & 242 \\ \text { UDEL } & 243 \\ \text { UDEL } & 244 \\ \text { UDEL } & 245 \\ \text { UDEL } & 246 \\ \text { UDEL } & 247 \\ \text { UDEL } & 248 \\ \text { UDEL } & 249 \\ \text { UDEL } & 250 \\ \text { UDEL } & 251 \\ \text { UDEL } & 252 \\ \text { UDEL } & 253 \\ \text { UDEL } & 254 \\ \text { UDEL } & 255 \\ \text { UDEL } & 256 \\ \text { UDEL } & 257 \\ \text { UDEL } & 258 \\ \text { UDEL } & 259 \\ \text { UDEL } & 260 \\ \text { UDEL } & 261 \\ \text { UDEL } & 262 \\ \text { UDEL } & 263 \\ \text { UDEL } & 264 \\ \text { UDEL } & 265 \\ \text { UDEL } & 266 \\ \text { UDEL } & 267 \\ \text { UDEL } & 268 \\ \text { UDEL } & 269 \\ \text { UDEL } & 270 \\ \text { UDEL } & 271 \\ \text { UDEL } & 272 \\ \text { UDEL } & 273 \\ \text { UDEL } & 274 \\ \text { UDEL } & 275 \\ \text { UDEL } & 276 \\ \text { UDEL } & 277 \\ \text { UDEL } & 278 \\ \text { UDEL } & 279 \\ \text { UDEL } & 280 \\ \text { UDEL } & 281 \\ \text { UDEL } & 282 \\ \text { UDEL } & 283 \\ \text { UDEL } & 284 \\ \text { UDEL } & 285 \\ \text { UDEL } & 286\end{array}$




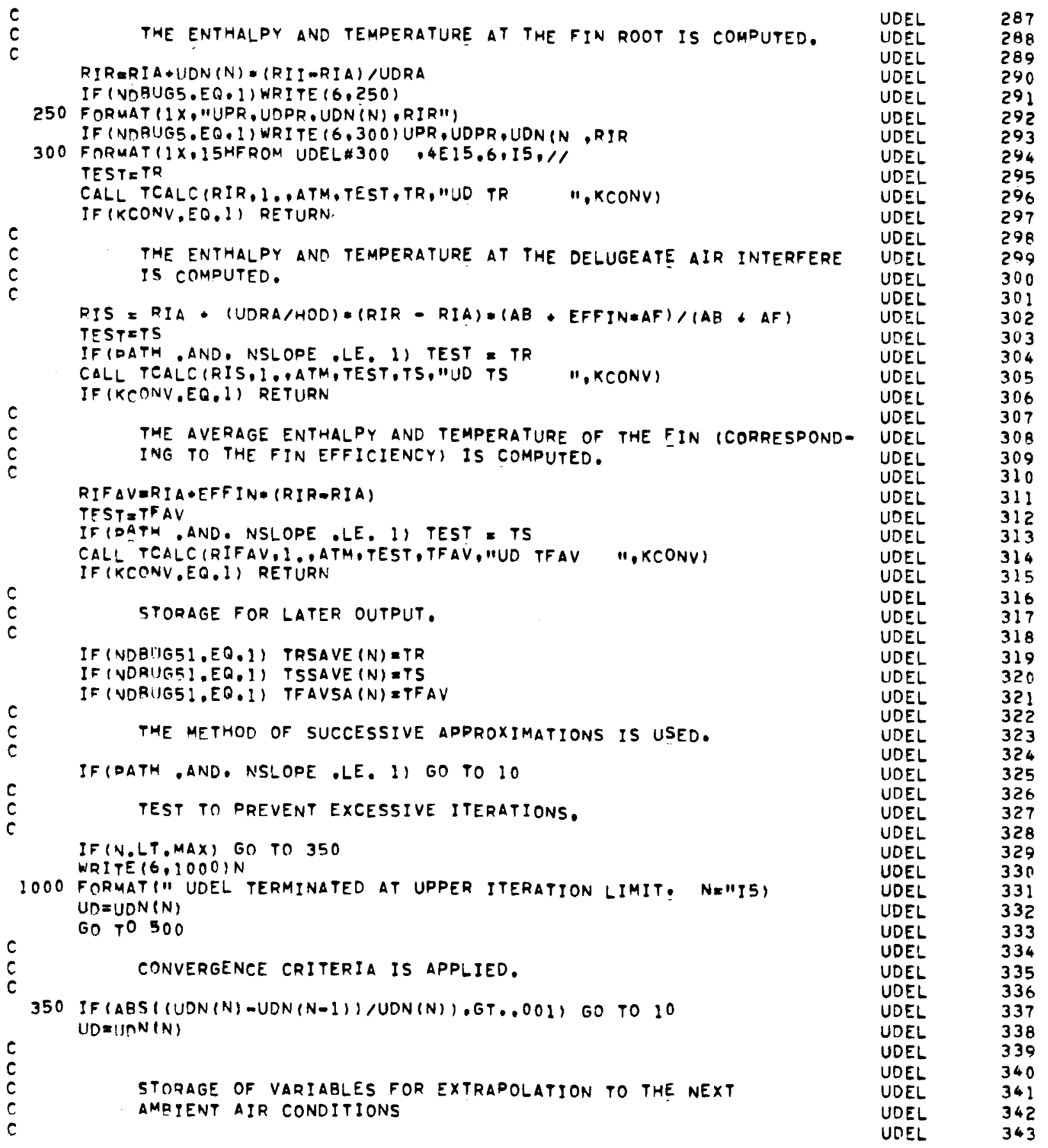




$\begin{array}{ll}\text { UDEL } & 344 \\ \text { UDEL } & 345 \\ \text { UDEL } & 346 \\ \text { UDEL } & 347 \\ \text { UDEL } & 348 \\ \text { UDEL } & 349 \\ \text { UDEL } & 350 \\ \text { UDEL } & 351 \\ \text { UDEL } & 352 \\ \text { UDEL } & 353 \\ \text { UDEL } & 354 \\ \text { UDEL } & 355 \\ \text { UDEL } & 356 \\ \text { UDEL } & 357 \\ \text { UDEL } & 358 \\ \text { UDEL } & 359 \\ \text { UDEL } & 360 \\ \text { UDEL } & 361 \\ \text { UDEL } & 362 \\ \text { UDEL } & 363 \\ \text { UDEL } & 364 \\ \text { UDEL } & 365 \\ \text { UDEL } & 366 \\ \text { UDEL } & 367 \\ \text { UDEL } & 368 \\ \text { UDEL } & 369 \\ \text { UDEL } & 370 \\ \text { UDEL } & 371 \\ \text { UDEL } & 372 \\ \text { UDEL } & 373 \\ \text { UDEL } & 374 \\ \text { UDEL } & 375 \\ \text { UDEL } & 376 \\ \text { UDEL } & 377 \\ \text { UDEL } & 378 \\ \text { UDEL } & 379 \\ \text { UDEL } & 380 \\ \text { UDEL } & 381 \\ \text { UDEL } & 382 \\ \text { UDEL } & 383 \\ \text { UDEL } & 384 \\ \text { UDEL } & 385 \\ \text { UDEL } & 386 \\ \text { UDEL } & 387 \\ \text { UDEL } & 388 \\ \text { UDEL } & 389 \\ \text { UDEL } & 390 \\ \text { UDEL } & 391 \\ \text { UDEL } & 392 \\ \text { UDEL } & 393 \\ \text { UDEL } & 394 \\ \text { UDEL } & 395 \\ \text { UDEL } & 396 \\ \text { UDEL } & 397 \\ \text { UDEL } & 398 \\ \text { UDEL } & 399 \\ \text { UDEL } & 400\end{array}$




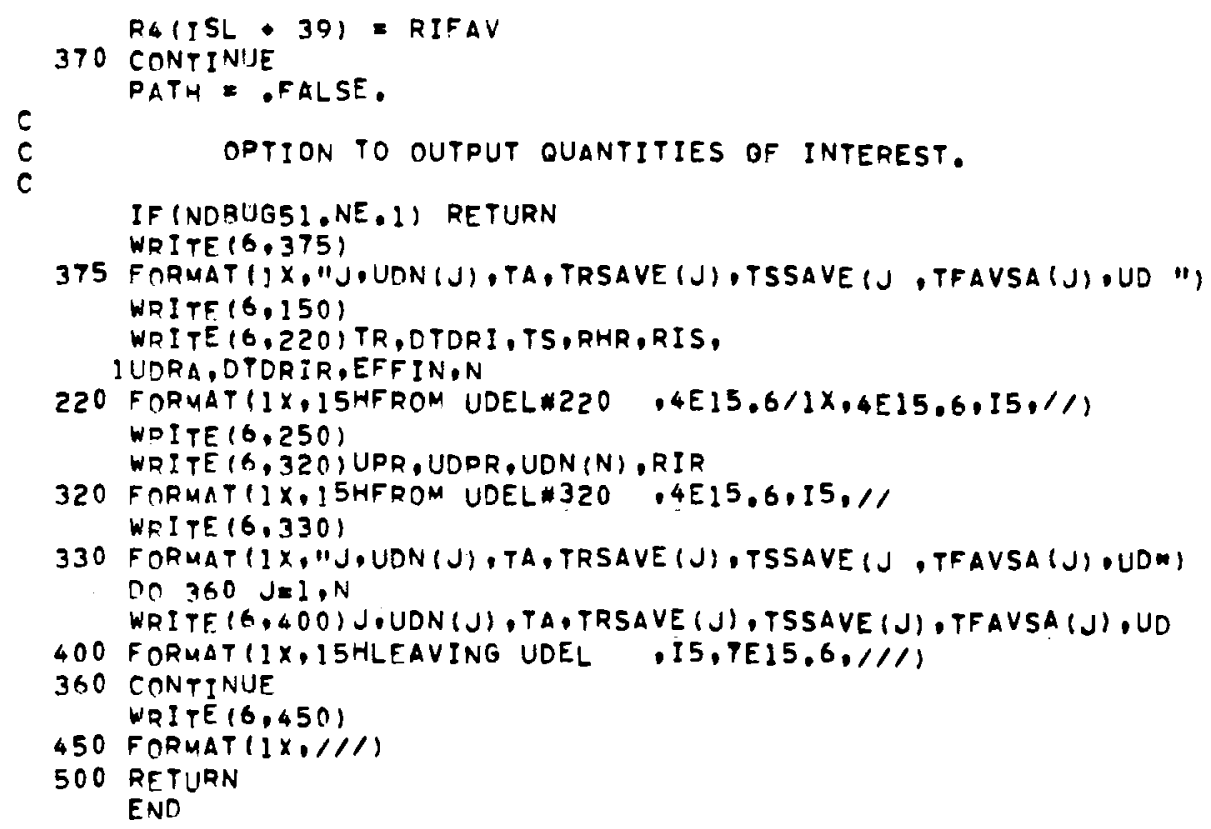

$\begin{array}{ll}\text { UDEL } & 401 \\ \text { UDEL } & 402 \\ \text { UDEL } & 403 \\ \text { UDEL } & 404 \\ \text { UDEL } & 405 \\ \text { UDEL } & 406 \\ \text { UDEL } & 407 \\ \text { UDEL } & 408 \\ \text { UDEL } & 409 \\ \text { UDEL } & 410 \\ \text { UDEL } & 411 \\ \text { UDEL } & 412 \\ \text { UDEL } & 413 \\ \text { UDEL } & 414 \\ \text { UDEL } & 415 \\ \text { UDEL } & 416 \\ \text { UDEL } & 417 \\ \text { UDEL } & 418 \\ \text { UDEL } & 419 \\ \text { UDEL } & 420 \\ \text { UDEL } & 421 \\ \text { UDEL } & 422 \\ \text { UDEL } & 423 \\ \text { UDEL } & 424 \\ \text { UDEL } & 425 \\ \text { UDEL } & 426 \\ & \end{array}$


$74 / 74 \quad$ OPT $=1$

FTN $4.5 * 414$

$06 / 05 / 78$

07.59 .59

SUBROUTINE VARITITA, TPER, PCST, KALL, CLSUM, FCSUM)

SUBROUTINE VARIT DETERMINES THE PERFORMANCE OF AN OPTIMUM POWER PLANT FOR A SPECIFIED AMBIENT TEMPERATURE THE SURROUTINE ALSO DETERMINES THE INCREMENTAL COST OF DPEDATING THE OPTIMUM DOWER PLANT AT THAT AMBIENT TEMPERATURE

DEFINITION OF VARIABLES

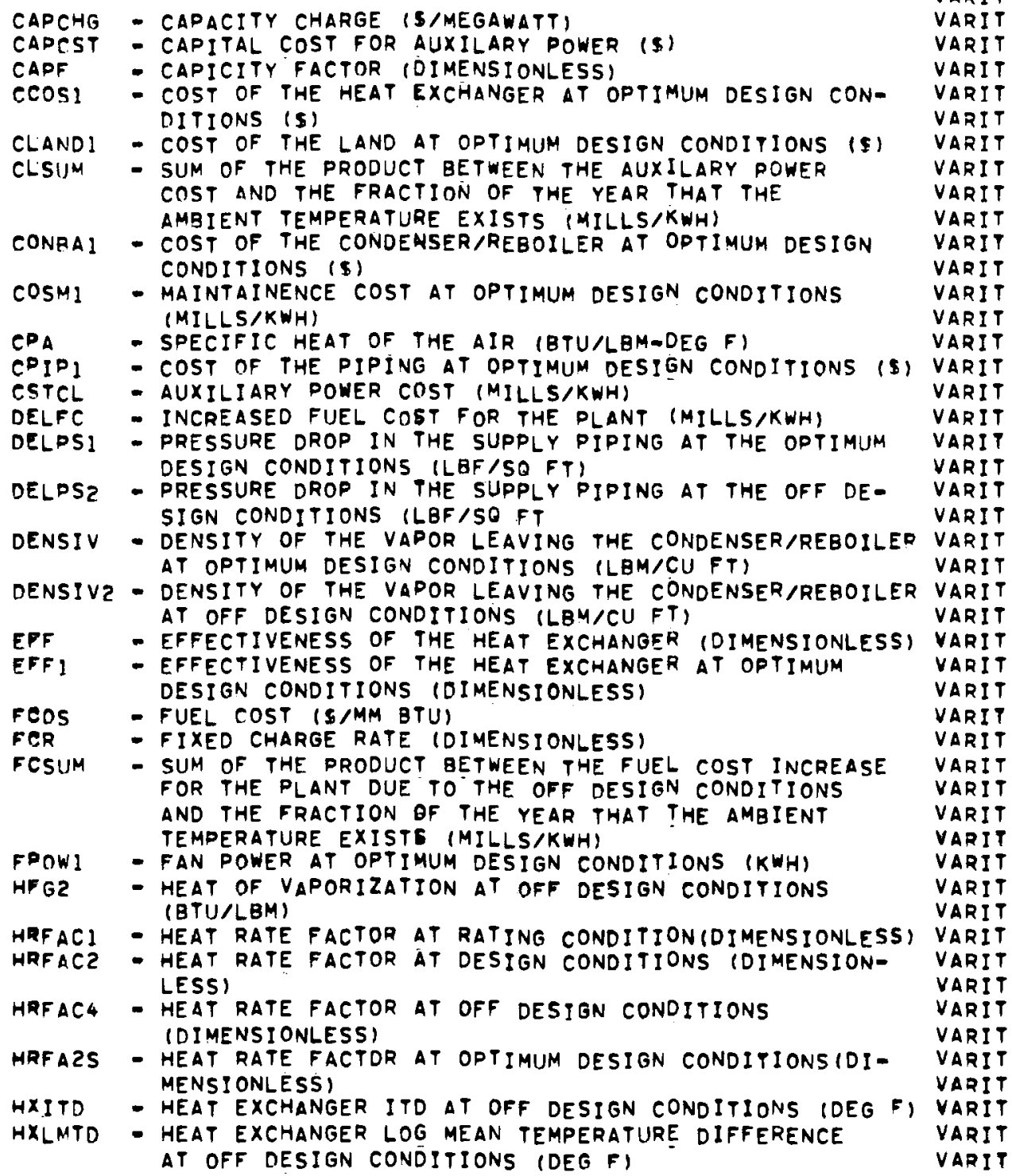

VARIT

VARIT

VARIT

VARIT

VARIT

VARIT

VARIT

VARIT

VARIT

VARIT

ARIT

VARIT

VARIT

ARIT

VARIT

VARIT

VARIT

VARIT

VARIT

VARIT

ARIT

VARIT

VARIT

VARIT

ARIT

VARIT

VARIT

VARIT

VARIT

VARIT

VARI

VARIT

ARIT

VARIT

VARIT

VARIT

VARIT 


\begin{tabular}{|c|c|c|c|}
\hline $74 / 74$ & FTN $4.5+414$ & $5 / 78$ & 1.50 \\
\hline OCST & $\begin{array}{l}\text { - BACK PRESSURE OF THE TURBINE IIN HGA) } \\
\text { - PERCENT OF THE YEAR AT THE SPECIFIED AMBIENT } \\
\text { TEMPERATURE TIMES THE INCREMENTAL COST AT THIS } \\
\text { TEMPERATURE (MILLG/KWH) }\end{array}$ & $\begin{array}{l}\text { VARIT } \\
\text { VARIT } \\
\text { VARIT } \\
\text { VARIT }\end{array}$ & \\
\hline 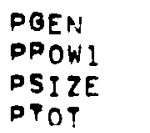 & $\begin{array}{l}\text { - POWER GENERATING Q9 THE PLANT (MEGAWATTS) } \\
\text { - PUMP POWER AT OPTIMUM DESIGN CONDITIONS (KWH) } \\
\text { - PLANT SIZE (MEGAWATTS) } \\
\text { - TOTAL POWER OUTPUT OF PLANT AT OFF DESIGN }\end{array}$ & $\begin{array}{l}\text { VARIT } \\
\text { VARIT } \\
\text { VARIT } \\
\text { VARIT }\end{array}$ & $\begin{array}{l}63 \\
64 \\
65\end{array}$ \\
\hline $\begin{array}{l}\text { DWCo } \\
112\end{array}$ & $\begin{array}{l}\text { CONDITIONS (MEGAWATTS) } \\
\text { - REPLACEMENT POWER COST (MILLS/KWH) } \\
\text { - AMMONIA PRESSURE AT THE CONDENSER/REBOILER (LBF) } \\
\text { SOFT) }\end{array}$ & & $\begin{array}{l}67 \\
68 \\
69\end{array}$ \\
\hline $\begin{array}{l}22 \\
\text { INI } \\
\text { DREJCTI }\end{array}$ & $\begin{array}{l}\text { - AMMONIA PRESSURE AT THE HEAT EXCHANGER (LBF/SQ FT) } \\
\text { - HEAT INPUT INTO THE OPTIMUM PRWER PLANT (PTU/HR) } \\
\text { - HEAT REJECTED AT THE OPTIMUM DESIGN CONDITIONS OF } \\
\text { THE PLANT (BTU/HR) }\end{array}$ & & $\begin{array}{l}71 \\
72 \\
73 \\
74\end{array}$ \\
\hline RE JCT2 & $\begin{array}{l}\text { - HEAT REJECTED AT THE OFF DESIGN CONDITIONS OF THE } \\
\text { PLANT }\end{array}$ & $\begin{array}{ll}\text { RIT } \\
\text { RIT }\end{array}$ & \\
\hline ANGE & $\begin{array}{l}\text { - TEMPERATURE RANGE OF THE AIR AT OPTIMUM DESIGN } \\
\text { CONDITIONS (DEG F) }\end{array}$ & & $\begin{array}{l}77 \\
78\end{array}$ \\
\hline ANGEZ & $\begin{array}{l}\text { - TEMPERATURE RANGE OF THE AIR AT THE OFF DESIGN } \\
\text { CONDITIONS (DEG F) }\end{array}$ & & $\begin{array}{l}79 \\
80\end{array}$ \\
\hline$P C D I$ & $\begin{array}{l}\text { - DIFFERENTIAL COST OF PLANT EXCLUDING THE COOLING } \\
\text { SYSTEM AT OPTIMUM DESIGN CONDITIONS (\$) } \\
\text { - TOTAL POMER OUTPUT OF PLANT AT OPTIMUM DESIGN }\end{array}$ & & $\begin{array}{l}81 \\
82 \\
83\end{array}$ \\
\hline A & $\begin{aligned} & \text { CONDITIONS (MEGAWATTŚ) } \\
& \text { - AMBIENT TEMPERATURE IDEG F }\end{aligned}$ & & 84 \\
\hline$A M M$ & $\begin{array}{l}\text { - TEMPERATURE OF THE AMMONIA LEAVING THE CONDENSER/ } \\
\text { REBOILER AT THE OPTIMUM DESIGN CONDITIONS FOR THE } \\
\text { PLANT (DEG F) }\end{array}$ & & $\begin{array}{l}86 \\
87 \\
88\end{array}$ \\
\hline Co & $\begin{array}{l}\text { - INCREMENTAL INCREASE IN COST OF THE POWER PRODUCEO } \\
\text { BY THE OPTIMUM POWER PLANT DUE TO THE COOLING } \\
\text { SYSTEM (MILLS/KWH) }\end{array}$ & & $\begin{array}{l}89 \\
89 \\
90\end{array}$ \\
\hline D & $\begin{aligned} & \text { SYSTEM (MILLS/KWH) } \\
& \text { - DESIGN AMBIENT TEMPERATURE (DEG F) }\end{aligned}$ & & \\
\hline $\begin{array}{l}E F \\
E F\end{array}$ & $\begin{array}{l}\text { - BASE THERMAL EFFICIENCY OF THE PLANT (DIMENSIONLESS) } \\
\text { - RATED THERMAL EFFICIENCY OF THE PLANT } \\
\text { (DIMENSIONLESS) }\end{array}$ & & $\begin{array}{l}93 \\
94 \\
95\end{array}$ \\
\hline EF & - DESIGN THERMAL EFFICIENCY OF THE OPTIMUM PLANT & & $\begin{array}{l}96 \\
97\end{array}$ \\
\hline EFI & $\begin{array}{l}\text { - OFF DESIGN THERMAL EFFICIENCY OF THE PLANT } \\
\text { (DIMENSIONLESS) }\end{array}$ & & $\begin{array}{r}98 \\
99\end{array}$ \\
\hline PER & $\begin{array}{l}\text { - TEMPERATURE OF THE AMMONIA AT THE INLET TO THE } \\
\text { HEATEXCHANGER IOEG F) } \\
\text { - FRACTION OF THE YEAR THAT THE CORRESPONDING }\end{array}$ & & $\begin{array}{lll}1 & 0 & 0 \\
1 & 0 & 1 \\
1 & 0 & 2\end{array}$ \\
\hline TD 1 & $\begin{aligned} & \text { AMBIENT TEMPERATURE EXISTS (DIMENSI DNLESS) } \\
& \text { - TEMPERATURE DIFFERENCE BETWEEN THE SATURATED }\end{aligned}$ & & $\begin{array}{l}103 \\
104\end{array}$ \\
\hline To11 & $\begin{array}{l}\text { STEAM AND AMMONIA IN THE CONDENSER/REBOILER } \\
\text { AT DESIGN CONDITIONS (DEG F) } \\
\text { - TEMPERATURE DIFFERENCE BETWEEN THE SATURATED }\end{array}$ & & $\begin{array}{l}06 \\
07\end{array}$ \\
\hline TD12 & $\begin{array}{l}\text { STEAM AND AMMONIA IN THE CONDENSER/REBOILER } \\
\text { AT OPTIMUM DESIGN CONDITIONS (DEG F) } \\
\text { - TEMPERTAURE DIFFERENCE BETWEEN THE SATURATED }\end{array}$ & $\begin{array}{l}\text { RIT } \\
\text { RIT }\end{array}$ & 10 \\
\hline & $\begin{array}{l}\text { STEAM AND AMMONIA IN THE CONDENSER/REBOILER } \\
\text { AT OFF DESIGN CONDITIONS (DEG F) }\end{array}$ & & \\
\hline TD2 & $\begin{array}{l}\text { - TEMPERATURE DROP OF THE AMMONIA IN THE SUPPLY } \\
\text { PIPING IDES FI } \\
\text { - TEMPERATURE DROP OF THE AMMONIA IN THE SUPPLY }\end{array}$ & $\begin{array}{l}\text { VARIT } \\
\text { VARIT } \\
\text { VARIT }\end{array}$ & $\begin{array}{l}113 \\
114 \\
115\end{array}$ \\
\hline
\end{tabular}




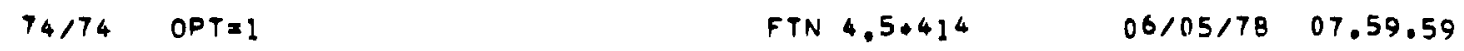

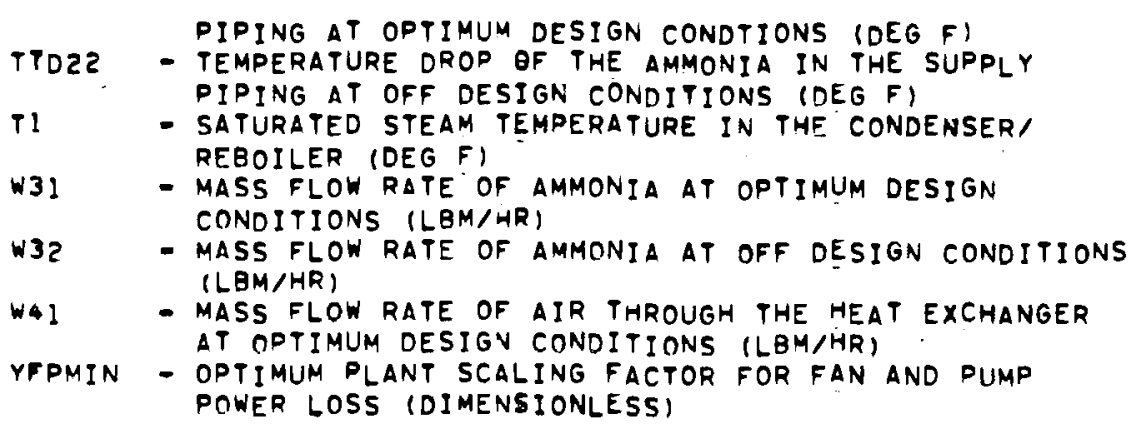

DEFINITION OF ARRAYS

DEPERQI - FRACTION OF THE HEAT LOAD THAT IS CARRIED OFF BY THE DELUGED PART OF THE HEAT EXCHANGER FOR EACH AMBIENT TEMPERATURE INCREMENT (DIMENSIONLESS)

DEPERI - FRACTION OF THE HEAT EXCHANGER THAT IS DELUGED FOR EACH AMBIENT TEMPERATURE INCREMENT (OIMENSI ONLESS)

DWD1 - WET/DRY BACK PRESSURE CURVE FOR THE AMBIENT TEMPERATURE RANGE (IN HGA)

TPO - ARRAY OF COEFFICIENTS FOR THE CUBIC CURVE FIT OF HEAT RATE FACTOR AS A FUNCTION OF TURBINE BACK PRESSURE (DIMENSIONLESS)

LOGICAL FIRST,FRST, FXTEMP,FXTVAR, FXTTTD,FXTLNG COMMON

\$ AFTR, ALPHA, ANG (3)

* BPLIM(16)

S CADF, CSSPKW, CONF, CONL, COSTL, CAPCHG, CONMAT, CONMAZ, CAF

S. CEJ, CYURB, CDANG, CLUVR, CHAILS, CVM

5 DEIN, DEEPL, DESVEL, DESVELV, DESVELD, DENSIF

S.EFFD, EFS

S. FCR, FIRST, FIXL, FCOS, FFHX, FXTEMP, FACTOR, FRST, FACIORD, FSHOT, FDELUG

S FIXVIFIXTTD,FXTVAR,FXTTTD,FXTLNG

5 . GSEFF

S. HXNP,HDCST,HCD

5 ITMAX, ITMIN

5 . JCANS

S KCONV, KALEXT

$5 . M \times E \times T$

S. NTA, NSIDES, NBUNHI

COMMON

5 ODR

5 .PSIZE, PER, PWCOS, PLANC, PFACT, POMDPL

- POHBAF, POHFAN, POHLEC, POHCIR. POHCND, POHSTC, POHSCl

5 RBP, PEDLICE, REDUCV, ROOFL, RHOUT

5 QREJ, OREDUCE, QREDUCV

S SIGMAG, SAAF, SEGL

\$ TD, TPO(4), TEFF, TLIM, THFIN, TLPRA, TFIX

T. TCD, TW

S. UCS. UWS

\$ WEV. WATCONA, WATCOST

S VAS (5)

S ,XOEPA, XW, XD 
COMMON /SCALER/HRFACI, ATUB, CPERA, SSCD.PLANCI, CASSSI, S GAIR, FAIR, HPAIR. HPWAT, WLRTP, PLANCTI COMYON /SUPPLY/ VAL (5). CPLN, CPIP, CCOS TEFF3=TEFF/HRFAZS

SCALER

SCALER

SUPPLY

SUPPLY

SUPPLY

SUPPLY

SUPPLY

SUPPLY

SUPPLY

SUPPLY

SUPPLY

SUPDLY

SUPDLY

SUPPLY

SUPPLY

SUPPLY

SUPPLY

SUPPLY

SUPPLY

SINK

SINK

SINK

SINK

SINK

SINK

SINK

SINK

SINK

SINK

SINK

SINK

SINK

SINK

SINK

SINK

SINK

VARIT

VARIT

VARIT

VARIT

VARIT

VARIT

VARIT

VARIT

VARIT

VARIT

VARIT

VARIT

VARIT

VARIT

VARIT

VARIT

VARIT

VARIT

VARIT

VARIT

VARIT 


\begin{tabular}{|c|c|}
\hline $\begin{array}{l}\text { VARIT } \\
\text { VARIT } \\
\text { VARIT }\end{array}$ & $\begin{array}{l}168 \\
169 \\
170\end{array}$ \\
\hline $\begin{array}{l}\text { VARIT } \\
\text { VARIT }\end{array}$ & $\begin{array}{l}171 \\
172\end{array}$ \\
\hline VARIT & 173 \\
\hline VARIT & 1 \\
\hline $\begin{array}{l}\text { VARIT } \\
\text { VARIT }\end{array}$ & \\
\hline VARIT & 177 \\
\hline VARIT & \\
\hline VARIT & \\
\hline VARIT & 18 \\
\hline $\begin{array}{l}\text { VARIT } \\
\text { VARIT }\end{array}$ & \\
\hline $\begin{array}{l}\text { VARIT } \\
\text { VARIT }\end{array}$ & $\begin{array}{l}183 \\
184\end{array}$ \\
\hline VARIT & 185 \\
\hline VARIT & 18 \\
\hline VARIT & $1 \mathrm{~A}$ \\
\hline VARIT & 18 \\
\hline VARIT & 18 \\
\hline VARIT & 19 \\
\hline VARIT & \\
\hline VARIT & \\
\hline VARIT & 130 \\
\hline $\begin{array}{l}\text { VARIT } \\
\text { VARIT }\end{array}$ & \\
\hline VARIT & 196 \\
\hline VARIT & \\
\hline VARIT & 19 \\
\hline VARIT & 9 \\
\hline VARIT & 20 \\
\hline VARIT & 20 \\
\hline VARIT & \\
\hline VARIT & \\
\hline VARIT & 20 \\
\hline VARIT & 20 \\
\hline VARIT & 20 \\
\hline VARIT & 20 \\
\hline VARIT & 20 \\
\hline VARIT & \\
\hline VARIT & 21 \\
\hline VARIT & \\
\hline VARIT & \\
\hline VARIT & \\
\hline VARIT & $c 1$ \\
\hline VARIT & \\
\hline VARIT & \\
\hline VARIT & \\
\hline VARIT & $c$ \\
\hline VARIT & \\
\hline VARIT & \\
\hline VARIT & \\
\hline VARIT & \\
\hline VARIT & \\
\hline VARIT & \\
\hline
\end{tabular}


IF (DEPER I (KALL) .EO.I.) GO TO 40

VARIT

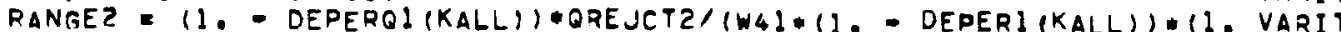

1 - YFOM $[N] * C P A)$

VARIT

POWER OUTPUT OF THE LOW-PRESSURE TURBINE AT THE SPECIFIC $\triangle M B I E N T$ TEMPERATURE

40 PTOT - QINI TEFF $4 / 3413 \cdot E+3$

VARIT 250

VARIT 230

VARIT 23I

VARIT 232

VARIT 233

DOWER OUTPUT OF THE PLANT AT THE SPECIFIC AMBIENT TEMPERATURE

PGEN = PTOT - PPOWI/1000. - FPOW1/1000.

TFFRDETEFF 4

$c$
$c$
$c$

IF (DGEN.GE.PSIZE) GO TO 50

COST OF THE AUXILIARY POWER REQUIRED TO MAKE UP FOR LOST CAPACITY ON HOT DAYS

CSTCL $=(P S I Z E-P G E N) / P S I Z E * P W C O S$

TEFFDETEFF 3

GO TO 60

c

c

INCREASED FUEL COST OF THE PLANT DUE TO OFF DESIGN CONDITIONS

60 DELFC * FCOS*3413.E-5*(1..YFPMIN)/TEFFD-1./TEFF)

IF (KALL.NE. I) GO TO 70

CAPCSTEO.0

IF (DSIZE.LE.PGEN\} GO TO 70

c

CAPITAL CHARGE FOR THE AUXILARY POWER CAPCSTECAPCHG (PSIZE-PGEN) 70 CONTINUE

INCREASE IN COST OF OPERATING THE PLANT AT THIS AMBIENT TEMPERATURE DUE TO THE USE OF THE COOLING SYSTEM

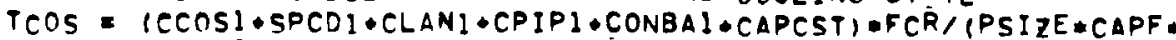
* A760.1 + COSMI + DELFC + CSTCL WATCONA*(.1203/1000.) WWATCOST/ STPSIZE CAPF $* 8750$.

FRACTION OF THE YEAR THAT THIS AMBIENT TEMPERATURE EXISTS TIMES THE INCREMENTAL COST OF THE COOLING SYSTEM AT THIS AMBIENT TEMPERATURE PESTETPER*TCOS

SUM OF THE PRODUCT BETWEEN THE AUXILARY POWER COST AND THE FRACTION OF THE YEAR THAT THE AMBIENT TEMPERATURE EXISTS

CLSUM ELSUM + CSTCL * TPER

SUM OF THE PRODUCT BETWEEN THE FUEL COST INCREASE FOR THE PLANT DUE TO THE OFF DESIGN CONDITIONS AND THE FRACTION OF THE YEAR THAT THE AMBIENT TEMPERATURE EXISTS FCSUM FCSUM - DELFC TPER $I D E G=0$ IF IINBG.EQ.0) GOTO 800

935 FORMATIT2,FG.1, T10,F6.1, T18,F5.2, T26,FT,4,T33.F7.3 $5 . T 41, F 6,3, T 47, F 7,2, T 54, F 7,2, T 62, F B .3, T 72, F 7,5, T 81,611.5$ 
$599, F 7,4, T 106,57,3, T 115,611.51$

VARIT

VARIT

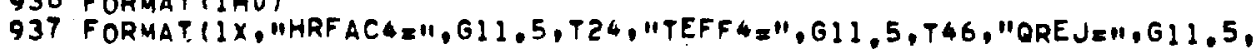

S T65,"RANGE=",G11,5,T85,"VAR(3)=", G11,5,T10B,"T11=",G11,5/

$51 X$, "PTOTAL=",GI1,5,T25, "PGEN=", G11,5,T46,"PPOW=11,G 11,5 ,

5 T66, "FPOWE", GI1,5,T87, "OINIE", GII,5,

$\$ 1 X, "$ TEFF $3=1, G 11,5, T 24$, "TEFF $1=11,611,5, T 44$, "YFPM $\} N=11, G 11,51$ WRITE $(6,936)$

WRITE $(6,937)$ HRFAC4, TEFF 4, QREJ, RANGE, VAR (3), TI I, PTOTAL,PGEN,

S PPOW,FPOW, OINI, TEFF3, TEFFI, YFPMIN

WRITE $(6,938)$ HRFAC2, TEFF, HRFAC, HRFACI, TEFFD, CCOS1, SPCDI, CLANI,

S CPIPI COSMI

938 FORMAT 1 X."HRFAC2 $=1, G 11,5, T 25$, "TEFF $=1, G 11,5, T 45$, "HRFAC =1, G 11,5 ,

5T64,"HRFACI=",G11,5,T86,"TEFFD=1,G11,5, T106,"CCOS1=11,G11.5/

$S 1 X, " S^{P} C D I=11, G 11,5, T 24, " C L A N I=11, G 11,5, T 45, " C P I P I=1 ", G 11,5, T 65$,

5 "COSMI=",GIl.5

939 FORMAT (T2, "TAI, TAO, TAMM, TII, RANGE, TTDI",6G15,6)

800 CONTINUE

TAMM = TI - TTDI2 - TTDZ2

HXITD=TI- TTDIZ - TTOZZ - TA

IF(HXITD.GT.0.0) HXLMTD = RANGEZ/ALOG(HXITO/(HXITD. RANGEZ))

5

BIO CONTINUE TD.LT.0.0) HXLMTO = 0.0

VARIT

VARIT

VARIT

VARIT

VARIT

VARIT

VARIT

VARIT

VARIT

VARIT

VARIT

VARIT

VARIT

VARIT

VARIT

VARIT

VARIT

VARIT

VARIT

VARIT

VARIT

IF (KALL.EQ.1) TBPMXZ =P IF (KALL:EQ.I) POWMAXIPGEN WRITE $(6,935)$ TA

S.TI

0

$S, D$

S, HRFAC4

S. DANGE?

S.TTDIZ

5

S.HXLMTD

S.HXITD

S.PGEN

S, DELFC

S.CSTCL

S, TCOS

SITPER * 100 .

S.PCST

IF (IDRG,EO.O) RETURN

RETURN

END

VARIT 305

VARIT 306

VARIT 307

VARIT 308

VARIT 309

VARIT 310

VARIT 311

VARIT 312

VARIT 313

VARIT 314

VARIT 315

VARIT 316

VARIT 317

VARIT 318

VARIT 319

VARIT 320

VARIT 321

VARIT 322

VARIT 323 
S, CSRD, WATERW, WSTRCT, CFOUND, HUBDIA, TOWLEN, NUMTOW, CTOWD

5. SORJ, SQR JS, ATUBC, CBA, TTDI, TTDZ, DELPS, CSTLVR, PIPCSTD

S , CSTHS, EYLRNG, CFANEL, PSTACS, PUFITC, PMPCST, RPTPL, SEPCST

\$ PWD (16) ,WATCWD (16), DEPER (16) ,DEPERQ (16)

\$ ,WAB,HTOW, NTOWDE, CSTIF, CSJ,CVF, CDP,WS

COMMON /TINE, XNS, TLA, XNTS, ARDS, TTDZOE, LINOR, XNP, XQUALY LOGICAL FIRST, FRST, FXTEMP, FXTVAR,FXTTTD, FXTLNG COMMON

S AFTR, ALPHA, ANG (3)

* Bolim(16)

- CAPF, CSSPKW, CONF, CONL, COSTL, CAPChg, CONMat, CONMAZ, CAF

5 , CRJ, CTURB, CDANG, CLUVR, CHAILS, CVM

5 ,DFIN,DEEPL,DESVEL, DESVELV,DESVELD, DENSIF

5 EFFO, EFS

S. FER, IF IRST, FIXL,FCOS,FFHX, FXTEMP, FACTOR, FRST, FACTORD, FSHOT , FDELUG

S, FIXVIFIXTTD,FXTVAR,FXTTTD, FXTLNG

c. GBEFF

S, HXNP, HDCST,HCD

S ITMAX, ITMIN

5 , JCONS

S KEONV, KALEXT

S.MXEXT

S NTA,NSIDES,NRUNHI

XTEND

XTEND

SINK

SINK

SINK

SINK

SINK

SINK

SINK

SINK

SINK

SINK

SINK

SI NK

SINK

SINK

SINK

SINK

SINK

SUPPLY

SUPPLY

SUPPLY

SUPPLY

SUPPLY

SUPPLY

SUPPLY

SUPPLY

SUPPLY

SUPPLY

SUPPLY

SUPPLY

SUPPLY

SUPPLY

SUPPLY

SUPPLY

SUPPLY

XTEND

BIGCOM

BIGCOM

BIGCOM

BI GCOM

BIGCDM

BI GCOM

BIGCOM

BIGCDM

BIGCOM

BIGCOM

BI GCOM

BI SCOM

BIGCOM

BIGCOM

BIGCOM

BIGCOM

BI GCOM 
SUBROUTINE XTEND T4/74 OPTEI FTN $4.5+414$

$06 / 05 / 78 \quad 07.59 .59$

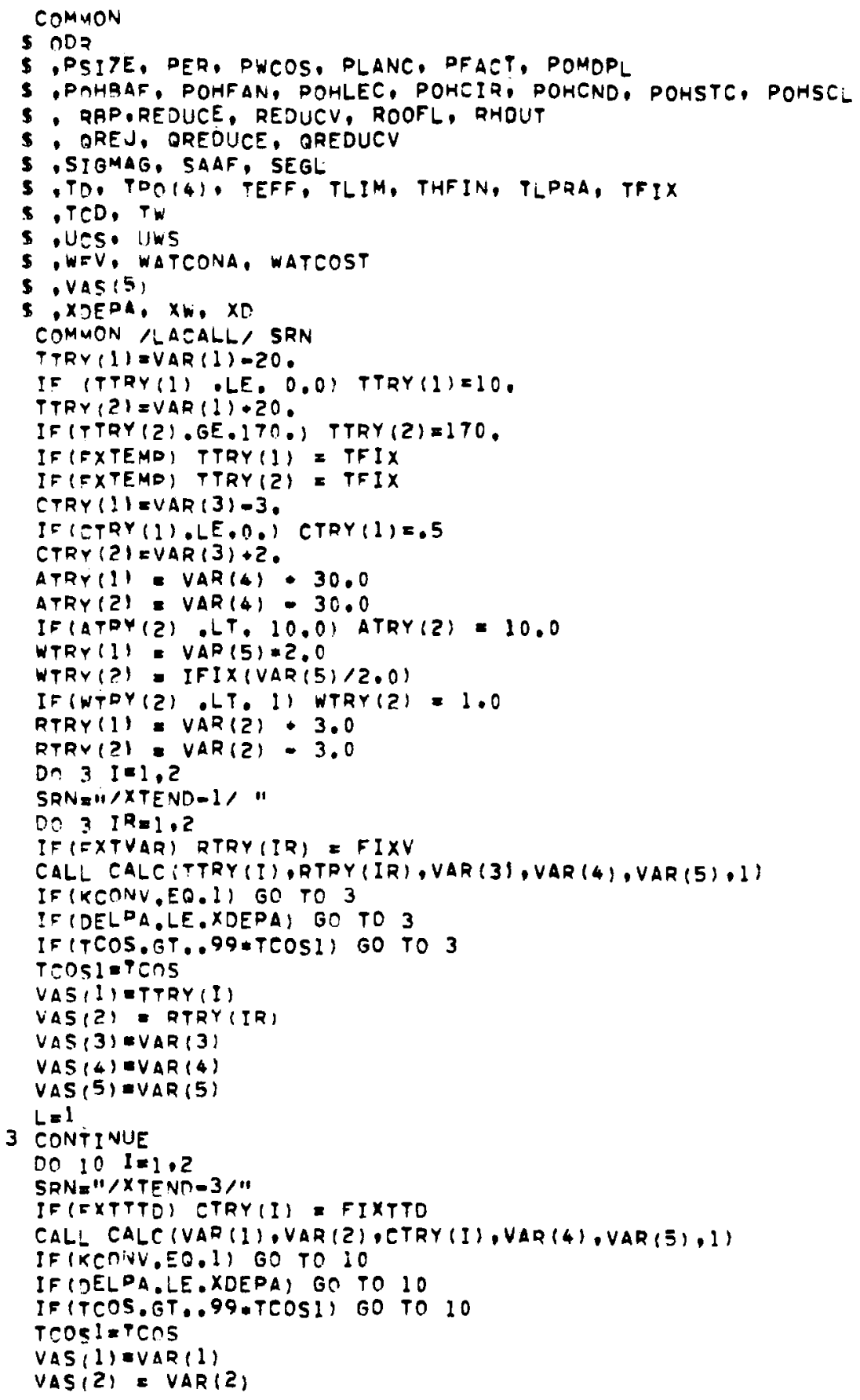

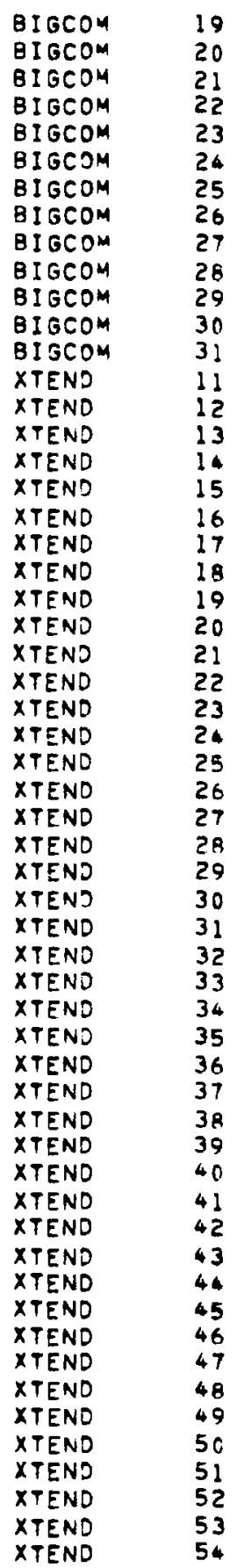


$V A S(3)=C T R Y(I)$

$V A S(4)=V A R(4)$

VAS $(5)=V A R(5)$

$L=1$

10 CONTINIJE

DO $11 I=1.2$

IF (FXTL'NG) GO TO II

CALL CALC (VAR(1), VAR (2) ,VAR(3), ATRY(I),VAR (5), 2*I)

IF (KCONV.EQ.1) GO TO 1 I

IF (DELPA.LE.XDEPA) GO TO 11

IF (TCOS.GT..99.TCOS1) GO TO 11

$T \operatorname{COS} 1=T \operatorname{COS}$

VAS $(1)=V A R(1)$

VAS $(?)=\operatorname{VAR}(2)$

VAS $(3)=\operatorname{VAR}(3)$

VAS $(4)=A T R Y(I)$

$V A S(5)=V A R(5)$

$L=1$

11 CONTINUE

SRN=" /XTEND-4/"

DO $12 \quad I=1,2$

IF (DEEPL.GT.0.0) GO TO 12

CALL CALC (VAR(I), VAR(2),VAR (3), VAR (4), WTRY (I), 3 + I)

IF (KCONV.EO.1) GO TO 12

IF (DELPA.LE, XDEPA) GO TO 12

IF (TCOS.GT. 99*TCOSI) GO TO 12

TCOS1 = TCOS

VAS (1) OVAR(1)

$\operatorname{VAS}(2)=V A R(2)$

VAS $(3)=V A R(3)$

VAS $(4)=V A R(4)$

VAS (5) =WTRY (I)

$L=1$

12 CONTINOE

SRN $" 1 \times T$ TEND-5/"

DO $14 I R=1.2$

IF (EXTVAR) GO TO 14

CALL CALC (VAR (1), RTRY (IR), VAR (3),VAR (4), VAR (5), I)

IF (KCONV. EQ. I) GO TO 14

IF (DELPA .LE. XDEPA) GO TO 14

IF (TCOS.GT. $0.99 * T C O S 1)$ GO TO 14

TCOSI - TCOS

VAS $(1)=\operatorname{VAR}(1)$

$V A S(2)=\operatorname{RTRY}(I R)$

$\operatorname{VAS}(3)=\operatorname{VAR}(3)$

$\operatorname{VAS}(4)=\operatorname{VAR}(4)$

$\operatorname{VAS}(5)=\operatorname{VAR}(5)$

$L=1$

14 CONTINUE

IF $(L, E Q, 1)$ WRITE $(6,13)$

13 FORMATI" XTEND CALLED. AND CHANGE MADE")

IF (L :EO, 1) KALEXT I KALEXT - I

IF (KALEXT .GT. MXEXT) STOP "CALLS TO S,R. EXTEND EXCEED MXEXT" RETURN

END

$\begin{array}{lc}\text { XTEND } & 55 \\ \text { XTEND } & 56 \\ \text { XTEND } & 57 \\ \text { XTEND } & 58 \\ \text { XTEND } & 59 \\ \text { XTEND } & 60 \\ \text { XTEND } & 61 \\ \text { XTEND } & 62 \\ \text { XTEND } & 63 \\ \text { XTEND } & 64 \\ \text { XTEND } & 65 \\ \text { XTEND } & 66 \\ \text { XTEND } & 67 \\ \text { XTEND } & 66 \\ \text { XTEND } & 69 \\ \text { XTEND } & 70 \\ \text { XTEND } & 71 \\ \text { XTEND } & 72 \\ \text { XTEND } & 73 \\ \text { XTEND } & 74 \\ \text { XTEND } & 75 \\ \text { XTEND } & 76 \\ \text { XTEND } & 77 \\ \text { XTEND } & 78 \\ \text { XTEND } & 79 \\ \text { XTEND } & 80 \\ \text { XTEND } & 81 \\ \text { XTEND } & 82 \\ \text { XTEND } & 83 \\ \text { XTEND } & 84 \\ \text { XTEND } & 85 \\ \text { XTEND } & 86 \\ \text { XTEND } & 87 \\ \text { XTEND } & 88 \\ \text { XTEND } & 89 \\ \text { XTEND } & 90 \\ \text { XTEND } & 91 \\ \text { XTEND } & 92 \\ \text { XTEND } & 93 \\ \text { XTEND } & 94 \\ \text { XTEND } & 95 \\ \text { XTEND } & 96 \\ \text { XTEND } & 97 \\ \text { XTEND } & 98 \\ \text { XTEND } & 99 \\ \text { XTEND } & 100 \\ \text { XTEND } & 101 \\ \text { XTEND } & 102 \\ \text { XTEND } & 103 \\ \text { XTEND } & 104 \\ \text { XTEND } & 105 \\ \text { XTEND } & 106 \\ \text { XTEND } & 107 \\ \text { XTEND } & 108 \\ \text { XTEND } & 109 \\ & \\ \text { XEND } & \end{array}$




\section{DISTRIBUTION}

No. of

Copies

OFFSITE

U.S. Department of Energy

A. A. Churm

Chicago Patent Group

9800 South Cass Avenue

Argonne, IL 60439

U.S. Department of Energy

Office of Assistant General

Counsel for Patents

Washington, DC 20545

27 DOE Technical Information Center

10 U.S. Department of Energy

I. Helms

Advanced Concepts Evaluation Branch - G-434

Division of Advanced Systems and Materials

Washington, DC 20545

U.S. Department of Energy

W. F. Savage

Chief, Advanced Concepts Evaluation Branch

Division of Advanced Systems and Materials

Washington, DC 20545

U.S. Department of Energy

D. C. Bauer

Director, Division of Advanced Systems and Materials Washington, DC 20545

U.S. Department of Energy

R. B. Morrow

Special Projects Branch

Division of Advanced Systems and Materials

Washington, DC 20545
No. of

Copies

U.S. Department of Energy

N. Gerstein

Chief, Special Projects Branch

Division of Advanced Systems and Materials

Washington, DC 20545

U.S. Department of Energy

W. E. Mott

Director, Energy Control Technology

Division of Biomedical and Environmental Research

Washington, DC 20545

U.S. Department of Energy

G. A. Newby

Assistant Director, Office of Technical Development

Division of Advanced Systems and Materials

Washington, DC 20545

Allied Chemical Company

B. R. Dickey

550 2nd Street

Idaho Falls, ID 8340 ]

All is-Chalmers Power Systems, Inc.

J. S. Joyce

1135 South 70th Street

West Allis, WI 53214

Aluminum Company of America

E. T. Wanderer

Alcoa Technical Center

Alcoa Center, PA 15069

American Electric Power

H. J. Janzon

2 Broadway

New York, NY 10004

American Electric Power Service Corporation

C. Swenson

2 Broadway

New York, NY 10004 
No. of

Copies

Aqua-Chem

R. M. Ahlgren

P. O. Box 421

Milwaukee, WI 53201

Arizona Public Service Co.

W. E. Campbe 11

P.0. Box 21666

Phoenix, AZ 85036

Arizona Public Service Co.

$T$. Woods

$2121 W$. Cheryl Drive

Phoenix, AZ 85021

Babcock \& Wilcox

M. W. Peterson

Fossil Power Division

20 South Van Buren

Barberton, OH 44203

Baltimore Aircoil Co., Inc.

E. Schinner

P.0. Box 7322

Baltimore, MD 21227

Baltimore Gas \& Electric Co.

G. C. Cree]

Gas \& Electric Building

Baltimore, MD 21203

Battelle-Geneva

J. P. Budliger

7 Route De Drizi

1227 Geneva, SWITZERLAND

Bechtel Corporation

$P$. Leung

P.0. Box 60860

Terminal Annex

Los Angeles, CA 90060

Bechtel Corporation

G. R. Reti

P.0. Box 3965

San Francisco, CA 94119
No. of

Copies

R. W. Beck and Associates

J. P. Rossie

400 Prudential Plaza

Denver, CO 80202

Betz Environmental Engineers

J. Soost

9317 J, Highway 99

Vancouver, WA 98665

Black, Crow and Eidsness, Inc.

C. G. Thompson

807 South McDonough

Montgomery, AL 35104

Black Hills Power and Light Company

B. Westre

P.0. Box 1400

Rapid City, SO 57701

Boeing Aerospace Division

D. Gilbert (M.S. 2ROO)

P.0. Box 3999

Seattle, WA 98124

Bonneville Power Administration E. H. Hall

1002 N.E. Holladay Street

Box 3621

Portland, OR 97208

Burns and Roe, Inc.

700 Kinder Kamack Rd.

Oradel, NY 07649

California Energy Commission

C. Webb

1111 Howe Avenue

Sacramento, CA 95825

California State Energy

Resources Conservation

and Development Commission

L. E. Stamets

1111 Howe Avenue

Sacramento, CA 95825 
No. of

Copies

Carolina Power \& Light Co. J. Sell

336 Fayettesville Street

Raleigh, NC 27602

Catalytic Construction Corp.

J. Morse

P.0. Box 11402

Charlotte, NC 28029

Ceramic Cooling Tower Co.

P. A. Frohwerk

P.0. Box 425

Fort Worth, TX 76101

Columbus and Southern Ohio Electric Co.

L. W. Meridith

General Manager, Generation Department

215 North Front Street

Columbus, $\mathrm{OH} 43215$

Combustion Engineering

H. H. Osborn

Air Preheater Company

Wellsville, NY 14895

Commonwealth Edison

R. H. Holyoak

One First Plaza

P.0. Box 767

Chicago, IL 60690

Consolidated Edison Co. of New York, Inc.

W. A. Messner

4 Irving Place

New York, NY 10003

Consolidated Edison Co. of New York, Inc.

C. L. Newman

4 Irving Place

New York, NY 10003
No. of

Copies

Cornell University

$F$. K. Moore

Ithaca, NY 14850

Curtiss-Wright Corporation

R. J. Haberski

One Passaic Street

Wood Ridge, NJ 07075

Dames \& Moore

L. Craton

Suite 1000

1100 Glendon Avenue

Los Angeles, CA 90024

Dames \& Moore

P. Gottlieb

Suite 1000

$1100 \mathrm{Glendon}$ Avenue

Los Angeles, CA 90024

Delmarva Power \& Light

F. Cook

800 King Street

Wilmington, DE 19801

Dow Chemical Company

E. Wagener

2800 Mitchell Drive

Walnut Creek, CA 94598

Duke Power Company

S. K. Blackley

P.0. Box 2178

Charlotte, NC 28201

Duquesne Light Co.

J. Latshaw

435 6th Avenue

Pittsburgh, PA 15219

Dynatech Company

E. Guyer

99 Erie Street

Cambridge, MA 02139 
No. of

Copies

Ecodyne

J. Slotnik

607 First Street, S.W.

Massilion, $\mathrm{OH} 44646$

Ecodyne Cooling Products Co.

J. K. Swindt

P.0. Box 1267

Santa Rosa, CA 95403

Ecodyne Cooling Products

K. D. Whitehead

P.0. Box 1267

Santa Rosa, CA 95403

Electric Power Research Institute

J. Maulbetsch

3412 Hillview Avenue

P.0. Box 10412

Palo Alto, CA 94304

Empire State Electric Energy Research Corp.

L. Geller

1271 Avenue of the Americas

New York, NY 10020

Environmental Protection Agency

T. G. Brna (MD-61)

IERL/RTP

Research Triangle Park, NC 27711

Environmental Protection Agency

A. Galley (WH-552)

401 M. Street SW

Washington, DC 20460

Environmental Protection Agency

M. Maxwell (MD-61)

IERL/RTP

Research Triangle Park, NC 27711
No. of

Copies

Environmental Protection Agency

F. H. Rainwater

Pacific Northwest water Laboratory

200 S.W. 35th Street

Corvallis, OR 97330

Environmental Protection Agency

F. A. Roberts

200 S.W. 35th Street

Corvaliis, OR 97330

Environmental Sciences and Services

W. G. Hoydysh

150 East 73rd Street

New York, NY 10021

Environmental Systems Corporation

K. Wilber

P. 0. Box 2525

Knoxville, TN 37901

ERG Incorporated

G. M. Benson

Lowell 57th Street

Oakland, CA 94608

Exxon Research Center

J. G. Stevens

Bldg. 1, Rm. 2048

P.0. Box 8

Linden, NJ 07036

Federal Power Commission

E. Sligh

825 N. Capitol Street

Washington, DC 20426

Florida Power \& Light Co.

$C$. Henderson

$9250 \mathrm{~W}$. Flagler Street

Miami, FL 33174 
No. of

Copies

Foster Wheeler Energy Co.

E. L. Damon

110 S. Orange Avenue

Livingston, NJ 07039

Frankl in Institute

A. M. Rub in

Twentieth \& Parkway

Philadelphia, PA 19103

GEA Airexchangers, Inc.

B. Davis

P.0. Box 1377

Thomasville, GA 31792

General Atomic Company

A. C. Eulberg

P.0. Box 81608

San Diego, CA 92138

General Electric Co.

E. H. Miller

Large Steam Turbine Division

300 Nott Street

Schenectady, NY 12301

General Motors Corp.

R. K. Shah

Harrison Radiator Division

Lockport, NY 14094

Georgia Power Co.

T. E. Byerley

P.0. Box 4545

Atlanta, GA 30302

Gilbert Associates, Inc.

J. F. Sebald

525 Lancaster Avenue

Reading, PA 19603

Heat Transfer Research Inc.

J. E. Taborek

1000 S. Fremont Avenue

Alhambra, CA 91802
No. of

Copies

Hudson Products

M. W. Larinoff

6855 Horwin Drive

Houston, TX 77036

Hudson Products

E. C. Smith

6855 Horwin Drive

P.0. Box 36100

Houston, TX 77036

H2M Corporation

H. D. Freudenthal

500 Broad Hollow Road

Melville, NY 11746

Ingersol1-Rand

W. R. Scott, Jr.

Phillipsburg, NJ 08865

Italimpianti-Societa Italiana Impianti p.a.

C. Rocco

Piazza, Piccapietra 9

18121 Genoe, ITALY

Los Angeles Department of Water and Power

J. L. Mulloy

111 N. Hope Street

Los Angeles, CA 90012

Louisiana Power \& Light Co.

D. L. Aswell 1

142 Delaronde Street

New Orleans, LA 70174

The Charles T. Main Co.

E. S. Miliaras

Southeast Tower

Prudential Center

Boston, MA 02199

Marley

C. A. Baird

12 S. 12th Street

Philadelphia, PA 19107 
No. of

Copies

Marley

J. D. Holmberg

5800 Fox Ridge Drive

Mission, KS 66202

Marley

R. Landon

5800 Fox Ridge Drive

Mission, KS 66202

Martin Marietta Laboratories

L. Bongers

1450 South Rolling Road

Baltimore, MD 21227

Massachusetts Institute of Technology

L. R. Glicksman

77 Massachusetts Avenue

Cambridge, MA 02139

Massachusetts Institute of Technology

M. W. Golay

77 Massachusetts Avenue

Cambridge, MA 02139

Massachusetts Institute of Technology

R. Harleman

Department of Civil Engineering

77 Massachusetts Avenue

Cambridge, MA 02139

McDonnell Douglas Astronautics Co. W. H. P. Drummond 5301 Balsa Avenue Huntington Beach, CA 92647

McDonnell Douglas Astronautics Co.

S. O'Hare

5301 Balsa Avenue

Huntington Beach, CA 92647
No. of

Copies

Minnesota Power Cooperative, Inc.

L. A. Hillier

Box 1318

Grand Forks, ND 58201

Montana Power Co.

$R$. Hofacher

40 E. Broadway

Butte, MT 59701

Niagara Blower

W. Kals

405 Lexington Avenue

New York, NY 10017

Northeast Utilities

R. H. Meyer

P.0. Box 270

Hartford, CT 06101

Northern States Power Co.

R. Stansfield

414 Nicollet Mall

Minneapolis, MN 55401

N.U.S. Corporation

$S$. Lefton

2 Palo Alto Square (Suite 624)

Palo Alto, CA 94304

Oak Ridge Nationa] Laboratory

J. W. Michel

OTEC Heat Exchange Project Activity

Box $Y$

Oak Ridge, TN 37830

Orange \& Rockland Utilities, Inc.

R. H. Metzger

Environmental Services Manager

75 West Route 59

Spring Valley, NJ 10977 
No. of

Copies

Oregon State University

L. P. Davis

Department of Mechanical

Engineering

Corvallis, OR 97330

Oregon State University

C. E. Wicks

Department of Chemical

Engineering

Corvallis, OR 97330

Oregon State University

J. G. Knudson

Engineering Experiment Station

Corvallis, OR 97330

Pacific Gas \& Electric

A. A. Ariey

77 Beale Street

San Francisco, CA 94106

Pacific Gas \& Electric

F. F. Mautz

77 Beale Street

San Francisco, CA 94106

Pacific Power \& Light Co.

W. C. Bruaer

Public Service Bldg.

Portland, OR 97204

Pennsylvania Power \& Light

W. Dussinger

2 North Ninth Street

Allentown, PA 18101

Pennsylvania Power \& Light

D. G. Pfeiffer

2 North Ninth Street

Allentown, PA 18101

PFR Engineering Systems, Inc.

$T$. Rozenmann, President

Suite 832

4676 Admiralty Way

Marina del Rey, CA 90291
No. of

Copies

Philadelphia Electric Co.

J. Allen

2301 Market Street

Philadelphia, PA 19101

Philadelphia Electric Co.

S. J. Kowalski

2301 Market Street, NZ-1

Philadelphia, PA 19101

Philadelphia Electric Co. J. B. Machel

2301 Market Street, $N Z-1$

Philadelphia, PA 19101

Philadelphia Electric Co. D. Marano

2301 Market Street, NZ-1

Philadelphia, PA 19101

Prof. Ing. Carlo Roma

Piazza delle Muse 8

Rome, ITALY

Pickard Low \& Garrick

L. Rust

1200 18th St. NW

Suite 612

Washington, DC 20036

Power Generation Cooling

Systems

G. L. Henderson

4714-52nd Street $S$.

Seattle, Wa 98118

Public Service of Colorado

R. F. Walker

5900 E. 39th Avenue

Denver, CO 80207

Public Service Company

of Indiana

S. W. Shields

V. P. Engineering

100 E. Main Street

Plainfield, IN 46168 
No. of

Copies

Public Service Company of New Mexico

C. D. Bedford

P. 0. Box 2267

Albuquerque, NM 87103

Publ ic Service Company of New Mexico

J. D. Maddox

Corporate Planning Dept.

Albuquerque, NM 87103

Quirk, Lawler and Mattusky, Eng.

J. Lawler

505 th Avenue

New York, NY 10017

Radian Corporation

F. B. Mesich

P.0. Box 9948

Austin, TX 78766

Research Cottrell

G. E. Collins

Hamon Cooling Tower Division

Box 750

Bound Brook, NJ 08805

Research Cottrell

R. H. Hannon

Hamon Cooling Tower Division

Box 750

Bound Brook, NJ 08805

Resources Conservation Company

$H$. Herrigel

P.0. Box 936

Renton, WA 98055

Resources for the Future

D. Abbey

1755 Massachusetts Avenue NW Washington, DC 20036
No. of

Copies

Reynolds Aluminum Co.

R. Lindberg

Reynolds Metallurgical

Research Laboratory

Richmond, VA 23261

Richmond Field Station

H. H. Sephton

47th and Hoffman Bivd.

Richmond, CA 94804

San Diego Gas \& Electric

R. G. Lacy

101 Ash Street

San Diego, CA 92107

Seattle City Light

T. R. Miller

Principal Mechanical Engineer

1015 Third Avenue

Seattle, WA 98104

Seattle City Light

R. L. Skone

1015 Third Avenue

Seattle, WA 98104

Southern Cal ifornia Edison

R. S. Currie

2244 Walnut Grove Avenue

Rosemead, CA 91770

Southern California Edison

W. C. Martin

2244 Walnut Grove Avenue

Rosemead, CA 91770

Southern California Edison

$F$. A. McCracken

2244 Walnut Grove Avenue

Rosemead, CA 91770

Southern Services, Inc.

C. H. Goodman

P.0. Box 2625

Birmingham, AL 35202 
No. of

Copies

P. Sporn

Consultant Engineer

74 Trinity PI., Suite 511

New York, NY 10006

Stanford University

A. L. London

Department of Mechanical

Engineering

Stanford, CA 94305

Stearns-Rogers, Inc.

J. Y. Parce

Box 5888

Denver, CO 80217

Stone \& Webster Engineering Corp.

D. H. Guild

225 Franklin Street

Boston, MA 02107

Tampa Electric Co.

H. I. Wilson

P.O. Box 111

Tampa, FL 33601

Tennessee Valley Authority

H. B. Flora, III

1320 Commerce Union Bank Bldg.

Chattanooga, TN 37401

Texas Electric Service Co.

W. Keel

$115 \mathrm{~W}$. Seventh Street

Fort Worth, TX 76102

Tucson Gas \& Electric

A. A. Ward

$220 \mathrm{~W}$. 6th Street

Tucson, AZ 85701

Union Carbide Corp.

J. A. Bartz

Linde Division

61 East Park Drive

Tonawanda, NY 14150
No. of

Copies

Union Carbide Corp.

G. J. Kidd

ORGDP - K-25

P.0. Box P, Mail Stop 197

Oak Ridge, TN 37803

Union Carbide Corp.

$F$. Notaro

Linde Division

61 East Park Drive

Tonawanda, NY 14150

United Engineers \&

Constructors

G. A. Englesson

30 S. 17th Street

Philadelphia, PA 19103

United Engineers \&

Constructors, Inc.

M. $\mathrm{Hu}$

$30 \mathrm{~S}$. 17th Street

Philadelphia, PA 19103

University of Bremen

K. Simhan

Bremen, WEST GERMANY

University of Iowa

J. F. Kennedy

Hydraulic Research Institute

Iowa City, IA 52240

University of Kentucky

T. E. Eaton

Mechanical Engineering

Department

Lexington, KY 40506

U.S. Congress

G. Haimes

214 Massachusetts Avenue NE Washington, DC 20510 
No. of

Copies

U.S. Fish and Wildlife Service

B. L. Foder

Information Transfer Special ist

National Power Plant Team

1451 Green Road

Ann Arbor, MI 48105

United States Steel Corp.

T. L. Myron

Research Laboratory

Monroeville, PA 15146

Utah Power \& Light

M. W. Russon

1407 W. N. Temple

Salt Lake City, UT 84103

Vermont Yankee Nuclear Power

E. Gaines, Jr.

77 Grove Street

Rutland, VT 05701

Virginia Electric \& Power Co.

S. Ragone

700 E. Franklin Street

Richmond, VA 23261

Washington Public Power

Supply System

J. Chasse

P.0. Box 968

300 George Washington Way

Richland, WA 99352

Washington State University

R. W. Crain, Jr.

Department of Mechanical

Engineering

Pullman, WA 99164

Washington Water Power Co.

D. L. Olson

E. 1411 Mission Avenue

Spokane, WA 99202
No. of

Copies

Water Purification Associates

H. Gold

238 Main Street

Cambridge, MA 02142

Western States Water Council

J. A. Barnett

Executive Director

220 South 2nd East

Suite 200

Salt Lake City, UT 84111

Westinghouse Electric Corp.

G. E. Jablonka

Power Generation Systems Engineering

700 Braddock Avenue, Room 9L51

East Pittsburgh, PA 15112

Westinghouse Electric Corp.

K. Kesavan

Advanced Reactor Division

P.O. Box 158

Madison, PA 15663

Westinghouse Electric Corp.

G. J. Silvestri

Steam Turbines Division - Gl08

Lester Branch

Box 9175

Philadelphia, PA 19113

Westinghouse Electric Corp.

K. A. Oleson

Steam Turbines Division - Gl08

Lester Branch

Box 9175

Philadelphia, PA 19113

L. E. Wilkinson

Consultant

6582 Ganon Street, S.E.

Salem, OR 97301 
No. of

Copies

William M. Rice University

W. G. Characklis

Environmental Science and

Engineering

Houston, TX 77001

ONSITE

DOE Richland Operations Office Energy Programs Division

H. E. Ransom

Westinghouse Hanford Company Hanford Engineering Development Laboratory

J. Fletcher

Pacific Northwest Laboratory

R. T. Allemann

J. A. Bamberger Daniel J. Braun

David J. Braun

J. W. Currie

D. E. Deonigi

R. L. Dillon

D. W. Dragnich

R. L. Drake

D. W. Faletti

J. W. Finnigan

T. J. Foley

B. C. Fryer

J. D. Goodenough

J. J. Hauth

A. J. Haverfield

C. H. Henager

A. B. Johnson

W. S. Kelly

R. S. Kemper

C. J. Knoll

W. V. Loscutoff

R. P. Marshall

D. E. Olesen
No. of

Copies

Pacific Northwest Laboratory (contd)

Y. Onishi

H. L. Parry

L. T. Pederson

H. C. Riches

J. R. Sheff

G. C. Smith

J. S. Stoakes

A. M. Sutey

R. D. Tokarz

D. S. Trent

M. Vagins

R. A. Walter

R. L: Watts

R. D. Widrig

L. E. Wiles

C. E. Willingham

F. R. Zaloudek

File - B. M. Johnson (30)

Technical Information (5)

Publishing Coordination (2) 
